\title{
In Vitro Lung Models to Assess the Mechanistic Genotoxicity of Characterised Few-Layer Graphene
}

\author{
Burgum, Michael J.
}

How to cite:

Burgum, Michael J. (2019) In Vitro Lung Models to Assess the Mechanistic Genotoxicity of Characterised Few-Layer Graphene. Doctoral thesis, Swansea University.

http://cronfa.swan.ac.uk/Record/cronfa51928

Use policy:

This item is brought to you by Swansea University. Any person downloading material is agreeing to abide by the terms of the repository licence: copies of full text items may be used or reproduced in any format or medium, without prior permission for personal research or study, educational or non-commercial purposes only. The copyright for any work remains with the original author unless otherwise specified. The full-text must not be sold in any format or medium without the formal permission of the copyright holder. Permission for multiple reproductions should be obtained from the original author.

Authors are personally responsible for adhering to copyright and publisher restrictions when uploading content to the repository.

Please link to the metadata record in the Swansea University repository, Cronfa (link given in the citation reference above.)

http://www.swansea.ac.uk/library/researchsupport/ris-support/ 


\section{In Vitro Lung Models to Assess the Mechanistic Genotoxicity of Characterised Few-Layer Graphene}

Michael John Burgum

Swansea University Medical School

Submitted to Swansea University in the fulfilment of the requirement for the degree of Doctor of Philosophy 
This work has not previously been accepted in substance for any degree and is not being concurrently submitted in candidature for any degree.

Signed (candidate)

Date

\section{STATEMENT 1}

This thesis is the result of my own investigations, except where otherwise stated. Where correction services have been used, the extent and nature of the correction is clearly marked in a footnote(s).

Other sources are acknowledged by footnotes giving explicit references. A bibliography is appended.

Signed (candidate)

Date

\section{STATEMENT 2}

I hereby give consent for my thesis, if accepted, to be available for photocopying and for interlibrary loan, and for the title and summary to be made available to outside organisations.

Signed (candidate)

Date 


\section{Dedication}

I would like to dedicate this thesis to the memory of my gran Martha June Davies who passed away on the $30^{\text {th }}$ April 2019. She will be sincerely missed and shall forever be in the thoughts of me and the family. 


\section{Acknowledgements}

Firstly, I must pay sincere thanks to my supervisor Professor Shareen H Doak for her continued guidance and breadth of knowledge which contributed so heavily to the success of this research. For her exceptional experience and tutoring I am greatly appreciative and utterly indebted to. To my second supervisor Dr Martin JD Clift I must also express sincere thanks for his consistently brilliant input to this project, providing me with opportunities (book chapters \& conferences) as well as introducing me to new avenues of research. I wish nothing but the best for both my supervisors and academic team on Floor 4. Finally, I would like to acknowledge Dr Afshin Tarat my company supervisor at Perpetuus Carbon Technologies for his expertise and guidance.

Sincere gratitude to all partners who collaborated within this research, namely; Andy Brown and Nicole Hondow for their TEM and plunge freeze contribution, Mark Miller and his team at the University of Edinburgh for performing EPR spectroscopy and Adam Williams and Sandra Bustamante Lopez who aided in Raman Spectroscopy and AFM respectively. Finally, a special thank you to Dr Ed Dudley who kindly contributed LC MS/MS to this project.

I would also like to express special thanks to my close friends who welcomed me warmly into their tight-knit group when I started 4 years ago namely my friend and mentor Dr Steve Evans as well as James Plant, Leanne Stannard and Stephanie Burnell. They have always been there to steer me in the right direction throughout the PhD.

To my parents Roger \& Annette and brother and sister Lee \& Rachel, I am forever grateful for everything you have done for me, teaching me the value of hard work and making sure I had a place to live, with a specific thank you to Rachel for that. Your guidance and support has never gone unnoticed.

This work has been supported by the European Social Fund (ESF) through the Welsh Government.
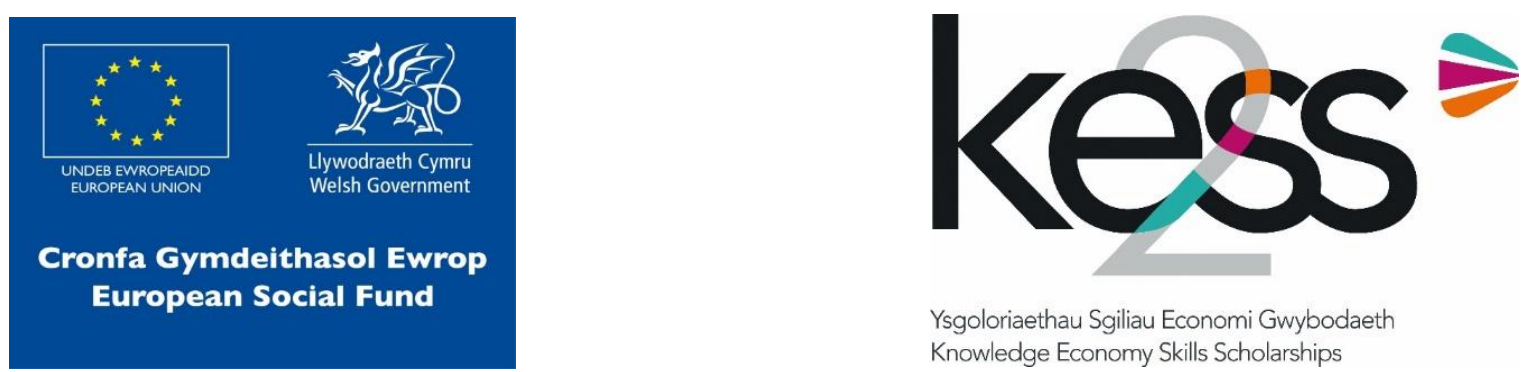

Ysgoloriaethau Sgiliau Economi Gwybodaeth Knowledge Economy Skills Scholarships 


\section{Summary}

Nanotechnology sits at the forefront of molecular and macromolecular medical science with tremendous applications worldwide. Paradoxically, as the number of applications for engineered nanomaterials (ENMs) increases the comparable knowledge of their toxicity may be limited. This could culminate in nano-safety issues relating to occupational, environmental and human exposure regarding ENM use without a full comprehension of their toxicological impact.

The aim of this thesis was firstly, to provide a comprehensive approach to characterising the physicochemical features of, few-layer graphene (FLG) engineered with i) no functionality (Neutral-FLG), ii) amine groups (Amine-FLG) or iii) carboxyl groups (Carboxyl-FLG) with a carbon black (CB) positive control. Secondly, the toxicological impact of these ENMs was investigated utilising relevant respiratory cell lines. This was performed using; in vitro monoculture exposures, allowing the detection of primary-indirect genotoxicity, then applying these ENMs to a dual-cell co-culture lung model to permit the detection of secondary genotoxicity.

By encompassing a comprehensive approach to ENM physico-chemical characterisation, a battery of complementary techniques was employed to gauge particle and agglomerate size, morphology, surface charge and surface area. Each ENM was then evaluated for their potential to promote cytotoxicity and genotoxicity, using relative population doubling and the micronucleus assay respectively in a monoculture of the human bronchial cell line, 16HBE140. A deeper investigation was then launched into the potential routes of oxidative and mitochondrial stress. Neutral-FLG, AmineFLG and CB particles promoted a genotoxic (predominantly clastogenic) response, however all tested ENMs induced oxidative \& mitochondrial stress with significant elevation of interleukin (IL)-8.

A transformed alveolar epithelial type-I (TT1) cell line and differentiated THP-1 (dTHP-1) macrophages formed the alveolar co-culture. This advanced cell model, which could detect secondary genotoxic mechanisms, highlighted the potential for each ENM to promote genotoxicity via secondary mechanisms. This was observed with a potency ranking of CB>Amine-FLG $>$ Neutral-FLG $>$ CarboxylFLG with overall 2-fold increases in chromosomal damage (ascertained from micronuclei frequencies) at $50 \mu \mathrm{g} / \mathrm{ml}$ following 24 hours of exposure. The potential mechanistic role of the oxidant: antioxidant balance as a contributor to the observed secondary genotoxicity was investigated by a 2-hour preincubation with $1.5 \mathrm{mM} \mathrm{N}$-acetyl-cysteine (NAC). This was found to reduce chromosomal damage to non-significant $(p<0.05)$ levels suggesting oxidative stress and secondary mediators to be strongly linked to the increased genotoxic response.

In conclusion, co-culture exposures revealed the capacity of each ENM to elevate the frequency of micronuclei in binucleated (BN/Mn) cells above monoculture levels, indicative of secondary mechanisms of genotoxicity. The co-culture system has therefore highlighted the importance of complex in vitro models as a means of improving upon 2D monocultures as predictors of potential in vivo toxicity, which would have otherwise been overlooked. 


\section{Table of Contents}

Declaration

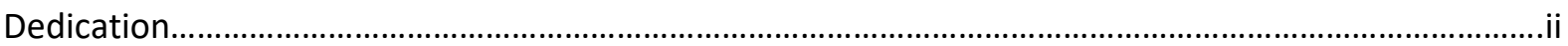

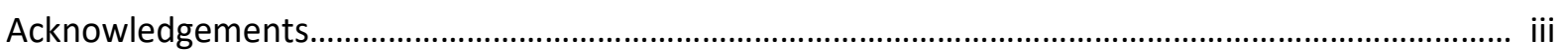

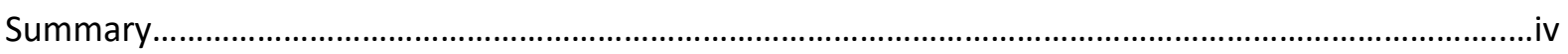

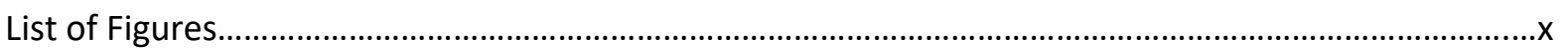

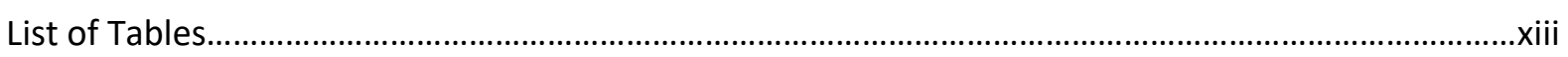

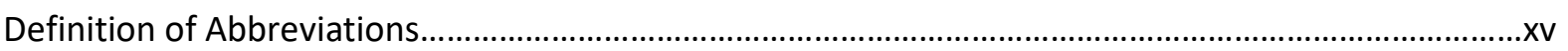

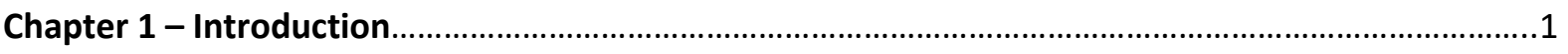

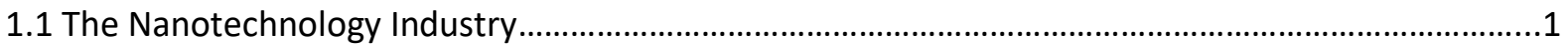

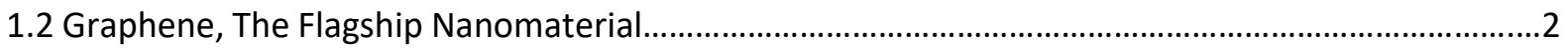

1.3 The Importance of Physico-Chemical Characterisation .........................................................................6

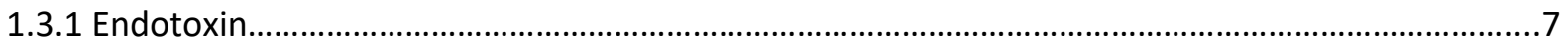

1.3.2 Size \& Shape

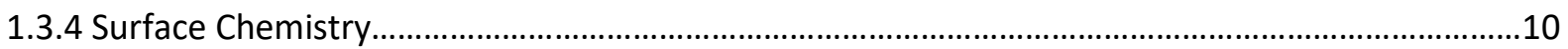

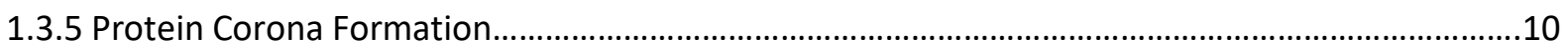

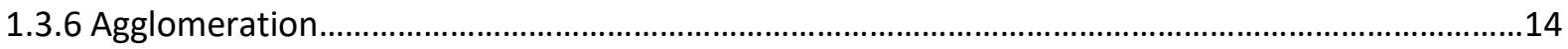

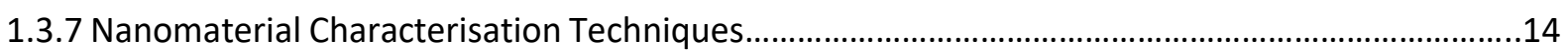

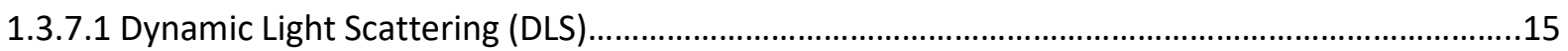

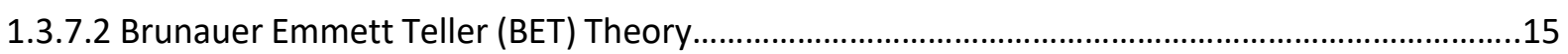

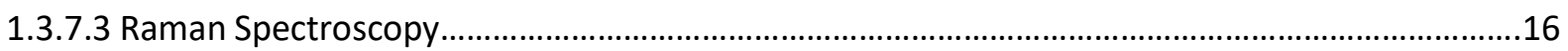

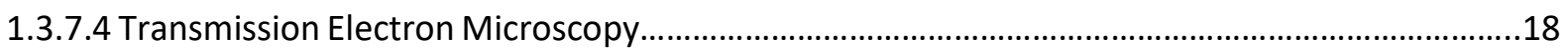

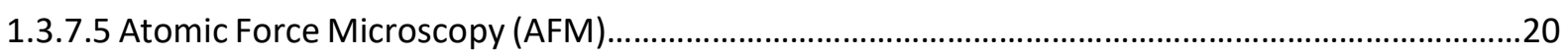

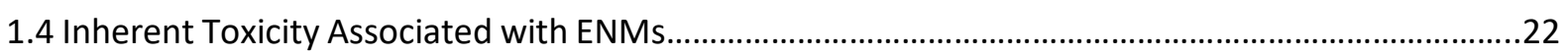

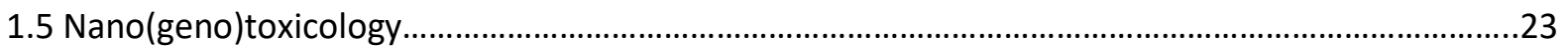

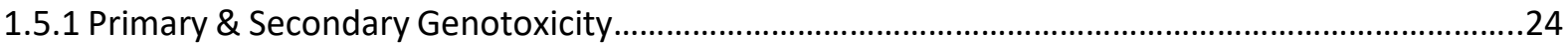

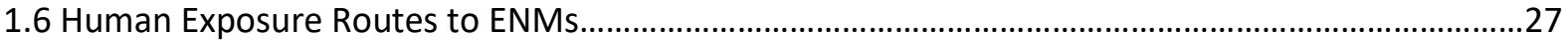

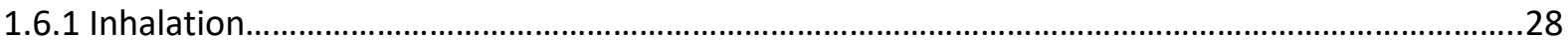

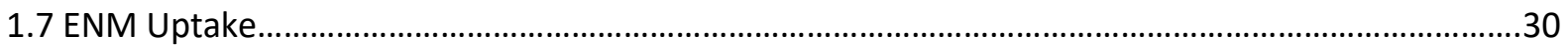

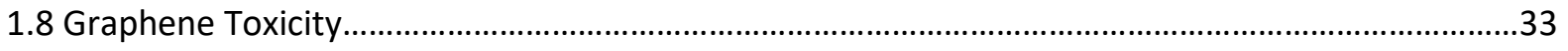

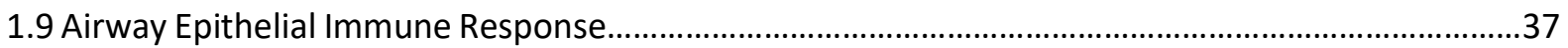

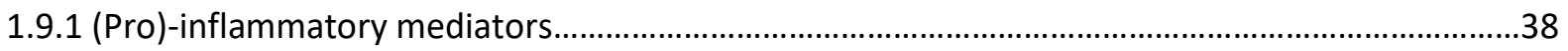

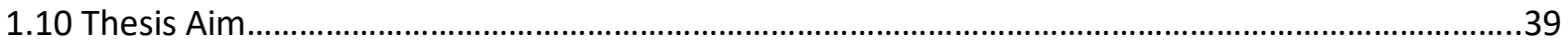

Chapter 2 - General Materials and Methods................................................................................... 


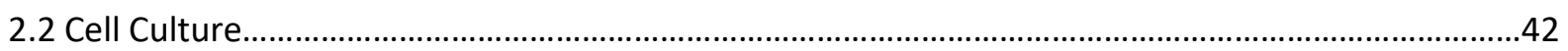

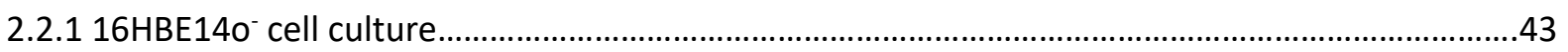

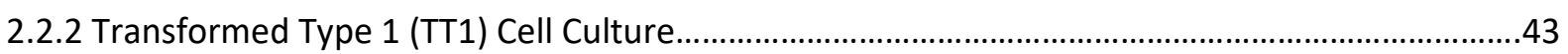

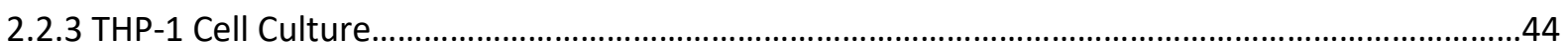

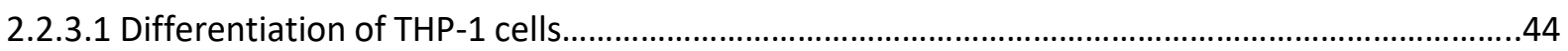

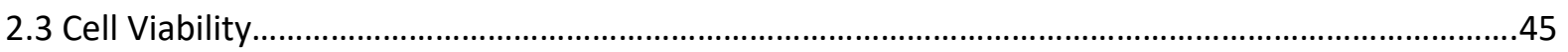

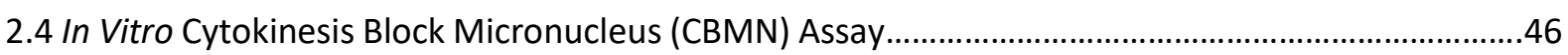

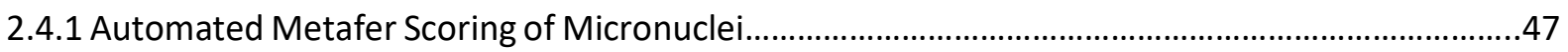

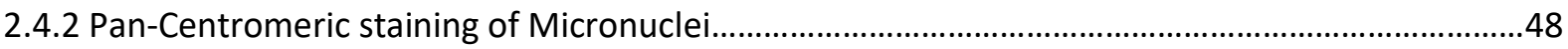

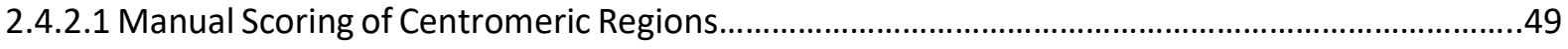

2.5 Quantification of (pro)-inflammatory mediators via enzyme linked immunosorbent assay.............49

2.6 Quantification of intracellular reduced Glutathione (GSH) .............................................................50

2.7 Determining Reactive Oxygen Species (ROS) .....................................................................................52

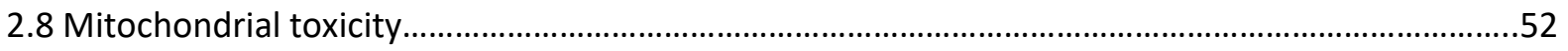

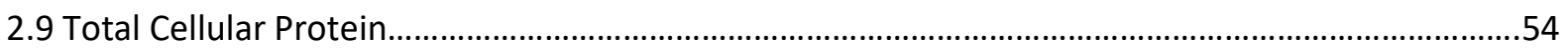

2.10 Sodium Dodecyl Sulfate Polyacrylamide Gel Electrophoresis (SDS-PAGE) .......................................55

2.11 Transmission Electron Microscopy (TEM) ..................................................................................56

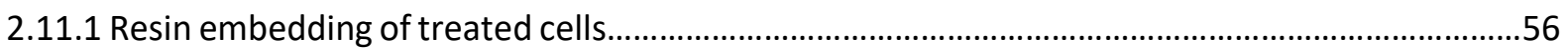

2.11.2 Ultramicrotomy sectioning of embedded samples........................................................................58

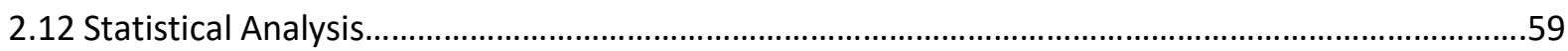

Chapter 3 - Quantitative and Qualitative Analysis of Few-Layer Graphene's Physico-Chemical

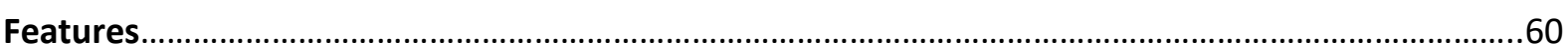

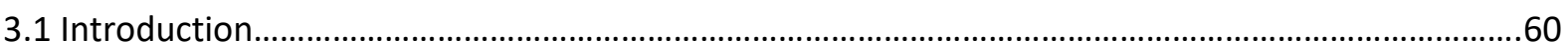

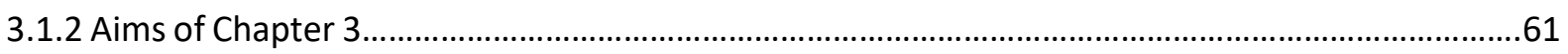

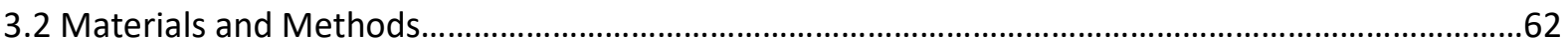

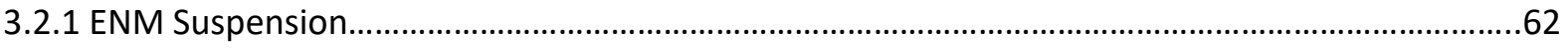

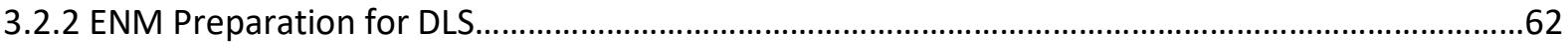

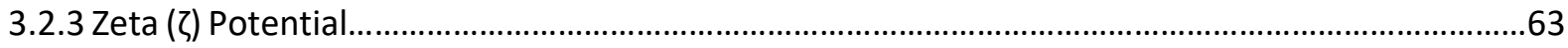

3.2.4 Scanning Electron Microscopy \& Energy Dispersive X-Ray Spectroscopy........................................63

3.2.5 Plunge Freeze Analysis of ENMs ..................................................................................................64

3.2.6 Atomic Force Microscopy..............................................................................................................64

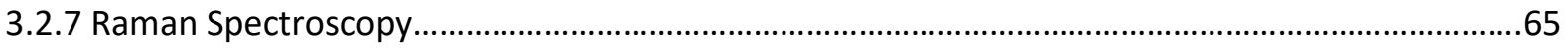

3.2.8 Acellular Superoxide Radical Detection via Electron Paramagnetic Resonance (EPR)...................65 
3.2.10 Limulus Amebocyte Lysate (LAL) Endotoxin Test....................................................................67

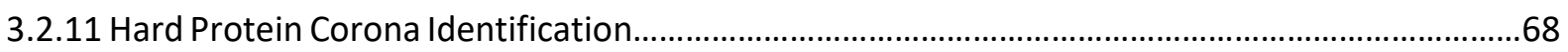

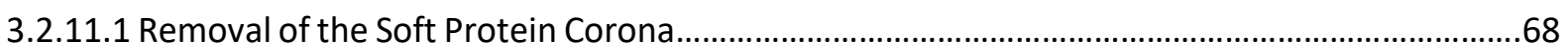

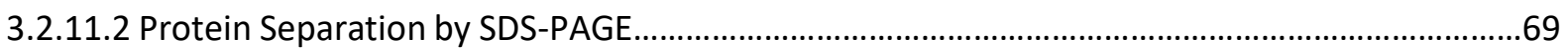

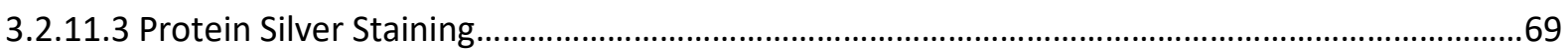

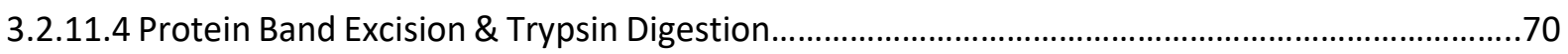

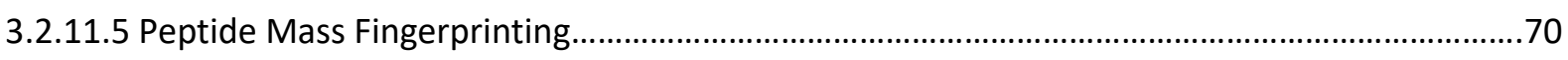

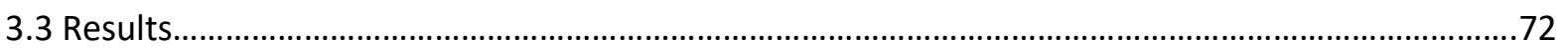

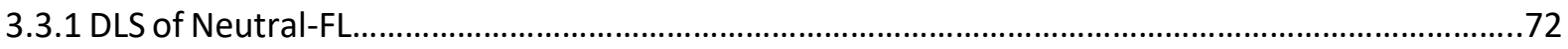

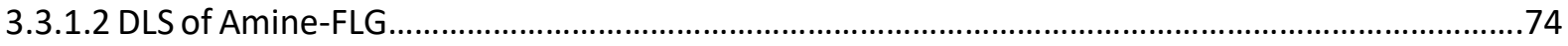

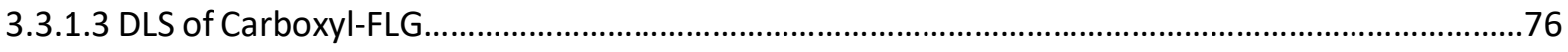

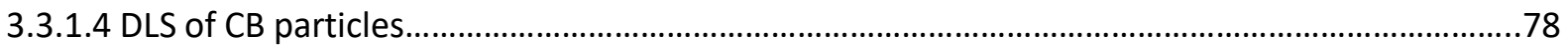

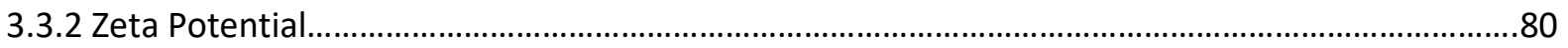

3.3.3 Scanning Electron Microscopy and Elemental Analysis of Primary ENM Characteristics................81

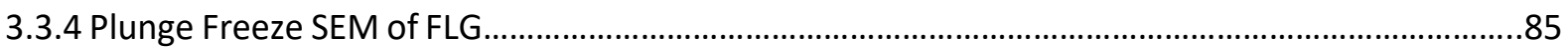

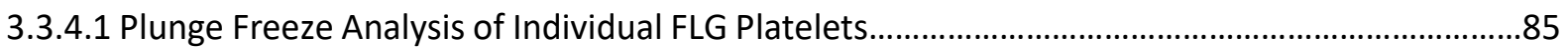

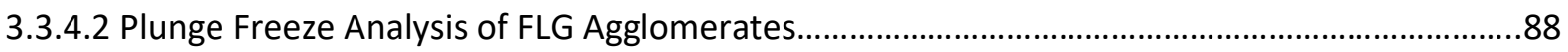

3.3.5 FLG Thickness Analysis by Atomic Force Microscopy.......................................................................92

3.3.5.1 Theoretically Calculated Aerodynamic Diameter \& Effective Density ..........................................96

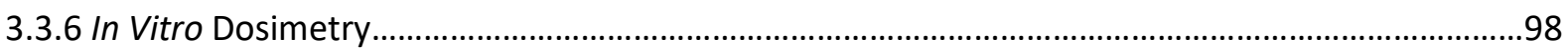

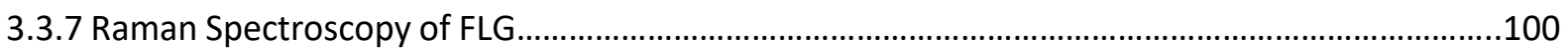

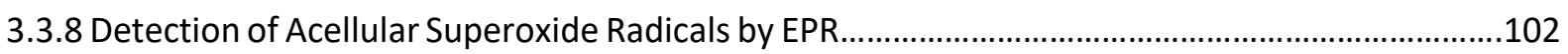

3.3.9 Determination of Primary ENMs Surface Area ...............................................................................104

3.3.10 Confirmation of Endotoxin Absence via LAL Gel Clot Test.........................................................106

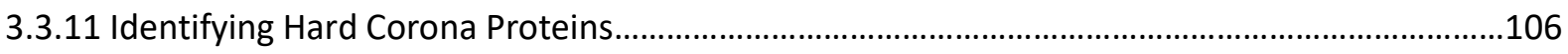

3.3.11.1 Peptide Mass Fingerprinting (Identification) of Hard Corona Proteins.....................................107

3.3.12 Summary of Physico-Chemical Features........................................................................................109

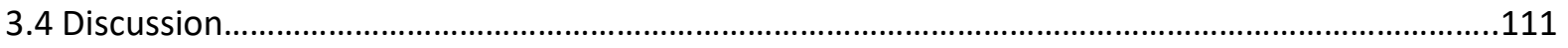

3.4.1 Physico-chemical Features of Neutral-FLG..............................................................................111

3.4.2 Effects of Additional Surface Chemistry (Amine \& Carboxyl Groups) Upon FLG...........................113

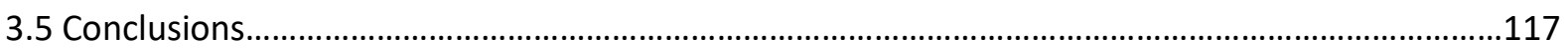


Chapter 4 - Investigating the Mechanisms Contributing to In Vitro Primary Genotoxicity in Bronchial Epithelial Cells

4.1 Introduction.

4.1.2 In Vitro Techniques to Assess Cytotoxicity, Genotoxicity, (Pro)-Inflammatory Response, Oxidative Stress and Mitochondrial Stress.

4.1.3 Cytotoxicity \& Genotoxicity Utilising the In Vitro CBMN Assay... 119

4.1.4 Sandwich ELISA (Pro)-Inflammatory Mediator Quantification 120

4.1.5 Oxidative Stress. 121

4.1.6 Mitochondrial Stress. 121

4.1.7 Chapter 4 Aims 123

4.2 Materials and Methods 124

4.2.1 16HBE140- Cell Culture. 124

4.2.2 In vitro Cytotoxicity \& Genotoxicity Assessment. 124

4.2.3 (Pro)-Inflammatory Response .124

4.2.4 Intracellular Reduced GSH. 124

4.2.5 Reactive Oxygen Species Detection .125

4.2.8 Mitochondrial Stress. .125

4.2.9 Cellular Protein Quantification. .126

4.2.10 Statistical Analysis 126

4.3 Results 127

4.3.1 Cytotoxicity \& Genotoxicity of 16HBE140`Following 24-hour Exposure to ENMs. .127

4.3.1.2 Pan-Centromeric Staining of Chromosomes to Highlight Clastogenicity / Aneugenicity..........129

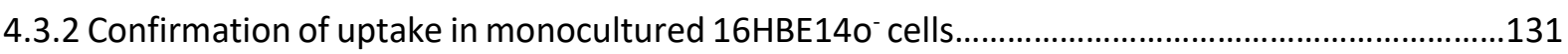

4.3.3 Quantification of IL-6 Release Following ENM Exposures........................................................136

4.3.3.1 Activation of an IL-8 (Pro)-Inflammatory Response Following ENM Exposure.......................137

4.3.4 Intracellular Glutathione (GSH) Depletion Following ENM Exposures......................................140

4.3.5 Quantification of Reactive Oxygen Species Generated by ENMs in 16HBE140- Cells..................142

4.3.5.1 Cell-free Reactive Oxygen Species Quantification...............................................................145

4.3.6 Oxygen Consumption and Extracellular Acidification Rates in 16HBE140- Cells Following ENM exposure

4.3.6.1 16HBE140- Respiratory Parameters as an Indicator of Mitochondrial Stress Following ENM exposure .150

4.4 Discussion. 155 
Chapter 5 - Evaluating Primary \& Secondary Mechanisms of In Vitro Genotoxicity in Alveolar Epithelial Models

5.1 Introduction .164

5.1.1 Minimising Animal Model Use .168

5.1.2 Lung Co-Cultures 169

5.1.3 Chapter 5 Aims. 172

5.2 Materials \& Methods 173

5.2.1. Basic Cell Culture 173

5.2.2 Construction of Bronchial \& Alveolar Co-Culture Model. 173

5.2.3 Pre-treatment of Co-Culture with N-acetyl-L-Cysteine (NAC) .................................................173

5.2.4 Fixation, Permeabilization and Fluorescent Labelling of Co-Culture..........................................174

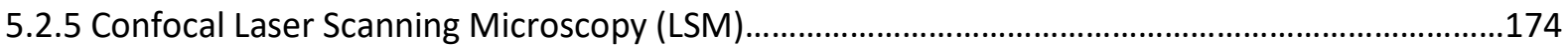

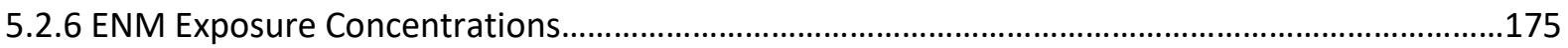

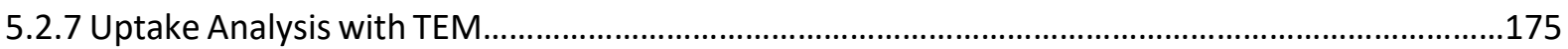

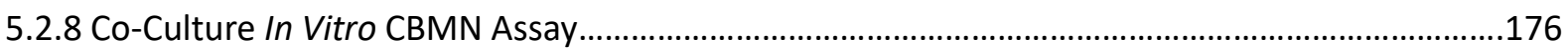

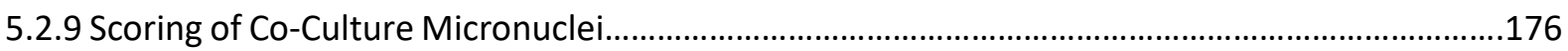

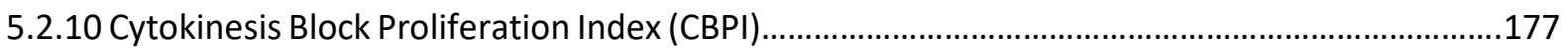

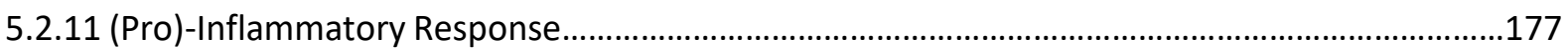

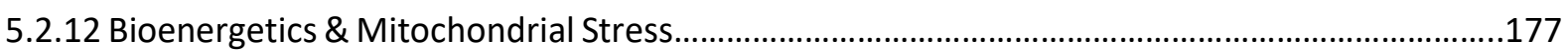

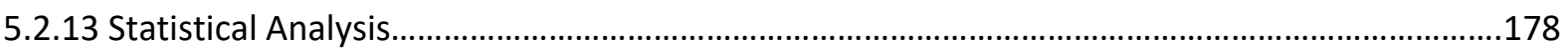

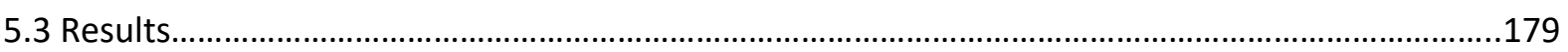

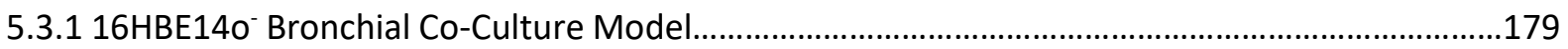

5.3.2 Quantifying the TT1/d.THP-1 (Pro)-Inflammatory Response.....................................................180

5.3.3 Cell Viability of d.THP-1 Following 24-hour ENM Exposure........................................................182

5.3.4 d.THP-1 (Pro)-Inflammatory Response Following ENM Exposure...............................................184

5.3.5 TT1 Cytotoxicity and Genotoxicity Following 24-hour ENM Exposure.......................................185

5.3.6 Confirmation of Uptake in Monocultured TT1 Cells Following 24-hour ENM Exposure...............187

5.3.7 Quantification of TT1 (Pro)-Inflammatory Mediators Following 24-hour ENM Exposure............192

5.3.8 Bioenergetic Response of TT1 Cells Following 24-hour ENM Exposure.......................................194

5.3.8.1 TT1 Mitochondrial Stress Following 24-hour ENM Exposure....................................................196

5.3.9 Confocal (LSM) Imaging of the TT1/d.THP-1 Co-Culture...........................................................199

5.3.10 Cytotoxicity \& Genotoxicity Evaluation of Co-Cultured TT1/d.THP-1 Cells Following 24-hour ENM Exposure. .201 
5.3.10.1 Evaluating Oxidative Stress in Alveolar Co-Cultures Pre-Incubated with NAC.

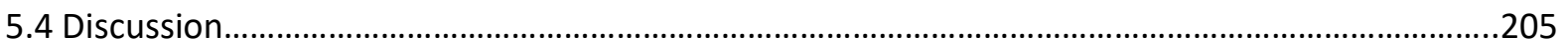

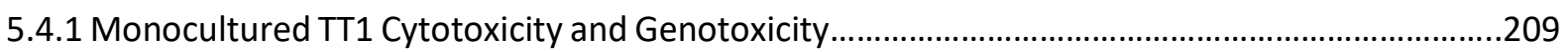

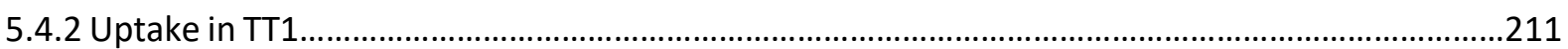

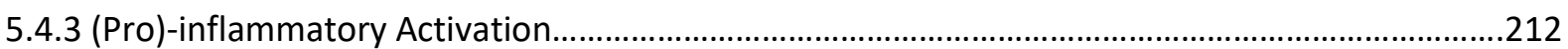

5.4.4 ENM effects upon Co-Cultured Cells.........................................................................................214

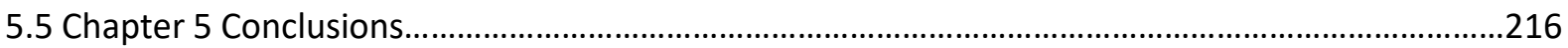

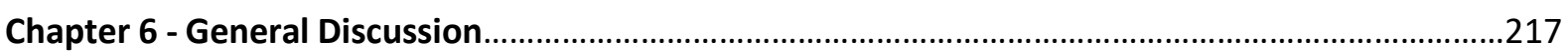

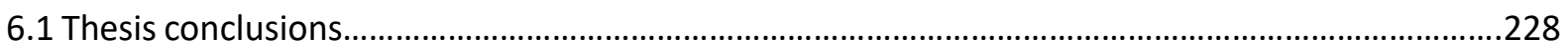

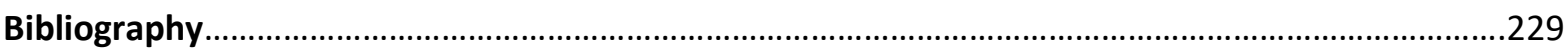

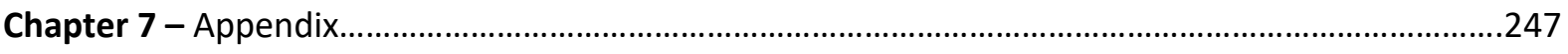

7.1 Total Protein Levels assessed by the DC Protein Assay.......................................................................247

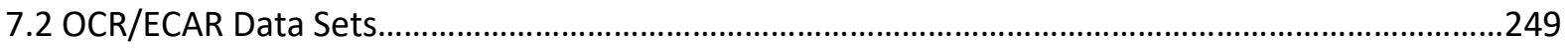

7.3 SDS-PAGE of BSA

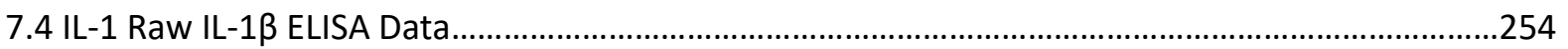

\section{List of Figures}

Figure 1.1 Ball \& stick model of graphene. .3

Figure 1.2 The top-down bulk manufacture of FLG introduces Stone-Wales defects into the monolayer structure .4

Figure 1.3 Graphene-based nanoplatelet induces frustrated phagocytosis in lung cells........................9

Figure 1.4 Differences in the corona formation on NPs depending on the site of administration.......11

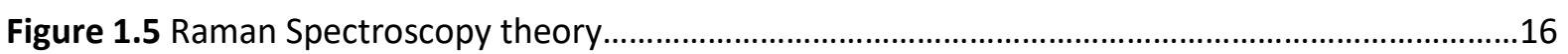

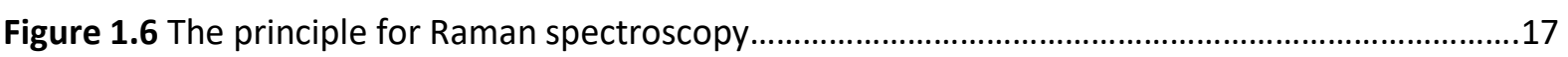

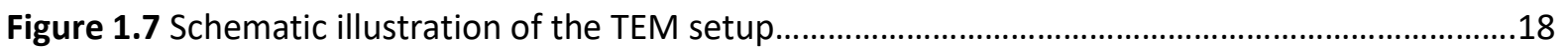

Figure 1.8 Illustration of the two possible edge characteristics displayed by graphene........................19

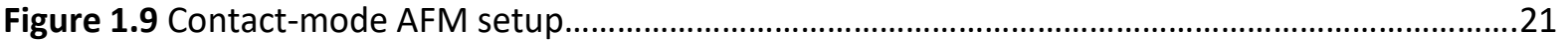

Figure 1.10 Prominent routes of ENM exposure, uptake, retention and translocation.........................27

Figure 1.11 ENM deposition in the respiratory tract........................................................................29

Figure 1.12 Active ENM internalisation by endocytosis........................................................................31

Figure 1.13 Internalisation routes of membrane-bound ENMs..........................................................33

Figure 1.14 The hypothesised role of IL-6 in mediating the switch between acute and chronic

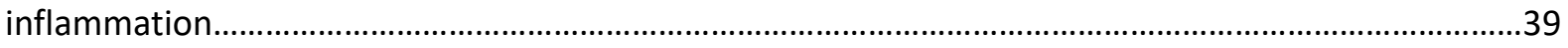

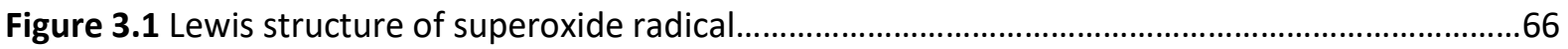


Figure 3.2 DLS measurement of Neutral-FLG. . .73

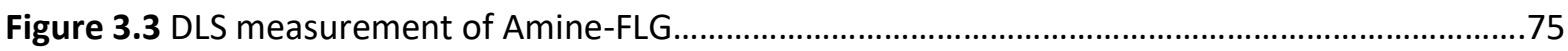

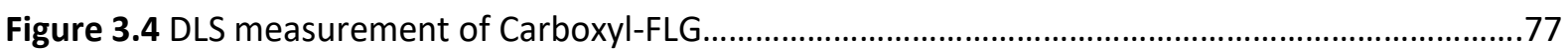

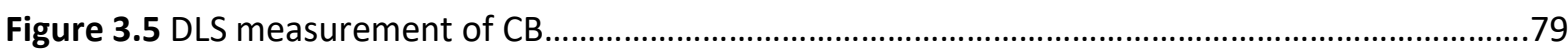

Figure 3.6 Size distribution histograms for Neutral- (A), Amine- (B), Carboxyl-FLG (C) and CB particles (D). . .83

Figure 3.7 Elemental composition for Neutral- (A), Amine- (B), Carboxyl-FLG (C) and CB particles (D)

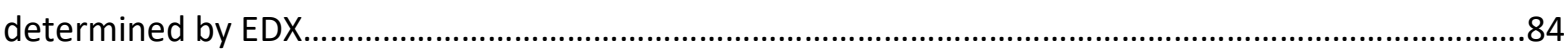

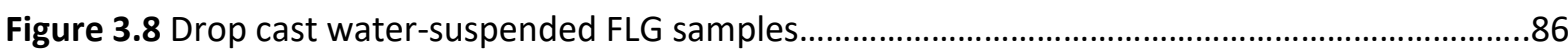

Figure 3.9 Drop cast serum-suspended FLG samples........................................................................8

Figure 3.10 Drop cast serum-suspended FLG samples.........................................................................90

Figure 3.11 Drop cast serum-suspended FLG samples..............................................................................91

Figure 3.12 Example depth data analysis using Bruker Nanoscope Analysis........................................93

Figure 3.13 Thickness analysis data acquired through Bruker Nanoscope Analysis Package (v 1.4) of

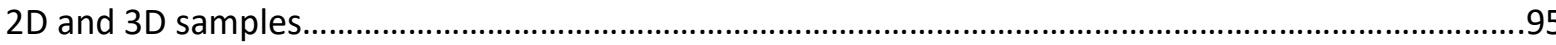

Figure 3.14 AFM images captured with the Bruker Dimension Icon AFM ...............................................96

Figure 3.15 Dosimetry plots modelling the final concentration to reach the adherent cell lines at the bottom of a T25 flask in $10 \mathrm{ml}$ of media . .99

Figure 3.16 Collective Raman Intensity Spectra of FLG samples, wavelength of incident laser set to $535 \mathrm{~nm}$

Figure 3.17 Superoxide generation of FLG quantified with EPR. Superoxide detection was evaluated in both Krebs Buffer (A) and distilled water (B) 103

Figure 3.18 SDS-PAGE, silver stained hard protein corona..................................................................107

Figure 3.19 Representative LC MS/MS ion spectra of Protein Argonaute-2. 109

Figure 4.1 Seahorse XFe cellular mitochondrial stress test profile indicating key respiratory parameters.

Figure 4.2 Seahorse XFe mitochondrial stress test modulators of the electron transport chain.......123

Figure 4.3 Cytotoxic and genotoxic assessment of ENM exposure to $16 \mathrm{HBE} 14 \mathrm{o}^{-}$cells.......................128

Figure 4.4 Stacked bar plots indicating clastogenic/aneugenic responses of $16 \mathrm{HBE} 14 \mathrm{o}^{-}$cells...........130

Figure 4.5 TEM micrographs of 16HBE14o- cells exposed to $20 \mu \mathrm{g} / \mathrm{ml}$ of Neutral-FLG.......................132

Figure 4.6 TEM micrographs of 16HBE140 cells exposed to $20 \mu \mathrm{g} / \mathrm{ml}$ of Amine-FLG.........................133

Figure 4.7 STEM/TEM micrographs of 16HBE140 cells exposed to $20 \mu \mathrm{g} / \mathrm{ml}$ of Carboxyl-FLG...........134

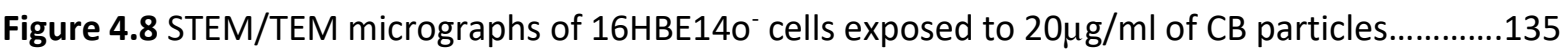

Figure 4.9 IL-6 (pro)-inflammatory response of 16HBE14o- cells after exposure to ENMs..................138 
Figure 4.10 IL-8 (pro)-inflammatory response of 16HBE14o- cells after exposure to ENMs 139

Figure 4.11 Intracellular reduced glutathione levels measured with monochlorobimane-GSH binding fluorescence after ENM exposures of 6 hours (A) and 24 hours (B). .141

Figure 4.12 ROS production quantified with DCFH-DA fluorescence in $16 \mathrm{HBE} 140^{\circ}$ cells over 0.5, 1, 1.5 and 2 hours

Figure 4.13 ROS production quantified with DCFH-DA fluorescence in 16HBE140- cells following over $4,8 \& 24$ hours

Figure 4.14 Acellular ROS DCFH-DA assay promoted by A) Neutral-FLG, B) Amine-FLG, C) CarboxylFLG and D) CB particles over 0.5, 1, 1.5 \& 2 hours

Figure 4.15 Acellular ROS DCFH-DA assay promoted by A) Neutral-FLG, B) Amine-FLG, C) Carboxyl-

FLG and D) CB particles over 4, 8 \& 24 hours .147

Figure 4.16 Oxygen consumption (A) and extracellular acidification rate (B) in $16 \mathrm{HBE} 140^{-}$cells following 24-hour exposure to Neutral-FLG

Figure 4.17 16HBE140- Proton Leak \& Non-Mitochondrial Respiration following a 24-hour exposure to Neutral-FLG (A), Amine-FLG (B), Carboxyl-FLG (C) and CB particles (D). 153

Figure 4.18 16HBE140 respiratory parameters following a 24-hour exposure to Neutral-FLG (A),

Amine-FLG (B), Carboxyl-FLG (C) and CB particles (D). .154

Figure 4.19 Hypothetical mechanism of ENM interference with the ETC 159

Figure 4.20 A representation of the electron recycling in complex III between iron-sulphur clusters which graphene ENMs have been reported to interfere with 160

Figure 4.21 Potential ROS clearance pathways linking GSH and mitochondrial stress. .161

Figure 5.1 Innate immunity against particle/fibre deposition in the respiratory tract 166

Figure 5.2 (Pro)-inflammatory response of co-cultured 16HBE140 cells following a 24-hour exposure to ENMs

Figure 5.3 (Pro)-inflammatory response of co-cultured TT1/d.THP-1 cells following a 24-hour exposure to ENMs at a concentration of $100 \mu \mathrm{g} / \mathrm{ml}$

Figure 5.4 d.THP-1 cell viability following 24-hour exposure to Neutral-FLG (A), Amine-FLG (B),

Carboxyl-FLG (C) \& CB particles (D).

Figure 5.5 d.THP-1 IL-8 (pro)-inflammatory response following a 24-hour exposure to ENMs

Figure 5.6 Cytotoxic and genotoxic evaluation of monocultured TT1 cells following a 24-hour exposure to ENMs.

Figure 5.7 TEM confirmation of uptake of ENMs in monocultured TT1 cells at $20 \mu \mathrm{g} / \mathrm{ml}$ following a 24-hour exposure period 188 
Figure 5.8 STEM confirmation of uptake of ENMs in monocultured TT1 cells at $20 \mu \mathrm{g} / \mathrm{ml}$ following a 24-hour exposure period.

Figure 5.9 Flow diagram illustrating the process of confirming the presence of graphitic material within TT1 cell vesicles 190

Figure 5.10 Flow diagram illustrating the process of confirmation for CB particle uptake within TT1 cells. .191

Figure 5.11 TT1 (pro)-inflammatory response following a 24-hour exposure to ENMs 193

Figure 5.12 OCR \& ECAR assessment of monocultured TT1 cells following a 24-hour exposure to Neutral-FLG 195

Figure 5.13 Metabolic parameters of monocultured TT1 cells following 24-hour exposure to ENMs. .198

Figure 5.14 LSM of co-cultured TT1/d.THP-1 macrophages grown on PET transwell inserts. 200

Figure 5.15 Cytotoxicity and chromosomal damage induced by ENMs within TT1/d.THP-1 cocultures.

Figure 5.16 Cytotoxicity and chromosomal damage induced by ENMs within TT1/d.THP-1 co-cultures pre-treated with NAC. 204

Figure 6.1 Hypothesised chemical structure of Amine-FLG 220

Figure 6.2 Mitochondrial enzymatic reactions

Figure 6.3 Potential mechanisms contributing to the genotoxicity observed in monocultured 16HBE140 and TT1 cells.

Figure 6.4 Potential co-culture secondary mechanisms of genotoxicity... 227

Figure 7.1 16HBE total protein levels

Figure 7.2 Total protein levels of 16HBE140 cells following 24-hour exposure to ENMs followed by bioenergetic analysis by the Seahorse Bioanalyzer XFe24

Figure 7.3 Cell line optimisation required prior to Seahorse XFe24 analysis 249

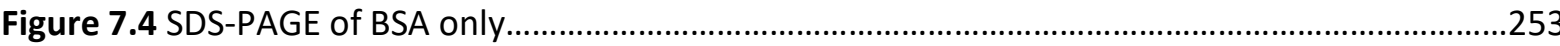

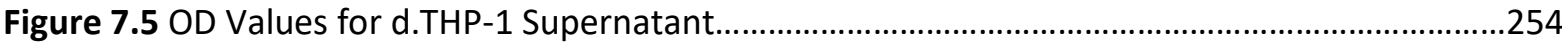

List of Tables

Table 2.1 ENMs dilution and dosing technique for mono-cultured cell lines; $16 \mathrm{HBE} 14 \mathrm{o}^{-}$and TT1 cells in T25 flasks, seeded at $1 \times 10^{5}$ .41

Table 2.2 Metafer microscope (Metafer 4, version 3.5) nuclei classifier settings.....................................47

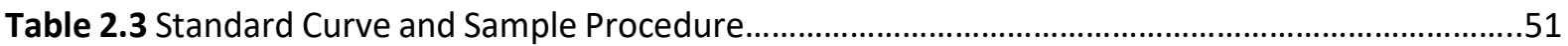

Table 2.4 Injection strategy for mitochondrial stress test .53 
Table 2.5 Protein Standard Curve setup for a 96-well half area clear plastic plate .55

Table 2.6 10\% Stacking and 4\% Resolving gel recipes for SDS-PAGE...................................................56

Table 3.1 Dispersant Characteristics required for DLS measurements....................................................63

Table 3.2 Detection of Endotoxin in ENMs utilising the LAL gel clot assay kit.......................................68

Table 3.3 Neutral-FLG characteristics defined by DLS.............................................................................

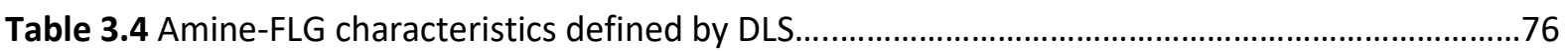

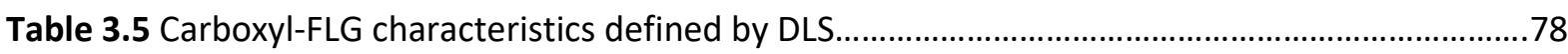

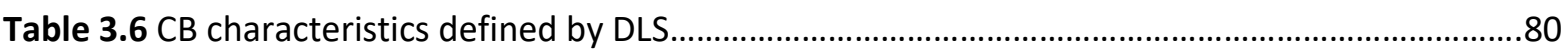

Table 3.7 Zeta Potential of carbonaceous ENMs suspended in both ultrapure double distilled water and cell culture media. .81

Table 3.8 Summary of FLG fibre size as determined from plunge freeze experiments and ImageJ analysis

Table 3.9 Summary of FLG agglomerate size as determined from plunge freeze experiments and ImageJ analysis

Table 3.10 Depth analysis on FLG using Bruker Nanoscope Analysis Package......................................94

Table 3.11 Summary of aerodynamic diameter and effective densities of FLG materials....................97

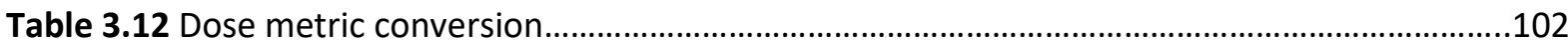

Table 3.13 Multipoint BET surface area and micropore features........................................................105

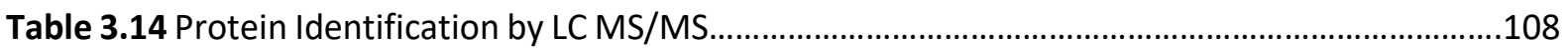

Table 3.15 Summary of the ENMs physico-chemical features.............................................................110

Table 4.1 Respiratory parameters of 16HBE140 - mitochondrion calculated from original OCR profiles of each ENM. .125

Table 4.2 Seahorse XFe24 injection compounds and corresponding effect on OCR..........................126

Table 5.1 Representative and commonly selected cell lines for ENM lung exposures........................171

Table 5.2 LSM fluorophores used in the visualisation of the co-culture model......................................175

Table 5.3 ENM dilutions to expose at the required concentration in co-cultures................................175

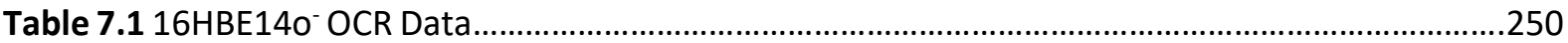

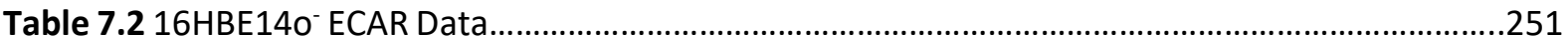

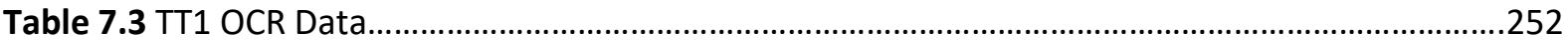

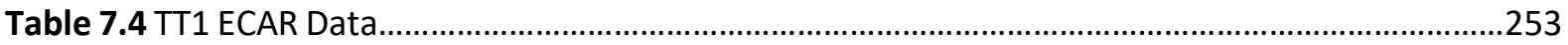




\section{Definitions of Abbreviations}

\begin{tabular}{|c|c|}
\hline$\sqrt{ }$ & Square Root (Mathematical Function) \\
\hline$\leq$ & Smaller than or equal to \\
\hline$\geq$ & Greater than or equal to \\
\hline$\circ$ & Degree Sign \\
\hline$\mu$ & Micro (Greek Letter) \\
\hline$\mu \mathrm{g}$ & Micro gram \\
\hline$\mu \mathrm{g} / \mathrm{ml}$ & Microgram per millilitre \\
\hline $16 \mathrm{HBE}^{2} 4 \mathrm{o}^{-}$ & Human Bronchial Epithelial Cell Line \\
\hline A549 & $\begin{array}{l}\text { Adenocarcinoma Human Alveolar-Basal } \\
\text { Epithelial Cell Line }\end{array}$ \\
\hline $\mathrm{ACN}$ & Acetonitrile \\
\hline AFM & Atomic Force Microscopy \\
\hline ALI & Air-Liquid Interface \\
\hline Amine-FLG & Amine Functionalised Few-Layer Graphene \\
\hline ANOVA & Analysis of Variance \\
\hline ATI & $\begin{array}{l}\text { Primary Human Alveolar Epithelial Type } 1 \text { Cell } \\
\text { Line }\end{array}$ \\
\hline ATII & $\begin{array}{l}\text { Primary Human Alveolar Epithelial Type } 2 \text { Cell } \\
\text { Line }\end{array}$ \\
\hline BEAS-2B & Human Bronchial Cell Line \\
\hline BET & Brunauer-Emmett-Teller \\
\hline $\mathrm{BN}$ & Binucleated Cell \\
\hline BSA & Bovine Serum Albumin \\
\hline Carboxyl-FLG & Carboxyl Functionalised Few-Layer Graphene \\
\hline CB & Carbon Black \\
\hline CBMN & Cytokinesis Block Micronucleus \\
\hline CBPI & Cytokinesis Block Proliferation Index \\
\hline $\mathrm{cm}$ & Centimetre \\
\hline $\mathrm{cm}^{2}$ & Centimetre squared \\
\hline CNT (s) & Carbon Nanotube (s) \\
\hline Control & $\begin{array}{l}\text { Un-Treated (Media Only) Cell Line Used in an } \\
\text { Experiment }\end{array}$ \\
\hline
\end{tabular}




\begin{tabular}{|c|c|}
\hline $\mathrm{COOH}$ & Carboxylate \\
\hline COPD & Chronic Obstructive Pulmonary Disease \\
\hline Cyto B & Cytochalasin B \\
\hline d.THP-1 & PMA-differentiated THP-1 Cell Line \\
\hline DAPI & 4'-6-diamidino-2-phenylindole \\
\hline DCFH-DA & $2^{\prime}, 7^{\prime}$-dichlorofluroscein diacetate \\
\hline DLS & Dynamic Light Scattering \\
\hline DMSO & Dimethylsulfoxide \\
\hline DTT & Dithiothreitol \\
\hline ECAR & Extracellular Acidification Rate \\
\hline EDX & Energy Dispersive X-Ray Spectroscopy \\
\hline EGTA & $\begin{array}{l}\text { Ethylene glycol-bis(2-aminoethylether)- } \\
\mathrm{N}, \mathrm{N}, \mathrm{N}^{\prime}, \mathrm{N}^{\prime} \text {-tetraacetic acid }\end{array}$ \\
\hline ELISA & Enzyme-Linked Immunosorbent Assay \\
\hline EM & Electron Microscopy \\
\hline $\operatorname{ENM}(\mathrm{s})$ & Engineered Nanomaterial (s) \\
\hline EPR & Electron Paramagnetic Resonance \\
\hline EU & European Commission \\
\hline FBS & Foetal Bovine Serum \\
\hline FCCP & $\begin{array}{l}\text { Carbonyl Cyanide 4- } \\
\text { (Trifluoromethoxy)Phenylhydrazone }\end{array}$ \\
\hline FITC & Fluorescein Isothiocyanate \\
\hline FLG & Non-Functionalised Few-Layer Graphene \\
\hline Glut & Glutamine \\
\hline GPx & Glutathione Peroxidase \\
\hline GRx & Glutathione Reductase \\
\hline GSH & Reduced Glutathione \\
\hline GSSG & Oxidised Glutathione \\
\hline GST & Glutathione S Transferase \\
\hline $\mathrm{H}_{2} \mathrm{O}_{2}$ & Hydrogen Peroxide \\
\hline HAADF & High Angle Annular Dark Field \\
\hline $\mathrm{HCl}$ & Hydrochloric Acid \\
\hline IL-1 $\beta$ & Interleukin-1 Beta \\
\hline
\end{tabular}




\begin{tabular}{|c|c|}
\hline IL-6 & Interleukin-6 \\
\hline IL-8 & Interleukin-8 \\
\hline $\mathrm{kDa}$ & Kilo Daltons \\
\hline LAL & Limulus Amebocyte Lysate \\
\hline LC MS/MS & Liquid Chromatography Mass Spectrometry \\
\hline LOEL & Lowest Observed Effect Level \\
\hline LPS & Lipopolysaccharide \\
\hline LSM & Laser Scanning Microscopy \\
\hline$M$ & Molar \\
\hline $\mathrm{m}^{2}$ & Metre Squared \\
\hline MDA & Malondialdehyde \\
\hline MEM & Minimum Essential Growth Medium \\
\hline $\mathrm{mg} / \mathrm{ml}$ & Milli gram per millilitre \\
\hline $\mathrm{Mi} / \mathrm{BN}$ & Micronucleus-Containing Binucleated Cell \\
\hline $\mathrm{ml}$ & millilitre \\
\hline $\mathrm{mM}$ & Milli Molar \\
\hline $\mathrm{mV}$ & milli Volts \\
\hline MW & Molecular Weight \\
\hline MWCNT (s) & Multi Walled Carbon Nanotubes \\
\hline NAC & $\mathrm{N}$-acetyl-L-cysteine \\
\hline $\mathrm{NaOH}$ & Sodium Hydroxide \\
\hline NCS & New-Born Calf Serum \\
\hline $\mathrm{NH}_{2}$ & Amino \\
\hline NLRP3 & $\begin{array}{l}\text { NACHT, LRR \& PYD domains containing Protein } \\
3\end{array}$ \\
\hline NM (s) & Nanomaterial (s) \\
\hline NP (s) & Nanoparticles (s) \\
\hline $\mathrm{O}_{2} \cdot{ }^{-}$ & Superoxide Radical \\
\hline OCR & Oxygen Consumption Rate \\
\hline OECD & $\begin{array}{l}\text { Organisation for Economic Co-Operation \& } \\
\text { Development }\end{array}$ \\
\hline $\mathrm{OH}$. & Hydroxyl Radical \\
\hline $\mathrm{P} / \mathrm{S}$ & Penicillin / Streptomycin \\
\hline
\end{tabular}




\begin{tabular}{|c|c|}
\hline PBS & Phosphate Buffered Saline \\
\hline PDI & Polydispersed Index \\
\hline PET & Polyethylene Terephthalate \\
\hline PET Solution & $\begin{array}{l}\text { Polyvinylpyrrolidone-Ethylene glycol-bis(2- } \\
\text { aminoether)-N,N,N',N',-tetra acetic acid Trypsin }\end{array}$ \\
\hline PFA & Paraformaldehyde \\
\hline PM & Particulate Matter \\
\hline PMA & Phorbol 12-Myristate 13-Acetate \\
\hline PSG & Penicillin / Streptomycin / Glutamine \\
\hline QD (s) & Quantum Dot (s) \\
\hline Raw 264.7 & $\begin{array}{l}\text { Transformed Abelson Murine Leukaemia Virus } \\
\text { Macrophage Cell Line }\end{array}$ \\
\hline RIPA Buffer & Radio Immunoprecipitation Assay Buffer \\
\hline RNS & Reactive Nitrogen Species \\
\hline ROS & Reactive Oxygen Species \\
\hline RPD & Relative Population Doubling \\
\hline RPM & Revolutions Per Minute \\
\hline RPMI & Roswell Park Memorial Institute 1640 Medium \\
\hline SDS & Sodium Dodecyl Sulphate \\
\hline SDS-PAGE & $\begin{array}{l}\text { Sodium Dodecyl Sulphate Polyacrylamide Gel } \\
\text { Electrophoresis }\end{array}$ \\
\hline SEM & Scanning Electron Microscopy \\
\hline SOD & Superoxide Dismutase \\
\hline SOP & Standard Operating Procedure \\
\hline SSC & Saline Sodium Citrate \\
\hline STEM & Scanning Tunnelling Electron Microscopy \\
\hline SWCNT (s) & Single Walled Carbon Nanotubes \\
\hline TBS & Tris-Buffered Saline \\
\hline TEM & Transmission Electron Microscopy \\
\hline TEMED & $\mathrm{N}, \mathrm{N}, \mathrm{N}^{\prime}, \mathrm{N}^{\prime}$-Tetrametheylethylenediamine \\
\hline Tempone-H & $\begin{array}{l}\text { 1-hydroxyl-2,2,6,6-tetramethyl-4-oxo- } \\
\text { piperidine }\end{array}$ \\
\hline TFA & Trifluoroacetic Acid \\
\hline
\end{tabular}




\begin{tabular}{|l|l|}
\hline THP-1 & $\begin{array}{l}\text { Human Monocyte Cell Line Derived from Acute } \\
\text { Monocytic Leukaemia }\end{array}$ \\
\hline THP-1 Macrophage & $\begin{array}{l}\text { Differentiated Human Monocyte Derived from } \\
\text { Acute Monocytic Leukaemia }\end{array}$ \\
\hline TLR & Toll-Like Receptor \\
\hline TNF $\alpha$ & Tumour Necrosis Factor Alpha \\
\hline Tris & Trisaminomethane \\
\hline TT1 & Transformed Type 1 Cell Line \\
\hline TT1/d.THP-1 & $\begin{array}{l}\text { Co-Cultured TT1 \& Differentiated THP-1 Cell } \\
\text { Lines }\end{array}$ \\
\hline Tween 20 & Polyethylene Glycol Sorbitan \\
\hline XPS & X-Ray Photoelectron Spectroscopy \\
\hline$\alpha$ & Alpha (Greek Letter) \\
\hline$\beta$ & Beta (Greek Letter) \\
\hline$\zeta$ & Zeta (Greek Letter) \\
\hline$\lambda$ & Lambda (Greek Letter) \\
\hline
\end{tabular}




\section{Chapter 1- Introduction}

Nanotechnology bridges various disciplines encompassing engineering, physics, biology and medicine with the purpose of manufacturing novel beneficial and revolutionary scientific concepts (Sahoo et al., 2007). Nanotechnology typically involves the manufacture of medical or engineering products at the nanoscale which represents one billionth of a metre $\left(10^{-9} \mathrm{~m}\right)$. Due to this uniquely small size with which nanomaterials (NMs) exist, they can interact at the molecular level of DNA (helix diameter of $2 \mathrm{~nm}$ ), prokaryotes $(200 \mathrm{~nm}$ in size) and are also capable of interacting on an intracellular level (eukaryotic cells typically several microns $\left(10^{-6} \mathrm{~m}\right)$ in size). The European Commission, as of 2011, defines a NM as: "A natural, incidental or manufactured material containing particles, in an unbound state or as an aggregate or as an agglomerate and where, for $50 \%$ or more of the particles in the number size distribution, one or more external dimensions is in the size range $1 \mathrm{~nm}-100 \mathrm{~nm}$ " (Bleeker et al., 2013). In a continuing statement the European Commission also defines that fullerenes, graphene flakes and Carbon Nanotubes (CNTs) with one or more external dimensions fewer than $1 \mathrm{~nm}$ should also be classed as a NM.

\subsection{The Nanotechnology Industry}

The nanotechnology industry is in a constant state of development with novel applications in medicine for the treatment of diseases in the human body, industrial uses involving pathogen detection, nanoencapsulation in the food industry and next-generation sequencing techniques (Agrawal et al., 2018, Rashidi and Khosravi-Darani, 2011, Thiruvengadam et al., 2018, Elingaramil et al., 2013). Paradoxically, the generation of NMs is not a new concept however, the manufacture of engineered nanomaterials (ENMs) with an intended use and carefully designed structure is what forms the foundation of the nanotechnology industry. Incidental or accidental NMs have been airborne since the discovery of fire, however due to the heightened industrial demand coupled to research opportunities the need for toxicology testing has become apparent owing to the potentially detrimental effects of these materials (Soares et al., 2018). ENMs can be sourced from a variety of bulk materials such as Carbon, Gold, Titanium, Silver and Iron to name a few. These materials in their current bulk state are relatively inert (in chemical terms, they offer little use) however manufacturing ENMs from either bottom-up (building ENMs from constituent parts, or top-down (manufacturing ENMs from bulk) drastically changes the electrical, physical and optical properties of bulk elements (Gatoo et al., 2014). This change in property from bulk to nano, provides the foundation of research in nanotechnology with the ENM counterpart of a bulk material, or example carbon being extremely useful at the nano-scale, as graphene for example. 


\subsection{Graphene, The Flagship Nanomaterial}

Graphene is the current flagship of nanomaterials since its isolation and discovery in 2004 by Andre Geim and Konstantin Novoselov (Geim and Novoselov, 2007). Graphene is a 2-dimensional monolayer of hexagonally arranged carbon atoms (Figure 1.1) with tremendous potential for its applications in material sciences (Bressan et al., 2014). Typically possessing $>90 \%$ carbon, graphene and graphenederivatives such as Few-Layer Graphene (FLG) containing upwards of three atomic layers of graphene are highly diverse in function and application (Burgum et al., 2018). Graphene carries high potential in the nanotechnology industry due to its electrical, thermal and physical properties which has put graphene-based research to the forefront of nanotechnology (Zhang et al., 2005, Yao et al., 2018, Lee et al., 2008). The unique physical properties graphene possesses has led to the emergence of many new fields in physics such as condensed matter physics and quantum relativistic phenomena (Geim and Novoselov, 2007). Electrical and conductivity testing on graphene has proved interesting and yielded promising results which in some instances have exceeded theoretically predicted values (Novoselov et al., 2012). Some examples of the physical properties of graphene include an exceptionally high thermal conductivity $\left(>3000 \mathrm{WmK}^{-1}\right)$, total non-permeability to gases and the potential to be chemically modified (Balandin, 2011, Bunch et al., 2008, Nair et al., 2010). Among other properties this is partly the reason graphene has been named the "miracle material" (Arvidsson et al., 2018). Graphene also has the potential to be manipulated into many new forms to change some of the properties discussed above, which give rise to novel features and uses. Graphene can be manufactured in several ways, each of which will have its own advantages and drawbacks depending on the quality and purpose required. High quality graphene is typically produced via graphite chemical exfoliation which offers many of the properties discussed above where the electrical and thermal qualities can be optimised (Novoselov et al., 2004). Other options for production of graphene include chemical vapour deposition and growing graphitic layers on silicon carbide chips, this process occurs by sublimating the silicon atoms ultimately yielding graphitic layers (Novoselov et al., 2012). The consequent drawbacks of the latter method are the high cost of acquiring the silicon carbide chips and reaching and maintaining the $1000^{\circ} \mathrm{C}$ temperature at which the silicon carbide becomes increasingly unsettled. Chemical vapour deposition also has cost drawbacks related to high energy usage and difficulty removing the copper foils on which the graphene is grown (Novoselov et al., 2012). 


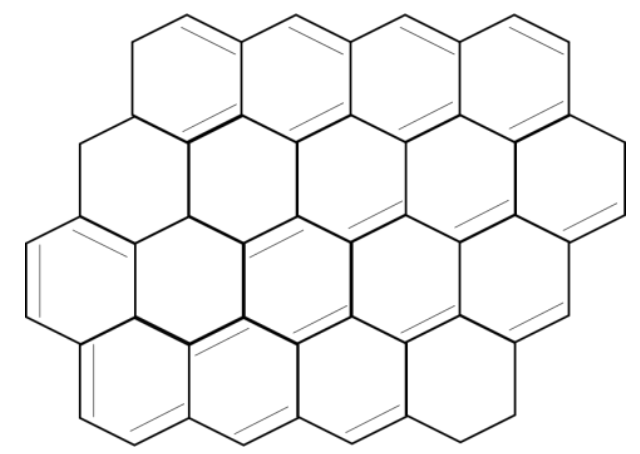

Figure 1.1 Ball \& stick model of graphene. The honeycomb lattice present in graphene and the $s p^{2}$ hybridised orbitals of the outer electrons gives graphene its superior strength, conductive, thermal and optical properties.

The physical parameters which make graphene unique are also subject to change, three of these features have been highlighted below, all of which can induce slightly different inflammatory and toxic responses. Monolayer graphene with $\mathrm{sp}^{2}$ hybridised carbon has a theoretical maximum surface area of $>2600 \mathrm{~m}^{2} / \mathrm{g}$. in comparison to most other nanomaterials this is at least one order of magnitude greater. The carbon atoms of the graphene monolayer are exposed on both sides of the sheet which provides the exceptionally high surface area (Sanchez et al., 2012). The disadvantage of the platelet form which graphene acquires means processes such as filtration become inefficient due to blockages. Additionally, the platelet shape makes dynamic light scattering (DLS) and zeta potential measurements (usually performed on spherical nanomaterials) difficult to measure, with precision, the agglomeration size in aqueous conditions (Sanchez et al., 2012). Stacking of monolayer graphene sheets into multi-layer stacks (Few-Layer Graphene (FLG)) will alter the material stiffness and as a result can influence the overall surface area (Figure 1.2). The elastic and breaking strength of graphene monolayers using nano-indentation with atomic force microscopy (AFM) has been investigated. In the study by Lee and colleagues, graphene was confirmed as the strongest material ever measured by this technique with a breaking strength of $43 \mathrm{~N} / \mathrm{m}$ representing the intrinsic strength of a pristine graphene sheet (Lee et al., 2008). Only one material has been theorised to surpass graphene in stiffness, that being carbyne, another potential allotrope of "sp" carbon hybridisation. Carbyne remains however a theoretical venture at this early stage in its development. Paradoxically, monolayer graphene and graphene oxide are quite deformable by water surface tension attractive and repulsive forces, which are inherently weak (Guo et al., 2011). It was also noted that water invasion between the layered sheets reduces the frictional forces and could be responsible for making stacked graphene more water-soluble. 

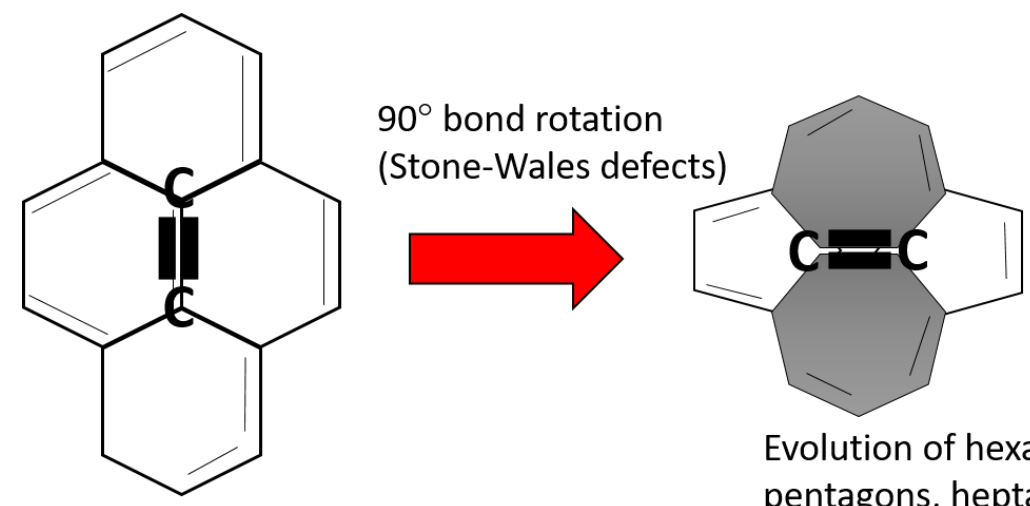

Evolution of hexagons into

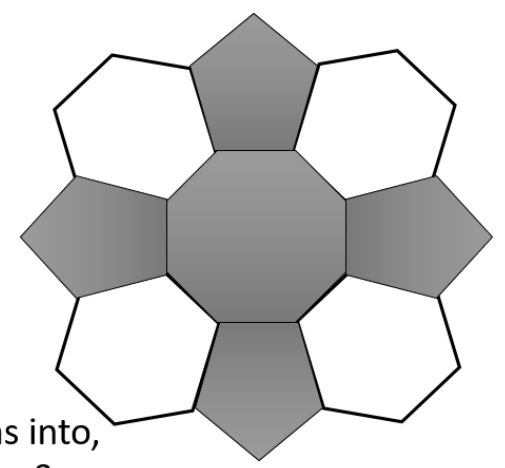
pentagons, heptagons \&

octagons

Figure 1.2 The top-down bulk manufacture of FLG introduces Stone-Wales defects into the monolayer structure. The greatest limitation on graphene's potential in the nanotechnology industry is the in-efficiency of bulk manufacture. FLG which can be bulk-manufactured will typically possess structural impurities characterised as Stone-Wales defects.

The lateral dimension of graphene and its derivatives does not alter surface area but is relevant in cellular uptake, clearance and transport. Cellular uptake of graphene is poorly documented but is thought to be largely influenced by the lateral dimension. The subsequent size of the endosome or lysosome which forms to degrade and remove the graphene may become unstable and lyse open releasing digestive enzymes in the cell. The lateral dimension of graphene products can vary indefinitely from $10 \mathrm{~nm}$ to as large as the cells at $>20 \mu \mathrm{m}$ (Sanchez et al., 2012). The flat molecular structure which graphene provides makes it an ideal carrier of therapeutic agents providing a niche environment for stem cell growth and graphene also has the potential to be rolled end to end to form single walled carbon nanotubes. Graphene has recently been introduced to regenerative medicine with testing still in the early phases, however interesting results have already been recorded. Graphene and a modified derivative known as graphene oxide were compared against a glass surface to support the growth of induced pluripotent stem cells (iPSC). The authors reported the graphene surfaces provided a strong niche environment for the stem cells and induced distinct cell differentiation and proliferation. Secondly the authors reported that cell adhesion and proliferation occurred at a faster rate on the graphene oxide surface, these findings should introduce both graphene and graphene oxide into further regenerative medicine based research to culture iPSC to be used in medical applications (Chen et al., 2012a). The idea that graphene could be incorporated into the cellular scaffold to offer elastic and mechanical support while also acting as a selective barrier to growing tissues may come to change the outlook of regenerative medicine and related biological applications (Nair et al., 2012). With other regards to biomedical applications graphene boasts a large 
surface area onto which chemicals and therapeutic drugs could be conjugated for use in targeted drug delivery. Owing to the single atom thickness, mechanical strength and surface area availability of graphene, it may be an ideal candidate for imaging targeted therapeutics using scanning and transmission electron microscopy (Wojcik et al., 2015). Again due to the large surface area of graphene-based materials such as graphene oxide $\sim 736.6 \mathrm{~m}^{2} / \mathrm{g}$ the chemical functionalisation potential makes this nanomaterial ideal for use in biosensors which could detect cancer biomarkers, glucose or haemoglobin (Montes-Navajas et al., 2013, Kuila et al., 2011). Graphene as a carrier of biological compounds has tremendous potential, the hexagonal carbon lattice which makes up the structure of graphene means the $s p^{2}$ arrangement of the $\pi$ electrons are delocalised which improves the graphene solubility and ability to bind molecules efficiently (Novoselov et al., 2012). Further to this, graphene is lipophilic which helps to overcome a barrier in targeted drug delivery which is pin point delivery to the desired area. It also increases the chances of the therapeutic to negate the cell membrane and be internalised and released from the graphene carrier (Novoselov et al., 2012). Naturally when a novel nanomaterial makes several breakthroughs in the medical area attention soon turns to treating cancer. Graphene has been utilised in tumour studies performed on mouse xenograft models where polyethylene glycol-modified graphene oxide was administered intravenously. When excited with a near infrared laser the graphene oxide demonstrated strong photothermal cancer treatment potential as the tumours showed cell death via irradiation from the graphene oxide (GO) (Yang et al., 2010).

Graphene has major applications in electronic equipment largely due to its high thermal and conductive nature but also due to its flexibility. The nanotechnology industry is finding uses for graphene in nano-electronics, and photonics. Due to the high optical transmittance of graphene being over $90 \%$ it has the potential to be used as a transparent coating for touch screen technology (Novoselov et al., 2012). With high quality samples, the resistance per square area is $30 \Omega$ and with exceptionally high transmittance values exceeding $97 \%$ per layer the graphene meets both electrical and optical demands required to be utilised as a transparent conductive coating material (Nair et al., 2008). Largely, graphene is in competition for this technology with indium tin oxide (ITO) which expresses marginally better properties than graphene. Graphene production is increasing in quality and thus physical properties, this ultimately means graphene could replace ITO as a surface coating (Lux et al., 2018). With advantageous and superior flexibility and durability, graphene encompasses the role of modern nanotechnology in favour of conventional flexible electronics (Li et al., 2018a). As discussed, the overall structure of graphene provides exciting prospects in science, however an issue remains regarding the physico-chemical features of graphene which may present a risk to cells and 
tissues. Fibrous shaped nanomaterials seem to pose a more significant inflammatory risk to cells when compared to spherical nanoparticles (Braakhuis et al., 2014). As a result, graphene, like other nanomaterials, must be subjected to the same rigorous in vitro testing before clinical trials commence to provide occupational and consumer protection. All ENMs should be tested for durability over time, protein corona interactions and critically, downstream toxicity testing, covering the whole spectrum of potential biological damage (Oberdorster et al., 2005a). Weathered ENMs represent a unique hazard and should warrant extensive toxicology testing given they would possess dramatically altered bio-distribution, bio-persistence and potentially different dose-responses (Scott-Fordsmand et al., 2017).

\subsection{The Importance of Physico-Chemical Characterisation}

ENMs possess unique physical characteristics which vary with each material, these can include properties such as:

- Shape

- Size

- Surface functionalisation

- Composition

- Surface charge distribution

Each of these properties can alter a materials toxic potential however it is currently unknown to what extent each individual characteristic plays in contributing to toxic potential. A strong knowledge of the physio-chemical properties of an ENM plays an important role in understanding the toxic effects that may be witnessed in experimental conditions (Sukhanova et al., 2018). The in vitro techniques provide a mechanistic opportunity to test and analyse the biological pathways which may be affected by a toxic nanomaterial which can be quantified with cytotoxic proliferation assays such as relative population doubling (RPD) (Manshian et al., 2015). Furthermore, in vitro techniques offer the best option in maintaining full control of the experimental conditions (Oberdorster et al., 2005a). The purpose of characterising the nanomaterial may then help distinguish if the material itself is toxic or if the combined presence of a corona, endotoxin or surface chemistry plays a role in governing material toxicity. 


\subsubsection{Endotoxin}

Endotoxins (lipopolysaccharide, LPS) are thermo-stable molecules which make up the cell wall of Gram negative bacteria such as Escherichia coli (Doreswamy and Peden, 2011). Endotoxin is among many molecules which can efficiently bind to nanomaterial surfaces, the lipid moiety provides a unique opportunity to bind to hydrophobic planes. The presence of endotoxin either introduced in the manufacturing process or through handling in the laboratory contains phosphate groups which confer a net negative charge also allows binding to positively charged materials and proteins (Jones and Grainger, 2009). Endotoxin is able to bind to lipopolysaccharide binding protein (LBP) and engage tolllike receptor 4 (TLR4) to initiate cellular activation, commonly observed in monocytes when responding to bacterial infection (Lu et al., 2008). A variety of cytokines are produced which confirm the presence of endotoxin including IL-1 $\beta$ and IL-6. This can cause complications when testing nanomaterials like graphene, which is linked with causing the induction of both cytokines in past studies (Han et al., 2015, Mukherjee et al., 2018). GO has been linked with inducing TLR4-dependent necrosis in macrophages combined with a strong TNF- $\alpha$ response; the same inflammatory mechanisms seen with endotoxin exposure (Qu et al., 2013). These results indicate GO and endotoxin can initiate similar inflammatory responses making it difficult to attribute the observed response to the nanomaterial or endotoxin contamination. Identifying the presence of endotoxin is required to prevent misconceptions when interpreting experimental data regarding an inflammatory response from a cell line in vitro. While many manufacturers attempt to synthesise nanomaterials in as pure manner as possible, contamination with endotoxin can still occur while in the manufacturing process or during handling of the nanomaterial in the laboratory (Smulders et al., 2012). Several assays exist to identify the presence of endotoxin; however, many utilise colorimetric or fluorometric dyes, which is problematic for nanomaterials. Some nanomaterials have the potential to interfere with the dyes that are central to these assays (Doak et al., 2009, Griffiths et al., 2011). To navigate around these issues, there are other assays to determine the presence of endotoxin in a sample, the Limulus Amebocyte Lysate (LAL) assay for example. Addition of this lysate to a sample at an appropriate concentration yields a gel which identifies the presence of endotoxin with minimal interference from the nanomaterial. If the sample is free of endotoxin, then any subsequent claims of toxicity can be attributed to the nanomaterial pending the results of other physio-chemical characterisation such as protein corona and surface chemistry analyses. The data collected can then be used to facilitate a risk assessment by characterising the nanomaterial to better inform the nanotechnology industry (Braakhuis et al., 2014). If a nanomaterial is contaminated with endotoxin the removal of this molecule is paramount to avoid misinterpretation of in vitro toxicity results. Endotoxin as discussed above is highly resistant to heat but certain conditions can be applied for safe removal such as dry heat 
incineration at high temperatures exceeding $250^{\circ} \mathrm{C}$ (Nakata, 1993). One study has suggested endotoxin de-pyrogenation could be carried out through ethylene oxide treatment (Tsuji and Harrison, 1979). The latter suggestion however may carry increased risk as the ethylene oxide is itself highly reactive and may interfere with the physio-chemical nature of the nanomaterial under investigation.

\subsubsection{Size \& Shape}

As well as the presence of endotoxin in the sample, the size and stability of the nanomaterial in solution may also have implications in the overall toxicity to viable cells (Hole et al., 2013). Nanomaterials, particularly nanoparticles react to their surroundings in a size dependent manner due to their large surface area to volume ratio which is able to interact with the nearby cells (Jiang et al., 2008, Tenzer et al., 2011). The size of NPs has a great influence on cellular uptake \& subsequent alteration of, signal processes in viable cells, including the mechanisms relating to cell death. Particles in the size range of $2-100 \mathrm{~nm}$ possessed this ability but particles in the size range of $40-50 \mathrm{~nm}$ seemed to induce the strongest effects (Jiang et al., 2008, Kettler et al., 2016). Further to this there seems to be a trend in results published which indicate that ultrafine particles have the potential to penetrate deeper into body portals, such as the alveolar region of the lungs (Hoet et al., 2004, Li et al., 2016). In the study by Nemmar and colleagues, ultrafine nanoparticles were used to explore the link between thrombosis formation and exposure to $60 \mathrm{~nm}$ polystyrene NPs. The nanoparticles were divided into groups for comparison, but the surprising results came from the carboxylate-functionalised polystyrene particles and the amine-functionalised particles. The results concluded that at high doses the carboxylate-polystyrene particles significantly inhibited thrombus formation and the aminepolystyrene particles enhanced thrombus formation at high doses (Nemmar et al., 2002). This seems to strengthen the resolve for successfully characterising all aspects of new nanomaterials as aspects such as type, and surface functionalisation could cause drastically different effects. It is already well established that the size of nanomaterials has a great impact upon cellular processes including uptake, endocytosis and processing of particles inside the cell (Nel et al., 2006). NP size therefore plays an important role in cellular uptake; however, this is also true of nano-sized fibres and platelets. Studies on CNT deposition and uptake can often place a strong emphasis on size-dependent cellular responses, with the cell line commonly an immune cell which facilitates the studies focus on ENM uptake. Fibres have proven to be central to toxicity studies given their similarities to asbestos which has prompted toxicology research to inform the nanotechnology industry on the correct procedures for handling, use and disposal of these ENMs (Boyles et al., 2015). In the study by Boyles and 
colleagues (2015), industrially manufactured MWCNTs were compared to CNTs and asbestos fibres for their ability to promote cytotoxicity, (pro)-inflammatory responses and (pro)-fibrotic responses in macrophages and A549 cells. The authors reported long MWCNTs promoted a significant cytotoxicity and activation of an immune response through frustrated phagocytosis. CNTs (short) did not induce cytotoxicity in the cells however they were considered potentially toxic for stimulating detrimental cellular responses greater than those seen in macrophages exposed to asbestos fibres on a mass-dose metric (Boyles et al., 2015).

The shape of a nanomaterial may also play a large role in its toxic potential. Molecules with high aspect ratios i.e. >3:1 may cause issues with degradation and subsequent clearance from cells (Journeay et al., 2008). Long, thin nanomaterials such as graphene may possess this property which could induce strong inflammatory responses from immune cells such as macrophages and dendritic cells due to the inability of the immune cells to successfully degrade and remove the material from the cellular environment (frustrated phagocytosis). This was suspected to be the case in a study by Schinwald and colleagues, where graphene-based nanoplatelets of $25 \mu \mathrm{m}$ diameter induced inflammatory responses in an in vitro lung model (Figure 1.3). In vitro THP-1 macrophages were exposed to the graphene-based nanomaterial and showed expression of IL-8 and IL-1 $\beta$ among others in the bronchoalveolar lavage (BAL) (Schinwald et al., 2012). The authors suggest that the physical structure of the platelets proved difficult for the macrophages to carry out phagocytosis despite having a strong 2-dimensional profile. The authors deemed the graphene-based nanoplatelets as hazardous ENMs, imparting their toxicity through size and shape predominantly.

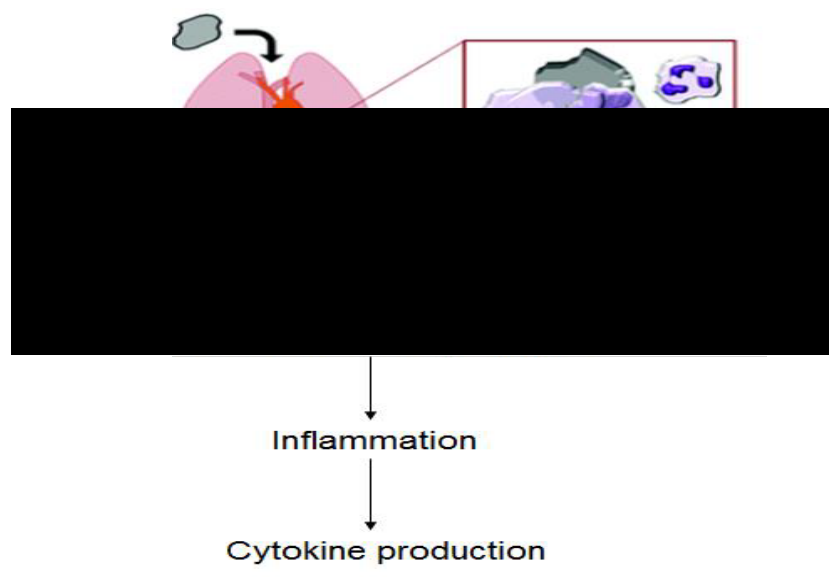

Figure 1.3 Graphene-based nanoplatelet induces frustrated phagocytosis in lung cells. This process may lead to the downstream immune response signalling and subsequent cytokine production. Image adapted from (Schinwald et al., 2012). 


\subsubsection{Surface Chemistry}

Modifying the surface chemistry of ENMs could prove to be an ingenious method of improving solubility, decreasing agglomeration or a means of drug delivery (Singh et al., 2009). In the case of graphene ENMs however, surface chemistry can play a pivotal role on toxicity. In the detailed review by Bussy and colleagues, the authors report that graphene bearing hydrophilic groups will disperse more readily (Bussy et al., 2013). The toxicity associated with these ENMs however often reflects the production methods used, this has been highlighted in the work by Chng and Pumera (2013), GO produced via top down manufacture was capable of a dose-dependent toxicity (measured with the MTT assay) and showed potential to promote mitochondrial stress (WST-8); this was reported for GO produced via four methods of manufacture (Chng and Pumera, 2013). Conversely, in the work by Bengtson and colleagues, who investigated the cytotoxic and genotoxic potential of graphene and GO in FE1 cells in vitro, the authors reported no cytotoxic or genotoxic events measured by proliferation and the comet assay respectively (Bengtson et al., 2016). There was however, a strong oxidative stress response promoted by $\mathrm{GO}$ which surpassed that of ROS induced by reduced $\mathrm{GO}(\mathrm{rGO})$ at doses up to $200 \mu \mathrm{g} / \mathrm{ml}$ over a 24 -hour exposure period. This effect was likely caused by the differing oxygencarbon ratios which Bengtson and colleagues also investigated where they detail $\mathrm{GO}$ having equimolar carbon, hydrogen and oxygen content with rGO having lower oxygen levels. This study may therefore hint at the importance of oxidation state on graphene ENMs, a simple yet obviously strong method of functionalisation via surface chemistry alterations. This effect has been evaluated in another study by Majeed and colleagues, whereby pristine graphene and GO ENMs had their surface oxygen content measured by X-ray photoelectron spectroscopy before assessing this effect on the toxicity of each material. The authors demonstrated pristine graphene promoted a dose-dependent cytotoxic trend which was not present with GO where the ENMs had surface oxygen contents of $2.5 \%$ and $31 \%$ respectively (Majeed et al., 2017). The role of oxidation upon graphene and GO ENMs is therefore highly specific and often dependent on the production methods used, the cell line, the cell culture environment, the oxidation state of the material, impurity content and many other chemically dynamic factors. These changes in oxidation state may also lead to changes in dispersion, solubility and the ability of proteins to adsorb to the surface of ENMs dispersed in cell culture medium.

\subsubsection{Protein Corona Formation}

In biological systems, a major component is fluid which primarily contains proteins. Once a nanomaterial is placed into this environment the outer surface can become coated with the fluid- 
based proteins to form a protein corona surrounding the nanomaterial (Figure 1.4) (Tenzer et al., 2011).

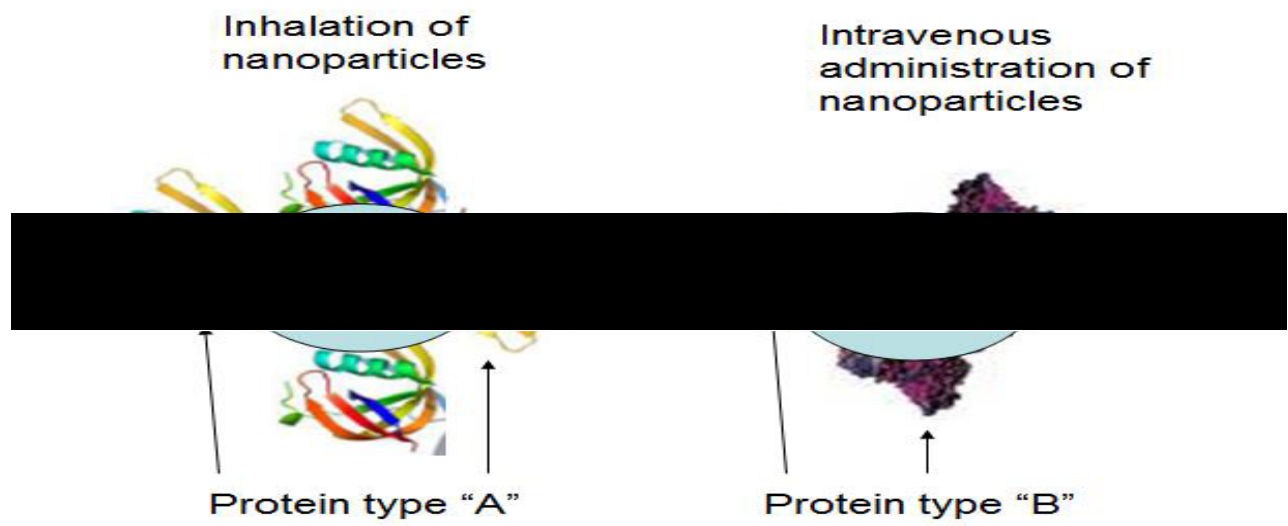

Figure 1.4 Differences in the corona formation on NPs depending on the site of administration. Protein adsorption may differ depending on the contact site and serum concentrations present in that specific region of tissue.

Understanding protein corona formation is of importance in nanotoxicology studies given its role in determining ENM physical properties (particle distribution \& surface charge) at biological interfaces (Foroozandeh and Aziz, 2015). The protein corona is a natural interface between a ENM and its biological environment (Monopoli et al., 2013). The resulting corona essentially instils a biological identity to the ENM which can be tested for using appropriate techniques. This can alter the ENMs biological interaction with cells and may have implications upon cellular uptake. The overall charge of the attached proteins can alter the uptake of ENMs, demonstrated by (Oh and Park, 2014). The authors demonstrated that polystyrene NPs of $100 \mathrm{~nm}$ in diameter underwent a higher rate of internalisation via phagocytosis if the particles were negatively charged as opposed to identical positively charged particles in human colon adenocarcinoma cells. Identification of the proteins contributing the NP corona is of interest in nanotoxicology. This can be achieved several ways however the most common being isolation via gel electrophoresis, trypsin digestion and peptide identification using inductively coupled mass spectrometry (ICP-MS) with size exclusion. This methodology provides accurate information regarding protein size (ascertained during gel electrophoresis) and peptide content (Fernandez-Iglesias and Bettmer, 2015). 
Gaining a thorough understanding of the protein corona may in fact help to inform the ENM internalisation pathways (Laurent et al., 2013). The numerous interactions of the ENM corona with the cellular may also be sufficient to trigger endocytosis and subsequently deliver the nanomaterial to the cell interior. Physiological systems tend to be in a strong dynamic state with biological interactions occurring constantly, it therefore follows that a regular time profile of corona formation and weathering should be taken into account in order to accurately predict ENM toxicity (Tenzer et al., 2013). The protein corona formation however is less documented despite being as important as its physiological presence, which is prompting future studies to investigate this phenomena to improve the current knowledge of these nanoparticle-corona complexes and how they contribute to inflammatory cellular responses (Monopoli et al., 2013). ENMs have a highly dynamic surface area onto which biological molecules adsorb easily when in contact with a biological interface (Nel et al., 2009). Rapid corona formation (<30s) was shown to affect NP uptake and endothelial cell death at early exposure times (Tenzer et al., 2013). The cellular interactions may have bio-adverse outcomes if the nanomaterial corona induces transformations or restructuring of the nanomaterial while inside the cell. To what extent the corona nanomaterials affects toxicity is yet to be confirmed and perturbing techniques may alter the chemistry of the system so efforts are underway to find non-perturbing means of monitoring the protein corona of nanomaterials ( $\mathrm{Nel}$ et al., 2009). Alterations to the corona may disturb the distribution of the nanomaterial and consequently could change the therapeutic or physiological influence it has on cells (Dobrovolskaia et al., 2009). Currently it is believed that the hard protein corona develops and evolves slowly and consists of an abundant number of proteins, albumin being the most common. The abundance of albumin at the surface of ENMs immersed in biological samples may indicate good biocompatibility of the nanomaterial in cell cultures primarily due to the proteins found naturally masking the foreign nature of any novel ENM (Casals et al., 2010). The soft corona by comparison is made up of highly interchangeable proteins which can alter within a space of 30 seconds. Logically it seems that the hard protein corona would be more responsible for important cellular functions relating to the nanomaterial such as internalisation while the interchangeable soft corona would undergo modification through the uptake process and become altered once inside a cell for example. In the paper published by Tenzer and colleagues a list of 20 of the most common corona proteins is listed, from there researching the isoelectric and kinetics of the proteins could yield helpful information for predicting corona formation in future experiments (Tenzer et al., 2013). The authors also dispute the hypothesis, in which ENM coronas are predicted to be positively charged to aid in the docking mechanisms with cell membranes (Huhn et al., 2013). Based on the perturbing methodology regarding the biological environments, the particle corona seems to be negatively charged predominantly which suggests that attractive force docking may be a simplified notion of the true 
mechanism. The commonality of apolipoproteins in the corona may also facilitate the uptake of nanoparticles into endothelial cells which has been witnessed in a number of studies (Zensi et al., 2010, Zensi et al., 2009). The study by Foroozandeh and Aziz, demonstrated a difference in the proteins that adsorb to the NP surface may vary depending on the route of administration. Inhaled nanoparticles may therefore inherit a different corona compared to NPs which are injected (Foroozandeh and Aziz, 2015). Injected nanoparticles which enter the bloodstream are likely to interact with blood plasma proteins which will comprise most of the corona in the early stages of its formation (Cedervall et al., 2007). Blood plasma proteins such as albumin, fibrinogen, and immunoglobulin $\mathrm{G}$ are highly common corona proteins and will likely be found on all nanoparticles which are suspended or dispersed in plasma (Aggarwal et al., 2009). The difference in proteins is likely caused by the initial biological interface the nanomaterial encounters, the lung environment may contain lower concentrations of blood plasma proteins such as fibrinogen and higher concentrations of glycoprotein for example which contributes to mucus production acting as a barrier to pathogens (Allen et al., 1984). The goal while performing experiments however is not simply to identify the presence of the corona but to quantify it. Techniques are now being sought out to measure the thickness, protein quantity and binding affinities of these proteins to nanomaterials (Walkey and Chan, 2012). As discussed above, the corona evolves over time as the proteins of the soft corona with lower binding affinity freely adsorb and release while the hard corona remains largely unchanged; methods are therefore now available to measure the exchange rates of the proteins. The hard corona should possess a slow exchange rate in comparison with the soft corona when dispersed in media. The reason behind this is now thought to be due to the proteins of the hard corona binding with high affinity directly to the nanomaterial surface while the proteins of the soft corona are relying on proteinprotein interaction to remain bound. This was confirmed in a recent study by Kokkinopoulou and colleagues (2017) who demonstrated for the first time, visualisation of the protein corona by TEM. The authors noted the hard protein corona measuring $~ 15 \mathrm{~nm}$ was strongly influenced by the host ENM surface chemistry. Further proteomic analysis revealed the outer corona (soft bound proteins) to be a loose, flowing network of proteins not the classical image of a protein shell (Kokkinopoulou et al., 2017). Consequently there are exchanges with other proteins dispersed in media or where ever the nanomaterial may be (Walkey and Chan, 2012). A theory put forward by Simberg and colleagues suggests that the initial proteins to adsorb to a nanomaterial surface can be referred to as primary binders which are then followed by secondary binders which bind through protein-protein interactions to the primary binders (Simberg et al., 2009). This concept could have large implications on the nanomaterial overall physio-chemical characteristics such as receptor binding to membranes. The secondary binders could essentially mask the affinities of the nanomaterial and the primary 
binders for the cellular membrane (Foroozandeh and Aziz, 2015). ENM surface chemistry will also likely impact upon biological recognition and significance once inside cells. More proteins are able to efficiently adsorb onto the surface of hydrophobic nanoparticles as opposed to hydrophilic ones, because of this the native structure of proteins adsorbing onto the hydrophobic surface will lose some of their functionality as they undergo denaturation (Roach et al., 2005).

\subsubsection{Agglomeration}

ENM agglomerates are an amalgam or group of singular ENMs bound together by van der Waals forces or electrostatic interactions involving dipoles at the NM surface. Agglomerates of ENMs should not be mistaken for a ENM aggregate which comprises strongly interacting singular NMs held together through sharing of electrons in a covalent bond or overlapping electron orbitals as in the case of metallic interactions (Baalousha, 2009). The formation of ENM agglomerates is generally an unwanted process however an inevitable one particularly where ENMs are concerned. ENM manufacture typically results in hydrophobic materials, graphene for example, and to enhance solubility to achieve the desired function coatings and chemical groups can be added (Yang et al., 2012). Agglomerate formation of ENMs can be achieved within a 30 minute time frame after which further agglomeration results in structures achieving micron-sized agglomerates ultimately causing sedimentation, an extreme issue in nanotoxicology which is often highlighted in publications as timed photographs of ENM stock solution over a 24-hour period (Hsiao and Huang, 2013). Nanotoxicology focuses on the impact of ENMs upon a specific cell line intended to model human exposure however most ENMs manufactured as a powder are hydrophobic and thus require a liquid carrier acting as the suspension (Ruge et al., 2012). As a result, in vitro nanotoxicology suspends dry powder ENMs in culture media to expose to cells in most liquid-liquid interface (LLI), a better approach however would be to nebulise the ENM and grow cells as an air-liquid interface (ALI) thus creating a more realistic approach, where the lung model is concerned.

\subsubsection{Nanomaterial Characterisation Techniques}

ENM characterisation requires an extensive approach and typically includes multiple techniques. Furthermore, techniques can be applied to pristine and suspended materials prompting a comparison to be drawn between the changes of ENMs immediately post manufacture and a 'weathered' ENM which has been suspended in culture media and undergone physico-chemical changes. 


\subsubsection{Dynamic Light Scattering (DLS)}

Perhaps the most common form of ENM characterisation is DLS, a powerful technique used to ascertain hydrodynamic diameter, agglomerate size distribution and the polydispersity index of a sample. The DLS principle is based upon Brownian Motion, a thermal process whereby molecules in a solution will randomly bump into a suspended ENM inducing a small but perceivable change in location of the ENM itself (Bendre et al., 2011). The Brownian Motion of larger agglomerates of ENMs will therefore be less when compared to smaller agglomerates; on this principle DLS calculates autocorrelation functions on the light-scattering signal of the solution (Nazarenus et al., 2014). Essentially DLS passes a laser of specific wavelength through the suspension and calculates the rate at which the intensity of light fluctuates. Correlation coefficients are crucial here, the non-random fluctuations are represented and plotted over time generating a correlation curve. This curve calculates a hydrodynamic diameter using the Stoke-Einstein Equation, whereby the hydrodynamic diameter can be defined as the diameter of a hypothetical sphere which possesses the same translational diffusion coefficient as the particle under analysis. In principle, larger hydrodynamic diameter scatter light much more than smaller agglomerates which means the size distribution will significantly depend on whether the intensity or number is displayed in publications, thus taking the mean hydrodynamic diameter is simply not sufficient with DLS (Nazarenus et al., 2014). DLS does however have limitations; firstly, the refractive index, suspension viscosity of the dispersant will need to be known beforehand - this is not always readily available and if incorrect can contribute to data skew. Supplemented protein in the suspension which is vital to nanotoxicology can often 'redshift' the average hydrodynamic diameter through the masking of the smallest agglomerates. DLS however suffers from the process of Rayleigh scattering, this unwanted electromagnetic process manifests as larger agglomerates masking the light scatter of smaller agglomerates which results in the data being skewed. Where DLS will come under scrutiny is ENM selection, as this principle works best for spherical particles. In contrast, platelets, cuboids, fibres, and flake-like ENMs will confound DLS to some degree due to the scattering angle fluctuations, consequently a more holistic and multi-modal approach is required for those ENMs.

\subsubsection{Brunauer Emmett Teller (BET) Theory}

The technique of BET measures the adsorption of gas (nitrogen) onto the surface of a material which includes porous structures. The volume of nitrogen gas adsorbed to the surface of the material is often measured accordingly with the boiling point of nitrogen $\left(-196^{\circ} \mathrm{C}\right)$, the calculation then utilises BET theory and the BET adsorption isotherm equation (Equation 1.1). Essentially the dissolution rate is 
determined which is equivalent to the surface area of the material. Traditionally nitrogen is used as the adsorbate gas however substitutes can be used permitting they are also relatively inert.

\section{Equation 1.1}

$1 /\left[\mathrm{V}_{\mathrm{a}} \cdot(\mathrm{P} / \mathrm{P}-1)\right]=\mathrm{C}-1 / \mathrm{V}_{\mathrm{m}} \mathrm{C} \cdot \mathrm{P} / \mathrm{P} 0+1 / \mathrm{V}_{\mathrm{m}} \mathrm{C}$

Where; $\mathrm{P}$ is the partial vapour pressure of adsorbate gas, $\mathrm{P}_{0}$ is the saturated pressure of gas, $\mathrm{V}_{\mathrm{a}}$ represents the volume of gas adsorbed at standard pressure and temperature $\left(273.15 \mathrm{~K}\right.$ and $1.013 \times 10^{5}$ $\mathrm{Pa}), \mathrm{V}_{\mathrm{m}}$ is the volume of gas adsorbed at standard temperature and pressure resulting in a surface monolayer and lastly $C$, an enthalpy of adsorption constant.

\subsubsection{Raman Spectroscopy}

Raman spectroscopy is based on the principle of monochromatic light scattering upon contact with a sample surface. It presents a useful, non-destructive methodology of investigating material thickness (Ni et al., 2007). A laser or light source of specific wavelength is fired at the sample of interest and the resulting wavelength of the scattered light is measured (Figure 1.5). This principle of Raman-scattered light can then be used to predict structural information regarding a certain material or sample (Figure 1.6). For this reason, Raman spectroscopy has become a fundamental tool for characterising structure and shape for cells, molecules (Ramos et al., 2015) and also ENMs such as graphene (Shtein et al., 2015). Raman spectroscopy is therefore not an absorption process; the light scattered by atoms is shifted in frequency by differences in the vibrational energy levels (Figure 1.6).
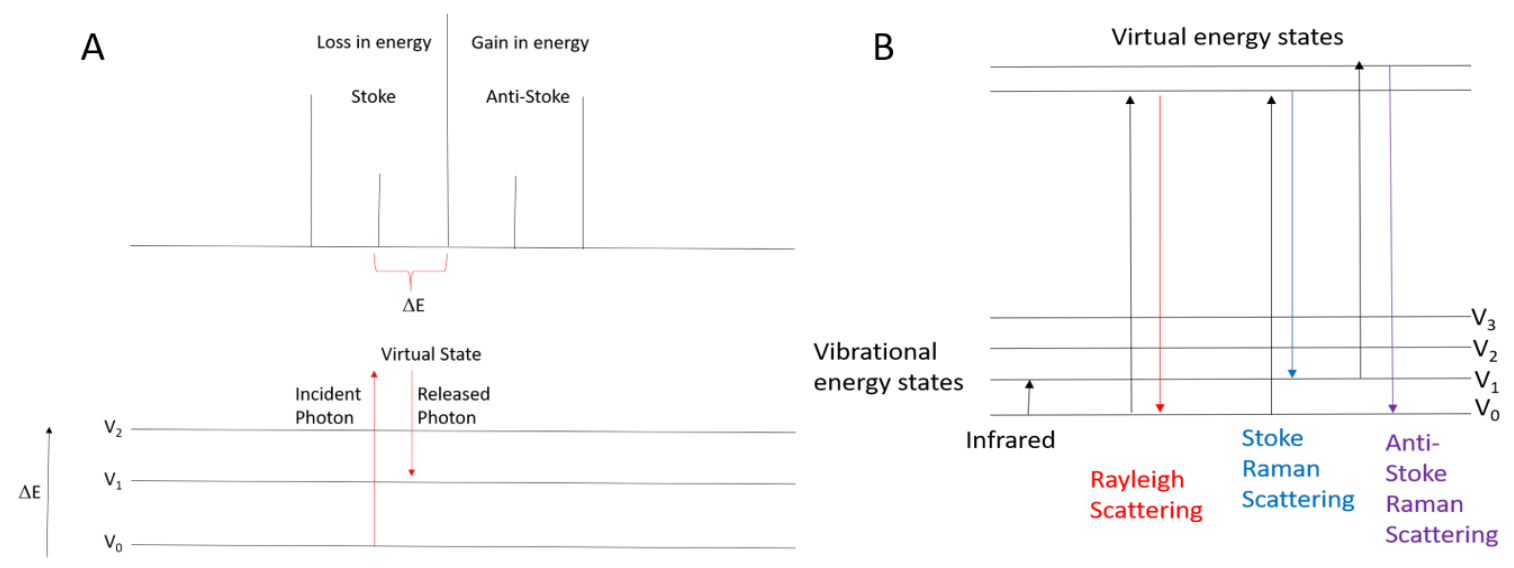

Figure 1.5 Raman Spectroscopy theory. Light enters with an oscillating transverse electromagnetic field contacting the sample and induces oscillations in the electron cloud of the sample. If the light which enters possesses a higher energy than the emitted light, this will manifest as a Stoke shift. 


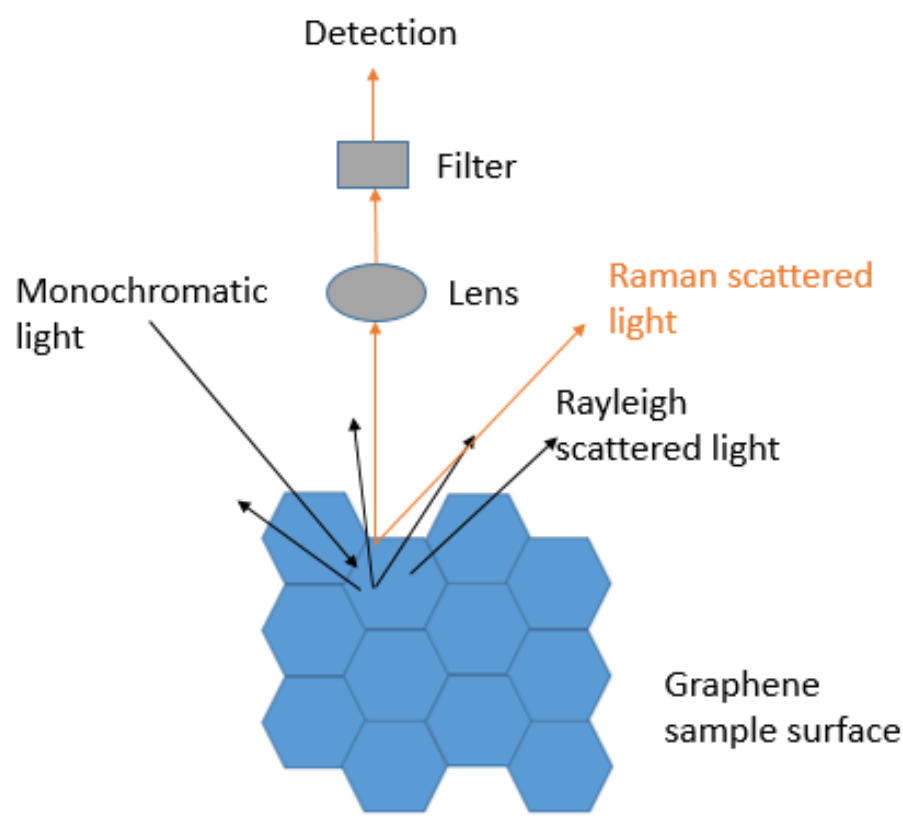

Figure 1.6 The principle for Raman spectroscopy focuses on the scattering of monochromatic light. The Rayleigh light scatter will have the same wavelength of light as the incident beam whereas the Raman-shifted light will have a longer wavelength and consequently a lower energy value. Due to the random direction of light scatter the longer wavelengths of light have a higher probability of reaching the lens and being detected which in this example will be the Raman-shifted light.

Raman spectroscopy has been applied to graphene analysis by determining the number of substrate layers and configuration of the stacked 2-D material under experimental conditions (Li et al., 2012). This technique holds high sensitivity and once coupled with the relative thickness of graphene samples (i.e. 3-5 layers) this technique becomes highly reproducible and accurate in determining the layer thickness of stacked nanomaterials (Li et al., 2012). To determine the thickness of FLG Raman spectroscopy can be employed in 2-D mode to visualise the spatial distribution of Bernal and rhombohedral stacking in tri and tetra-layer graphene (Lui et al., 2011). Using Raman spectroscopy individual carbon planes can be visually mapped to determine whether the planes are stacked "in" or stacked "out". In this particular form of analysis the Raman spectroscopy can be coupled with transmission electron microscopy (TEM) as a means of transferring the platelets of FLG samples onto a TEM grid resulting in a far more powerful analytical tool (Cong et al., 2013). When analysing single layer graphene, the orientation of the edges can also be characterised by Raman spectroscopy in $G$ mode. With this analysis Cong and colleagues demonstrated that the edges of the graphene single layer exhibited polar behaviour and differed in configuration according to the polarisation of the 
incident laser (Cong et al., 2010). The spectral peaks obtained from carrying out Raman provide an instant indicator as to whether the graphene sample is a single layer or stacked, by taking a scanning confocal approach Graf and colleagues were able to distinguish between bilayer and few-layer graphene samples from the dimensions of the " $D$ " band on the spectra. If a bilayer of graphene is abundant the $D$ band splits from a single peak into multiple peaks, the intensity of the peak also provides material defect information (Graf et al., 2007).

\subsubsection{Transmission Electron Microscopy}

TEM has been making crucial contributions to the field of biology for over 50 years (Winey et al., 2014). The technique involves passing electrons through a thin section of embedded cells to provide a detailed image with incredibly high resolution. The TEM is setup with four main sections, the electron source, an electromagnetic lens, the sample holder and lastly the imaging system, as illustrated in Figure 1.7.

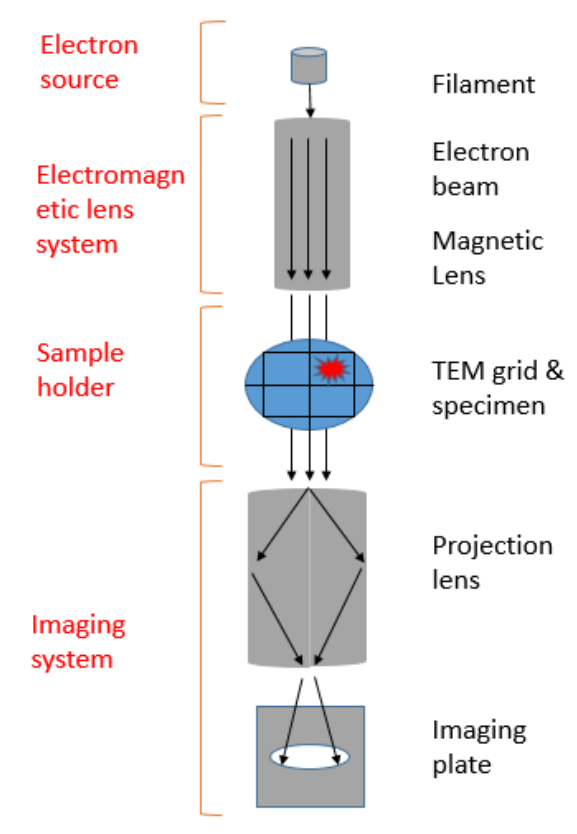

Figure 1.7 Schematic illustration of the TEM setup. The filament electron source consists of a tungsten cathode which emits electrons once heated, the electrons are then accelerated towards the specimen through the anode which acts to focus the beam directly at the sample. The electromagnetic lens meanwhile focuses the beam so that each electron only possesses a well-defined energy range. Lastly the imaging system consists of a double-lens system, one for refocusing electrons and the second for enlarging the TEM image on the screen. 
Due to the short wavelength of the electron beam with respect to high energy photons, the resolution encompasses the sub-nm range (Winey et al., 2014). The sample preparation for TEM is a laborious process which involves chemical fixation with osmium typically which acts to confer some electron density to the sample membrane. Sample dehydration is then employed utilising a solvent of choice at increasing concentrations, ethanol or acetone are common. Once the sample is sufficiently dehydrated it is then embedded in resin for sectioning. To provide an image in the microscope the sections cut need to be between $60-80 \mathrm{~nm}$ thick which are harvested onto TEM grids (Winey et al., 2014). Given TEM images are high resolution, this technique is often the gold standard to investigate nanomaterial uptake and internalisation within various cell lines. Current advances in the technique permit image capture with resolution as precise as $0.11 \mathrm{~nm}$, however image resolution typically reflects sample quality (Gai and Boyes, 2009). Graphene and few layer graphene has been imaged using TEM to investigate theoretically-predicted ripples in the lattice structure which could be a source of material fractures when under high stress (Wang et al., 2012). Using tilting TEM experiments the edges of single layer graphene and bilayer graphene can be successfully characterised as either zigzag or armchair configurations (Figure 1.8) and that the edges are mostly closed between layers (Liu et al., 2009). The closed edges of the stacked material can be visualised as a curved edge as opposed to multiple stacked sheets which lay adjacent to one another (Liu et al., 2009). From the study by Wang and colleagues, the authors concluded that such ripples may always exist in ultra-thin materials for which TEM should provide a successful detection system. Since graphene uptake has been proven, as in the study by Mullick Chowdhury and colleagues with TEM images in several cell lines including HeLa cells and MCF7 cells at concentrations as low as $20 \mu \mathrm{g} / \mathrm{ml}$ (Mullick Chowdhury et al., 2013).

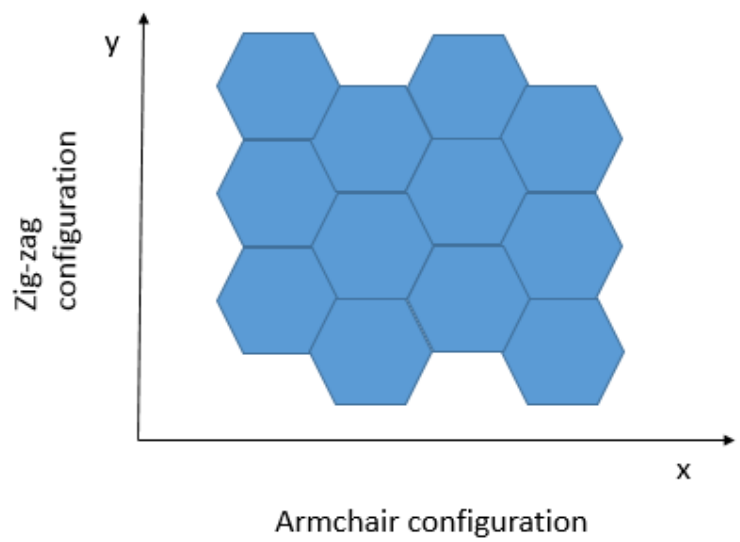

Figure 1.8 Illustration of the two possible edge characteristics displayed by graphene. Typically, along one edge of the material there will be the armchair arrangement while the adjacent axis will take on the zig-zag arrangement of carbon atoms. 


\subsubsection{Atomic Force Microscopy (AFM)}

AFM, sometimes referred to as scanning force microscopy (SFM), operates by moving a small, needlelike tip (fixed to a cantilever) across a sample's surface and measuring the resulting attractive and repulsive forces (Robson et al., 2018). AFM is currently an irreplaceable technique for inspecting the topographical nature of materials as well as analysing physical structures (de Pablo, 2011). The advantages of this technique are that it can be applied to a wide number of nanomaterials, particles, spheroids and liquids alike (Baalousha and Lead, 2012). A particular advantage of AFM over traditional electron microscopy is the capacity to measure samples in air, vacuum or liquid (de Pablo, 2011). This key feature of analysing biological samples while in liquid provides a useful insight into replicating natural biological environments while under experimental conditions. The high resolution of this technique permits inspection of ENM interactions with cells and investigate potential accumulation points at the cell surface, which crucially, can be conducted on live cells (Taatjes et al., 2013). The process by which AFM operates is based on the adhesion forces between the sample surface and the probe tip of the cantilever (Trache and Meininger, 2008). The probe tip is positioned above the sample surface (Figure 1.9) while the configuration can take up one of three imaging settings, contact, tapping or non-contact mode. While in contact mode the probe tip is dragged along the sample surface, the deflections of the cantilever are detected, and an image is formed regarding the surface topography. Commonly however a constant feedback signal is applied to maintain the probe tip position and any alteration indicated results in a deflection of the cantilever. Currently the only limitation with this form of AFM is imaging rate which can be laborious, however new high speed AFM machines can combat this issue (Casuso et al., 2009). 


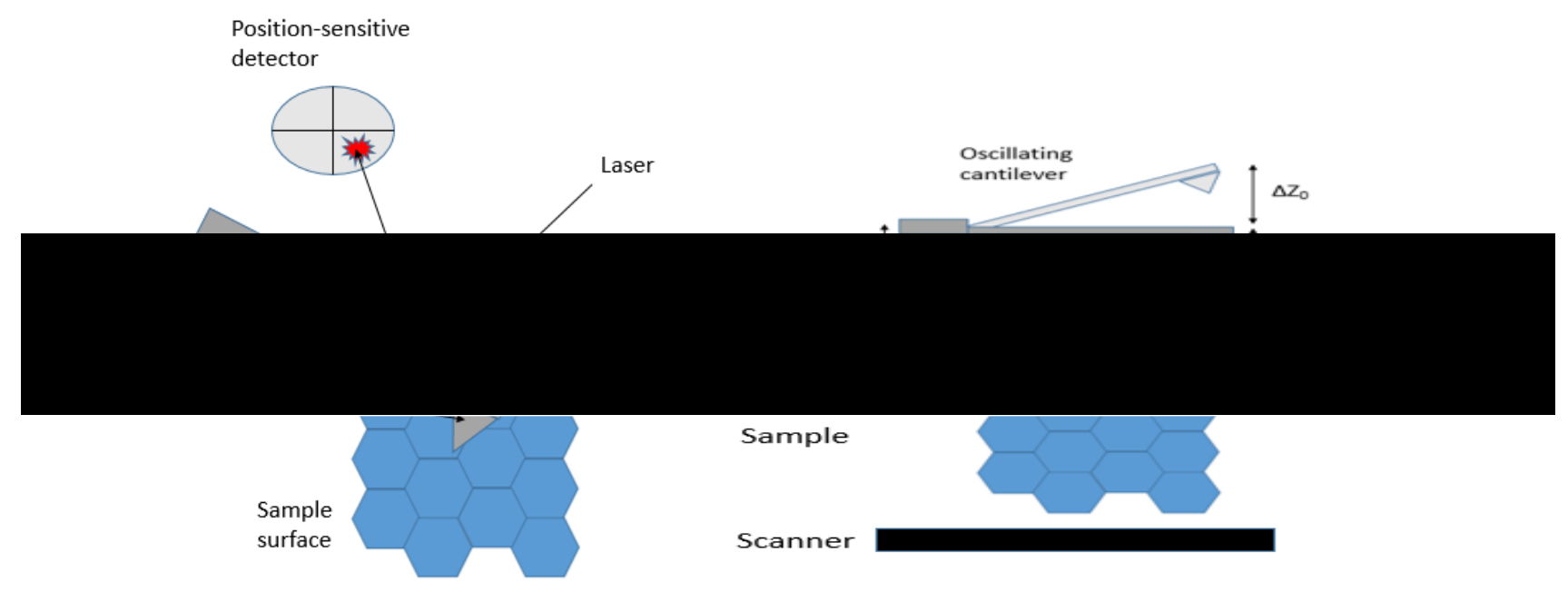

Figure 1.9 Contact-mode AFM setup. The piezo-ceramic cantilever is first positioned with an applied voltage, the probe is then moved across the surface of the material to create a 3D representation of the material surface. Cantilever dynamics in tapping mode are shown where $D_{0}$ is the resting cantilever position, secondly, $Z_{0}$ is the position of the cantilever with respect to the sample surface and lastly $\Delta Z_{0}$ is the amplitude of the cantilever, image adapted from (Legleiter, 2009).

Tapping mode can be employed typically for use on liquid samples where contact mode may be hampered by adhesion forces of the liquid medium. Tapping mode therefore reduces the time the probe tip is in contact with the liquid medium thus providing a more accurate representation of the biological sample. In tapping mode the cantilever is encouraged to oscillate near the resonant frequency (Figure 1.10), the amplitude of the oscillations can vary (10-40nm) (Legleiter, 2009). The resolution of tapping mode AFM on protein surfaces at the vertical axis can be as small as $0.1 \mathrm{~nm}$ while lateral dimensions can lie in the range of 1-1.5nm (Moller et al., 1999). The resolution of modern AFM sample analysis can reach sub-nm levels however this is typically found in liquid samples (Miller et al., 2016). The primary advantage to tapping mode as opposed to contact mode is the reduced contact of the probe tip with the sample surface. This feature both elongates the shelf life of the probe tip and is minimally intrusive to the sample which, if biological, shouldn't introduce structural damage. Tapping mode is therefore the preferred methodology for analysing delicate structures such as membranes, fluid samples and most recently liposomes (Roiter and Minko, 2005, Robson et al., 2018). Additionally, by using a less perturbing method of AFM for single molecule analysis the sample is likely to remain stable for longer, whereas full contact mode may introduce structural defects into the honeycomb crystal lattice of graphene. Finally, there is the non-contact mode of AFM in which the probe tip is never in contact with the sample surface. In this form of sample analysis the probe tip is 
again oscillated at near the resonant frequency slightly above the sample at a distance of $<10 \mathrm{~nm}$ typically (Gross et al., 2009, Altman et al., 2015). From here the van der Waals forces influence the cantilever oscillation through attractive forces, the subsequent decrease in the resonant frequency is measured by the software and accounted for when constructing the sample image. Non-contact AFM therefore remains a high-ranking technique for characterising graphene surface topography, mechanical and physical properties in a non-perturbing approach, it is currently the method of choice for imaging large scale productions of defect-free graphene (Paton et al., 2014).

\subsection{Inherent Toxicity Associated with ENMs}

Nanotoxicology has become a necessity due to the increasing commercial demand for ENMs and as a result the current understanding of the potential hazards surrounding the use of ENMs has improved dramatically (Doak and Pfuhler, 2012). Many ENMs differ greatly to their bulk counterpart (micronsized materials), gold nanoparticles for example possess enhanced conductivity and optical properties at the nano scale (Huang et al., 2007). The nano counterparts of bulk materials such as gold and silver are being sought out for their exceptional conductive and highly sensitive nature ( $\mathrm{Nel}$ et al., 2006). ENM production is set to increase as the demand for novel therapeutics and replacement nanotechnology increases (Ramachandraiah et al., 2015). Consequently, the requirement for human health impact (safety) testing is paramount and one quick and extensively used model in nanotoxicology is the in vitro monoculture system (Clift et al., 2014). A preferred choice over in vivo models which are more expensive, complex, time and labour consuming, and ethically demanding, the in vitro system therefore provides a strong knowledge foundation on which to build upon when screening the human health and environmental impact of ENMs.

The need for holistic toxicology on ENMs can sometimes be overlooked given the lack of toxic effects of the bulk material which crucially, do not possess the same properties (Franklin et al., 2007). Given the risk associated with ENMs, which was significantly highlighted when CNTs were under scrutiny due to their similarities to Asbestos (Boyles et al., 2015), the nanotechnology industry has relied on nanotoxicology research to inform industrial, occupational and consumer nano-safety. Natural airborne nano-sized particles such as $C B$ have been in the atmosphere and exposed to humans since the dawn of fire however there is now increasing exposure with the demand for ENMs (Oberdorster et al., 2005b). Much of the data surrounding nanotoxicology has been collected from in vitro studies where a number of valuable assays can be employed to analyse cellular damage (Arora et al., 2012). 
Due to the decreased desire to conduct animal testing, the results from in vitro experiments are often extrapolated to in vivo models with the use of pharmacokinetic studies performed in silico (Krewski et al., 2010). The importance of thorough genotoxic testing in toxicology is profound, especially with the increased number of ENMs being proposed in future technologies where human exposure will become increasingly common. Furthermore, discovering DNA damage early on during in vitro exposures could provide vital information on the potential of ENMs to induce carcinogenesis.

\subsection{Nano(geno)toxicology}

Genotoxicity plays a significant role in novel chemical, pharmaceutical and nanomaterial (among others) research and manufacture on a global scale. The identification of ENMs which are genotoxic is of vital importance given that if misused could potentially promote carcinogenesis. This becomes a greater concern when considering carcinogenesis has been shown to stem from chronic oxidative stress and chronic inflammation (Morgillo et al., 2018). These two contributing factors are strongly associated with ENMs in the literature. Consequently, ENMs must undergo strict hazard testing before they can be targeted for mass production in biomedical, electrical or physical applications, as is the case for any other novel chemical or product destined for the market-place. Genotoxicity testing aims to identify and quantify DNA damage induced following exposure to an exogenous agent and requires a battery of assays that evaluate the different forms of damage that may arise specifically point mutations and/or, chromosomal damage. Measuring the genotoxicity of a material requires strict protocols for both in vitro and in vivo testing using a battery of tests which must be suitably adaptable to encompass ENMs (Elespuru et al., 2018). The role of utilising multiple assays not only covers all forms of DNA damage that may arise, but also eliminates the occurrence of mis-leading positive/negative results.

The exposure risk to humans and the environment therefore requires constant monitoring and extensive testing, of which genotoxic testing is of vital importance. Given that ENMs possess highly unique features, their nano-safety evaluations present challenges. There are a panel of in vitro genotoxicity tests currently in use including the HPRT and micronucleus assays which play a crucial role in determining the risk associated with ENMs by quantifying DNA damage; however these assays require adaptation to incorporate ENM assessment (Doak et al., 2012b). Consequently, the Genetic Toxicology Technical Committee of the International Life Sciences Institute's Health and Environmental Sciences Institute has addressed potential sources for improvement and/or adaptation 
to the current ENM genotoxic assessment criteria (Elespuru et al., 2018). As no single genotoxicity test exists which could assess all areas of DNA damage, a panel of tests is required. In the review by Elespuru and colleagues (2018) the authors highlight a collection of common tests employed in genotoxicology including; the in vitro Ames test, chromosomal aberrations and the micronucleus assay and, in vivo tests such as the transgenic mutation assays. The authors reported a substantial methodological variation when the tests were applied to the evaluation of ENMs, many of which were not upstanding to the criteria required for valid genetic toxicology testing (Elespuru et al., 2018). Thus, owing to the highly specialised physio-chemical characterisation of ENMs they must be treated differently to chemical compounds, and where necessary, specific adaptations to standard test methods are required to ensure the output of hazard-based assays are relevant (Doak et al., 2009, Doak et al., 2012b, Elespuru et al., 2018).

\subsubsection{Primary \& Secondary Genotoxicity}

Mechanisms underlying the genotoxicity of ENMs can be divided up into two classes, primary and secondary. Primary genotoxicity describes the mechanisms of genotoxicity imparted the single cell level, this requires ENM internalisation and subsequent biomolecular interactions. Secondary genotoxic damage however involves the recruitment of macrophages or neutrophils (in vivo) to promote ROS production resulting in a chronic inflammatory response (Doak et al., 2012a). One example of a common oxidative radical being superoxide, a highly reactive oxygen species commonly related to an ENMs influence on toxicity (Schins and Knaapen, 2007). Standard in vitro culture systems only support the identification of genotoxicity induced by primary mechanisms, as typically they are based on the culture of a single cell-type. Only the construction of more relevant in vitro culture systems incorporating multiple cell types (at minimum an epithelial and inflammatory cell line) could potentially result in the identification of secondary mechanisms of genotoxicity in vitro such as the release of (pro)-inflammatory mediators and oxygen-centred radicals.

Primary genotoxicity can in fact be further classified into direct-primary genotoxic damage and indirect-primary genotoxic damage (Scassellati Sforzolini et al., 2004). Direct-primary involves the exogenous agent inducing DNA damage through direct physical interaction with the DNA molecule itself. GO for example has been shown to induce DNA cleavage through generation of free radicals during in vitro studies (Ren et al., 2010). Further to this, evidence from fluorescence spectroscopy and thermal DNA denaturation confirmed the GO could intercalate into DNA molecules and when 
combined with copper ions, induce DNA scission (Ren et al., 2010). In contrast, indirect-primary genotoxicity is caused by the exogenous agent damaging key molecules involved in DNA replication and/or fidelity; for example, DNA repair enzymes, replication machinery, cell cycle proteins or the spindle apparatus. Indirect-primary damage can also be induced via mitochondrial damage as well as ROS production, two factors that contribute to oxidative DNA damage. Indirect mechanisms have been studied sparsely with CNTs which have been linked with the depletion of glutathione, a key antioxidant that protects cellular DNA from oxidative damage (Liu et al., 2011). Liu and colleagues suggested that the large surface area of the CNTs and GO contributed to the oxidant: antioxidant imbalance which depleted glutathione levels. It was proposed that dioxygen was reacting with active sites in the graphitic surfaces resulting in modifications which then reacted heterogeneously with the glutathione (Liu et al., 2011). Other indirect mechanisms of primary genotoxic damage include depletion of the mitochondrial membrane potential releasing ROS (Bhattacharjee et al., 2012). NADPH oxidase-ENM interactions have also been investigated (Frohlich, 2013). Finally, damage to DNA repair proteins has been investigated by $\mathrm{Qu}$ and colleagues as well as damage to cell cycle proteins. Furthermore cytoskeletal damage which may expose regions of DNA to excessive stress from oxidative damage has been explored with fluorescence microscopy through the tagging of specific proteins associated with cytoskeleton formation and recruitment (Qu et al., 2013). ENMs may have their surfaces modified through the addition of chemical groups, like polyethylene glycol (PEG) or simple chemical groups such as amine functionalisation. These modifications may be used to enhance biological compatibility, to aid the intended industrial/medical application or for added efficacy, or may be a source of highly energetic radicals (Duch et al., 2011, Subbiah et al., 2010, Fenoglio et al., 2006).

Secondary genotoxicity involves a chronic immune response followed by; immune cell activation, an increased flux of (pro)-inflammatory mediators and then increased ROS production which culminates in oxidative DNA damage (Elespuru et al., 2018). In a study by Bengtson and colleagues, GO and reduced graphene oxide ( $>2$ layers thick) were exposed via intratracheal administration in mice to assess the pulmonary inflammation, acute phase response - a biomarker for cardiovascular disease and genotoxicity. The toxicity induced in the mice was assessed over; 1, 3, 28 and 90 days at 18, 54 and $128 \mu \mathrm{g} / \mathrm{mouse}$. Genotoxicity was assessed in BAL cells, liver and the lung whereby DNA strand breaks were investigated using the comet assay which found that GO induced significant DNA damage in bronchoalveolar lavage (BAL) cells as early as day 3 and 28 at $18 \mu \mathrm{g} / \mathrm{mouse}$. Secondly, it was reported that rGO induced significant DNA damage at the same dose of $18 \mu \mathrm{g} / \mathrm{mouse}$ at days 29 and 90 compared to vehicle control. Interestingly, no significant DNA damage was reported in lung and liver 
following exposure to $\mathrm{GO}$ and $\mathrm{rGO}$, however there was significant inflammation, activation of the acute phase but no induction of fibrosis (Bengtson et al., 2017). In vivo CB particle exposures have also been performed in mice, as in the study by Bourdon and colleagues in 2012. Here, the authors also investigated inflammation, acute phase responses, DNA strand breaks and oxidative stress in C57BL/6 mice over 28 days with an exposure dose of $0.018,0.054$, or $0.162 \mathrm{mg}$ of Printex 90 carbon black nanoparticles (CBNPs). The measure of genotoxicity was characterised by strand breaks in BAL cells, lung and liver using the alkaline comet assay whilst Formamidopyrimidine DNA glycosylase (FPG) sensitive stress sites provided biomarkers of oxidative stress (Bourdon et al., 2012). The authors reported inflammation was strongest at days 1 and 3 post-exposure and remained significantly elevated at the two highest doses of 0.054 and $0.162 \mathrm{mg}$. Significant strand breaks were detected at the same high doses and the level of FPG sensitive sites in the lung was significantly increased throughout with the highest levels recorded on days 1 and 3. Where differences occurred between the lung and liver was the expression of serum amyloid protein (Saa3) mRNA (crucial for systemic inflammation regulation) which was increased in the lung tissue on day 1 at all doses but this was not seen in the liver. The deposition of CBNPs however, clearly demonstrated the ability to promote inflammatory and genotoxic events in both primary tissues and in lung BAL cells, these effects were also observed in the liver, a secondary tissue (Bourdon et al., 2012). SWCNTs in lung trials have induced inflammatory responses but have not been directly linked with free radical damage (Fenoglio et al., 2006). In the same investigation MWCNTs in aqueous suspension did not generate oxygen-containing radicals (elucidated with spin trap experiments) and instead the authors discovered the MWCNTs exhibit a strong radical scavenging behaviour (Fenoglio et al., 2006). Whilst genotoxicity was not evaluated by Fenoglio and colleagues, they concluded that given the MWCNTs utilised in their study demonstrated a scavenger effect of oxygen-centred radicals, any cellular responses particularly in vivo would likely be a result of the fibrous shape and morphology or the CNTs not the commonly reported theory that they impart oxidative stress through ROS production. ROS are molecules of one or more oxygen atoms which are highly reactive, more so than molecular $\mathrm{O}_{2}$ (Frohlich, 2013). The average halflife of these molecules can be as short as nano to micro seconds in duration and furthermore the halflife appears to be inversely related to molecular reactivity, thus if hydroxyl radicals are not removed via enzymatic redox reactions it can cause serious damage in a short timeframe (Frohlich, 2013). Reactive nitrogen species (RNS) exert nitrosative stress which adds to inflammatory response induced by the oxygen species (Mittal et al., 2014). The nitrogen radicals tend to affect different cellular pathways, nitric oxide signalling for example. Currently however there is little in the literature to correlate graphene ENMs with RNS production and subsequent genotoxicity in vitro. 


\subsection{Human Exposure Routes to ENMs}

The inhalation of ENMs is now believed to be one of the primary routes of exposure (Figure 1.11), particularly in the working environment (Christensen et al., 2010). The interaction of ENMs with tissues and cells of the body and consequently the response observed greatly depends on the site of contact. There are four main portals to the human body which ENMs and their unique size can exploit. Inhalation represents one of the four main portals of entry to the body, other common sources include dermal exposure, oral, and ocular. It should also be noted that ENMs can translocate following ingestion and absorption through the digestive tract (Oberdorster et al., 2005a). EMNs, particularly in an occupational environment, can indeed be ingested or otherwise enter the body through one of the body portals illustrated in Figure 1.10. However, given that inhalation via the nasal-pharyngeal or directly into the respiratory tract represents the most prominent route, inhalation will be the focus in subsequent sections where ENM interaction, uptake and toxicity are concerned.

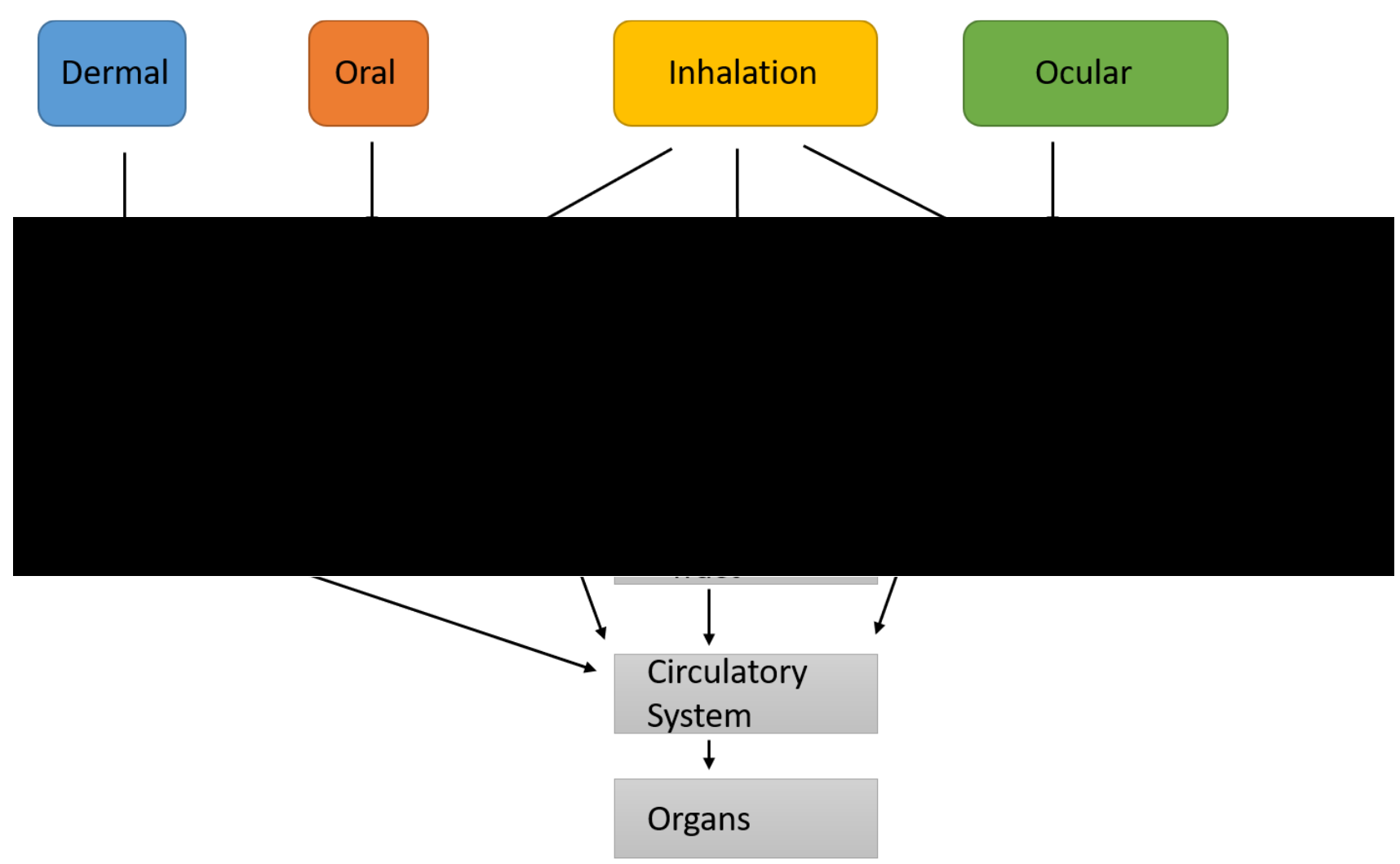

Figure 1.10 Prominent routes of ENM exposure, uptake, retention and translocation. The coloured boxes represent the initial exposure route commonly associated with ENM occupational and environmental conditions. Following the arrow trajectory downwards represents the potential translocation routes, which are typically dependent upon the ENM physico-chemical status. Image adapted from (Yokel and Macphail, 2011). 


\subsubsection{Inhalation}

Once ENMs have gained access to the respiratory tract comprising up to 40 cell types (Sorokin, 1970), bypassing the mucosal defences of the posterior and anterior nasal passages they have potential to deposit in multiple locations. This deposition relies heavily on the physico-chemical features of the inhaled particle or fibre which, briefly, can manifest as inertial impaction or gravitational sedimentation. ENM persistence (unsuccessful clearance by the immune system) could promote cellular damage over time through the recruitment of immune cells to the infected site, resulting in a chronic inflammatory response. Persistent lung inflammation can cause (but is not limited to) genetic damage through oxidative stress, mitochondrial dysfunction, immune activation and further downstream aberrant signalling exacerbating the initial tissue damage (Park et al., 2017a, Coin et al., 1994). The deposition of ENMs can be influenced by several mechanisms, however upon removing the mechanisms detailed in Figure 1.11 the final deposition and resting place for ENMs is driven by their physico-chemical features, with the consensus being the smaller the ENM the greater the deposition achieved. During the development of the combustion engine and subsequent production of particulate matter such as Diesel Exhaust Particles (DEPs) aerodynamic diameter governed the lung deposition potential of these materials. Principally the deposition of ENMs can be attributed to their aerodynamic diameter but far more commonly their physico-chemical features play a larger role which can fall into several classifications. For example a graphene platelet possessing a lateral diameter of $20 \mu \mathrm{m}$ can display an aerodynamic diameter of $\leq 2.5 \mu \mathrm{m}$ which represents a fraction of particulate matter hypothesised to be respirable in the alveolar region (Schinwald et al., 2012). Prior to the study by Schinwald and colleagues, subsequent developments in dosimetry have shown the aerodynamic diameter of ENMs plays an important role when considering airway deposition, especially where bio-distribution and translocation of ENMs from the alveolar beds is concerned. 


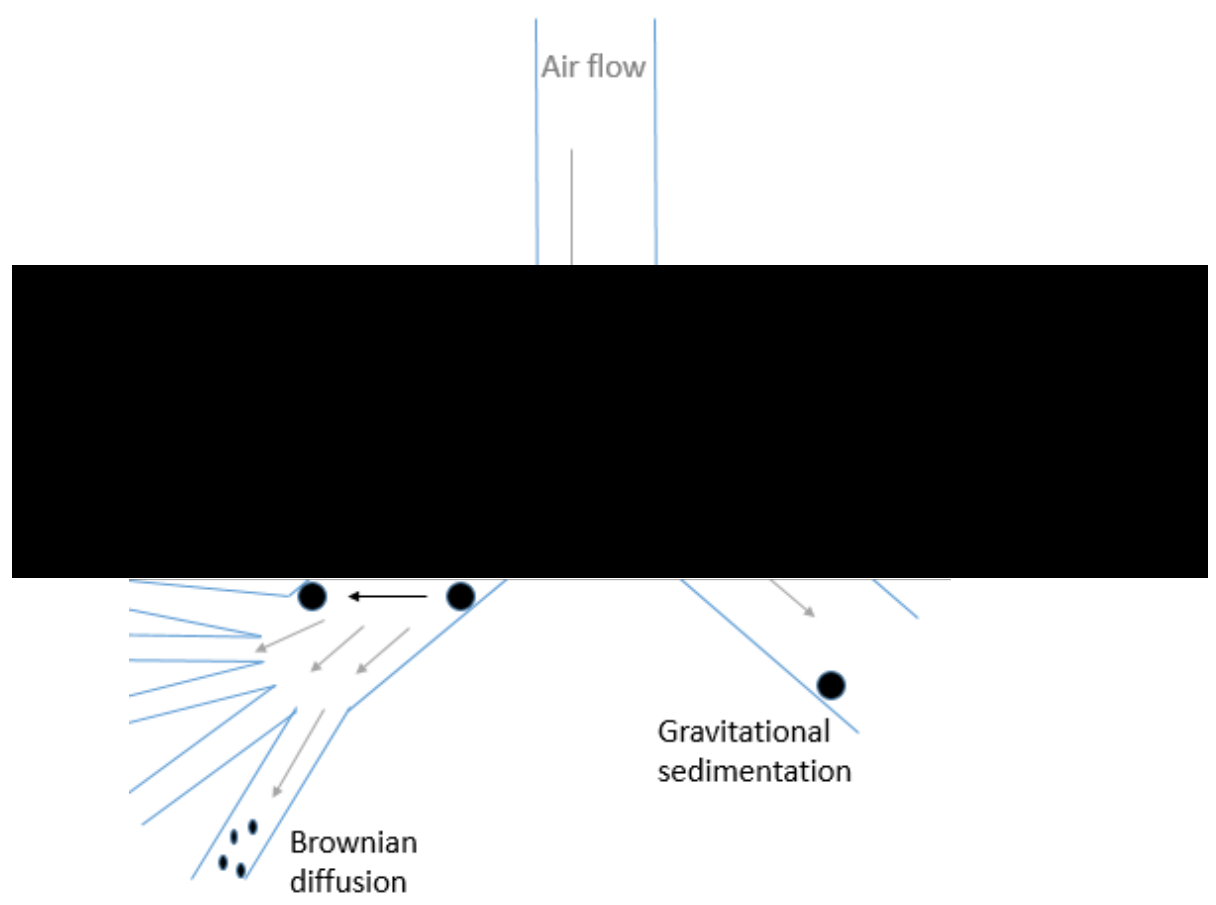

Figure 1.11 ENM deposition in the respiratory tract. Inertial impaction commonly relates to larger ENMs becoming embedded in the epithelial layer of cells of the main bronchus. Slightly smaller ENMs bearing functional groups may then navigate the branching airway further to reach the bronchioles through electrostatic deposition, relying on surface charge to migrate through the respiratory network. To clear ENMs the host organism utilising air flow may exacerbate the movement of the particle or fibre resulting in gravitational sedimentation and interception where air flow aids the particle migration deeper into the tissues. Finally, respirable fractions bearing an aerodynamic diameter of $\leq 2.5 \mu \mathrm{m}$ can utilise Brownian diffusion to reach the alveolar region of epithelial cells where gaseous exchange occurs. Image adapted from (Frohlich and Salar-Behzadi, 2014).

Cells immortalised and grown in vitro as monocultures however may lose this architecture and phenotypic trait to secrete surfactants as defences against particle contact, the $16 \mathrm{HBE} 14 \mathrm{O}^{-}$cell line is one example of this but still remain an ideal barrier cell line for ENMs to overcome, especially when other studies performed on competing cell lines report inconsistencies within data sets of A549 and Beas-2B cytotoxicity (Singh, 2016, Chatterjee et al., 2015). Unlike the competing cell lines mentioned, the 16HBE140- maintain comparably strong immune function and stable genetics. The respiratory epithelium maintain a vast arsenal of innate immune receptors, toll-like receptors and nucleotide oligomerisation domain-like receptors which are crucial to immune function and the secretion of (pro)-inflammatory mediators (Parker and Prince, 2011). Aside from specialised mucous-secreting (Goblet, Clara) cells, the last line of immunity are barrier-forming epithelial cells, some of which 
possess the ability to secrete pulmonary surfactants to aid in the overall immunity to inhaled particulate matter. Given that the majority of ENM clearance in the lung is governed by alveolar macrophages, mucociliary escalation and he epithelial barrier layer (Patton and Byron, 2007, Geiser and Kreyling, 2010). The study of the pulmonary surfactants expressed by alveolar epithelial cells is crucial to understanding ENM uptake and translocation across this barrier. In the peripheral deep lung, the alveolar-lining fluid, a thin hypophase and surface-active lipid-protein complex is known colloquially as pulmonary surfactant which covers the alveolar epithelial layer (Ruge et al., 2012). This pulmonary surfactant is spread via cilia from the distal to the proximal lungs and is positioned at the air-interface which means it lies on the outer side of the air-blood barrier (Bastacky et al., 1995), (Goerke, 1998). Pulmonary surfactant is a composite of approximately $90 \%$ lipid and $10 \%$ protein this can of course vary between types of pulmonary surfactant of which there are four. Surfactant protein (SP) can exist as four isotypes SP-A, - B, - $C$ and $-D$ where each isotype performs a specific function. SP$B$ and SP-C group together as small peptides which interact with surfactant lipids which allows them to contribute to biophysical surfactant functionality (Serrano and Perez-Gil, 2006). SP-A (630kD) and SP-D (520kD) sometimes referred to as collectins (collagen-containing, calcium-dependent lectins) group together with an exceeding biological interest in bio-nano interactions act as opsonins and scavenger molecules (Kishore et al., 2006). The existence of these collectins at the air-liquid interface of the alveoli ideally permits them to bind inhaled particulate matter able to achieve deep lung deposition and making initial contact with this surfactant lining (Seaton et al., 2010). Furthermore the binding of SP-A, -D to inhaled particulate matter also trigger's the activation of alveolar macrophages to internalise inhaled particles as another defence mechanism in an attempt of matter clearance (Wright, 2005). Surfactant protein (SP) A and D both calcium-dependent glycoproteins, have been shown to offer some form of innate immunity through phospholipid binding (Mason et al., 1998). While SP B and C, components of the cell membrane appear to play roles in cellular signalling relating to the recycling of SP packaging and secretion from lamellar bodies (Whitsett et al., 1995). These lines of defence against intrusion from ENMs plays a crucial role upon internalisation.

\subsection{ENM Uptake}

By nature, a cell represents a porous structure allowing the diffusion and active transport of useful ions, metabolites and proteins among many other molecules required for growth and proliferation. This sponge-like nature is present in all cells, immune cells such as macrophages are uniquely diverse and specialised in their uptake capacity, removing foreign bodies from host organisms. Epithelial cells while not specialised for uptake, will be the first contact point for inhaled ENMs and so their ability to 
internalise particles through voltage-gated ion channels, endocytosis or passive diffusion could be crucial to their toxicity (Oh and Park, 2014). Given the scalar size increase from proteins to ENMs with one dimension measuring $\leq 100 \mathrm{~nm}$ the likely internalisation mechanism would be energeticallydemanding endocytosis (Figure 1.12) (Oberdorster et al., 2005b).

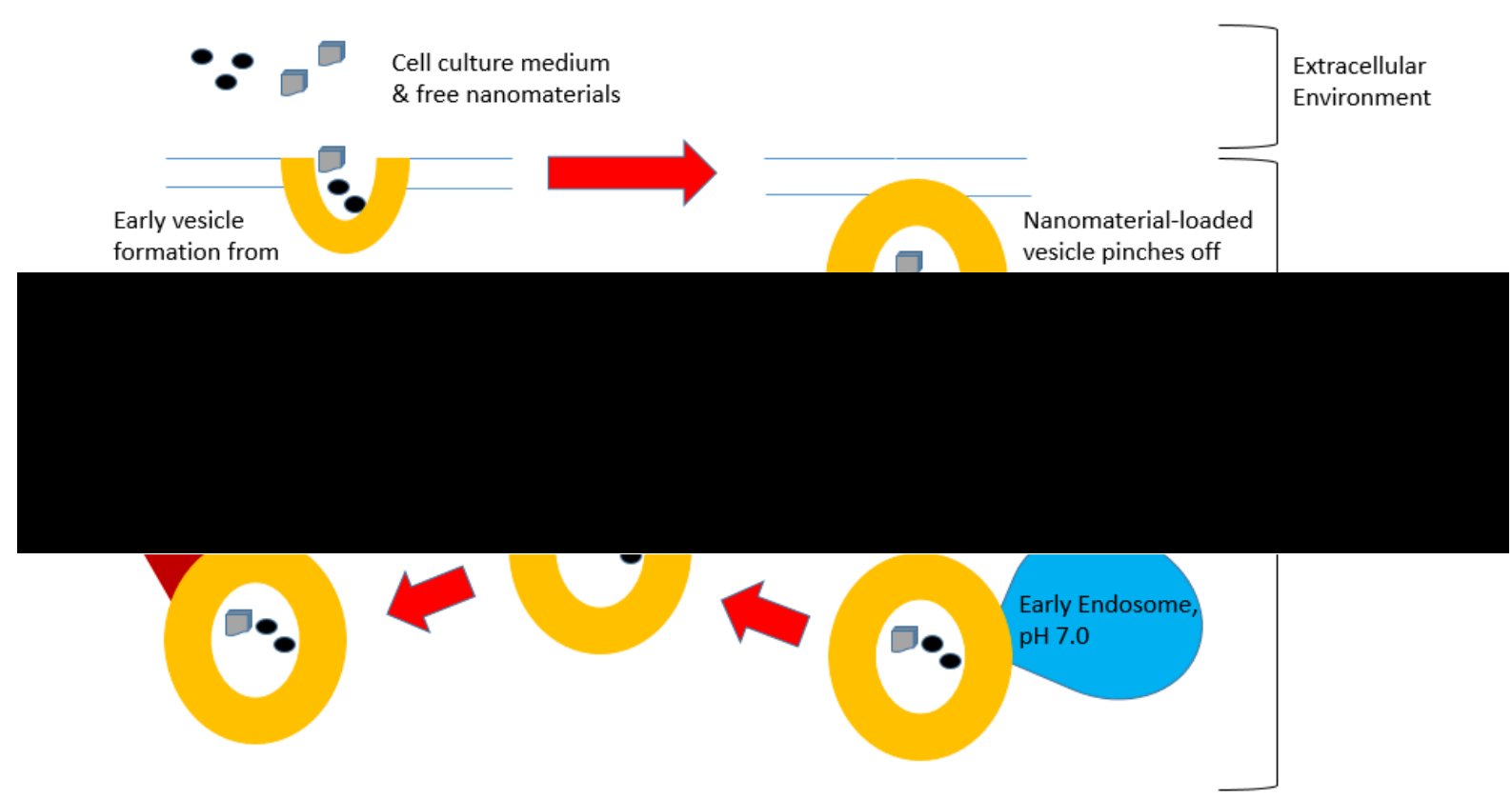

Figure 1.12 Active ENM internalisation by endocytosis. Once bound in endocytotic vesicles a loaded (nanomaterial-containing) vesicle will fuse with an early endosome, typically located at the cell periphery to receive surface bound receptors. Materials tagged or destined for lysosomal degradation, common example being EGFR recycling, will then pass to late endosomes with a slightly acidic $\mathrm{pH}$ of 5.5 , if further protein or ENM tagging is required these can then pass to the lysosome with a pH of 4.5 for extremely acidic breakdown. Lysosomal degradation also represents one of the fundamental excretion pathways with the others being vesicle-related and non-vesicle-related secretion.

Clathrin-mediated endocytosis initially involves a foreign object or in this case ENM approximately 100 $\mathrm{nm}$ in diameter into a region of membrane containing clathrin protein, upon receptor binding this region of membrane undergoes a morphological change into a clathrin-coated pit, slowly enveloping the ENM until it is completely enclosed in membrane, becoming a pinched vesicle (Rejman et al., 2004, Zhang et al., 2015). Caveolin-mediated endocytosis / clathrin independent endocytosis, utilises energy for specific, size-dependent endocytosis, this time accommodating biomolecules $\leq 100 \mathrm{~nm}$. In some cases molecules exceeding $60-80 \mathrm{~nm}$ in diameter and $10-50 \mathrm{~nm}$ bottle-neck can be internalised, 
indicative of a compliant property to this process and to some extent non-selective uptake based on size alone (Wang et al., 2009). Pelkmans and colleagues also reported that this route of endocytosis is favoured when biomolecules are coated with albumin, a common component of blood serum and FBS used to condition culture media. The caveolae is a product of caveolin- 1 and -2 oligomerisation required for the curvature initiation of budding vesicles, a process used in the transcytosis of antibodies (Pelkmans, 2005). Given the range of particle sizes caveolin-mediated endocytosis may accommodate, several studies have investigated the time differences as uptake relates to size. Wang and colleagues reported 20 and 40nm particle uptake was 5-10 times greater than that of $100 \mathrm{~nm}$ particles in endothelial cells, which commonly express caveolin proteins (Wang et al., 2009). The authors concluded that, albumin-coated nanoparticles promoted internalisation, and that caveola size is the primary determinant restricting internalisation. In the comprehensive review by An and Zhang (2017), nanoparticle coating with albumin was proposed to facilitate the uptake of potential nanomedicines used in bio-imaging and drug delivery (An and Zhang, 2017).

Phagocytosis is a specialised form of endocytosis, typically associated with immune cell ingestion of foreign bodies. Macrophage phagocytosis of pathogens represents the initiation of innate immunity which in turn informs adaptive immunity. Macrophages express mannose receptors adapted to recognise specific extracellular motifs on pathogens in a lock-and-key type of reaction (Aderem and Underhill, 1999). The typical size of an engulfed pathogen for instance can be excessively large even for endocytic capabilities, with macrophages accepting molecules as large as 500nm in diameter (Oh and Park, 2014). This actin-dependent mechanism will utilise mannose and scavenger-receptormediated mechanisms to internalise foreign particles tagged for destruction. Engulfment of an ENM by a macrophage results in phagosome formation which upon actin skeleton rearrangement can fuse with an acidic lysosome resulting in a hybrid phagolysosome (Hartwig et al., 1977). The remaining uptake mechanisms fall under the blanket term pinocytosis. Macropinocytosis, the internalisation of extracellular fluids and molecules of the order $200 \mathrm{~nm}$ to $>1 \mu \mathrm{m}$, and in some instances $5 \mu \mathrm{m}$, occurs non-specifically through membrane ruffling. This can be observed microscopically and appears as one large 'arm' of the membrane which extends over the receptor-bound material (Figure 1.13). 


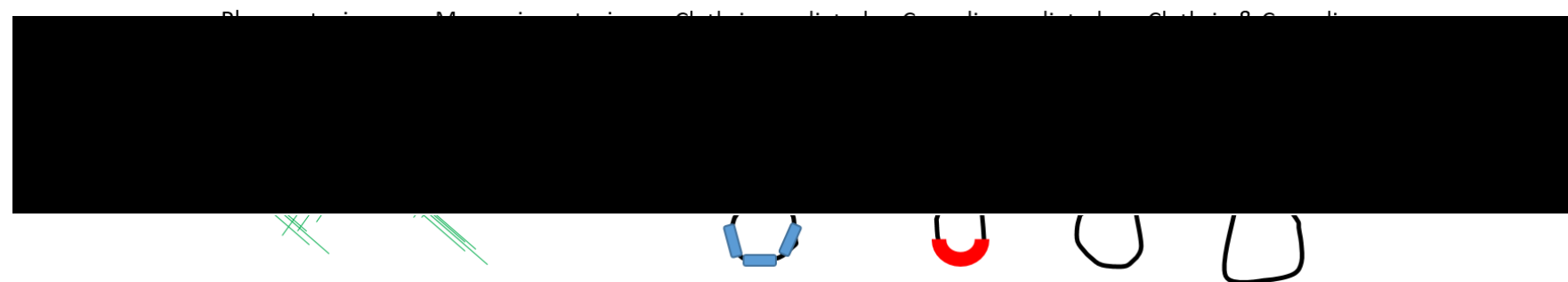

Figure 1.13 Internalisation routes of membrane-bound ENMs. The uptake route largely depends upon the size of the cargo, cell type and the ligand and receptor binding energetics. Image adapted from (Conner and Schmid, 2003).

Where phagocytosis and macropinocytosis differ is the method of membrane engulfment of particles and the sizes associated to those materials. Phagocytosis, typically a specialised process native to immune cells and epithelial cells possessing immune function, tends to engulf biologically tagged molecules and is based upon actin movement and two 'arms' forming from the cell membrane. Macropinocytosis also utilises actin cytoskeleton re-shuffling but here the membrane forms ruffles whereby one 'arm' develops to overlay onto the membrane-bound material. Cellular uptake processes which are independent of both clathrin, caveolin and phagocytosis may also occur via pit formation. This typically specifies materials with diameters of approximately $60 \mathrm{~nm}$ and utilises no specific protein machinery to facilitate uptake. Once an object with specific size enters a membrane pit, the budding process matures often taking the shape of a lipid raft which utilises cholesterol-rich domains (lipid rafts) (Panariti et al., 2012). The literature regarding in vitro endocytosis of graphene ENMs is currently sparse however, Li and colleagues did manage to demonstrate graphene microsheets entering cells at the edge asperities with TEM (Li et al., 2013). The precise mechanisms of endocytosis; phagocytosis, clathrin or caveolin remains unknown and will likely be highly dependent upon particle and agglomeration sizes which varies significantly across the field of graphene ENM manufacture.

\subsection{Graphene Toxicity}

Material science has been revolutionised by graphene and the graphene family of ENMs (GFN), developing a toxicity profile is therefore of vital importance (Bianco, 2013). Each member of this group of nanomaterials can elicit different biological responses from cells. Cytotoxicity and genotoxicity have 
been correlated in some investigations while others report no significant health risks. Nanomaterials with their unique dimensions pose a significant threat if inhaled, the nasal passage, trachea, bronchi and alveolar are all at risk if nanomaterials should sediment at any of these locations (Itoh et al., 2004). The retention time of the material will depend on the site of deposition as well as the speed of the immune response in an attempt to clear the contaminant (Sanchez et al., 2012). Much of initial in vitro testing on novel nanomaterials involves cells of the respiratory tract as they are likely to be the first cells to contact airborne ENMs. Many published results regarding graphene inflammation and toxicity to cells in vitro were gathered using variants of the parent molecule. GO is widely used owing to its increased solubility and dispersible nature in water compared to pristine, non-functionalised graphene (Guo and Mei, 2014a). GO is typically produced from graphene in the presence of concentrated nitric acid as well as a powerful oxidiser such as potassium permanganate (Chng and Pumera, 2013).

An investigation addressing the potential of GO-induced genotoxic damage, treated adenocarcinoma human alveolar basal epithelial (A549) cells with GO and found that oxidative stress was induced in concentrations as low as $10 \mu \mathrm{g} / \mathrm{ml}$ (Chang et al., 2011). Chang and colleagues also discovered that while oxidative stress was significantly elevated, there was no entry of GO into A549 cells and no significant cytotoxicity or genotoxicity was reported. Published findings have already demonstrated that derivatives of graphene such as GO cause cytotoxicity and genotoxicity to human lung fibroblasts. The genotoxic damage was shown to be induced by ROS production which contributed to the cytotoxic response (Wang et al., 2013). Wang and colleagues utilised methyl thiazolyl tetrazolium (MTT) to analyse the cytotoxicity, the comet assay to provide an indicator of genetic damage and the sub-G1 assay measuring on a flow cytometer to analyse cell cycle abnormalities. The investigation demonstrated a concentration - dependent cytotoxicity and genotoxicity relationship with GO administered at concentrations between $1-100 \mu \mathrm{g} / \mathrm{ml}$ (Wang et al., 2013). The extra oxygen functional groups of GO radically affect its toxicity to cells compared to pristine graphene. It follows therefore that functionalising GO to make it more biologically compatible should be considered before use in commercial applications with expected human exposure. This has already been investigated by Zhi and colleagues during an in vitro study which sought to compare the immunogenicity of graphene oxide and polymer coated graphene oxide (Zhi et al., 2013). The polymer used in the study was Polyvinylpyrrolidone (PVP) which had a lower immunogenicity profile than the non-modified graphene oxide. The PVP polymer coating may have been large enough to impede the process of macrophage phagocytosis, which is an issue that needs to be addressed in future studies to overcome a chronic immune response (Zhi et al., 2013). The overall toxicity of GO is now believed to lie in the oxidation 
process used to manufacture the nanomaterial. Aspects of the functional groups and structural defects may alter the inflammatory and toxic potential of GO. This information has come to light after the in vitro study by Chng and Pumera suggested that varying methods used to produce GO can affect the oxygen/carbon content ratio which then proceeds to alter the toxic potential (Chng and Pumera, 2013). The study, which used adherent lung cells (A549), demonstrated a dose-dependent cytotoxic response from the four graphene-production methods used (Hoffman, Staudenmaier, Tour and Hummers) to produce the GO after 24-hour exposure. The authors concluded there was a correlation between oxygen content within the functional groups and the toxicological significance to the lung cells while stating further studies should aim to replicate the findings to reinforce this claim (Chng and Pumera, 2013).

GO with its large surface creates an opportunity for many proteins to adsorb to the surface. To provide further information regarding this matter $\mathrm{Hu}$ and colleagues carried out a study to determine the effect of increasing the foetal bovine serum (FBS) concentration from 1-10\% on GO cytotoxicity to alveolar basal epithelial cells. FBS is commonly included in cell culture medium at $10 \%$ to imitate in vivo conditions during in vitro studies, at low concentrations of $1 \%$ FBS the alveolar cells showed concentration-dependent cytotoxicity in the presence of GO (Hu et al., 2011). Increasing the FBS concentration to $10 \%$ greatly reduced the cell cytotoxicity, a promising result to consider for future in vivo testing with GO. The authors concluded the cytotoxicity was due to direct contact and breaching of the cell membrane by the GO which caused structural cellular damage. The high protein adsorption potential of the graphene oxide at higher FBS concentrations may have increased the bio-compatibility of the nanomaterial and masked the asperity of the graphene oxide reducing the direct ENMmembrane contact sites. Graphene sheets and GO cytotoxicity has been explored in adherent skin fibroblasts by Liao and colleagues who measured mitochondrial activity as an indicator of cytotoxic damage (Liao et al., 2011). Initially the authors employed the methylthiazolyldiphenyl-tetrazolium bromide (MTT) assay, a standard assay performed in nanotoxicology testing. The MTT assay is a colorimetric assay which assesses the activity of NADPH oxidase enzymes and thus viable cells. During the investigation however, the graphene nanomaterials spontaneously reduced the MTT which generated false positives which prompted alternative methods to be used. The authors turned to trypan blue staining and the reactive oxygen species assay which revealed compact graphene sheets were more damaging to the adherent skin fibroblasts than the less-dense graphene oxide (Liao et al., 2011). Further to this, the authors concluded that the exposure environment i.e. suspension or adherent cell types greatly affects the toxicity of these graphene products. The unique physical structure of graphene and graphene derivatives means the dimensions and surface modifications 
make it a hazard to cells in the body. As discussed the purity of graphene products can greatly affect the inflammatory profile, smaller sized nanosheets with a lower surface area may present a decreased risk to macrophages and dendritic cells which could internalise the material efficiently and eliminate the risk to cells (Mogharabi et al., 2014).

The toxicity of a nanomaterial can be exacerbated by bio-persistence which presents a problem where graphene and graphene derivatives are concerned. These ENMs are renowned for their strength and durability which indicates they should be difficult for cells to degrade and remove thus decreasing the damage caused to the surrounding tissue. However new avenues are being pursued to encourage biodegradation of graphene-based products such as single walled carbon nanotubes (SWCNT) to enable the nanomaterial to safely carry therapeutic agents in the body and deliver them to cells. Once the payload has been delivered the enzymatic degradation of the nanomaterial can begin to eradicate the potential secondary genotoxic mechanisms from being initiated (Kotchey et al., 2013). The enzymatic degradation of graphene and all graphene based medical products may be the future of safe targeted drug delivery. By using several analytical techniques such as Raman, Fourier transform mass spectrometry and atomic force microscopy Kotchey and colleagues were able to show that horseradish peroxidase (HRP) could successfully breakdown and oxidise a single layer of graphene oxide while in the presence of $\sim 40 \mu \mathrm{M}$ hydrogen peroxide. The catalysis resulted in holes forming in the basal plane of the structure which the authors attributed to the close proximity of the heme group in the peroxidase enzyme to the GO (Kotchey et al., 2011). As a close comparison the investigation was also carried out on $\mathrm{rGO}$ which proved to be more resilient to the peroxidase as the heme group was unable to attain proximity when compared to the GO. To depict with accuracy, the binding mechanism of the heme group of the HRP enzyme with the GO and the rGO a molecular modelling system was employed. The software package used in this study was AutoDock Vina, a relatively new program which presents information regarding molecular docking with virtual screening (Trott and Olson, 2010). The molecular modelling revealed that HRP exhibits only one site for rGO binding but GO could bind in two locations which was amino acid specific. The rGO could also bind at a closer distance to the heme group at $11.5 \AA$ compared to the 8.7 and $12.8 \AA$ distances with which the GO could bind. The data from the molecular modelling program suggests that the increased number of hydroxyl groups of GO may have contributed to more favourable binding with the HRP heme group and thus caused the greater oxidative stress observed by the nanomaterial (Kotchey et al., 2011). The higher degree of catalysed oxidation to $\mathrm{GO}$ as opposed to $\mathrm{rGO}$ the authors concluded was due to the higher affinity of the HRP heme group for the GO which was in closer proximity and possessed double the number of binding sites. These factors coupled together made the GO more susceptible to carbon- 
carbon bond damage (Kotchey et al., 2011). Further to this, proving that graphene products can be degraded by naturally occurring enzymes it follows that graphene could be carried forward as a candidate for future drug carriers in biomedical applications. With regards to other means of biodegradation as a removal system for graphene-based products few options have been explored thus far, the effects of hypochlorite and radical intermediates of the neutrophil enzyme myeloperoxidase have been briefly studied on single walled CNTs, with attention now turning to graphene derivatives . The study by Kagan and colleagues found that when lung cells treated with single-walled carbon nanotubes were exposed to the myeloperoxidase the enzyme acted as a catalyst in the biodegradation of the nanomaterial which reduced pulmonary inflammation (Kagan et al., 2010). Furthermore, once biodegraded the SWCNTs did not induce any pulmonary inflammation when aspirated into the lungs of mice (Kagan et al., 2010). The biodegradation of the nanomaterial during the in vitro experiment and in the neutrophils provides promising results for future studies and highlights crucial safety information with respect to graphene-based products. However, the investigation also included a macrophage experiment which demonstrated a lesser ability to degrade and clear the CNTs which should prompt further research to be carried out in this area. Confirmation graphene and FLG biodegradation can be confirmed by both Raman spectroscopy and microscopy as was observed by Kurapati and colleagues. In the latter study, the authors noted the ability of degranulating human neutrophils and human myeloperoxidase mediated catalysis (Kurapati et al., 2018). In the recent publication by Fadeel and colleagues, the authors note the importance of the number of graphene layers, the $\mathrm{C}: \mathrm{O}$ ratio and finally the lateral dimensions which determine the biodegradation of graphitic ENMs by immune cells. It is important to state however, as stated by Fadeel and colleagues, graphene materials engineered with PEG and BSA coatings showed resistance to biodegradation by horseradish peroxidase (Fadeel et al., 2018).

\subsection{Airway Epithelial Immune Response}

Upon ENM inhalation, the first point of contact will be the epithelial cells of the trachea, bronchus, bronchioles and branching network leading towards the alveolar region of the lung. Much like immune cells, lung epithelial cells possess a potent innate immune function, able to release (pro)-inflammatory mediators as a host defence against inhaled ENMs (Hiemstra et al., 2015). To counteract the threat of invading pathogens or inhaled ENMs, airway epithelial cells possess membrane-bound pattern recognition receptors (PRRs) such as TLRs and C-type lectin receptors (CTLRs). These act to recognise bound molecules and through defence mechanisms; mucociliary clearance, production of reactive oxygen/nitrogen species ROS/RNS and interferon I and III coupled to chemokine and cytokine 
production result in adaptive and innate immunity which contribute to host defence (Hiemstra et al., 2015). The generation of chemokine (chemoattractant) IL-8 plays a role in the distinct recruitment and activation of neutrophils, immune cells with phagocytic capabilities designed to engulf and clear foreign particles. Cytokine IL-6 acting in the acute phase response of inflammation (generation of acute phase proteins) in conjunction with soluble IL-6 receptor (sIL-6R) dictates the transition from the acute phase of inflammation to chronic (Hurst et al., 2001, Gabay, 2006). This process involves the chemical signalling of leucocyte infiltration altering from polymorphonuclear neutrophils to monocytes/macrophages (Gabay, 2006). IL-6 may also possess a favourable B- and T-cell activation thus favouring the prolonged response. Numerous studies have highlighted that murine epithelial cells react to airborne ENMs administered at $5 \mathrm{mg} / \mathrm{m}^{3}$ for 4-hour periods over the course of four days through promotion of (pro)-inflammatory signalling (Rouse et al., 2008).

\subsection{1 (Pro)-inflammatory mediators}

The role of the cytokine IL- 6 is multifunctional, it regulates many aspects of immune response, acute phase inflammation and haematopoiesis (blood component production) (Kopf et al., 1994). The relative role of IL- 6 however has been noted to change when moving from in vitro to in vivo where in the latter IL-6-sIL6R complexes activated endothelial cells to secrete IL-8 and monocyte chemoattractant protein-1 (MCP-1), inducing the expression of adherent cells (Romano et al., 1997). A breakdown of IL- 6 signalling can be seen below in Figure 1.14 where the switch between acute and chronic stages of inflammation can be hypothesised. The true switch for this mechanism, the phagocytosis of PMNs by monocyte-derived macrophages, stimulates an increase in transforming growth factor $\beta$ and MCP-1 while also decreasing IL-8 production, this leads to a chemokine shift which favours monocyte recruitment (Gabay, 2006). IL-8 is a chemo-attracting cytokine produced by a number of immune cells such as macrophages (Bickel, 1993). Crucially IL-8 is also produced by epithelial cells including $16 \mathrm{HBE} 140^{-}$cells. IL-8 reacts to inflammatory stimuli such as IL-1 and tumour necrosis factor-alpha (TNF- $\alpha$ ) (Baggiolini and Clark-Lewis, 1992). The primary function of IL-8 is to recruit and activate neutrophils as promote subsequent endocytosis by the same cells. Gene expression of IL-8 has been sourced to many cytokines including TNF- $\alpha$ and IL-1 $\beta$ (Tobe et al., 2002). 


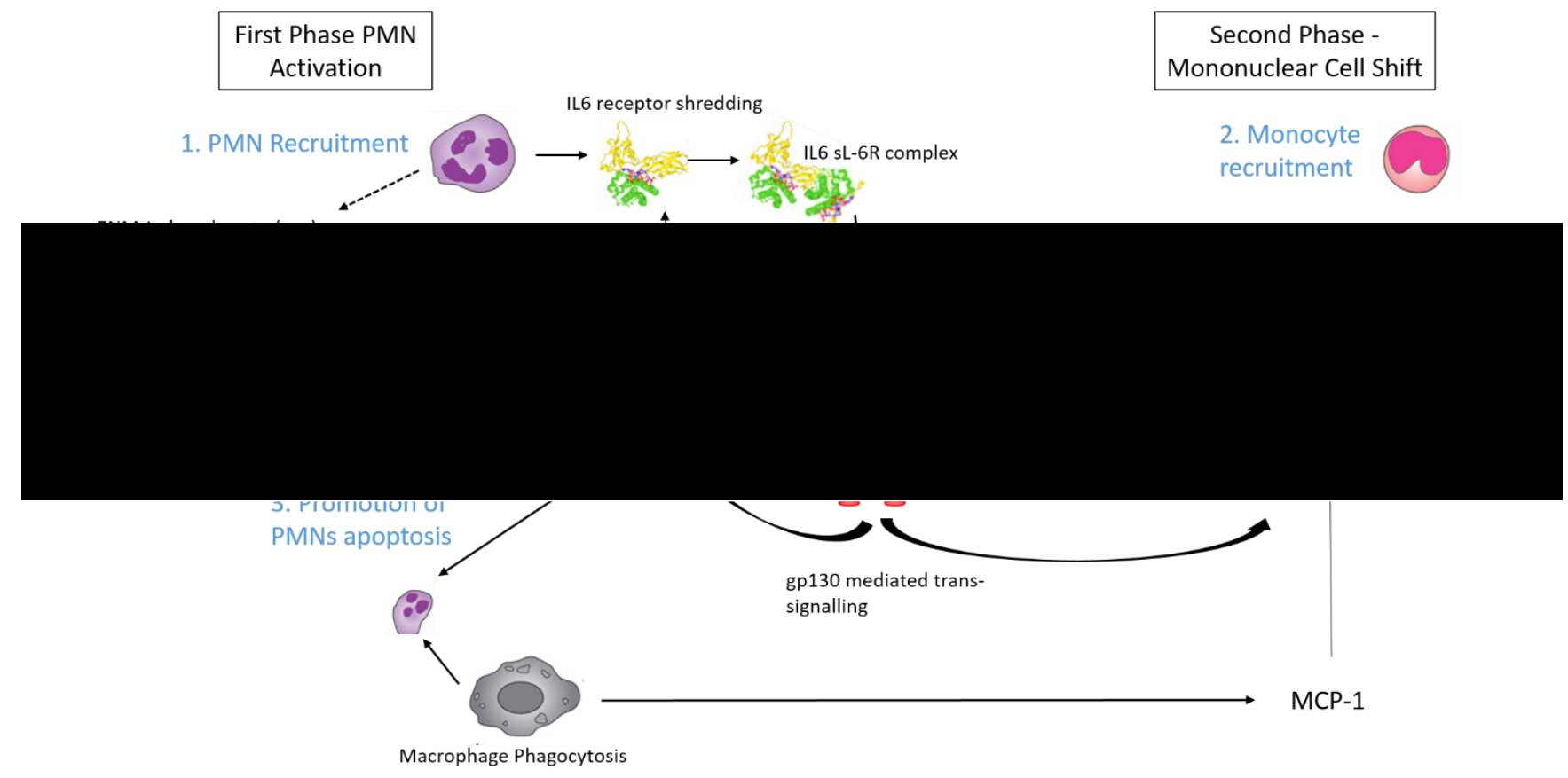

Figure 1.14 The hypothesised role of IL-6 in mediating the switch between acute and chronic inflammation. At Stage-1 the binding of IL- 6 with sIL-6R is initiated by an acute inflammatory event this triggers Stage-2, gp130 signalling and recruitment of monocytes/ macrophages. Lastly Stage-3 sustained inflammation results in neutrophil apoptosis, phagocytosis and monocyte accumulation at injury site, image adapted from (Gabay, 2006).

\subsection{Thesis Aim}

This thesis has investigated the potentially toxic effects of industrially-relevant carbonaceous ENMs; namely, Neutral-FLG, Amine-FLG and Carboxyl-FLG. Consumer-relevant CB particles (a Type IA Carcinogen), commonly used as printer toner, were also introduced providing a direct genotoxic comparison to the graphene ENMs. The potential of these ENMs to promote a genotoxic response in relevant cell lines and in vitro culture models was assessed over three results chapters $(3,4$ and 5$)$. Prior to the study, FLG surface area and surface chemistry were assumed to play a large role on the toxicity of each material, with the hypothesis that additional surface groups could relieve toxicity through radical scavenging mechanisms. Chapter 3 was concerned with extensive physico-chemical characterisation of each ENM incorporating measurements and analyses of both the primary and secondary features where applicable. Given that these ENMs pose a great risk in the work place as an occupational inhalation hazard, an array of relevant lung cell types and culture models were used in this thesis to provide essential toxicological response data following 24-hour exposures to these materials. A deep understanding of the physico-chemical features of the ENMs would also provide a 
means of discriminating between the materials based on their observed respective biological responses. Chapter 4 investigated the potential of all four ENMs to promote genotoxicity in a 2D monoculture of bronchial epithelial (16HBE140') cells. This in vitro set-up would highlight the ability of the ENMs to promote primary genotoxicity, a DNA damage response elicited in the absence of a chronic immune response. Chapter 4 also extensively investigates the potential mechanisms associated with primary genotoxic responses, exploring (pro)-inflammatory mediators, oxidative stress and mitochondrial stress. Chapter 5 then investigated the effects of these ENMs upon a coculture of TT1 and differentiated THP-1 macrophages, to detect the presence of secondary mechanisms of genotoxicity which may be present following ENM exposures. 


\section{Chapter 2 - General Materials and Methods}

This section details the common materials and methods utilised throughout Chapters 3-5. Variations, if any, to the methodologies are specifically described in the materials and methods sections of each result chapter.

\subsection{Engineered Nanomaterials (ENMs)}

Neutral-, Amine-, and Carboxyl-FLG were manufactured via dielectric barrier discharge (plasma exfoliation) of mined graphite supplied by Perpetuus Carbon Technologies (PCT, UK) whilst the CB particles (FLAMMRUSS 101, Lamp Black \#8235102) were supplied by Evonik Degussa Inorganic Materials, Frankfurt. All ENMs were supplied as powder and were placed into suspension at Swansea University to a stock concentration of $10 \mathrm{mg} / \mathrm{ml}$ in double distilled water. Prior to cell exposures or physico-chemical characterisation where applicable, ENMs were sonicated in a 90W Ultrasonic Bath (Fisher Scientific \#FB15046) for 20 minutes at $37^{\circ} \mathrm{C}$ to encourage destabilisation of agglomerate material. During cell exposures, ENMs were always added in $200 \mu$ l total volumes to the cell flasks / plates, detailed in Table 2.1.

Table 2.1 ENMs dilution and dosing technique for monocultured cell lines; $16 \mathrm{HBE} 140^{-}$and TT1 cells in T25 flasks, seeded at $1 \times 10^{5}$.

\begin{tabular}{|l|l|l|l|}
\hline ENM Dose $(\mu \mathrm{g} / \mathrm{ml})$ & $\begin{array}{l}\text { Required volume of } \\
10 \mathrm{mg} / \mathrm{ml} \text { stock }(\mu \mathrm{l})\end{array}$ & $\begin{array}{l}\text { Required volume of } \\
\text { media }(\mu \mathrm{l})\end{array}$ & $\begin{array}{l}\text { Volume added to } 10 \\
\mathrm{ml} \text { cells }(\mu \mathrm{l})\end{array}$ \\
\hline 0 & 0 & 200 & 200 \\
\hline 2 & 2 & 198 & 200 \\
\hline 4 & 4 & 196 & 200 \\
\hline 8 & 8 & 192 & 200 \\
\hline 10 & 10 & 190 & 200 \\
\hline 20 & 20 & 180 & 200 \\
\hline 50 & 50 & 150 & 200 \\
\hline 100 & 100 & 100 & \\
\hline
\end{tabular}




\subsection{Cell Culture}

Cell culturing and assays requiring sterile conditions were performed in a Class II tissue culture hood (Scanlaf Mars, Denmark) which, during the night is UV-sterilised for 6 hours and immediately prior to use was washed with $70 \%$ ethanol. Incubation conditions were maintained throughout at $37^{\circ} \mathrm{C}$ at $5 \%$ $\mathrm{CO}_{2}$ atmospheric pressure in a NU-5510 DHD Autoflow incubator (Nuaire, UK). Culture plates, flasks, pipettes were sourced from Greiner Bio-One (Gloucester, UK). Approximately 30 minutes prior to use, all tissue culture reagents were warmed in a water bath at $37^{\circ} \mathrm{C}$ before being sterilised with $70 \%$ ethanol and placed inside the class II tissue culture hood. Cell cultures were always checked for functionality and confluence via an Axiovert light microscope (Carl Zeiss, Cambridge, UK). Epithelial and macrophage cell lines utilised in this study underwent long-term storage in liquid nitrogen, frozen down in $2 \mathrm{ml}$ Cryovials (Elkay Lab Products, Basingstoke, UK) at $1 \times 10^{6}$ at passage numbers below 10 in a suspension of $10 \%$ dimethyl sulfoxide (DMSO) (Sigma, UK) 90\% serum, either FBS or HS depending on cell line. Initially cells were transferred to a $-80^{\circ} \mathrm{C}$ freezer inside an isopropanol-lined container for 24-48 hours. Cryovials were then moved to liquid nitrogen for long term storage. Sub-culturing and cell seeding for assays required the use of the chemical equation $C_{1} V_{1}=C_{2} V_{2}$, repurposed to determine the seeding density of $16 \mathrm{HBE} 14 \mathrm{o}^{-}$cells and macrophages. This technique was supported with the use of the Z1 Coulter Counter (Beckman Coulter, UK) where 100 $\mu$ l of re-suspended cells was pipetted into $10 \mathrm{ml}$ of coulter diluent (Beckman, UK) with parameters set to count a size range of 10-17 $\mu \mathrm{m}$. Seeding densities were subsequently calculated using Equation 2.1. Centrifuge steps were typically performed in revolution per minute (RPM) however converting to relative centrifugal force (RCF) with units (g) was calculated using Equation 2.2.

\section{Equation 2.1}

Concentration of stock cells $\left(C_{1}\right) \times$ Volume of stock cells required $\left(V_{1}\right)=$ Concentration of cells to be seeded $\left(C_{2}\right) \times$ Total volume of cells required $\left(V_{2}\right)$

\section{Equation 2.2 (RPM $\rightarrow$ RCF)}

$\mathrm{g}=1.118 \times 10^{-5} \times \mathrm{r} \times \mathrm{S}^{2}$ where; $1.118 \times 10^{-5}$ is a unit conversion factor, $\mathrm{r}$ is the radius of the rotor $\operatorname{arm}(\mathrm{cm})$ and $S$ is the speed in RPM. 


\subsubsection{HBE140 cell culture}

The immortalized bronchial epithelial cell line $16 \mathrm{HBE} 140^{\circ}$ was selected as a model cell line to assess the inhalation hazard posed by the ENMs, featured in Chapter 4 of this study, under investigation in the present study. The cell line kindly provided by Professor D. C. Gruenert at the University of California, San Francisco, USA. These cells provided an ideal barrier model in vitro and offer an opportunity to model the effects of bronchial respiratory hazards with carbonaceous ENMs, in comparison against an alveolar cell line (Transformed Type I, TT1). When needed, 16HBE140 were thawed from liquid nitrogen and placed into a fibronectin-coated T25 flask (88\% LHC Basal Media (Invitrogen, UK), 10\% $1 \mathrm{mg} / \mathrm{ml} \mathrm{BSA} \mathrm{(Invitrogen,} \mathrm{UK),} \mathrm{1 \%} \mathrm{3mg/ml} \mathrm{bovine} \mathrm{collagen} \mathrm{(Invitrogen,} \mathrm{UK)} \mathrm{and}$ $1 \% 1 \mathrm{mg} / \mathrm{ml}$ human fibronectin (BD, UK)). Once the cells had proliferated they were transferred to $\mathrm{T75}$ flasks for sub-culturing. The 16HBE140 cells were sub-cultured with MEM media (Invitrogen, UK) supplemented with 10\% FBS (Sigma, UK), 1\% L-Glutamine, 1\% Penicillin $(100 \mu \mathrm{g} / \mathrm{ml})$ / 1\% Streptomycin $(100 \mu \mathrm{g} / \mathrm{ml})$ (Sigma, UK). The epithelial cell line was cultured to $70-80 \%$ confluency before being subcultured or seeded for experiments. Epithelial cells were split as following; existing media was first removed and discarded, adherent cells were then washed twice with pre-warmed PBS (Corning, UK), following the second wash, PBS was discarded and trypsinised with $4 \mathrm{ml}$ of PolyvinylpyrrolidoneEthylene glycol-bis(2-aminoethylether-tetra-acetic acid (EGTA) trypsin (PET) solution (72\% HBS, 10\% polyvinylpyrrolidine (Sigma, UK), 10\% EGTA (Sigma, UK) 8\% trypsin (Invitrogen, UK)) and placed flat in a $37^{\circ} \mathrm{C}, 5 \% \mathrm{CO}_{2}$ incubator for 7 minutes. Flasks were inspected under a light microscope to ensure cells had detached thoroughly before being transferred to a $15 \mathrm{ml}$ Falcon tube with $6 \mathrm{ml}$ of complete media (deactivate Trypsin-EDTA) and centrifuged at 290g for 5 minutes. Lastly, epithelial cells were seeded at a new passage at $1.5 \times 10^{5}$ cells $/ \mathrm{ml}$ and returned to the incubator. Sub-culturing of cells was required every 3 days, cells were passaged multiple times before use in toxicological assays, and additionally cells which exceeded 20 passages were not used in any toxicological assays. Lastly, cells were used in toxicological assays within the range of 1-10 passages to maintain a low genetic mutation frequency and maintain a healthy cell population.

\subsubsection{Transformed Type 1 (TT1) Cell Culture}

The immortalized alveolar type-1 (ATI)-like, TT1 cell line was kindly donated by Professor Terry Tetley of Imperial College London, UK. This alveolar cell line, featured in Chapter 5 of this study, had been previously transformed, immortalised and characterised by Kemp and colleagues as a model for ATI cells. TT1 cells when required, were thawed from liquid nitrogen and transferred to T25 flasks to initiate proliferation for 48 hours or until reaching $70-80 \%$ confluency before sub-culturing into a T75. 
TT1 cells were cultured with DCCM-1 Media (Geneflow Ltd, UK) supplemented with 1\% Penicillin / Streptomycin / L-Glutamine (Sigma, UK) and 10\% New-born Calf Serum (NCS) (Sigma, UK). Subculturing of TT1 cells began with removing existing media and discarding, washing the adherent cells twice with pre-warmed PBS before adding $4 \mathrm{ml}$ of thawed Trypsin-EDTA (detailed above (2.9.1)) and incubated flat at $37^{\circ} \mathrm{C}$ for 5 minutes to disassociate. Once cells had lifted, complete media was added $(6 \mathrm{ml})$ to de-activate the Trypsin-EDTA solution and the cells transferred to $15 \mathrm{ml}$ Falcon tubes to be centrifuged at $340 \mathrm{~g}$ for 10 minutes. The resulting supernatant was discarded, cells re-suspended and seeded at $1 \times 10^{5}$ and returned to the incubator at $37^{\circ} \mathrm{C}$. When seeding TT1 cells for toxicological assays, cells were counted by re-suspending and taking $10 \mu \mathrm{l}$ and adding to $90 \mu \mathrm{l}$ of fresh media then pipetting $10 \mu l$ into a Neubauer chamber. Cells in the four corners of the chamber were counted, averaged and multiplied by $10^{4}$ for the number of cells per ml. TT1 cells were sub-cultured every 3 days, reaching $90-100 \%$ confluency in that time. TT1 cells were not used in toxicological assays once exceeding 10 passages.

\subsubsection{THP-1 Cell Culture}

Suspension peripheral blood monocyte THP-1 cells, used in Chapter 5, were purchased from the American Tissue Culture Collection (ATCC). Cells when required, were thawed from liquid nitrogen and transferred to T25 flasks to begin proliferation. Culture media required for THP-1 growth was RPMI 1640 (Invitrogen, UK) supplemented with 10\% FBS, 1\%, 1\% L-Glutamine, 1\% Penicillin $(100 \mu \mathrm{g} / \mathrm{ml}) / 1 \%$ Streptomycin $(100 \mu \mathrm{g} / \mathrm{ml})$ (Sigma, UK). THP-1 cells required passaging every $2-3$ days and not allowed to proliferate beyond $8 \times 10^{5}$ cells $/ \mathrm{ml}$ in T75 flasks, similarly THP-1 cells were not used in toxicological assays once they had reached and exceeded 20 passages of sub-culturing. THP-1 cells were cultured by transferring the cells to a $50 \mathrm{ml}$ Falcon tube and centrifuged at $129 \mathrm{~g}$ for 5 minutes at room temperature, supernatant discarded and then re-suspended in fresh, supplemented RPMI media and placed into a T75 flask.

\subsubsection{Differentiation of THP-1 cells}

To perform the Chapter 5 co-culture (bi-culture) experiments, THP-1 cells required differentiation into macrophage-like cells with an immune antagonist, namely phorbol 12-myristate 13-acetate (PMA). PMA was selected over lipopolysaccharide due to the potency at which differentiation was achieved, at low concentrations while also providing a large yield of differentiated THP-1 cells. 
To differentiate, THP-1 cells were centrifuged at $129 \mathrm{~g}$ for 5 minutes at room temperature, immediately after which the cell pellet was re-suspended at $5 \times 10^{5}$ cells $/ \mathrm{ml}$ in a $15 \mathrm{ml}$ Falcon tube, $50 \mathrm{nM}$ (optimised to provide the greatest yield of differentiated THP-1 cells and maintain viability) of freshly thawed PMA was then added to the cell suspension before being transferred to a T75 flask for 24 hours. Cells were checked after the incubation period via light microscopy, differentiation was evident by the nowadherent cells on the bottom of the flask which didn't return to suspension over gentle agitation of the flask. The PMA-containing media was then discarded, the cells washed three times with prewarmed PBS and freshly thawed RPMI media was added, the cells were then incubated for a further 24 hours to recover from PMA-stimulated (pro)-inflammatory stimulation. Cells were typically differentiated a day or two in advance of co-culture setup which allowed (pro)-inflammatory cytokine / chemokine levels (induced by PMA) to return to basal levels as to not warrant the transfer of (pro)inflammatory mediators into the co-culture setup. Lastly, once THP-1 cells had been differentiated, they were not maintained for more than two days, with the only exception being co-culture construction whereby the nature of the cytokinesis block micronucleus assay required them to be nurtured for the duration of the assay.

\subsection{Cell Viability}

In vitro ENM-cell testing was performed in accordance with the Organisation for Economic CoOperation and Development (OECD) guidelines, a descript characterisation of screening the potential health effects of chemicals in an industrial or academic setting. These guidelines however were adapted to the health effects of industrially-relevant carbonaceous ENMs instead of chemicals as described in detail by Doak and colleagues (Doak et al., 2012b). Assessing the cell viability of the epithelial cell lines TT1 and 16HBE140 ${ }^{-}$was performed using relative population doubling (RPD) and Equation 2.3 below. To begin, an initial cell count was performed prior to ENM exposures to act as the initial cell count. Following an exposure time of 24 hours a second cell count was performed to complete the equation. This was performed in triplicate for all doses.

\section{Equation 2.3}

RPD = No of population doubling of exposed cells / No of population doubling within control $x 100$

Where; Population Doubling $=[\log ($ Post Treatment Count $/$ Initial Count $)] \times \log ^{2}$ 


\subsection{In Vitro Cytokinesis Block Micronucleus (CBMN) Assay}

As alluded to in section 2.11, the genotoxicity testing as written in the OECD guidelines had to be adapted here for the incorporation of ENMs, supported by the detailed research and information written by Doak and colleagues in 2012 (Doak et al., 2012b). Both epithelial cell lines, TT1 and 16HBE140- after being seeded in T25 flasks at $1 \times 10^{5}$ cells $/ \mathrm{ml}$ and treated with ENMs and a positive control of Mitomycin C (MMC), an alkylating agent at a concentration of $0.01 \mu \mathrm{g} / \mathrm{ml}$. This concentration was selected as it provided a clearly detectable and reproducible increase over control micronuclei frequencies. Following the 24-hour exposure, treatment media was removed and stored for subsequent analysis. Adherent cell lines were then washed twice with pre-warmed PBS, fresh supplemented media was then provided with the addition of Cytochalasin (Cyto) B at $3 \mu \mathrm{g} / \mathrm{ml}$ for a complete cell cycle, 22 hours for $16 \mathrm{HBE}^{-14 \mathrm{o}^{-}}$and 24 hours for TT1 cells (TT1 required more than one complete cell cycle to produce $>50 \%$ BN cells). Following the cell cycle incubation with Cyto $B$, the media was discarded, and the cells washed twice with pre-warmed PBS. Cells were then lifted from T25 flasks with Trypsin-EDTA ( $2 \mathrm{ml}$ per flask), neutralised with $4 \mathrm{ml}$ of fresh media, transferred to $15 \mathrm{ml}$ Falcon tubes and centrifuged at 290g for 5 minutes. The cell pellet was then re-suspended in $5 \mathrm{ml}$ of $\mathrm{KCl}$ prepared at $0.56 \%$ and centrifuged at $4^{\circ} \mathrm{C}$ at $290 \mathrm{~g}$ for 10 minutes. Following this, supernatant was discarded, and the pellet re-suspended in $5 \mathrm{ml}$ of fixative 1 (a 5:6:1-part solution of methanol, acetic acid and freshly prepared $0.9 \% \mathrm{NaCl}$ respectively). The cells were incubated at $4^{\circ} \mathrm{C}$ for 10 minutes before being centrifuged for 10 minutes at 129g. The supernatant was discarded, and the cell pellet re-suspended in $5 \mathrm{ml}$ of fixative 2 (a 5:2-part solution of methanol and acetic acid respectively), again left at $4{ }^{\circ} \mathrm{C}$ for ten minutes prior to centrifugation and then centrifuged at $129 \mathrm{~g}$ for 10 minutes, this step was repeated three times. Cells could then be stored at $4^{\circ} \mathrm{C}$ for a maximum of 2 weeks or slides could be prepared immediately. Slide preparation required the slides (Academy Microscope $76 \mathrm{x}$ $26 \mathrm{~mm}$ (frosted at one end, both sides)) to be pre-soaked in fixative 2 (in a glass container) for at least 24 hours prior to making, on the day of which the fixative 2 was replaced with double distilled water. Slides were then wiped clean with slide tissue paper (Kimberly-Clark Professional) and left to air dry standing vertically. Cells previously incubated in $5 \mathrm{ml}$ of fixative 2 were centrifuged as detailed above and re-suspended in $1 \mathrm{ml}$ of fixative 2 . Following this $100 \mu \mathrm{l}$ of re-suspended cells were drop-pipetted onto the labelled slides. To ensure cells were evenly dispersed the reconstitution volume of $1 \mathrm{ml}$ of fixative 2 could be altered with each slide checked via light microscopy for over-crowding. For each concentration, 3-5 slides were prepared for true biological replicates. Slides were air dried vertically standing after which, slides could be stored at $-20^{\circ} \mathrm{C}$ for subsequent scoring. 
Scoring of slides at room temperature required a nuclear stain; $30 \mu \mathrm{l}$ of $4^{\prime} 6$, diamido-2-phenylindole (DAPI-Vectorshield) (Sigma, UK). Stained slides received coverslips of dimensions; $22 \mathrm{~mm} \times 60 \mathrm{~mm}$ and $0.17 \mathrm{~mm}$ thickness, these were placed in the dark to incubate for 20 minutes prior to scoring.

\subsubsection{Automated Metafer Scoring of Micronuclei}

To evaluate the genotoxicity of the carbonaceous ENMs, the automated Metafer microscope (Axioimager Z2 fluorescent microscope) (Carl Zeiss, UK) with an 8-slide mounting stage was employed and operated in darkness. A total of 2000 binucleated cells were scored per replicate (performed in triplicate), totalling 6000 binucleated (BN) cells per dose, this was performed on the Metafer 4 software (Table 2.2), version 3.5 (MetaSystems, Germany). Slides were scanned through an objective magnification of $x 10$ and checked more thoroughly at $x 40$ for the presence of abnormal genotoxic events such as nuclear bridges as well as correctly identifying / classifying a micronucleus which were counted with a handheld UPGREEN counter.

Table 2.2 Metafer microscope (Metafer 4, version 3.5) nuclei classifier settings

\begin{tabular}{|l|l|l|}
\hline Nuclei Parameter & Nuclei Classifier & Micronuclei Classifier \\
\hline Minimum Area & $20 \mu \mathrm{m}^{2}$ & $1.5 \mu \mathrm{m}^{2}$ \\
\hline Maximum Area & $400 \mu \mathrm{m}^{2}$ & $55 \mu \mathrm{m}^{2}$ \\
\hline $\begin{array}{l}\text { Maximum Relative Concave } \\
\text { Depth }\end{array}$ & 0.9 & 0.9 \\
\hline Maximum Aspect Ratio & 1.5 & 4 \\
\hline Maximum Distance & $30 \mu \mathrm{m}^{2}$ & $25 \mu \mathrm{m}^{2}$ \\
\hline
\end{tabular}




\subsubsection{Pan-Centromeric staining of Micronuclei}

To provide a quantitative measure of the clastogenic/aneugenic damage observed in the CBMN assay, pan-centromeric staining of the micronuclei and binucleated cells was performed. This technique has warranted a large degree of popularity when coupled to the CBMN assay for rapidly identifying aneugens and clastogens, which other assays such as the chromosome aberration does not accomplish unless specifically modified (Doak et al., 2012b). Centromeric staining provided an indicator as to whether the observed effect originated from clastogenic (double-strand DNA fragmentation) or aneugenic damage, based on the assumption an entire chromosome has been lost during cell division. For this, a second positive control had to be introduced to run against the MMC detailed earlier in section 2.12, this new positive control a published aneugenic compound Carbendazim at $1.6 \mu \mathrm{g} / \mathrm{ml}$ which provided a $40 \%$ reduction in RPD to $16 \mathrm{HBE} 140^{-}$cells. To perform the pan-centromeric staining, cells which had undergone the CBMN standard operating procedure (SOP) and had been lifted from their T25 flasks, instead of being re-suspended in $\mathrm{KCl}$, were instead resuspended in $5 \mathrm{ml}$ of pre-warmed PBS. Meanwhile microscope slides soaked in $90 \%$ methanol for a minimum of 20 minutes at $-20^{\circ} \mathrm{C}$ were air dried standing vertically and labelled accordingly. Cells were centrifuged at $4^{\circ} \mathrm{C}$ for 10 minutes at $129 \mathrm{~g}$ and then re-suspended in $1 \mathrm{ml}$ of ice-cold PBS, of which $100 \mu \mathrm{l}$ was centrifuged at $290 \mathrm{~g}$ for 1 minute onto respective slides and then dropped into ice cold $90 \%$ methanol fixative. Fixed slides could be stored at $-20^{\circ} \mathrm{C}$ or used immediately. Cells were first permeabilised with several drops (directly onto the cytodot) of $30 \mu \mathrm{g} / \mathrm{ml} \mathrm{Pepsin}$ (Sigma, UK) suspended in $0.01 \mathrm{M} \mathrm{HCl}(\mathrm{pH} 2.7-3)$ (Sigma, UK) pre-warmed to $37^{\circ} \mathrm{C}$. Slides were left with Pepsin/ $\mathrm{HCl}$ for 1 minute before being immersed in PBS for 5 minutes to arrest the Pepsin treatment and then immersed in $50 \mathrm{mM} \mathrm{MgCl}_{2} / \mathrm{PBS}$ solution for a further 5 minutes to protect the chromosomes from further chemical degradation. Fixed cells were then dehydrated in a series of ethanol washes of $70 \%, 80 \%$ and $95 \%$ for 2 minutes each, during which time the pan-centromeric probes (Cambio Ltd, UK) were thawed and mixed well by vortex at $37^{\circ} \mathrm{C}$ for 5 minutes. After air drying the slides, $5 \mu$ l of probe per slide was added to a microcentrifuge tube whilst the slides were immersed in $70 \%$ Formamide ( $>99.5 \%$, Sigma, UK)/ $2 x$ saline sodium citrate (SSC) where; $20 x$ SSC $-87.6 \mathrm{~g} \mathrm{NaCl}, 44.1 \mathrm{~g}$ Sodium Citrate, $500 \mathrm{ml}$ distilled water had been diluted $10 \mathrm{x}$, this step was performed for 2 minutes at $70^{\circ} \mathrm{C}$. Slides were then immersed in ice cold $70 \%$ ethanol and dehydrated through a series of ethanol washes at $70 \%, 90 \%$ and $100 \%$ before being air dried vertically standing. Pan-centromeric probes were de-natured at $85^{\circ} \mathrm{C}$ for 10 minutes and then immediately chilled on ice in microcentrifuge tubes. $5 \mu$ l of probe was applied to the cytodot on each slide and allowed to hybridise for 16 hours at $37^{\circ} \mathrm{C}$ in a humidified chamber (Raymond A Lamb Windosr Incubator) with coverslips applied. The following day, coverslips were removed, and the slides washed in 2x SSC solution for 5 minutes before a wash step in 50\% Formamide / 2x SSC for 5 
minutes each at $37^{\circ} \mathrm{C}$, this was repeated one more time. Slides were then counterstained with $30 \mu \mathrm{l}$ of DAPI-VECTASHIELD ${ }^{R}$ mounting medium for 20 minutes in the dark with coverslips applied.

\subsubsection{Manual Scoring of Centromeric Regions}

Scoring of centromere-stained cells was performed using the same microscope described in section 2.12.1, this time substituting the Metafer software for the Trackball to manually move through the slides in 10x objective magnification to locate BN/Mn cells and then using the ISIS software which allows the user to toggle between scanning the cells with two filters; DAPI (nuclear material stained blue) and FITC (centromeres stained green). To capture an image, the ISIS software first captured the cell through the blue filter then toggle to the green before overlapping them to provide one final image of the $\mathrm{Mn} / \mathrm{BN}$ cell. A micronucleus of a binucleated cell containing a minimum of one centromere was deemed an aneugenic response. A total of 105 micronuclei were scored per dose (35 per biological replicate), to compensate for this number, up to 5 slides were prepared per biological replicate to ensure the total number of micronuclei required to be scored was achieved.

\subsection{Quantification of (pro)-inflammatory mediators via enzyme linked immunosorbent assay (ELISA)}

Post ENM treatment as well as positive control (LPS at 100ng/ml) (\#L2630 Sigma, UK) of all cell culture models tested supernatants were harvested and stored at $-80^{\circ} \mathrm{C}$. The exposure media, containing both (pro)-inflammatory mediators and suspended carbonaceous ENM was then centrifuged $(16,000 \mathrm{~g}$ for 1 minute) to remove as much ENM as possible to avoid interference with the duo-set ELISA kits (R\&D Systems, UK). All ELISA experiments were performed in 96-well half area clear plastic bottom plates (Sigma, UK) which when required were first coated with capture antibody diluted down to a working concentration detailed in the manufacturer's certificate of analysis for cytokine interleukin (IL)- 6 and chemokine IL-8. Briefly, capture antibody was diluted down from $720 \mu \mathrm{g} / \mathrm{ml}$ to $4 \mu \mathrm{g} / \mathrm{ml}$ in $5 \mathrm{ml}$ of PBS and $50 \mu \mathrm{l}$ loaded into each of the 96-wells via multi-channel pipette and covered with parafilm and stored at $4^{\circ} \mathrm{C}$ for no longer than one week. Following at least 24 hours, capture antibody was aspirated and washed three times with $150 \mu$ l of ELISA wash buffer $\left(0.05 \%\right.$ Tween $^{20}$ (Sigma, UK)) in PBS. Following a thorough aspiration of wash buffer onto paper towels, $50 \mu$ l of block buffer (1\% BSA in PBS) was added to the plate for one hour whilst being gently rocked out of direct sunlight. During this time, frozen supernatant samples were thawed at room temperature and standards were prepared. To prepare a high standard working concentration of $2000 \mathrm{pg} / \mathrm{ml}$ (IL-8) or $600 \mathrm{pg} / \mathrm{ml}$ (IL-6), $10 \mu$ l of $100 \mathrm{ng} / \mathrm{ml}$ standard was diluted in $490 \mu \mathrm{l}$ of double distilled water. For a seven-point standard curve, a 
series of serial dilutions were performed as described in the manufacturer's guide. Following a onehour antibody block, the block buffer (1\%BSA PBS) was aspirated and the plate washed as described above. Subsequently $50 \mu \mathrm{l}$ of standards and samples were loaded into the plate and rocked at room temperature for a further two hours. During the remaining 30 minutes of the previous step, detection antibody was prepared by diluting stock antibody $(3.6 \mu \mathrm{g} / \mathrm{ml})$ to a working concentration $(20 \mathrm{ng} / \mathrm{ml})$ in $5 \mathrm{ml}$ of freshly prepared reagent diluent. After the two-hour sample incubation, the plate was aspirated and washed three times before $50 \mu \mathrm{l}$ of detection antibody was applied and the plate left to incubate for a further two hours. Following aspiration and washing of the plate, $50 \mu \mathrm{l}$ of freshly prepared streptavidin-HRP diluted down 200x from the stock concentration was pipetted to the plate and incubated at room temperature for 20 minutes. The plate was aspirated and washed for a final time before $50 \mu$ l of Substrate (R\&D Systems, UK) Reagents A \& B (1:1) was added and left for colour to develop in the dark for approximately 20 minutes or until colour had visibly developed across the entire standard range. This incubation was stopped by the addition of $50 \mu \mathrm{l}$ of $1 \mathrm{M}$ of (hydrochloric or sulfuric) acid to each well. Finally, within one hour of completing the assay, the optical density (OD) was recorded at a wavelength set to $450 \mathrm{~nm}$ with an Omega Multimode microplate reader (BMG LABTECH Ltd, UK). A standard curve was utilised to determine the concentration $(\mathrm{pg} / \mathrm{ml})$ of the samples using Microsoft Excel. IL-6 and IL-8 levels were quantified from biological replicates (triplicate).

\subsection{Quantification of intracellular reduced Glutathione (GSH)}

To quantify the intracellular GSH content of $16 \mathrm{HBE}^{-140^{-}}$cells, T25 flasks were setup accordingly with $1 \times 10^{5}$ cells $/ \mathrm{ml}$ and exposed to ENMs for 6 and 24 hours. A positive control of Staurosporine (diluted in cell culture medium $(1 \mu \mathrm{g} / \mathrm{ml})$ ) (Sigma, UK) was employed and exposed to the cells for 2 hours. This assay utilised a GSH kit purchased from Sigma, UK (\#CS1020). After this the exposure media was removed and the cells washed twice with pre-warmed PBS. Cells were lifted from flasks with TrypsinEDTA, neutralised with media and transferred to $15 \mathrm{ml}$ Falcon tubes, centrifuged at $600 \mathrm{~g}$ for 5 minutes. Cells were re-suspended with PBS and centrifuged for $600 \mathrm{~g}$ for another 5 minutes. The supernatant was discarded, the pellet re-suspended in $1 \mathrm{ml}$ of PBS and transferred to microcentrifuge tubes and spun down at $600 \mathrm{~g}$ for 5 minutes at room temperature. The supernatant was then discarded before $150 \mu$ l of $1 x$ Lysis Buffer (Sigma, UK) was added to the cell pellet and vortexed (IKA Vortex Genius 3) before being incubated on ice for 15 minutes and then centrifuged at 16,000g for 10 minutes. The supernatant was retained while the cell pellet was discarded, some of the supernatant $(5-10 \mu l)$ was retained and stored for total protein measurements either later or run alongside the GSH experiment. 
Once the supernatant had been obtained, a black, flat-bottom 96-well plate (Corning, UK) was setup as described in Table 2.3. The reaction was initiated by the final addition of the GST enzyme, the plate was left to incubate at $37^{\circ} \mathrm{C}$ for 1 hour. The plate was then read on a FLUOstar OMEGA Multimode microplate reader (BMG, LABTECH Ltd, UK) with the $\lambda$ excitation set to $390 \mathrm{~nm}$ and the $\lambda$ emission at $478 \mathrm{~nm}$. A standard curve was then used to convert arbitrary units of fluorescence to nmoles of intracellular GSH for the cell samples using Microsoft Excel.

Table 2.3 Standard Curve and Sample Procedure

\begin{tabular}{|l|l|l|l|l|l|}
\hline Sample & $\begin{array}{l}1 \mathrm{mM} \text { rGSH } \\
\text { Standard }(\mu \mathrm{l})\end{array}$ & Sample $(\mu \mathrm{l})$ & $\begin{array}{l}\text { Assay Buffer } \\
(\mu \mathrm{l})\end{array}$ & $\begin{array}{l}\text { GST Enzyme } \\
(\mu \mathrm{l})\end{array}$ & $\begin{array}{l}\text { Substrate } \\
\text { Solution }(\mu \mathrm{l})\end{array}$ \\
\hline Blank & - & - & 92.5 & 5 & 2.5 \\
\hline $\begin{array}{l}\text { GSH Standard } \\
1.3 \text { nmoles }\end{array}$ & 1.3 & - & 91.0 & 5 & 2.5 \\
\hline $\begin{array}{l}\text { GSH Standard } \\
2.5 \text { nmoles }\end{array}$ & 2.5 & - & 90.0 & 5 & 2.5 \\
\hline $\begin{array}{l}\text { GSH Standard } \\
5.0 \text { nmoles }\end{array}$ & 5 & - & 87.5 & 5 & 2.5 \\
\hline $\begin{array}{l}\text { GSH Standard } \\
10 \text { nmoles }\end{array}$ & 10 & - & 82.5 & 5 & 2.5 \\
\hline Sample & - & 5 & 87.5 & 5 & 2.5 \\
\hline Where; GST - Glutathione & Transferase, catalyses the & bonding of poorly fluorescent \\
\hline
\end{tabular}

monochlorobimane (probe) to reduced GSH to become a strongly fluorescent adduct. 


\subsection{Determining Reactive Oxygen Species (ROS)}

Intracellular detection of ROS was undertaken with the fluorescent 2', 7'-dichlorofluorescein (DCFHDA) assay (Abcam, UK). This isotype of the chemical was selected for its enhanced retention within the cell (Tetz et al., 2013). This assay was applied to 16HBE140 adherent cells which were seeded at $100 \mu \mathrm{l}$ of $1 \times 10^{5}$ per well in black sided, clear bottom 96 well plates (Corning, Germany). Cells were incubated overnight for 24 hours at $37^{\circ} \mathrm{C}$. The following day, the media was aspirated, and the cells washed with pre-warmed PBS by multi-channel pipette delivering $100 \mu$ l per well. Once aspirated the cells were incubated with $100 \mu \mathrm{l}$ of $20 \mu \mathrm{M}$ of DCFH-DA fluorescent marker (re-suspended on the day of the assay in PBS and diluted down 400x with media) the cells were then incubated for a further 1 hour at $37^{\circ} \mathrm{C}$, to allow time for the passive uptake of the dye into the cells. Following this the cells were washed once again with PBS and treated with ENMs across a concentration range and maintaining a positive control of freshly opened hydrogen peroxide $\left(\mathrm{H}_{2} \mathrm{O}_{2}\right)$ at $100 \mu \mathrm{M}$. Intracellular ROS activity was measure at timed intervals beginning at 30 minutes and including a measurement at 24 hours posttreatment. Acellular detection of ROS was performed by deacetylation with $10 \mathrm{mM}$ of sodium hydroxide at room temperature in the dark for 30 minutes prior to dilution to working concentration of $20 \mu \mathrm{M}$ of DCFH-DA and plated for assay. Measurements were performed on the FLUOstar OMEGA Multimode microplate reader (BMG, LABTECH Ltd, UK) with the $\lambda$ excitation set to $485 \mathrm{~nm}$ and the $\lambda$ emission at $520 \mathrm{~nm}$.

\subsection{Mitochondrial toxicity}

To identify whether the ENMs elicit a stress response in the mitochondria of the $16 \mathrm{HBE}^{-14 \mathrm{O}^{-}}$and TT1 cells, a series of experiments were set up to investigate this biological response. Prior to mitochondrial toxicity testing, several parameters were optimised those being the seeding densities for both epithelial cells lines and the injection concentrations. The initial optimisation step began with a cell titration over $20,40,60,80,100$ and $120 \times 10^{3}$ cells $/ \mathrm{ml}$ seeded $(100 \mu \mathrm{l})$ into the XFe24 tissue culture plates (Seahorse Bioscience) allowing 3 spaces free for blank (media only) measurements. On the same day, $1 \mathrm{ml}$ of Seahorse Calibrant (Seahorse Bioscience) was placed into the XFe24 Flux plate and placed into a non- $\mathrm{CO}_{2}$ oven until required (no more than 72 hours). The following day, cells were topped up with growth media $(150 \mu \mathrm{l})$, it was at this stage cells were treated with ENMs and incubated for a further 24 hours. On the third day, injections were made up with XF Assay Media supplemented with $1 \mathrm{mM}$ sodium pyruvate (Sigma, UK), 5.5mM D-(+)-glucose (Sigma, UK) and 1mM L-Glutamine (ThermoFisher) (Oligomycin $(1 \mu \mathrm{M})$, FCCP $(1 \mu \mathrm{M})$ and Rotenone/Antimycin A $(0.5 \mu \mathrm{M})$ using the MitoStress Test Kit (Seahorse Bioscience) as follows in Table 2.5. XF Assay media had undergone pH 
equilibration to $7.34+/-0.05$ and filter sterilised through a $0.22 \mu \mathrm{m}$ filter (Millipore, UK) with a $20 \mathrm{ml}$ syringe. Mitochondrial stress was measured via the initial addition of Oligomycin to block ATP synthase. Then, carbonyl cyanide-p-trifluoromethoxyphenylhydrazone (FCCP), a proton ionophore which acts to remove proton gradient regulatory mechanisms, is injected allowing the maximum respiration rate to be determined. Finally, Rotenone and Antimycin A inhibit complex I and III, respectively, of the electron transport chain. XF Assay media (Seahorse Bioscience) was supplemented with $5.5 \mathrm{mM}$ glucose and $1 \mathrm{mM}$ pyruvate as pre-optimized for use with the Mito-Stress kit (Seahorse Bioscience). Once optimised, $120 \times 10^{3}$ (16HBE14o-) or $100 \times 10^{3}$ (TT1) cells $(100 \mu \mathrm{l} /$ well) formed an evenly dispersed monolayer (inspected via light microscopy). On test day, $200 \mu$ l growth media was removed and the cells washed twice with $600 \mu$ l pre-warmed XF assay media before a final $475 \mu$ I was added to each well (final volume $525 \mu \mathrm{l}$ ). The tissue culture plate was then incubated in a non- $\mathrm{CO}_{2}$ oven for 1 hour at $37^{\circ} \mathrm{C}$. After one-hour incubation the cells were processed using the $\mathrm{XFe} 24$ Extracellular Flux Analyser (Seahorse Bioscience). Mitochondrial function was measured as oxygen consumption rate (OCR) and extracellular acidification rate (ECAR) after $75 \mu$ injections of $1 \mu \mathrm{M}$ Oligomycin (ATP synthase inhibitor), $1 \mu \mathrm{M}$ FCCP (electron transport chain accelerator) and $0.5 \mu \mathrm{M}$ Antimycin A (complex III inhibitor) plus $0.5 \mu \mathrm{M}$ rotenone (complex I inhibitor), according to the manufacturer's instructions (Table 2.4).

Table 2.4 Injection strategy for mitochondrial stress test.

\begin{tabular}{|l|l|l|l|}
\hline Injection & $\begin{array}{l}\text { Assay media added to } \\
\text { stock pellet }(\mu \mathrm{l})\end{array}$ & $\begin{array}{l}\text { Volume of stock } \\
\text { removed to prepare } \\
\text { final injection }(\mu \mathrm{l})\end{array}$ & $\begin{array}{l}\text { Volume of assay media } \\
\text { required to prepare } \\
\text { final injection }(\mu \mathrm{l})\end{array}$ \\
\hline Oligomycin & $630(100 \mu \mathrm{M})$ & 240 & $2760(1 \mu \mathrm{M})$ \\
\hline FCCP & $720(100 \mu \mathrm{M})$ & 270 & $2730(1 \mu \mathrm{M})$ \\
\hline Antimycin A/Rotenone & $540(500 \mu \mathrm{M})$ & 300 & $2700(0.5 \mu \mathrm{M})$ \\
\hline
\end{tabular}

Once the injection compounds had been pipetted into the flux plate this was inserted into the Seahorse XFe24 Bioanalyser and calibrated for 20 minutes. After this was completed, the Flux plate tray was removed and swapped with the tissue culture plate removed from the non- $\mathrm{CO}_{2}$ oven and run in the seahorse for approximately 96 minutes. Data was collected and transferred to Microsoft Excel for i) normalising against protein content and ii) subsequent analysis on metabolic parameters, OCR 
and ECAR. Once the optimum seeding density had been achieved for both epithelial cell lines, the injection compound concentrations were then optimised in a new series of experiments to identify any significant changes to the bioenergetics of the cell lines. The other injection concentrations investigated during the optimisation were Oligomycin $0.5 \& 2 \mu \mathrm{M}$ as well as FCCP at $0.5 \& 2 \mu \mathrm{M}$, the concentration of Antimycin A/Rotenone however did not require optimisation. The seeding density was considered optimised and used for future experiments when the basal rates of OCR and ECAR were not significantly promoted or decreased and the signal was deemed to have reached a plateau whilst the injection compound concentration which produced the greatest effect was selected for future experiments (Nicholls, 2002). To normalise the data sets, protein content per well was selected for a simple and rapid analysis of total protein. To perform this, the tissue culture plate was removed from the Seahorse Bioanalyser and the cells were rinsed twice with $200 \mu$ lice cold PBS, following this $50 \mu$ l of RIPA Buffer was added to each well and mixed thoroughly via pipette. The cells were then incubated at $4^{\circ} \mathrm{C}$ for 5 minutes before the suspension was transferred to a microcentrifuge tube and centrifuged at $10,000 \mathrm{~g}$ at $4^{\circ} \mathrm{C}$, the resulting supernatant was then used for DC Protein assays detailed in Section 2.9.

\subsection{Total Cellular Protein}

Total cellular protein measurements were required for data sets to be normalised, namely the GSH and mitochondrial toxicity data. The DC Protein assay kit (Bio-Rad, UK) was selected for a rapid and simple procedure of determining the protein concentrations of sample supernatants harvested during the SOP's. Protein supernatants which had been harvested from sections 2.14 and 2.16 respectively were thawed from a $-20^{\circ} \mathrm{C}$ storage when required. To measure the concentration, a series of protein standards were prepared using a protein standard of BSA (Bio-Rad, UK) stock concentration $2.5 \mathrm{mg} / \mathrm{ml}$ and a diluent of RIPA buffer (ThermoFisher, UK). Standards were set up as detailed in Table 2.5. To perform the DC assay, working reagent $A^{\prime}$ was prepared by adding $20 \mu$ l of Reagent S per ml of Reagent A. Then $5 \mu$ l of standards and protein supernatant samples were added to the plate in triplicate before adding $25 \mu \mathrm{l}$ of working reagent $A^{\prime}$ to every well followed by $100 \mu$ l of Reagent $B$ to every well. Each well was gently agitated with a hand-held pipette tip before the plate was incubated and left to develop a cyan-blue colour change for 15 minutes. The plate was then read inside the hour at $750 \mathrm{~nm}$ on an Omega Multimode microplate reader (BMG LABTECH Ltd, UK). Cellular protein data can be viewed in the Appendix Section 7.1. 
Table 2.5 Protein Standard Curve setup for a 96-well half area clear plastic plate (Sigma, UK)

\begin{tabular}{|l|l|l|}
\hline Final Concentration $(\mathrm{mg} / \mathrm{ml})$ & BSA stock at $2.5 \mathrm{mg} / \mathrm{ml}(\mu \mathrm{l})$ & RIPA Buffer $(\mu \mathrm{l})$ \\
\hline 0 & 0 & 20 \\
\hline 0.5 & 4 & 16 \\
\hline 1.0 & 8 & 12 \\
\hline 1.5 & 12 & 8 \\
\hline 2.0 & 16 & 4 \\
\hline 2.5 & 20 & 0 \\
\hline
\end{tabular}

\subsection{Sodium Dodecyl Sulfate Polyacrylamide Gel Electrophoresis (SDS-PAGE)}

To identify the serum protein corona of the ENMs, SDS-PAGE gels were constructed from the chemical recipe listed in Table 2.6. Prior to ENM preparation, glass plates were pre-washed with ethanol and assembled in a casting stand. First the resolving gel was prepared by adding the chemical ingredients to a $50 \mathrm{ml}$ Falcon tube at room temperature and pipetted into the glass plate mould with a $10 \mathrm{ml}$ pipette, leaving $\sim 1$ inch of space for the stacking gel. Finally, $5 \mu$ l of isopropanol were added to eliminate air bubbles at the surface. Once polymerised (checked by slightly angling the casting stand) the stacking gel was prepared, again with a $50 \mathrm{ml}$ Falcon tube and overlaid onto the resolving gel and a 10-well comb (Bio-Rad 1.5mm) inserted and left to polymerise for one hour. Once prepared, gels could be stored in damp paper towels at $4^{\circ} \mathrm{C}$ for up to two weeks. If gels were being run the same day, they were placed into an electrode assembly tank (Bio-Rad, UK) topped up with Running Buffer (25mM Tris, $192 \mathrm{mM}$ glycine and $0.1 \%$ SDS). To estimate the protein sizes as they appear, $2 \mu \mathrm{l}$ of a protein kaleidoscope was purchased (from Bio-Rad, UK) and always occupied lane 1 of the gel whilst $15 \mu$ l of samples occupied the other lanes. SDS-PAGE gels were always run at $150 \mathrm{~V}$ and stopped when the band-front had reached the bottom of the glass mould. 
Table 2.6 10\% Resolving and 4\% Stacking gel recipes for SDS-PAGE.

\begin{tabular}{|l|l|l|}
\hline Ingredient List & $10 \%$ Resolving Gel & $4 \%$ Stacking Gel \\
\hline Double distilled water & $6 \mathrm{ml}$ & $3 \mathrm{ml}$ \\
\hline 1.5 M Tris (Sigma, UK) & $3.75 \mathrm{ml}$ & N/A \\
\hline $1 \mathrm{M}$ Tris (Sigma, UK) & N/A & $1.25 \mathrm{ml}$ \\
\hline $\begin{array}{l}\text { 30\% Acrylamide } \\
\text { (ThermoFisher, UK) }\end{array}$ & $5 \mathrm{ml}$ & $650 \mu \mathrm{l}$ \\
\hline $\begin{array}{l}10 \% \text { SDS (Sigma, UK) } \\
\begin{array}{l}10 \% \text { Ammonium Persulfate } \\
\text { (APS) (Sigma, UK) }\end{array}\end{array}$ & $75 \mu \mathrm{l}$ & $50 \mu \mathrm{l}$ \\
\hline $\begin{array}{l}\text { Tetramethylethylenediamine } \\
\text { (TEMED) (ThermoFisher) }\end{array}$ & $15 \mu \mathrm{l}$ & $25 \mu \mathrm{l}$ \\
\hline
\end{tabular}

Note; TEMED was always added last and performed in a Class II Fume Hood.

\subsection{Transmission Electron Microscopy (TEM)}

TEM analysis on ENM-cell interactions was performed at Leeds University in a collaboration with $\mathrm{Dr}$ Andy Brown and Dr Nicole Hondow at the institute of Material Research, School of Process, Environmental and Materials Engineering, University of Leeds, Leeds. The analysis was performed with a FEI Titan3 Themis G2 operating at 300kV fitted with 4 EDX silicon drift detectors, and a Gatan OneView CCD. EDX spectroscopy and mapping was undertaken using Bruker Esprit v1.9 software.

\subsubsection{Resin embedding of treated cells}

Cells treated with ENMs required fixing, embedding and sectioning (70nm sections) prior to TEM analysis, these stages were performed at Swansea University. Following ENM treatment of epithelial cell lines and co-cultures, cells were washed and trypsinised in maintenance buffer before being placed into $0.5 \mathrm{ml}$ microcentrifuge tubes. 
Phosphate buffered components

- $200 \mathrm{mM}$ di-sodium hydrogen orthophosphate dihydrate $\left(\mathrm{Na}_{3} \mathrm{HPO}_{4} \mathrm{H}_{2} \mathrm{O}\right)$ (Sigma, UK) dissolved in $250 \mathrm{ml}$ double distilled water

- $200 \mathrm{mM}$ sodium dihydrogen orthophosphate monohydrate $\left(\mathrm{NaH}_{2} \mathrm{PO}_{4} \mathrm{H}_{2} \mathrm{O}\right)$ (Sigma, UK) dissolved in $100 \mathrm{ml}$ double distilled water

Maintenance Wash Buffer

- $34.22 \mathrm{~g}$ sucrose (Sigma, UK) dissolved in $50 \mathrm{ml}$ double distilled water, $62.5 \mathrm{ml}$ of sucrose solution then transferred to a $500 \mathrm{ml}$ vessel and $187.5 \mathrm{ml}$ double distilled water added.

- Following this $128 \mathrm{ml}$ of $200 \mathrm{mM} \mathrm{Na} \mathrm{HPO}_{4} \mathrm{H}_{2} \mathrm{O}$ and $38 \mathrm{ml}$ of $\mathrm{NaH}_{2} \mathrm{PO}_{4} \mathrm{H}_{2} \mathrm{O}$ were added to a second $500 \mathrm{ml}$ vessel which was then adjusted to $\mathrm{pH} 7.3$.

- Lastly, $165 \mathrm{ml}$ of the freshly prepared phosphate buffer was added to the sucrose solution, mixed for 20 minutes before being stored at $4^{\circ} \mathrm{C}$.

Primary Fixative (2.5\% Glutaraldehyde / 100mM phosphate buffer)

- $77 \mathrm{ml}$ of $\mathrm{Na}_{3} \mathrm{HPO}_{4} \mathrm{H}_{2} \mathrm{O}, 23 \mathrm{ml}$ of $\mathrm{NaH}_{2} \mathrm{PO}_{4} \mathrm{H}_{2} \mathrm{O}$ and $100 \mathrm{ml}$ of double distilled water were added to a $100 \mathrm{ml}$ beaker, stirred and $\mathrm{pH}$ adjusted to 7.35 .

- Following this $190 \mathrm{ml}$ of this freshly prepared solution was transferred to $500 \mathrm{ml}$ vessel plus $10 \mathrm{ml}$ of vacuum distilled EM grade $50 \%$ glutaraldehyde (Agar Scientific) added, mixed and stored at $4^{\circ} \mathrm{C}$ in the dark wrapped in aluminium foil.

Secondary Fixative (1\% Osmium Tetroxide)

- Solution $1-2.26 \%$ sodium dihydrogen orthophosphate (Sigma, UK) prepared by dissolving $0.565 \mathrm{~g}$ in $25 \mathrm{ml}$ double distilled water

- Solution $2-2.52 \%$ sodium hydroxide (Sigma, UK) was prepared by dissolving $0.63 \mathrm{~g}$ in $25 \mathrm{ml}$ double distilled water

- Solution 3-5.4\% glucose was prepared by dissolving $0.135 \mathrm{~g}$ in $2.5 \mathrm{ml}$ distilled water

- Finally, $20 \mathrm{ml}$ of solution 1 and $4.25 \mathrm{ml}$ of solution B was added to a $100 \mathrm{ml}$ bottle, mixed and $\mathrm{pH}$ adjusted to 7.3 . Then $2.5 \mathrm{ml}$ of Solution 3 was added to make the final precursor solution. Following this, a $0.25 \mathrm{~g}$ vial of osmium tetroxide was shattered in a $250 \mathrm{ml}$ Duran before $25 \mathrm{ml}$ of the precursor solution was added. This final osmium fixative was incubated in the dark for four hours at $4^{\circ} \mathrm{C}$ before being filtered into a fresh bottle and stored appropriately at $4^{\circ} \mathrm{C}$ in the dark until required (stable for 1 month). 
Cell pellets were later re-suspended in $100 \mathrm{mM}$ phosphate buffered $2.5 \%$ glutaraldehyde fixative at pre-warmed to $37^{\circ} \mathrm{C}$ for 15 minutes. Cells were centrifuged at $290 \mathrm{~g}$ for 10 minutes and re-suspended in fresh glutaraldehyde fixative at $4^{\circ} \mathrm{C}$ for 4 hours before the fixative was aspirated and $0.5 \mathrm{ml}$ of maintenance buffer placed over cell pellets and left to incubate overnight. The following day, cells were centrifuged at $290 \mathrm{~g}$ for 10 minutes and washed in maintenance buffer. Cells were later fixed in $1 \%$ osmium tetroxide fixative for 90 minutes at $4^{\circ} \mathrm{C}$ rocking in the dark. Following this fixation step, cells were pelleted at $400 \mathrm{~g}$ for 10 minutes with the fixative aspirated once complete. TAAB Premix resin (TAAB Laboratory and Microscopy Reagents, UK) was prepared by adding the polymerising agent to the resin and placed on a roller for 1 hour, after which an accelerating agent could be added. Cells were then dehydrated through a series of ethanol washes at $10 \%$ for 10 minutes, $70 \%$ for 30 minutes and lastly $100 \%$ ethanol for 20 minutes each with occasional mixing throughout. Cell samples were placed into $100 \%$ propylene oxide for 20 minutes and performed twice in total before adding 1:1 resin and propylene oxide for a 90-minute incubation. Cells were finally placed into $100 \%$ resin and left overnight and chilled at $4^{\circ} \mathrm{C}$. The next day, fresh resin was brought to room temperature and placed onto the cells before transferring the cells to an oven set at $60^{\circ} \mathrm{C}$ for 24 hours, allowing for evaporation by leaving the caps of the microcentrifuge tubes open.

\subsubsection{Ultramicrotomy sectioning of embedded samples}

To excise the resin cylinders from their $0.5 \mathrm{ml}$ microcentrifuge tubes, glass knives were prepared using an EM-KMR3 (Leica Microsystems, UK) to shape right-angled triangle glass knives from a block of glass. The glass knives were mounted to an EM-UC7 ultramicrotome (Leica Microsystems, UK) and used to cut the $0.5 \mathrm{ml}$ microcentrifuge tubes away from the resin cylinders. Resin blocks were trimmed at 100 $\mathrm{mm} /$ second with distance set to $150 \mathrm{~nm}$, continuing this approach until the resin bore a flat edge at the forefront (i.e. encompassing the cell pellet). From this now-squared face, dimensions to be cut were as follows; $750 \mu \mathrm{m}$ length $\times 750 \mu \mathrm{m}$ width $\times 50 \mu \mathrm{m}$ in depth at $100 \mathrm{~mm} / \mathrm{second}$ with a $100 \mathrm{~nm}$ approach speed. Final sections were cut at $70 \mathrm{~nm}$ thickness using an Ultra $45^{\circ}$ diamond knife (Diatome, Switzerland) with a $1 \mathrm{~mm} /$ second cutting speed. Subsequent sections could float away from the diamond knife into the water basin of the Ultra $45^{\circ}$ diamond knife and retrieved with tweezers holding a 150-mesh copper grid (Agar Scientific, UK). Copper sample grids bore a 3.5nm carbon film coating previously installed with a Q150-TE carbon coater (Quorum Technologies, UK). Sections were then stored for transport to Leeds University for imaging as detailed in section 2.11. 


\subsection{Statistical Analysis}

All data is shown as the mean +/- standard deviation. Statistical analysis was performed in SPSS statistics software (v.20 IBM, UK) where a one-way analysis of variance (ANOVA) was performed with post hoc Dunnett's multiple comparisons applied to evaluate pairwise statistical significance between control and exposure concentration data sets, the alpha value was set to 0.05 . All data sets were analysed beforehand for normality (Shapiro-Wilk test, $p \leq 0.05$ ) and for equal variance $p \leq 0.05$ ) all biological replicates were $\mathrm{N}=3$, unless otherwise stated. 


\section{Chapter 3 - Quantitative and Qualitative Analysis of Few-Layer Graphene's Physico-Chemical Features}

\subsection{Introduction}

Due to the overwhelming interest in ENMs owing to their novel uses in biomedical, mechanical and physical sciences, therein lies a requirement to understand the physico-chemical properties. Novel ENMs are manufactured with a desired function in mind, which typically relies on the physico-chemical features to achieve the desired end result, such as drug delivery to a specific cell type or location in the body (Aggarwal et al., 2009). ENMs can also be designed to resist mechanical strain or enhanced therapeutic tracking in vivo (Bae et al., 2010). The importance of physico-chemical characterisation has become paramount in the determination of ENM toxicology, particularly in the last decade where studies have screened the effect of nano versus micron-sized particles (Kohane, 2007). The scientific community agree that size is a crucial factor where toxicology is concerned; the consensus being smaller ENMs can gain access to cellular regions that are otherwise guarded against larger micronsized ENMs. This plays an ever important role in ENM-lung toxicology which has been extensively reported in the literature (Braakhuis et al., 2014), (Ferin et al., 1992), (Su et al., 2016). Whilst there exist some studies which contradict the assumption that smaller ENMs pose the greater toxicological risk, what is universally agreed on is the importance of fully characterising novel ENMs ideally with a panel of synergistic and supportive techniques.

One of the obstacles with measuring ENMs physico-chemical features is that there exist two states. Primary physico-chemical features (are those that reflect the initial state of matter immediately post manufacture). Secondary physico-chemical features, are those that result following, ENMs immersion in a test system in situ such as in vitro cell culture media, micro-tissue fluid, blood plasma etc (Sayes and Warheit, 2009). Thus, the primary features to be characterised include; size surface area, surface charge and surface chemistry; and then these same features must be monitored and recorded in secondary states as they can all be subjected to weathering and change in a biological environment (Scott-Fordsmand et al., 2017). Ideally, physico-chemical characterisation of ENMs should be monitored at 4 key stages during their shelf life; 1) Production 2) Transport 3) Use and 4) Disposal. This constant monitoring could shed new light on the weathering of unique ENMs and highlight unwanted effects during the weathering process such as degradation or oxidation (Nowack et al., 2016, Scott-Fordsmand et al., 2017). 


\subsubsection{Aims of Chapter 3}

The purpose of Chapter 3 was to characterise; Neutral-, Amine-, Carboxyl-FLG and CB particles (human type-1A carcinogen) in relation to their primary and secondary physico-chemical characteristics. Understanding the secondary physico-chemical characteristics was of great importance in this study as suspension of the ENM powder in physiological buffer (water, cell culture media) caused dramatic changes to the parameters measured in the primary state. Defining the physico-chemical features of these materials is of paramount importance as they can directly dictate cytotoxicity and genotoxicity under in vitro conditions. Thus, defining the characteristics which implicate the ENMs in adverse biological harm could ultimately lead to safer-by-design manufacturing. Given the awkward nature of a near-two-dimensional (2D) material such as FLG, characterising the material required a battery of both chemical and physical techniques to provide a robust definition of the features exhibited by these ENMs. 


\subsection{Materials and Methods}

\subsubsection{ENM Suspension}

FLG and CB particle suspension used during the characterisation process has been explicitly detailed in the Materials and Methods section (Chapter 2 section 2.1). Samples were maintained at $37^{\circ} \mathrm{C}$ prior to measurements if ENM suspension in a physiological buffer was required.

\subsubsection{ENM Preparation for DLS}

DLS was performed using the Malvern Zetasizer Nano ZS (Malvern Instruments, UK). All ENMs were initially diluted to $100 \mu \mathrm{g} / \mathrm{ml}$ and incubated for one hour at $37^{\circ} \mathrm{C}$ prior to performing measurements. ENM dilutions were performed using either $10 \% \mathrm{FBS}$ media or double distilled water to a final volume of $1 \mathrm{ml}$ in $1.5 \mathrm{ml}$ eppendorf vessels. The standard operating procedure (SOP) for DLS measurements was setup on the Malvern Zetasizer software version 7.02 coupled to the device which required the input of ENM optical properties such as refractive index, and diluent characteristics including density and viscosity, outlined below in Table 3.1. At the time of measurement, $100 \mu$ l of sample was transferred to plastic-grade micro cuvettes (Sigma-Aldrich, UK) and placed into the measurement chamber and allowed to equilibrate for two minutes. Once initiated, each replicate $(n=3)$ measurement consisted of 15 runs, which were averaged. Analysis of data collected was performed using the Malvern Zetasizer software v.7.02. This software would provide average data sets of Size and Zeta Potential with an option to extract raw data which was required to calculate mean, median and range values. 
Table 3.1 Dispersant Characteristics required for DLS measurements (MEM \& DCCM-1)

\begin{tabular}{|c|c|c|}
\hline $\begin{array}{l}\text { Physico-chemical feature of } \\
\text { test system }\end{array}$ & Double distilled water & $\begin{array}{l}\text { 10\% Serum, } 1 \% \text { glut, } 1 \% \mathrm{P} / \mathrm{S} \text {, } \\
88 \% \text { Media }\end{array}$ \\
\hline Density $\left(\mathrm{g} / \mathrm{cm}^{3}\right)$ & 1.004 & 1.011 \\
\hline Viscosity (cP) & 0.696 & 0.753 \\
\hline Refractive Index & 1.340 & 1.343 \\
\hline
\end{tabular}

Where: Serum - Foetal Bovine Serum (FBS) Minimum Essential Medium (MEM) or New-born Calf Serum (NCS) (DCCM-1); L-Glu - Tissue Culture-grade Glutamine; P/S - Penicillin/Streptomycin.

\subsubsection{Zeta (Ъ) Potential}

Investigation into the zeta potential across each ENM began with identical handling of materials as described in Section 2.2 for DLS measurements. First, $500 \mu$ l of ENM was injected into a plastic-grade U-bend, folded capillary cell (\# DTS1060, Malvern, UK) with a $1 \mathrm{ml}$ syringe and sealed at both ends. The cell was then loaded into the same chamber detailed above and allowed to equilibrate for 2 minutes before measurements. To measure the zeta potential, a different experimental SOP was loaded, performing 3 measurements of 15 runs each with the dielectric current maintained throughout at $74.5 \mathrm{mV}$ and Henry's function set to the Smoluchowski-Kramers approximation ( $F=1.5)$ with intent to describe Brownian motion (erratic particle movement gained from the microscopic collisions of surrounding particles in fluid). Data was collected and analysed using the Malvern Zetasizer software version 7.02 .

\subsubsection{Scanning Electron Microscopy \& Energy Dispersive X-Ray (EDX) Spectroscopy}

Investigation into the lateral dimensions of the dry ENMs began by performing SEM. Firstly, the dry powder ENMs were carefully loaded (by sterile spoon) onto a disposable adhesive pad before being mounted onto an aluminium stub (SEM Clip; $32 \mathrm{~mm}$ x 10mm x M4 (3 clips)) (Agar Scientific). The sample was then loaded into the SEM vacuum chamber before being positioned into the path of the electron beam. Analysis was performed using the Hitachi Ultra High-Resolution field emission (FE)SEM model number: S-4800 run at $15 \mathrm{kV}$. EDX was performed by preparing and mounting the sample as described above, however once mounted the sample was subsequently angled and analysis performed at a tilt of $20^{\circ}$ using the INCA EDX energy software coupled to the SEM interface. 
Measurements were performed by either analysing an entire area of material as viewed through the microscope or through highlighting a single particle and firing multiple beams at specific regions either the periphery or the centre. Multiple measurements were performed to quantify the percentage (\%) composition of key elements such as carbon, oxygen, nitrogen while also scanning for contaminant heavy metals. Images were transferred to $12 x$ CD-RW (Verbatim) and analysed with ImageJ software by focusing on one image and subsequently investigating the lateral diameter of individual particles $(\mathrm{N}=121)$.

\subsubsection{Plunge Freeze Analysis of ENMs}

Plunge freeze SEM analysis of FLG samples was performed at Leeds University in a collaboration with Dr Andy Brown and Dr Nicole Hondow at the Institute of Material Research, School of Process, Environmental and Materials Engineering, University of Leeds, Leeds, LS2 9JT. The plunge freeze SEM provided a unique "real-time" image capture of the particles/agglomerates as they would be perceived in suspension. The technique utilises such a rapid freezing in liquid ethane $\left(-182^{\circ} \mathrm{C}\right)$, even crystalline structure which could form via liquid nitrogen freezing techniques are inhibited from forming and corrupting the agglomerate integrity. Liquid Nitrogen is avoided in this analysis due to the boiling off process by which rapid freezing of copper grids will induce cubic crystals to form. Samples (all FLG ENMs) were prepared by mixing $990 \mu$ l of stock FLG with $10 \mu \mathrm{l}$ of FBS. After 20 minutes sonication the suspension was prepared for SEM by the following route. Continuous carbon films supported by copper TEM grids (\#AGS160-4, Agar Scientific) were plasma cleaned for 10s before loading with $3.5 \mu \mathrm{l}$ droplets of the FLG-media suspension inside the preparation chamber of the Mark IV Vitrobot ${ }^{\mathrm{TM}}(\mathrm{FEI})$, which was maintained $22^{\circ} \mathrm{C}$ and $100 \%$ humidity. $\mathrm{A} 30$ s delay was applied to ensure the droplet was equilibrated before blotting and vitrification through rapid plunge freezing into liquid ethane. Prepared grids were transferred into a vacuum desiccator and then allowed to warm to room temperature thereby preserving the original particle agglomeration state. Samples were imaged by SEM using a Hitachi SU8230 cold field emission gun SEM operating at 15kV. Sizing data was generated using the measure function of the ImageJ software, data output was then transferred to Excel to plot histograms for each ENM.

\subsubsection{Atomic Force Microscopy}

AFM analysis of FLG ENMs was performed in Swansea University, Institute of Life Sciences 1 (ILS1) by Dr Sandra B.C. Lopez in collaboration with the Head of Physics, Professor Ken Meissner of the College 
of Engineering. To produce topographical images of the FLG and investigate the depth of agglomerates, water-suspended stock ENM was diluted down (with ultrapure $\mathrm{H}_{2} \mathrm{O}$ ) to $100 \mu \mathrm{g} / \mathrm{ml}$ before $10 \mu \mathrm{l}$ was pipetted onto an air-brushed mica slide and analysed using a Bruker Dimension Icon AFM optimised for scanning and obtaining depth and optional lateral dimensions of the FLG agglomerates. A diamond tapping tip (Bruker tip MPP21000 RSFP 3N/m) and the SCANASYST-AIR, an image-optimization scanning (tapping) mode were used to image the samples. Utilising the SCANASYST-AIR, the Bruker Dimension Icon AFM could monitor progression and movement through the sample and feedback image quality allowing optimised data acquisition. The probe tip used for this analysis was the SCANASYST-AIR and cantilever parameters for all measurements were; Thickness $(T): 650 \mathrm{~nm}$, Length (L): $115 \mu \mathrm{m}$, Width $(\mathrm{W}): 25 \mu \mathrm{m}$, resonance frequency $\left(\mathrm{f}_{0}\right): 70 \mathrm{kHz}$, spring constant (k): $0.4 \mathrm{~N} / \mathrm{m}$. Topographical images were analysed for differences between materials and quantifying layer number with the Bruker Nanoscope Analysis Package version 1.4.

\subsubsection{Raman Spectroscopy}

Image capture of FLG ENMs utilising the Raman Spectroscopic technique was performed by Dr Adam Williams, formerly of Swansea University College of Engineering ILS2. Data produced was then graphed using the coding package Wolfram Mathematica software. FLG ENMs were investigated for their layer number, surface impurities and structural integrity via Raman Spectroscopy (Renishaw inVia Reflex Spectrometer System for Raman spectral / imaging analysis at 533nm). Through rapid acquisition, data collection and live feedback was provided with spatial resolution ranging from $>1 \mathrm{~mm}$ to $<1 \mu \mathrm{m}$. Prior to Raman spectroscopy, all three variants of the stock FLG; Neutral-, Amine- and Carboxyl-FLG were diluted down to $100 \mu \mathrm{g} / \mathrm{ml}$ with ultrapure water and dried onto silicon chips and placed inside the Raman spectrometer sample chamber. Raman spectra of each material was produced highlighting the characteristic peaks common to all graphitic ENMs namely the $D, G$ and 2D peaks with some peaks uncommon to graphene and FLG produced via chemical vapour deposition (CVD).

\subsubsection{Acellular Superoxide Radical Detection via Electron Paramagnetic Resonance (EPR)}

The detection of acellular superoxide radicals (Figure 3.1) was performed in collaboration with the University of Edinburgh and Dr Mark Miller, with experiments performed by Dr Lorraine Bruce at the Centre for Cardiovascular Science, Queens Medical Research Institute, Edinburgh. 
EPR, sometimes referred to as Electron Spin Resonance (ESR) is a magnetic resonance based technique capable of measuring oxygen levels in biological samples either in vitro or in vivo (Ahmad and Kuppusamy, 2010). This technique required the addition of a spin trap molecule Tempone-H (1hydroxyl-2,2,6,6-tetramethyl-4-oxo-piperidine) which shows selectivity for superoxide radicals (Miller et al., 2009). The purpose of the spin trap in this study was to covalently react with the short-lived superoxide radical to form a stable adduct which possesses paramagnetism, a fundamental aspect of EPR detection.

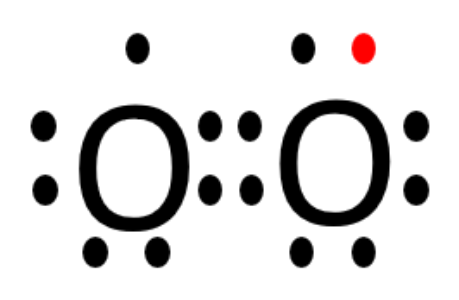

Figure 3.1 Lewis structure of superoxide radical. The seven outer electrons of each atom are shaded black, one electron pair is shared at the center, the unpaired electron is on the upper left atom and the additional electron is shown in red which confers the overall anionic dipole.

To provide an accurate measure of the FLG ENMs reactivity, EPR was used to establish oxygen-centred free radical generation by the method developed by (Miller et al., 2009). Stock FLG samples for EPR were suspended in $500 \mu$ l physiological saline solution (Krebs buffer: $118.4 \mathrm{mM} \mathrm{NaCl}, 25 \mathrm{mM} \mathrm{NaHCO}{ }_{3}$, $11 \mathrm{mM}$ glucose, $4.7 \mathrm{mM}$ potassium chloride $(\mathrm{KCl}), 1.2 \mathrm{mM} \mathrm{MgSO}_{4}, 1.2 \mathrm{mM} \mathrm{KH}_{2} \mathrm{PO}_{4}, 2.5 \mathrm{mM} \mathrm{CaCl}_{2}$ ) at concentrations of $3.2-180 \mu \mathrm{g} / \mathrm{ml}$. The FLG samples were then incubated with the spin-trap, Tempone$\mathrm{H}$ (1mM; Enzo Life Sciences, Exeter, UK), immediately before the initial measurement. Tempone-H is a highly sensitive spin-trap that shows selectivity for superoxide, forming a stable product that can be

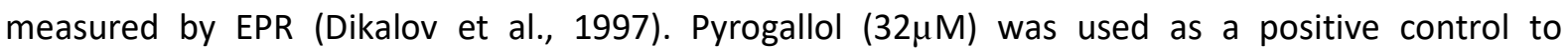
spontaneously generate superoxide radicals in Krebs buffer (Taylor et al., 2004). The FLG samples were kept at $37^{\circ} \mathrm{C}$ throughout and measurements were taken after 60 minutes by drawing $50 \mu$ l of sample into a capillary tube (VWR International, Lutterworth, UK) and sealing with a plug of soft sealant (Cristaseal, VWR International). An X-band EPR spectrometer (Magnettech MS-200, Berlin, Germany) was used with the following parameters: microwave frequency, $9.3-9.55 \mathrm{~Hz}$; microwave power, 20 $\mathrm{mW}$; modulation frequency, $100 \mathrm{kHz}$; modulation amplitude, 1500mG; center field, 3365G; sweep width, 50G; sweep time, 30s; number of passes, 1. Baseline signals (Tempone-H in buffer alone with the appropriate volume of media (9\%) from the FLG stock concentrations) were subtracted from that 
of experimental readings. Free radical generation was quantified using the first derivative of the initial peak of the spectra obtained from the reaction of the Tempone-H spin-trap with superoxide.

\subsubsection{Surface Area Measurements}

To produce an accurate measure of the Brunauer, Emmett and Teller (BET) surface area of all four ENMs the NOVA 200e was utilised operating both A and B stations through the adjoining computer running the NovaWin software. Prior to handling the ENMs, two $9 \mathrm{~mm}$ (bulb-ended long, BET cells were purchased (Quantachrome Ltd, UK) for running the experiments. Firstly, the Nova 200e was started up, vacuum pump switched on and nitrogen gas with 1 bar of pressure was passed through the system. Secondly, once the NovaWin software had been set up, the cells were calibrated in stations $A$ and $B$ simultaneously and the thermal delay was set to 600 s. Next the dewar was filled with liquid nitrogen at 77K below the cells and then initiated. For sample preparation, FLG and CB particles were weighed on a weigh-safe balance after the empty cells had been weighed thus providing sample weight. Following this, the samples were de-gassed for two hours at room temperature under a vacuum before the samples were weighed again to ascertain their de-gassed weight. Using the NovaWin software; surface area, pore radius and volume were selected, following this the dewar was filled with liquid nitrogen and placed beneath the cells to begin. The experiment was run for 10 hours overnight and the data output was collected the following day.

\subsubsection{Limulus Amebocyte Lysate (LAL) Endotoxin Test}

To assess the quality of the manufactured ENMs with regards to contamination, the E-Toxate LAL gel clot assay (Sigma, UK) was utilised. The core rationale for this kit was that it is not reliant on a colorimetric output which is notoriously associated with interactions and interference when analysing ENMs. The assay was performed using endotoxin-free $10 \mathrm{ml}$ glass vials (Lonza, Switzerland), with each ENM receiving its own vial. To perform the assay, E-Toxate working reagent was prepared from stock by the aseptic addition of $2 \mathrm{ml}$ of pyrogen-free water and swirled to dissolve at room temperature. Stock ENMs following sonication (Chapter 2 Section 2.1), were prepared at a suitably low concentration of $10 \mu \mathrm{g} / \mathrm{ml}$ (avoiding strong agglomeration) using pyrogen-free water at a final volume of $100 \mu \mathrm{l}$. The LAL gel clot assay was prepared as detailed in Table 3.2. Immediately after sample preparation, glass vials were covered with parafilm and incubated in a still water bath set to $37^{\circ} \mathrm{C}$ and left for one hour. To identify a negative / positive result, immediately after the one-hour incubation the glass vials were removed from the water bath and inverted $180^{\circ}$ whilst observing the gel clot 
formation at the bottom of the vial. To confirm a negative endotoxin result, the gel clot displayed disruption upon inversion; conversely an endotoxin-positive sample displayed a solid gel clot which did not move upon inversion. This assay was performed in triplicate.

Table 3.2 Detection of Endotoxin in ENMs utilising the LAL gel clot assay kit.

\begin{tabular}{|l|l|l|l|l|}
\hline Sample & ENMs 10 $(\mu \mathrm{g} / \mathrm{ml})$, & $\begin{array}{l}\text { Pyrogen-free } \\
\text { water }(\mu \mathrm{l})\end{array}$ & $\begin{array}{l}\text { E-Toxate } \\
\text { Standard }(\mu \mathrm{l})\end{array}$ & $\begin{array}{l}\text { Endotoxin } \\
\text { Positive Control } \\
(\text { LPS })(\mu \mathrm{l})\end{array}$ \\
\hline Negative Control & 0 & 100 & 100 & 0 \\
\hline ENMs & 100 & 0 & 100 & 0 \\
\hline Inhibition & 100 & 0 & 100 & $100(4 \mathrm{EU} / \mathrm{ml})$ \\
\hline
\end{tabular}

Where $\mathrm{EU} / \mathrm{ml}$ is Endotoxin Units/ml of which the LAL gel clot assay (Sigma, UK) can detect down to $0.03 \mathrm{EU} / \mathrm{ml}$. As of 2012, the Food and Drug Administration guidelines for novel nano products which either directly or indirectly contact the lymphatic or cardiovascular system the requirement is for the Endotoxin Units to be below 0.5EU/ml (Mukherjee et al., 2016).

\subsubsection{Hard Protein Corona Identification}

The protein's which displayed a strong affinity with the ENMs after suspension in cell culture media designated the hard protein corona, was initially isolated with centrifugation at room temperature.

\subsubsection{Removal of the Soft Protein Corona}

Initially, stock ENMs were sonicated and then suspended at a diluted concentration of $100 \mu \mathrm{g} / \mathrm{ml}$ in supplemented MEM cell culture media in $1.5 \mathrm{ml}$ eppendorf tubes which were then incubated for one hour in a heated block set to $37^{\circ} \mathrm{C}$. Following this the samples were transferred to a bench-top centrifuge and centrifuged at $4000 \mathrm{~g}$ for 40 minutes. The use of the Ultra Centrifuge to achieve higher centrifugal forces $>4000 \mathrm{~g}$ was ultimately not used in this SOP to avoid the sedimentation of biomolecules interfering with the endpoint (hard protein corona identification). Following centrifugation in cell culture media, the media was carefully aspirated and discarded and $1 \mathrm{ml}$ of PBS used to re-suspend the pellet, taking care to fully break up the sediment before being centrifuged 
again for 20 minutes at 4000g in the first of three wash steps. The final pellet following three PBS washes, was then prepared for SDS-PAGE, the process of separating the proteins based on molecular weight (MW) before LC MS/MS was performed to identify proteins. Pellets constituting the hard protein corona were re-suspended in $20 \mu$ I SDS-PAGE loading buffer (nine-parts loading buffer (Cell Signalling Technologies, UK) one-part dithiothreitol (DTT) (Sigma, UK) and 40 $\mu$ I PBS. This suspension then underwent heat-shock treatment at $90^{\circ} \mathrm{C}$ in a heat block for 10 minutes.

\subsubsection{Protein Separation by SDS-PAGE}

To construct the SDS-PAGE gels required for this stage the recipe was prepared according to Chapter 2 Section 2.10 Table 2.6. Briefly, a 10\% resolving and 4\% stacking gel were prepared to isolate individual proteins based on MW alone with the addition of a Protein Kaleidoscope as described by Bio-Rad, UK.

\subsubsection{Protein Silver Staining}

Upon completion of the SDS-PAGE, gels were removed from their glass moulds and placed in clean glass containers for silver staining of protein bands utilising the Pierce Silver Stain Kit (ThermoFisher, UK). The following SOP was performed with the gel kept in the glass container and shaken during incubation stages on an orbital shaker, a final volume of $25 \mathrm{ml}$ of reagent was used per gel / per stage was used. Immediately after removing the gels from their glass moulds they were washed twice with ultrapure water twice for five minutes each. The proteins were then fixed with $30 \%$ ethanol/acetic acid solution overnight. The following day, the fixative was discarded, and the gel washed with $10 \%$ ethanol and two subsequent washes with ultrapure water. The gels then required a one-minute incubation with Pierce Silver Stain Sensitizer (one-part silver stain sensitizer: 500 parts ultrapure water) (prepared in a 50ml Falcon tube)), following this step, two washes with ultrapure water were performed. Silver stain was then prepared in a $50 \mathrm{ml}$ Falcon tube (one-part Pierce Silver Stain Enhancer: 50 parts Pierce Silver Stain) and incubated with the gel for 30 minutes allowing time for the silver stain to develop. Following the 30-minute incubation, the gel was washed with ultrapure water before finally the protein bands were visualised with developer working solution. Bands could develop sufficiently until this process was stopped by the addition of $5 \%$ acetic acid. 


\subsubsection{Protein Band Excision \& Trypsin Digestion}

Prior to peptide mass fingerprinting, silver stained protein bands had to be excised from the SDS-PAGE gels, washed and then chemically digested. Bands were cut from the gel with an ethanol-sterilised scalpel (a new one for each protein) and placed into $1.5 \mathrm{ml}$ microcentrifuge tubes which had been cleaned with Acetonitrile (ACN) (Sigma, UK). The protein bands were then de-stained with $50 \mu \mathrm{l} /$ band of a 1:1 solution of $3 \mathrm{mM}$ potassium ferricyanide (Sigma, UK) and $100 \mathrm{mM}$ sodium thiosulphate (Sigma, UK), the protein samples were then incubated at room temperature on an orbital shaker until the brown stain was no longer visible, protein bands subsequently acquired a faint yellow colour. The protein bands were then washed 2-3 times with $50 \mu \mathrm{l}$ of ultrapure water to remove as much of the residual yellow colour as possible with centrifugation between each wash step at $800 \mathrm{~g}$ for 1 minute. Following the final wash step, ultrapure water was aspirated, and the protein bands incubated with $100 \mu \mathrm{l}$ of $200 \mathrm{mM}$ ammonium bicarbonate $\left(\mathrm{NH}_{4} \mathrm{HCO}_{3}\right)$ (Sigma, UK) for 20 minutes on an orbital shaker. Mass spectrometry-grade trypsin (ThermoFisher, UK) was prepared next by reconstituting $20 \mu \mathrm{g}$ of Trypsin with $100 \mu$ l of reconstitution buffer $(50 \mathrm{mM}$ acetic acid), this was then aliquoted into 10 vials of $10 \mu \mathrm{l}$ for future use/need, stored at $-20^{\circ} \mathrm{C}$. One aliquot was used, whereby $500 \mu \mathrm{l}$ of $50 \mathrm{mM} \mathrm{NH}_{4} \mathrm{HCO}_{3}$ was added, resulting in the final trypsin solution. Following this $50 \mu l$ of trypsin solution was added to each band, incubated at $37^{\circ} \mathrm{C}$ overnight while shaking on an orbital shaker. The following day, peptide recovery took place by first centrifuging the samples at $800 \mathrm{~g}$ for 1 minute. The subsequent supernatant was then transferred to fresh, labelled sample tubes whilst $30 \%$ and $70 \%$ ACN / $0.2 \%$ Trifluoroacetic acid (TFA) (Sigma, UK) solutions were prepared in labelled bottles in a fume hood (where $70 \%$ ACN - 7ml ACN: $200 \mu$ I TFA: $2.8 \mathrm{ml}$ ultrapure water). Finally, $100 \mu \mathrm{l}$ of $30 \%$ ACN was added to each sample tube and incubated at $37^{\circ} \mathrm{C}$ for 10 minutes, followed by $100 \mu \mathrm{l}$ of $70 \% \mathrm{ACN}$ for a further 10 minutes at $37^{\circ} \mathrm{C}$. The samples were spun down briefly at $800 \mathrm{~g}$ for 30 seconds before allowing the volume to reduce in an Eppendorf, UK speedy vac until protein bands were subsequently dehydrated resulting in a dry powder.

\subsubsection{Peptide Mass Fingerprinting}

Peptide mass fingerprinting was performed by Dr Ed Dudley of Swansea University Medical School. The dehydrated protein samples were re-suspended in $0.1 \%$ formic acid (Sigma, UK) and prepared for electrospray liquid chromatography mass spectrometry (LC MS/MS). Firstly, buffer solutions of $0.1 \%$ formic acid and $100 \%$ ACN/0.1\% formic acid were prepared for high performance liquid chromatography (HPLC) (Surveyor, ThermoFinnigan, CA). The samples were then run utilising a 72minute gradient with the electrospray electrohydrodynamic flow rate set to $150 \mu \mathrm{l} /$ minute. Peptide 
identification was performed by analysing the sample spectra on BioWorksBrowser version 3.3.1 SP1 (ThermoFisher Scientific) using Sequest Uniport/Swiss-Prot database. 


\subsection{Results}

\subsubsection{DLS of Neutral-FLG}

DLS was first performed on water-dispersed Neutral-FLG, which demonstrated a large size range of 78.82-1990nm indicative of a polydisperse sample within which the average size (Z-average) was $290.8 \mathrm{~nm}$, and the median value within this range was $63.42 \mathrm{~nm}$. The Neutral-FLG suspensions was highly polydispersed with an average PDI of 0.365 , where a typical well-monodispersed sample displays a PDI value of $\leq 0.2$. The Neutral-FLG water-dispersed sample displayed a broad polydispersed peak which appears to exhibit 2 peaks weakly joined at $2500 \mathrm{~nm}$, suggesting the presence of two separate monodispersed agglomerate regions within the Neutral-FLG suspension (Figure 3.2). The Neutral-FLG sample exhibited a double peak with each peak representing a separate Z-average of $361.8 \mathrm{~nm}$ and $4557 \mathrm{~nm}$, however the majority of the polydispersed sample falls within peak 1 accordingly with the Z-average of $290.8 \mathrm{~nm}$. DLS analysis of the media-dispersed Neutral-FLG revealed a size range of $190.1-1990 \mathrm{~nm}$, within this range a Z-average of $504.7 \mathrm{~nm}$ was recorded and a median value of $75.01 \mathrm{~nm}$. Crucially the media-dispersed suspension displayed an additional third peak indicative of high degrees of polydispersity, these three peaks exhibited a Z-average of; 89.8, 663.5 and $4569 \mathrm{~nm}$ respectively with the bulk of the suspension inhabiting peak two ultimately contributing the greatest to the observed Z-average of $504.7 \mathrm{~nm}$. This dramatic difference in suspension between water and serum-containing media indicated a 'redshift' in the average peak intensity. This introduced a more polydisperse sample with the Neutral-FLG forming three distinct families of agglomerates and increasing the Z-average by $213.9 \mathrm{~nm}$ possibly indicative of the surfactant properties of proteins bound to ENMs in suspension, summarised by Table 3.3 . 

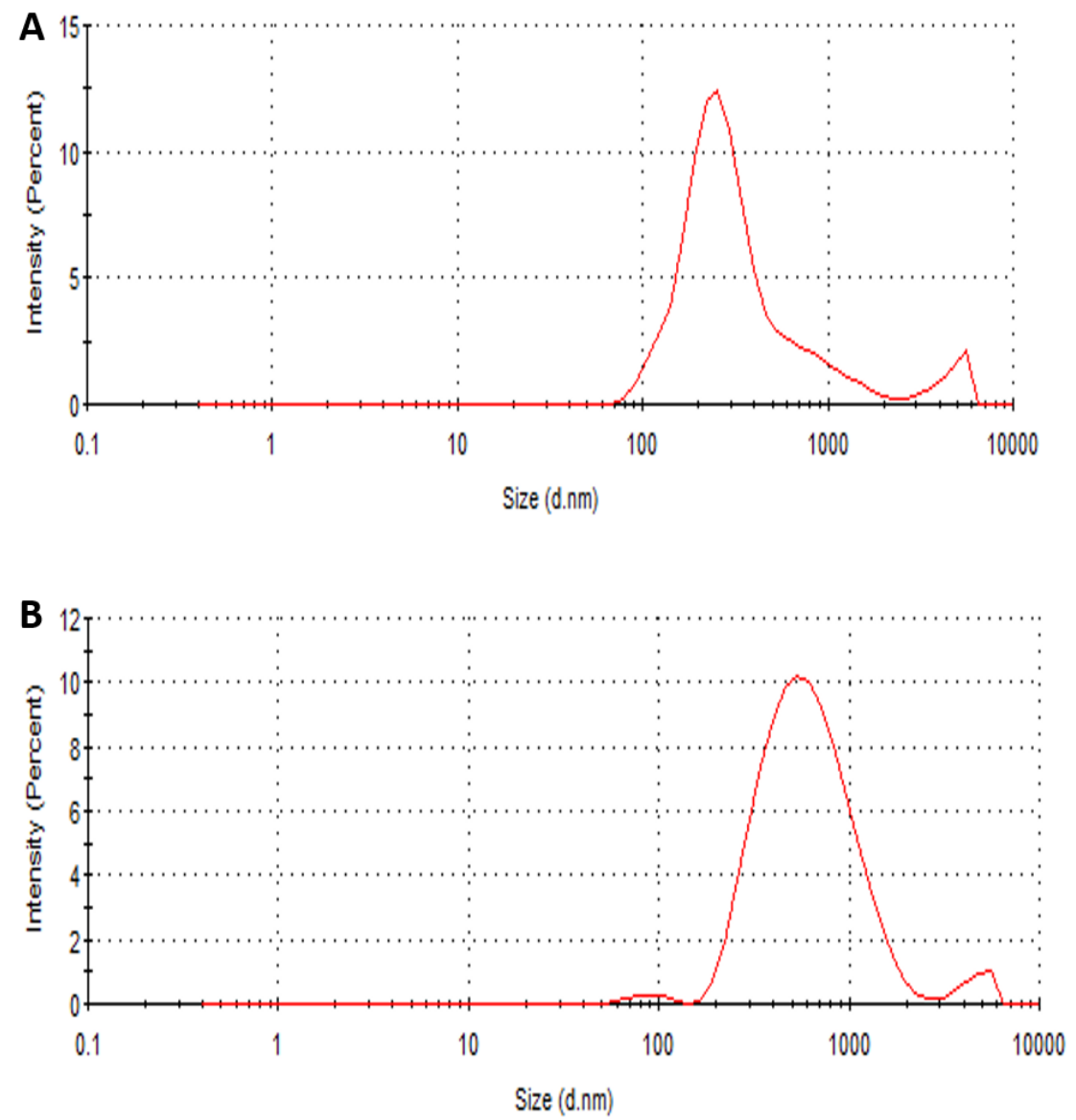

Figure 3.2 DLS measurement of Neutral-FLG. Water-dispersed Neutral-FLG (A) and media-dispersed Neutral-FLG (B) respectively, the data is displayed as the percentage intensity size range at $37^{\circ} \mathrm{C}$. 
Table 3.3 Neutral-FLG characteristics defined by DLS. Data presented pertains to the percentage intensity of the material in both ultrapure double distilled water and cell culture media (10\% FBS, $1 \%$ $\mathrm{P} / \mathrm{S}, \mathrm{MEM})$.

\begin{tabular}{|l|l|l|l|l|l|l|l|l|}
\hline Dispersant & $\begin{array}{l}\text { Size } \\
\text { Range } \\
(\mathrm{nm})\end{array}$ & $\begin{array}{l}\text { Z-average } \\
(\mathrm{nm})\end{array}$ & PDI & Median & $\begin{array}{l}\text { Standard } \\
\text { Deviation } \\
(\mathrm{nm})\end{array}$ & $\begin{array}{l}\text { Average } \\
\text { Peak }\end{array}$ & $\begin{array}{l}\text { Average } \\
\text { Size } \\
(\mathrm{nm})\end{array}$ & $\begin{array}{l}\text { Average } \\
\text { Peak 2 } \\
\text { Size } \\
(\mathrm{nm})\end{array}$ \\
\hline Water & $\begin{array}{l}78.82- \\
1990\end{array}$ & 290.8 & 0.365 & 63.42 & 302.6 & 361.8 & $\begin{array}{l}\text { Peak 3 } \\
\text { Size } \\
(\mathrm{nm})\end{array}$ \\
\hline Media & $\begin{array}{l}190.1- \\
1990\end{array}$ & 504.7 & 0.293 & 75.01 & 364.7 & 663.5 & 4659 & N/A \\
\hline
\end{tabular}

\subsubsection{DLS of Amine-FLG}

Amine-FLG samples dispersed in water exhibited a smaller size range compared to the Neutral-FLG suspension, varying between $43.82-531.2 \mathrm{~nm}$ with a Z-average of $170.1 \mathrm{~nm}$ and a more stable PDI value of 0.186 . These values are indicative of the water-dispersed Amine-FLG demonstrating a more uniform, less polydisperse suspension compared to the Neutral-FLG. The medina value was $55.56 \mathrm{~nm}$ and only a single peak was observed with a total size of $211.3 \mathrm{~nm}$. Furthermore, the water-dispersed Amine-FLG revealed a monodispersed single peak under the Zetasizer software analysis as in Figure 3.3. Media-dispersed Amine-FLG however (Figure 3.3B) displayed a greatly increased size range of $164.2-1718 \mathrm{~nm}$ with a new Z-average of 426.4 indicating a greater average agglomerate size however the sample appears relatively monodisperse all be it in a 'redshifted' manner, evidenced by the greater median value of $94.04 \mathrm{~nm}$ and greater PDI value (0.261). Crucially, whilst the bulk of the mediadispersed Amine-FLG remains largely uniform in agglomerate size, there were 3 peaks observed each with a total size of; $542.3,4646$ and $89.8 \mathrm{~nm}$ as summarised in Table 3.4. 

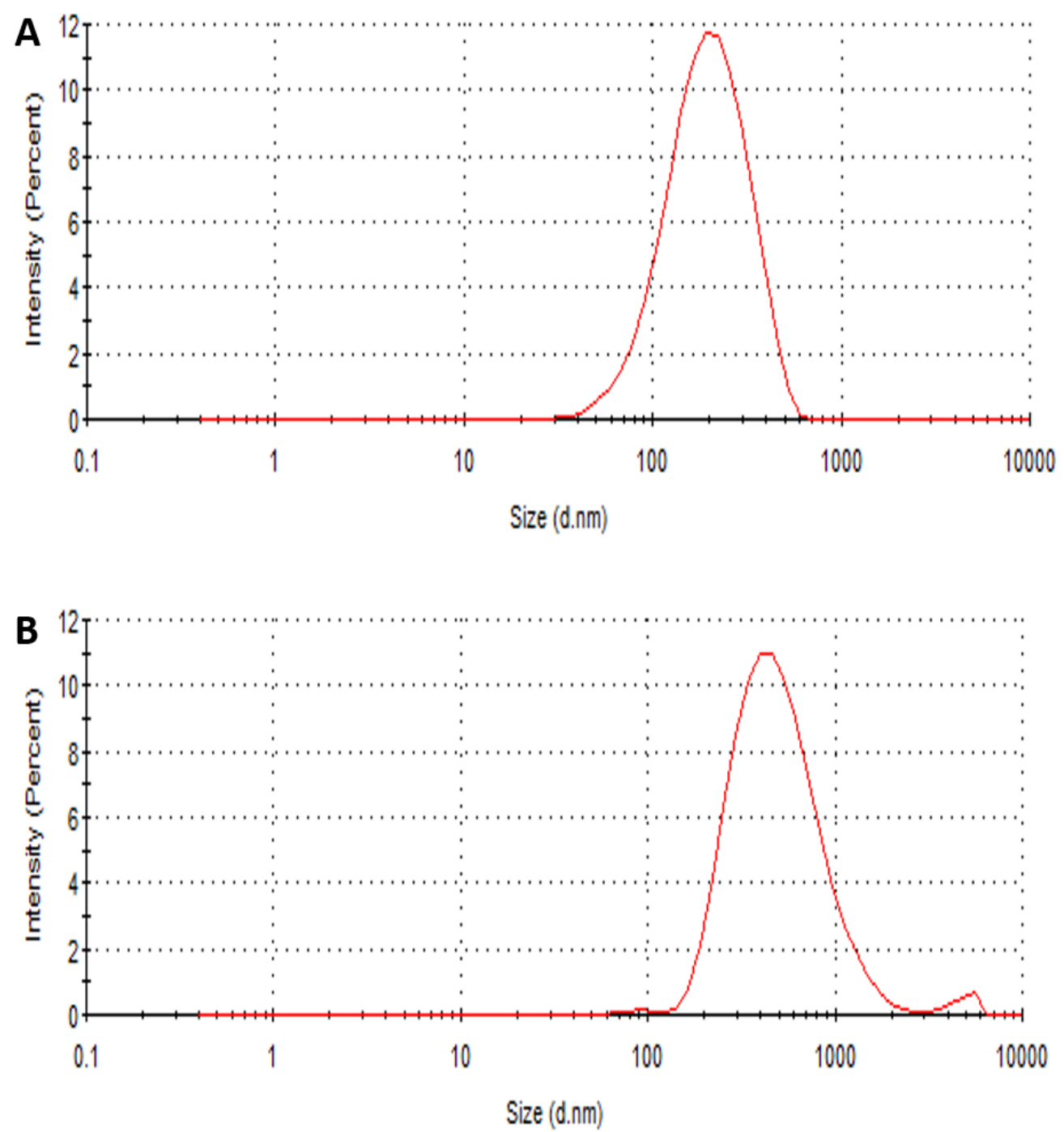

Figure 3.3 DLS measurement of Amine-FLG. Water-dispersed Amine-FLG (A) and media-dispersed Amine-FLG (B) respectively, the data is displayed as the percentage intensity size range at $37^{\circ} \mathrm{C}$. 
Table 3.4 Amine-FLG characteristics defined by DLS. Data presented pertains to the percentage intensity of the material in both ultrapure double distilled water and cell culture media (10\% FBS, $1 \%$ $\mathrm{P} / \mathrm{S}, \mathrm{MEM})$.

\begin{tabular}{|c|c|c|c|c|c|c|c|c|}
\hline Dispersant & $\begin{array}{l}\text { Size } \\
\text { Range } \\
(\mathrm{nm})\end{array}$ & $\begin{array}{l}\text { Z-average } \\
(\mathrm{nm})\end{array}$ & PDI & Median & $\begin{array}{l}\text { Standard } \\
\text { Deviation } \\
(\mathrm{nm})\end{array}$ & $\begin{array}{l}\text { Average } \\
\text { Peak } 1 \\
\text { Size } \\
(\mathrm{nm})\end{array}$ & $\begin{array}{l}\text { Average } \\
\text { Peak } 2 \\
\text { Size } \\
(\mathrm{nm})\end{array}$ & $\begin{array}{l}\text { Average } \\
\text { Peak } 3 \\
\text { Size } \\
(\mathrm{nm})\end{array}$ \\
\hline Water & $\begin{array}{l}43.82- \\
531.2\end{array}$ & 170.1 & 0.186 & 55.56 & 97.92 & 211.3 & N/A & N/A \\
\hline Media & $\begin{array}{l}164.2- \\
1718\end{array}$ & 426.4 & 0.261 & 94.04 & 310.8 & 542.3 & 4646 & 89.84 \\
\hline
\end{tabular}

\subsubsection{DLS of Carboxyl-FLG}

The water-dispersed Carboxyl-FLG analysis by DLS revealed a highly polydispersed suspension supported by a single sized peak with a range of $68.06-458.7 \mathrm{~nm}$ (Figure 3.4A) a Z-average of $169.6 \mathrm{~nm}$ and a PDI value of 0.141 , the smallest PDI of any tested ENM in this study. The water-dispersed sample also exhibited a mean value of 55.99 with peak 1 showing a total size intensity of $197.3 \mathrm{~nm}$. Once suspended in media however the Carboxyl-FLG suspension adopts a slightly less monodisperse agglomerate formation, with a smaller second family of agglomerates evident by peak two in Figure 3.4B with a size intensity of 5103nm. The media-dispersed Carboxyl-FLG possessed a size range of $141.8-1106 \mathrm{~nm}$ with a Z-average of $348.2 \mathrm{~nm}$ and median value of $77.26 \mathrm{~nm}$. Supporting the formation of the second peak distribution is a higher PDI value of 0.229 , up from the water dispersed value of 0.141 (summarised in Table 3.5). 

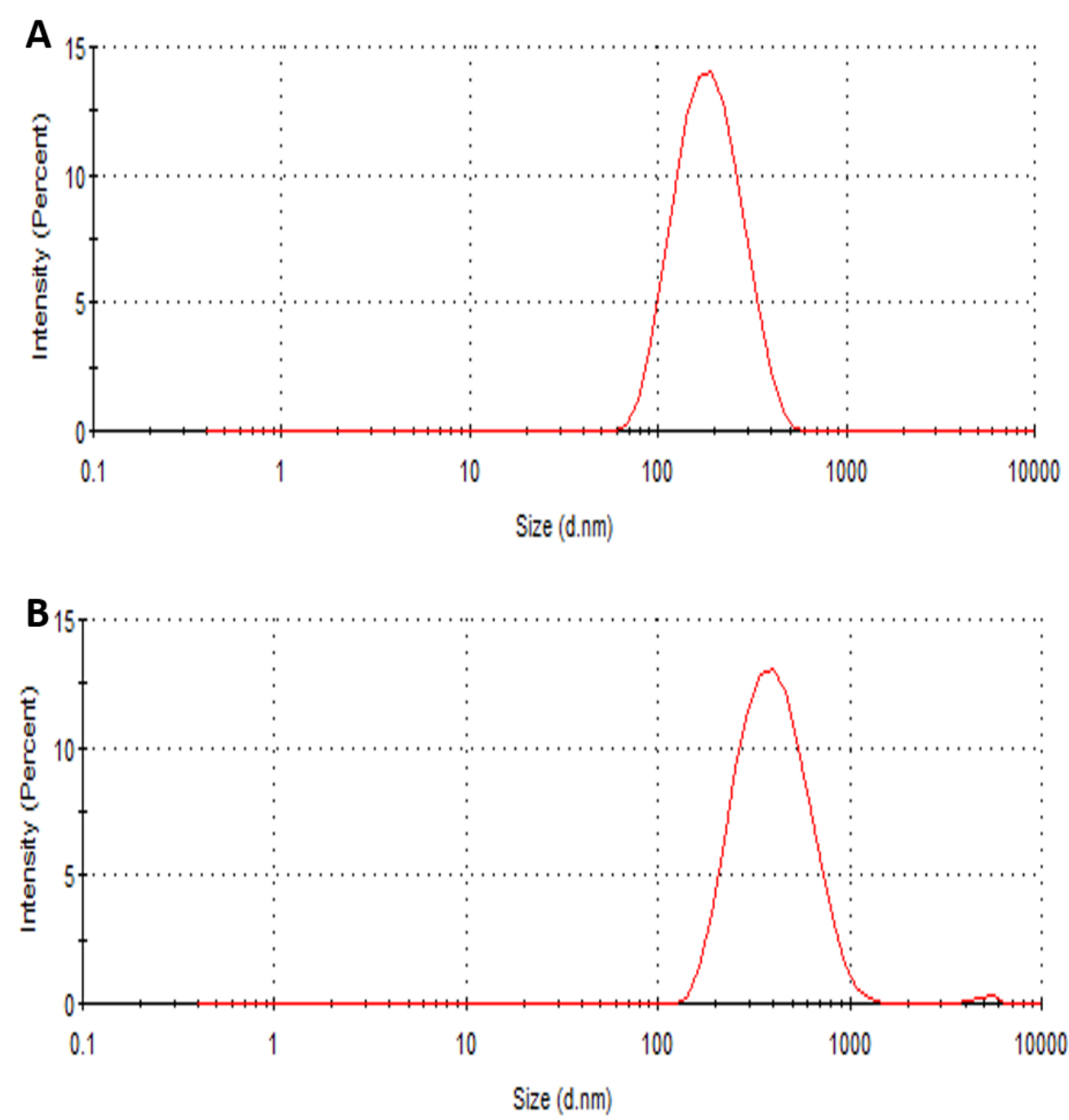

Figure 3.4 DLS measurement of Carboxyl-FLG. Water-dispersed Carboxyl-FLG (A) and mediadispersed Carboxyl-FLG (B) respectively, the data is displayed as the percentage intensity size range at $37^{\circ} \mathrm{C}$. 
Table 3.5 Carboxyl-FLG characteristics defined by DLS. Data presented pertains to the percentage intensity of the material in both ultrapure double distilled water and cell culture media (10\% FBS, $1 \%$ $\mathrm{P} / \mathrm{S}, \mathrm{MEM})$.

\begin{tabular}{|c|c|c|c|c|c|c|c|c|}
\hline Dispersant & $\begin{array}{l}\text { Size } \\
\text { Range } \\
(\mathrm{nm})\end{array}$ & $\begin{array}{l}\text { Z-average } \\
(\mathrm{nm})\end{array}$ & PDI & Median & $\begin{array}{l}\text { Standard } \\
\text { Deviation } \\
(\mathrm{nm})\end{array}$ & $\begin{array}{l}\text { Average } \\
\text { Peak } 1 \\
\text { Size } \\
(\mathrm{nm})\end{array}$ & $\begin{array}{l}\text { Average } \\
\text { Peak } 2 \\
\text { Size } \\
(\mathrm{nm})\end{array}$ & $\begin{array}{l}\text { Average } \\
\text { Peak } 3 \\
\text { Size } \\
(\mathrm{nm})\end{array}$ \\
\hline Water & $\begin{array}{l}68.06- \\
458.7\end{array}$ & 169.6 & 0.141 & 55.99 & 76.88 & 197.3 & N/A & N/A \\
\hline Media & $\begin{array}{l}141.8- \\
1106\end{array}$ & 348.2 & 0.229 & 77.26 & 183.9 & 426.1 & 5103 & $\mathrm{~N} / \mathrm{A}$ \\
\hline
\end{tabular}

\subsubsection{DLS of CB particles}

The CB analysis with DLS revealed counter intuitive results to the graphene-based samples. The FLG samples appeared more monodispersed in the water buffer but gained more polydispersity and a greater range of agglomerate sizes when in the media buffer. Water-dispersed CB particles however exhibited a triple peak intensity in water, the only ENM to do so. The average size range of this material in a water suspension was $105-6439 \mathrm{~nm}$, vastly larger than the FLG samples. The large PDI of 0.402 does suggest that CB particles may not be well suited to DLS measurements but this PDI value was still within cumulant analysis range befitting the expert report on the Zetasizer software. As seen in Figure 3.5B below, the CB particles gained more stability once suspended in culture media, evident from the now-monodisperse single peak ranging from $141.8-1106 \mathrm{~nm}$. This significant reduction in average agglomerate size $(513.3 \mathrm{~nm}$ to $347.7 \mathrm{~nm}$ ) is supported in part by the reduced PDI of 0.225 , detailing a far more suitable sample suspension for DLS analysis. Whilst the average agglomerate size is reduced the median value $(96.28 \mathrm{~nm})$ suggests the particles have been 'redshifted' and find more stability forming larger agglomerates in the culture media buffer, data summarised in Table 3.6. 

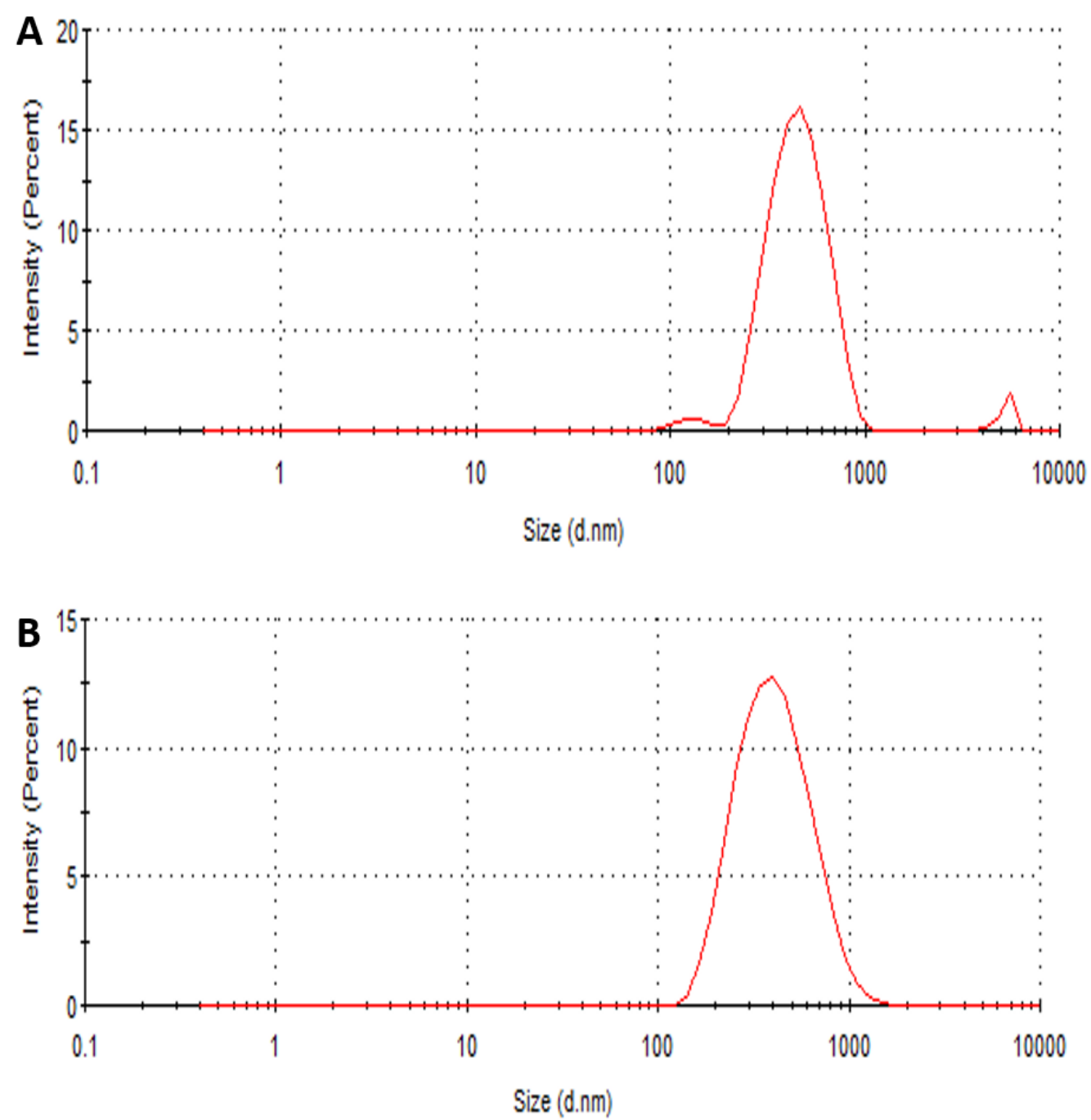

Figure 3.5 DLS measurement of CB. Water-dispersed $C B(A)$ and media-dispersed $C B(B)$ respectively, the data is displayed as the percentage intensity size range at $37^{\circ} \mathrm{C}$. 
Table 3.6 CB characteristics defined by DLS. Data presented pertains to the percentage intensity of the material in both ultrapure double distilled water and cell culture media (10\% FBS, $1 \% \mathrm{P} / \mathrm{S}, \mathrm{MEM})$.

\begin{tabular}{|c|c|c|c|c|c|c|c|c|}
\hline Dispersant & $\begin{array}{l}\text { Size } \\
\text { Range } \\
(\mathrm{nm})\end{array}$ & $\begin{array}{l}\text { Z-average } \\
(\mathrm{nm})\end{array}$ & PDI & Median & $\begin{array}{l}\text { Standard } \\
\text { Deviation } \\
(\mathrm{nm})\end{array}$ & $\begin{array}{l}\text { Average } \\
\text { Peak } 1 \\
\text { Size } \\
(\mathrm{nm})\end{array}$ & $\begin{array}{l}\text { Average } \\
\text { Peak } 2 \\
\text { Size } \\
(\mathrm{nm})\end{array}$ & $\begin{array}{l}\text { Average } \\
\text { Peak } 3 \\
\text { Size } \\
(\mathrm{nm})\end{array}$ \\
\hline Water & $\begin{array}{l}105- \\
6439\end{array}$ & 513.3 & 0.402 & 62.99 & 421.2 & 468.6 & 5323 & 126 \\
\hline Media & $\begin{array}{l}141.8- \\
1106\end{array}$ & 347.7 & 0.225 & 96.28 & 195.1 & 434.7 & $\mathrm{~N} / \mathrm{A}$ & $N / A$ \\
\hline
\end{tabular}

\subsubsection{Zeta Potential}

ENM surface charge was assessed by measuring the zeta potential across the suspended samples, dispersed in both ultrapure double distilled water and $10 \%$ serum containing media ( $10 \% \mathrm{FBS}, 1 \% \mathrm{P} / \mathrm{S}$, MEM). ENMs where dispersed in water showed highly stable suspensions with zeta potentials indicating strong stability with surface charges exceeding $-30 \mathrm{mV}$ indicating high electrostatic repulsion between particles / agglomerates. Additionally, the role of engineered surface charge to the FLG samples proved to be significant with the Amine-FLG sample $(-41.96 \mathrm{mV})$ holding a stronger dispersion in water compared to the Neutral- $(-31.72 \mathrm{mV})$ or Carboxyl-FLG $(-34.36 \mathrm{mV})$ samples. CB particles however demonstrated the greatest zeta potential at $-43.7 \mathrm{mV}$, indicative of an extremely stable dispersion in water. CB particles suspended in cell culture media however exhibited drastically different surface charges (summarised in Table 3.7). The ENMs suspended in water exhibited an order of stability; $\mathrm{CB}>$ Amine-FLG $>$ Neutral-FLG $>$ Carboxyl-FLG, whilst in cell culture media the latter two ENMs were reversed so the order now reads; $C B>A$ mine-FLG $>$ Carboxyl-FLG $>$ Neutral-FLG, indicative that in cell culture media the Neutral-FLG has become the most destabilised of all materials. All ENMs however exhibited significant reductions in stability as measured by zeta potential when moved from ultrapure double distilled water to cell culture media. 
Table 3.7 Zeta Potential of carbonaceous ENMs suspended in both ultrapure double distilled water and cell culture media.

\begin{tabular}{|c|c|c|c|}
\hline ENM & Dispersant & Zeta Potential (mV) & $\begin{array}{ll}\text { Standard } & \text { Deviation } \\
(+/-\mathrm{mV}) & \end{array}$ \\
\hline Neutral-FLG & $\begin{array}{ll} & \mathrm{H}_{2} \mathrm{O} \\
\text { - } & \text { Media }\end{array}$ & $\begin{array}{ll} & -31.72 \\
\text { - } & -9.801\end{array}$ & $\begin{array}{ll}- & 1.95 \\
- & 0.66\end{array}$ \\
\hline Amine-FLG & $\begin{array}{ll}\text { - } & \mathrm{H}_{2} \mathrm{O} \\
\text { - } & \text { Media }\end{array}$ & $\begin{array}{ll}\text { - } & -41.96 \\
\text { - } & -9.971\end{array}$ & $\begin{array}{ll} & 0.86 \\
\text { - } & 0.71\end{array}$ \\
\hline Carboxyl-FLG & $\begin{array}{ll} & \mathrm{H}_{2} \mathrm{O} \\
\text { - } & \text { Media }\end{array}$ & $\begin{array}{ll} & -34.36 \\
\text { - } & -9.76\end{array}$ & $\begin{array}{ll}- & 3.06 \\
\text { - } & 0.72\end{array}$ \\
\hline CB & $\begin{array}{ll}\text { - } & \mathrm{H}_{2} \mathrm{O} \\
\text { - } & \text { Media }\end{array}$ & $\begin{array}{ll}-43.7 \\
\text { - } & -12.4\end{array}$ & $\begin{array}{ll}\text { - } & 5.40 \\
\text { - } & 0.83\end{array}$ \\
\hline
\end{tabular}

\subsubsection{Scanning Electron Microscopy and Elemental Analysis of Primary ENM Characteristics}

Primary size characterisation of both FLG materials and CB particles was performed on dry samples to provide an indication as to the diameter of the ENMs under investigation. Approximately $1 \mathrm{mg}$ of stock ENM was spread carefully onto an adhesive pad and analysed with the SEM and EDX using the specifications detailed in Section 3.2.3. Primary sizing characteristics of Neutral-FLG immediately post manufacture revealed an average diameter of $1.03 \mu \mathrm{m}$ with a range of $0.102-4.1 \mu \mathrm{m}$ (Figure 3.6A) whilst elemental composition of this material was found to be $97.38 \%$ Carbon and $1.93 \%$ Oxygen by weight (Figure 3.7A). Interestingly, trace quantities of Sulphur were also detected however the trace values remained far below background intensities and were largely masked by aluminium and silicon, constituents of the SEM shuttle used for sample loading. FLG functionalised with amine groups meanwhile exhibited an average size of $0.98 \mu \mathrm{m}$ inside a range spanning from $0.068-4.13 \mu \mathrm{m}$ (Figure 3.6B). The Amine-FLG therefore displayed a smaller diameter and exhibited a slightly reduced carbon content under EDX analysis with an $88 \%$ carbon content by weight with characteristic Nitrogen at a relatively low percentage of $1.74 \%$ and finally a $9.66 \%$ oxygen content (Figure $3.7 \mathrm{~B})$. This data achieved by EDX analysis may suggest the presence of amide bonds in addition to amine bonds which could account for the higher oxygen content present in the sample. The remaining FLG sample functionalised with carboxyl groups represented a size range of $0.13-5.84 \mu \mathrm{m}$ with an average size of $1.14 \mu \mathrm{m}$ (Figure $3.6 \mathrm{C})$, the largest of the FLG samples with regards to fibre diameter post manufacture. Elemental 
composition of the Carboxyl-FLG revealed $92.16 \%$ carbon and $7.32 \%$ oxygen by weight (Figure 3.7C). This sample also bore trace values of Nitrogen, likely a source of accidental nitrogen doping from ambient air during the manufacture process. Finally, CB particles possessed an average diameter of $129.4 \mathrm{~nm}$ but ranged from $87.9-190 \mathrm{~nm}$ (Figure 3.6D), proving to be individually smaller than the graphene-based ENMs. CB particles exhibited a simple EDX spectra consisting of $98.94 \%$ carbon and $1.06 \%$ oxygen by weight (Figure 3.7D), similar by \%weight content as Neutral-FLG. 

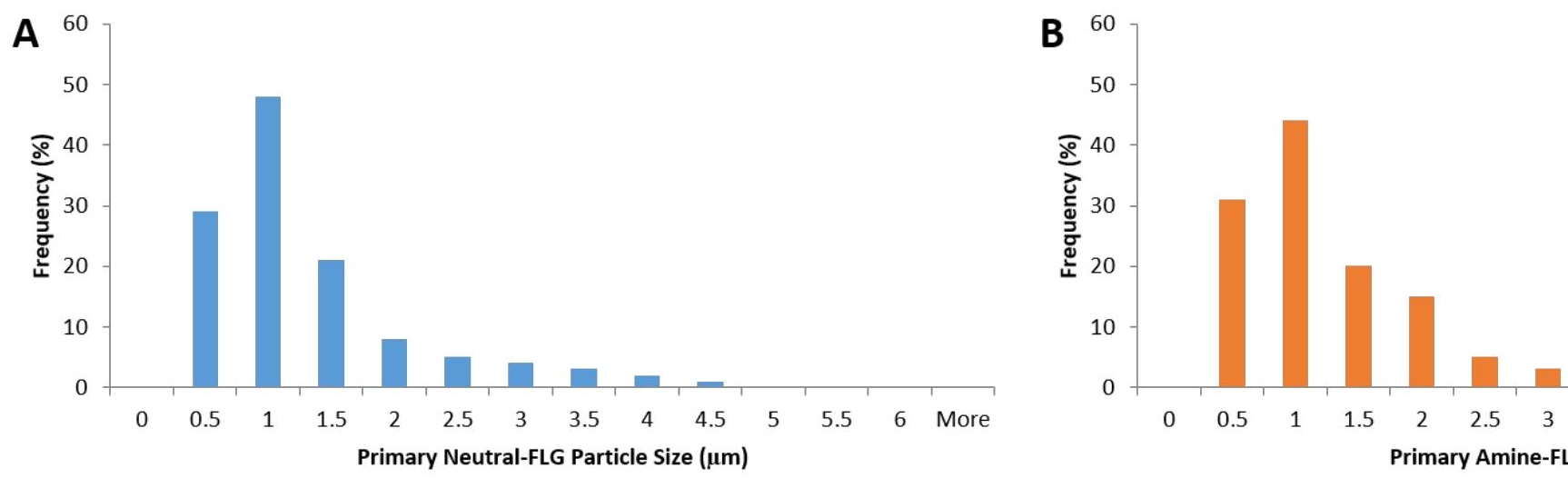

\section{C}
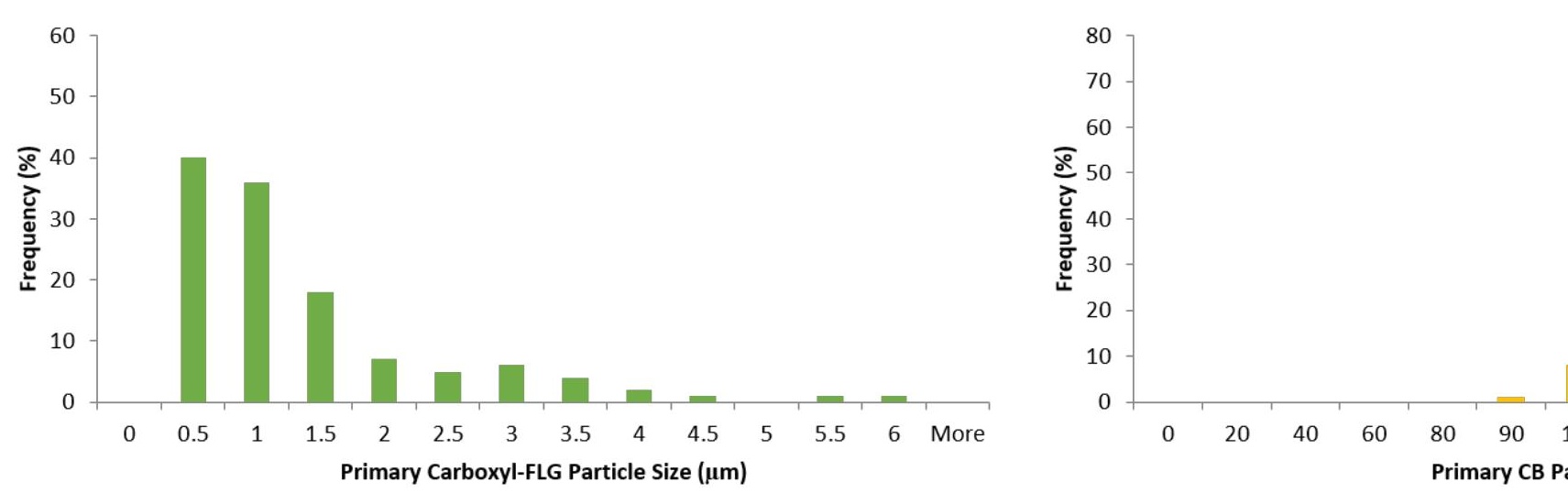

Figure 3.6 Size distribution histograms for Neutral- (A), Amine- (B), Carboxyl-FLG (C) and CB particles (D). Each ENN diameter, with FLG materials primarily averaging one micron whilst CB particles averaged between 120 and $130 \mathrm{~nm}$. N 

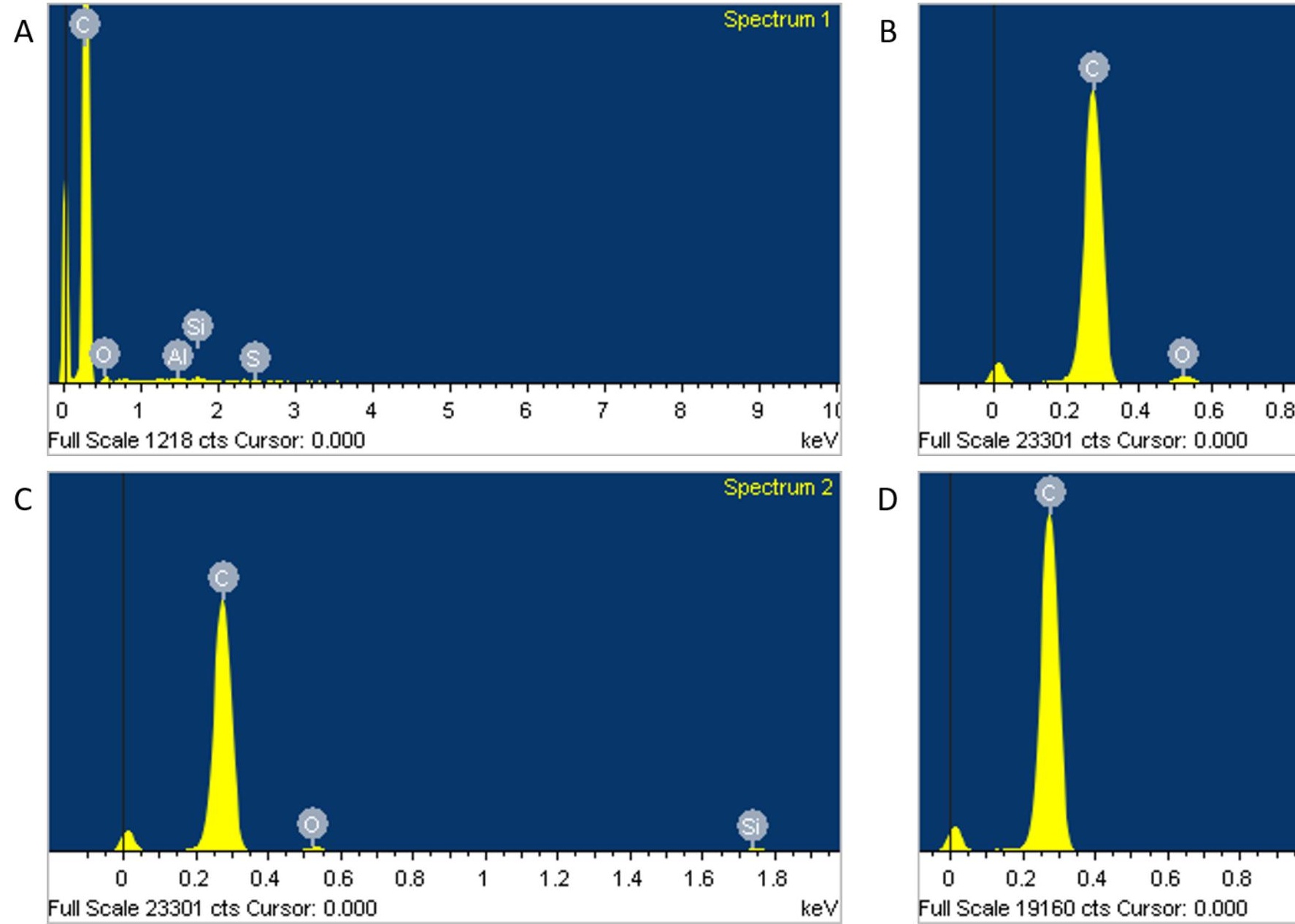

Figure 3.7 Elemental composition for Neutral- (A), Amine- (B), Carboxyl-FLG (C) and CB particles (D) determined by metal contamination and variable carbon: oxygen ratios. 


\subsubsection{Plunge Freeze SEM of FLG}

The technique of drop casting the FLG into liquid ethane $\left(-182^{\circ} \mathrm{C}\right)$ acted to cryogenically preserve the sample by freezing the water buffer suspension rapidly enough to prevent water crystals forming, allowing the electron beam to hit the sample unhindered. By adhering to this technique of snapfreezing the FLG a true representation of the agglomerate and individual particle size in suspension could be obtained.

\subsubsection{Plunge Freeze Analysis of FLG Platelets}

Brightfield SEM images were captured after FLG samples had been drop cast onto TEM grids allowing size, morphology and 2D aspect ratio to be calculated. Particle sizes are described with regards to average diameter +/- standard deviation. This assessment was carried out on particles suspended in both ultrapure water (Figure 3.8) and 10\% serum containing cell culture media (Figure 3.9). Initial ImageJ analysis of Neutral-FLG suspended in water revealed a particle size range of $106-207 \mathrm{~nm}$, an average diameter of $153.9 \mathrm{~nm}$ with a median value of $155.3 \mathrm{~nm}$ (Figure 3.8 Ai-Aii). Analysis of AmineFLG revealed a slightly larger size ranging from $112.8-240.9 \mathrm{~nm}$ with average and median values of 163.8 and $162.9 \mathrm{~nm}$ respectively (Figure 3.8 Bi-Bii). Finally, Carboxyl-FLG (Figure 3.8 Ci-Cii) suspended in water exhibited a size range of $104.7-210.7 \mathrm{~nm}$ with average and median values of 158.5 and $157.4 \mathrm{~nm}$ respectively. Therefore Amine-FLG suspended in water proved to be the largest of the three graphene-based ENMs. This was supported by calculating the 2D aspect ratio with a descending order of; 1.36 (Amine-FLG) >1.34 (Neutral-FLG) >1.30 (Carboxyl-FLG). Carboxyl-FLG therefore poses the lowest internalisation hazard to cells (by aspect ratio) with a marginally smaller aspect ratio which was calculated by dividing the major by minor axis of overlaid masks in ImageJ. This data has been summarised further in Table 3.7. FLG analysis of media-suspended samples revealed interesting results whereby the particle size was reduced. Neutral-FLG, Amine-FLG and Carboxyl-FLG (Figure 3.9) all showed size reductions when suspended in media as opposed to water with respective ranges being: 71.4-177.6, 99.8-152.4 and 77.8-172nm. As summarised in Table 3.8, there was also a reduction in $2 \mathrm{D}$ aspect ratio for two of the FLG samples namely the Neutral-FLG and most notably so in CarboxylFLG with a decrease from $1.30-1.17 \mathrm{~nm}$. 

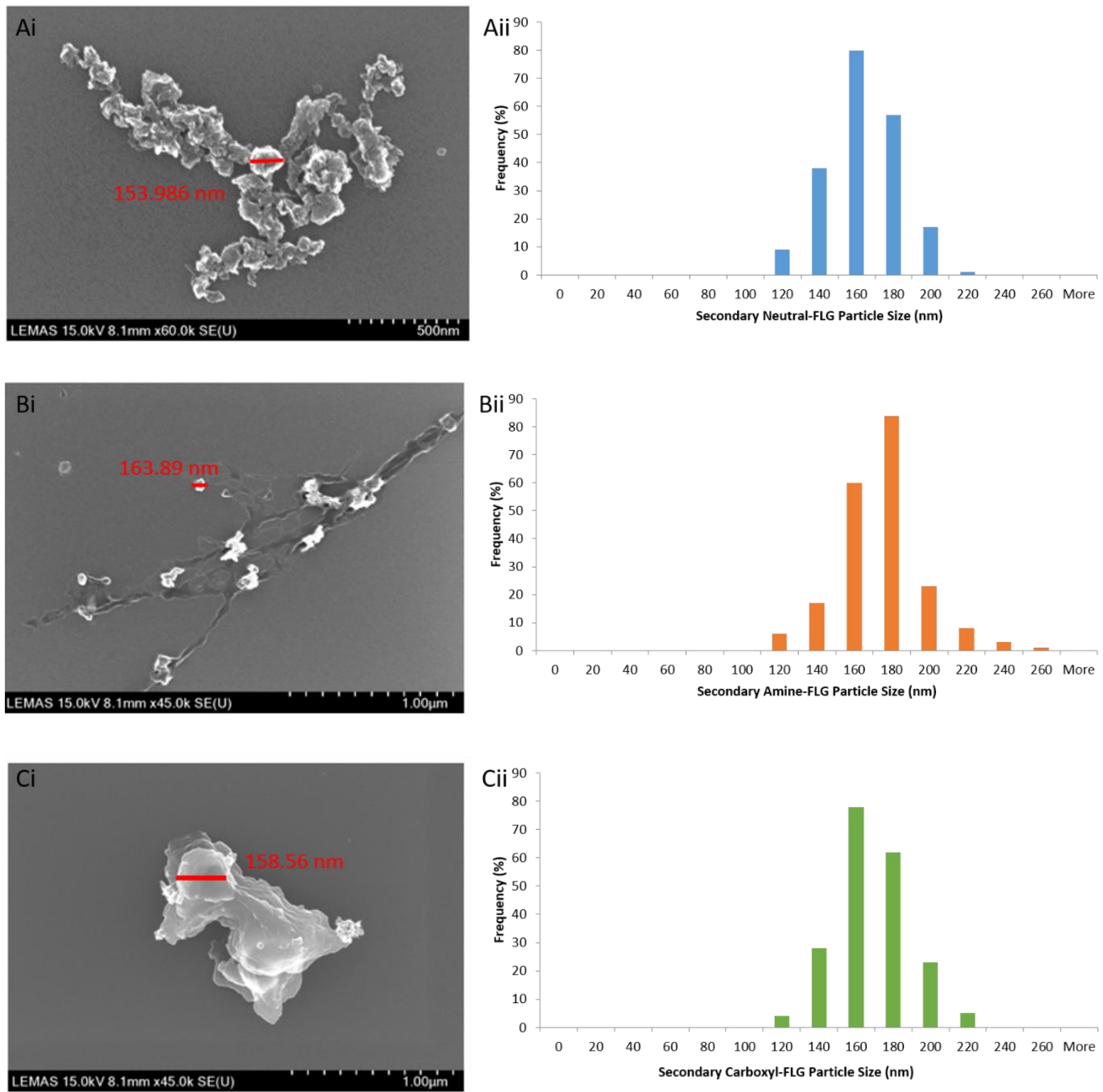

Figure 3.8 Drop cast water-suspended FLG samples. Average size $(\mathrm{nm})$ was calculated using ImageJ analysis to measure particle diameter where Neutral-FLG (Ai-A ii), Amine-FLG (Bi-B ii) and CarboxylFLG (Ci-C ii). (n=200) 

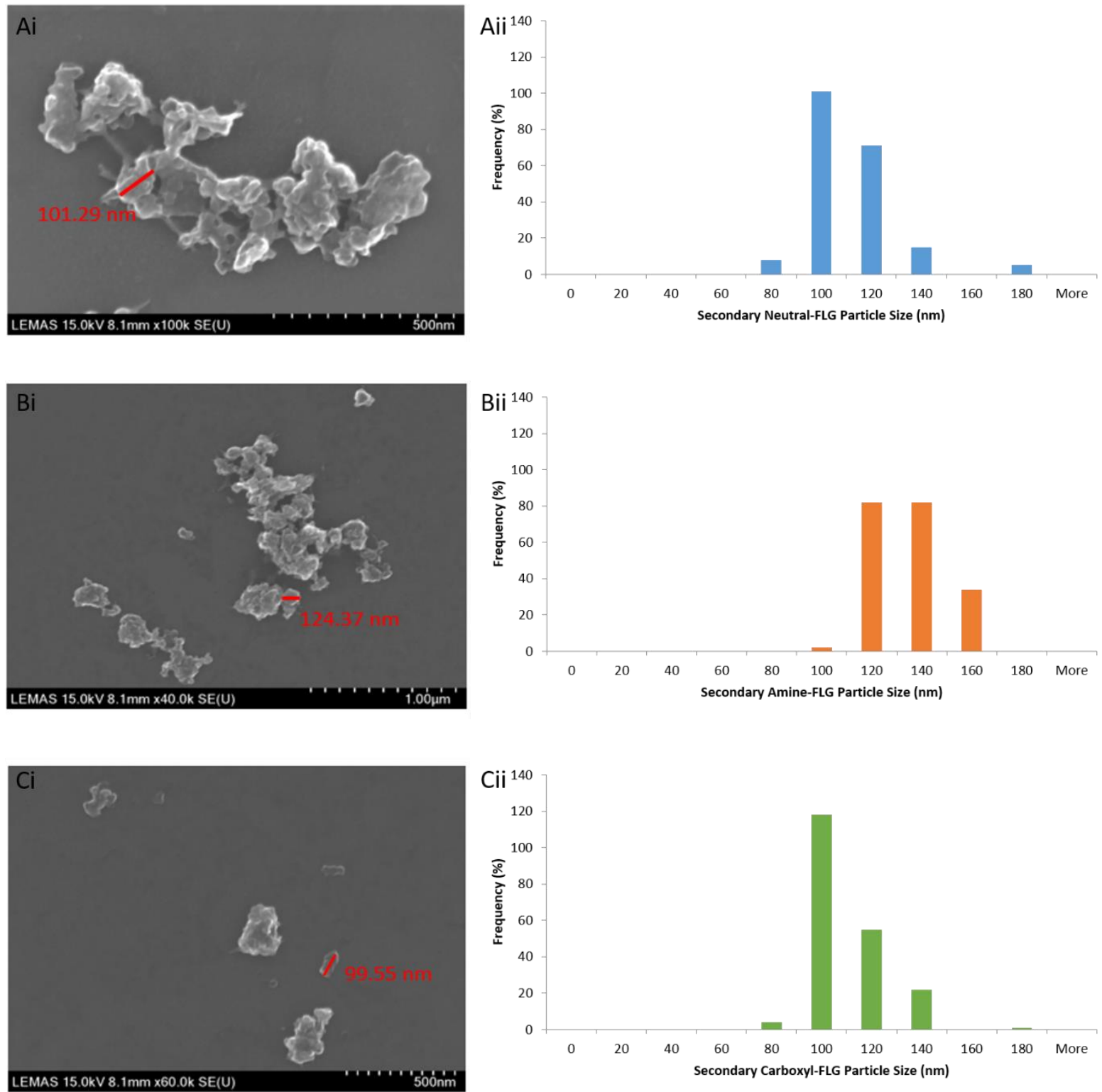

Figure 3.9 Drop cast serum-suspended FLG samples. Average size $(\mathrm{nm})$ was calculated using Image J analysis to measure particle diameter where Neutral-FLG (Ai-A ii), Amine-FLG (Bi-B ii) and CarboxylFLG (Ci-C ii). $(n=200)$ 
Table 3.8 Summary of FLG size as determined from plunge freeze experiments and ImageJ analysis.

\begin{tabular}{|l|l|l|l|l|l|l|l|l|}
\hline & \multicolumn{3}{|c|}{ Water } & \multicolumn{3}{c|}{ Media } \\
\hline Sample & $\begin{array}{l}\text { Range } \\
(\mathrm{nm})\end{array}$ & $\begin{array}{l}\text { Mean } \\
\text { Diameter } \\
+/- \text { SD } \\
(\mathrm{nm})\end{array}$ & $\begin{array}{l}\text { Median } \\
(\mathrm{nm})\end{array}$ & $\begin{array}{l}\text { Aspect } \\
\text { Ratio } \\
(\mathrm{AU})\end{array}$ & $\begin{array}{l}\text { Range } \\
(\mathrm{nm})\end{array}$ & $\begin{array}{l}\text { Mean } \\
\text { Diameter } \\
+/- \text { SD } \\
(\mathrm{nm})\end{array}$ & $\begin{array}{l}\text { Median } \\
(\mathrm{nm})\end{array}$ & $\begin{array}{l}\text { Aspect } \\
\text { Ratio } \\
(\mathrm{AU})\end{array}$ \\
\hline $\begin{array}{l}\text { Neutral- } \\
\text { FLG }\end{array}$ & $\begin{array}{l}106.5- \\
207.2\end{array}$ & $\begin{array}{l}153.9+/- \\
19.2\end{array}$ & 155.3 & 1.34 & $\begin{array}{l}71.4- \\
177.6\end{array}$ & $\begin{array}{l}101.3+/- \\
16.8\end{array}$ & 97.9 & 1.31 \\
\hline $\begin{array}{l}\text { Amine- } \\
\text { FLG }\end{array}$ & $\begin{array}{l}112.8- \\
240.9\end{array}$ & $\begin{array}{l}163.8+/- \\
21.7\end{array}$ & 162.9 & 1.36 & $\begin{array}{l}99.8- \\
157.4\end{array}$ & $\begin{array}{l}124.4+/- \\
13.9\end{array}$ & 122.3 & 1.36 \\
\hline $\begin{array}{l}\text { Carboxyl } \\
\text {-FLG }\end{array}$ & $104.7-$ & $\begin{array}{l}158.5+/- \\
19.6\end{array}$ & 157.4 & 1.30 & $\begin{array}{l}77.8- \\
172.4\end{array}$ & $\begin{array}{l}99.5+/- \\
15.3\end{array}$ & 95.4 & 1.17 \\
\hline
\end{tabular}

\subsubsection{Plunge Freeze Analysis of FLG Agglomerates}

As a direct comparison to DLS, agglomerate sizes of the graphene-based ENMs were analysed with drop casting onto copper TEM grids and the images processed using ImageJ. FLG agglomerate analysis like the DLS approach allowed a direct comparison between water and $10 \%$ supplemented media comparisons with regards to size. Some interesting differences were observed when comparing across techniques however. For example, Neutral-FLG agglomerates (which increased in size when dispersed in media as opposed to water) when measured with DLS were conversely revealed to be smaller in size (in media as opposed to water) when analysing drop cast SEM images. Similarities between the techniques were observed whereby Amine- and Carboxyl-FLG agglomerates were recorded as larger in media than in water across both DLS and plunge freeze SEM techniques. Thus, highlighting the requirement for multiple techniques to be employed and the multi-modal data to be interpreted collectively. Agglomerate analysis of plunge freeze SEM can be seen through Figures 3.10 and 3.11. Neutral-FLG suspended in water exhibited a size range of $167-1349 \mathrm{~nm}$ averaging $729.4 \mathrm{~nm}$ and a median of $802 \mathrm{~nm}$ suggesting stable near-micron scale agglomerates at the higher end of the size range. Amine-FLG agglomerates displayed slightly smaller trends ranging from 309-905nm averaging $557.4 \mathrm{~nm}$ with a median at $556 \mathrm{~nm}$. Lastly, the Carboxyl-FLG water-suspended samples ranged from $131-1183 \mathrm{~nm}$ and averaged $543.1 \mathrm{~nm}$ with a median at $519 \mathrm{~nm}$. From this data, the Carboxyl-FLG 
exhibited the smallest average agglomerate size (Table 3.9), which agrees with previously recorded DLS data for FLG samples (Table 3.5). Additionally, the Neutral- and Amine-FLG represented the largest agglomerates in descending order in both DLS and plunge freeze analyses. There was however a significant difference between the average water-suspended agglomerate sizes between techniques. Analysis of media-suspended FLG agglomerates revealed a size increase in two of the FLG. However Neutral-FLG exhibited smaller agglomerates in media as opposed to water which may indicate a preference of FBS proteins. The order of descending agglomerate size initially recorded by DLS of; Neutral-, Amine-, Carboxyl-FLG was then reversed when analysing the drop cast SEM images now showing Carboxyl-FLG as forming the larger agglomerates and Neutral-FLG the smallest. However, the large standard deviation of the drop cast analysis clearly highlights the requirement of considering DLS as a supportive technique to plunge freezing ENMs as this technique presents data of one dimension only, making analysis through ImageJ difficult. 

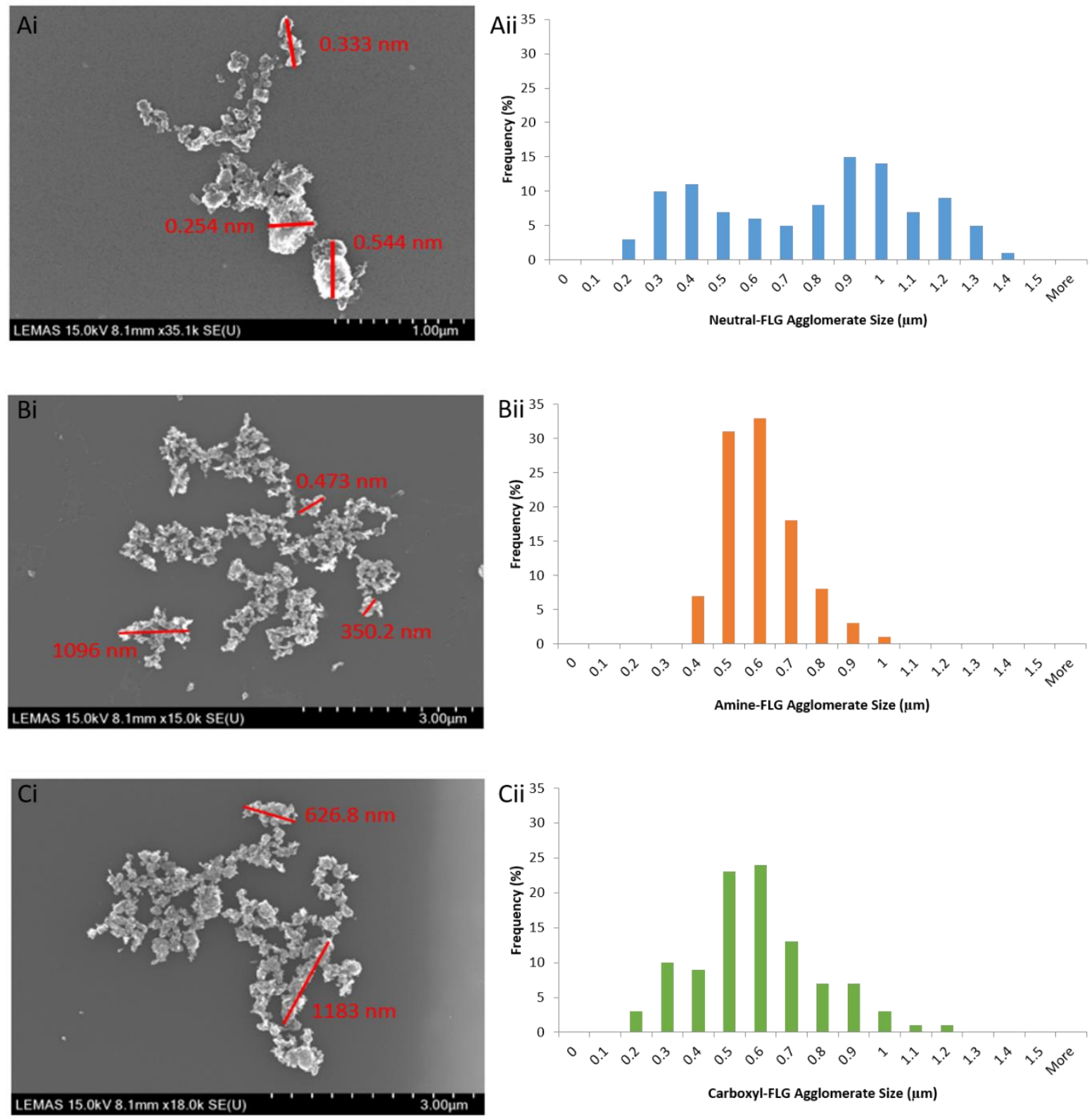

Figure 3.10 Drop cast serum-suspended FLG samples. Average size was calculated using ImageJ analysis to measure particle diameter where Neutral-FLG (Ai-A ii), Amine-FLG (Bi-B ii) and CarboxylFLG (Ci-C ii). ( $n=100)$ 

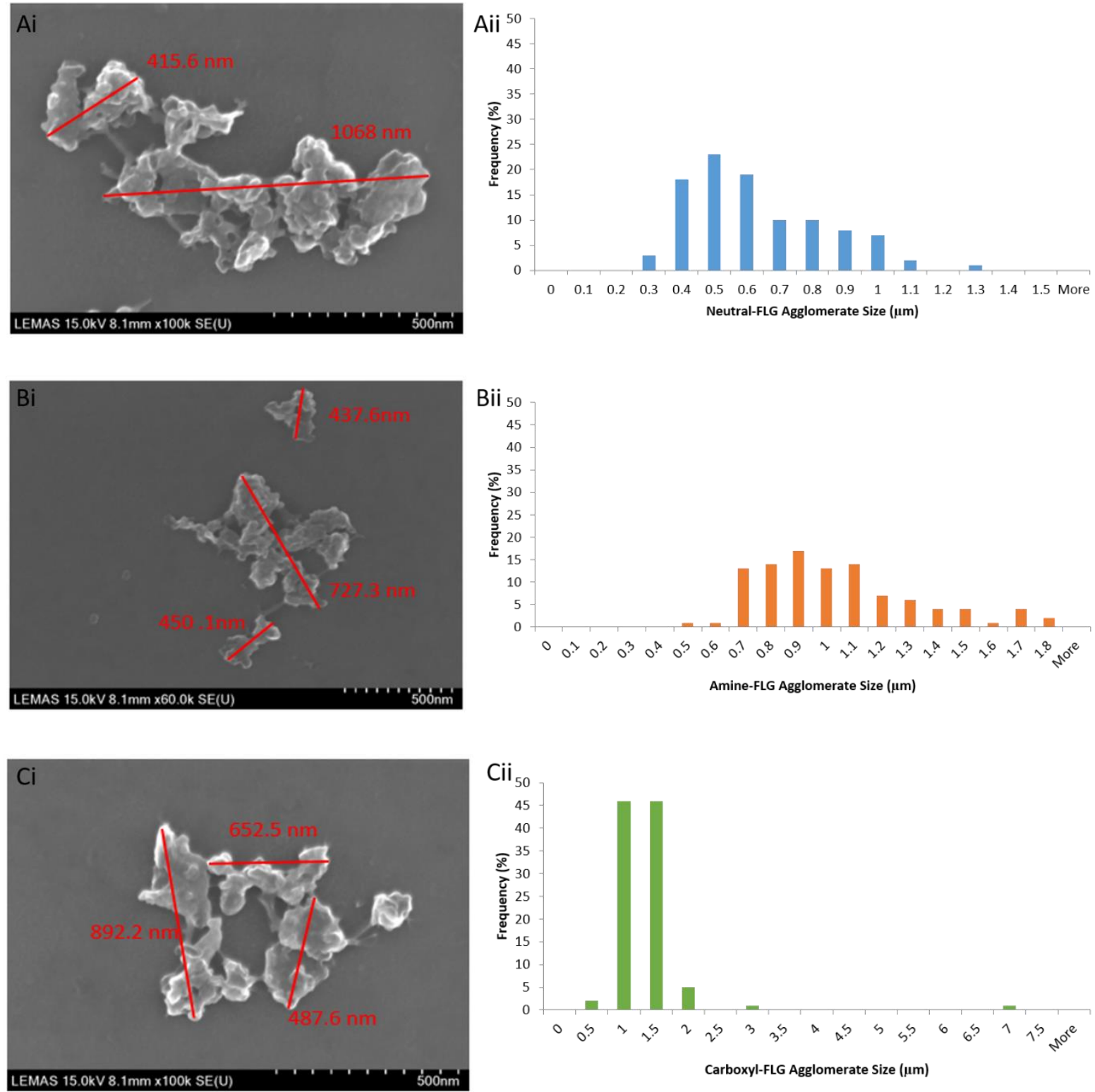

Figure 3.11 Drop cast serum-suspended FLG samples. Average size was calculated using ImageJ analysis to measure particle diameter where Neutral-FLG (Ai-A ii), Amine-FLG (Bi-B ii) and CarboxylFLG (Ci-C ii). ( $n=100)$ 
Table 3.9 Summary of FLG agglomerate size as determined from plunge freeze experiments and ImageJ analysis.

\begin{tabular}{|l|l|l|l|l|l|l|}
\hline & \multicolumn{3}{|c|}{ Water } & \multicolumn{3}{c|}{ Media } \\
\hline Sample & $\begin{array}{l}\text { Range } \\
(\mathrm{nm})\end{array}$ & $\begin{array}{l}\text { Mean } \\
\text { Diameter } \\
+/- \text { SD } \\
(\mathrm{nm})\end{array}$ & $\begin{array}{l}\text { Median } \\
(\mathrm{nm})\end{array}$ & $\begin{array}{l}\text { Range } \\
(\mathrm{nm})\end{array}$ & $\begin{array}{l}\text { Mean } \\
\text { Diameter } \\
+/- \text { SD } \\
(\mathrm{nm})\end{array}$ & $\begin{array}{l}\text { Median } \\
(\mathrm{nm})\end{array}$ \\
\hline $\begin{array}{l}\text { Neutral- } \\
\text { FLG }\end{array}$ & $\begin{array}{l}167- \\
1349\end{array}$ & $\begin{array}{l}729.4+/- \\
317\end{array}$ & 802 & $\begin{array}{l}269- \\
1240\end{array}$ & $\begin{array}{l}583.7+/- \\
212\end{array}$ & 537 \\
\hline Amine- & $309-$ & $\begin{array}{l}557.4+/- \\
119.9\end{array}$ & 556 & $450-$ & $\begin{array}{l}988.3+/- \\
285.3\end{array}$ & 934 \\
\hline Carboxyl & $131-$ & $543.1+/-$ & 519 & $413-$ & $1083+/-$ & 1029 \\
- FLG & 1183 & 202.3 & & 1516 & 626.6 & \\
\hline
\end{tabular}

\subsubsection{FLG Thickness Analysis by Atomic Force Microscopy}

Acquiring the third dimension of FLG required Atomic Force Microscopy (AFM), the preparation of which was discussed in detail in Section 3.2.5. Briefly, stock FLG was diluted down to $100 \mu \mathrm{g} / \mathrm{ml}$ in ultrapure water before $10 \mu \mathrm{l}$ was pipetted and dried onto a mica slide for analysis. The AFM was operated in scanning (tapping) mode with images captured and analysed with the Bruker Nanoscope Analysis Package version 1.4 (Figure 3.12). This analysis provided detail regarding the approximate height of the material. The data in this section has been represented as average thickness of the FLG materials, determined from 3D figures of the landscape of the sample slide (Figure 3.13) as well as live AFM acquisition (Figure 3.14). 


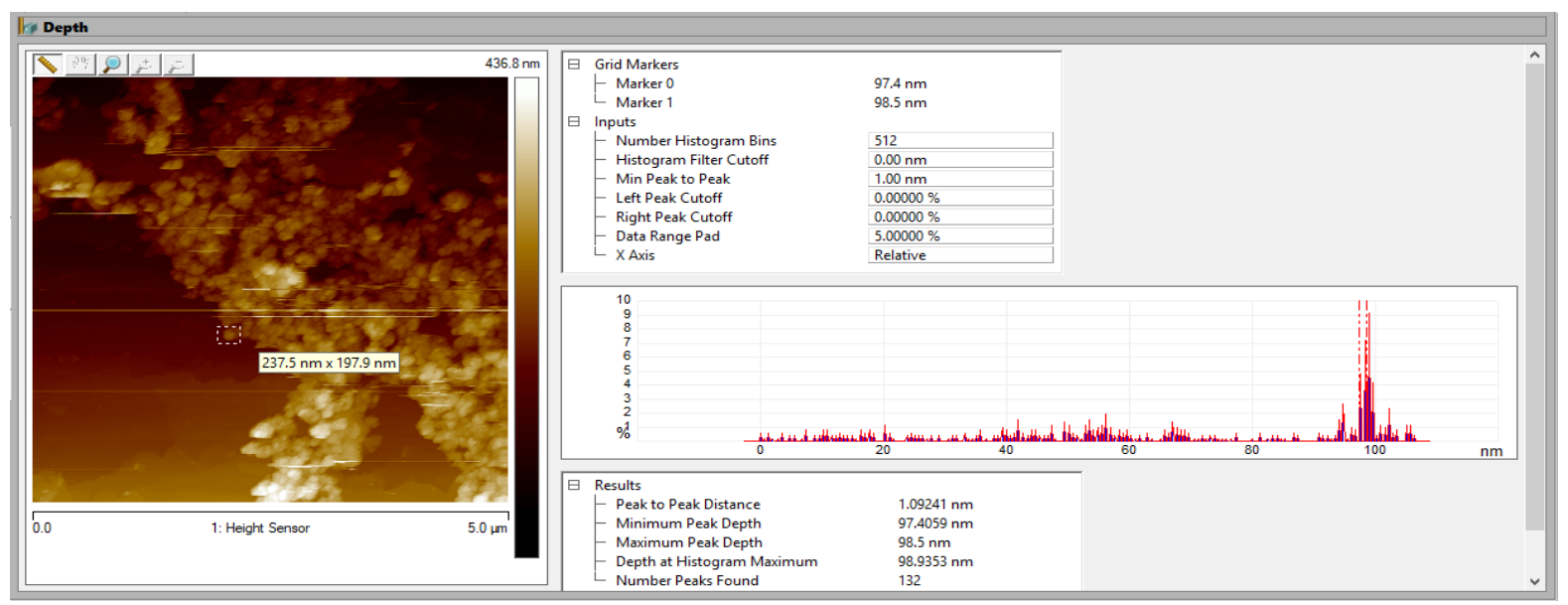

Figure 3.12 Example depth data analysis using Bruker Nanoscope Analysis Package version 1.4.

Individual FLG particles were measured and the raw data extracted from the results tab to plot both the average, median, standard deviation and the depth size range. $(n=100)$

Cell culture media resulted in crystallisation (Figure 3.13E), which rendered the analysis impossible, hence 3D depth analysis was performed on water-suspended FLG samples. This analysis revealed Neutral-FLG as the thickest of all three samples with an average depth of $94.725 \mathrm{~nm}$. Amine-FLG averaged $86.197 \mathrm{~nm}$ and Carboxyl-FLG was $55.16 \mathrm{~nm}$ summarised in Table 3.10. The range of particle depths revealed striking agglomerate size differences ranging from a few atomic layers of graphene up to bulk quantities. Neutral-FLG being a prime example at $17.3-494 \mathrm{~nm}$. Median values of all three FLG samples displayed a wide range overall with Amine-FLG at $92.75 \mathrm{~nm}$ and Carboxyl-FLG at $42.75 \mathrm{~nm}$, suggesting the most stable third dimension for the water-suspended FLG samples would be sub-100 $\mathrm{nm}$. AFM can also be utilised to calculate the number of layers from different sized agglomerates with Equation 3.1. In doing so, and using the smallest estimate of the size distribution, the Neutral-FLG again proves to be the largest in terms of layer number at 50 atomic sheets of graphene (graphite). In contrast, Carboxyl-FLG particles were the smallest exhibiting the lowest possible conformation of four atomic layers of graphene (Table 3.10).

\section{Equation 3.1}

$N=\left(t_{\text {measured }}-0.4\right) / 0.335$

Where; $\mathrm{N}$ is the calculated layer number, $\mathrm{t}_{\text {measured }}$ is the thickness (depth) measured by AFM, 0.4 is arbitrary and 0.335 represents the inter-layer spacing between graphene sheets (Shearer et al., 2016). 
Table 3.10 Depth analysis on FLG using Bruker Nanoscope Analysis Package (v 1.4). Number of layers (N) was calculated using Equation 3.1 and the smallest (most accurate) representation of thickness measured by AFM.

\begin{tabular}{|l|l|l|l|l|l|}
\hline ENM & Range $(\mathrm{nm})$ & Average $(\mathrm{nm})$ & Median $(\mathrm{nm})$ & $\begin{array}{l}\text { Standard } \\
\text { Deviation }(\mathrm{nm})\end{array}$ & $\begin{array}{l}\text { Calculated } \\
\text { layer } \\
\text { number }(\mathrm{N})\end{array}$ \\
\hline Neutral-FLG & $17.3-494$ & 94.73 & 74.90 & 67.94 & 50 \\
\hline Amine-FLG & $4.5-201$ & 86.20 & 92.75 & 42.16 & 12 \\
\hline Carboxyl-FLG & $1.73-177$ & 55.16 & 42.75 & 42.22 & 4 \\
\hline
\end{tabular}



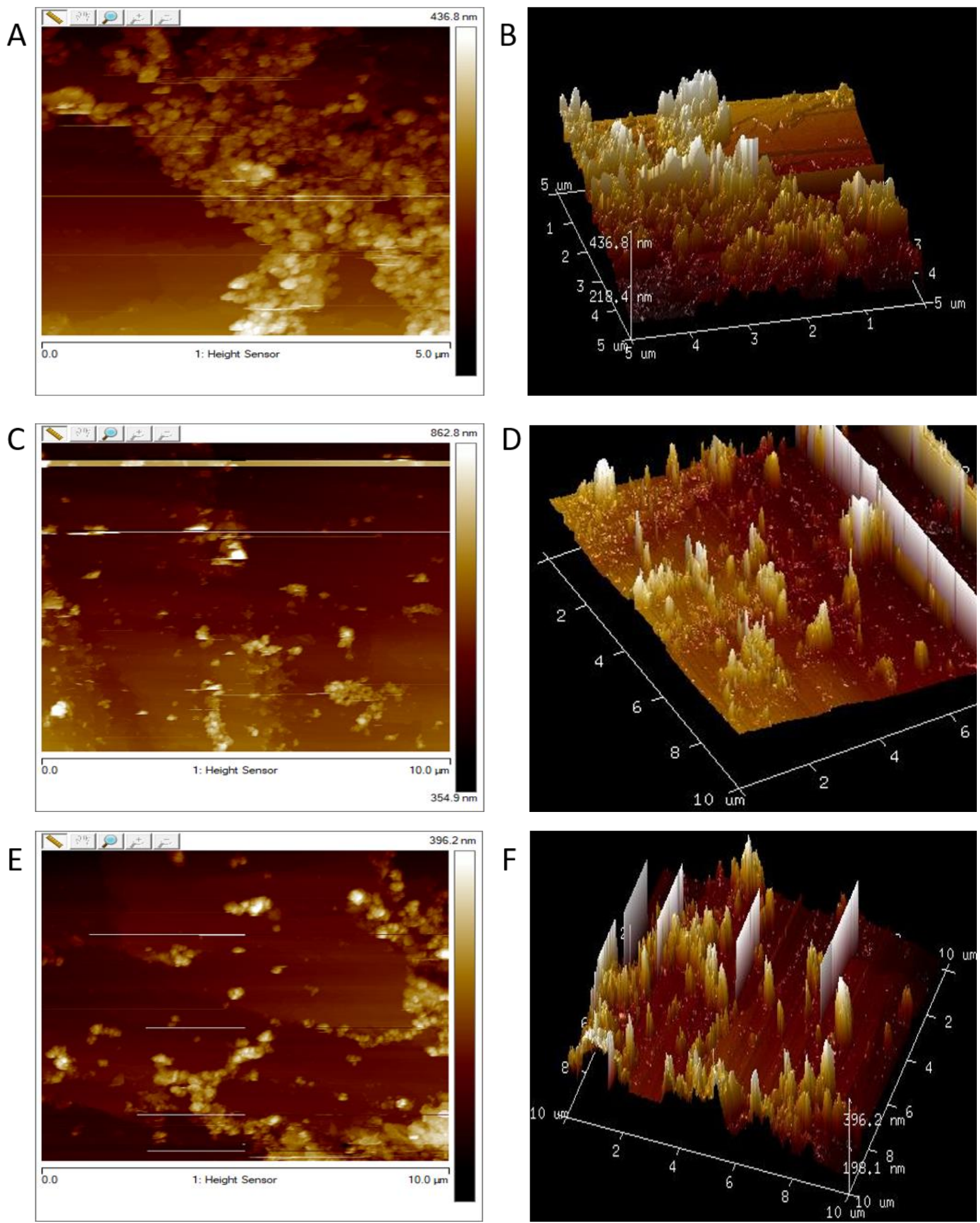

Figure 3.13 Thickness analysis data acquired through Bruker Nanoscope Analysis Package (v 1.4) of 2D and 3D samples (Neutral-FLG (A-B), Amine-FLG (C-D) and Carboxyl-FLG (E-F)). A total of 100 particles were scanned and analysed per material, software permitted conversion of live 2D images into a 3D landscape. Additionally, AFM when viewed in the 3D animation permits the detection and visualisation of small and large agglomerates within each sample. 

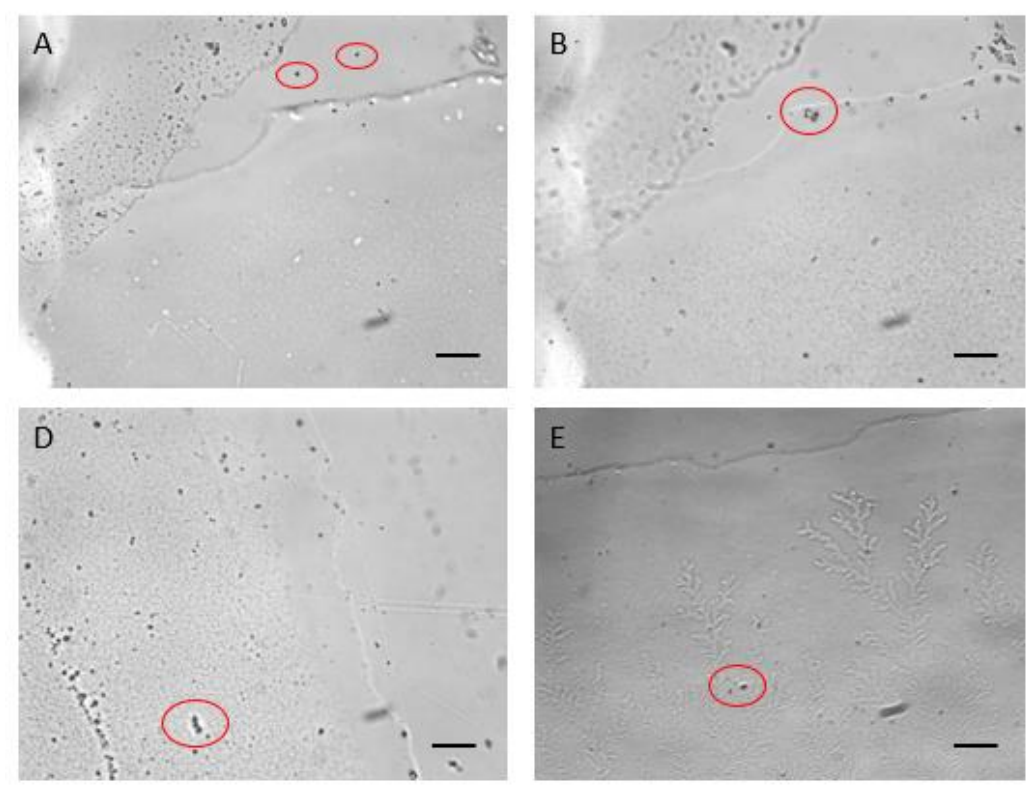
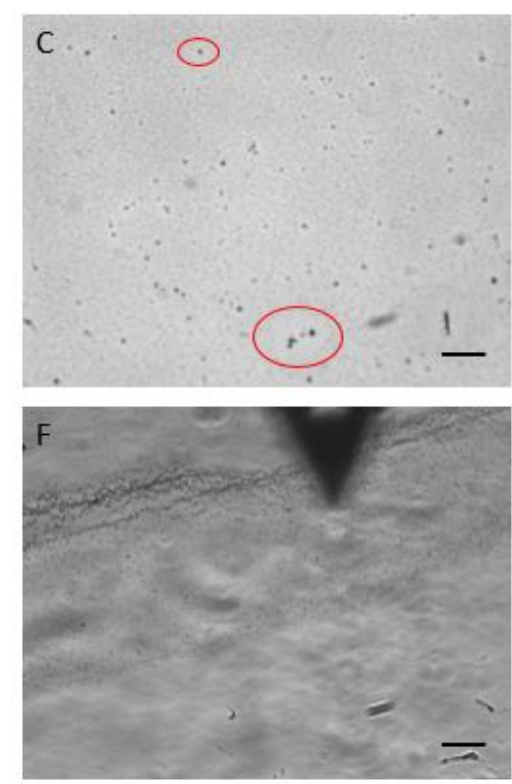

Figure 3.14 AFM images captured with the Bruker Dimension Icon AFM. Image capture revealed individual FLG particles as well as agglomerates. Example particles/agglomerates have been highlighted in red (Neutral-FLG (A, B), Amine-FLG (C, D), Carboxyl-FLG (E, F)). Analysis of media suspended samples, evident in image $\mathrm{E}$ was not undertaken due to sample crystallisation. (Scale bar $5 \mu \mathrm{m})$.

\subsubsection{Theoretically Calculated Aerodynamic Diameter \& Effective Density}

Utilising AFM as a tool for calculating the thickness of the FLG materials combined with the accuracy of plunge freeze analysis of particle diameter, these values can be inserted into Equation 3.2 derived by Schinwald and colleagues to calculate the aerodynamic diameter of non-spherical nanoparticles. The only issue with this equation being it relies on the known unit and bulk density of graphene. Since the density of true graphene (one single sheet) is experimentally impossible to derive, theoretical graphene densities were used as highlighted by Park and colleagues (Park et al., 2017b). The authors define the unit density of graphene nano-platelets between $0.03-0.1 \mathrm{~g} / \mathrm{cm}^{3}$ and bulk graphite between $2.09-2.23 \mathrm{~g} / \mathrm{cm}^{3}$. 


\section{Equation 3.2}

$D_{a e}=\sqrt{ } 9 \pi / 16 \times \rho / \rho_{0} \times$ diameter $\times$ thickness

Where; $\rho$ is the unit density, $\rho_{0}$ is the bulk density, average diameter in $10 \%$ serum (calculated from plunge freeze analysis of individual particles) and average thickness (calculated using AFM).

Effective Density was calculated using Equation 3.3 derived by DeLoid and colleagues (DeLoid et al., 2014). With this value, a dosimetry script was utilised, written by the same author to simulate the sedimentation of the FLG materials in $10 \%$ supplemented culture media, in effect determining what concentration of the original dose of $100 \mu \mathrm{g} / \mathrm{ml}$ deposits onto the adherent bronchial or alveolar cell lines utilised in later Chapters.

\section{Equation 3.3}

$\left.\rho_{\mathrm{EV}}=\rho_{\text {media }}+\left[\left(\mathrm{M}_{\mathrm{ENM}}-\mathrm{M}_{\mathrm{ENMsol}} / \mathrm{V}_{\text {pellet }} \mathrm{SF}\right)\right] .\left(1-\rho_{\text {media }} / \rho_{\mathrm{ENM}}\right)\right]$

Where; $\rho$ is density, $\mathrm{M}$ is mass, $\mathrm{V}_{\text {pellet }}$ (volume of FLG pellet at $100 \mu \mathrm{g} / \mathrm{ml}$ )

Table 3.11 Summary of aerodynamic diameter and effective densities of FLG materials.

\begin{tabular}{|l|l|l|}
\hline Material & Calculated $\mathrm{D}_{\mathrm{ae}}(\mathrm{nm})$ & $\begin{array}{l}\text { Calculated Effective Density } \\
\left(\mathrm{g} / \mathrm{cm}^{3}\right)\end{array}$ \\
\hline Neutral-FLG & $19.23-33.9$ & 1.008 \\
\hline Amine-FLG & $18.92-33.45$ & 1.008 \\
\hline Carboxyl-FLG & $14.89-26.32$ & 1.008 \\
\hline
\end{tabular}

The calculated aerodynamic diameter and effective density of the FLG materials used in this research can be supported by the findings of Su and colleagues (2016). Which were of a similar order to the data generated and summarised in Table 3.11. Su and colleagues also demonstrate, using a script simulation, that increasing the aerodynamic diameter decreases the effective density. 


\subsubsection{In Vitro Dosimetry}

Following the successful calculation of the FLG aerodynamic diameter and effective density, a dosimetry script written by DeLoid and colleagues modelling the in vitro sedimentation of ENMs at $100 \mu \mathrm{g} / \mathrm{ml}$ (highest tested concentration) was used to predict how much of the initial $100 \mu \mathrm{g} / \mathrm{ml}$ reaches the adherent epithelial cells (DeLoid et al., 2014). This effect can be seen in Figure 3.15 whereby the lowest and the highest theoretical densities of FLG were used matched with the plunge freeze fibre diameter to complete the script analysis. The dosimetry script was run using MATLAB (R2015b). The trend shown by all FLG materials in Figure 3.15 is that over the course of the 24-hour exposure the adherent cells at the bottom of the exposure vessel (whether it be a T25 culture flask or 96-well plate) are never truly exposed to the theoretical test concentration. This effect becomes more apparent as the unit density of the FLG is increased from 0.03 to $0.1 \mathrm{~g} / \mathrm{cm}^{3}$. The dosimetry modelling of these materials however has demonstrated great efficiency with in vitro dosing of a liquid - liquid interface. Approximately $1.8 \mu \mathrm{g} / \mathrm{ml}$ is lost during the exposure period over 24 hours in FLG materials with a density of $0.03 \mathrm{~g} / \mathrm{cm}^{3}$ whereas $3.3 \mu \mathrm{g} / \mathrm{ml}$ is lost in FLG materials with a density of $0.1 \mathrm{~g} / \mathrm{cm}^{3}$ according to the MATLAB script performed on the highest tested concentration of ENMs used in this study $(100 \mu \mathrm{g} / \mathrm{ml})$. 

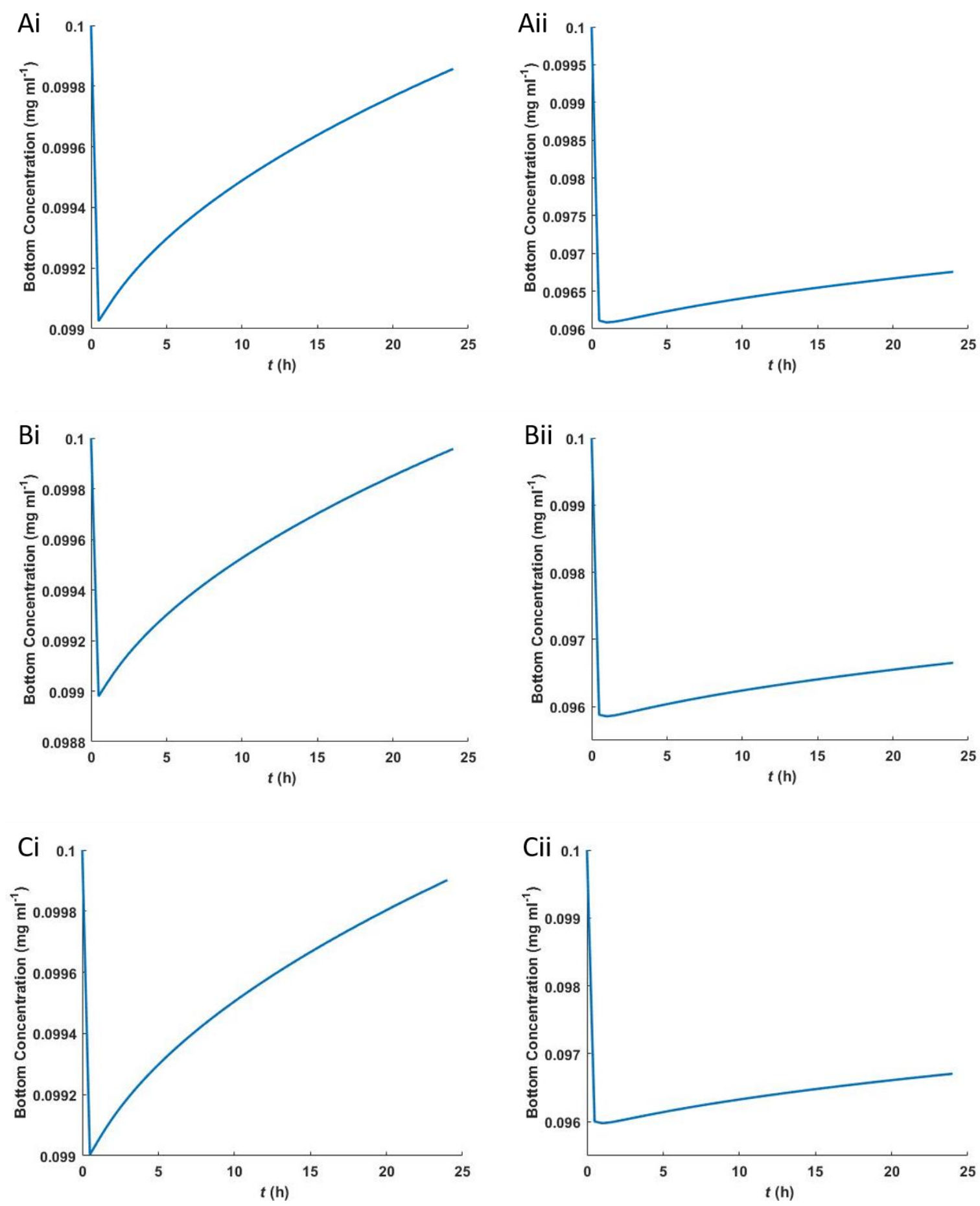

Figure 3.15 Dosimetry plots modelling the final concentration to reach the adherent cell lines at the bottom of a T25 flask in $10 \mathrm{ml}$ of media from an initial $100 \mu \mathrm{g} / \mathrm{ml}$ exposure concentration of FLG. To account for the range of graphene densities put forward by Park and colleagues (2017), both the minimum and maximum values $\left(0.03-0.1 \mathrm{~g} / \mathrm{cm}^{3}\right)$ were modelled for each FLG material. Neutral-FLG (Ai-Aii), Amine-FLG (Bi-Bii) and Carboxyl-FLG (Ci-Cii). All graphs denoted by (i) represent FLG ENMs modelled with a density of $0.03 \mathrm{~g} / \mathrm{cm}^{3}$, whereas graphs denoted by (ii) represent FLG ENMs modelled with a density of $0.1 \mathrm{~g} / \mathrm{cm}^{3}$. 


\subsubsection{Raman Spectroscopy of FLG}

In addition to calculating the layer number with Equation 3.1 in section 3.3.5 by use of AFM, Raman spectroscopy is perhaps the most widely considered technique for determining the layer number in CVD and exfoliated graphene samples. As described in section 3.2.6, FLG was diluted down to $100 \mu \mathrm{g} / \mathrm{ml}$ and dried onto silicon chips for analysis, a representative spectrum for each FLG material can be seen in Figure 3.16. All three FLG materials exhibited a peak originating at $1350 \mathrm{~cm}^{-1}$ constituting the $\mathrm{D}$ (disorder) peak, this was far less prominent however in the Amine-FLG. Both Neutral-FLG and Carboxyl-FLG showing a 4-fold intensity increase in this band alone suggesting greater structural defects as compared to Amine-FLG. Showing a sharp signal at $1583 \mathrm{~cm}^{-1}$ is the $\mathrm{G}$ band which again is unique among the samples with Amine-FLG exhibiting a sharp concise $G$ band that splits at the base into a second order (double-resonance)-induced D' band. The broader $\mathrm{G}$ band of both Neutral- and Carboxyl-FLG is highly indicative of lower frequency $E_{2 G}$ phonon interactions at $\Gamma$ of the carbon rings. The source of the Amine-FLG $D^{\prime}$ band is an intra-valley process connecting two points of the same Dirac Cone surrounding $\mathrm{K}$ or $\mathrm{K}^{\prime}$. The $\mathrm{G}^{*}$ band arising as a double resonant process at $2450 \mathrm{~cm}^{-1}$ has been subject to much debate, sometimes termed $\left(D+D^{\prime}\right)$ and has been assigned a combination of a $D$ phonon and an acoustic in plane longitudinal (LA) phonon. Meanwhile the $2 \mathrm{D}$ band at $2700 \mathrm{~cm}^{-1}$ present in all samples arises not from a disordered structure but from two transverse optical (TO) phonons near the $\mathrm{K}$ points of the Brillouin Zone. The 2D peak is unique to $\mathrm{FLG}$ and provides an indicator to layer number. The greater the number of layers the broader the peak becomes ultimately splitting. The ratio of $2 \mathrm{D}$ to $\mathrm{G}$ is often a rapid determination of how many atomic sheets of graphene are present in a sample which in this instance appears to be 3-4 in each sample. Bespoke to the FLG in this study however and not observed in the literature are the peaks present at $2350 \mathrm{~cm}^{-1}$. which is weakest by intensity in Amine-FLG, and is possibly a result of the silicon substrate. 


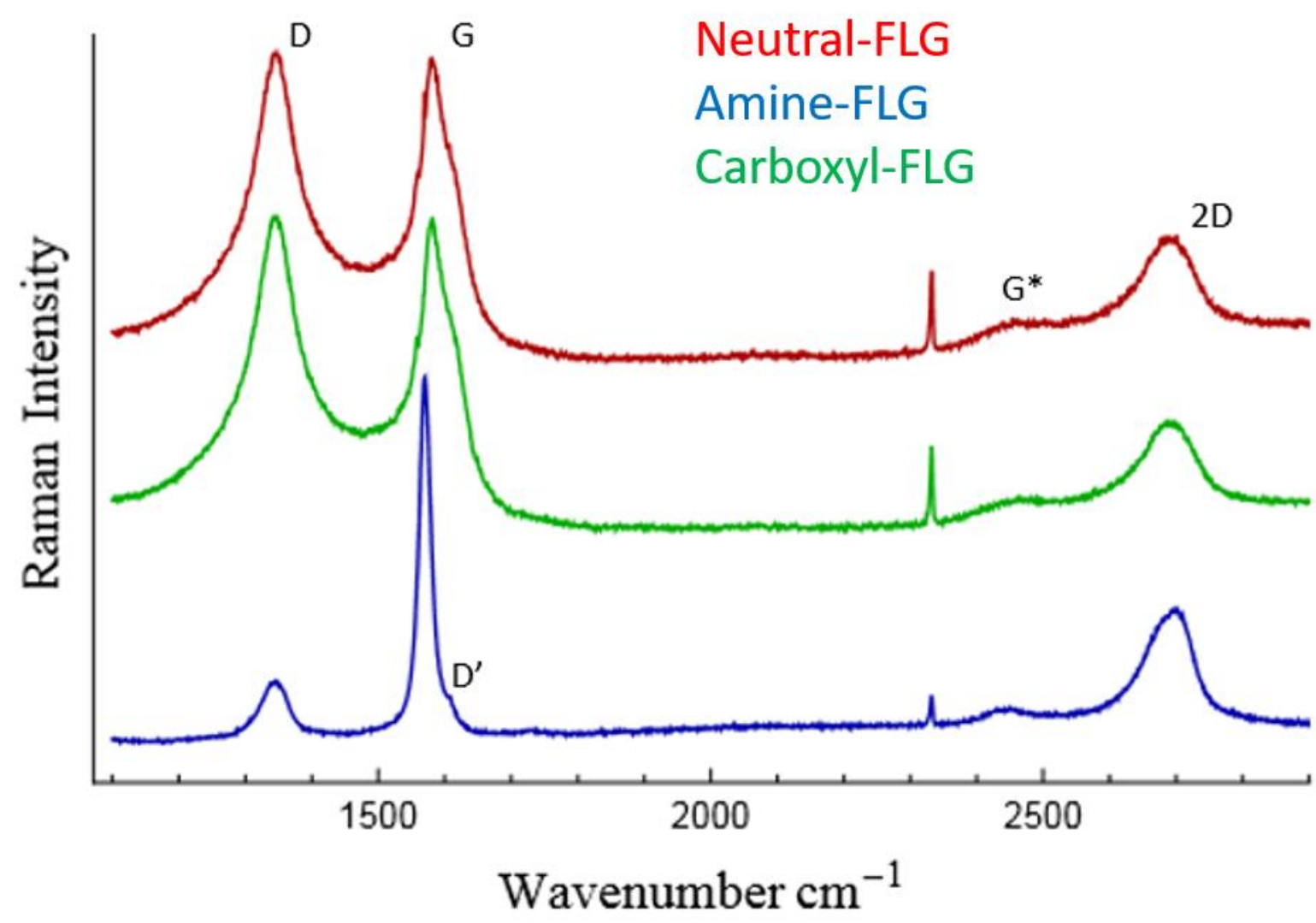

Figure 3.16 Collective Raman Intensity Spectra of FLG samples, wavelength of incident laser set to $533 \mathrm{~nm}$. FLG samples exhibited the D, G and 2D peaks representing structural disorder, carbon-carbon bond stretching (vibrational mode) and layer number respectively. Key; Neutral-FLG (Red), Amine-FLG (Blue) and Carboxyl-FLG (Green). 


\subsubsection{Detection of Acellular Superoxide Radicals by EPR}

Measurements (represented as $\mathrm{g} / \mathrm{ml}$, Table 3.12) of oxygen-centred free radical generation via spintrap EPR revealed a significant $(p<0.05)$ concentration-dependent trend (Figure 3.17). Initially, this trend was witnessed in water-suspended samples at $55 \mu \mathrm{g} / \mathrm{ml}$ with Neutral- and Carboxyl-FLG ENMs. Meanwhile the highest non-significant recorded response was induced by FLG at $30 \mu \mathrm{g} / \mathrm{ml}$. ENMs were incubated with Tempone-H a highly sensitive spin-trap which selectively targets superoxide radicals $\left(\mathrm{O}_{2}{ }^{-*}\right)$. Amine-FLG was only capable of producing $\mathrm{O}_{2}{ }^{-*}$ at a higher concentration of $100 \mu \mathrm{g} / \mathrm{ml}$. EPR intensity of the highest tested concentration of $180 \mu \mathrm{g} / \mathrm{ml}$ was highly significant in all FLG samples showing 70-fold, 23-fold and 25-fold changes over control levels with Neutral-FLG, Amine-FLG and Carboxyl-FLG respectively. A near-identical trend was observed with samples suspended in Krebs Buffer however due to $n=1$ further analysis was unable to be performed.

Table 3.12 Dose metric conversion.

\begin{tabular}{|l|l|}
\hline Dose log [particle] $(\mathrm{g} / \mathrm{ml})$ & Dose $(\mu \mathrm{g} / \mathrm{ml})$ \\
\hline-5.5 & 3.2 \\
\hline-5.25 & 6 \\
\hline-5 & 10 \\
\hline-4.75 & 18 \\
\hline-4.5 & 30 \\
\hline-4.25 & 55 \\
\hline-4 & 100 \\
\hline-3.75 & 180 \\
\hline
\end{tabular}



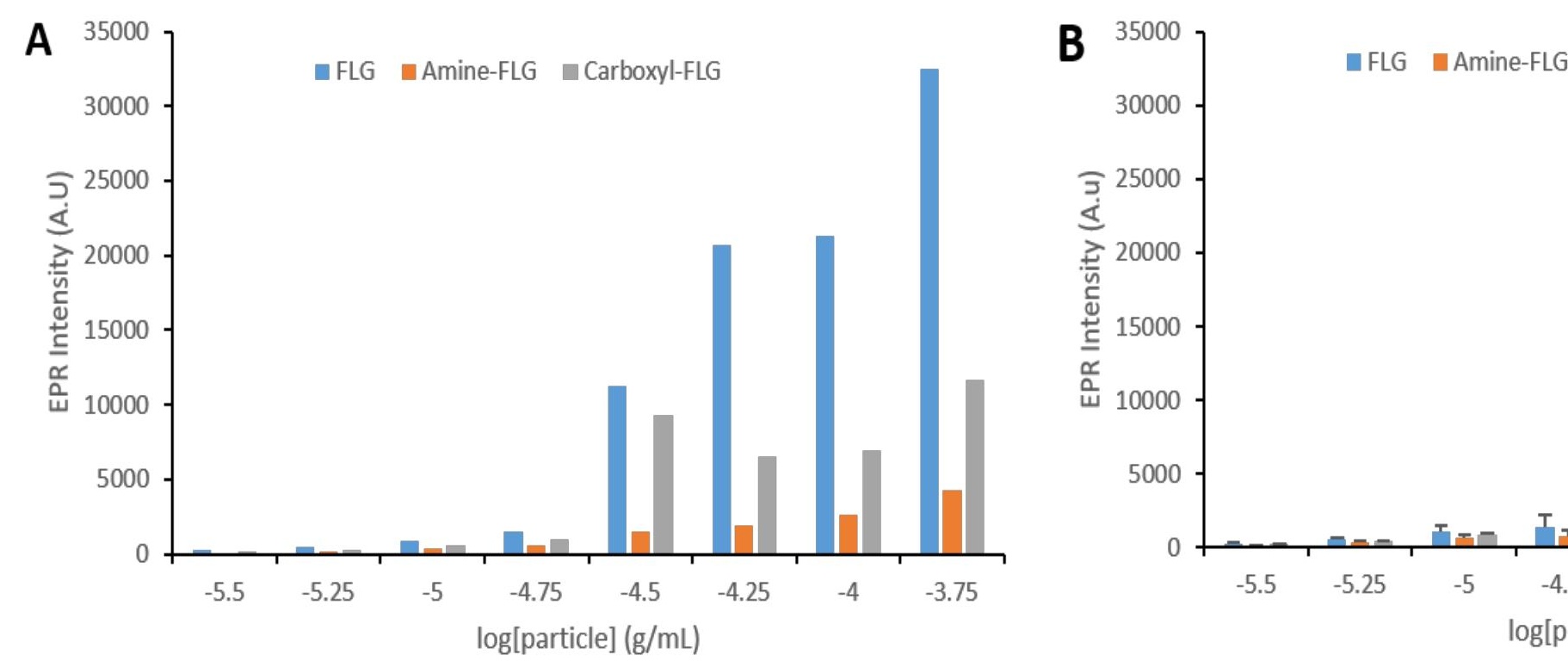

Figure 3.17 Superoxide generation of FLG quantified with EPR. Superoxide detection was evaluated in both Krebs Bu diluted in Krebs Buffer $(n=1)$ and samples diluted in water $(n=3)$. There was a significant elevation in superoxide rad a concentration-dependent manner. Neutral-FLG demonstrated the greatest capacity to generate harmful oxidative a more potent superoxide production over Amine-FLG. Results were considered statistically significant $\left({ }^{*}\right)$ when $p<0$. 


\subsubsection{Determination of Primary ENMs Surface Area}

The specific surface area of each ENM used in this study was evaluated by BET, coupled to pore size analysis of the FLG samples. The benefit of this technique when applied to ENMs is that the dissolution rate may be predicted, which acts as an indicator of bioavailability, a crucial aspect of ENM reactivity. BET measures the adsorption of gas (Nitrogen) onto the surface of a material which includes porous structures thus typically providing a larger value than other conventional techniques such as air permeability. The volume of Nitrogen gas adsorbed to the surface of the material is often measured accordingly with the boiling point of Nitrogen $\left(-196^{\circ} \mathrm{C}\right)$, the calculation then utilises BET theory and the BET adsorption isotherm equation (Equation 3.4).

\section{Equation 3.4}

$1 /\left[\mathrm{V}_{\mathrm{a}} \cdot(\mathrm{P} / \mathrm{P}-1)\right]=\mathrm{C}-1 / \mathrm{V}_{\mathrm{m}} \mathrm{C} \cdot \mathrm{P} / \mathrm{P}_{0}+1 / \mathrm{V}_{\mathrm{m}} \mathrm{C}$

Where; $\mathrm{P}$ is the partial vapour pressure of adsorbate gas, $\mathrm{P}_{0}$ is the saturated pressure of gas, $\mathrm{V}_{\mathrm{a}}$ represents the volume of gas adsorbed at standard pressure and temperature $\left(273.15 \mathrm{~K}\right.$ and $1.013 \times 10^{5}$ $\mathrm{Pa}), \mathrm{V}_{\mathrm{m}}$ is the volume of gas adsorbed at standard temperature and pressure resulting in a surface monolayer $(\mathrm{ml})$ and $\mathrm{C}$, is an enthalpy of adsorption constant.

The results from the BET analysis of Neutral-, Amine-, and Carboxyl-FLG and CB particles revealed a range of surface areas between all ENMs (Table 3.13). Upon inspection of the FLG samples which undergo the same manufacture process of plasma exfoliation, there appears to be a slight difference in their BET surface areas, namely the Neutral-FLG which displays an approximate $20 \mathrm{~m}^{2} / \mathrm{g}$ increase over the Amine- and Carboxyl-FLG materials. This change in surface area however is not sustained where pore characteristics are concerned. The incorporation of nano-sized pores into the FLG materials was an addition included by the manufacturers, (PCT) to aid in one of their targeted applications in hydrogen storage. This data here however suggests the minor 1.7-fold change is not enough to warrant a change in pore size characteristics. Moreover, the analyses on FLG pore size has revealed, not specific pore sizes, but rather surface structure defects of the order of single carbon atoms. The requirement of both techniques (HK and SF) in this analysis was essential to cover the nano-pore features which can manifest as micropores $(<2 \mathrm{~nm})$ or mesopores (2-50nm). However, both techniques when applied to the FLG materials provided identical values for each material. In the work published by Kostoglou and colleagues (2015) the FLG materials like the ones used in this study were manufactured with nanopores ranging from $0.7-0.8 \mathrm{~nm}$. In stark contrast to this study however, both 
the HK ad SF methods, routinely employed to evaluate microporous structures revealed $0.1838 \mathrm{~nm}$ and $0.1754 \mathrm{~nm}$ pore radii respectively. These values are likely not indicators of nanoporous structures in the FLG but more likely attributed to the Stone-Wales defects in the graphene planes. CB particles can exist in a multitude of surface areas due to the fractions of carbon which exist in CB products, these changes can include amorphous and crystalline carbon allotropes, however the manufacturers provide a range of existing surface areas for Lampblack particles $\left(20-95 \mathrm{~m}^{2} / \mathrm{g}\right)$, which appears to agree with the data exhibited in Table 3.13.

Table 3.13 Multipoint BET surface area and micropore features. Two techniques were selected to analyse pore parameters; Horvoth Kawazoe (HK) and Saito and Foley (SF).

\begin{tabular}{|c|c|c|c|}
\hline Material & Surface Area $\left(\mathrm{m}^{2} / \mathrm{g}\right)$ & Pore Volume (cc/g) & Pore Size $(\AA)$ \\
\hline Neutral-FLG & 55.2 & $\begin{array}{ll} & \mathrm{HK}-1.091 \\
\text { - } & \mathrm{SF}-1.140\end{array}$ & $\begin{array}{ll}\text { - } & \mathrm{HK}-1.838 \\
\text { - } & \mathrm{SF}-1.754\end{array}$ \\
\hline Amine-FLG & 31.2 & $\begin{array}{ll}\text { - } & \mathrm{HK}-1.091 \\
\text { - } & \mathrm{SF}-1.140\end{array}$ & $\begin{array}{ll}\text { - } & \mathrm{HK}-1.091 \\
\text { - } & \mathrm{SF}-1.140\end{array}$ \\
\hline Carboxyl-FLG & 33.7 & $\begin{array}{ll}\text { - } & \mathrm{HK}-1.091 \\
\text { - } & \mathrm{SF}-1.140\end{array}$ & $\begin{array}{ll}\text { - } & H K-1.091 \\
\text { - } & \mathrm{SF}-1.140\end{array}$ \\
\hline CB & 33.2 & N/A & $N / A$ \\
\hline
\end{tabular}




\subsubsection{Confirmation of Endotoxin Absence via LAL Gel Clot Test}

The production of ENMs can often introduce unwanted contaminations that, can manifest commonly as heavy metals or components of bacterial cell membranes known as endotoxins (Smulders et al., 2012). This can then be exacerbated with careless handling leading to unpredictable biological testing. To assess the endotoxin levels in the PCT-supplied FLG samples and the Evonik Industriesmanufactured CB particles the E-Toxate kit (Sigma, UK) was employed. All ENMs were incubated with E-Toxate working solution in a water bath at $37^{\circ} \mathrm{C}$ for 1 hour before inversion where a positive result was determined only if a solid clot had formed. All ENMs under investigation in this study provided a negative result for endotoxin presence and were therefore applicable for toxicological testing. To ensure the samples did not interfere with the assay, each ENM was incubated with LPS (Sigma, UK) and E-Toxate solution and upon inversion these samples did clot, confirming the LPS was responsible.

\subsubsection{Identifying Hard Corona Proteins}

ENMs were incubated in 10\% FBS MEM media for 1 hour to permit the formation of a protein corona. Following the initial incubation, each ENM suspension was centrifuged and washed three times with PBS to remove weakly-associated proteins constituting the soft corona. The final suspension consisting of ENM-hard protein corona were chemically treated, heat-shocked and analysed with SDSPAGE and silver stained alongside a Protein Kaleidoscope to estimate protein size. The gels processed (Figure 3.18) after silver staining demonstrated the difference in protein corona formation between the graphene-based samples and the CB particles. The hard corona of Neutral-, Amine- and CarboxylFLG (Figure 3.18A) stained 9 identical proteins with extremely minor differences in silver stain intensity. Arguably, the Carboxyl-FLG exhibited a weaker band intensity of proteins $>75 \mathrm{kDa}$ when compared to Neutral- and Amine-FLG. CB particles shown in duplicate in Figure 3.17B exhibited only one protein band, evident between 50 and $75 \mathrm{kDa}$. This same size protein is stained in the FLG samples albeit to a higher degree, which was assumed to be Bovine Serum Albumin (BSA), commonly associated with supplemented media and is a major component of FBS. Upon closer inspection of each gel, the FLG samples were deemed to possess a hard corona consisting of 9 serum proteins as opposed to the 1 protein of CB particles (Figure 3.18B). A gel of BSA (Sigma, UK) only was also run to identify the location of the protein on the protein kaleidoscope, this can be found in the Appendix Section 7.3. 

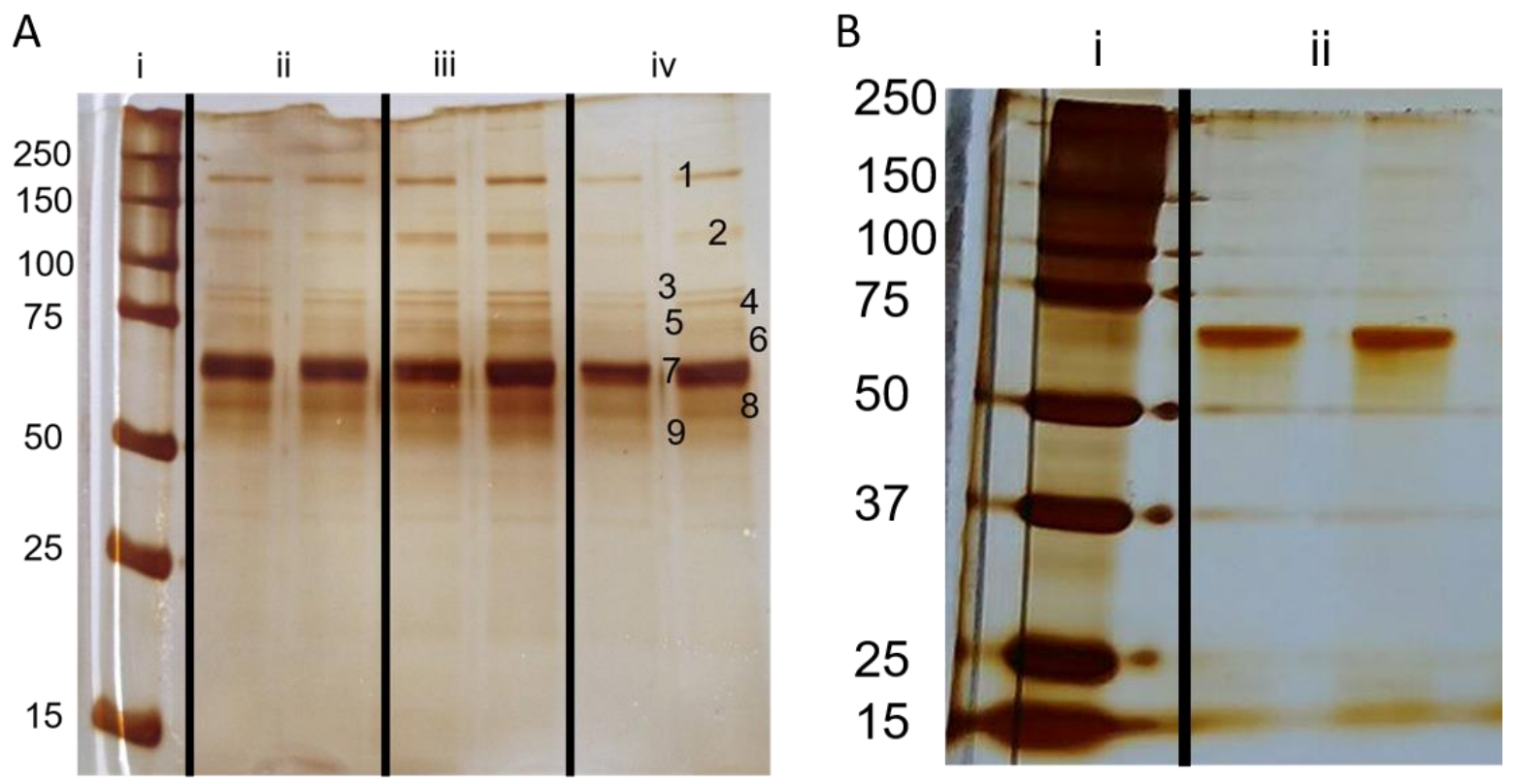

Figure 3.18 SDS-PAGE, silver stained hard protein corona. FLG samples (A) and CB particles (B) suspended in $10 \%$ serum containing media, $\mathrm{N}=2$. Key: Lane (i) of each gel occupied the Protein (MW kDa) Kaleidoscope (Bio-Rad, UK), (ii), (iii) and (iv) of Figure (A) represents the corona of Neutral-, Amine- and Carboxyl-FLG respectively, lastly in Figure (B), the duplicates of lane B are CB particle corona proteins.

\subsubsection{Peptide Mass Fingerprinting (Identification) of Hard Corona Proteins}

Proteins constituting the hard corona of each ENM were determined by LC MS/MS by Dr Ed Dudley, Swansea University School of Medicine. Briefly, proteins were excised from the SDS-PAGE gels and chemically treated to remove silver stain before being digested with Trypsin. The resulting peptide supernatant was dehydrated and analysed via mass spectrometry. Resulting peptides were fragmented by MS/MS to determine the amino acid sequence of the peptides and use these sequences to identify the protein they originated from. Upon fragmentation, peptides lose amino acids to produce ions which can be either $b$ or $y$ with the net charge of the remaining peptide being retained by either the $\mathrm{N}$ (amine) terminus or the $\mathrm{C}$ (carboxyl) terminus respectively. Each $\mathrm{b}$ and $\mathrm{y}$ ion are then numbered depending on its position in the peptide. A representative LC MS/MS spectrum can be seen in Figure 3.19, with the peptide sequence of the protein (VGDTVLGMATQCVQMK) identified below under Sequence 3.1, highlighted in red. The peptide showed no identity, but nearsignificant $(p<0.059)$ homology to Protein Argonaute-2 which was assigned as the protein identified by SDS-PAGE. Each peptide has been highlighted in Table 3.14. 
Table 3.14 Protein Identification by LC MS/MS

\begin{tabular}{|c|c|c|}
\hline $\begin{array}{l}\text { Protein \# identified in SDS- } \\
\text { PAGE }\end{array}$ & Protein Name & $\begin{array}{l}\text { Monoisotropic mass of neutral } \\
\text { peptide (Mr) }\end{array}$ \\
\hline 1 & Protein Argonaute-2 & 1695.8 \\
\hline 2 & $\begin{array}{lr}\text { Arginine } & \text { Biosynthesis } \\
\text { Bifunctional } & \text { Protein } \\
\text { (mitochondrial) } & \end{array}$ & 1125.6 \\
\hline 3 & $\begin{array}{l}\text { UDP-N-acetylglucosamine } 1 \text { - } \\
\text { carboxyvinyltransferase }\end{array}$ & 612.4 \\
\hline 4 & $\begin{array}{l}\text { Apolipoprotein } \\
\text { acyltransferase }\end{array}$ & 1854.9 \\
\hline 5 & Acetylcholinesterase & 1726.9 \\
\hline 6 & $\begin{array}{ll}\text { Queine } & \text { tRNA } \\
\text { ribosyltransferase } & \end{array}$ & 711.4 \\
\hline 7 & $\begin{array}{l}\text { 3-phosphoshikimate } \\
\text { carboxyvinyltransferase }\end{array}$ & 825.5 \\
\hline 8 & $\begin{array}{l}\text { Pyrrolidine-carboxylate } \\
\text { peptidase }\end{array}$ & 611.3 \\
\hline 9 & $\begin{array}{l}\text { O-acetyl-ADP-ribose } \\
\text { deacetylase } 2\end{array}$ & 1854.9 \\
\hline
\end{tabular}


Table 3.15 Summary of the ENMs physico-chemical features. By assuming Neutral-FLG as the control / bare ENM, any feature of the functionalised FLGs which appears in green indicates an increase and conversely red indicates a decrease in that specific physico-chemical feature. Thus, highlighting the effects of surface functionalisation upon graphene ENMs.

\begin{tabular}{|c|c|c|c|c|c|c|}
\hline Properties & Technique & Unit & Neutral-FLG & Amine-FLG & Carboxyl-FLG & CB \\
\hline Primary Diameter & SEM & $\mu \mathrm{m}$ & $1.03+/-0.8$ & $0.98+/-0.7$ & $1.14+/-1.1$ & $0.129+/-0.3$ \\
\hline Composition & EDX & $\%$ & $\begin{array}{l}C(97.4) \\
O(1.93) \\
S(0.31)\end{array}$ & $\begin{array}{l}\mathrm{C}(88) \\
\mathrm{O}(9.66) \\
\mathrm{N}(1.74)\end{array}$ & $\begin{array}{l}\mathrm{C}(92.16) \\
\mathrm{O}(7.32) \\
\mathrm{N}(0.52)\end{array}$ & $\begin{array}{l}\text { C (98.94) } \\
\text { O (1.06) }\end{array}$ \\
\hline $\begin{array}{l}\text { Surface Charge } \\
\left(\mathrm{H}_{2} \mathrm{O}\right)\end{array}$ & Zetasizer Nano ZS & $\mathrm{mV}$ & $-31.72+/-1.95$ & $-41.96+/-0.86$ & $-34.36+/-3.06$ & $-43.7+/-5.4$ \\
\hline $\begin{array}{l}\text { Surface Charge } \\
\text { (10\% FBS MEM) }\end{array}$ & & $\mathrm{mV}$ & $-9.801+/-0.66$ & $-9.971+/-0.71$ & $-9.76+/-0.72$ & $-12.4+/-0.83$ \\
\hline $\begin{array}{l}\text { Hydrodynamic } \\
\text { Diameter } \mathrm{H}_{2} \mathrm{O}\end{array}$ & DLS & $\mathrm{nm}$ & $290.8+/-302.6$ & $170.1+/-97.92$ & $169.6+/-76.88$ & $513.3+/-421.2$ \\
\hline $\begin{array}{l}\text { Hydrodynamic } \\
\text { Diameter 10\% FBS } \\
\text { MEM }\end{array}$ & & $\mathrm{nm}$ & $504.7+/-364.7$ & $426.4+/-310.8$ & $348.2+/-183.9$ & $347.7+/-195.1$ \\
\hline $\begin{array}{l}\text { Presence of } \\
\text { Endotoxin }\end{array}$ & LAL Gel Clot Assay & N/A & Negative & Negative & Negative & Negative \\
\hline Thickness & AFM & $\mathrm{nm}$ & $94.73+/-67.94$ & $86.20+/-42.16$ & $55.16+/-42.22$ & N/A \\
\hline Layer Number & AFM & $\mathrm{nm}$ & 50 & 12 & 4 & N/A \\
\hline Surface Area & MultiPoint BET & $\mathrm{m}^{2} / \mathrm{g}$ & 55.2 & 31.72 & 33.7 & 33.2 \\
\hline $\begin{array}{l}\text { Aerodynamic } \\
\text { Diameter }\end{array}$ & Calculated & $\mathrm{nm}$ & $19.2-33.9$ & $18.9-33.5$ & $14.9-26.3$ & N/A \\
\hline $\begin{array}{l}\text { Number of Corona } \\
\text { Proteins }\end{array}$ & SDS-PAGE & N/A & 9 & 9 & 9 & 1 \\
\hline Particle Size $\mathrm{H}_{2} \mathrm{O}$ & Plunge Freeze SEM & $\mathrm{nm}$ & $153.2+/-19.2$ & $163.8+/-21.7$ & $158.5+/-19.6$ & N/A \\
\hline Particle Size Media & & & $101.3+/-16.8$ & $124.4+/-13.9$ & $99.5+/-15.3$ & N/A \\
\hline $\begin{array}{l}\text { Agglomerate Size } \\
\mathrm{H}_{2} \mathrm{O}\end{array}$ & & & $729.4+/-317$ & $557.4+/-119.9$ & $543.1+/-202.3$ & N/A \\
\hline $\begin{array}{l}\text { Agglomerate Size } \\
\text { Media }\end{array}$ & & & $583.7+/-212$ & $988.3+/-285.3$ & $1083+/-626.6$ & $\mathrm{~N} / \mathrm{A}$ \\
\hline
\end{tabular}




\subsection{Discussion}

Chapter 3 has highlighted the necessity of characterising the physico-chemical features of carbonaceous ENMs utilised in the present study; Neutral-, Amine-, Carboxyl-modified FLG and CB particles. The physico-chemical characteristics determined for each of these ENMs has been summarised (Table 3.15). The physico-chemical features of ENMs are central to better understanding their hazard potential; for this reason, it is imperative that a robust and multi-modal approach is taken to fully characterise the ENMs under study prior to in vitro or in vivo exposures. Due to the complex 3D structure of the FLG samples, far more emphasis was placed on characterising these ENMs as opposed to CB the particles, which have been extensively evaluated in the published literature. This chapter has therefore implemented a host of characterisation techniques to provide a useful screening approach to holistically analyse all the features of graphene-based ENMs. This chapter has focused on graphene characterisation for primary features including the use of BET, AFM and Raman Spectroscopy. Secondary features were then assessed with techniques such as DLS, Zeta Potential and plunge freezing in liquid ethane to evaluate the hydrodynamic diameter, surface charge and agglomerate diameter. Endotoxin tests were performed on all ENMs as well as isolating and identifying the proteins that constitute the hard corona when suspended in serum containing cell culture media.

\subsubsection{Physico-chemical Features of Neutral-FLG}

The first of the carbonaceous ENMs to be investigated, Neutral-FLG immediately post-manufacture showed no presence of endotoxin contamination which was confirmed with the LAL gel clot assay. All other materials tested in this study were, negative for endotoxin. Utilising plunge freeze SEM, individual particles were sized and found to be $153.9 \mathrm{~nm}$ in water and $101.3 \mathrm{~nm}$ when suspended in cell culture media, indicative of potentially some material degradation or enhanced dispersion. This was somewhat reflected in a marginal decrease in aspect ratio which in water was 1.34 and dropped to 1.31 in media. Whilst these measurements do not depict the true airborne hazard of Neutral-FLG as they are liquid suspensions, even the raw material (dry powder) possesses a diameter of $1.03 \mu \mathrm{m}$ which is small enough to achieve deep deposition in the respiratory tract. Plunge freeze SEM images of Neutral-FLG reveal a stacked structure forming small agglomerates. These appear to be hydrostatically linked to other smaller agglomerates culminating in larger disjointed structures. This pattern appears to be repeated amongst all FLG samples. The stacking of Neutral-FLG was confirmed with Raman Spectroscopy and AFM respectively. Raman spectroscopy revealed a minimum of tri-layer graphene within the Raman spectra ratio of 2D/G peaks, and graphitic stacking of FLG clusters, however when analysed with AFM an average thickness of $94.7 \mathrm{~nm}$ was observed. By combining these 
techniques, it could be assumed that Neutral-FLG when dried and under analysis forms tri-layer graphene (from Raman 2D peak) which then hydrostatically interacts with other tri-layer fibres to form nano-graphite clusters. Where Neutral-FLG greatly differs from the other FLG samples and CB particles was the BET surface area value of $55.2 \mathrm{~m}^{2} / \mathrm{g}$. The adjoining analysis of pore size and volume was identical for all materials where they exhibited an average pore volume of $1.115 \mathrm{cc} / \mathrm{g}$ and average pore size of $1.796 \AA$. This may indicate the presence of sub-nm engineered pores in the planar structure or far more likely the presence of structural defects (evidenced by Raman), known as Stone-Wales defects. These defects are purposely delivered during the manufacture process to exfoliate sheets of graphene which augments the $\mathrm{C}-\mathrm{C}$ bonds by rotating them $90^{\circ}$ thus evolving the hexagonal lattice into pentagons, heptagons and octagonal structures, described in Figure 1.2.

Although the BET surface area calculated with BET appears relatively low, as demonstrated by Nikolaos Kostoglou in 2015, the surface area of these FLG materials after sheer mixing and liquid suspension can dramatically increase to $700 \mathrm{~m}^{2} / \mathrm{g}$ (Kostoglou Nikolaos, 2015). As stated in the literature, surface area is highly regarded as the preferred dose metric where in vitro and in vivo exposures are concerned in nanotoxicology (Herzog et al., 2013). This is particularly important with graphene-based ENMs, where due to the low density of the material far more particles are required to amount to an equivalent mass concentration as compared to other more conventional ENMs such as iron oxide or titanium NPs. Another primary feature of Neutral-FLG physico-chemical feature is the elemental composition which was explored with EDX spectroscopy to highlight the presence of possible heavy metal contaminants and degree of oxygen/nitrogen saturation which could be a source of free radical generation once suspended in media and under sonication. Whilst EDX analysis revealed carbon, oxygen, aluminium, silicon and sulphur, the latter three elements represent the components of the SEM shuttle. EDX could not reveal how the oxygen and carbon were bonded nor the concentration of any additional dopants on the surface of the material, this data however could be retrieved by XPS as demonstrated by other studies in the literature (Susi et al., 2015). XPS which focuses on the binding energy of atoms within molecules could provide useful additional information regarding the degree of C-O bonds within Neutral-FLG as it is currently hypothesised that any excess oxygen on the structure exists at the edge asperity as dangling bonds (Liu et al., 2009). These dangling bonds are ideal locations for subsequent graphene layers to bond with ambient oxygen amongst other elements (Ridene et al., 2016). Neutral-FLG exhibited the greatest thickness as evaluated by AFM which when compared to Raman Spectroscopy may show how the Neutral-FLG forms graphitic structures, through stacking of several tri-layer lattices. The tri-layer graphene then through hydrostatic agglomeration forms large stacks which, at their maximum height were $\sim 490 \mathrm{~nm}$. 


\subsubsection{Effects of Additional Surface Chemistry (Amine \& Carboxyl Groups) Upon FLG}

This section will highlight key differences in the physico-chemical features introduced during the manufacturing process by surface functionalisation with amine or carboxyl groups. Immediate measurements of the lateral diameter by SEM indicates a slight difference between Neutral-FLG $(1.03 \mu \mathrm{m})$ and the Amine-FLG $(0.98 \mu \mathrm{m})$ and Carboxyl-FLG $(1.14 \mu \mathrm{m})$ sizes however this appeared to be negligible and due to standard deviation within the sample size. Elemental differences, which were determined with EDX spectroscopy revealed that the addition of amine groups reduced the carbon content from $97.4 \%$ in Neutral-FLG samples to just $88 \%$. Oxygen content in the Amine-FLG increased to $9.66 \%$ whilst the nitrogen forming the basis of the amine grouping was $1.74 \%$. Conversely, the Carboxyl-FLG sample showed slightly weaker oxygen and nitrogen signals at 7.32 and $0.52 \%$ respectively, both of which were markedly increased over the Neutral-FLG sample. The addition of amine groups to the surface of FLG increased the zeta potential from -31.72 to $-41.96 \mathrm{mV}$, substantially increasing the stability of the material in water, whilst in $10 \%$ serum-containing culture media, the increase in stability was marginal with only a $0.17 \mathrm{mV}$ increase to $-9.971 \mathrm{mV}$. Crucially these measurements reveal that the surface amine groups when dispersed in water adorning the surface of FLG in water or supplemented culture media does not confer a cationic surface charge, consequently the net negative charge must originate from another set of chemical groups. Given this net negative charge is significantly lowered in culture media it should be assumed the corona of proteins confer a net positive charge to the FLG material enabling a decrease in overall zeta potential.

Carboxyl groups offered little stability to FLG, only increasing stability in water by $2.64 \mathrm{mV}$ and decreasing colloidal stability in culture media by lowering the zeta potential from -9.801 to $-9.76 \mathrm{mV}$ when moving from Neutral-FLG to Carboxyl-FLG respectively. The similarity between the zeta potential of all three variants of FLG appears to be a result of the protein corona cancelling out the effects of the surface charge, an effect which has been documented (Huhn et al., 2013). Given the overall symmetry in the corona of each FLG material it could be assumed that the net charge in serummedia would be similar. Upon suspension in $10 \%$ supplemented culture media, both FLG ENMs and CB particles underwent a significant change in surface charge (Table 3.15), with an average $28 \mathrm{mV}$ drop in surface charge from water suspended samples. This effect of serum protein upon ENM surface charge has been reported heavily in the literature (Huhn et al., 2013), (Kokkinopoulou et al., 2017) (Monopoli et al., 2013). Many studies indicate the effect of serum proteins tends to bring the surface charge towards a neutral net charge. This effect has been highlighted within the data of Table 3.15, whereby the two highest negatively charged ENMs; Amine-FLG and CB at -41.96 and $-43.7 \mathrm{mV}$ were 
reduced to -9.971 and $-12.4 \mathrm{mV}$ respectively. Thus, the protein corona they have adopted must therefore largely be positively charged. This data agrees with the theories put forward by Monopoli and colleagues 2012, and Nel and colleagues (2009). Therefore, the significant reduction in surface charge of each FLG ENMs has been caused by a multitude of proteins, likely taking advantage of the large surface area of FLG when dispersed in media whilst the CB particles have acquired only one strongly associated protein. One protein is the same on both the FLG and CB ENMs and is most likely $B S A$, the most abundant serum protein contained within FBS and often associates with ENM protein coronas (Sengupta et al., 2015). The presence of BSA on both ENMs is likely to have a large impact upon ENM-cell interactions in vitro. Multiple cell lines have been identified as possessing albumin receptors; gp60 at the endothelium, gp30 on macrophages, and gp18 on fibroblasts (Merlot et al., 2014). The presence of BSA (among other proteins in the case of FLG) has the potential to offer biological recognition at the cell surface with cell uptake a likely possibility. Huhn and colleagues made a similar observation whereby the serum corona can mask the sign charge on ENMs. Proteins within serum-containing media contain biologically-relevant molecules and ions such as $\mathrm{Ca}_{2}{ }^{+}, \mathrm{Na}^{+}$, and $\mathrm{K}^{+}$ commonly which will be electrostatically attracted to anionic ENMs (Zhang et al., 2011). This effect was then replicated in separate studies by Riedinger and colleagues in 2010 and by Zhang and colleagues a year earlier in 2009 (Zhang et al., 2010, Riedinger et al., 2010). Conversely, if the net charge on the FLG had been cationic, negatively charged biomolecules would be attracted and adsorbed to the surface which could include; $\mathrm{Cl}^{-}$. Other biomolecules that could adsorb to a cationic dipole of FLG may include nucleic acids such as mRNA (Xia et al., 2009, Su et al., 2011). However, measurements of the hydrodynamic diameter utilising DLS highlights subtle differences between the materials with each material exhibiting greater hydrodynamic diameters when dispersed in serumcontaining media as opposed to water, increasing across each material by $\sim 216 \mathrm{~nm}$. Clearly the additional amine and carboxyl groupings do not confer surfactant chemistry but rather enhance agglomeration when measured with DLS, albeit not an ideal technique for evaluating FLG hydrodynamic diameters. The reason graphitic ENMs do not suit DLS is based upon DLS calculating autocorrelation functions of scattered light signals in a solution. This process assumes the free diffusion of spherical particles, not sheet-like materials (Nazarenus et al., 2014). Whilst functionalisation appears to have minimal effects when evaluated by DLS, AFM analysis on depth (3D) demonstrated that both amine and carboxyl groups lower the layer number by factors of 4 and 12.5 respectively. From this, it could be hypothesised that steric hinderance plays a role at the plane of graphene sheets both above and below which could account for the decrease in layer number when amine and carboxyl groups are added, essentially the additional surface groups act as intercalating agents to increase dispersion. Theoretically in a solution of water (Equation 3.5) details the chemical 
effects of electrostatic repulsion between Amine-FLG layers and Equation 3.6 Carboxyl-FLG layers (Sun et al., 2018).

\author{
Equation 3.5 $\mathrm{R}-\mathrm{NH}_{2}+\mathrm{H}_{2} \mathrm{O} \leftrightarrow \mathrm{R}-\mathrm{NH}_{3}+\mathrm{OH}^{-}$ \\ Equation 3.6 R-COOH $\leftrightarrow \mathrm{R}-\mathrm{COO}^{-}+\mathrm{H}^{+}$
}

One issue remains however; the degree of electrostatic repulsion in Carboxyl-FLG in this hypothesis was three times greater than in Amine-FLG. It therefore follows that either Amine-FLG contains other functional groups which contribute to an attractive force and thus explains the higher layer number, or the concentration of the chemical functionalisation's are not equal. Size and surface area play a crucial role in the interactions of ENMs and biological systems with the consensus being that as size decreases relative to volume the surface area increases and with it the surface reactivity of the ENM (Gatoo et al., 2014). The surface area of Neutral-FLG being $55.2 \mathrm{~m}^{2} / \mathrm{g}$ appeared changed when the material was functionalised, where Amine-, and Carboxyl-FLG exhibited noticeably lower surface areas of 31.72 and $33.7 \mathrm{~m}^{2} / \mathrm{g}$ respectively. Surface area has been extensively reported to be a leading factor in lung genotoxicity mediated through inflammatory mechanisms in rodents and being a highly relevant dose metric where ENMs are concerned (Holgate, 2010, Herzog et al., 2013). It appears the effect of additional functionalisation, in this instance the addition of amine and carboxyl groups to the FLG, may have increased the material size thus, explaining the inverse relationship with surface area. Following this, it may be expected that Neutral-FLG with the higher surface area could prove more toxic under in vitro experiments even when an identical mass dose metric is used for Amine- and Carboxyl-FLG, this effect has been detailed extensively by Donaldson and Stone who investigated the effects of surface area toxicity regarding particulate matter (Donaldson and Stone, 2003). Similarly, Risom and colleagues reported on the decrease in size relative to surface area increases, leading to a dose dependent elevation in oxidative and DNA damaging potential (Risom et al., 2005). The surface area of graphene ENMs plays a pivotal role in their suspension state and has been assessed in this chapter (Figure 3.14). Given that FLG can have a range of densities, the rate at which these materials disperse, suspend and crucially sediment at is important when considering their toxicity. As modelled in Figure 3.14 over a 24-hour period, FLG with an extremely low density of $0.03 \mathrm{~g} / \mathrm{cm}^{3}$ as hypothesised by Park and colleagues (2017) sediments to a far lesser extent than the same material with a density of $0.1 \mathrm{~g} / \mathrm{cm}^{3}$. This effect was so strong in the dosimetry script used in this investigation that presumably, not all the administered concentration of FLG would reach an adherent cell line. The 
additional amine groups appeared to show the most dissimilar trend to that of the Neutral-FLG material which displayed similar models to that of Carboxyl-FLG. Given that the surface area of Neutral-FLG is $55.2 \mathrm{~m}^{2} / \mathrm{g}$ in comparison to 31.72 and $33.7 \mathrm{~m}^{2} / \mathrm{g}$ for Amine- and Carboxyl-FLG respectively the agglomeration state will be different. Agglomeration state of ENMs also plays an important role on effective density and size in suspension (DeLoid et al., 2015). As stated in the work by DeLoid and colleagues, the relationship between sedimentation rate of a particle and size is based upon the following assumptions, whereby; particle sedimentation rate is equivalent to the square of its diameter, which means a 10 -fold difference in size $=$ a 100 fold difference in sedimentation rate (DeLoid et al., 2015). This also applies for in vitro doses, however in the present study the size evaluation of each material proved to be of the same order concluding with broadly similar dosimetry models.

FLG and CB particle purity showed no signs of heavy metal contamination which can prove a common source of mis-leading positives where toxicity testing is concerned. Whilst the EDX spectroscopy does provide a measure of elemental composition, the bonding states of those elements is not deciphered. This analysis would be highly useful in discriminating between the three FLG materials in the present study given the large number of bonding possibilities of carbon, oxygen and nitrogen. A technique such as XPS could prove useful in ascertaining if those materials are bonded as $\mathrm{C}=\mathrm{O}, \mathrm{C}-\mathrm{OH}, \mathrm{C}-\mathrm{NH}_{2}$ and thus highlight at what percentage are those groupings present at. This has been performed recently on GO ENMs by Muzyka and colleagues (2018), whereby XPS identified with precision the presence of alcohol bonds $(\mathrm{C}-\mathrm{OH})$, R-groupings $(\mathrm{C}-\mathrm{O}-\mathrm{C})$, carbonyl bonds $(\mathrm{C}=\mathrm{O})$ and ester linkages $(\mathrm{O}=\mathrm{C}-\mathrm{OH})$ (Muzyka et al., 2018). These chemical groups have drastically different reactivities in solution, it is therefore highly important to identify if these are present on the FLG materials used in the present study to better understand their toxicity. As detailed in the work by Chua and colleagues (2012), the production method used to manufacture graphene, specifically the oxidation/ reduction, can greatly impact the type of functional group on the final product. Typically, sulfuric acid is used in conjunction with i) nitric acid fumigation oxidation with potassium chlorate ii) concentrated nitric acid followed by potassium chlorate, iii) sodium nitrate in the presence of potassium permanganate and iv) concentrated phosphoric acid with potassium permanganate (Chua et al., 2012). The characterisation of graphene materials in future could therefore be based upon the production method used during manufacture. One of the most prominent techniques used to analyse graphene structure post manufacture is Raman spectroscopy. Raman spectroscopy, both non-destructive and rapid, allows the surface of the material to be probed for structural defects and impurities. From the D band exhibited by both Neutral- and Carboxyl-FLG at $\sim 1350 \mathrm{~cm}^{-1}$, there appears to be large number of structural 
defects, these can manifest as either dislocations between carbon bonds or as surface vacancies (Kumar et al., 2014). Strikingly, the Amine-FLG D band was extremely low in intensity indicating good structural integrity. Exploiting these defects, which include Stone-Wales defects, single vacancy and divacancies (all of which have been studied with high resolution TEM by Vicarelli and colleagues, 2015) could be a possible avenue of research where nanomedicine is concerned. The spacing of a divacancy within a graphene structure is the perfect placement for atoms such as platinum (Vicarelli et al., 2015). Differences between the three materials was again evident by the shape of the $G$ band at $~ 1580 \mathrm{~cm}^{-1}$ whereby the G peak of both Neutral- and Carboxyl-FLG are less distinct than that of Amine-FLG. This relates to the in-plane vibration of $\mathrm{sp}^{2}$ carbon atoms and could suggest it is more prominent with Amine-FLG which displayed a far more distinct peak. The final prominent band is the 2D peak which offers information on the number of layers in samples. Whilst this is similar across each FLG sample, the information which alludes to the presence of tri-layer graphene is not in agreement with the layer number calculated via AFM following depth analysis. The latter analysis, which accounts for the interlayer spacing, indicates that Neutral-, Amine- and Carboxyl-FLG have 50, 12 and 4 layers of atomic graphene respectively. While the two techniques vary extremely in their experimental approach, similar trends in the data should be observed and are not in this instance. This discrepancy between Raman and AFM could be attributed to the physics of each technique, whilst Raman spectroscopy is collecting data related to the vibration and stretching of $\mathrm{sp}^{2} \mathrm{C}-\mathrm{C}$ bonds, AFM is directly probing the surface of the ENMs, and is most likely giving the true three-dimensional analysis which could be further elucidated with 3D rendering of plunge freeze SEM. This third dimension to analysing the agglomerate size via plunge freezing would likely reveal depth diameters of the order seen by twodimensional analysis performed in this chapter.

\subsection{Conclusions}

This chapter has demonstrated the importance of using a multi-modal approach to comprehensively evaluate the physico-chemical characterisation of graphene ENMs. From Table 3.15 it was apparent that the primary features of the graphene were largely similar irrespective of surface chemistry modifications. Deeper investigation into the secondary physico-chemical features highlighted several crucial differences however, including a higher carbon: oxygen ratio in Amine- and Carboxyl-FLG, providing greater surface charges and stability in water as well as larger hydrodynamic diameters when compared to Neutral-FLG. Modified FLG materials also showed a lower stacking number calculated from measurements performed utilising AFM which neatly correlated to minor decreases in the aerodynamic diameters of Amine-FLG and Carboxyl-FLG. When utilising plunge-freeze SEM, a 
superior technique for investigating graphene agglomerate size in water and cell culture medium, the presence of amine and carboxyl groups decreased agglomerate size in water. Interestingly, this effect was reversed in serum-containing culture media whereby the additional surface groups of both Amine- and Carboxyl-FLG increased agglomerate size to approximately twice that of the Neutral-FLG agglomerates. This increase in agglomeration may be the result of increase in surface charge (lower $\mathrm{mV}$ ) thus a more negative dispersion when intercalated with positive ions (the effect of proteins in $10 \%$ serum) would produce greater agglomerate diameters. Overall, DLS revealed an all-round decrease in hydrodynamic diameter size when surface modifications are introduced. Conversely, plunge freeze SEM (arguably a more robust approach) demonstrated the ability of amine and carboxyl groups to lower agglomerate size in a water suspension. However, in cell culture media the amine and carboxyl groups were able to increase agglomerate size. Peptide mass fingerprinting highlighted the difference between the highly ordered and crystalline graphene ENMs and the amorphous CB particles with clear differences in the number of serum proteins bound to the surface of each material. A total of nine proteins were observed in the hard corona of each FLG material whereas only one protein (likely BSA) was observed at the surface of CB particles. Clearly, this research has implied the use of multi-modal graphene characterisation to be a necessity not just a recommendation. Gathering as much useful information pertaining to FLG physico-chemical features would greatly enhance the knowledge surrounding its behaviour under in vitro exposure conditions. 


\section{Chapter 4 - Investigating the Mechanisms Contributing to In Vitro Primary Genotoxicity in Bronchial Epithelial Cells}

\subsection{Introduction}

Understanding the genotoxic impact of carbonaceous ENMs is of vital importance given their highly dynamic and varied applications (Burgum et al., 2018). However, determining the genotoxic impact provides just one endpoint and in an ideal world a more holistic approach should be taken whereby mechanisms contributing to a genotoxic response are elucidated. The most rapid and reproducible way of completing this is by first selecting a representative cell line; in this Chapter they were a

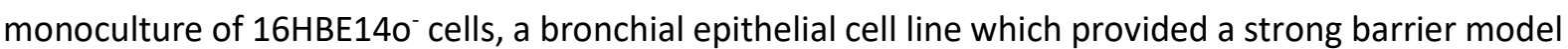
which also represented a key deposition site for ENMs with the physico-chemical features present in FLG and CB particles. Following exposures to ENMs, the $16 \mathrm{HBE} 14 \mathrm{O}^{-}$cells were screened for chromosomal damage using the in vitro CBMN assay adapted for ENMs as detailed by Doak and colleagues (Doak et al., 2012b). Following this a deeper investigation into the underlying causes associated with ENM genotoxicity including an extensive oxidative stress paradigm exploring ROS, depletion of intracellular GSH and mitochondrial stress was conducted.

\subsubsection{In Vitro Techniques to Assess Cytotoxicity, Genotoxicity, (Pro)-Inflammatory Response, Oxidative Stress and Mitochondrial Stress}

This section of Chapter 4 provides an overview of the techniques routinely employed in nanotoxicology and in this study to investigate the toxicity endpoints measured following ENM exposure.

\subsubsection{Cytotoxicity \& Genotoxicity Utilising the In Vitro CBMN Assay}

The in vitro CBMN assay provided a robust and quantitative method of detecting DNA (chromosomal) damage. Chromosomal aberrations detected utilising the CBMN assay are a result of unrepaired double strand breaks whereby regions of chromosome are excluded into a mini nuclear body in the cytoplasm, hence the term micronucleus (Iliakis et al., 2004). Whilst the CBMN assay is adept for quantifying clastogenic damage as it does in this regard, it will also detect aneuploidy. The CBMN version of the assay utilises cytochalasin $B$ and a test agent to determine genotoxic impact. Cytochalasin B reactivity is noted for its inhibition of actin polymerisation which blocks the formation 
of actin filaments in dividing cells hence it's use in this assay (MacLean-Fletcher and Pollard, 1980). The ability to hold a cell mid-mitotic division maintains cells in a state of anaphase inhibiting further cell division (telophase) and culminates in bi-nucleated (BN) cells. This is extremely important in this study as it will focus solely on the cells which have undergone cell division. Given that the frequency of micronuclei in BN cells can be used as a measure of genotoxicity, further classification of the chromosomal damage can be performed with pan-centromeric staining to ascertain whether the imparted DNA damage is clastogenic or aneugenic. The exclusion of a micronucleus from the parent nuclear body may also provide further genetic information with pan-centromeric staining with fluorescent markers, providing useful information on clastogenic (chromosome double strand breaks) or aneugenic (abnormal chromosome number) damage (Fenech, 2007). Commonly a ratio of clastogenic: aneugenic damage can be extrapolated from data sets to elucidate which mechanism predominates as micronucleus frequency increases with test agent dose. The use of cyto $B$ in the CBMN assay provides an issue however as it inhibits endocytosis and interferes with cellular exocytosis which presents a problem when investigating ENMs (Davies and Allison, 1978). To overcome this issue, ENM exposures can be performed prior to cyto B addition allowing for sufficient ENM uptake and subsequent formation of BN cells without interference from cyto B (Doak et al., 2009). Running parallel with genotoxicity testing is cytotoxicity evaluation which can be performed in several ways; relative population doubling (RPD), relative increase in cell count (RICC) and cytokinesis block proliferation index (CBPI) being the most common. RPD measurements work particularly well with the CBMN assay which are quick to perform and include a measure of proliferation. RPD measurements have been validated useful for use with the CBMN assay through OECD guidelines where it is characterised as; $\mathrm{RPD}=$ number of population doubling (treated cells) / number of population doubling (control cells) And; Population doubling $=\log ($ Post treatment cell count $/$ initial cell count) $\times \log$ [2] (Doherty, 2012).

\subsubsection{Sandwich ELISA (Pro)-Inflammatory Mediator Quantification}

The sandwich ELISA focuses on a colour change detection of (pro)-inflammatory cyto/chemokines produced by certain cell types, in this chapter, epithelial cells. This unique sandwich ELISA utilises a capture antibody which first binds to the 96 -well plate surface to bind the target protein molecule secreted into the extracellular content of initial treated flask media. Upon capture antibody and antigen binding a secondary, detection antibody is added conjugated to an enzyme, finally substrate is added to detect the colour change catalysed by the bound enzyme. The use of ENMs with ELISAs can be an issue due to the interference of some ENMs with colorimetric assays (Ong et al., 2014). 
Where possible centrifugation of the supernatant-containing ENM should be employed to remove as much debris as possible before committing to the ELISA in addition to the use of appropriate positive and negative controls and subtraction of background readings.

\subsubsection{Oxidative Stress}

Oxidative stress in this study was characterised through two independent mechanisms; Intracellular GSH depletion, an indirect measure of oxidative stress and secondly, quantification of ROS production with the DCFH-DA assay. Firstly the measure of intracellular GSH, utilises a thiol probe (monochlorobimane) which can freely diffuse across the plasma membrane and fluoresces once bound to reduced glutathione (GSH), a reaction catalysed by glutathione $S$ transferase (GST) (Kamencic et al., 2000). Similarly, the measurement of ROS was performed with a fluorescence plate reader, this time utilising the conversion of $2^{\prime} 7^{\prime}$-dichlorofluorescein (DCF) a non-fluorescent molecule to a fluorescent dye in the presence of ROS. Esterase enzymes convert the DCF once internalisation has taken place, typically after 30 minutes of incubation. The DCFH-DA assay detects a wide number of oxidative species such as $\mathrm{RO}_{2}$, and $\mathrm{RO}$ which in some cases the user can specify and target one species however issues are raided in this assay pertaining to ENM interference with fluorescence. DCF has an extremely unstable chemical structure allowing oxidation slowly over time and can be prone to photo-oxidation by laser frequencies thus potentially leading to false positives (Doak et al., 2009). Doak and colleagues also demonstrated the ability of dextran-coated iron oxide NPs to interfere and decrease probe fluorescence which was observed in a dose-dependent manner over concentrations of $0.1 \mu \mathrm{g} / \mathrm{ml}$. Cell-free versions of the assay can be performed and are typically adapted by the user thereby the di-acetate section of the probe is cleaved manually with esterase enzymes or strong bases (Sodium Hydroxide) prior to plating.

\subsubsection{Mitochondrial Stress}

To assess mitochondrial stress the Seahorse XFe24 Bioanalyser was employed to monitor cellular oxygen consumption rate (OCR) (Figure 4.1) and extracellular acidification rate (ECAR) over a period of approximately 96 minutes in the cells real-time. From this initial data the bioenergetics and respiratory parameters could be calculated, and further analysis performed. Firstly, a series of injections are required which will directly impact upon cellular respiration during the assay. These injections of; Oligomycin, FCCP and a 1:1 mix of Rotenone and Antimycin A will impact respiration at set times during the assay displayed in Figure 4.1. Briefly, Oligomycin will act to inhibit complex $\mathrm{V}$ 
decreasing the conversion of ADP to ATP acting to lower basal respiration. FCCP inhibits complex III, a proton pump which moves protons to the intermembrane space giving the respiratory effect of an overload causing respiratory stress. Lastly, the Rotenone/Antimycin A compound injection which acts as an extremely potent poison effectively shutting down complex I of the electron transport chain. The injections themselves play a key role in the bioenergetics measurements, detailed in Figure 4.2, which highlight the bioenergetic inhibitory / stimulatory effects of the injected compounds.

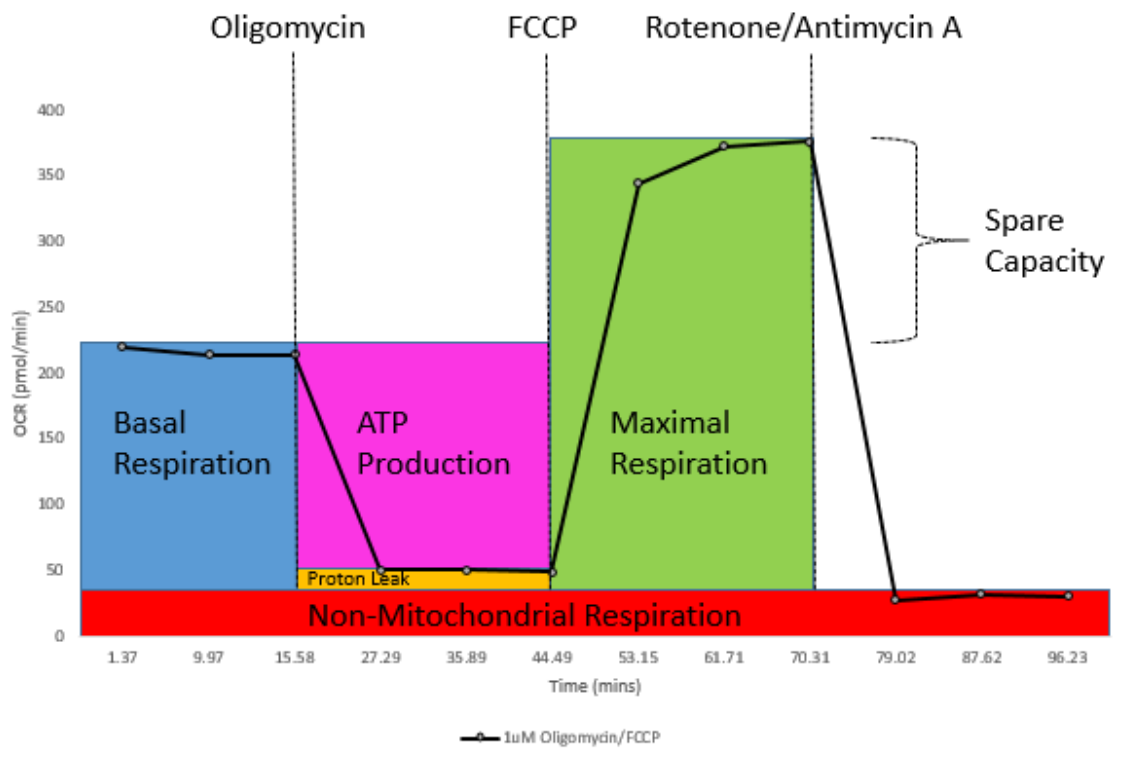

Figure 4.1 Seahorse XFe cellular mitochondrial stress test profile indicating key respiratory parameters. Sequential injections are administered into each test well to measure basal respiration, non-mitochondrial respiration, proton leak, ATP production, maximal respiration and respiratory reserve capacity. Image adapted from Seahorse Bioscience. 


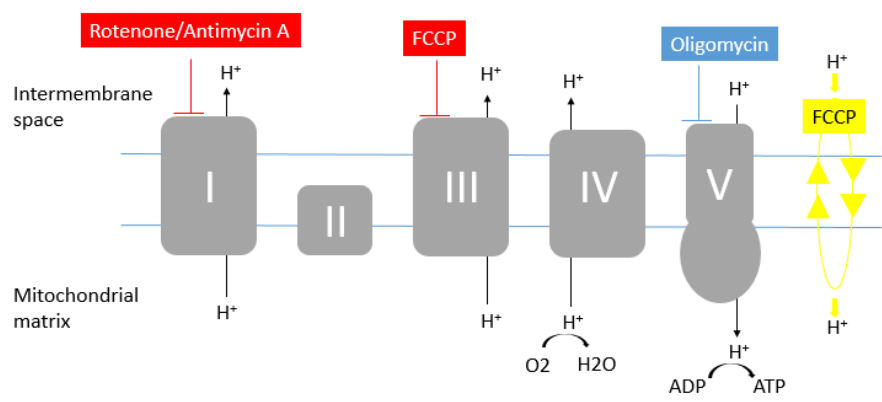

Figure 4.2 Seahorse XFe mitochondrial stress test modulators of the electron transport chain (ETC). This figure illustrates the effect each injection compound has on the complexes of the ETC. Initially at $\sim 16$ minutes of the assay Oligomycin is injected to deplete basal respiration levels through the inhibition of complex V. Following this at $\sim 45$ minutes of the assay FCCP is injected which inhibits the proton pumping of protons into the intermembrane space which has the effect of causing energetic demand on the cells evident by the increase in oxygen consumption. Lastly, Rotenone/Antimycin A completely shut down electron transport at stage one through inhibition of complex I.

\subsubsection{Chapter 4 Aims}

This chapter aimed to evaluate, quantify and understand the in vitro genotoxicity of $16 \mathrm{HBE} 14 \mathrm{o}^{-}$cells elicited by Neutral-FLG, Amine-FLG, Carboxyl-FLG and CB particles. Cytotoxicity and genotoxicity were assessed with RPD and the CBMN assay coupled to pan-centromeric staining respectively. The promotion of a (pro)-inflammatory response was quantified with IL-6 and IL-8 ELISAs. As oxidative stress was believed to be a prime protagonist of the observed genotoxic responses, ROS production was evaluated with the DCFH-DA assay. Glutathione depletion was also measured as an indicator of antioxidant levels in the cells, while mitochondrial stress and bioenergetics were evaluated with the Seahorse XFe24 Bioanalyser. Following the characterisation of each ENM in Chapter 3, Neutral-FLG was presumed to be the most potent promoter of genotoxicity due to the larger agglomerates (in media) and higher layer number contributing to more sedimentation and therefore interaction. The additional surface groups of Amine-FLG and Carboxyl-FLG providing enhanced dispersion were presumed to interact with the cells less contributing to a lower genotoxic response. Uptake of each ENM was also theorised to be size-dependent leading to the conclusion that endocytosis would be observed with smaller agglomerates of each FLG material. 


\subsection{Materials and Methods}

\subsubsection{HBE140- Cell Culture}

The $16 \mathrm{HBE}_{14 \mathrm{O}^{-}}$cell line was cultured as detailed in Chapter 2 section 2.2.1.

\subsubsection{In vitro Cytotoxicity \& Genotoxicity Assessment}

In accordance with the CBMN assay, satellite flasks were seeded in parallel to allow a cytotoxicity assessment by RPD analysis detailed in Chapter 2 section 2.3. The CBMN assay was performed as detailed in Chapter 2 section 2.4 with all four ENMs at concentrations of 0, 2, 4, 8, 10, 20, 50 and $100 \mu \mathrm{g} / \mathrm{ml}$. To quantify clastogenic and/or aneugenic mechanisms of genotoxicity, pan-centromeric paints were used as detailed in Chapter 2 section 2.4.2. Imaging of the micronuclei-containing / absent centromeres was done on the Metafer microscope detailed specifically in Chapter 2 section 2.4.1. All biological replicates were $\mathrm{N}=3$.

\subsection{3 (Pro)-Inflammatory Response}

An in vitro (pro)-inflammatory response was quantified with sandwich-based ELISAs on the supernatant harvested from treated cells outlined in Chapter 2 section 2.5. IL-6 ELISA used a reagent diluent of $0.1 \%$ BSA, $0.05 \%$ Tween $^{20}$ in Tris-Buffered-Saline (TBS) solution (2.4g Tris, $8.7 \mathrm{~g} \mathrm{NaCl}, 1 \mathrm{~L}$ distilled water). IL-6 standards were prepared initially at the top standard concentration of $600 \mathrm{pg} / \mathrm{ml}$ and then using serial dilutions in reagent diluent the following standards $(\mathrm{pg} / \mathrm{ml})$ were prepared for standard curve analysis; $300,150,75,37.5,18.75,9.375$, and a blank of reagent diluent. IL-8 standards were prepared initially at the top standard concentration of $2000 \mathrm{pg} / \mathrm{ml}$ and then using serial dilutions in reagent diluent the following standards $(\mathrm{pg} / \mathrm{ml})$ were prepared for standard curve analysis; 1000, $500,250,125,62.5,31.25$ and a blank of reagent diluent. All biological replicates were $\mathrm{N}=3$.

\subsubsection{Intracellular Reduced GSH}

To quantify the intracellular reduced GSH, treatments with all four ENMs were performed as detailed in Chapter $\mathbf{2}$ section $\mathbf{2 . 6}$ utilising the Sigma monochlorobimane kit. Prior to loading cell lysates into the 96-well plate a series of reduced GSH standards were prepared to calculate a standard curve for analysis. Top standard was prepared followed by serial dilutions to reach a final nmoles concentration of; $10,5,2.5,1.3$ and a blank of assay buffer. All biological replicates were $\mathrm{N}=3$. 


\subsubsection{Reactive Oxygen Species Detection}

To assess the production of ROS both in an acellular and cellular environment, the DCFH-DA assay was performed as described in Chapter 2 section 2.7. All biological replicates were $\mathrm{N}=3$.

\subsubsection{Mitochondrial Stress}

OCR and ECAR bioenergetics were first calculated with the Seahorse XFe24 Bioanalyser, cells were then treated with RIPA buffer and prepared for the detergent compatible (DC) Assay employed to normalise cells against protein content per well. Following normalisation, OCR data was re-organised in Microsoft Excel for calculation of respiratory parameters - proton leak, basal respiration, nonmitochondrial respiration, ATP production, maximal respiration and reserve respiratory capacity detailed in Chapter 2 section 2.8. Respiratory parameters were calculated utilising the calculations in Table 4.1, and the compound injections listed in Table 4.2. All biological replicates were $\mathrm{N}=3$.

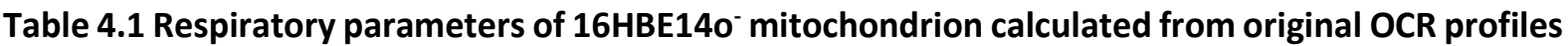
of each ENM.

\section{Respiratory Parameter}

Proton leak

Non-mitochondrial respiration

Basal respiration

ATP-linked respiration

Maximal respiration

Reserve respiratory capacity

\section{Calculation}

Subtract Rotenone/Antimycin A injection from the Oligomycin injection

The three data-points following

Rotenone/Antimycin A injection

Initial three OCR data-points

Basal respiration data-points subtracted from proton leak data-points

Subtract the three data-points following Oligomycin injection from the three data-points following FCCP injection

Subtract the three basal respiration data-points from the three data-points following FCCP injection 
Table 4.2 Seahorse XFe24 injection compounds and corresponding effect on OCR.

\begin{tabular}{|l|l|l|}
\hline Injection Compound(s) & $\begin{array}{l}\text { Electron Transport Chain (ETC) } \\
\text { Target }\end{array}$ & Effect on OCR \\
\hline Oligomycin $(1 \mu \mathrm{M})$ & Complex V & Decrease \\
\hline FCCP $(1 \mu \mathrm{M})$ & Complex III & Increase \\
\hline Rotenone/Antimycin A $(0.5 \mu \mathrm{M})$ & Complex I \& III respectively & Decrease \\
\hline
\end{tabular}

\subsubsection{Cellular Protein Quantification}

Where needed (GSH and OCR, ECAR) data sets required normalising, this was done through quantification of cellular protein utilising the DC Assay and a series of BSA standards beginning with 2 $\mathrm{mg} / \mathrm{ml}$ and using serial dilutions the following concentrations were made for standard curve interpretation of cellular protein concentration; 1.5, 1, 0.5, and a RIPA buffer blank, detailed in full in Chapter 2 section 2.9). All biological replicates were $\mathrm{N}=3$.

\subsubsection{Statistical Analysis}

All data is shown as the mean +/- standard deviation. Statistical analysis was performed in SPSS statistics software (v.20 IBM, UK) where a one-way analysis of variance (ANOVA) was performed with post hoc Dunnett's multiple comparisons applied to evaluate pairwise statistical significance between control and exposure concentration data sets, the alpha value was set to 0.05 . All data sets were analysed beforehand for normality (Shapiro-Wilk test, $p \leq 0.05$ ) and for equal variance $p \leq 0.05$ ) all biological replicates were $\mathrm{N}=3$, unless otherwise stated. 


\subsection{Results}

\subsubsection{Cytotoxicity \& Genotoxicity of 16HBE140- Following 24-hour Exposure to ENMs}

The cytotoxic \& genotoxic potential of these four carbonaceous ENMs to promote cell death and chromosomal damage in $16 \mathrm{HBE} 140^{-}$was assessed with RPD and the in vitro CBMN assay respectively. This cyto-/genotoxic evaluation was performed in triplicate over a concentration range of $0,2,4,8$, $10,20,50$, and $100 \mu \mathrm{g} / \mathrm{ml}$, a positive control was also run for the genotoxic assessment where Mitomycin C (MMC), a chemotherapeutic compound capable of promoting high clastogenicity was employed at a concentration of $0.01 \mu \mathrm{g} / \mathrm{ml}$. No statistically relevant data was recorded for RPD of any of the carbonaceous ENMs, with the data plateauing consistently in Neutral-FLG and Amine-FLG. Genotoxic assessment of Neutral-FLG upon 16HBE140- (Figure 4.3A) demonstrated a concentrationdependent increase in chromosomal damage with all concentrations above the LOGEL of $10 \mu \mathrm{g} / \mathrm{ml}$. A NOEL was therefore witnessed at concentrations of $2-8 \mu \mathrm{g} / \mathrm{ml}$. At the LOGEL concentration there was a 2 -fold increase over control with the top three $\% \mathrm{Mn} / \mathrm{BN}$ frequencies reaching $0.43 \%, 0.6 \%$ and $0.7 \%$ at 20,50 and $100 \mu \mathrm{g} / \mathrm{ml}$ respectively; at the top concentration of $100 \mu \mathrm{g} / \mathrm{ml}$, this represented a 3-fold increase. Cells treated with Amine-FLG (Figure 4.3B) showed two significant responses to ENM exposure at high concentrations of 50 and $100 \mu \mathrm{g} / \mathrm{ml}$ with respective $\% \mathrm{Mn} / \mathrm{BN}$ frequencies of $0.45 \%$ and $0.51 \%$ representing a 2 -fold increase at the highest concentration. Thus, the LOGEL with AmineFLG treated cells was higher than with Neutral-FLG at 50 $\mu \mathrm{g} / \mathrm{ml}$ and a NOEL of $20 \mu \mathrm{g} / \mathrm{ml}$. Carboxyl-FLG exposures demonstrated little effect on the $16 \mathrm{HBE} 140^{-}$cell line promoting no significant cytotoxic or genotoxic responses at any of the tested concentrations (Figure 4.3C). Lastly, CB particles (Figure 4.3D) promoted a strong genotoxic response with a LOGEL value of $20 \mu \mathrm{g} / \mathrm{ml}$ and a NOEL of $10 \mu \mathrm{g} / \mathrm{ml}$. The top three concentrations of $\mathrm{CB}$ particles demonstrated respective $\% \mathrm{Mn} / \mathrm{BN}$ frequencies of $1.4 \%, 1.4 \%$, $1.63 \%$, representing a 3 -fold increase over control at $100 \mu \mathrm{g} / \mathrm{ml}$. The ability of Neutral-FLG, Amine-FLG and $\mathrm{CB}$ particles to promote significant chromosomal damage in the $\mathrm{CBMN}$ assay allows these materials to be ranked in order of their mass-dose metric genotoxic inhalation hazard; $C B=$ NeutralFLG >Amine-FLG >Carboxyl-FLG. Although Neutral-FLG demonstrated a lower LOGEL than CB particles, the greater frequency of micronuclei induced by the latter ENM seems to offer greater genotoxic damage especially considering it has a lower surface area compared to the FLG materials. 
A
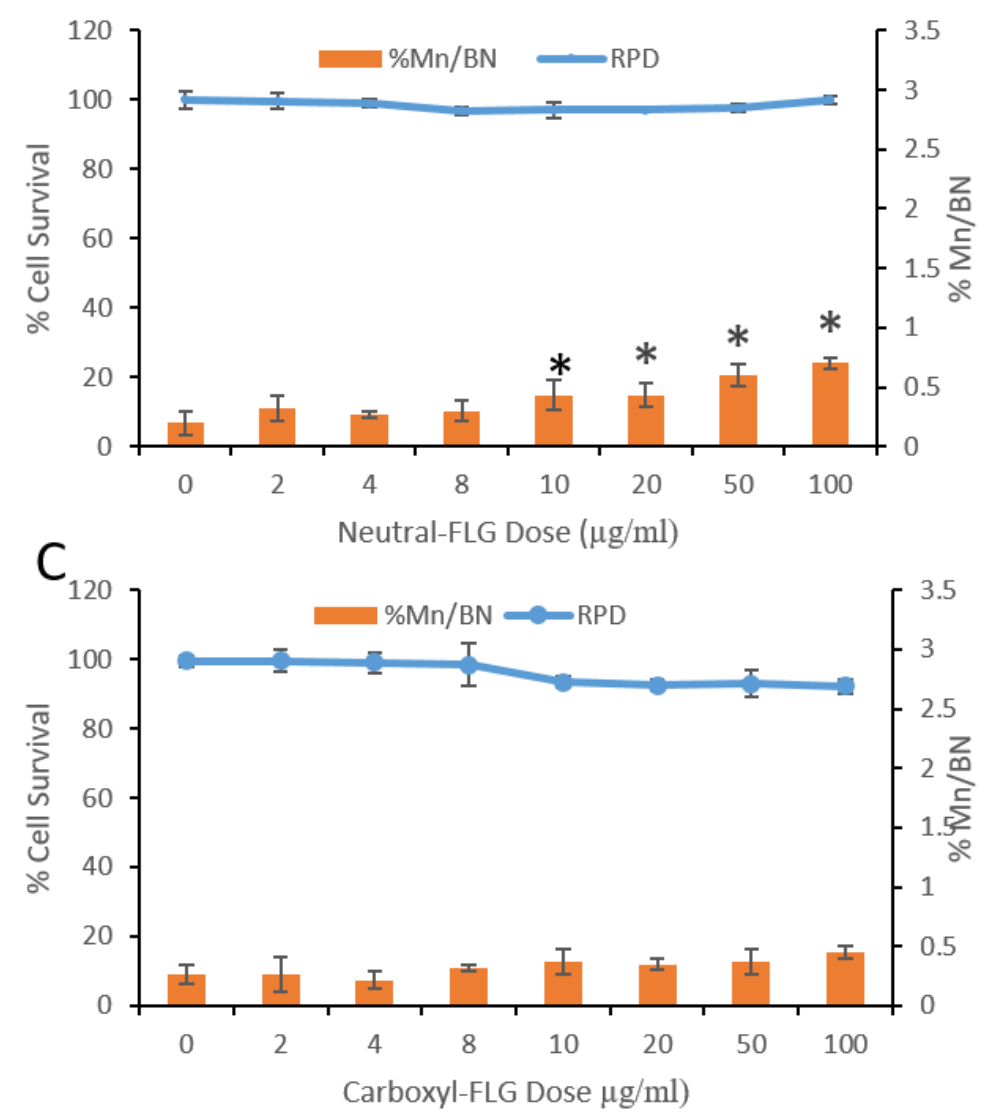

B
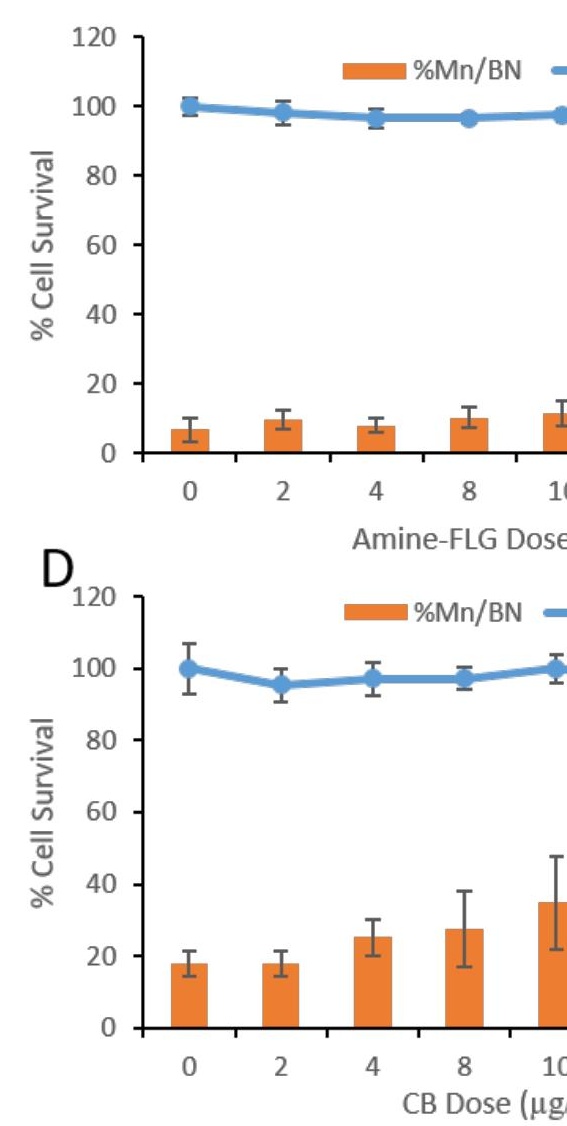

Figure 4.3 Cytotoxic and genotoxic assessment of ENM exposure to 16HBE140- cells. Cytotoxicity, evaluated th assessment of $16 \mathrm{HBE} 140^{-}$cells utilising the in vitro CBMN assay following exposures to carbonaceous ENMs A) Neut and $D)$ CB particles. Results were considered significant $\left({ }^{*}\right)$ when $p<0.05$. MMC positive control demonstrated a 2.7-f( 


\subsubsection{Pan-Centromeric Staining of Chromosomes to Highlight Clastogenicity / Aneugenicity}

Of the ENM doses which promoted a significant genotoxic response in $16 \mathrm{HBE} 140^{-}$cells, pan centromeric paints were employed to highlight the presence / absence of centromeric regions within the micronucleus of a BN cell. This method of detection relies on FITC fluorescence (green) overlapped against the DAPI (blue). Carboxyl-FLG which promoted no significant genotoxic responses was omitted from this analysis and only significant concentrations of other ENMs were selected: Neutral-FLG (10, $20,50$ and $100 \mu \mathrm{g} / \mathrm{ml})$, Amine-FLG (50 and $100 \mu \mathrm{g} / \mathrm{ml}$ ) lastly CB $(20,50$ and $100 \mu \mathrm{g} / \mathrm{ml})$. Control cells $(0 \mu \mathrm{g} / \mathrm{ml})$ exhibited a balanced $0.1 \%$ and $0.09 \%$ ratio of clastogenic and aneugenic response; with no treatment this result is as expected where genetic mutation is a spontaneous and random process during cell division. Hence, unless a genetic predisposition exists the split of clastogenic and aneugenic responses would be roughly 50-50 (Figure 4.4A). Following the control however with sequentially incremental doses of Neutral-FLG the clastogenic potential marginally overcomes the aneugenic response suggesting a greater induction of double strand DNA lesions as the predominant cause of genotoxicity within cells treated with Neutral-FLG. At $100 \mu \mathrm{g} / \mathrm{ml}$ the clastogenic levels were 4 -fold over control levels whereas the aneugenic levels at $100 \mu \mathrm{g} / \mathrm{ml}$ are 3 -fold over control levels. The trend shown by cells treated with Amine-FLG (Figure 4.4B) shows a slight switch between the dose responses of 50 and $100 \mu \mathrm{g} / \mathrm{ml}$. At the $50 \mu \mathrm{g} / \mathrm{ml}$ concentration a ratio of $50: 50$ aneugenicity to clastogenicity exists. However, at the top concentration, an approximate 4-fold increase in clastogenic damage was observed with the \%C-Mn elevated from 0.1 to $0.38 \%$. This was the only significant elevation of clastogenicity over anuegenicity recorded for any concentration of all tested ENMs. Finally, 16HBE140cells treated with CB particles (Figure 4.4C) showed an incremental increase in the clastogenicity with baseline levels being elevated from 0.3 to $0.84 \%$ however there was no mechanism, either clastogenic or aneugenic, which stood out as the predominant process contributing to the observed genotoxic responses. 
A

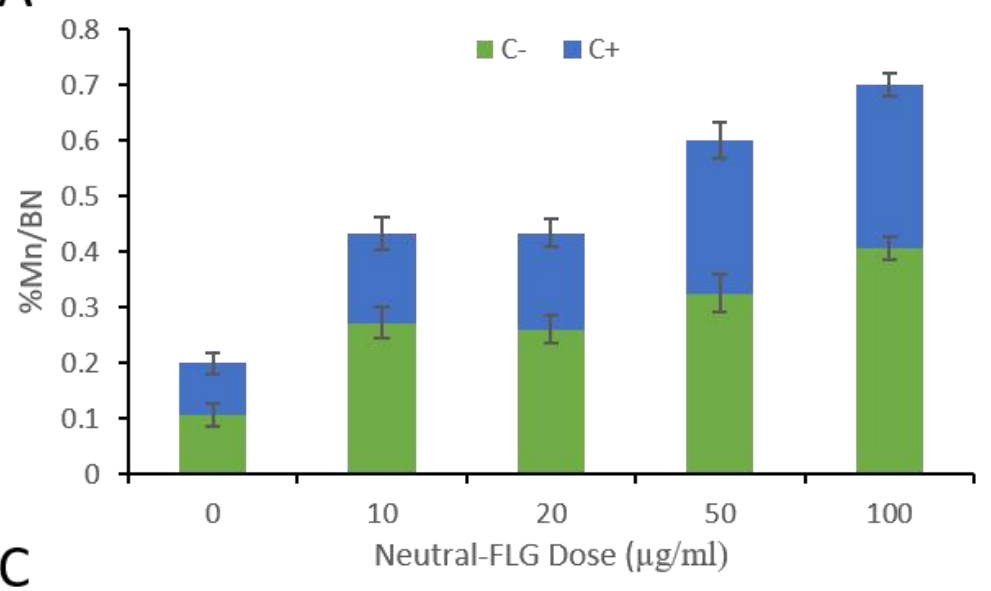

C

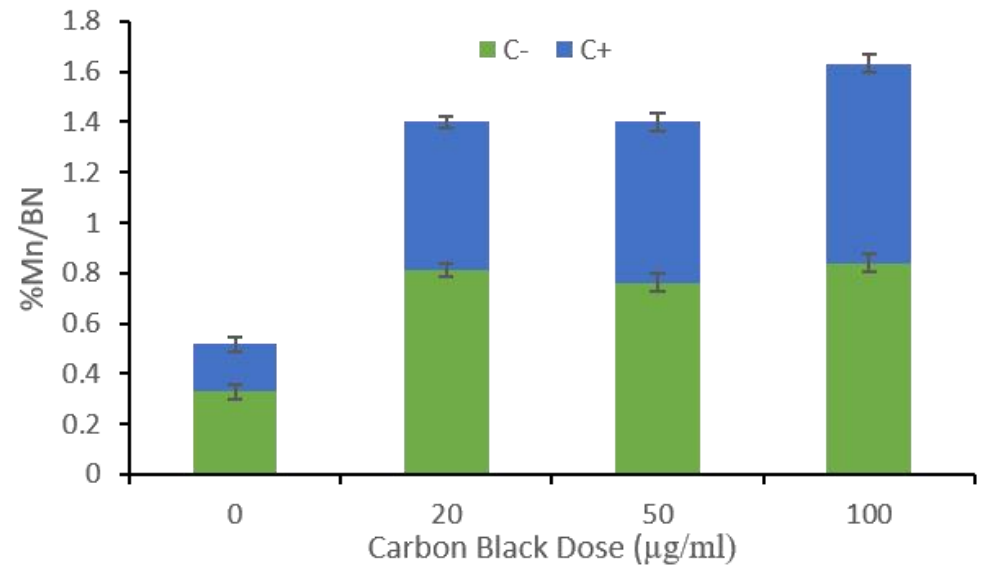

B

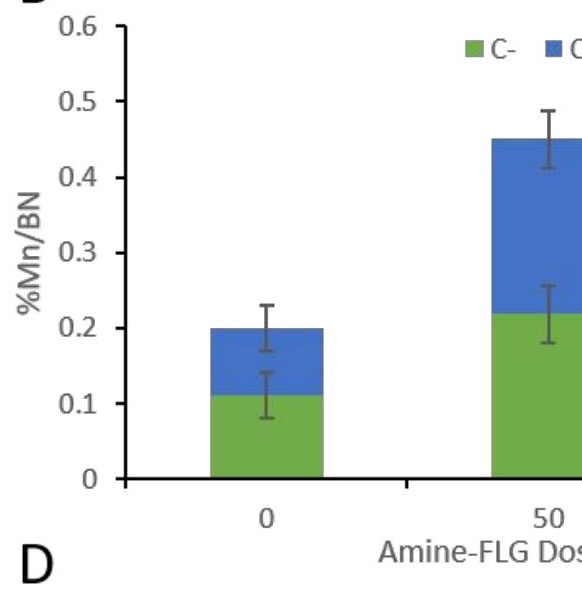

Clastogenic Response

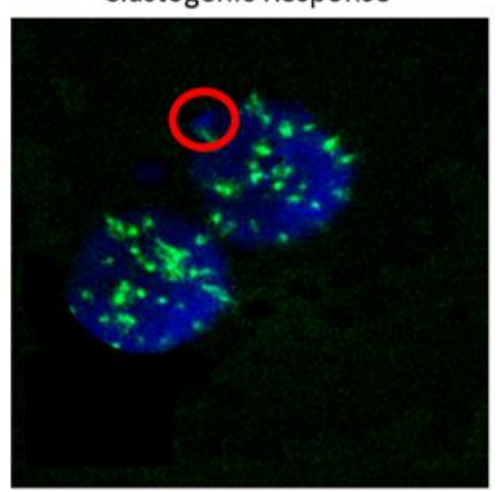

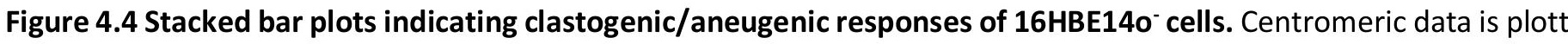
data; Neutral-FLG (A), Amine-FLG (B), CB particles (C), a representative centromere-negative and positive Mn/BN cell positive control at $1.6 \mu \mathrm{g} / \mathrm{ml}$ which promoted aneugenicity to $80 \%$ of total $\mathrm{Mn} / \mathrm{BN}$ cells scored. Results were cons $p<0.05$. Biological replicates $(\mathrm{N}=3)$. 


\subsubsection{Confirmation of uptake in monocultured 16HBE140 cells}

Uptake of each ENM used in this study was confirmed with TEM (Figures 4.5-4.8), the concentration of ENMs used for confirming TEM was $20 \mu \mathrm{g} / \mathrm{ml}$, thus representing the LOGEL concentration. The observed quantity of internalised ENMs within endosomal vesicles was extremely high for each graphene variant material, whilst CB particles showed less uptake with much smaller vesicle diameters. Vesicle diameters for internalised graphene ENMs in some instances was more than $2 \mu \mathrm{m}$ whereas $\mathrm{CB}$ particle exposures generally appeared smaller with vesicles measuring approximately $1 \mu \mathrm{m}$. Furthermore, none of the ENMs appeared free in the cell cytoplasm, thus indicating passive diffusion was not taking place and thus the uptake process could therefore have been phagocytosis and/or macropinocytosis, both of which will result in sizeable endosomes within the cell.

The size and morphology of the ENMs, particularly the graphene materials appeared drastically different during the uptake process as the agglomerates appeared during the Chapter 3 evaluation of agglomerate size using plunge-freezing SEM. Whereas before (in plunge-freeze SEM images) large agglomerates would appear as chain-like structures, here when observed using TEM, the agglomerates became severely compacted into large concentrations. This process of compaction, likely due to the internalisation process of vesicle pinching and subsequent formation appeared to be enough to encase the ENMs without the FLG or CB particles puncturing the membrane-bound vesicles. Sample crystallinity was confirmed with high magnification images of each ENM, whereby the lattice planes could provide a unique sample identification, in the case of the FLG samples, the interlayer spacing. 

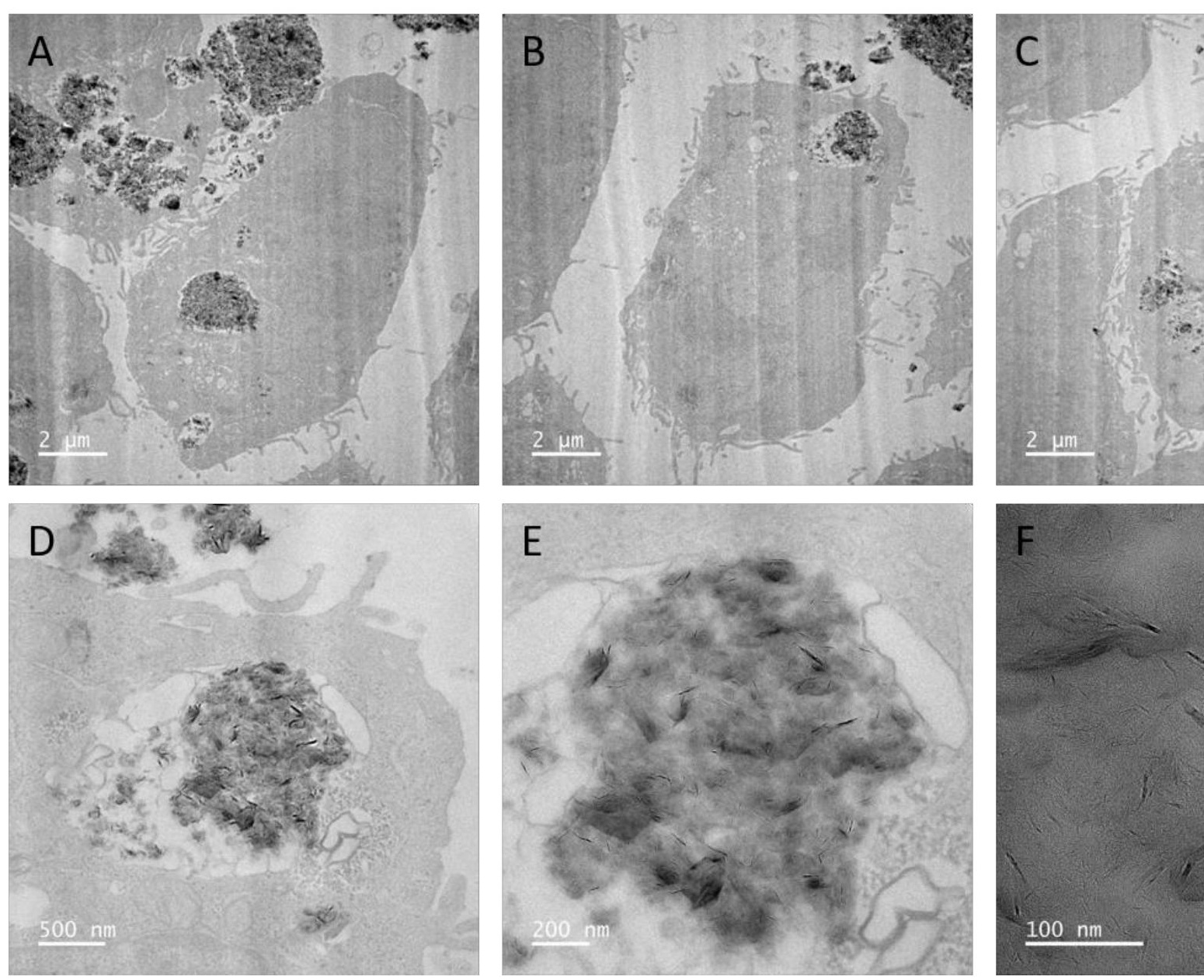

Figure 4.5 TEM micrographs of $16 \mathrm{HBE} 140$ - cells exposed to $20 \mu \mathrm{g} / \mathrm{ml}$ of Neutral-FLG. Where A-C represents thre represents higher magnification images of the ENM. 

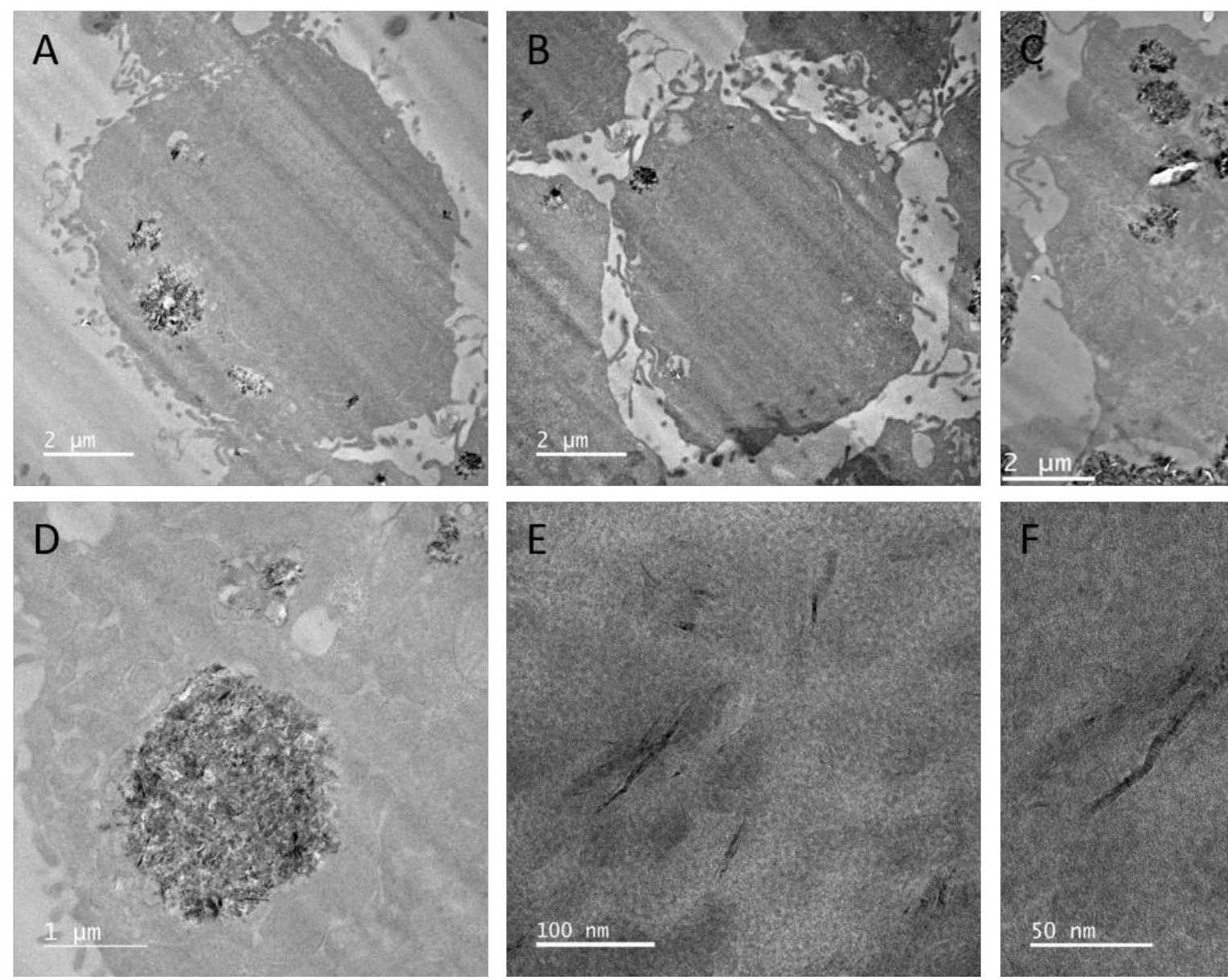

Figure 4.6 TEM micrographs of $16 \mathrm{HBE} 140^{-}$cells exposed to $20 \mu \mathrm{g} / \mathrm{ml}$ of Amine-FLG. Where A-C represents three represents higher magnification images of the ENM. 

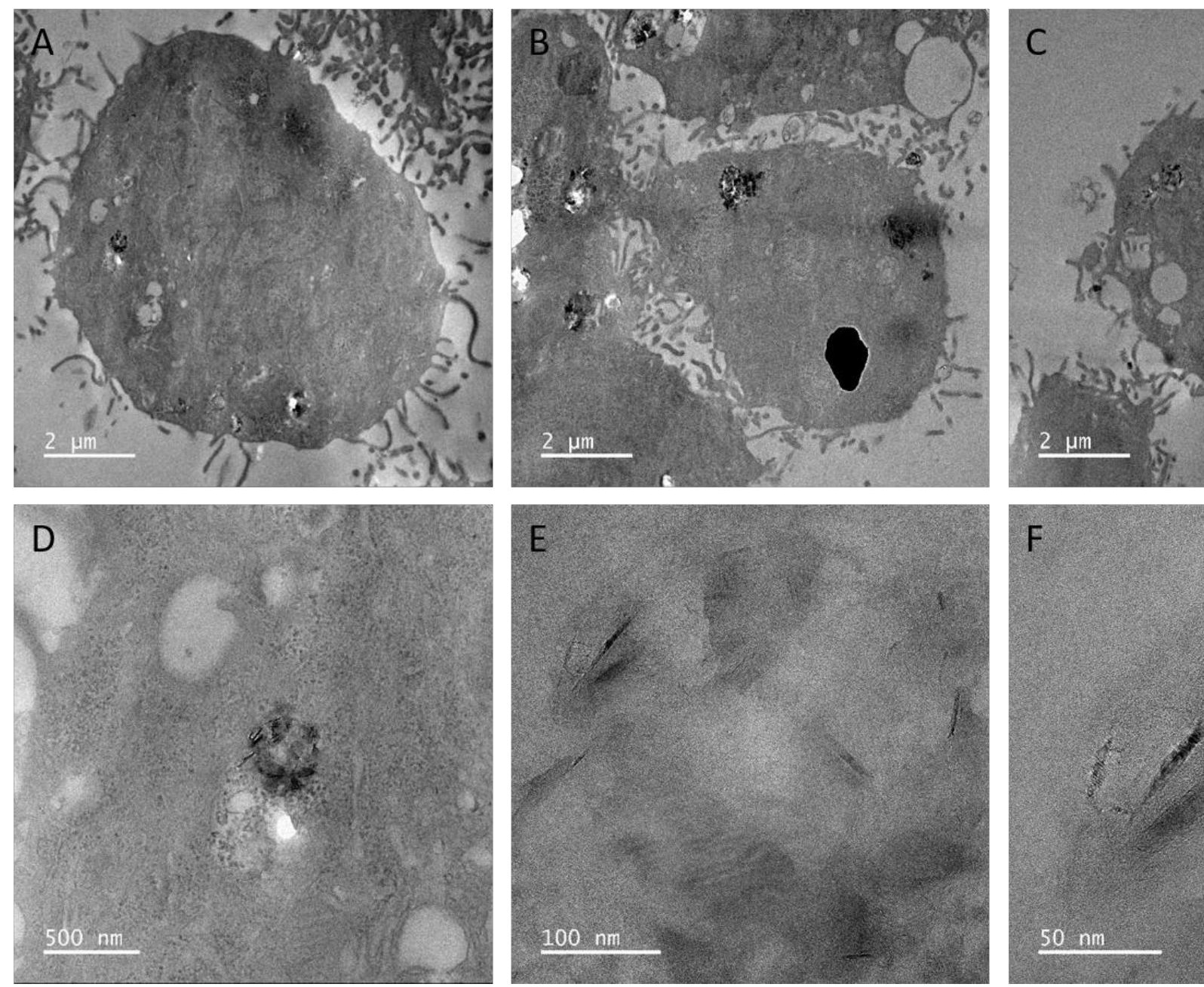

Figure 4.7 TEM micrographs of $16 \mathrm{HBE} 140^{-}$cells exposed to $20 \mu \mathrm{g} / \mathrm{ml}$ of Carboxyl-FLG. Where A-C represents thre represents higher magnification images of the ENM. 

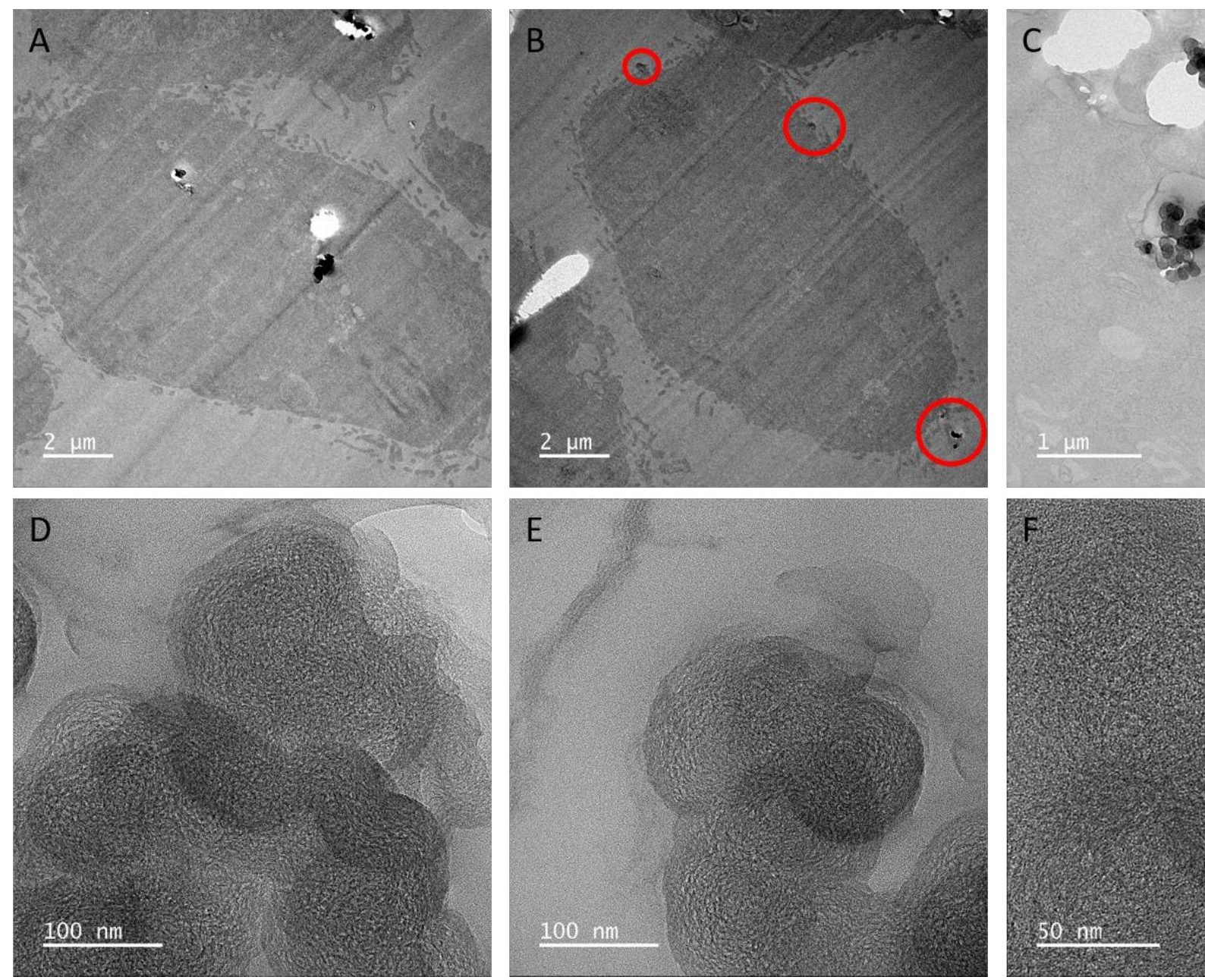

Figure 4.8 STEM/TEM micrographs of $16 \mathrm{HBE} 14 \mathrm{o}^{-}$cells exposed to $20 \mu \mathrm{g} / \mathrm{ml}$ of CB particles. Where A-C represents th represents higher magnification images of the ENM. 


\subsubsection{Quantification of IL-6 Release Following ENM Exposures}

Following the 24-hour ENM exposures, the cellular media was harvested and investigated to quantify the (pro)-inflammatory response of the $16 \mathrm{HBE} 140^{-}$cells focusing on cytokine IL- 6 . The $16 \mathrm{HBE} 140^{-}$cells were exposed to Neutral-FLG, Amine-FLG, Carboxyl-FLG and CB particles at 0, 2, 4, 8, 10, 20, 50 and $100 \mu \mathrm{g} / \mathrm{ml}$ for 6 and 24 hours. Following exposures, supernatants were aspirated, and the IL-6 levels quantified by IL-6-specific ELISA as a measure of the acute-phase (pro)-inflammatory response activation. Initially, supernatant harvested after 6 hours (Figure 4.9A) of exposure showed no indication of an acute phase response with any of the ENMs. Control levels of IL-6 after 6 hours were $98.95 \mathrm{pg} / \mathrm{ml}$ and peaked after carboxyl-FLG exposures at $100 \mu \mathrm{g} / \mathrm{ml}$ reaching $123.67 \mathrm{pg} / \mathrm{ml}$. Within the concentration range the ENM treatment showed a slight decline in IL-6 levels highlighting a partial but beneficial anti-(pro)-inflammatory effect which was interrupted by an overall increase in IL-6 protein levels at higher concentrations of 50 and $100 \mu \mathrm{g} / \mathrm{ml}$. IL-6 protein levels quantified after 24 hours (Figure 4.9B) of exposure revealed no change in IL-6 protein levels, similarly the baseline levels of IL-6 had increased by approximately $50 \mathrm{pg} / \mathrm{ml}$. Control levels of IL-6 increased from 98.95 to $162.6 \mathrm{pg} / \mathrm{ml}$ as over the 18 hour time frame between ELISA measurements which may indicate cellular stress. However, at the highest concentration of ENMs i.e. $100 \mu \mathrm{g} / \mathrm{ml}$ the IL-6 levels at 6 hours for NeutralFLG, Amine-FLG, Carboxyl-FLG and CB were; 112.9, 83.56, 123.7 and $118.4 \mathrm{pg} / \mathrm{ml}$ respectively, measured again at 24 hours the values had increased to $160.9,163.02,161.2$ and $164.3 \mathrm{pg} / \mathrm{ml}$. The three FLG materials therefore, over an 18-hour timeframe, elevated the IL-6 protein levels in the cells which could suggest a delayed acute-phase response. 


\subsubsection{Activation of an IL-8 (Pro)-Inflammatory Response Following ENM Exposure}

Following the 24-hour ENM exposures, the cellular media was harvested and investigated to quantify the (pro)-inflammatory response of the 16HBE140 cells, this time focusing on the chemokine IL-8. Initially 16HBE140 cells were treated with carbonaceous ENMs at concentrations of $0,2,4,8,10,20$, 50 and $100 \mu \mathrm{g} / \mathrm{ml}$ before supernatant was aspirated. IL-8 protein levels were subsequently quantified with an ELISA to measure the chronic phase (pro)-inflammatory response within 16HBE140- cells at 16 and 24 hours of exposure, which would investigate both the peak protein levels post-activation, and the protein levels correlating to the genotoxicity data sets at 24 hours. Firstly, ENM treatment of $16 \mathrm{HBE} 140^{-}$cells for 16 hours (Figure 4.10A) promoted a concentration-dependent incremental response becoming significant for all materials at $50 \mu \mathrm{g} / \mathrm{ml}$ and elevating the IL-8 activation further at $100 \mu \mathrm{g} / \mathrm{ml}$. Control levels of IL-8 $(899 \mathrm{pg} / \mathrm{ml})$ were elevated to 2365 (Neutral-FLG), 1767 (Amine-FLG), 1994 (Carboxyl-FLG) and 1784pg/ml (CB particles) at $100 \mu \mathrm{g} / \mathrm{ml}$ showing an approximate 2-fold increase in IL-8 activation at 16 hours. At the extended 24-hour exposure time point (Figure 4.10B) the IL-8 response indicated a slight elevation in control levels to $1259 \mathrm{pg} / \mathrm{ml}$ meanwhile the top concentration of ENMs maintained their 2-fold activation over baseline levels with Neutral-FLG, Amine-FLG, Carboxyl-FLG and CB particles promoting IL-8 baseline levels to 2161, 2064, 2134 and $2615 \mathrm{pg} / \mathrm{ml}$ respectively. This suggests an average trend of increasing the IL- 8 activation over time for all ENMs apart from Neutral-FLG which showed a marginal decrease in protein levels. At $100 \mu \mathrm{g} / \mathrm{ml}$ the Amine-FLG showed the smallest IL-8 protein expression of all tested ENMs whilst the Neutral-FLG at 16 hours and CB at 24 hours showed the greatest IL-8 protein expression respectively. 
A

B
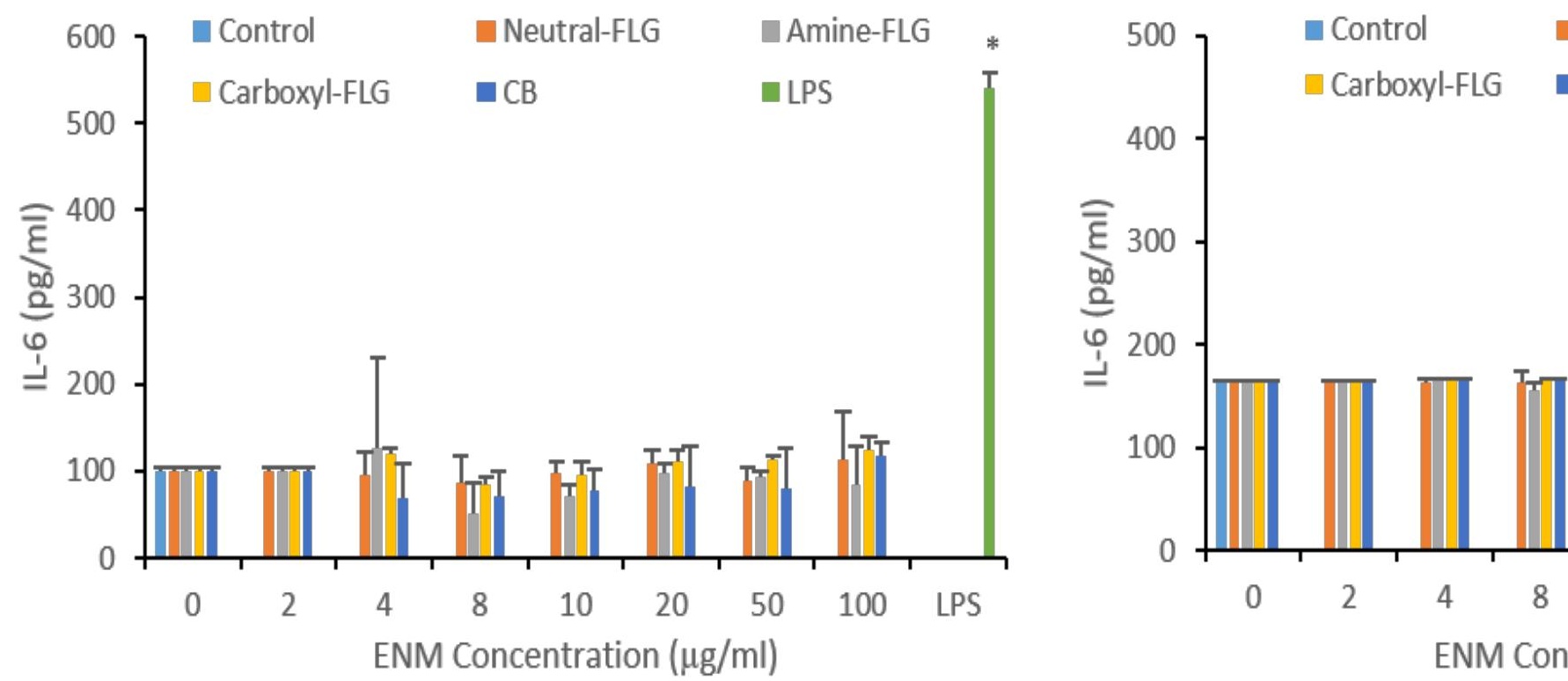

Figure 4.9 IL-6 (pro)-inflammatory response of 16HBE140' cells after exposure to ENMs. IL-6 protein levels after 6 hou was used as the positive control throughout and negative control (media only). Results were considered statistically s 

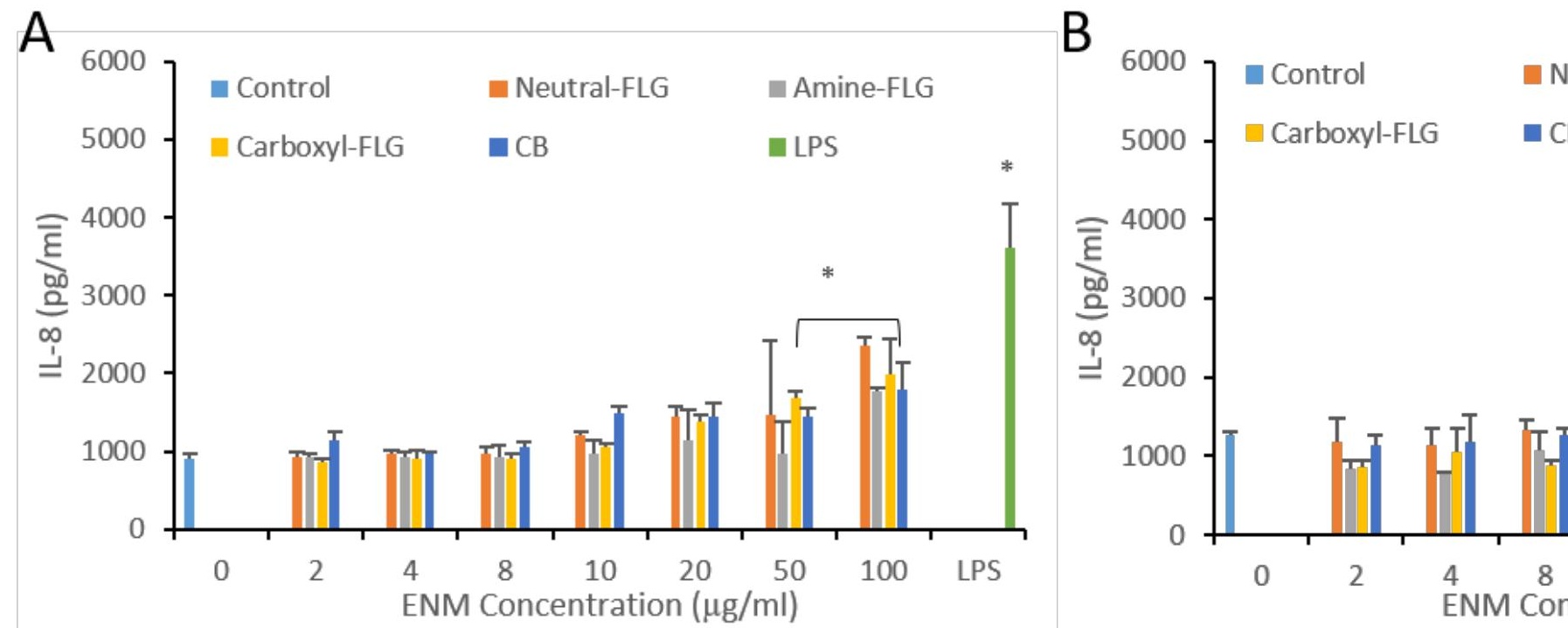

Figure 4.10 IL-8 (pro)-inflammatory response of 16HBE140 cells after exposure to ENMs. IL-8 protein levels after $100 \mathrm{ng} / \mathrm{ml}$ was used as the positive control throughout and negative control (media only). Results were considered $(\mathrm{N}=3)$. 


\subsubsection{Intracellular Glutathione (GSH) Depletion Following ENM Exposures}

To assess the effect of the carbonaceous ENMs on intracellular antioxidant levels as an indirect measure of oxidative stress, the $16 \mathrm{HBE} 14 \mathrm{o}^{-}$cell line was treated with $0,2,4,8,10,20,50$ and $100 \mu \mathrm{g} / \mathrm{ml}$ of ENMs. Following this and a series of washes with PBS the cell lysates were harvested after 6 and 24-hour exposure periods to determine the initial effects on intracellular GSH and comparably longterm effects respectively. To measure the antioxidant depletion a thiol probe (monochlorobimane) which fluoresces once bound to GSH was employed, the basis of this assay relies on hypothesis that ENMs will deplete the GSH levels, in the process converting more to oxidised glutathione (GSSG) reducing the fluorescence. Initially, the GSH levels in the $16 \mathrm{HBE} 14 \mathrm{o}^{-}$cells were quantified after a 6hour (Figure 4.11A) exposure period revealed a significant depletion in the antioxidant at the higher concentrations of 50 and $100 \mu \mathrm{g} / \mathrm{ml}$ with all test ENMs. However, the initial doses of 2 and $4 \mu \mathrm{g} / \mathrm{ml}$ revealed a slightly beneficial role, promoting the GSH levels in the cells to an average of 6-7 nmoles/mg protein from the control level of approximately 5 nmoles/mg protein. In contrast, concentrations of 8$100 \mu \mathrm{g} / \mathrm{ml}$ initiated a concentration-dependent decrease in GSH levels which at 50 and $100 \mu \mathrm{g} / \mathrm{ml}$ represented a 2 and 4-fold depletion from control levels at $\sim 2.8$ and $\sim 1.6$ nmoles $/ \mathrm{mg}$ protein respectively across all ENMs. Following a 24 hour exposure period to ENMs (Figure 4.11B), the GSH levels in the $16 \mathrm{HBE} 140^{-}$cells showed no sign of recovery after the initial depletion from control levels with $\mathrm{CB}$ acting to lower GSH levels even further with a 5 -fold depletion at $1 \mathrm{nmoles} / \mathrm{mg}$ protein at $100 \mu \mathrm{g} / \mathrm{ml}$. Differences at $100 \mu \mathrm{g} / \mathrm{ml}$ can be noted between ENMs with Neutral-FLG and CB administering a greater oxidising potential by lowering the GSH levels from 1.6 and $1.02 \mathrm{nmoles} / \mathrm{mg}$ protein to 1.3 and $1.01 \mathrm{nmoles} / \mathrm{mg}$ protein whilst the carboxyl-FLG appeared the least oxidising with GSH levels rising from 1.98 to $2.3 \mathrm{nmoles} / \mathrm{mg}$ protein at $100 \mu \mathrm{g} / \mathrm{ml}$, this could suggest the oxidising potential of Carboxyl-FLG is exerted early in the exposure period and is rendered chemically less reactive as time increases towards the 24-hour mark. Finally, at the 24-hour mark, CB has lowered GSH levels beyond the positive control Staurosporine with respective reduced GSH levels of 1.01 and $1.1 \mathrm{nmoles} / \mathrm{mg}$ protein highlighting a strong oxidative potential over the course of a 24-hour exposure period. 


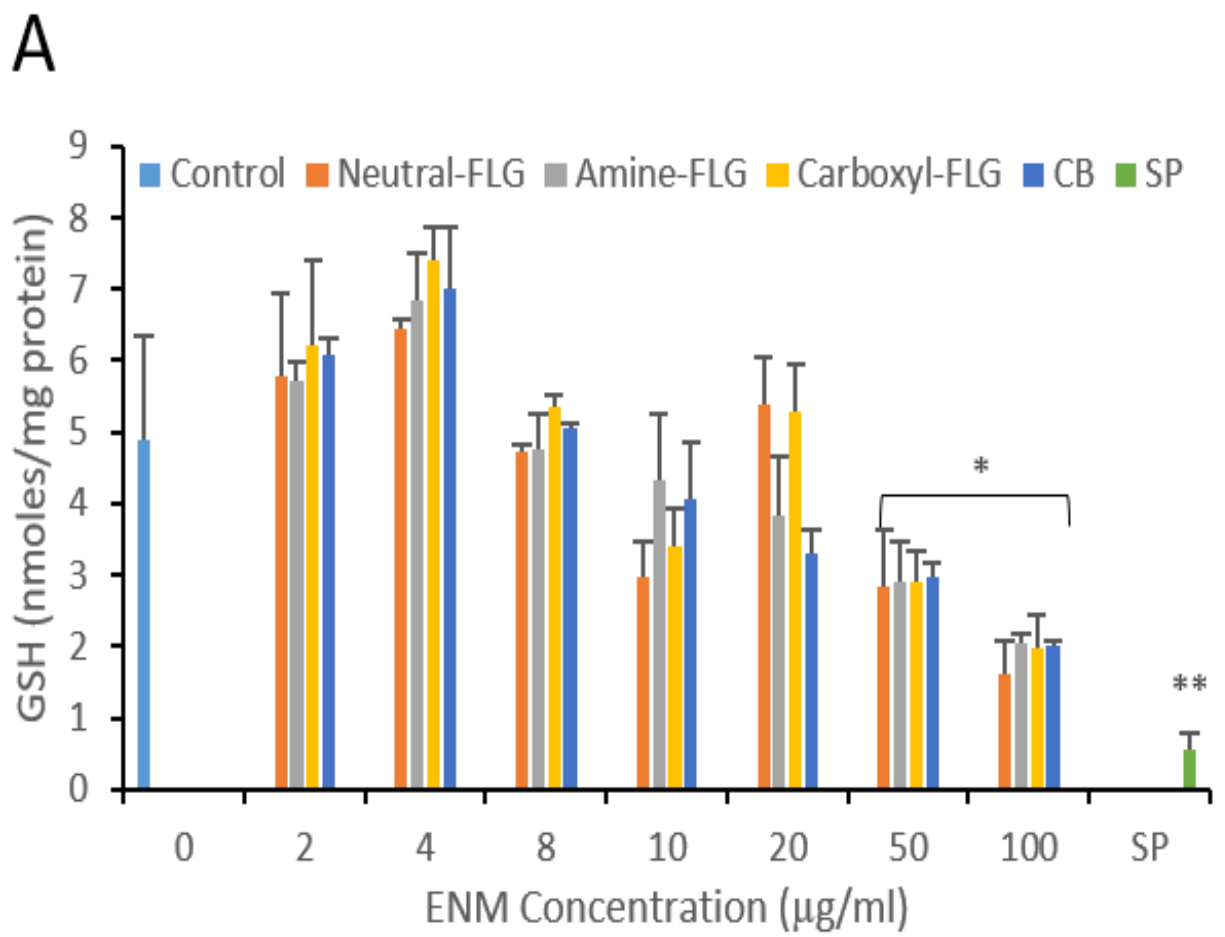

B

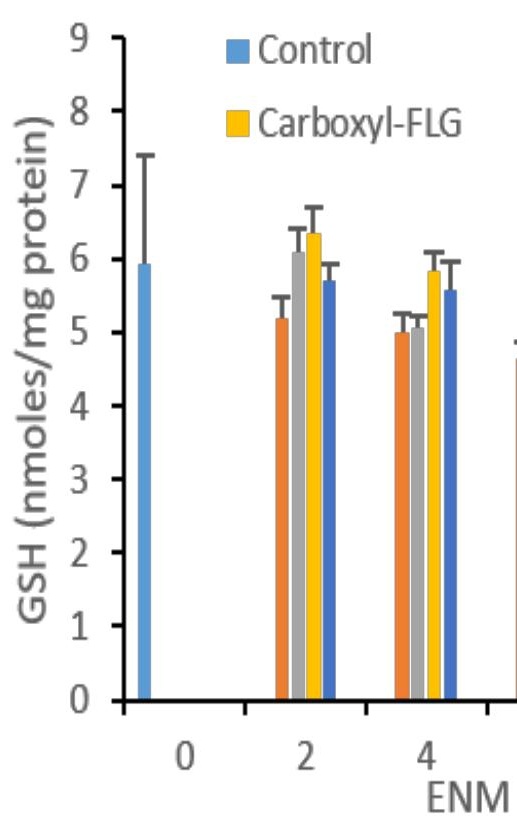

Figure 4.11 Intracellular reduced glutathione levels measured with monochlorobimane-GSH binding fluorescence a 24 hours (B). Staurosporine (SP) was used as a positive control at $1 \mu \mathrm{g} / \mathrm{ml}$ inducing apoptosis in the cells as rapidly a: considered statistically significant $\left({ }^{*}\right)$ when $p<0.05,{ }^{* *} p<0.01 \mathrm{~N}=3$. 


\subsubsection{Quantification of Reactive Oxygen Species Generated by ENMs in 16HBE140`Cells}

To quantify ROS production in a cellular environment a full concentration range $(0-100 \mu \mathrm{g} / \mathrm{ml})$ was performed and the significant concentrations have been plotted to stream-line the larger data set. Neutral-FLG demonstrated a potent ability to promote ROS in $16 \mathrm{HBE}^{-140^{-}}$in a concentrationdependent manner from $10 \mu \mathrm{g} / \mathrm{ml}$ in accordance with the LOEL observed during the CBMN genotoxic evaluation. At this relatively low concentration the peak fluorescence was witnessed at 24 hours of exposure where fluorescence was significantly elevated by $17 \%$, following this a dose-dependent increase in ROS was observed (Figures 4.12A \& 4.13A) where concentrations of 20,50 and $100 \mu \mathrm{g} / \mathrm{ml}$ induce the greatest fluorescence at 8 hours with 19, 25 and 25\% percent increases over control levels. 16HBE140 cells treated with Amine-FLG (Figures 4.12B \& 4.13B) demonstrated significant ROS production at concentrations of 50 and $100 \mu \mathrm{g} / \mathrm{ml}$ with respective 15 and $20 \%$ increases in DCFH fluorescence over the 24-hour period. Unlike the Neutral-FLG-induced fluorescence which clearly spiked at the 8-hour time-point, Amine-FLG-treated cells demonstrated a less prominent timedependent response, with ROS production at a similar level across the 24-hour time-period, possibly indicative of a slightly less reactive material in the cellular environment. DCFH fluorescence obtained from cells exposed to Carboxyl-FLG (Figures 4.12C \& 4.13C) showed an initial significant elevation $(16 \%)$ over control levels at $20 \mu \mathrm{g} / \mathrm{ml}$ at 1 hour of exposure. The 1 -hour time point marked the highest fluorescence recorded for Carboxyl-FLG treated cells at $100 \mu \mathrm{g} / \mathrm{ml}$ with a $26 \%$ increase over control levels. Interestingly, Carboxyl-FLG demonstrated strong potential to promote oxidative stress through ROS production in the absence of a genotoxic response in 16HBE140 cells which suggests other mechanisms (non-oxidative stress) could be attributed to the overall genotoxicity of carbonaceous ENMs. Lastly, CB-treated cells (Figures 4.12D \& 4.13D) which promoted a LOGEL in the CBMN assay of $20 \mu \mathrm{g} / \mathrm{ml}$ behaved similarly with regards to ROS production as Neutral-FLG. CB induced a significant response at $20 \mu \mathrm{g} / \mathrm{ml}$ at 24 hours (20\% over control) followed by strong responses at higher concentrations of 50 and $100 \mu \mathrm{g} / \mathrm{ml}$ with an approximate $17 \%$ rise in fluorescence over control for both concentrations. In summary, the strongest fluorescence intensity was generated by Neutral-FLG and Carboxyl-FLG after 8 hours and 1-hour exposures respectively further highlighting the difference which surface chemistry appears to play during in vitro ENM cellular exposures. 

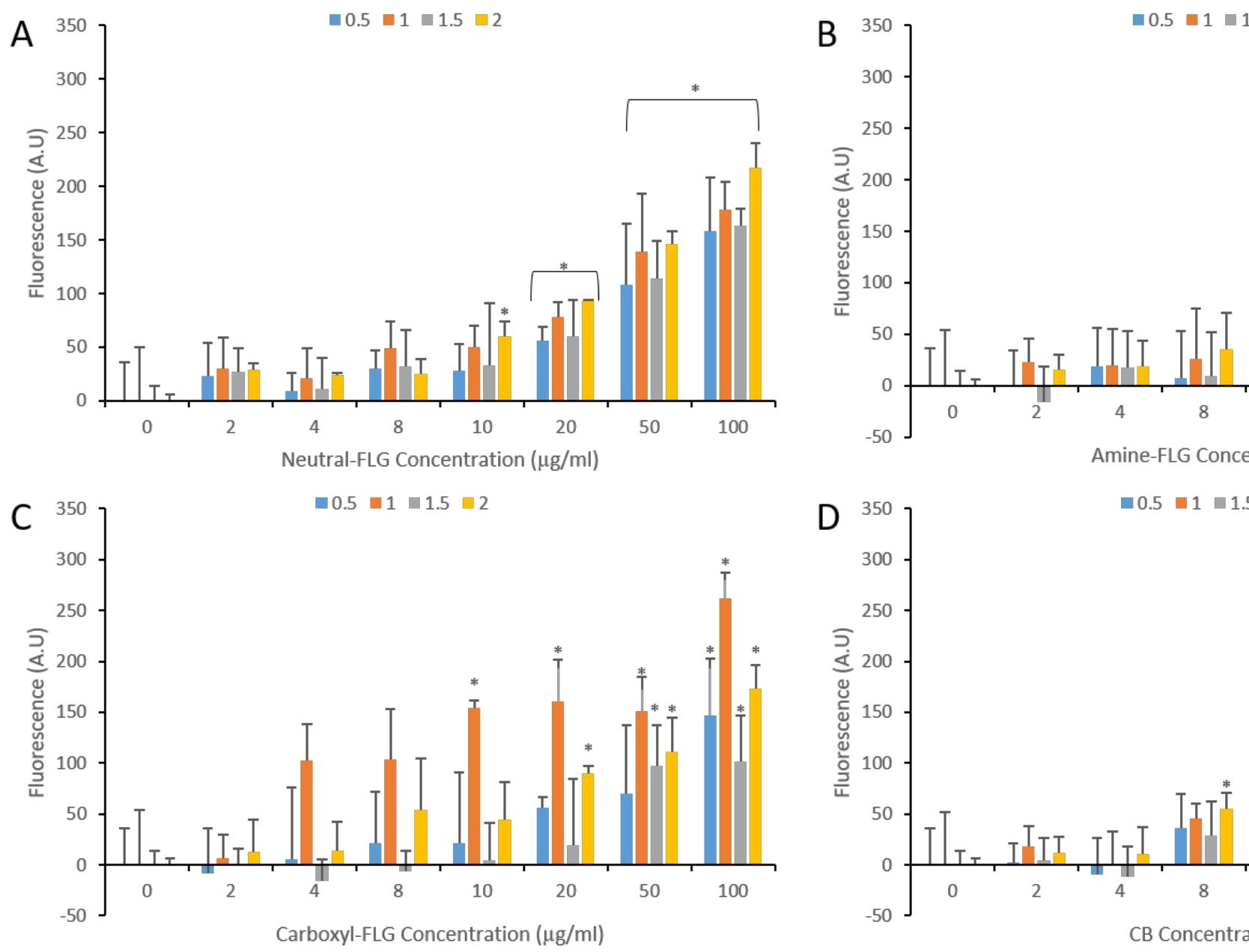

Figure 4.12 ROS production quantified with DCFH-DA fluorescence in 16HBE140' cells following A) Neutral-, B) Amin over $0.5,1,1.5$ and 2 hours. Fluorescence was normalised against control $(0 \mu \mathrm{g} / \mathrm{ml})$. Results were considered statistic 

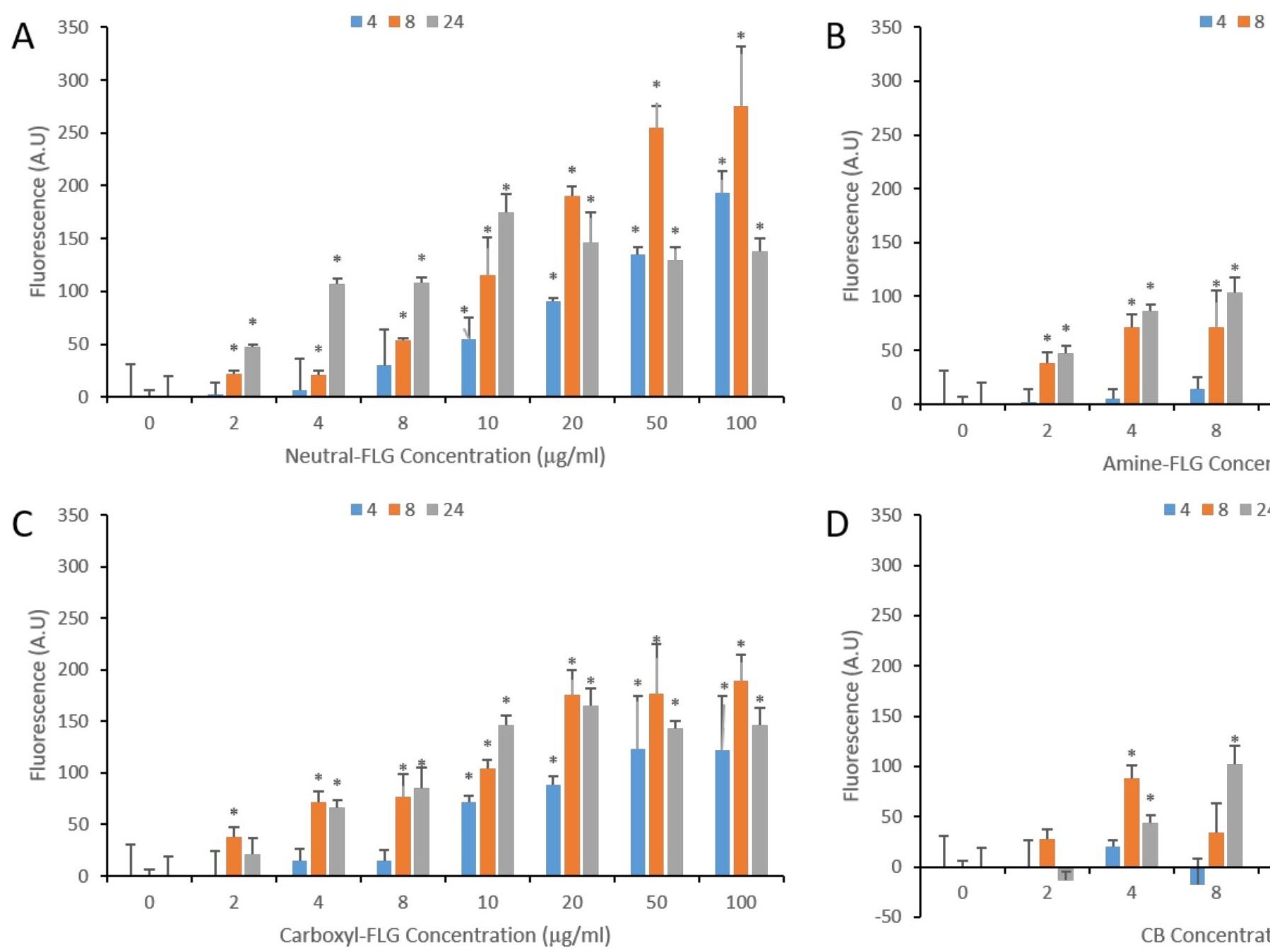

Figure 4.13 ROS production quantified with DCFH-DA fluorescence in 16HBE140 cells following A) Neutral-, B) Amin over 4, 8 \& 24 hours. Fluorescence was normalised against control $(0 \mu \mathrm{g} / \mathrm{ml})$. Results were considered statistically sig 


\subsubsection{Cell-free Reactive Oxygen Species Quantification}

The acellular version of the DCFH-DA assay was performed to investigate the potential of the tested ENMs to promote ROS production in the absence of cells, thus replicating the extracellular ROS production which would have been present during routine cell exposures. The adapted SOP required chemical cleavage of the di-acetate group via Sodium Hydroxide prior to plating and ENM addition. Once again, this data set has been stream-lined from the original concentration range of $0-100 \mu \mathrm{g} / \mathrm{ml}$. Neutral-FLG promoted significant ROS fluorescence initially at $10 \mu \mathrm{g} / \mathrm{ml}$ (Figures 4.14A \& 4.15A) which was sustained from 1 hour of exposure to 24 hours at doses of 10,20, 50 and $100 \mu \mathrm{g} / \mathrm{ml}$. This result indicated a shift in reactivity from the cell-based assay where fluorescence spiked at 8 hours, whereas here the average there appeared to be a time-dependent increase up to 8 hours and a reduction at 24 hours as expected due to the short-lived nature of oxidising radicals. The ROS production from Amine-FLG (Figures 4.14 \& 4.15B) showed an initial significant $7 \%$ rise at concentrations of $20 \mu \mathrm{g} / \mathrm{ml}$ and $50 \mu \mathrm{g} / \mathrm{ml}$ with a sharp increase in fluorescence at $100 \mu \mathrm{g} / \mathrm{ml}$ with ROS production rising to approximately $20 \%$. This cell-free reactivity becomes significant prior to the LOGEL concentration of $50 \mu \mathrm{g} / \mathrm{ml}$ in the CBMN assay which may signify the $16 \mathrm{HBE} 14 \mathrm{O}^{-}$ability to tolerate mild oxidative stress from this ENM. Carboxyl-FLG in a cell-free environment (Figures 4.14C \& 4.15C) behaves similarly with regards to ROS production as in the cell-based DCFH assay whereby concentrations of 20,50 and $100 \mu \mathrm{g} / \mathrm{ml}$ showed a significant elevation of ROS. The cell-free assay revealed peak fluorescence at $20-$ $100 \mu \mathrm{g} / \mathrm{ml}$ as was observed at 4 hours with 12,14 and $17 \%$ increases respectively. In the absence of cells, CB particles showed the lowest degree of ROS production (Figures 4.14D \& 4.15D) peaking again at 8 hours with only an $11 \%$ elevation above control levels. 

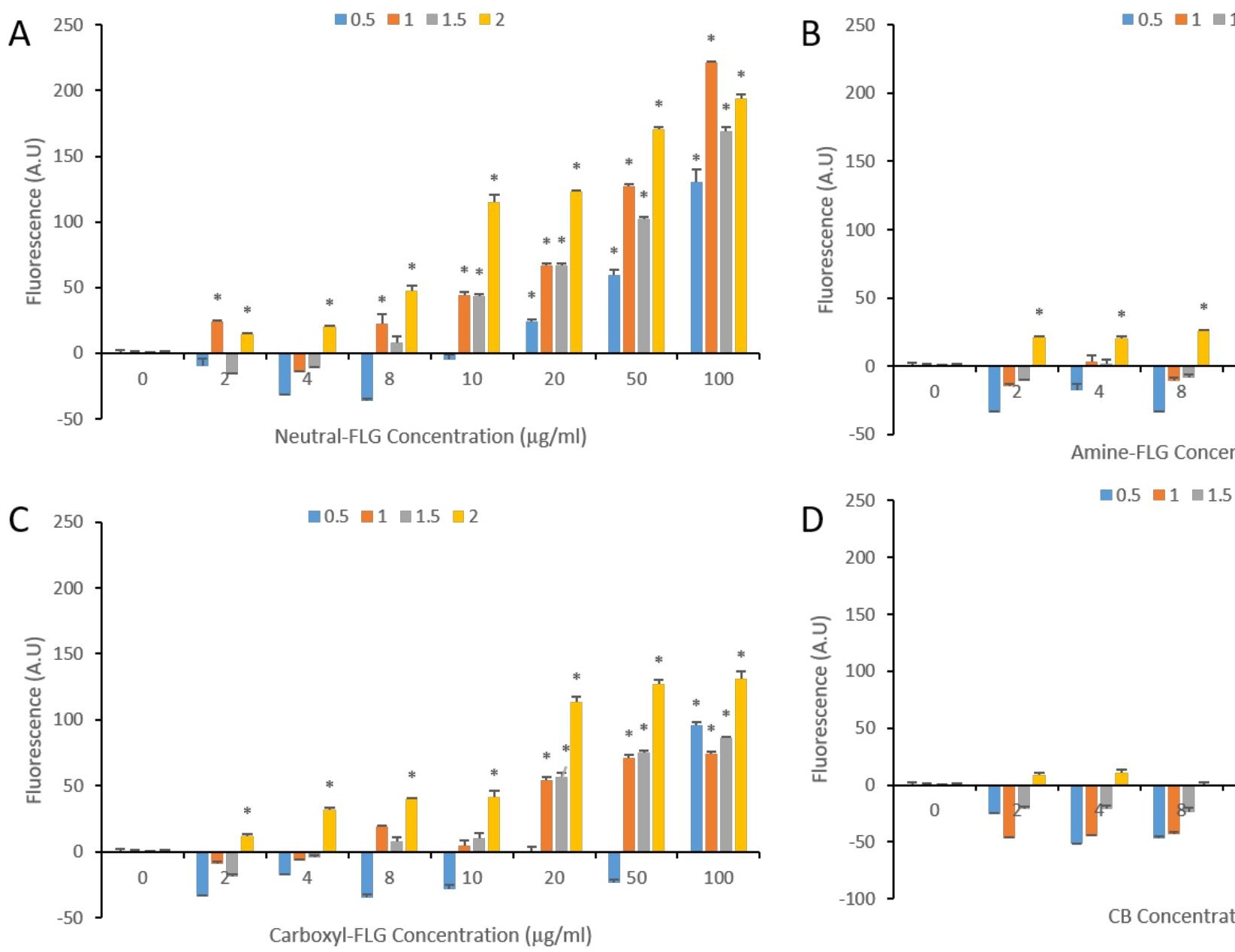

Figure 4.14 Acellular ROS DCFH-DA assay promoted by A) Neutral-FLG, B) Amine-FLG, C) Carboxyl-FLG and D) C Fluorescence was normalised against control $(0 \mu \mathrm{g} / \mathrm{ml})$. Results were considered statistically significant $(*)$ when $p<0$. 

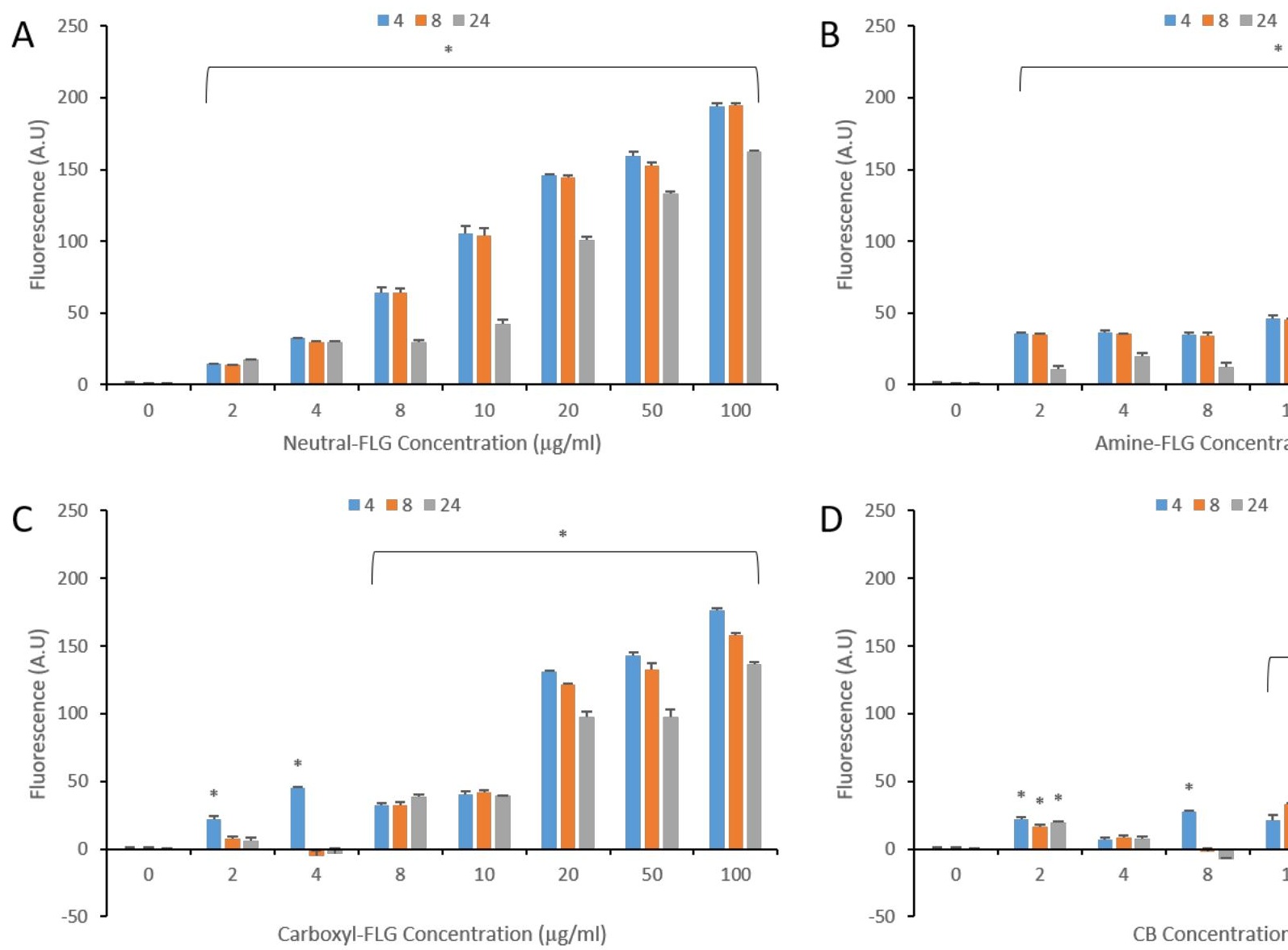

Figure 4.15 Acellular ROS DCFH-DA assay promoted by A) Neutral-FLG, B) Amine-FLG, C) Carboxyl-FLG and D) CB part was normalised against control $(0 \mu \mathrm{g} / \mathrm{ml})$. Results were considered statistically significant $\left({ }^{*}\right)$ when $p<0.05 \mathrm{~N}=3$. 


\subsubsection{Oxygen Consumption and Extracellular Acidification Rates in 16HBE140' Cells Following ENM exposure}

To assess the effect of Neutral-, Amine-, Carboxyl-FLG and CB particles upon 16HBE140- mitochondrial function, the epithelial cells were seeded into XFe24 plates and treated with concentrations of $0,2,4$, $8,10,20,50$ and $100 \mu \mathrm{g} / \mathrm{ml}$ for 24 hours. Optimal seeding concentration was determined to be 120,000 cells per well and the injection concentrations optimised at $1 \mu \mathrm{M}$ for Oligomycin and FCCP and a Rotenone/Antimycin A concentration of $0.5 \mu \mathrm{M}$. Following this, the plate was loaded into a Seahorse XFe24 Bioanalyser and the mitochondrial stress was monitored for $~ 96$ minutes. OCR and ECAR data for each ENM can be found in the Appendix Section 7.2 which displayed a similar to trend to the Neutral-FLG displayed below, whereby all doses with the exception of $4 \mu \mathrm{g} / \mathrm{ml}$ induced a depletion of oxygen consumption (Figure 4.16A) and also decreased the extracellular acid production (Figure $4.16 \mathrm{~B}$ ) in $16 \mathrm{HBE} 140^{\circ}$ cells following 24 hours exposure. Firstly, dealing with the OCR in 16HBE140', the Neutral-FLG showed no specific trend in which concentrations depleted the OCR to the greatest extent however the concentration of $50 \mu \mathrm{g} / \mathrm{ml}$ in all tested ENMs proved to be the most potent in depleting the OCR. This effect was supported with the ECAR data whereby $50 \mu \mathrm{g} / \mathrm{ml}$ depleted extracellular proton production without however this result did not become significant. Interestingly, $4 \mu \mathrm{g} / \mathrm{ml}$ showed the opposite effect on OCR and ECAR by elevating both above control levels with ECAR becoming significant 27,35 and 44 minutes into the analysis. The effect was not sustained, extracellular acidification dropped below significant levels until 87 and 96 minutes into the analysis. ECAR profiles of both the control cells and cells exposed to ENMs generally increased over the course of the experiment however differences arose between ENM concentrations. At $4 \mu \mathrm{g} / \mathrm{ml}$ there was a 1.3 -fold increase from 108 to $147 \mathrm{pmoles} / \mathrm{min} / \mathrm{mg}$ protein. This trend then underwent a steady decline before rising 1.3-fold above control once more becoming significant until the end of the assay. 
A

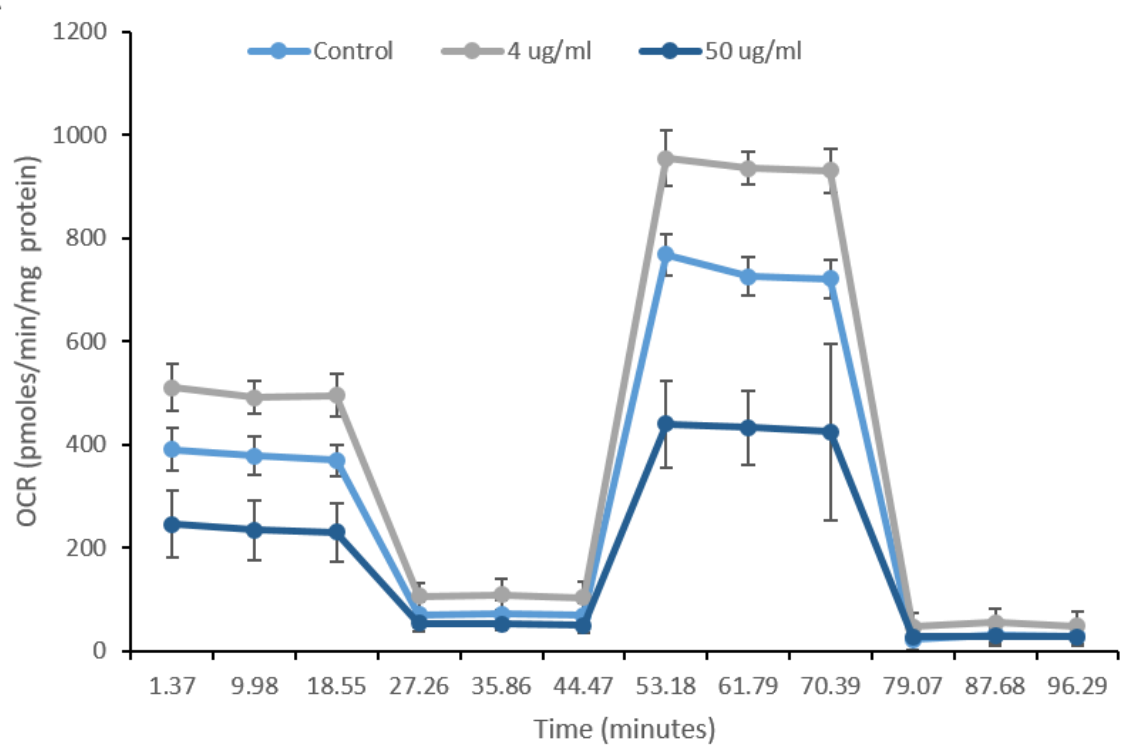

B

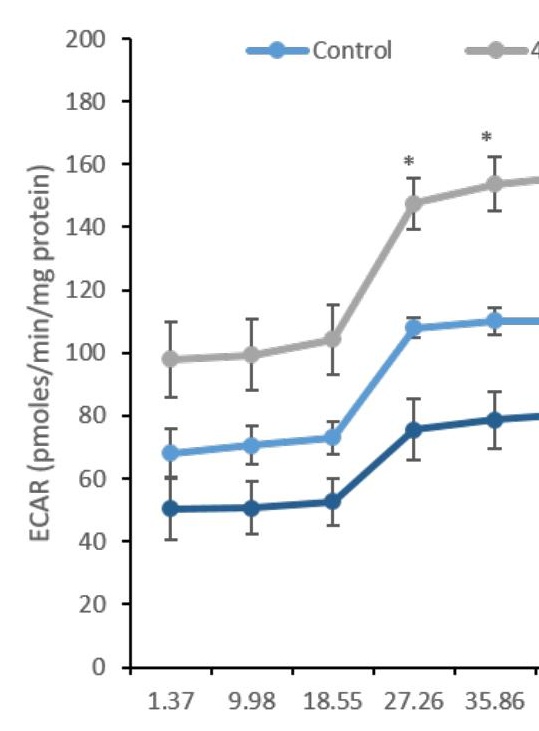

Figure 4.16 Oxygen consumption (A) and extracellular acidification rate (B) in $16 \mathrm{HBE}^{-140^{-}}$cells following 24 -hour ex material). OCR and ECAR values were normalised against protein content per well (DC Assay). Results were considered $\mathrm{N}=3$ 


\subsubsection{16HBE140 Respiratory Parameters as an Indicator of Mitochondrial Stress Following ENM exposure}

Following the evaluation of OCR and ECAR, further analysis was performed on the OCR profile of each ENM following 24-hour exposures with $16 \mathrm{HBE}^{-140^{-}}$cells. The trend observed for the proton leak in cells exposed to Neutral-FLG (Figure 4.17A) initially underwent a significant increase at $4 \mu \mathrm{g} / \mathrm{ml}$ before then succumbing to the concentration-dependent decrease which reached significant levels at 8,20 and $50 \mu \mathrm{g} / \mathrm{ml}$. Non-mitochondrial respiration showed an increase at low concentrations of 2, 4, 8 and $20 \mu \mathrm{g} / \mathrm{ml}$ with significant elevation over control levels of $27 \mathrm{pmoles} / \mathrm{min} / \mathrm{mg}$ protein to respective values of $40,50,38.5$ and $40 \mathrm{pmoles} / \mathrm{min} / \mathrm{mg}$ protein. This effect was not sustained into the higher concentrations however. Basal levels of respiration in $16 \mathrm{HBE}^{-140^{-}}$cells showed a concentrationdependent decrease which reached a significant depletion at 8,10 and $50 \mu \mathrm{g} / \mathrm{ml}$. Basal levels of respiration (379pmoles/min/mg protein) spiked at $4 \mu \mathrm{g} / \mathrm{ml}$ following Neutral-FLG exposure (Figure 4.18A) with a 'beneficial' effect on the $16 \mathrm{HBE} 14 \mathrm{O}^{-}$cells where basal levels peaked at $499 \mathrm{pmoles} / \mathrm{min} / \mathrm{mg}$ protein. The concentrations of 8,10 and $50 \mu \mathrm{g} / \mathrm{ml}$ saw approximate drops in basal levels by 100,80 and 140 pmoles/min/mg protein respectively. Some recovery of the basal levels was observed at the highest concentration of $100 \mu \mathrm{g} / \mathrm{ml}$ at $343 \mathrm{pmoles} / \mathrm{min} / \mathrm{mg}$ protein. ATP production following Neutral-FLG exposure for 24 hours at $4 \mu \mathrm{g} / \mathrm{ml}$ underwent significant depletions at 8,10 and $50 \mu \mathrm{g} / \mathrm{ml}$ with a recovery of ATP production at $100 \mu \mathrm{g} / \mathrm{ml}$. Control $(0 \mu \mathrm{g} / \mathrm{ml})$ levels of ATP production (309pmoles $/ \mathrm{min} / \mathrm{mg}$ protein) was depleted at a low concentration of $2 \mu \mathrm{g} / \mathrm{ml}$ by $\sim 40 \mathrm{pmoles} / \mathrm{min} / \mathrm{mg}$ protein whereas at significant levels the drop was $\sim 82,70$ and, 124pmoles/mg protein for concentrations of 8,10 and $50 \mu \mathrm{g} / \mathrm{ml}$ respectively. Maximal respiration in $16 \mathrm{HBE} 140^{-}$cells displayed similar trends with a concentration-dependent depletion becoming significant at 8,20 and $50 \mu \mathrm{g} / \mathrm{ml}$, a spike at $4 \mu \mathrm{g} / \mathrm{ml}$ and a recovery response induced at the highest concentration of $100 \mu \mathrm{g} / \mathrm{ml}$. Control levels of $711 \mathrm{pmoles} / \mathrm{min} / \mathrm{mg}$ protein initially underwent a depletion at $2 \mu \mathrm{g} / \mathrm{ml}$ which became significant at 528, 516 and $404 \mathrm{pmoles} / \mathrm{min} / \mathrm{mg}$ protein representing respective $0.3,0.4$ and 0.75 -fold decreases from control levels at 8,20 and $50 \mu \mathrm{g} / \mathrm{ml}$. Maximal respiration showed a recovery towards control levels at $100 \mu \mathrm{g} / \mathrm{ml}$ with a concentration of $629 \mathrm{pmoles} / \mathrm{min} / \mathrm{mg}$ protein. Reserve respiratory only underwent a significant drop from control levels at 20 and $50 \mu \mathrm{g} / \mathrm{ml}$ with marginal fold-decreases respective fold-decreases ( $<1$-fold).

Amine-FLG promoted a significant depletion in proton leak (Figure 4.17B) from control levels (42pmoles $/ \mathrm{min} / \mathrm{mg}$ protein) at concentrations of 20,50 and $100 \mu \mathrm{g} / \mathrm{ml}$ with respective concentrations of 31,26 and $27 \mathrm{pmoles} / \mathrm{min} / \mathrm{mg}$ protein. Non-mitochondrial respiration showed a significant increase 
as concentration of Amine-FLG increased initially at $2 \mu \mathrm{g} / \mathrm{ml}(0.7$-fold increase) also at $20 \mu \mathrm{g} / \mathrm{ml}(0.8-$ fold increase) over control levels. Other concentrations showed similar increases albeit not to significant levels, except for $10 \mu \mathrm{g} / \mathrm{ml}$ which declined from 27 to 23 pmoles $/ \mathrm{min} / \mathrm{mg}$ protein. Basal respiration levels in 16HBE140 cells post treatment with Amine-FLG (Figure 4.18B) showed an initial increase in concentration between $2-8 \mu \mathrm{g} / \mathrm{ml}$ which at $4 \mu \mathrm{g} / \mathrm{ml}$ was (significant) $0.9-$ fold over control levels of $379 \mathrm{pmoles} / \mathrm{min} / \mathrm{mg}$ protein. Subsequent doses of $10,20,50$ and $100 \mu \mathrm{g} / \mathrm{ml} \mathrm{marked} \mathrm{a}$ depletion in basal respiration levels with $0.3,0.1,0.4$ and 0.35 -fold decreases from control levels respectively. ATP production post Amine-FLG treatment showed an initial increase at concentrations of 2,4 and $8 \mu \mathrm{g} / \mathrm{ml}$ which was significant at $4 \mu \mathrm{g} / \mathrm{ml}$. The maximal respiration of $16 \mathrm{HBE} 14 \mathrm{o}^{-}$cells post treatment with Amine-FLG displayed an increase from control levels (711pmoles/min/mg protein) to 749 and $872 \mathrm{pmoles} / \mathrm{min} / \mathrm{mg}$ protein at respective doses of 2 and (significant) $4 \mu \mathrm{g} / \mathrm{ml}$. From 8 to $100 \mu \mathrm{g} / \mathrm{ml}$ a decreasing trend can be seen with significant values at 10, 50 and 100 where the fall from control was $0.2,0.3$ and 0.22 -fold respectively. Finally, the reserve respiratory capacity, the only significant concentration proved to be $50 \mu \mathrm{g} / \mathrm{ml}$ where the fall from control levels of $358 \mathrm{pmoles} / \mathrm{min} / \mathrm{mg}$ protein was 0.14 -fold with an overall trend showing a depletion as concentration increased from $8-100 \mu \mathrm{g} / \mathrm{ml}$ with a small elevation in reserve capacity between the concentrations of $2-8 \mu \mathrm{g} / \mathrm{ml}$.

Carboxyl-FLG appeared to promote greater stress on the $16 \mathrm{HBE} 14 \mathrm{O}^{-}$mitochondrion when compared to the two ENMs discussed previously. Relatively small impacts were observed for proton leak (Figure 4.17C), initially a significant depletion (0.5-fold) was observed before rising above control levels at $4 \mu \mathrm{g} / \mathrm{ml}$ to $45 \mathrm{pmoles} / \mathrm{min} / \mathrm{mg}$ protein followed by a significant decrease in proton leak at concentrations of 8,20 and $100 \mu \mathrm{g} / \mathrm{ml}$ with $0.57,0.4$ and 0.42 -fold depletions observed. Nonmitochondrial respiration was significantly depleted at $4 \mu \mathrm{g} / \mathrm{ml}$, the only significant effect on this respiratory parameter showing a 0.5 -fold decrease this effect was not sustained and the remaining concentrations plateaued in accordance with control levels of $\sim 25 \mathrm{pmoles} / \mathrm{min} / \mathrm{mg}$ protein. Basal respiration was significantly depleted by Carboxyl-FLG treatment (Figure 4.18C) at concentrations of $2,8,10,20$ and $100 \mu \mathrm{g} / \mathrm{ml}$ with $0.5,0.48,0.45,0.42$ and 0.42 -fold decreases from control concentrations. ATP production also fell in accordance with increasing concentration of Carboxyl-FLG with significant levels first seen at $2 \mu \mathrm{g} / \mathrm{ml}$ and sustained at concentrations of 8,10 and $100 \mu \mathrm{g} / \mathrm{ml}$ with respective fold decreases of $0.5,0.5$ and 0.47 . Maximal respiration was considerably depleted in 16 HBE140 $0^{-}$cells treated with Carboxyl-FLG initially observed as early as $2 \mu \mathrm{g} / \mathrm{ml}$ with a 2.9 -fold decrease. The 2.98 -fold decrease at $2 \mu \mathrm{g} / \mathrm{ml}$ proved to be the lowest fall in maximal respiration with concentrations of $8,10,20$ showing respective 2.95, 2.76 and 2.5-fold decreases from 711 
pmoles $/ \mathrm{min} / \mathrm{mg}$ protein (control), concentrations of 50 and $100 \mu \mathrm{g} / \mathrm{ml}$ were marginally recovering to non-significant concentrations. Finally, the reserve respiratory capacity, from control levels of $358 \mathrm{pmoles} / \mathrm{min} / \mathrm{mg}$ protein declined sharply at $2 \mu \mathrm{g} / \mathrm{ml}$ to $60.9 \mathrm{pmoles} / \mathrm{min} / \mathrm{mg}$ protein showing a 5.9fold significant decrease which sustained across the 8,10 , and $20 \mu \mathrm{g} / \mathrm{ml}$ concentration range before recovering some respiratory capacity at 50 and $100 \mu \mathrm{g} / \mathrm{ml}$ (105.7 and $115 \mathrm{pmoles} / \mathrm{min} / \mathrm{mg}$ protein) respectively.

$\mathrm{CB}$ treated $16 \mathrm{HBE}^{-} \mathrm{O}^{-}$cells showed a concentration-dependent trend in proton leak only becoming significant at $10 \mu \mathrm{g} / \mathrm{ml}$ (Figure 4.17D) and was sustained at subsequent concentrations up to and including $100 \mu \mathrm{g} / \mathrm{ml}$. A 2.7 -fold decrease was observed at $10 \mu \mathrm{g} / \mathrm{ml}$ which at $100 \mu \mathrm{g} / \mathrm{ml}$ decreased to a 2.1-fold indicating marginal recovery of proton leak and correlated to a small decline in the ECAR of CB treated cells. Non-mitochondrial respiration showed a general dose-dependent increase rising from control levels at $27 \mathrm{pmoles} / \mathrm{min} / \mathrm{mg}$ protein to a peak at $50 \mu \mathrm{g} / \mathrm{ml}$ of $41.8 \mathrm{pmoles} / \mathrm{min} / \mathrm{mg}$ protein before declining at $100 \mu \mathrm{g} / \mathrm{ml}$. Crucially, and unlike the graphene ENMs CB did not induce a significant non-mitochondrial response in $16 \mathrm{HBE} 140^{-}$cells which may offer insight into the mechanisms promoting the mitochondrial stress. CB particles promoted a concentration-dependent depletion in basal respiration levels in 16HBE140 cells (Figure 4.18D), initial significant responses were recorded at $8 \mu \mathrm{g} / \mathrm{ml}$ which was exacerbated at 10,50 and $100 \mu \mathrm{g} / \mathrm{ml}$ but not at $20 \mu \mathrm{g} / \mathrm{ml}$ where basal levels increased marginally to just a 1.7 -fold depletion from control levels whereas at $10 \mu \mathrm{g} / \mathrm{ml}$, the concentration which promoted the strongest effect on basal respiration, a 2.7-fold decrease was observed. The effect of CB particles upon ATP production promoted an initial significant depletion at $8 \mu \mathrm{g} / \mathrm{ml}$ which was repeated at 10 and $100 \mu \mathrm{g} / \mathrm{ml}$ with respective fold decreases of $1.9,3.2$ and 2.4

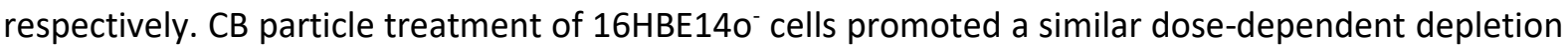
of maximal respiration levels. The fall at significant concentrations of 810,50 and $100 \mu \mathrm{g} / \mathrm{ml}$ by 438 , 517, 457 and $469 \mathrm{pmoles} / \mathrm{min} / \mathrm{mg}$ protein were indicative of a 1.3-fold depletion when maximal respiration was most heavily influenced $(10 \mu \mathrm{g} / \mathrm{ml})$. The effect of CB particles upon reserve respiratory capacity indicated a concentration-dependent depletion initiated at $8 \mu \mathrm{g} / \mathrm{ml}$ and sustained at 10,50 and $100 \mu \mathrm{g} / \mathrm{ml}$. A concentration of $8 \mu \mathrm{g} / \mathrm{ml}$ proved to be the most influential whereby the respiratory reserve fell from 358 to $81 \mathrm{pmoles} / \mathrm{min} / \mathrm{mg}$ protein indicating a $\sim$-fold depletion. Respiratory reserve capacity was however restored from this initial 4-fold reduction with levels rising to 97 and 94 pmoles $/ \mathrm{min} / \mathrm{mg}$ protein at 50 and $100 \mu \mathrm{g} / \mathrm{ml}$ respectively. 

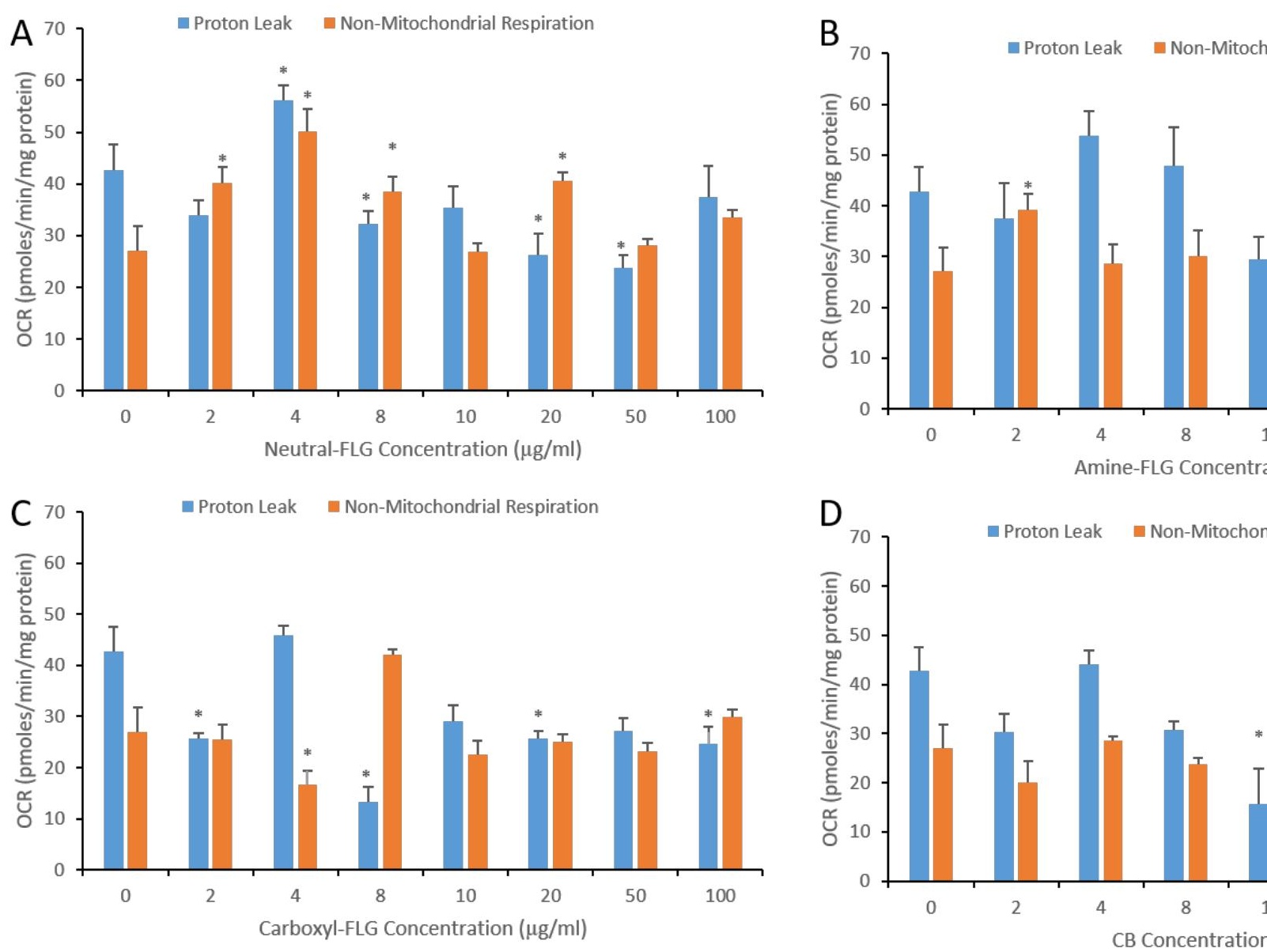

Figure 4.17 16HBE140- Proton Leak \& Non-Mitochondrial Respiration following a 24-hour exposure to Neutral-FLC and CB particles (D). Results were considered statistically significant $(*)$ when $p<0.05$. $N=3$ 


\section{A}

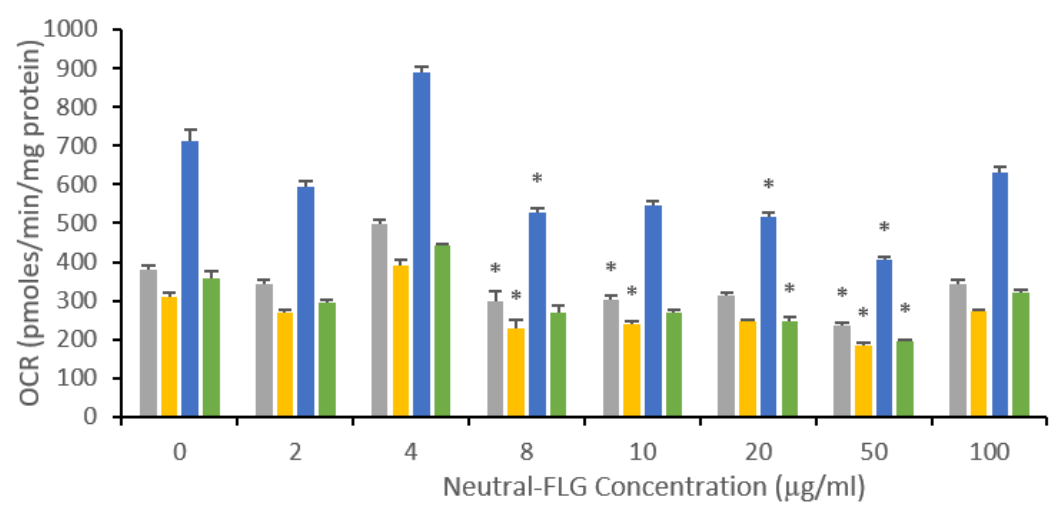

C Basal Respiration

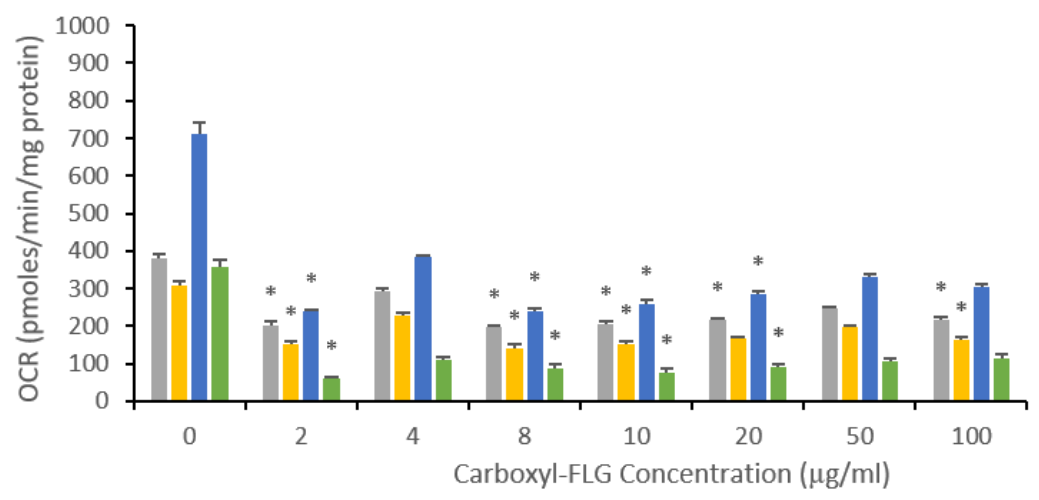

- Basal Respiration

- Maximal Respiration
ATP Production

neserve Respiratory Capacity
B

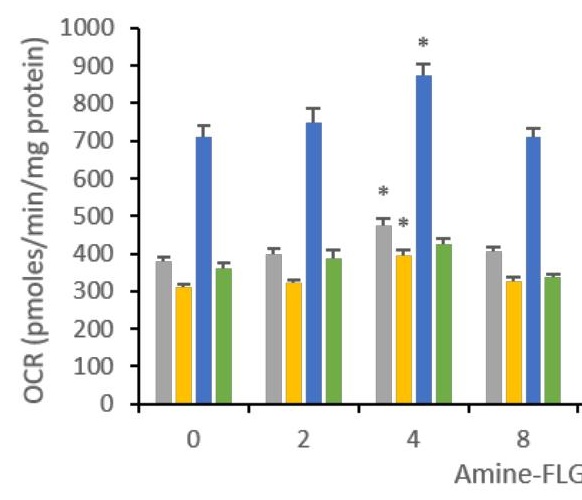

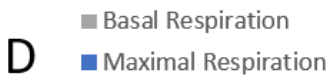

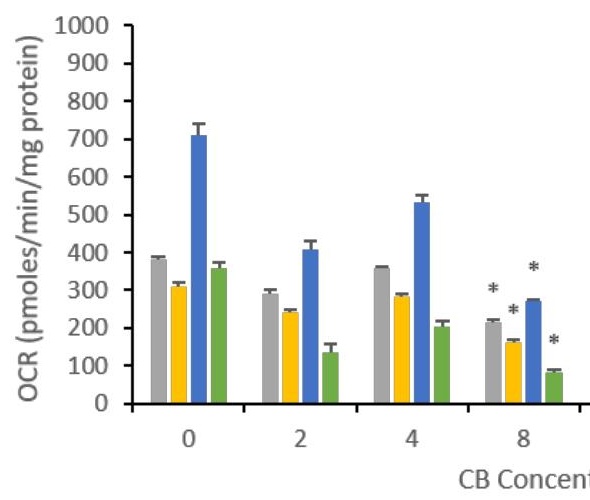

Basal Respiration

n Maximal Respiration

Figure 4.18 16HBE140 respiratory parameters following a 24-hour exposure to Neutral-FLG (A), Amine-FLG (B), Results were considered statistically significant $(*)$ when $p<0.05$. N=3 


\subsection{Discussion}

This chapter aimed to characterise the genotoxic impact of Neutral-FLG, Amine-FLG, Carboxyl-FLG and $\mathrm{CB}$ particles upon a monoculture of $16 \mathrm{HBE} 140^{-}$cells. Additionally, mechanisms contributing to the observed genotoxic response were explored by investigating (pro)-inflammatory cyto/chemokines, oxidative stress and mitochondrial stress responses. The in vivo inhalation of ENMs represents a potential source of lung inflammation, following inhalation ENMs will interact with the epithelial barrier cells and immune cells such as macrophages. If the macrophages strongly interact with and phagocytose these ENMs, an inflammatory response is initiated (Mukherjee et al., 2017). This issue can be exacerbated if the inhaled ENMs is contaminated with sources of endotoxin, typically promoting the release of inflammatory mediators such as TNF $\alpha$ (Mukherjee et al., 2016). Internalisation can also be induced in epithelial cells which has been conclusively demonstrated in vitro to replicate in vivo conditions (Ruenraroengsak and Tetley, 2015). Given that most ENMs including the graphene family of ENMs will be capable of promoting a chronic immune response in vivo (Bianco, 2013). Investigating these effects outside the use of animal models is therefore of paramount importance. ENMs are engineered to fulfil applications in biomedical or electromechanical devices; this is particularly true for graphene-based nanomaterials as well as CB products, however their toxicity in a number of cell lines cultured in vitro has not been consistently demonstrated due to the wide distribution in physico-chemical features and coatings in each of the studies, which has resulted in lacking correlations in the literature (Mittal et al., 2016). Following the comprehensive characterisation of all ENMs evaluated in this thesis (Chapter 3), the first biological endpoints to be assessed were cytotoxicity and genotoxicity, measured in parallel with RPDs and the CBMN assay respectively. The data revealed no significant cytotoxicity at any concentration of ENM up to and including $100 \mu \mathrm{g} / \mathrm{ml}$, which was consistent with numerous studies on graphene and graphene-family of nanomaterials (GFNs) (Bengtson et al., 2016, Chang et al., 2011) where no obvious cytotoxicity (assessed with the Alamar Blue Assay) has been observed in A549 cells with doses as high as $200 \mu \mathrm{g} / \mathrm{ml}$. The same result is true of CB particles where no significant cytotoxicity has been reported at low concentrations of $16 \mu \mathrm{g} / \mathrm{ml}$ in A549, alveolar cells and in THP-1 immune cells (Don Porto Carero et al., 2001). The lack of a cytotoxic response could be attributed to the serum-containing media, which in other studies reportedly decreased the cytotoxic effects of nano and micon-sized materials through the formation of a protein corona (Duan et al., 2015). One example of the serum effect on A549 cells was performed by Hsiao and Huang using zinc oxide nano- $(50-70 \mathrm{~nm})$ and sub-micron particles to treat the alveolar cells in media containing 5 or 10\% FBS (Hsiao and Huang, 2013). The authors noted the highest degree of cytotoxicity in serum-free media in contrast to 5 and 10\% FBS-media. The ZnO particles in serum-free media would therefore have a higher dose-to-cell ratio resulting from larger 
particles agglomerates, greater rate of sedimentation, absence of protein corona which can enhance biocompatibility, and lower cell-growth rate (Hsiao and Huang, 2013). In the recent work by Wills and colleagues (2017) it was demonstrated with high precision and definition, utilising TEM and DLS as prime characterisation techniques, that the number of nanoparticles per agglomerate will affect the mass-dose delivered to in vitro cell cultures. This study was performed using 20 and 40nm carboxylated polystyrene-latex and 16 and $85 \mathrm{~nm}$ silicon dioxide NPs. Wills and colleagues used 3D reconstruction of agglomerates from TEM micrographs to calculate particle number per agglomerate and conclude that tipping points can form during mass-dose deliveries subsequently effecting cellular interactions and increasing toxicity. Moreover, the authors noted this resulted in a direct shift in the nano-safety dose-response and demonstrated that $90 \%$ of a delivered dose of NPs corresponds with $2 \%$ of the largest NP agglomerates (Wills et al., 2017). The lack of a cytotoxic response in the present study could also be attributed to the serum effect and the large protein corona the FLG materials acquire when suspended in full culture media. Culture media however is not the only effective coating which can mitigate toxicity of carbon-based ENMs. In the work by Tabet and colleagues in 2011, MWCNTs were delivered to RAW 264.7 macrophages in vitro and via intratracheal administration in Balb/c mice to investigate the effect of 50/50 acid-base coated-MWCNTs, polystyrene-coatedMWCNTs and uncoated MWCNTs upon toxicity. Raw 264.7 macrophages underwent MWCNT exposures between 6 and 24 hours to $0.1-100 \mu \mathrm{g} / \mathrm{ml}\left(0.02-20 \mu \mathrm{g} / \mathrm{cm}^{2}\right)$ in serum-free media. Macrophages showed considerable uptake of ENMs bound in vesicles with a dose-dependent decrease in mitochondrial metabolism. The authors noted that functionalization with an acidic polymer, whilst reducing specific surface area five-fold, enhanced dispersion and aided macrophage uptake both in vitro and in vivo (Tabet et al., 2011). The polystyrene polymer acted by supressing cytotoxicity and oxidative stress and inflammation in contrast to the acidic-polymer functionalization. MWCNTs have also been shown to be cytotoxic in A549 cells by the MTT assay by Tabet and colleagues in 2009. In this study, MTT values underwent a significant depletion from 10-100 $\mu \mathrm{g} / \mathrm{ml}$ of MWCNTs reaching $60 \%$ viability of the control levels at $100 \mu \mathrm{g} / \mathrm{ml}$ following 48 hours of exposure. This was achieved with no uptake of the ENMs which was confirmed by TEM, indicating these MWCNTs strongly interact with the alveolar cell surface to impart toxicity. Interestingly, when investigating toxicity using the Neutral-Red Assay, no significant A549 cytotoxicity was noted at any time-point from 6-72 hours following MWCNT incubation (Tabet et al., 2011). Issues surrounding the industrial production of CNTs and their disputed toxicity has since moved onto graphene ENMs (Bussy et al., 2013).

In the present study it has been demonstrated that both Neutral-FLG and CB particles can promote a strong genotoxic effect in $16 \mathrm{HBE} 14 \mathrm{o}^{-}$cells and that the addition of amine groups to the surface of FLG 
can diminish this effect. Additionally, the presence of anionic carboxyl groups lowered the micronuclei frequency to non-significant levels across the entire concentration range. The genotoxicity of carbonaceous ENMs however is greatly varied in the literature which can be attributed to numerous factors including the cell line used, the material physico-chemical features, the assay used to investigate the response and the exposure time. While CB is quite well established as a genotoxic carcinogen in lung cells the GFNs show slightly more variability in promoting a genotoxic response (Don Porto Carero et al., 2001, Hussain et al., 2010, Jacobsen et al., 2008). The FLG ENMs investigated in this study demonstrated the potentially detrimental effects of FLG engineered with and without surface chemistry. Neutral-FLG promoted a strong genotoxic effect with a LOGEL of $10 \mu \mathrm{g} / \mathrm{ml}$ while Amine-FLG elicited a LOGEL of 50 $\mu \mathrm{g} / \mathrm{ml}$. Finally, 16HBE140 cells treated with CB particles promoted a LOGEL response at $20 \mu \mathrm{g} / \mathrm{ml}$.

Neutral-FLG was the most genotoxic of the FLG ENMs and possesses distinguishing physico-chemical characteristics which may be related to the genotoxic response observed. This material had not undergone surface functionalization and appeared to pose the greater genotoxic risk, this is supported by studies reporting oxidative stress (imparted by additional oxygen functionalisation) as a large contributory factor (Wang et al., 2013, Duch et al., 2011). Neutral-FLG has the largest agglomerate size as measured via plunge freeze SEM (the most reliable form of characterisation in this study); possesses the greatest number of layers according to AFM; appears to be the least stable (assumed from the zeta potential); and is the least oxidised FLG structures evaluated (highlighted in Chapter 3 by EDX). The effect of oxidising hydrophilic graphene oxide nanosheets ( $>300 \mathrm{~nm}$ ) to increase culture media stability was performed by Hong and colleagues in 2011, where they concluded that through stabilization greater cellular uptake would be achieved (Hong et al., 2012). These physico-chemical features could facilitate the sedimentation of Neutral-FLG in cell exposures, being the least stable and most dense the degree of sedimentation is greater thus increasing the exposure time directly at the cell surface. Materials such as Amine- and Carboxyl-FLG with their more hydrophilic chemistry theoretically sediment less rapidly and would not be exposed to the cell surface for the same amount of time as Neutral-FLG. Correlating the genotoxicity of CB particles to their physico-chemical features is perhaps more difficult. However, the clear difference between the FLG and CB ENMs would be their serum corona of tightly bound proteins (hard corona). CB simply acquires far less proteins (just BSA) at its surface, which has been theorised to offer biological protection during in vitro exposures of cells to ENMs (Wu et al., 2018). The greater number of corona proteins adorning the FLG samples may be providing biological recognition to the cells contributing to a lowered genotoxic response. The FLG samples appear therefore to interact with the $16 \mathrm{HBE} 140^{-}$cells in a unique way based on their physico- 
chemical features. Given that primary indirect genotoxicity and oxidative stress are a key feature of each material, the physico-chemical features of Neutral-FLG may be facilitating a more direct interaction with the cells.

Carbonaceous ENMs have been proven to promote both clastogenic and aneugenic DNA damage (Muller et al., 2008). Muller and colleagues for instance, demonstrated the potential of MWCNTs to induce both clastogenic and aneugenic events during both an in vivo $\mathrm{MN}$ assay in rats and utilising the in vitro CBMN assay. In the study, they administered doses of 0.5 and $2 \mathrm{mg}$ in vivo and a concentration range of 10,25 and $50 \mu \mathrm{g} / \mathrm{ml}$ in the in vitro element of the investigation. The authors targeted type II pneumocytes and rat lung epithelial cells where they reported at $50 \mu \mathrm{g} / \mathrm{ml}$ a 2 -fold increase in micronuclei, involving both clastogenic and aneugenic mechanisms. In the present study, clastogenicity was the stronger contributor to the observed genotoxicity, however only Amine-FLG promoted a significant level of clastogenicity which was three-fold over control levels. The only other study by focusing on the clastogenic/aneugenic effects of carbonaceous ENMs was an in vivo study on Swiss-Webster mice and a comparative study of functionalised ( $\mathrm{COOH}$ groups) vs non-functionalised MWCNTs (Patlolla et al., 2010). The authors investigated the mitotic index, chromosomal aberrations and micronuclei formation in leukocytes. Intraperitoneal administered doses of $0.25,0.5$ and $0.75 \mathrm{mg} / \mathrm{kg}$ were delivered to the mice with a positive control of $\mathrm{CB}$. Genotoxicity was evaluated by the comet assay where the authors declared that functionalised MWCNTs were significantly clastogenic, more so than non-functionalised MWCNTs. These results conflict with the genotoxicity observed in the present study which generally indicated that additional surface coatings act to lower the genotoxic response with Carboxyl-FLG promoting no significant responses at any tested concentration. In accordance with the data in this study the authors also monitored the ROS production and found that with the DCFH-DA assay there was significant fluorescence at the highest doses which is mimicked in the present study whereby all ENMs promoted significant oxidative stress through ROS production. Numerous publications have highlighted the potential of graphene ENMs to promote oxidative stress, which can be achieved through antioxidant depletion, increase in ROS, lipid peroxidation or mitochondrial stress. One such reason for the highly significant effect of ENMs on the mitochondria of $16 \mathrm{HBE} 14 \mathrm{o}^{-}$cells is the ability of i) these ENMs to gain access to the cells and ii) once within the cytosol can potentially interfere with electron transfer through the ETC (Figure 4.19) thus lowering the energy output of the cell. The mechanisms of graphene toxicity within mammalian cells have been suggested to be driven by an overproduction of ROS linked to mitochondrial depolarisation, which is exacerbated with pristine isotypes of graphene ENMs as opposed to functionalised variants (Jarosz et al., 2016). The region of the ETC where electrons can be most susceptible to interference is 
within complex III where iron-sulphur clusters work with the recycling system of the q-pool (Figure 4.20) to move electrons towards complex IV where ATP can be synthesised (Zhang et al., 2016).

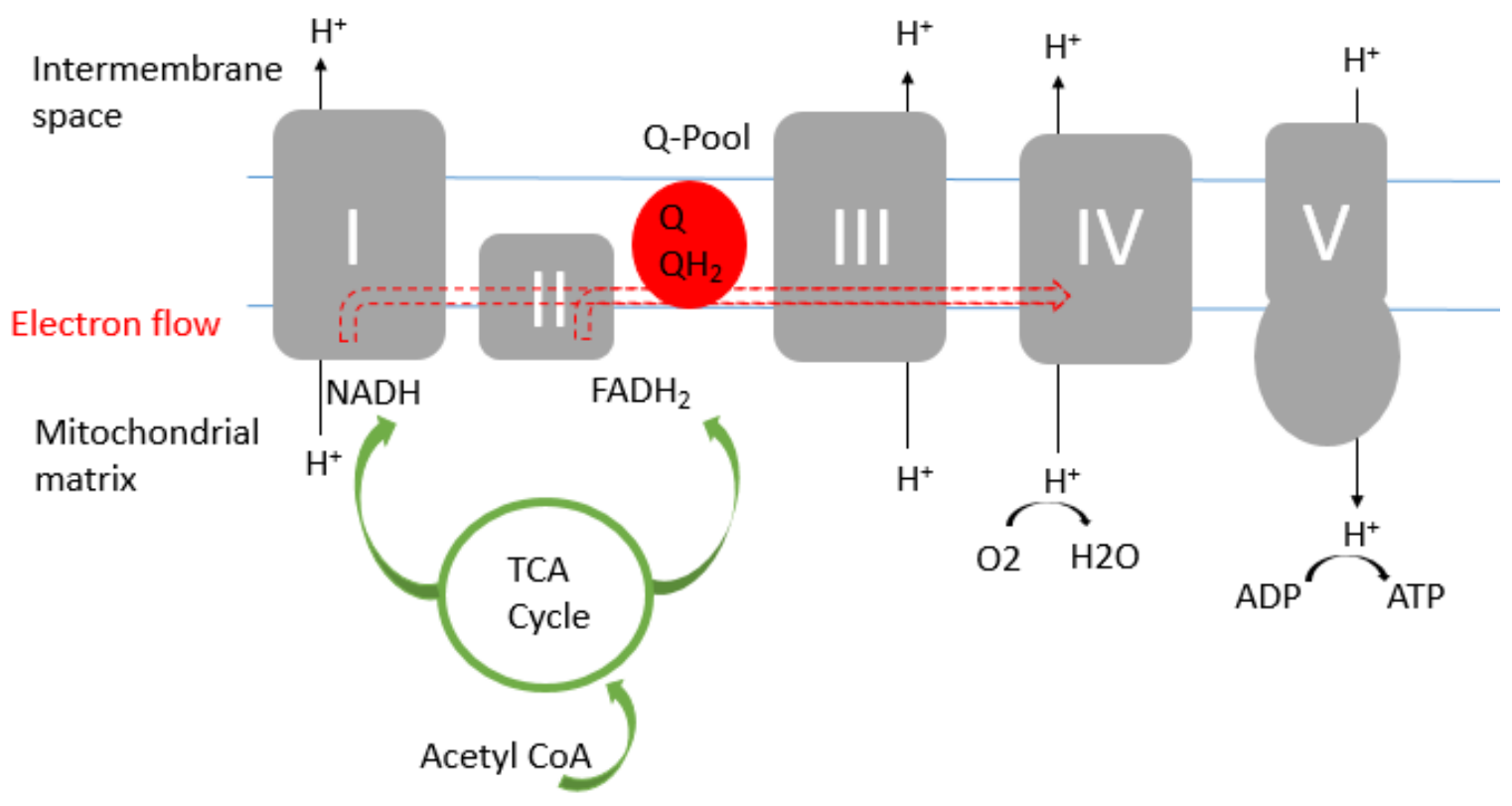

Figure 4.19 Hypothetical mechanism of ENM interference with the ETC. The electron source of the ETC are two carriers, NADH and $\mathrm{FADH}_{2}$ which funnel electrons into complexes I and II respectively. From there electrons move through the Q-pool, where the redox states of the ENMs may interfere through a conduction of electrons out of this system. Except for cellular uptake via non-passive mechanisms, ENM interference at this juncture in the ETC could be one possible cause of ATP depletion. 


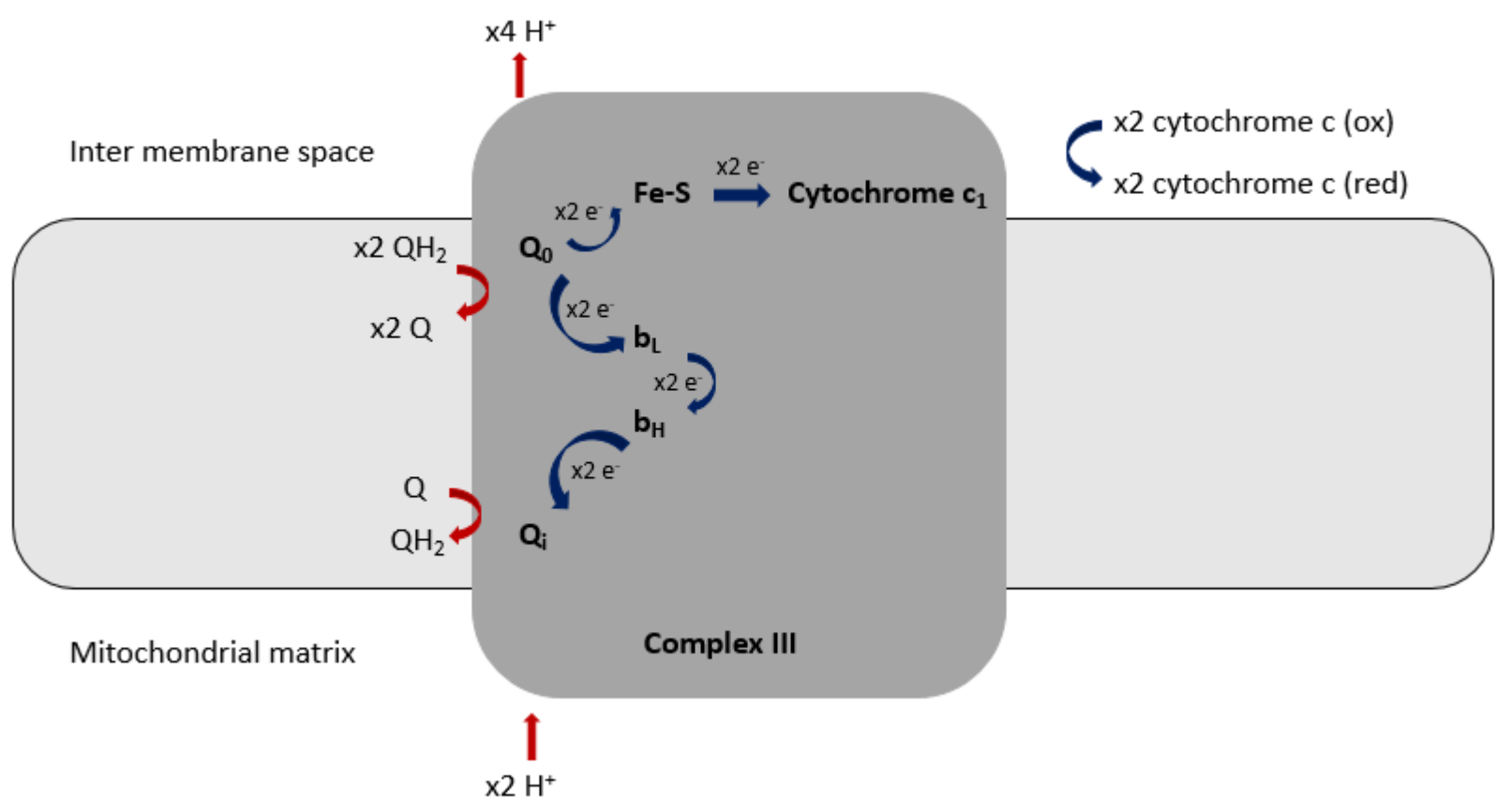

Figure 4.20 A representation of the electron recycling in complex III between iron-sulphur clusters which graphene ENMs have been reported to interfere with. The disruption of electron transfer ultimately leads to a mitochondrial membrane potential drop and reduced ATP synthesis.

Theoretical calculations on graphene sheet diameters demonstrated that a diameter greater than $6.7 \mathrm{~nm}$ of graphene would conduct electrons better than electron acceptor sites (Zhou et al., 2014). The 4Fe4S iron-sulphur clusters (Figure 4.21) for example which require reduction may be impaired by graphene conduction of electrons. This would explain the impairment of ATP production specifically where Carboxyl-FLG exposure is concerned, given it is the more oxidised structure compared to Neutral-FLG. The underlying mechanisms contributing to ATP production impairment and mitochondrial membrane potential decrease have been explored by Zhang and colleagues (2016) who hypothesise that graphene ENMs can act as electron donors, ramping up the supply of electrons through complexes I and II of the ETC thus accelerating the supply of ROS to the cell (Zhang et al., 2016). This does not appear to be the case in the present study where FLG and CB treatment of 16HBE140 cells depleted oxygen consumption indicating a different mechanism may be leading to oxidative stress. Furthermore, a study by Zhang and colleagues in 2012 utilised the nematode as an ideal model to test the hypothesis that graphene oxide with low oxidation states (highly reduced) proved more damaging via oxidative stress than highly oxidised graphene. This appeared to be true, with $\mathrm{COOH}$ groups demonstrating a critical effect on the decomposition of $\mathrm{H}_{2} \mathrm{O}_{2}$ into hydroxyl radicals contributing largely to indirect oxidative stress, determined by electron spin resonance spectrometry. 
These findings correlate with the respiratory parameter data in the present study whereby carboxylFLG proved more effective in depleting mitochondrial functions than Neutral- or Amine-FLG ENMs. From the EDX spectra (illustrated in Chapter 3), the oxidation state of each material from high to low appears as; Amine-FLG $>$ Carboxyl-FLG $>$ CB $>$ Neutral-FLG. This suggests Neutral-FLG being the most reduced ENM under investigation. Subsequently, as the most reduced material it is the least ready to accept electrons out of the ETC, conversely as Carboxyl-FLG shows the greatest oxidative potential, it can be postulated that Amine-FLG possesses additional hydrogen groups which would lower its oxidation state and produce the observed mitochondrial profiles seen in the present study. Further characterisation would be necessary to confirm this theory with XPS the most prominent technique appearing in the literature (Susi et al., 2015). This has been supported in the present study by EPR data (Chapter 3) which highlighted that the more reduced Neutral-FLG demonstrated a higher superoxide radical-producing potential while the oxidised Carboxyl-FLG indicated a lower radical-producing potential experimentally. The ability of these lung epithelial cells to deal with an enhanced state of oxidising radicals therefore plays a crucial role, many pathways of which rely on the antioxidant enzymes of glutathione redox chemistry. The lung employs crucial antioxidant mechanisms for neutralising potentially deleterious ROS, both enzymatic and non-enzymatic (Figure 4.21) (Thorley and Tetley, 2007). In the present study, the focus has been on the non-enzymatic intracellular glutathione levels to provide an indicator of oxidative stress, however future studies would benefit from investigating the enzymatic activity of superoxide dismutase, catalase and glutathione peroxidase.

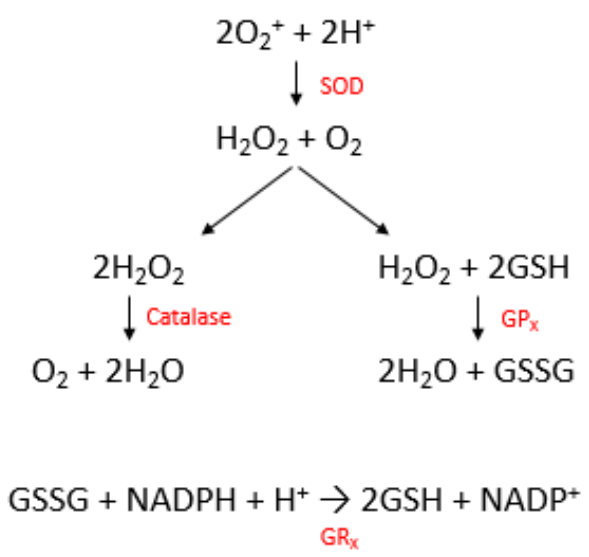

Figure 4.21 Potential ROS clearance pathways linking GSH and mitochondrial stress. This pathway of highly reactive radical clearance via enzymatic degradation and dismutation could potentially be the link in the present study between the observed depletion of glutathione and mitochondrial stress. Here the hydrogen peroxide is degraded by catalase and glutathione peroxidase (GPx). Finally, GSH is regenerated from its oxidised state (GSSG) by NADPH, catalysed by glutathione reductase. Image adapted from (Thorley and Tetley, 2007). 
Graphene ENMs have been linked to the depletion of intracellular glutathione, which correlated with a significant increase in intracellular ROS and mitochondrial membrane destabilisation (Mittal et al., 2016). This effect was however stronger in alveolar A549 cells as opposed to BEAS-2B cells indicating i) the levels of intracellular GSH differs between lung cell types, and/or ii) alveolar cells are more readily affected by the oxidative capabilities of graphene ENMs. The present study investigated the concentration-dependent effect of carbonaceous ENMs on intracellular glutathione to discover a depletion of the antioxidant as early as 6 hours from which there is very little recovery 18 hours later. The significant depletion of GSH at 50 and $100 \mu \mathrm{g} / \mathrm{ml}$ demonstrated the potential of the ENMs to interfere with the reactions displayed in Figure 4.21. However, since there appears to be no recovery of GSH at 24 hours from the initial loss at 6 hours of exposure a likely possibility would be the final reaction whereby GSH is replenished by NADPH and glutathione reductase. The prevailing theory in relation to the present study (GSH depletion, ROS generation, EPR production and ATP depletion (at the higher concentrations)) and the enzymatic reactions discussed above seems to suggest that $\mathrm{H}_{2} \mathrm{O}_{2}$ is abundant in the cells (a result of superoxide over-production by the ENMs themselves driving the dismutation reaction) indicative of glutathione peroxidase functioning normally. This also means the dismutation reaction which produces $\mathrm{H}_{2} \mathrm{O}_{2}$ is also occurring normally; oxidative stress may therefore be oversaturating catalase with $\mathrm{H}_{2} \mathrm{O}_{2}$ in the cell. In accordance to the depletion of GSH this cell saturation with $\mathrm{H}_{2} \mathrm{O}_{2}$ may be the prevailing factor in $\mathrm{GSH}$ consumption and peroxidation via glutathione peroxidase. Finally, due to the inefficiency of the $16 \mathrm{HBE} 14 \mathrm{o}^{-}$cells to restore the GSH levels at 24 hours (after the initial 6-hour depletion) the activity of glutathione reductase may be impacted whereby the restoration of GSH from GSSG is reduced. This would be exacerbated in cells exposed to Carboxyl-FLG and CB particles (the ENMs with the theoretically greater reducing potential (not confirmed in this study)) as they would deplete NADPH electron carriers the most.

With regards to the promotion of (pro)-inflammatory mediators in $16 \mathrm{HBE} 14 \mathrm{o}^{-}$cells, there does appear to be a correlation with significant IL-8 release and glutathione depletion. Determining which event precedes the other could be investigated further with a simply designed experiment whereby $16 \mathrm{HBE} 14 \mathrm{O}^{-}$cells are seeded at the same density but follow a pre-incubation with glutathione prior to ENM exposures. This would allow the mechanisms to be elucidated by identifying if additional glutathione can pre-emptively block or diminish the (pro)-inflammatory response and lower oxidative stress. In the study by Schinwald and colleagues in 2012, the administered dose of platelet-like graphene initiated a signalling pathway which the authors categorised as; (pro)-inflammatory response >oxidative stress >cytotoxicity and genotoxicity. This concept has been explored, in an unrelated study, where the additional supplementation of glutathione decreased the serum levels of 
TNF- $\alpha$, IL-6, IL-8 and TGF- $\beta$ in patients with chronic hepatitis B after 1200g of glutathione had been intravenously administered indicating a favourable effect of increased serum levels of glutathione (Qian et al., 2017). This minor effect of increased glutathione can be briefly witnessed in the present study when comparing the oxidative potential of the ENMs under investigation. $16 \mathrm{HBE} 14 \mathrm{o}^{-}$cells treated with ENMs after 6 hours (Fig.4.12) at $4 \mu \mathrm{g} / \mathrm{ml}$ indicates a slight increase in intracellular GSH/mg protein which later correlates to a favourable effect in Figure 4.17 representing the OCR and ECAR levels in the cells. At the same concentration of $4 \mu \mathrm{g} / \mathrm{ml}$ an increase in the metabolic parameters is shown while every other concentration tested promoted a decline in respiratory parameters in the cells. This small elevation in metabolic activity could be indicative of cellular defence mechanism activation in response to low concentrations of ENM whereas the higher concentration range could prove detrimental to the metabolic function.

\subsection{Chapter 4 Conclusions}

The findings of this chapter have demonstrated the significance of FLG ENMs surface chemistry and the importance of morphology with regards to CB particles, in governing biological interactions and responses. Neutral-FLG (non-functionalised) possessed the greatest genotoxic hazard on air-way cells by promoting significant DNA damage quantified with the in vitro CBMN assay. Furthermore, the additional surface carboxyl groups appear to offer bio-compatibility, given that Carboxyl-FLG did not induce a genotoxic effect. However, all tested ENMs were able to promote a significant (pro)inflammatory response increasing IL-8 generation, produce ROS, deplete antioxidant (GSH) levels and demonstrate a potent ability to interfere with mitochondrial function in $16 \mathrm{HBE}^{-140^{-}}$cells. The oxidative stress however was not always a contributing factor to the genotoxicity of the ENMs. Carboxyl-FLG, whilst able to deplete mitochondrial function and promote ROS production, induced no significant DNA damage. CB particle exposure, seemingly acting via separate mechanisms to the FLG samples (substantially less uptake demonstrated whilst promoting the greatest genotoxic response) demonstrated similar effects on mitochondrial stress but simultaneously promoted a strong genotoxic effect. Pan-centromeric labelling of micronuclei induced in 16HBE140- following exposures to Neutral, Amine-FLG and CB particles demonstrated both clastogenic and aneugenic mechanisms, indicative of oxidative stress. This was supported by an increase in ROS during the exposure period along with a depletion of intracellular GSH and subsequent mitochondrial stress. Therefore, in the 16HBE140monoculture system, primary indirect genotoxicity was promoted by $C B$ particles, $>$ Neutral- and >Amine-FLG ENMs. 

Epithelial Models

\subsection{Introduction}

The field of in vitro toxicology produces a vast number of publications each year with the focus of improving our current understanding of cellular interactions with accidental or ENMs. The problem however, is that the bulk of these publications centre on the use of in vitro monocultures, cultured in 2D format, which does not accurately reflect in vivo tissue structure. 2D monocultures provide useful models for large dose-response assays, understanding mechanisms and high-throughput analysis. However, there is recognition that the sensitivity and specificity of such simple in vitro test systems suffer limitations when trying to understand the impact of exposure to an exogenous agent in humans (Clift et al., 2011). Consequently, the current push in the hazard assessment field has been to move towards more complex culturing techniques (3D models) that can more closely replicate in vivo tissue, to produce data with enhanced physiological relevance.

Cytotoxicity is one major endpoint for hazard assessment and numerous mechanistic routes (oxidative stress, antioxidant depletion, and mitochondrial stress) can be further explored to identify which pathways, organelles, or characteristics are leading to the toxic response. The use of 2D monoculture systems allows multiple endpoints including cytotoxicity, (genotoxicity, oxidative stress and (pro)inflammatory responses) to be characterised and quantified relatively quickly providing empirical datasets which may then inform future in vivo testing (Edmondson et al., 2014). Utilising a single cell line however, eliminates a key concept of genetic toxicology, the importance of secondary mechanisms of genotoxicity (Schins, 2002). As outlined in Chapter 1 Section 1.5.1 of this thesis and in the recent publication by Evans and colleagues (2019), 2D monocultures can be limited in their predictive capability of in vivo genotoxic outcomes relating to chronic inflammation, owing to their capacity to only detect primary genotoxic mechanisms (Evans et al., 2019). Several cellular interactions can be missed when only considering a single cell line as opposed to the responses observed in in vivo tissues including; the expression of cellular receptors and the differences which arise between gene and protein expression (Baharvand et al., 2006). The net result of these key cellular processes would almost certainly equate to a differing toxic response in a complex tissue environment than when compared to a single cell-line. Where in vitro ENM toxicity testing is concerned, the impact and reliability of the study will depend firstly on the cell-type selection and secondly the suitability of the 
screening tools, more specifically the endpoints under investigation and their association with the chosen cell line. An ENM designed to penetrate the skin as a novel therapeutic would therefore be partnered with a suitable skin model with stable genetic background. ENMs such as FLG and CB particles manufactured as fine powders and available in nanocomposites and printer toner respectively and are best suited to a lung model utilising a suitable nasal, bronchial or alveolar cell line (Jones and Grainger, 2009).

The respiratory tract (nasal, tracheal, and pharyngeal tissues) in humans, consists of highly specific cell types (illustrated in Figure 5.1) which offer immunity to the inhalation of foreign bodies such as pathogens, dust and ENMs (Parker and Prince, 2011). Goblet and clara cells in vivo can secrete (celltype dependent) mucus to coat the lining of the bronchi and bronchioles enabling easier clearance of inhaled particles. This mucus lining, generated by pseudostratified epithelia and swept by cilia from distal to proximal airways, is essentially an extracellular fluid up to $50 \mu \mathrm{m}$ thick composed of water and highly glycosylated protein. This mucus acts as a trap for inhaled toxins with the intent to aid their translocation back up the respiratory tract to be swallowed or spat out (Fahy and Dickey, 2010). Further down this branching network is the $140 \mathrm{~m}^{2}$ alveolar region (Hermanns et al., 2004), (Gehr et al., 1978). These beds contain surfactant-secreting alveolar type-2 (AT-II) cells which provide the first form of protection from inhaled particulate matter. The alveolar epithelium consists of two specialised cells namely alveolar type-1 (ATI) and ATII. The terminally differentiated squamous ATI cells cover $93 \%$ of the alveolar region by surface area; however, they do not secrete pulmonary surfactant. The cuboidal ATII cells while only covering $7 \%$ of the alveolar region by surface area actually makes up $67 \%$ of the total cell number within the alveoli (Crapo et al., 1982). ATII also offer the lung innate immunity through secretion of pulmonary surfactant proteins $A, B, C$ and $D$ which can bind numerous pathogens, particulates, pollen and other allergens (Crouch, 1998). 


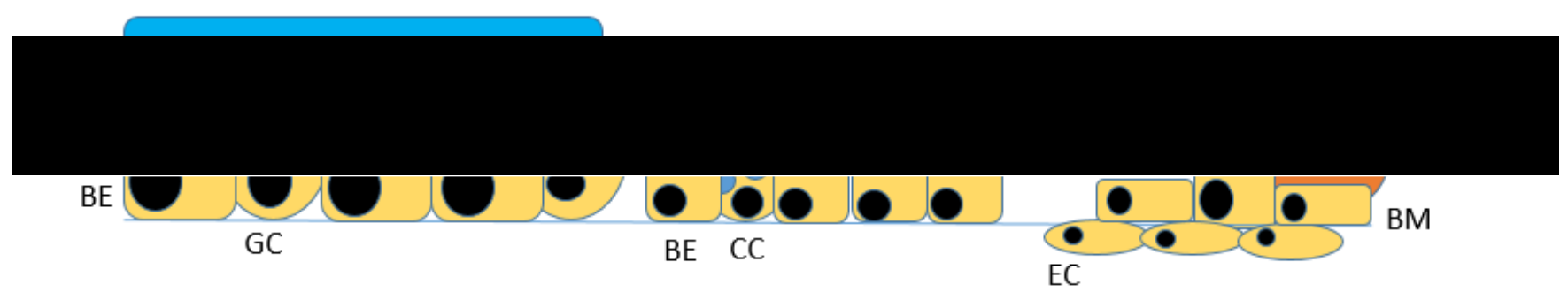

Figure 5.1 Innate immunity against particle/fibre deposition in the respiratory tract. The cells of the human airway are highly specific in each region of respiratory system, the cells of the bronchus for example is where most of foreign materials will deposit. The two cell types occupying the lining of the bronchus are epithelial cells and mucus-secreting goblet cells, which through the adaptation of cilia, the epithelial cells will disperse across the lining of the bronchus as a means of immunity against inhaled foreign bodies. The bronchioles, consisting of epithelial cells and mucus-secreting Clara cells again utilise cilia to disperse mucus as a protective barrier. Lastly, the cells of the alveolar region form a tight network of immune cells, dendritic cells and pulmonary surfactant-secreting type II alveolar cells providing the final defensive barrier against inhaled materials. Key; Bronchial epithelial cell (BE), Goblet cell (GC), Clara cell (CC), Basement membrane (BM), Macrophage (M), Endothelial cell (EC), Alveolar epithelial cell type I (AT-I), Alveolar epithelial cell type II (AT-II). Image adapted from (Frohlich and Salar-Behzadi, 2014).

The respiratory epithelium maintain a vast arsenal of innate immune receptors, toll-like receptors (TLR9) and nucleotide oligomerisation domain-like receptors which are crucial to immune function and the secretion of (pro)-inflammatory mediators (Parker and Prince, 2011). Aside from specialised mucous-secreting (Goblet, Clara) cells, the last line of immunity are barrier-forming epithelial cells, some of which possess the ability to secrete pulmonary surfactants to aid in the overall immunity to inhaled particulate matter. The majority of ENM clearance in the lung is governed by mucociliary escalation and the epithelial barrier layer (Patton and Byron, 2007, Geiser and Kreyling, 2010). Therefore, the study of the pulmonary surfactants expressed by alveolar epithelial cells is crucial to understanding ENM uptake and translocation across this barrier. The alveolar-lining fluid, a thin hypophase and surface-active lipid-protein complex is known colloquially as pulmonary surfactant which covers the alveolar epithelial layer (Ruge et al., 2012). This pulmonary surfactant is spread via epithelial cilia from the distal to the proximal lungs and is positioned at the air-interface which means it lies on the outer side of the air-blood barrier (Bastacky et al., 1995, Goerke, 1998). Pulmonary surfactant is a composite of approximately $90 \%$ lipid and $10 \%$ protein and they can exist as four 
isotypes SP-A, -B, - C and -D where each isotype performs a specific function (Goerke, 1998). SP-B and SP-C group together as small peptides which interact with surfactant lipids allowing them to contribute to biophysical surfactant functionality (Serrano and Perez-Gil, 2006). SP-A (630kD) and SP-D (520kD) sometimes referred to as collectins (collagen-containing, calcium-dependent lectins) group, act as opsonins and scavenger molecules (Kishore et al., 2006). The existence of these collectins at the airliquid interface of the alveoli has been postulated to allow the binding of inhaled particulate matter (Seaton et al., 2010). Furthermore, the binding of SP-A or -D to inhaled particulate matter also trigger's the activation of alveolar macrophages causing them to internalise inhaled particles as another defence mechanism to attempt clearance of the foreign material (Wright, 2005). SP-A and SP-D are both calcium-dependent glycoproteins; and have been shown to offer some form of innate immunity through phospholipid binding (Mason et al., 1998). While SP-B and SP-C, components of the cell membrane appear to play roles in cellular signalling relating to the recycling of surfactant protein packaging and secretion from lamellar bodies (Whitsett et al., 1995). Although currently unproven, these lines of defence against intrusion from ENMs may play a crucial role upon internalisation through endocytic mechanisms.

Standard 2D culturing techniques are largely based upon high throughput-handling of cells grown as a flat monolayer (in the case of adherent cells) or as suspension cells. In this manner, data can be gathered relatively quickly while also providing an opportunity to delve into the underpinning mechanisms giving rise to a response whether that be cytotoxicity, chromosomal damage or oxidative stress, as three common hazard endpoints. Monocultures pertaining to the lung, often an epithelial cell line, provide reproducible cytotoxic and genotoxic data which is required for screening potentially toxic chemicals and novel ENMs and lastly, monocultured cells exposed to a concentration range can often be used to model in vivo doses (Clift et al., 2014). The limitations however begin with, but are not limited to, the tendency of genotoxicity to be associated with chronic inflammation (Savolainen et al., 2010). This tendency to link inflammatory processes with genotoxicity warrants the use of animal models or at the very least a dual-cell co-culture as these can provide more robust data pertaining to the mechanisms and mediators present between two or more cell types. Finally, basic in vitro conditions (not incorporating air flow or fluid movement, etc) provide little information on the direct human exposure scenario, an endpoint which can be met with more rigour when utilising in vivo approaches (Clift et al., 2011). 


\subsubsection{Minimising Animal Model Use}

Following a positive in vitro test, animal models have been used as the gold standard for assessing the mutagenic or genotoxic potential of novel therapeutics, cancer treatments and more recently ENMs (Speit, 2009). However, in vivo testing has moral issues associated with it and it is also not possible for all industries to use. For example, the stance taken by the Seventh Amendment to the European Cosmetics Directive bans the use of marketed cosmetics which have undergone animal trials within Europe to back the development of more rigorous in vitro techniques. In vivo animal models persist however within all other industrial sectors due to the number of false and or misleading positive results obtained with in vitro models, an issue which can be overcome with use of more relevant cell lines (Pfuhler et al., 2014). The Cosmetics Europe Genotoxicity Task Force therefore funded three projects aimed at addressing these false positive results obtained from optimised mammalian cell assays and concluded that the predicative capacity of the in vitro micronucleus assay was enhanced dramatically just by incorporating more relevant cell lines (Pfuhler et al., 2014). Undertaking animal testing introduces several socio-cultural, ethical and economically-viable issues such as animal cruelty/suffering, reproducibility and budget constraints. Firstly, the ethical issue at hand - is the animal's limited lifespan and suffering worthy of the knowledge which may be gained by the end of the study? Secondly, can the experiments be reproduced in other laboratories attempting to duplicate the data or partake in a transferability study? If the answer is no to the latter, how can the ethics be justified from the initial testing? Test animals can also vary greatly within the same species and so acquiring a useable and reliable data set may be challenging. Lastly, can these experiments be performed within a certain budget, and often within a tight timeframe? In vivo animal testing introduces a raft of financial considerations from the culturing and maintenance of the test animal, feeding requirements, maintaining optimal living conditions as well as the cost of the scientific endeavour. One last concern could be how does the test subject; rat, monkey, mouse, rabbit, etc relate to human exposures i.e. how relevant is the animal model to the human condition? If the animal model bares no common physiology with humans, the model should be substituted with specific humanderived cell lines cultured to replicate target tissues.

Since it has been established that in vitro monocultures have their limitations and in vivo animal models can prove problematic for the reasons discussed, development of relevant advanced in vitro models has gained substantial momentum over the last 10 years. These models are typically 3D microtissues, co-cultured cell lines from at least two differing but architecturally and anatomically relevant primary cells or cell lines, spheroids and the most recent advances in lab-on-a-chip 
technology. These independent advanced cell models each have their 'pros' and 'cons' which have been highlighted in great depth by Evans and colleagues (Evans et al., 2017). Briefly, these models attempt to replicate specific tissues, or organs of the body. Within nanotoxicology this can often mean target organs most susceptible to exposure i.e. the lungs via inhalation, the skin via dermal contact or the liver which models ENM translocation from exposure sites. Microtissues, for example spheroids, are considered highly representative of in vivo tissue as they can imitate anatomical architecture. However can sometimes lack multicellular components as most spheroids are comprised of a single cell line only, and spheroids can also be time-consuming and costly to generate (Shah et al., 2018). Crucially, the development and use of more advanced co-culture models are a necessity to the close the gap between in vitro and in vivo science, this would contribute greatly towards minimising animal testing.

\subsubsection{Lung Co-Cultures}

Since the lung represents the primary source of human exposure, lung co-culture models are of significant interest. Perhaps one of the most well-known co-culture models is that developed by Rothen-Rutishauser and colleagues where monocyte-derived macrophages (MDM) and monocytederived dendritic cells (MDDC) were differentiated from human blood and cultured with alveolar epithelial (A549) cells (Rothen-Rutishauser et al., 2005). The triple-cell culture, which was grown on PET membranes was developed by growing A549 cells on the apical side of a transmembrane insert for 7 days, after which dendritic cells were added to the basal underside of the PET membrane before the macrophages were applied to the apical layer to replicate the air-liquid interface during exposures. Similar co-culture models have been employed to study the deposition of particulate matter in the respiratory tract. In the case of Diabate and colleagues (2008), the authors investigated the effects of ultrafine incinerator fly ash administered using the CULTEX system at $100 \mathrm{ml} / \mathrm{min}$ for $1-6$ hours on a co-culture of BEAS-2B and differentiated macrophages seeded on transwell inserts. Under investigation was the effect on IL-8, glutathione, and haem oxygenase-1 expression. IL-8 release was considered a dose-dependent response with the authors noting the deposited mass of particles positively correlated with time of exposure, whilst no effect was witnessed on glutathione or viability with the test aerosol (Diabate et al., 2008). Performing exposures in this manner correctly models particulate deposition in the lung as opposed to submerged liquid cultures negating the issue of sedimentation due to agglomeration (Diabate et al., 2008). 
Further development of lung co-cultures is also attempting to mimic blood flow to strengthen the coculture concept. In the work by Luyts and colleagues a co-culture was established using 16 HBE140 cells, differentiated THP-1 cells and human lung microvascular endothelial cells (HLMVEC) whereby the epithelial and endothelial cells were grown on opposite sides of a transwell insert membrane prior to differentiated THP-1 macrophage addition (Luyts et al., 2015). The authors used confocal and electron microscopy to confirm the integrity of the co-culture, noting tight junction formation between 16HBE140 cells (TEER values); however the authors noted a 73\% TEER reduction following the addition of the PMA-differentiated monocytes (Luyts et al., 2015). The application of lung cocultures in nano(geno)toxicology is currently lacking in the literature with limited resources available which have conclusively demonstrated their advantageous potential to screen secondary mechanisms of genotoxicity. Diesel Exhaust Particles (DEP) have been associated with inducing genotoxicity in lung cell DNA primarily via oxidative stress (Moller et al., 2008). Upon investigation the genetic lesion resulting from sustained oxidative stress was the pre-mutagenic 8-oxo 7, 8-dihydro-2-deoxyguanosine (8-oxodG) (Moller et al., 2003). This is hypothesised to be a direct result of ROS elevation, quinone compounds, or through activated (pro)-inflammatory mediators such as neutrophils (Donaldson and Tran, 2002). Li and colleagues added further support on the detrimental effect of DEP with the identification of DEP within the inner mitochondrial membrane of cultured macrophages (Li et al., 2003). Macrophages within co-cultures appear to provide rudimentary protection over the epithelial monolayer, this was confirmed in the study by Jantzen and colleagues whereby the genotoxic effects induced by DEP witnessed in monocultured type II pneumocytes (A549 cells) was mitigated when cocultured with differentiated macrophages (Jantzen et al., 2012). The cell densities of A549: THP-1 were either 1:1 or 9:1 respectively, derived from the average cell volume of macrophages $\left(2492 \mu \mathrm{m}^{3}\right)$ and pneumocytes $\left(2653 \mu \mathrm{m}^{3}\right)$ for a 1:1 whilst the 9:1 ratio corresponded to the comparative number of macrophages $\left(22 \times 10^{9}\right)$ to the total cell number of the alveolar region $\left(230 \times 10^{9}\right)$ (Jantzen et al., 2012). Seemingly, the only limitation of co-cultures focusing upon the human respiratory tract in modern genotoxicity assays is determining which cell line (Table 5.1) has provided the observed response to either (pro)-inflammatory mediators, observed cytotoxicity and genotoxicity and other such endpoints. Hence, many studies have attempted to include monoculture data of the respective cell lines prior to co-culture assays. Additionally, tagging cells with fluorescent markers for identification during adapted hazard assessment assays provides a clear and concise method for evaluating cocultures in vitro. 
Table 5.1 Representative and commonly selected cell lines for ENM lung exposures.

\begin{tabular}{|c|c|c|}
\hline Cell Line & Pros & Cons \\
\hline A549 & $\begin{array}{l}\text { - Can be sub-cultured routinely without loss in phenotype or } \\
\text { morphology } \\
\text { - Can model ATIl cuboidal structure and use for in vitro toxicology } \\
\text { testing without ethical issues or primary cell line extraction } \\
\text { - Express surfactant proteins at the ALI }\end{array}$ & $\begin{array}{l}\text { - Do not form tight juı } \\
\text { - Cand ENM interaction } \\
\text { upon cancer cell line } \\
\text { - Limited hepatic cyto }\end{array}$ \\
\hline $16 \mathrm{HBE}^{\prime} 4 \mathrm{o}^{-}$ & $\begin{array}{l}\text { - Can form tight junctions when cultured in vitro and are } \\
\text { represent a suitable barrier model } \\
\text { - Can be sub-cultured routinely without losing phenotypic traits }\end{array}$ & $\begin{array}{l}\text { - Can become activate } \\
\text { resulting in an unwaı }\end{array}$ \\
\hline ATI & $\begin{array}{l}\text { - Represent primary alveolar cells and retain their in vivo } \\
\text { morphological and phenotypic traits }\end{array}$ & $\begin{array}{l}\text { - } \quad \text { Ethically demanding } \\
\text { - } \quad \text { Primary cell lines are }\end{array}$ \\
\hline ATII & $\begin{array}{l}\text { - Can be used to model ENM interactions with primary cell } \\
\text { characteristics }\end{array}$ & $\begin{array}{l}\text { - } \text { Ethically demanding } \\
\text { - Hard to maintain, pr } \\
\text { and as a primary cell } \\
\text { of morphological anc }\end{array}$ \\
\hline TT1 & $\begin{array}{l}\text { - Immortalised ATII cells with an ATI-like morphology } \\
\text { - Can be sub-cultured for a much longer period than primary } \\
\text { alveolar epithelial cells }\end{array}$ & $\begin{array}{l}\text { - Relatively novel cell I } \\
\text { - Lacking some of the }\end{array}$ \\
\hline
\end{tabular}




\subsubsection{Chapter 5 Aims}

The aim of this chapter was to determine if the ENMs characterised in Chapter 3 could induce secondary genotoxic mechanisms. To achieve this aim, an alveolar lung model of TT1 and d.THP-1 cells were selected for co-culture (in favour over the 16HBE140- bronchial co-culture, which was also evaluated). Prior to the construction of the co-culture model, both monocultured TT1 and d.THP-1 cell-lines were exposed to the test ENMs individually to evaluate their ability to induce (pro)inflammatory mediator release, cytotoxicity and genotoxicity using the techniques detailed in Chapter 4. Mitochondrial stress was also evaluated in monocultured TT1 cells prior to co-culturing. To understand the importance of secondary genotoxic mechanisms, the alveolar co-culture was subsequently exposed to the test ENMs both with and without NAC pre-incubation to investigate the possible role of oxidative stress in driving DNA damage induction. Given that in Chapter 4 oxidative stress was clearly demonstrated with each test ENM, the Chapter 5 hypothesis was that primaryindirect genotoxicity would occur in TT1 monocultures and that secondary genotoxic mechanisms could be detected in a co-culture model. 


\subsection{Materials \& Methods}

\subsubsection{Basic Cell Culture}

Cell culture of TT1 cells and d.THP-1 cells including the differentiation into macrophages protocol was performed as described in Chapter 2 Section 2.2.2.

\subsubsection{Construction of Bronchial \& Alveolar Co-Culture Model}

The alveolar lung epithelial co-culture model was comprised of TT1 alveolar cells and d.THP-1 macrophages. These co-cultures were constructed upon the apical side of PET track-etched $4.2 \mathrm{~cm}^{2}$ transwell inserts ( $3 \mu \mathrm{m}$ pores) supported by a 6 -well companion plate (Corning, Germany). Transwell inserts were handled during media changes with ethanol sterilised forceps and a second companion plate to hold inserts during the media change. The initial stage of co-culture construction focused on the growth of a monolayer of epithelial cells, in this study - TT1 cells. TT1 cells were cultured from Greiner flasks until required and transferred to inserts at an initial seeding density of $5 \times 10^{5} \mathrm{cell} / \mathrm{s} / \mathrm{ml}$ (final concentration per well, $1 \times 10^{6}$ ). The TT1 monolayer was given 7 days to form a stable monolayer during which time, 3 complete media changes were performed. Cells were cultured with $2 \mathrm{ml}$ of complete DCCM-1 media in the apical side of the inserts and $3 \mathrm{ml}$ in the basolateral companion plate. To prevent cell migration across the PET track-etched membrane a cell scraper was used gently on the underside of the insert at day 7 as performed by Clift and colleagues (2017). At day 5 of the co-culture growth week, THP-1 monocytes were differentiated with 50nM of PMA, day 6 the PMA-containing d.THP-1 media was aspirated and replaced with fresh d.THP-1 media and incubated for a 24-hour recovery period. On day 7 the newly differentiated THP-1 macrophages were resuspended at a concentration of $1 \times 10^{5} \mathrm{cells} / \mathrm{ml}$ in DCCM-1 media, following this all the media ( $2 \mathrm{ml}$ ) was removed from the apical inserts and 500 $\mu$ l of THP-1 cell stock was placed onto each insert. Macrophages were subsequently incubated at $37^{\circ} \mathrm{C}$ for 2 hours to adhere to the epithelial monolayer. Following the incubation period, any unbound macrophages were removed as the $500 \mu \mathrm{l}$ was aspirated and replaced with complete DCCM-1 media and the entire co-culture model incubated at $37^{\circ} \mathrm{C}$ for 24 hours prior to ENM exposures.

\subsubsection{Pre-treatment of Co-Culture with N-acetyl-L-Cysteine (NAC)}

To assess the role of oxidative stress in the co-culture model, on the day of ENM exposure the lung model was pre-treated for 2 hours with NAC prepared in complete DCCM-1 media at a final 
concentration of $1.5 \mathrm{mM}$. Following the NAC incubation period, a complete media change was performed prior to ENM exposures.

\subsubsection{Fixation, Permeabilization and Fluorescent Labelling of Co-Culture}

To investigate the structural integrity of the co-culture model developed as described in section 5.2.2, CLSM was performed. After the successful growth of the co-culture model to day 8 , the PET tracketched membranes were excised (using a freshly opened scalpel) from their plastic insert chambers whilst inverted on a sterilised surface. Using forceps, the inserts were placed into ordinary (noncompanion) 6-well plates for all subsequent steps. The cells were washed 3 times in ice cold PBS. Cells were then fixed in $3 \%$ PFA for 24 hours and incubated at $4{ }^{\circ} \mathrm{C}$ sealed in parafilm. Following fixation cells were washed three times with ice cold PBS prior to permeabilization with $0.2 \%$ TRITON X100 in PBS for 15 minutes. Following permeabilisation cells were washed three times in ice cold PBS then stained with $1 \mu \mathrm{g} / \mathrm{ml}$ Alexa Fluor Phalloidin 633 (BioLegend, San Diego CA) and incubated at room temperature in the dark for 45 minutes. Cells were washed three times with ice cold PBS before $3 \mu \mathrm{g} / \mathrm{ml} \mathrm{CD11b-}$ FITC (Biolegend, San Diego CA) was incubated with the cells for 45 minutes in the dark at room temperature. Cells were washed three times with ice cold PBS one last time prior to mounting to clean glass slides and dried in the dark overnight. On the day of analysis, $20 \mu \mathrm{l}$ of DAPI/Vectashield (Vector Laboratories, USA) was placed ( $10 \mu \mathrm{l}$ below and $10 \mu \mathrm{l}$ on top) on the cell insert and coverslips mounted and fixed in place with nail varnish to dry overnight in the dark at $4^{\circ} \mathrm{C}$.

\subsubsection{Confocal Laser Scanning Microscopy (LSM)}

Confocal microscopy was performed using an LSM-710 microscope coupled with ZEN black software (v2.3) using either 40x or 63x apochromatic oil immersion DIC objectives possessing apertures of 1.3 and 1.4 (Carl Zeiss, UK). Fluorophores (conjugated to distinct antibodies) were specifically selected (Table 5.2) based on their excitation and emission wavelengths and listed in Table 5.2. Image analysis and Z-stacking image acquisition was performed on ZEN Lite Black (v2.3) (Carl Zeiss, UK). 
Table 5.2 LSM fluorophores used in the visualisation of the co-culture model.

\begin{tabular}{|l|l|l|l|}
\hline Wavelength & Fluorophore & $\begin{array}{l}\text { Excitation Wavelength } \\
(\mathrm{nm})\end{array}$ & $\begin{array}{l}\text { Emission Wavelength } \\
(\mathrm{nm})\end{array}$ \\
\hline 488 & Cd11b-FITC & 488 & 520 \\
\hline 633 & $\begin{array}{l}\text { Alexa Fluor Phalloidin- } \\
633\end{array}$ & 632 & 647 \\
\hline 405 & DAPI & 350 & 470 \\
\hline
\end{tabular}

\subsubsection{ENM Exposure Concentrations}

ENM handling, suspension and sonication was performed as detailed in Chapter 2 section 2.1. ENM stock at $10 \mathrm{mg} / \mathrm{ml}$ was diluted to an appropriate concentration using complete DCCM-1 media prior to exposures highlighted in Table 5.3.

Table 5.3 ENM dilutions to expose at the required concentration in co-cultures.

\begin{tabular}{|l|l|l|l|}
\hline ENM Dose $(\mu \mathrm{g} / \mathrm{ml})$ & $\begin{array}{l}\text { Required volume of } \\
10 \mathrm{mg} / \mathrm{ml} \text { stock }(\mu \mathrm{l})\end{array}$ & $\begin{array}{l}\text { Required volume of } \\
\text { media }(\mu \mathrm{l})\end{array}$ & $\begin{array}{l}\text { Volume added to } 2 \mathrm{ml} \\
\text { cells }(\mu \mathrm{l})\end{array}$ \\
\hline 0 & 0 & 200 & 200 \\
\hline 4 & 0.8 & 199.2 & 200 \\
\hline 10 & 2.0 & 198 & 200 \\
\hline 20 & 4.0 & 196 & 200 \\
\hline 50 & 10 & 190 & 200 \\
\hline
\end{tabular}

\subsubsection{Uptake Analysis with TEM}

To assess the internalisation of the carbonaceous ENMs in both the monocultured TT1 and co-culture cells were exposed to $20 \mu \mathrm{g} / \mathrm{ml}$ of ENMs, following exposures the cells were washed three times in PBS, fixed with $2.5 \%$ glutaraldehyde and transferred to TEM maintenance buffer to be embedded and sectioned as detailed in Chapter 2 Section 2.11. 


\subsubsection{Co-Culture In Vitro CBMN Assay}

The CBMN assay was performed as a measure of chromosomal damage in both monocultured and cocultured TT1 cells. The monoculture version of the assay was performed identically to the CBMN assay detailed in Chapter $\mathbf{2}$ Section $\mathbf{2 . 4}$ for monocultured cells. This section focuses on the changes required to adapt this assay for co-cultured cells.

Co-culture exposures were performed as detailed in Section 5.2.6 for 24 hours after which the cells were washed three times with PBS, complete media change and then incubated with $3 \mu \mathrm{g} / \mathrm{ml}$ cytochalasin B for 24 hours (1.5 TT1 cell cycles) placed in both the apical insert and basolateral companion plate. Following the cytochalasin B exposure, cells were washed three times with PBS before $5 \mathrm{ml}$ of Acutase was added to each well ( $2 \mathrm{ml}$ apical, $3 \mathrm{ml}$ basal) whilst incubated at $37^{\circ} \mathrm{C}$ for 10 minutes to gently lift the cells. Acutase-cell suspension was then pipetted up and down to ensure the maximal cell harvest per insert. Acutase was then deactivated with the addition of complete DCCM-1 media and aspirated into $15 \mathrm{ml}$ Falcon tubes. Cells were washed three times with PBS and pelleted once more at $340 \mathrm{~g}$ for 10 minutes (same speed throughout). Cell pellets were re-suspended in $1 \mathrm{ml}$ of 3\% PFA in PBS for 15 minutes followed by centrifugation and permeabilization with $1 \mathrm{ml} 0.2 \%$ TRITON X100 in PBS for 15 minutes at room temperature. Following centrifugation, cells were washed three times with PBS, pelleted then resuspended in 100 $\mu$ l of CD324 (E-Cadherin)-FITC (BioLegend, San Diego CA) at a concentration of $1 \mu \mathrm{g} / \mathrm{ml}$ (diluted with PBS). The cells were then incubated at room temperature in the dark for 45 minutes. Following the staining incubation, cells were washed with PBS three times and re-suspended in $1 \mathrm{ml}$ of PBS for $100 \mu$ to be drop cast via pipette onto ethanol-cleaned, air-dried glass microscope slides. Slides were air-dried horizontally prior to $30 \mu \mathrm{l}$ of DAPI counterstaining and coverslip mounting, these were then left to incubate at room temperature in the dark for 20 minutes.

\subsubsection{Scoring of Co-Culture Micronuclei}

Scoring of micronuclei frequency in binucleated TT1 cells was performed using an Axioimager Z2 fluorescent microscope coupled to an 8-slide mounting stage (Carl Zeiss, UK). Scoring of TT1 Mn/BN cells was performed by identifying 500BN per replicate per concentration (1500 per concentration). TT1 cells were identified based on their FITC fluorescence (attributed to E-Cadherin expression) which would not be present with THP-1 macrophages (only displaying DAPI fluorescence). 


\subsubsection{Cytokinesis Block Proliferation Index (CBPI)}

The assessment of cytostasis was employed in this adaptation to the in vitro CBMN assay due to the inclusion of cytochalasin B. In this instance where cell proliferation and cytochalasin B are concerned the CBPI can be used to quantify cytostasis whereby a CBPI of $1=100 \%$ cytostasis. Cytostasis was calculated from the following formula;

$\%$ Cytostasis $=100-100\left(\left(C B P I_{T}-1\right) /\left(C B P I_{C}-1\right)\right)$

Where $\mathrm{CBPI}_{\mathrm{T}}$ is the test chemical and $\mathrm{CBPI}_{\mathrm{C}}$ is the control and;

CBPI = (Number of mononucleate Cells + (2xNumber of binucleate cells) $+(3 x$ Number of multinucleate cells $)) /$ Total number of cells

Cell viability was calculated based on $\%$ cytostasis i.e. $20 \%$ cytostasis $=20 \%$ cytotoxicity thus cell viability $=80 \%$.

\subsubsection{1 (Pro)-Inflammatory Response}

The investigation into (pro)-inflammatory markers was performed on monocultured TT1 monocultures (IL-6 \& IL-8), monocytes (IL-8 \& IL-1 $\beta$ ) and co-cultured cells (IL-6 \& IL-8) as detailed in Chapter 2 section 2.5. Following 24-hour exposures, cell supernatant was harvested, and the supernatant stored at $-20^{\circ} \mathrm{C}$ until required.

\subsubsection{Bioenergetics \& Mitochondrial Stress}

Quantifying the mitochondrial impact of each ENM on monocultured TT1 cells was carried out identically as described in Chapter 2 section 2.8. As discussed in Chapter 4, data gathered via the Seahorse Bio Analyzer required normalising to cellular protein content per well. This was performed with the DC Protein Assay (Bio-Rad) using the methodology detailed in Chapter $\mathbf{2}$ section 2.9. 


\subsubsection{Statistical Analysis}

All data is shown as the mean +/- standard deviation. Statistical analysis was performed in SPSS statistics software (v.20 IBM, UK) where a one-way analysis of variance (ANOVA) was performed with post hoc Dunnett's multiple comparisons applied to evaluate pairwise statistical significance between control and exposure concentration data sets, the alpha value was set to 0.05 . All data sets were analysed beforehand for normality (Shapiro-Wilk test, $p \leq 0.05$ ) and for equal variance $p \leq 0.05$ ) all biological replicates were $\mathrm{N}=3$, unless otherwise stated. 


\subsection{Results}

\subsubsection{HBE140- Bronchial Co-Culture Model}

To detect the potential secondary mechanisms of genotoxicity a bronchial co-culture model was constructed using $16 \mathrm{HBE} 14 \mathrm{o}^{-}$and d.THP-1 cells. This co-culture was exposed to the highest concentration of ENMs $(100 \mu \mathrm{g} / \mathrm{ml})$ for 24 hours, the supernatant was harvested, and an IL-8 response was recorded (Figure 5.2). Each ENM proved capable of eliciting a strong (pro)-inflammatory response in this co-culture with respective readings at $100 \mu \mathrm{g} / \mathrm{ml}$ for Neutral-, Amine-, Carboxyl-FLG \& CB particles of; $1894.79,1812.94,2096.67 \& 1949.6 \mathrm{pg} / \mathrm{ml}$. This data however was matched with an exceptionally large negative control $(0 \mu \mathrm{g} / \mathrm{ml})$ of $2356.12 \mathrm{pg} / \mathrm{ml}$ demonstrating the capacity of the $16 \mathrm{HBE} 14 \mathrm{o}^{-}$to become activated when co-cultured. Given the capability of the $16 \mathrm{HBE} 14 \mathrm{o}^{-}$cell line to become activated with a two-fold negative control increase over monocultured; 16HBE140$(1259 \mathrm{pg} / \mathrm{ml})$ and a 35 -fold increase over monocultured THP-1 cells $(72.4 \mathrm{pg} / \mathrm{ml})$, it was decided not to continue with this co-culture model, instead moving onto a more relevant alveolar model comprised of TT1/d.THP-1.

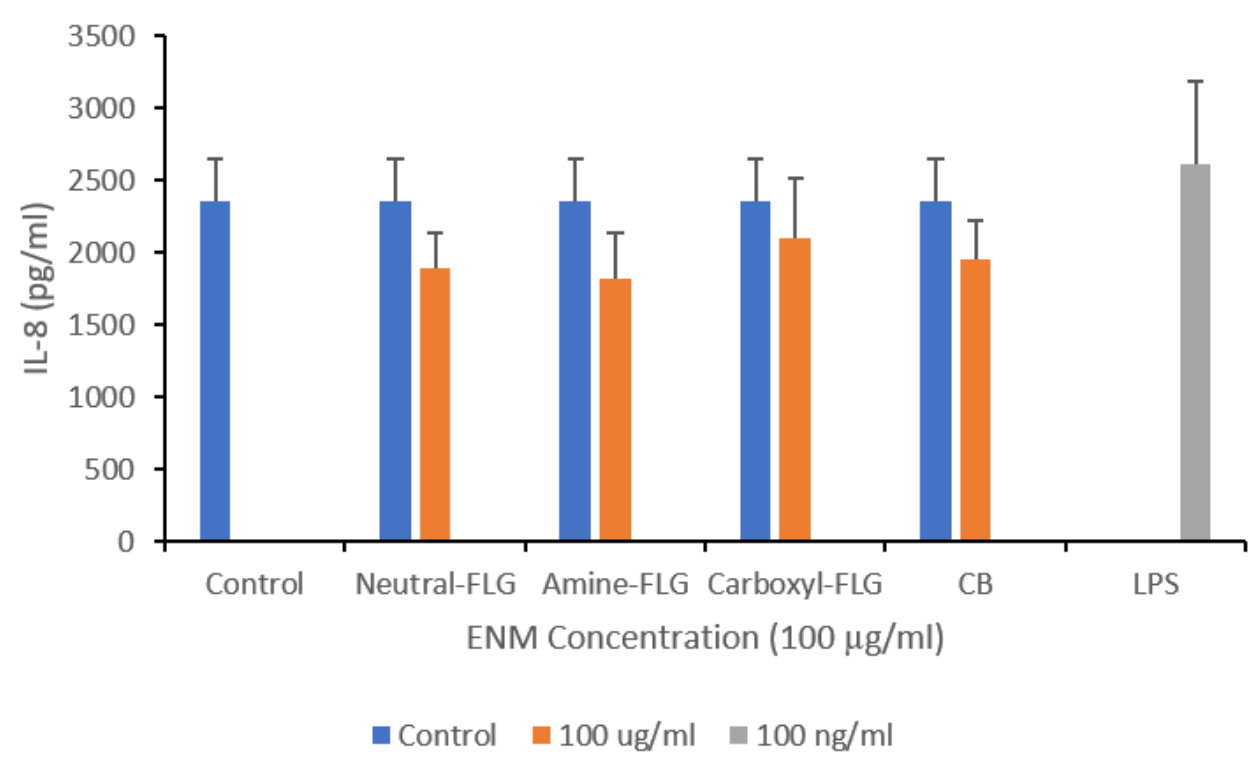

Figure 5.2 (Pro)-inflammatory response of co-cultured 16HBE140 cells following a 24-hour exposure to ENMs. IL-8 protein of the negative control $(0 \mu \mathrm{g} / \mathrm{ml})$ exceeded the highest concentration of ENMs, highlighting the potential issues of undertaking cytotoxicity and genotoxicity evaluations in future work. LPS (the positive control) provided an IL-8 response of $2605.86 \mathrm{pg} / \mathrm{ml}$ at an exposure concentration of $100 \mathrm{ng} / \mathrm{ml}(\mathrm{N}=3)$. 


\subsubsection{Quantifying the TT1/d.THP-1 (Pro)-Inflammatory Response}

Given the bronchial co-culture model showed a considerable (pro)-inflammatory response minus the addition of ENMs, a comparable alveolar model was designed whereby TT1 and d.THP-1 cells were cocultured together. The cells were exposed to ENMs at a concentration of $100 \mu \mathrm{g} / \mathrm{ml}$ for 24 hours, the supernatant was then harvested, and the (pro)-inflammatory response quantified with IL-6 (Figure 5.3A) and IL-8 (Figure 5.3B) ELISAs. Negative control protein levels of IL-6 and IL-8 were 163.4 and $69.73 \mathrm{pg} / \mathrm{ml}$, and LPS (at an exposure concentration of $100 \mathrm{ng} / \mathrm{ml}$ ) provided responses of 492.6 and $1245.3 \mathrm{pg} / \mathrm{ml}$ respectively. The ENMs (at $100 \mu \mathrm{g} / \mathrm{ml}$ ) proved capable of eliciting a highly significant (pro)-inflammatory response with both mediators. Firstly, IL-6 was elevated by 3.5-, 3.3-, 2.5-, and 3.5fold over control levels following a 24-hour exposure to Neutral-, Amine-, Carboxyl-FLG and CB particles respectively. Comparatively, IL8 underwent a greater elevation with Neutral-, Amine-, Carboxyl-FLG and CB particles promoting a 16-, 12-, 17-, and 11-fold increase over control protein levels. Peak IL-6 levels were obtained following exposure to Neutral-FLG with a final measurement of $581.5 \mathrm{pg} / \mathrm{ml}$ whereas IL-8 levels reached a maximum average of $1227.9 \mathrm{pg} / \mathrm{ml}$. 

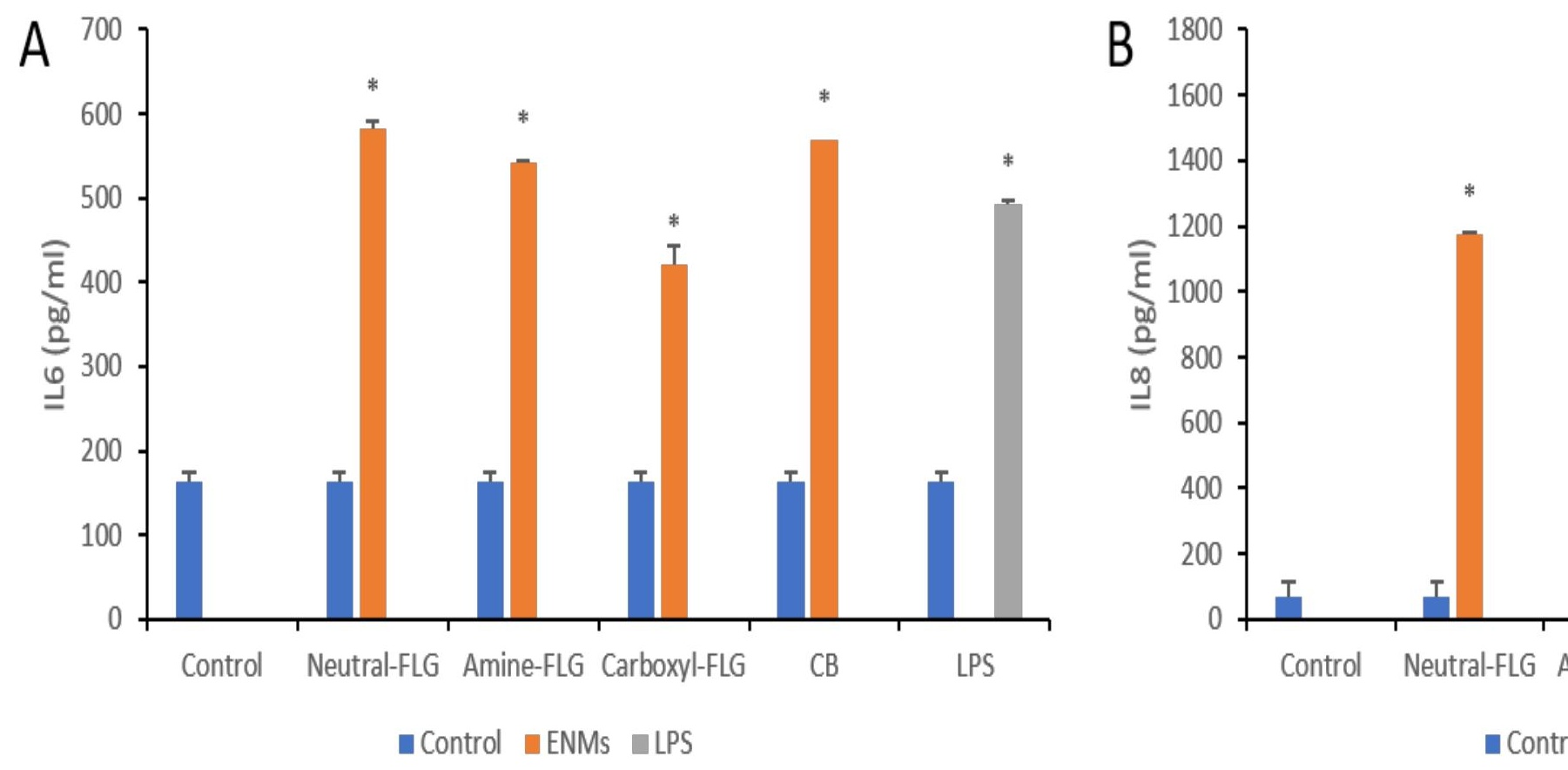

Figure 5.3 (Pro)-inflammatory response of co-cultured TT1/d.THP-1 cells following a 24-hour exposure to ENMs at and IL-8 protein levels (B) after 24 hours ENM exposure. LPS at $100 \mathrm{ng} / \mathrm{ml}$ was used as the positive control and nega considered statistically significant $(*)$ when $p<0.05(\mathrm{~N}=3)$. 


\subsubsection{Cell Viability of d.THP-1 Following 24-hour ENM Exposure}

Prior to undertaking cytotoxicity and genotoxicity testing of the alveolar co-culture, it was important to verify any toxic effects of the ENMs on monocultured d.THP-1 cells. Briefly, d.THP-1 cells were seeded at $1 \times 10^{5}$ cells $/ \mathrm{ml}$ in T25 flasks, exposed to ENMs for 24 hours and the cell viability evaluated with Trypan Blue exclusion. Neutral-FLG exposure to d.THP-1 (Figure 5.4A) cells revealed a nonsignificant concentration-dependent decrease in viability up to $10 \mu \mathrm{g} / \mathrm{ml}$, however concentrations of 20,50 and $100 \mu \mathrm{g} / \mathrm{ml}$ promoted a 1.4, 1.4 and 1.6-fold decrease from control levels with cell viability falling to 72,70 and $62 \%$. This effect was also observed with exposures to Amine-FLG (Figure 5.4B) however the final \% viability here was 73,71 and $64 \%$ at concentrations of 20,50 and $100 \mu \mathrm{g} / \mathrm{ml}$ respectively. THP-1 cells exposed to Carboxyl-FLG for 24-hours (Figure 5.4C) showed greater sensitivity with the initial significant decrease in viability at $10 \mu \mathrm{g} / \mathrm{ml}$ whereby the cell viability was at $77 \%$. A concentration-dependent trend was observed with viability recorded at 59 and $51 \%$ at 50 and $100 \mu \mathrm{g} / \mathrm{ml}$, thus demonstrating the most potent effect on d.THP-1 viability with a 2-fold decrease at the highest exposure concentration. Lastly, d.THP-1 cells exposed to CB particles (Figure 5.4D) revealed little-to-no cytotoxicity which may indicate a slower, more complex mechanism underlying the mode of action of $C B$ as alluded to in the literature. 

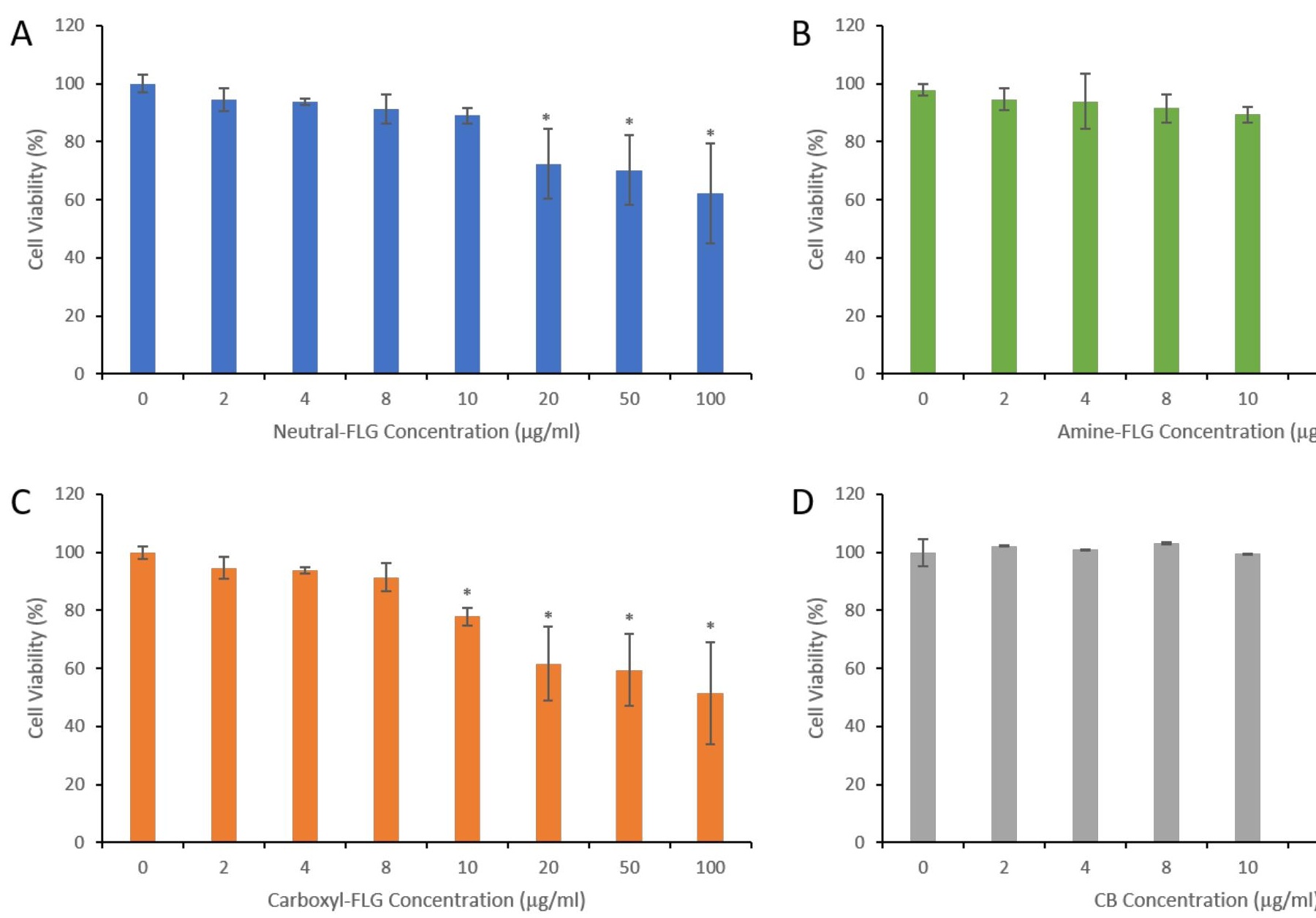

Figure 5.4 d.THP-1 cell viability following 24-hour exposure to Neutral-FLG (A), Amine-FLG (B), Carboxyl-FLG (C evaluated through Trypan Blue Exclusion; results were considered significant $\left({ }^{*}\right)$ when $p<0.05(\mathrm{~N}=3)$. 


\subsection{4 d.THP-1 (Pro)-Inflammatory Response Following ENM Exposure}

Following the 24-hour exposure to ENMs, d.THP-1 cell supernatant was aspirated and stored for the purposes of quantifying IL-8. IL-8 protein levels (Figure 5.5) revealed a concentration-dependent response however only at the highest exposure concentration for the FLG materials and CB particles. The CB particles and the Carboxyl-FLG however also engaged a significant IL-8 response at $50 \mu \mathrm{g} / \mathrm{ml}$ where the respective fold changes over control IL-8 levels of $72.4 \mathrm{pg} / \mathrm{ml}$ were 11.8 and $3.7-$ fold. The exposure concentration of $100 \mu \mathrm{g} / \mathrm{ml}$ however promoted a significant IL-8 response, rivalling the positive LPS control $(1055.9 \mathrm{pg} / \mathrm{ml})$. At this top concentration, Neutral-, Amine-, Carboxyl-FLG \& CB particles elicited IL-8 responses of $932.8,768.8,953.6$ and $993.8 \mathrm{pg} / \mathrm{ml}$ respectively and demonstrated $13,11,13$ and 13.8 respective fold changes over control levels. Supernatant was tested for IL-1 $\beta$ but OD values fell below standard curve (raw data can be viewed in Appendix Section 7.4) and thus the data could not be considered reliable.

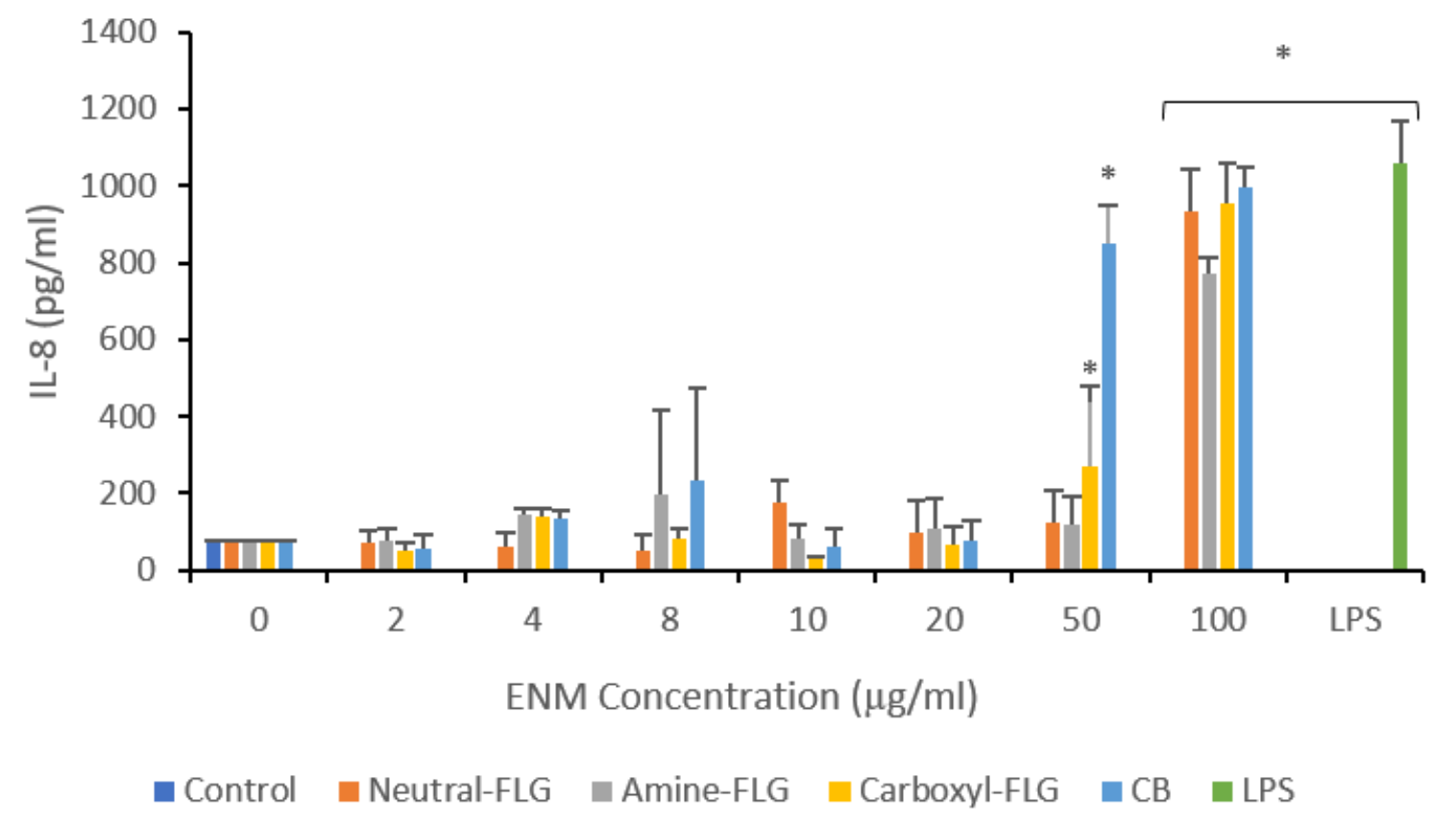

Figure 5.5 d.THP-1 IL-8 (pro)-inflammatory response following a 24-hour exposure to ENMs. IL-8 levels were significantly engaged at $100 \mu \mathrm{g} / \mathrm{ml}$, with only two ENMs (Carboxyl-FLG \& CB) inducing significant responses at $50 \mu \mathrm{g} / \mathrm{ml}$. LPS at $100 \mathrm{ng} / \mathrm{ml}$ was used as the positive control and negative control was media only; results were considered significant $(*)$ when $p<0.05(\mathrm{~N}=3)$. 


\subsubsection{TT1 Cytotoxicity and Genotoxicity Following 24-hour ENM Exposure}

TT1 cells were monocultured and underwent 24-hour ENM exposures to provide a comparison to the 16HBE140 cell line utilised in Chapter 4, this would demonstrate the suitability of the TT1 cells as an optimal cell line for co-culturing. To assess the cytotoxic and genotoxic potential of the ENMs following a 24-hour exposure to TT1 cells, RPD and the in vitro CBMN assay were employed respectively. This was performed in triplicate over the concentration range $0-100 \mu \mathrm{g} / \mathrm{ml}$, with MMC acting as a positive clastogenic control at $0.01 \mu \mathrm{g} / \mathrm{ml}$. TT1 cells exposed to Neutral-FLG (Figure 5.6A) displayed significant cytotoxicity at concentrations of 20,50 and $100 \mu \mathrm{g} / \mathrm{ml}$, with only an average $7 \%$ decrease in cell viability at these concentrations. The CBMN assay revealed significant micronuclei frequencies over control levels (1.2\% Mn/BN) at the same concentration range of 20,50 and 100 $\mu \mathrm{g} / \mathrm{ml}$ with \%Mn/BN frequencies of 1.8, 2.2 \& $3 \%$ resulting in an overall 1.5-fold, 1.8-fold, and 3-fold increase respectively. TT1 cells exposed to Amine- and Carboxyl-FLG (Figures 5.6B \& 5.6C respectively) displayed significant responses at $8 \mu \mathrm{g} / \mathrm{ml}$ and increased to $100 \mu \mathrm{g} / \mathrm{ml}$. Secondly, the $\% \mathrm{Mn} / \mathrm{BN}$ frequencies showed initial significant frequencies at $8 \mu \mathrm{g} / \mathrm{ml}$ for both ENMs. Thus, and unlike NeutralFLG, Amine-, and Carboxyl-FLG displayed LOGEL's of $8 \mu \mathrm{g} / \mathrm{ml}$ which at the highest exposure concentration reached 3.15\% and 2.7\% $\mathrm{Mn} / \mathrm{BN}$, resulting in a 2.6- and 2.3-fold increase over control levels respectively. Therefore, the surface functionalisation has lowered the LOGEL (effectively halved the necessary concentration required to promote significant $\% \mathrm{Mn} / \mathrm{BN}$ ) and the NOEL which was $10 \mu \mathrm{g} / \mathrm{ml}$ (Neutral-FLG) became $4 \mu \mathrm{g} / \mathrm{ml}$ with the addition of amine and carboxyl chemical groups. CB particles also promoted a significant cytotoxic response within the concentration range $10-100 \mu \mathrm{g} / \mathrm{ml}$ with viability decreasing by $9.1 \%$ at the highest concentration (Figure 5.6D). This cytotoxic response showed a positive correlation with the $\% \mathrm{Mn} / \mathrm{BN}$ frequencies whereby the earliest significant genotoxic response was observed at $10 \mu \mathrm{g} / \mathrm{ml}$ and increased at $50 \& 100 \mu \mathrm{g} / \mathrm{ml}$. The $\% \mathrm{Mn} / \mathrm{BN}$ frequencies observed in TT1 cells following 24-hour CB particle exposure were 2.1, 1.9, 2.3 and 2.5\%, the highest concentration demonstrating a 2-fold increase over control levels, making the $C B$ exposure at $100 \mu \mathrm{g} / \mathrm{ml}$ the lowest response of all tested ENMs. 

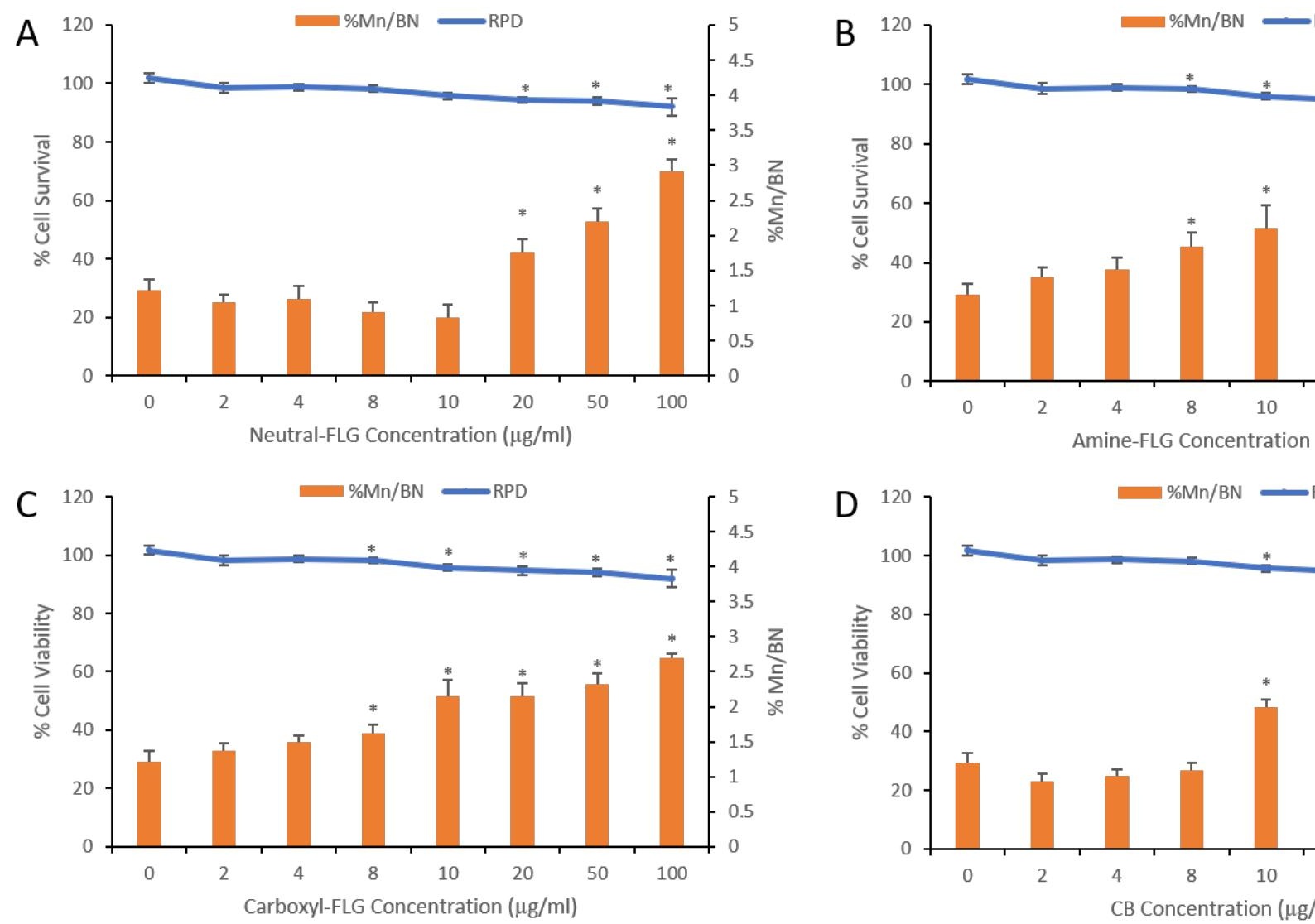

Figure 5.6 Cytotoxic and genotoxic evaluation of monocultured TT1 cells following a 24-hour exposure to ENMs. C chromosomal damage assessment of TT1 cells utilising the in vitro CBMN assay following exposures to ENMs A) Neut $\mathrm{CB}$ particles. Results were considered significant $(*)$ when $p<0.05$. MMC positive control demonstrated a $6.2 \% \mathrm{Mn} / \mathrm{BN}$ 


\subsubsection{Confirmation of Uptake in Monocultured TT1 Cells Following 24-hour ENM Exposure}

To evaluate the cellular uptake of each ENM at $20 \mu \mathrm{g} / \mathrm{ml}$ within TT1 monocultures, TEM \& STEM were employed to provide high resolution images. Exposures of TT1 cells to ENMs resulted in substantial uptake with all tested materials (Figure 5.7 \& Figure 5.8). Since both the FLG materials and CB particles were comprised of over 90\% Carbon, EDX spectroscopy would not provide intricate detail of the internalised ENMs, instead EDX was employed to rule out the presence of potentially toxic heavy metal contamination within the ENM suspensions. This approach was utilised when imaging CB particles, the FLG materials, once located within membrane-bound vesicles were analysed with Fast Fourier Transform (FFT). The resulting image (Figure 5.9E) displays the electron densities of the FLG sheets which is strongest at the atomic positions whereby using the cursor and ImageJ the atomic spacing between the sheets can be calculated. When performing uptake analysis of $C B$ particles, it was crucial to employ EDX (Figure 5.9D) as FFT analysis was not possible since the CB particles were amorphous and lacked a crystal structure. Instead of FFT to confirm the identity of CB within the TT1 cells, EDX mapping was employed (Figure 5.9C) whereby elemental Carbon featured in red and trace amounts of other contaminant materials would be highlighted in contrasting colours. This however did not occur with CB EDX mapping and only a strong Carbon signal was obtained. An EDX spectra has been included which highlights only elements present in the TEM fixation and embedding steps of the SOP (Os, $\mathrm{Cu}, \mathrm{O}, \mathrm{C}$ ) and other elements present within the TEM sample chamber ( $\mathrm{Cu}, \mathrm{Si})$. Trace amounts of Sulphur were witnessed in the EDX spectra (Figure 5.10); however, this is present in extremely small trace quantities and did not feature in the EDX elemental map. 

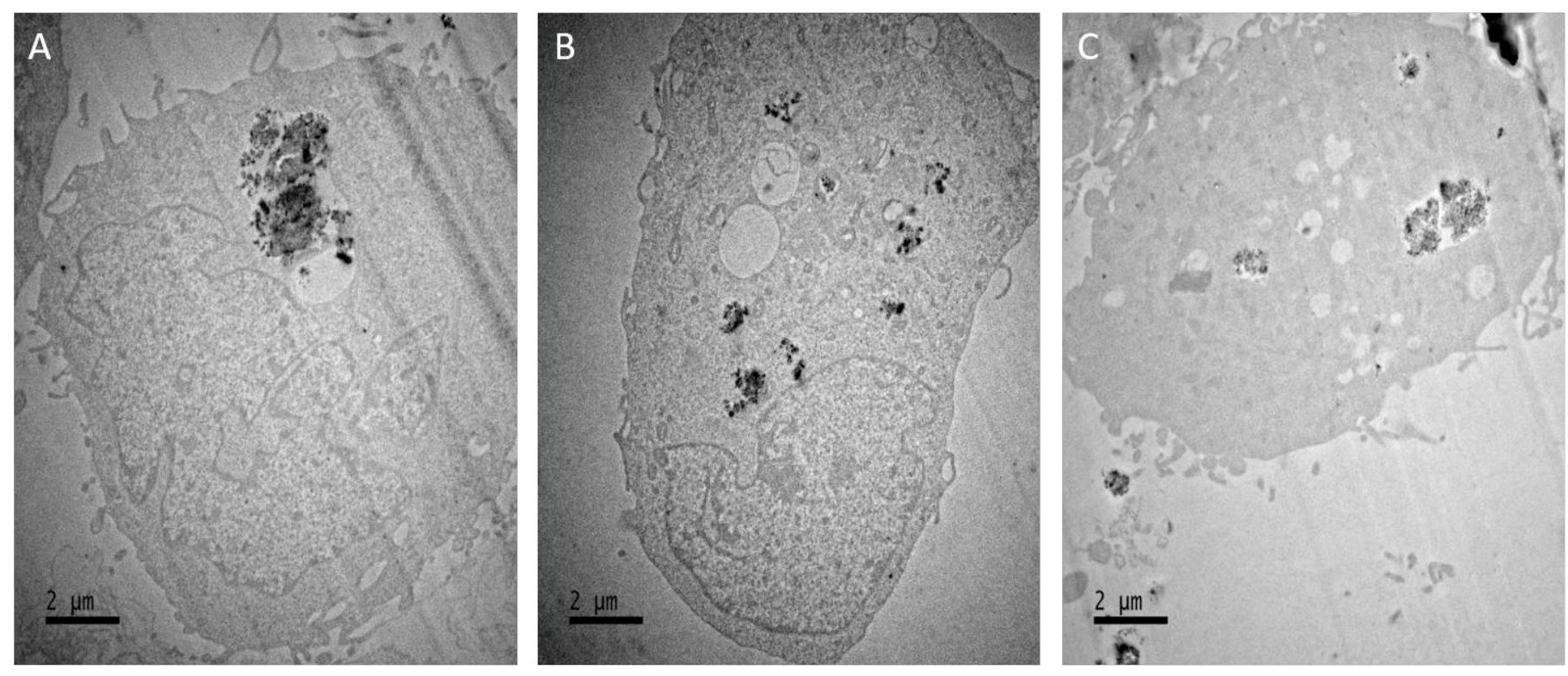

Figure 5.7 TEM confirmation of uptake of ENMs in monocultured TT1 cells at $20 \mu \mathrm{g} / \mathrm{ml}$ following a 24-hour exposur FLG (A), Amine-FLG (B), Carboxyl-FLG (C) \& CB (D)-exposed TT1 cells. Approximately 20 cells were evaluated per copp ENM showed significant agglomeration $(>2 \mu \mathrm{m})$ into tightly packed vesicles within the TT1 cells comparatively, CB however it should be noted CB particles were also contained within vesicles where present in the TT1 cells, no ENM wa 

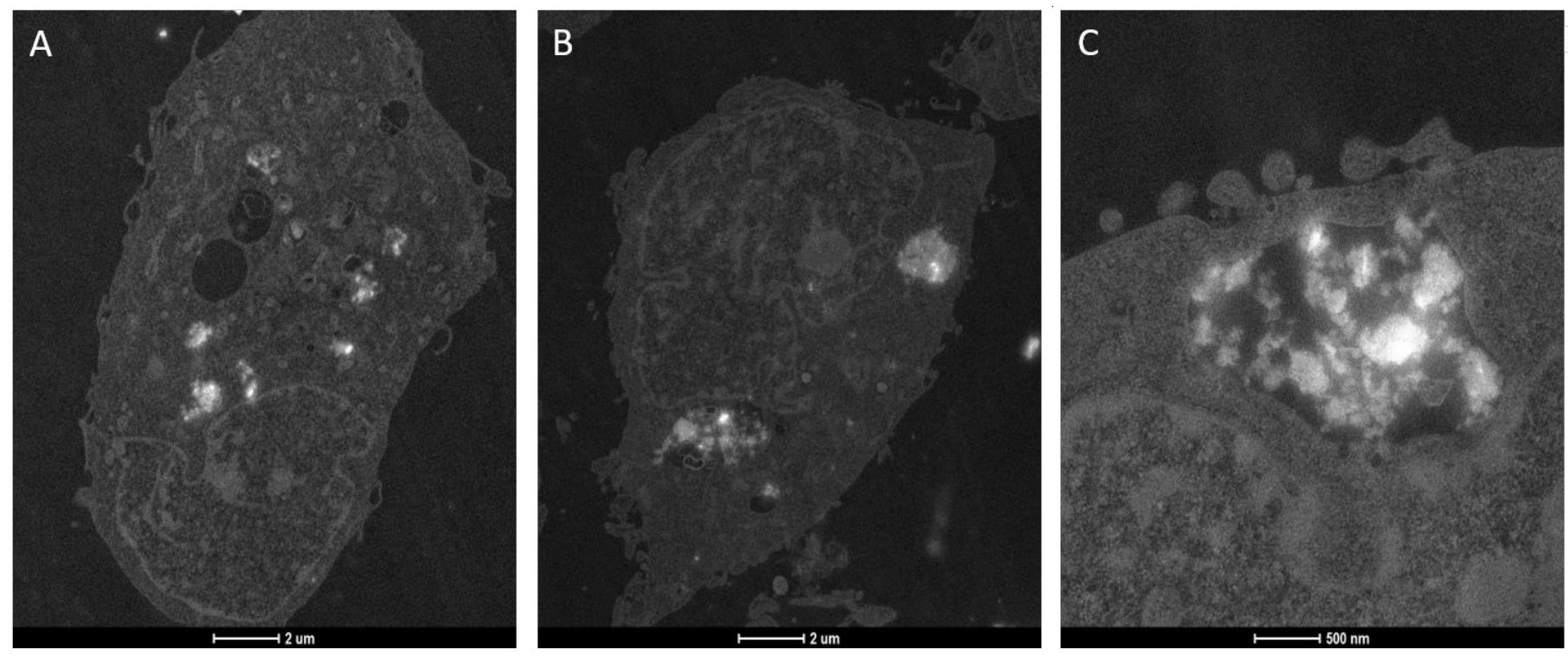

Figure 5.8 STEM confirmation of uptake of ENMs in monocultured TT1 cells at $20 \mu \mathrm{g} / \mathrm{ml}$ following a 24-hour exposur captured whereby Neutral-FLG (A), Amine-FLG (B), Carboxyl-FLG (C)\& CB (D)-exposed TT1 cells. Each FLG ENM show into tightly packed vesicles within the TT1 cells comparatively. 

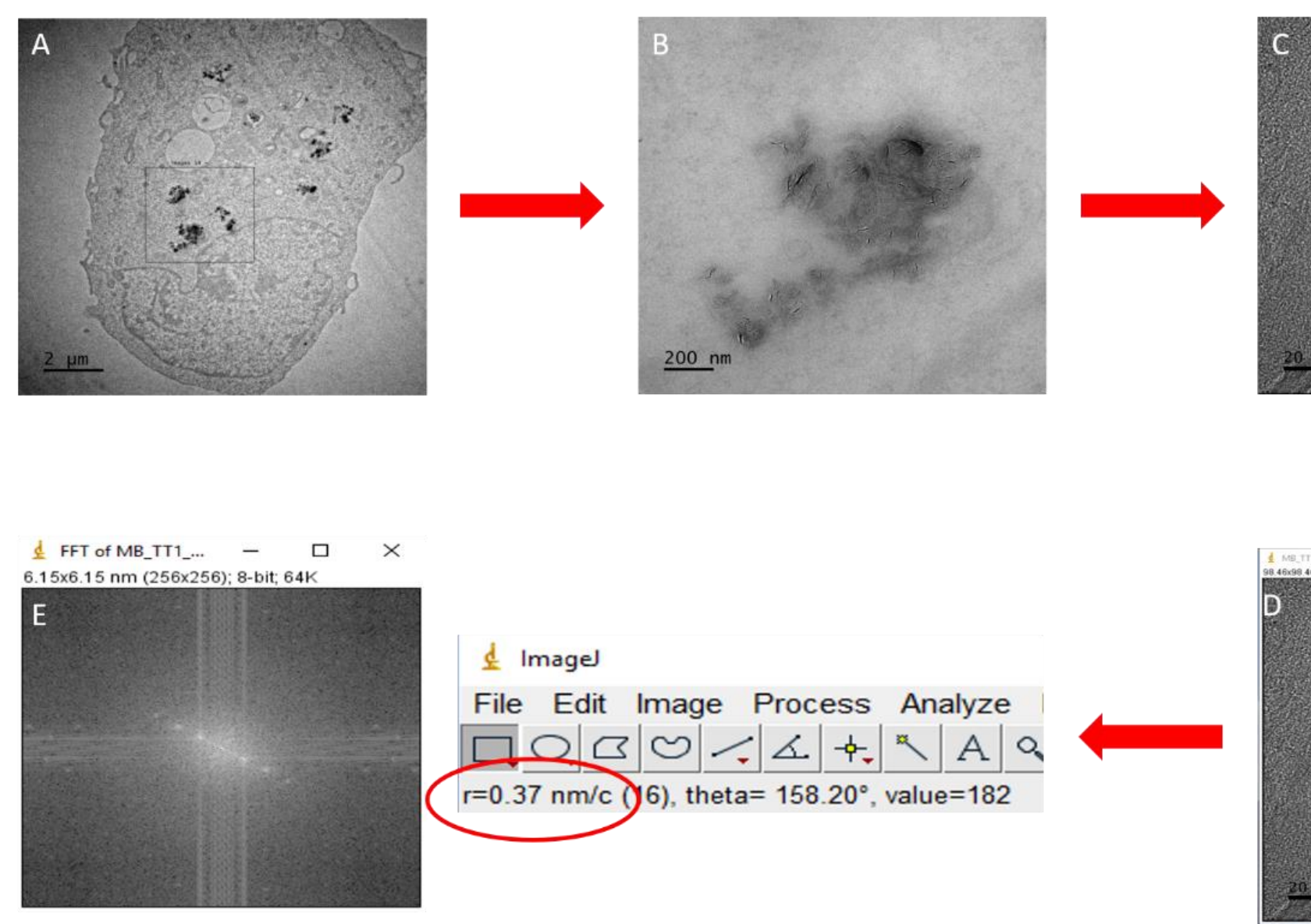

Figure 5.9 Flow diagram illustrating the process of confirming the presence of graphitic material within TT1 cell (example material) was located (A), the vesicle was magnified and focused upon (B), a FLG stack / crystal which was ti FFT analysis was then performed using the ImageJ software (E). The FFT output produces a crystallographic image relates the atomic spacing between graphene sheets. As highlighted in a red circle in image $E$ of the flow diagram, the (3.7Å). 

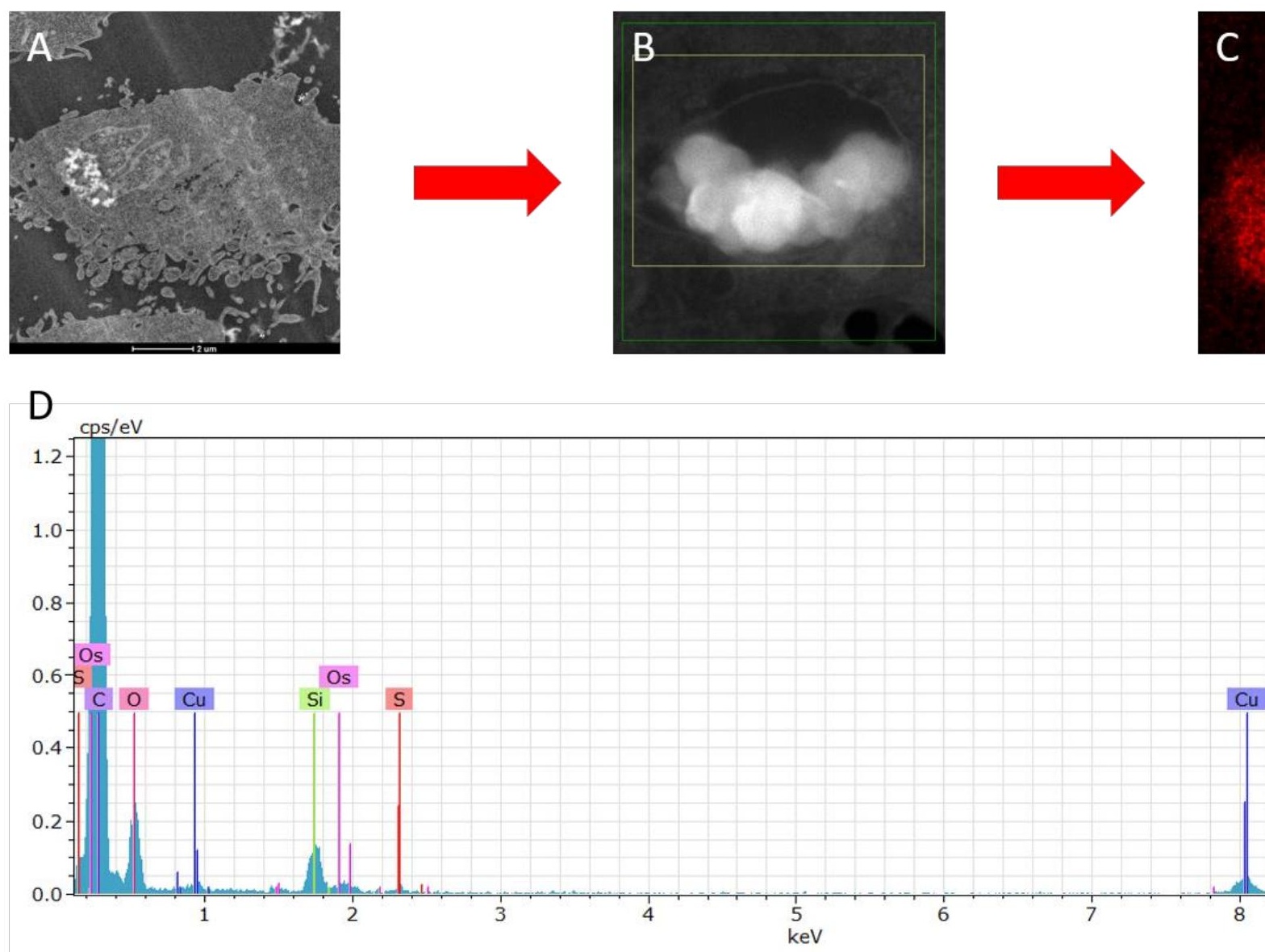

Figure 5.10 Flow diagram illustrating the process of confirmation for CB particle uptake within TT1 cells. The proce vesicles was firstly, find a cell containing CB particles (A), focus in on the particle-containing vesicle (B), finally map spectroscopy. Carbon-mapping highlighted in red (C), did reveal a strong Carbon signal corresponding to the geomet with FLG materials, EDX on CB particles provided little information on the elemental composition (carbon-based) However, EDX spectroscopy did demonstrate there were no contaminant heavy metals (D). 


\subsubsection{Quantification of TT1 (Pro)-Inflammatory Mediators Following 24-hour ENM Exposure}

Following the 24-hour exposure to ENMs over the concentration range $0-100 \mu \mathrm{g} / \mathrm{ml}$, TT1 cell supernatant was aspirated and stored for the purposes of quantifying IL-6 and IL-8 (pro)-inflammatory mediators. TT1 cell supernatant harvested and investigated for the release of IL-6 (Figure 5.11A) revealed a concentration-dependent increase in the (pro)-inflammatory mediator, revealing an initial significant response at $8 \mu \mathrm{g} / \mathrm{ml}$ induced by Amine-FLG at $66.2 \mathrm{pg} / \mathrm{ml}$ with a 12 -fold increase over control levels $(5.32 \mathrm{pg} / \mathrm{ml})$. The concentration of IL-6 protein then steadily increased over the concentration range of $10-100 \mu \mathrm{g} / \mathrm{ml}$ with Amine- and Carboxyl-FLG the greatest release of the mediator at $100 \mu \mathrm{g} / \mathrm{ml}$ with values of 458.3 and $458.7 \mathrm{pg} / \mathrm{ml}$ respectively, a 90-fold change. Cells challenged with LPS at $100 \mathrm{ng} / \mathrm{ml}$ also produced significant IL-6 protein expression $(432.2 \mathrm{pg} / \mathrm{ml})$.

Supernatant evaluated for IL-8 content (Figure 5.11B) displayed a similar trend to IL-6 whereby a concentration-dependent increase in (pro)-inflammatory protein was observed over the course of the $0-100 \mu \mathrm{g} / \mathrm{ml}$ range. With IL-8 however, the initial significant response was attributed to exposure with Neutral-FLG at $10 \mu \mathrm{g} / \mathrm{ml}$ before all ENMs elicited significant IL-8 release at $20-100 \mu \mathrm{g} / \mathrm{ml}$. Once again TT1 cells challenged with LPS produced a significant increase of IL-8 protein (1909.2pg/ml), equalling the highest IL-8 concentration used in the standard curve. At $20 \mu \mathrm{g} / \mathrm{ml}$ Neutral-, Amine, Carboxyl-FLG and CB particles had elicited an IL-8 protein concentration within the supernatant of $822.9,930$, $1038.5 \& 1035.7 \mathrm{pg} / \mathrm{ml}$ respectively, demonstrating a 7-, 8-, 9- and 9-fold increase over control IL-8 levels $(106.34 \mathrm{pg} / \mathrm{ml})$ respectively. A concentration-dependent increase subsequently continued whereby Neutral-, Amine-, Carboxyl-FLG and CB particles had gained a 13-fold increase in IL-8 protein at $100 \mu \mathrm{g} / \mathrm{ml}$ with Carboxyl-FLG producing the slightly greater response with an average $1396.7 \mathrm{pg} / \mathrm{ml}$ of IL-8 protein. 

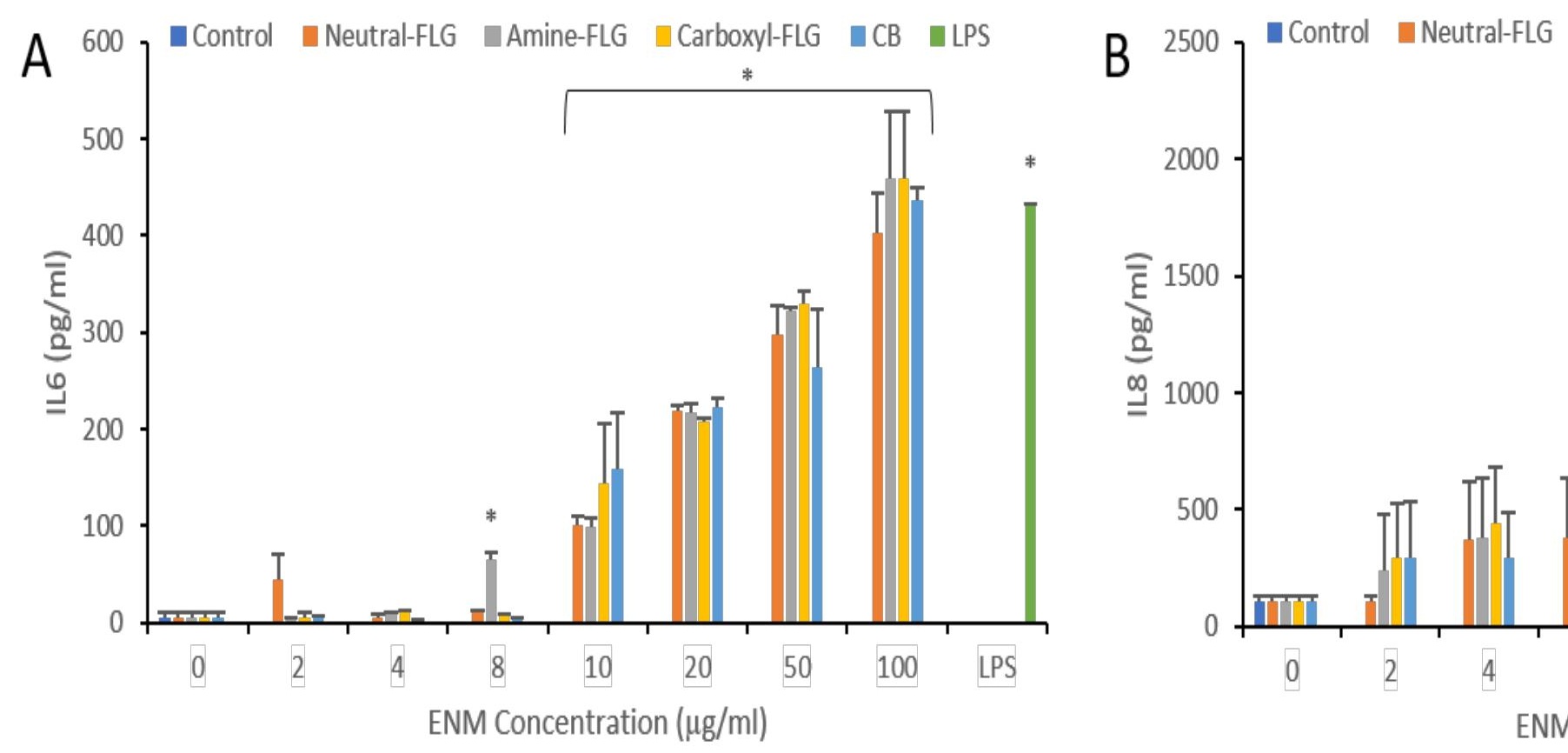

Figure 5.11 TT1 (pro)-inflammatory response following a 24-hour exposure to ENMs. Both IL-6 (A) and IL-8 (B) show in protein expression with IL- 6 showing significant increases from concentrations of $8 \& 10 \mu \mathrm{g} / \mathrm{ml}$. IL-8 showed one in Neutral-FLG before all ENMs induced significant IL-8 release at concentrations of $20-100 \mu \mathrm{g} / \mathrm{ml}$. LPS at $100 \mathrm{ng} / \mathrm{ml}$ was u control was media only; results were considered significant $(*)$ when $p<0.05(\mathrm{~N}=3)$. 


\subsubsection{Bioenergetic Response of TT1 Cells Following 24-hour ENM Exposure}

To assess the effect of Neutral-, Amine-, Carboxyl-FLG and CB particles upon TT1 mitochondrial function, the alveolar cells were seeded into XFe24 plates and treated with concentrations of $0,2,4$, $8,10,20,50$ and $100 \mu \mathrm{g} / \mathrm{ml}$ for 24 hours. Optimal seeding concentration was determined to be 100,000 cells per well and the injection concentrations optimised at $1 \mu \mathrm{M}$ for Oligomycin and FCCP and a Rotenone/Antimycin A concentration of $0.5 \mu \mathrm{M}$ (Appendix). Following this, the plate was loaded into a Seahorse XFe24 Bioanalyser and the OCR/ECAR monitored for 96 minutes. Example OCR \& ECAR data $(N=1)$ seen in Figure 5.12A \& B respectively, reveals the effect of Neutral-FLG (representative material) on the $\Pi T 1$ OCR, whereby exposure concentrations of $2-50 \mu \mathrm{g} / \mathrm{ml}$ heightened the OCR and ECAR over the course of the experiment. Whilst not a significant elevation in both OCR and ECAR, the effect was evident with each ENM elevating both OCR and ECAR above control levels. The effect on OCR and ECAR appears to be concentration-dependent with the lower concentrations of $4 \& 10 \mu \mathrm{g} / \mathrm{ml}$ only showing minor deviations from control $(0 \mu \mathrm{g} / \mathrm{ml})$ levels whereas the highest tested concentration of $50 \mu \mathrm{g} / \mathrm{ml}$ demonstrated a potent effect by raising the OCR and ECAR of TT1 cells following a 24-hour exposure period. 

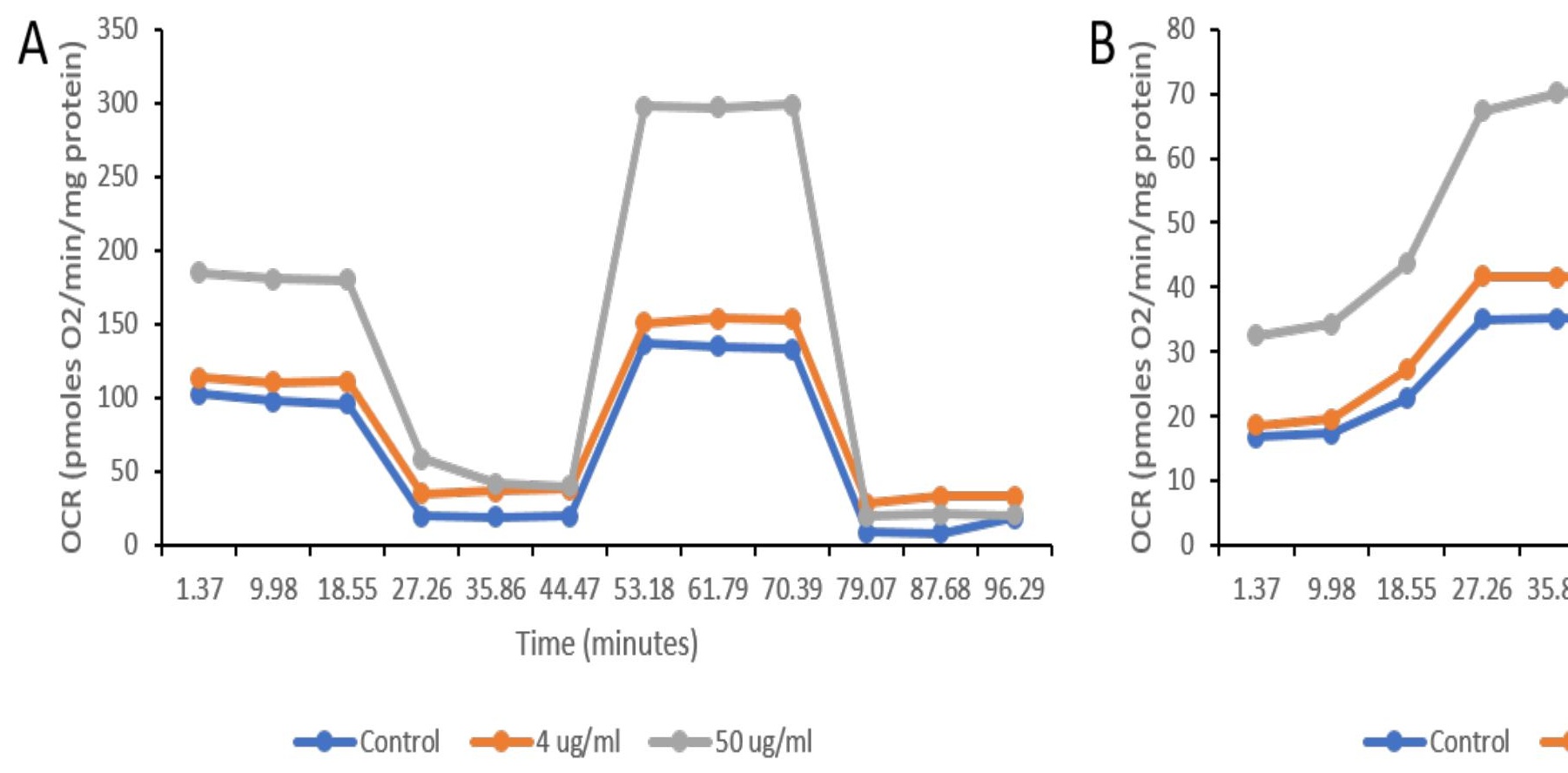

Figure 5.12 OCR \& ECAR assessment of monocultured TT1 cells following a 24-hour exposure to Neutral-FLG (repre were normalised against protein content per well (DC Assay). ( $N=1$ displayed here for clarity, however all experiment sets can be viewed in the Appendix Section 7.2. 


\subsubsection{TT1 Mitochondrial Stress Following 24-hour ENM Exposure}

TT1 cells exposed to the ENMs showed a small (non-significant $(*))$ trend in their metabolic parameters whereby an increase was observed as the concentration of ENMs increased to $20 \mu \mathrm{g} / \mathrm{ml}$ for each ENM. At the highest tested concentration of $50 \mu \mathrm{g} / \mathrm{ml}$ Neutral-FLG (Figure 5.13A) and CB particles (Figure 5.13D) elicited a decrease in OCR, conversely Amine- (Figure 5.13B) and Carboxyl-FLG (Figure 5.13C) depleted the OCR levels at $50 \mu \mathrm{g} / \mathrm{ml}$. The effect of the ENMs on proton leak was to increase in a concentration dependent manner for all ENMs up to $20 \mu \mathrm{g} / \mathrm{ml}$ where the average fold increase over control ( $8.3 \mathrm{pmoles} / \mathrm{min} / \mathrm{mg}$ protein) across each tested material was 3 -fold. However, at $50 \mu \mathrm{g} / \mathrm{ml}$ this effect was depleted where the final proton leak averaged at $22.3 \mathrm{pmoles} / \mathrm{min} / \mathrm{mg}$ protein. This observation was repeated in non-mitochondrial respiration whereby a concentration of $20 \mu \mathrm{g} / \mathrm{ml}$ produced the greatest fold change increase particularly with Neutral-FLG. TT1 cells exposed to Neutral-FLG underwent a 5 -fold increase in non-mitochondrial respiration rising to $50.1 \mathrm{pmoles} / \mathrm{min} / \mathrm{mg}$ protein from control levels $(0 \mu \mathrm{g} / \mathrm{ml}$, of $11.6 \mathrm{pmoles} / \mathrm{min} / \mathrm{mg}$ protein). Amine-, Carboxyl and CB particles meanwhile raised the non-mitochondrial respiration levels to 26.7, 21.9 and $17.3 \mathrm{pmoles} / \mathrm{min} / \mathrm{mg}$ protein respectively, an average 2 -fold increase from control levels.

Initial observations of basal respiration revealed a concentration-dependent increase from control $(98.7 \mathrm{pmoles} / \mathrm{min} / \mathrm{mg}$ protein) to $20 \mu \mathrm{g} / \mathrm{ml}$. At $20 \mu \mathrm{g} / \mathrm{ml}$ basal levels were raised to $198.7,198.2,219.9$ pmoles/min/mg protein when exposed to Neutral-, Amine- and Carboxyl-FLG, representing average 2 -fold increases. Exposures at $50 \mu \mathrm{g} / \mathrm{ml}$ revealed a depletion in basal respiration levels with respect to $20 \mu \mathrm{g} / \mathrm{ml}$ which dropped to $181.7,143.8$ and $132.4 \mathrm{pmoles} / \mathrm{min} / \mathrm{mg}$ protein for Neutral-, Amine-, and Carboxyl-FLG respectively. CB particle exposures revealed basal respiration was raised at 20 and $50 \mu \mathrm{g} / \mathrm{ml}$ with respective basal levels of 150.3 and $169.5 \mathrm{pmoles} / \mathrm{min} / \mathrm{mg}$ protein. Control levels (79.3pmoles/min/mg protein) of ATP production was raised by all but one of the ENMs (Neutral-FLG) raised this function at $4 \mu \mathrm{g} / \mathrm{ml}$. At $10 \mu \mathrm{g} / \mathrm{ml}$ a depletion in ATP production from control levels was observed for each ENMs with an average OCR decrease of $10 \mathrm{pmoles} / \mathrm{min} / \mathrm{mg}$ protein, most prominent with cells exposed to Neutral-FLG. ATP production spiked at $20 \mu \mathrm{g} / \mathrm{ml}$ for all ENMs reaching 127.9, $145.8,171.8$ and $111.4 \mathrm{pmoles} / \mathrm{min} / \mathrm{mg}$ protein in cells exposed to Neutral-, Amine-, Carboxyl-FLG and CB particles respectively indicating Carboxyl-FLG elicited the greatest response with a 2-fold increase. Amine- and Carboxyl-FLG exposures at $50 \mu \mathrm{g} / \mathrm{ml}$ produced a decline in ATP production with average OCR values falling 38.6 and $71.9 \mathrm{pmoles} / \mathrm{min} / \mathrm{mg}$ protein respectively. 
Maximal respiration (123.1 pmoles/min/mg protein) was increased by ENM exposure at $4 \mu \mathrm{g} / \mathrm{ml}$. Carboxyl-FLG and CB particle exposures produced the greatest increase in maximal respiration at this concentration with respective OCR values of 167.9 and $153.8 \mathrm{pmoles} / \mathrm{min} / \mathrm{mg}$ protein. ENM exposures at $10 \mu \mathrm{g} / \mathrm{ml}$ produced a maximal respiration resembling control levels with an average of $122.3 \mathrm{pmoles} / \mathrm{min} / \mathrm{mg}$ protein. This observation was not seen at $20 \mu \mathrm{g} / \mathrm{ml}$ which yielded a 2-, 2.4-, 3and 2-fold increase over control levels following respective exposures to Neutral-, Amine-, CarboxylFLG and CB particles. Neutral-FLG and CB particles at $50 \mu \mathrm{g} / \mathrm{ml}$ elevated the $T T 1$ maximal respiration by 53.1 and $49.2 \mathrm{pmoles} / \mathrm{min} / \mathrm{mg}$ protein whereas Amine- and Carboxyl-FLG depleted by 89.8 and $200.4 \mathrm{pmoles} / \mathrm{min} / \mathrm{mg}$ protein respectively.

The reserve respiratory capacity was raised initially at $4 \mu \mathrm{g} / \mathrm{ml}$ from 36.1 to $40.7,45.5,67.5$ and $50.2 \mathrm{pmoles} / \mathrm{min} / \mathrm{mg}$ protein following respective exposures to Neutral-, Amine-, Carboxyl-FLG and CB particles. This effect was largely unchanged at $10 \mu \mathrm{g} / \mathrm{ml}$ with only Amine-FLG increasing the reserve respiratory capacity by $2.6 \mathrm{pmoles} / \mathrm{min} / \mathrm{mg}$ protein. Neutral-, Carboxyl-FLG and CB particles elicited a depletion with averages of $40.4,43.3$ and $39.8 \mathrm{pmoles} / \mathrm{min} / \mathrm{mg}$ protein respectively. At $20 \mu \mathrm{g} / \mathrm{ml}$ an elevation was observed in respiratory capacity for all ENMs with 2-, 3-, 4-, and 3-fold increases over control levels following Neutral-, Amine-, Carboxyl-FLG and CB particle exposure respectively. At $50 \mu \mathrm{g} / \mathrm{ml}$, Neutral-FLG and CB particles demonstrated a concentration-dependent increase by raising the reserve respiratory capacity by 40.3 and $38.8 \mathrm{pmoles} / \mathrm{min} / \mathrm{mg}$ protein respectively. Conversely, Amine- and Carboxyl-FLG depleted the reserve respiratory capacity by 40.9 and $87 \mathrm{pmoles} / \mathrm{min} / \mathrm{mg}$ protein with respect to data recorded at $20 \mu \mathrm{g} / \mathrm{ml}$. 

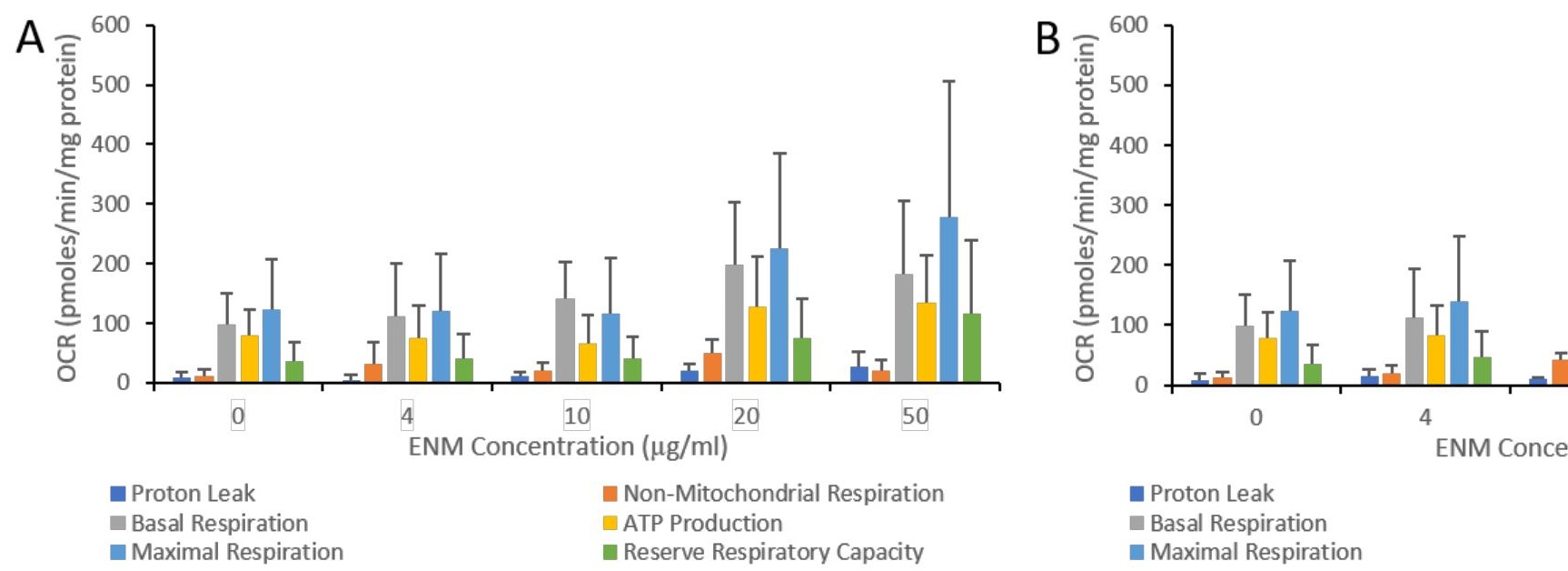

- Proton Leak

- Basal Respiration

- Maximal Respiration
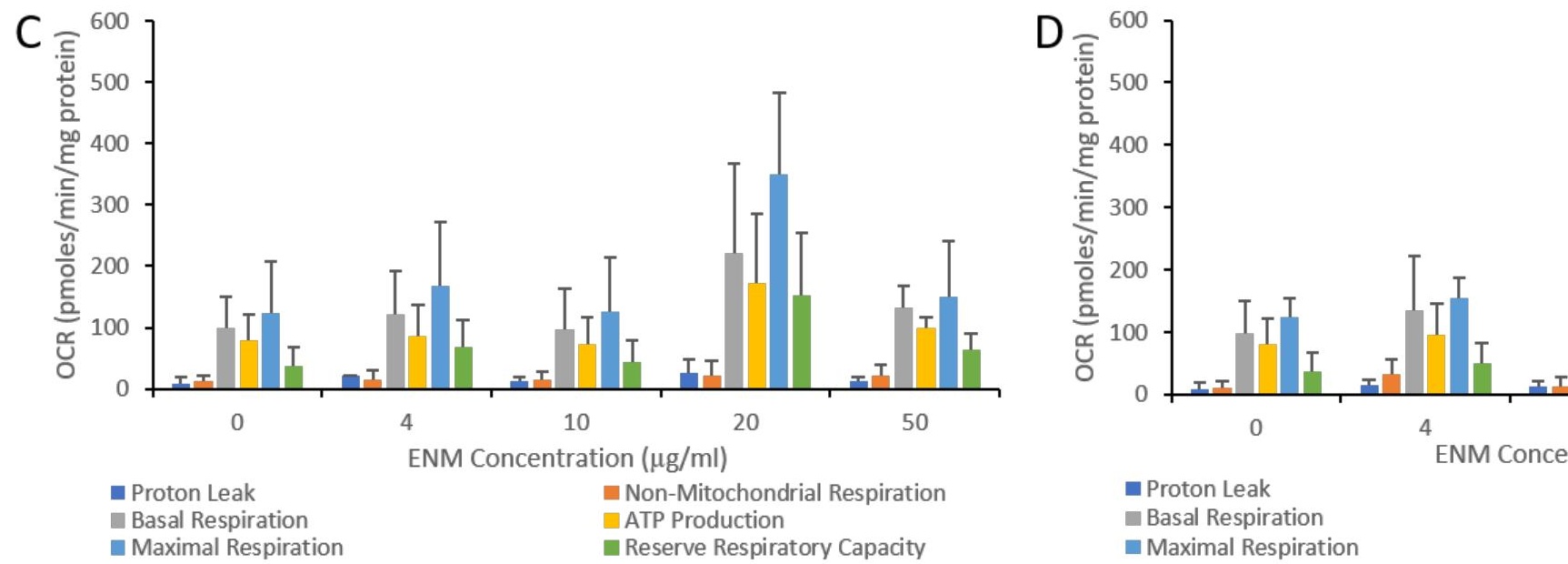

- Proton Leak

Basal Respiration

Maximal Respiration

Figure 5.13 Metabolic parameters of monocultured TT1 cells following 24-hour exposure to ENMs where Neutralparticles (D). $\mathrm{N}=3$ 


\subsubsection{Confocal (LSM) Imaging of the TT1/d.THP-1 Co-Culture}

When constructing the alveolar co-culture, it was necessary to permit direct cell to cell contact between the cell types, a monolayer of TT1 alveolar epithelial cells and differentiated THP-1 macrophages (10:1 ratio). Direct cell to cell contact was important to establish in the co-culture, enabling the exchange of cellular mediators. Whilst the model was prepared to assess the genotoxic impact of the FLG and CB ENMs it was necessary to qualitatively inspect the model for authenticity, structure and integrity, this was achieved with Confocal microscopy. To provide an idea of cell-cell interplay, firstly representative images were captured of the TT1 monolayer before moving onto the co-cultured images. TT1 monolayer image (Figure 5.14A) capture was performed near the periphery of the PET transwell insert due to the fluorescence intensity being excessive near the centre, this may be evidence of TT1 cells beginning to establish a second layer of cells on the formed monolayer. Imaging with co-cultured cells however illustrated an interesting morphological occurrence whereby the THP-1 macrophages were not just present on the surface of the monolayer but showed potential to migrate between the TT1 monolayer (Figure 5.14D). The strong CD11b signal indicated no substantial loss in differentiated THP-1 macrophages, highlighting the co-culture construction was successful. Whilst some areas of the co-culture showed some non-specific fluorescence for CD11b (Figure 5.14B), much of the co-culture insert demonstrated intense singular regions of FITC fluorescence, a characteristic of CD11b successful binding and efficient wash steps when the coculture was constructed. 3D imaging of the co-culture as in Figure 5.14D, highlighted the presence of two cell types towards the periphery, a membrane of epithelial TT1 cells mounted by differentiated THP-1 macrophages displaying a strong FITC, CD11b signal. Excess FITC fluorescence could be considered an artefact due to the lack of strong association with cell morphology based upon the DAPI fluorescence representing the nuclei. However, non-specific binding of the CD11b antibody may also be attributed to non-specific binding. 

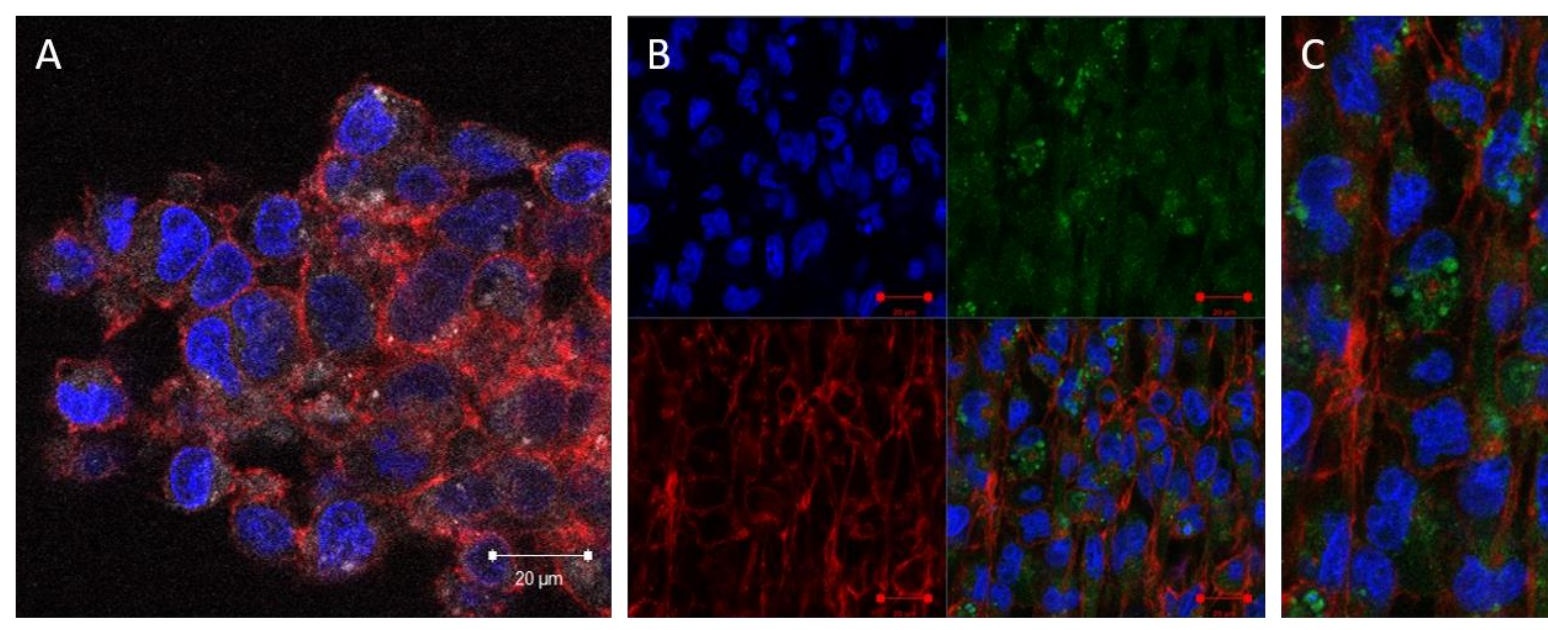

D

Figure 5.14 LSM of co-cultured TT1/d.THP-1 macrophages grown on PET transwell inserts. The TT1 monolayer (A) cultured cells with individual fluorescent signals seperated (B), combined 2D co-culture image (C) and lastly a 3D Z-s images illustrated here, cell nuclei (Blue), actin filaments (Red), and Cd11b integrin protein (Green). 


\subsubsection{Cytotoxicity \& Genotoxicity Evaluation of Co-Cultured TT1/d.THP-1 Cells Following 24-hour ENM Exposure}

The cytotoxic and genotoxic potential of Neutral-, Amine-, Carboxyl-FLG and CB particles to promote cell death and chromosomal damage in the dual-cell lung co-culture was assessed with CBPI and the in vitro CBMN assay adapted for co-cultures respectively. This evaluation was performed in triplicate over a concentration range of $0,4,10,20$, and $50 \mu \mathrm{g} / \mathrm{ml}$ and a positive control of Mitomycin C (MMC) was employed at a concentration of $0.01 \mu \mathrm{g} / \mathrm{ml}$. Utilising the CBPI as a means of calculating cell viability it was observed that no significant cell death was induced by the FLG ENMs at any tested concentration from $4-50 \mu \mathrm{g} / \mathrm{ml}$. Carboxyl-FLG did appear to induce a small decrease in viability at concentrations of 20 and $50 \mu \mathrm{g} / \mathrm{ml}$ as seen in Figure 5.15, but this did not achieve statistical significance.

With respect to chromosomal damage, the background frequency of $\mathrm{Mn} / \mathrm{BN}$ was observed to be $1.73 \%$, and upon exposure to ENMs for 24-hours, each ENM elicited a significant increase in \%Mn/BN. Neutral-, Amine-FLG and CB particles (Figures 5.15A, B and D respectively) produced initial significant increases over control levels at $10 \mu \mathrm{g} / \mathrm{ml}$ (LOGEL) with 3.27, 3 and $2.6 \% \mathrm{Mn} / \mathrm{BN}$ respectively whereas Carboxyl-FLG produced a significant response (3.73\%) initially at $20 \mu \mathrm{g} / \mathrm{ml}$ (LOGEL). At concentrations of $20 \mu \mathrm{g} / \mathrm{ml}$, generally a 2-fold increase over control levels was observed for each ENM this response was enhanced at $50 \mu \mathrm{g} / \mathrm{ml}$ for two ENMs, whereby Neutral-FLG and CB particles raised the \%Mn/BN a further 0.17 and $0.33 \%$ respectively. This was not observed with co-cultures exposed to $50 \mu \mathrm{g} / \mathrm{ml}$ of Amine-FLG which showed no average increase in $\% \mathrm{Mn} / \mathrm{BN}$. Co-cultures exposed to $50 \mu \mathrm{g} / \mathrm{ml}$ of Carboxyl-FLG showed a decline of $0.26 \%$ in $\% \mathrm{Mn} / \mathrm{BN}$ from cells exposed to $20 \mu \mathrm{g} / \mathrm{ml}$. However, it should be noted that even with no significant rise in $\% \mathrm{Mn} / \mathrm{BN}$ at $50 \mu \mathrm{g} / \mathrm{ml}$ when compared to $20 \mu \mathrm{g} / \mathrm{ml}$ this still represented an average 2 -fold increase in micronuclei induction compared to control level. 

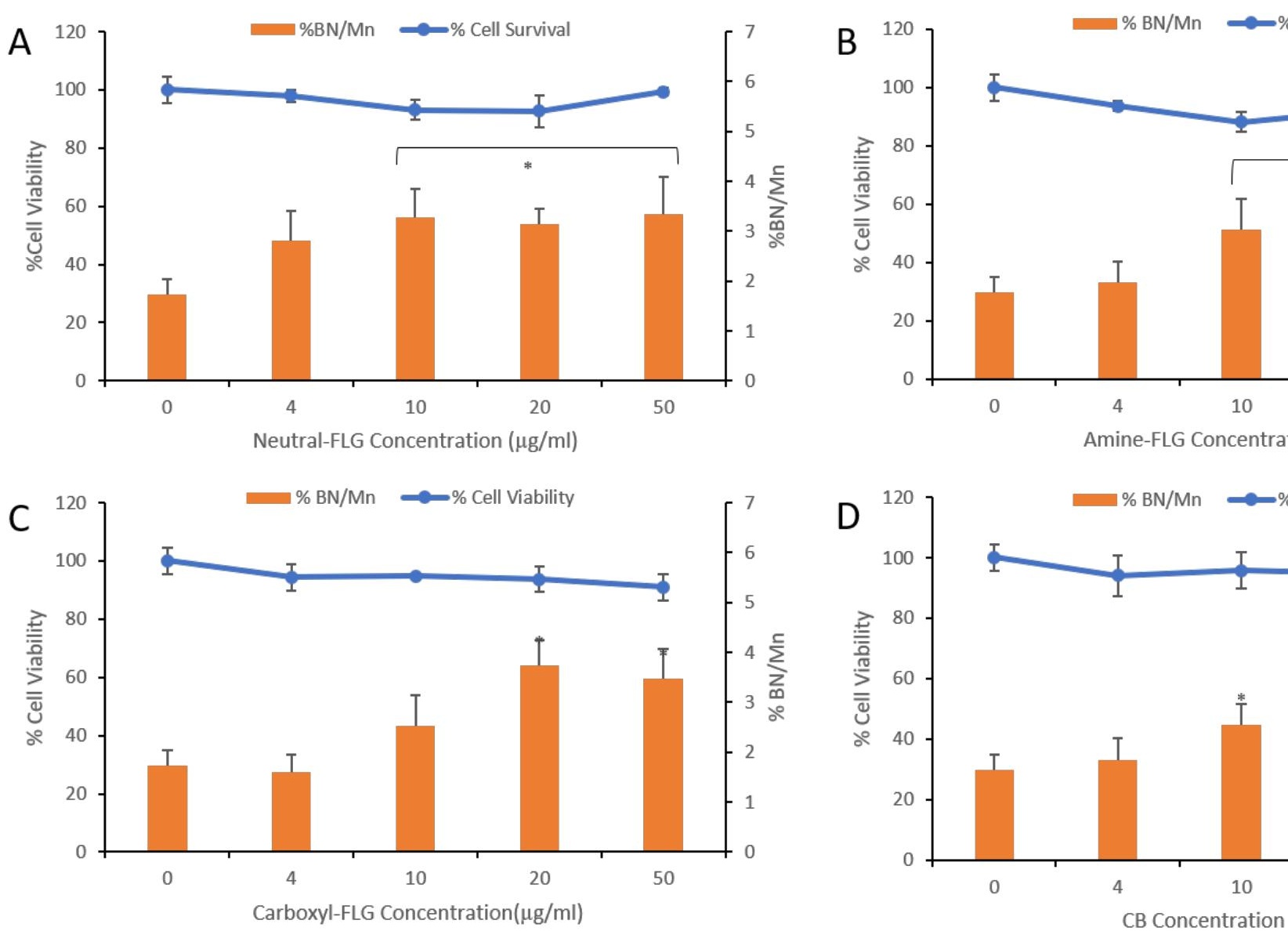

Figure 5.15 Cytotoxicity and chromosomal damage assessment induced by ENMs within TT1/d.THP-1 co-cultures chromosomal damage assessment of TT1 cells utilising the in vitro CBMN assay adapted for co-cultures following ex (B), Carboxyl-FLG (C) and CB particles (D). Results were considered significant $\left({ }^{*}\right)$ when $\left.p<0.05\right)$. The MMC positive col 


\subsubsection{Evaluating Oxidative Stress in Alveolar Co-Cultures Pre-Incubated with NAC}

To assess the role of oxidative stress as a potential mediator of secondary genotoxicity within the coculture, a 2-hour pre-incubation with NAC was performed. Following the NAC pre-incubation, a complete media change was performed prior to the exposure to ENMs for 24 hours. CBPI and cell viability calculations performed on the co-cultures following ENM exposures revealed no significant decrease in viability (Figure 5.16 A-D). Interestingly however, no significant data was obtained with regards to an increase in \% $\mathrm{Mn} / \mathrm{BN}$ from control levels (1.73\%) was observed over the concentration range of $4-50 \mu \mathrm{g} / \mathrm{ml}$ for any of the tested ENMs. Even though there appears to be a slight positive correlation between increasing concentration and the frequency of $\% \mathrm{Mn} / \mathrm{BN}$, this did not reach statistical significance.

NAC therefore appears to have limited the potentially harmful oxidative radicals present in the media or from causing DNA damage. It should also be noted that MMC (positive control agent) micronuclei frequencies were lower, however due to the nature of the MMC mode of action (alkylating agent of DNA helix) this could be due to random data sampling and not the action of NAC itself. Exposures of dual-cell lung co-cultures of TT1/d.THP-1 macrophages to Neutral-, Amine-, Carboxyl-FLG and CB particles over $0-50 \mu \mathrm{g} / \mathrm{ml}$ revealed no genotoxicity suggesting the important role of free radicals in the observed genotoxic response observed in Figure 5.15. 

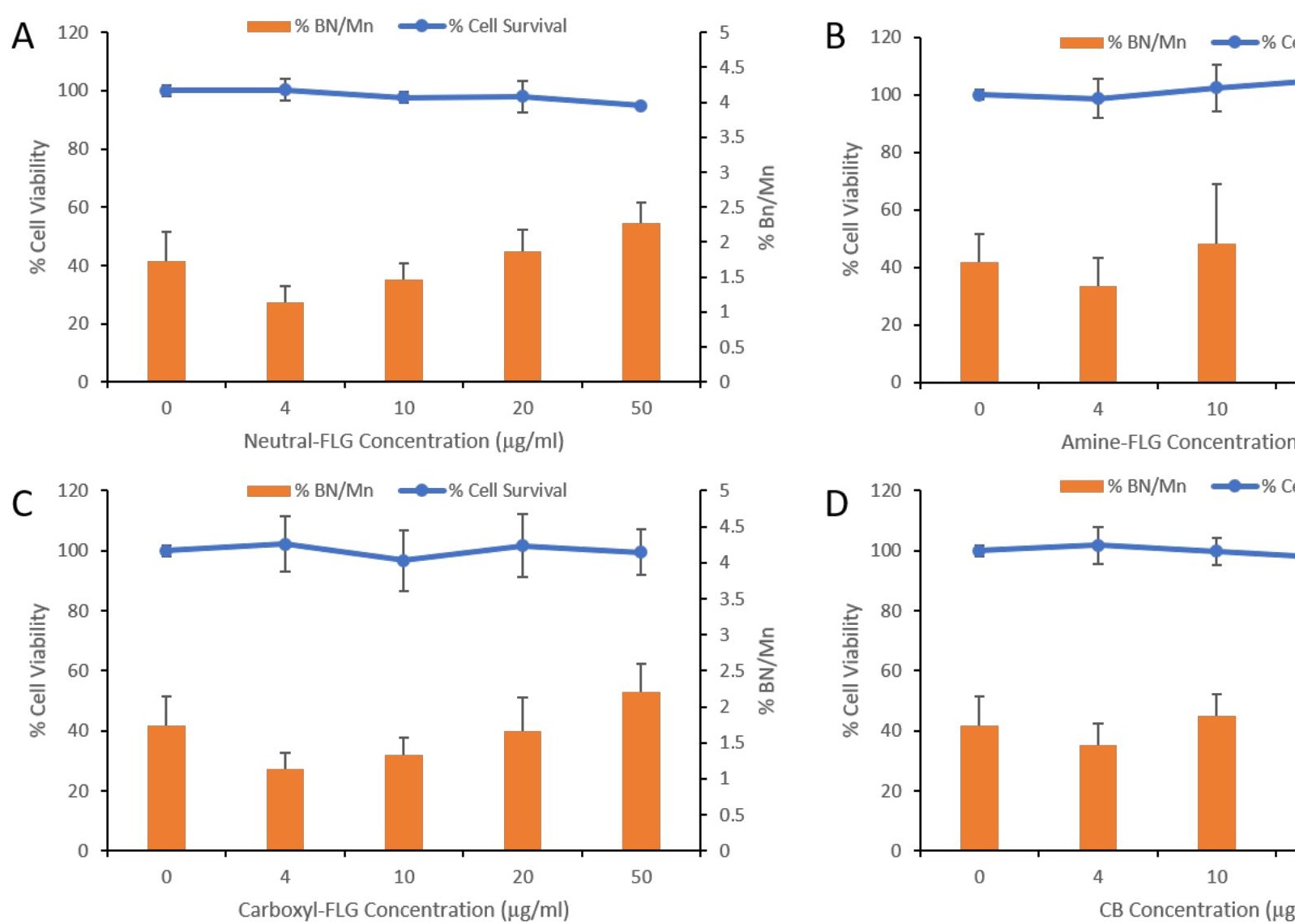

Figure 5.16 Cytotoxicity and chromosomal damage assessment induced by ENMs within TT1/d.THP-1 co-cultures pre through CBPI and chromosomal damage assessment of TT1 cells utilising the in vitro CBMN assay was adapted for co-C FLG (A), Amine-FLG (B), Carboxyl-FLG (C) and CB particles (D). Results were considered significant $(*)$ when $p<0.05)$. Th $\mathrm{Mn} / \mathrm{BN}(\mathrm{N}=3)$. 


\subsection{Discussion}

Hazard safety data is largely built upon monocultured cell lines, primarily due to their relatively simple design, high-throughput capacity, necessity for regulatory approvals and ease of handling. However due to severe limitations in modelling complex cell-cell signalling and architecture, more robust models are being developed to advance our current understanding of ENM toxicity (Evans et al., 2017). To determine whether the test ENMs could induce secondary genotoxicity, a bronchial co-culture was set up using the 16HBE140 cell line with differentiated THP-1 macrophages. Upon investigation into the suitability of this model for detecting secondary genotoxicity, the (pro)-inflammatory response was briefly investigated. Whilst uncommon for epithelial cells to become activated and release (pro)inflammatory mediators during co-culture construction, this does appear to be the case for the $16 \mathrm{HBE} 14 \mathrm{O}^{-}$cell line in this investigation. The un-treated co-culture had a base-level IL-8 reading of $2356 \mathrm{pg} / \mathrm{ml}$, exceeding the top standard used in the experiment. Thus, this preliminary experiment demonstrated potential issues which may be encountered with co-culturing the $16 \mathrm{HBE}^{-14 \mathrm{O}^{-}}$cell line and highlighted the need for a more robust and representative cell line. The bronchial co-culture showed considerable activation prior to ENM exposure and thus could not be used. Having established the genotoxic effects of Neutral-FLG, Amine-FLG and CB upon 16HBE140 cells in Chapter 4 it was logical to explore the potentially detrimental effects these ENMs may have on the alveolar region. Therefore, TT1 cells were selected to explore the genotoxicity of each ENM upon an alveolar cell line before attempting a co-culture with d.THP-1 cells. Similarly, the (pro)-inflammatory response of the co-culture prior to ENM exposures was investigated and revealed no activation in comparison to the 16HBE140\% $/$.THP-1 model.

The alveolar co-culture model was examined by confocal microscopy for integrity, cell to cell contact and architecture, allowing for multicellular communication to more closely replicate in vivo conditions. To demonstrate the role of oxidative stress in secondary mechanisms of genotoxicity the co-cultures were exposed to ENMs both with and without the pre-incubation with $1.5 \mathrm{mM}$ of NAC. The importance of ENM interactions with immune cells has been extensively highlighted by Evans and colleagues. Briefly, upon ENM-immune cell interaction a plethora of outcomes are possible, the most likely of which are oxidative stress through excess free radical production and the other being a sustained inflammatory response (Evans et al., 2017). NAC acts as a cysteine precursor in the production of glutathione and free radical neutralizer, balancing the unpaired electrons of oxygen and nitrogen. NAC has other hypothesised roles such as providing anti-inflammatory relief which may impact upon the co-culture model in a potentially beneficial way (Matera et al., 2016). Immune cells have a tendency 
to promote highly reactive nitric oxide through the up-regulation of nitric oxide synthase, a process requiring common (pro)-inflammatory mediators such as IL-1 $\beta$ and TNF $\alpha$ (Tripathi, 2007). As a result, many studies such as the recent publication by Lategan and colleagues (2018) choose to evaluate the role of ENMs upon immune cells by investigating nitric oxide production as compared to a positive control (Lategan et al., 2018).

Currently, the line between data on genotoxicity generated in vivo and in vitro with ENM do not always correlate. As reported by Downs and colleagues (2012), amorphous silica NPs (15 and 55nm) were tested for the capacity to induce DNA damage in liver, lung and blood cells. The authors reported no genotoxicity at low doses confirmed by the in vitro micronucleus assay. Downs and colleagues then hypothesised silica NPs would promote secondary genotoxicity through release of inflammatory cellderived oxidants. This was supported by neutrophilic infiltration, liver cell apoptosis and TNF- $\alpha$ and IL6 release into plasma, concluding that silica NPs promoted secondary genotoxicity through mechanisms driven by inflammation (Downs et al., 2012). Ultimately, this issue needs to be resolved using suitable cell lines but more importantly advanced in vitro culture models. The suitability of cell lines to model primary cells in vitro has been extensively questioned (Swain et al., 2010). The human lung consists of various cell lines, and dependent on their location within the branching network of the respiratory tract could be vulnerable to ENM interactions. The alveolar bed of the human lung consists of two types of primary alveolar cell types, type I and type II. The ATI and ATII cells offer some innate immunity to the alveolar region of the lung through secretion of pulmonary surfactant protein, the role of ATII cells whilst ATI act to enhance the dispersion of pulmonary surfactant through microvilli to coat the alveolar epithelium. In vitro cultures of primary cells can prove problematic however, for example culturing ATII cells in vitro over a period of 1-2 weeks can undergo spontaneous differentiation resulting in the expression of cellular features such as proteins and morphology resembling the characteristics of ATI cells (Fuchs et al., 2003). To overcome these issues the correct selection of cell line must be made to suit the individual requirement of the experiments. This can be achieved by performing Raman spectroscopy for example, which can be implemented to discriminate between the biochemistry of ATI and ATII cells cultured in vitro (Kemp et al., 2008). The TT1 cell line is derived from ATII cells but adopt an ATI cell morphology with similar protein receptor expression. These represent an important cell line to model ATI cells, which cover $95 \%$ of the alveolar epithelial surface area (Sweeney et al., 2015). 
The human adenocarcinoma cell line (A549) has been used as a lung model of ATII cells, which as discussed previously are highly specialised cells with unique functions. They produce pulmonary surfactant, a multifunctional lubricant which reduces surface tension within the alveolar region and inhibits alveolar collapse during ventilation (Swain et al., 2010). A549 cells, derived from a type II pneumocyte lung tumour by Giard et al in 1972, express similar traits to ATII cells such as phospholipid synthesis cytoplasmic lamellar bodies and apical microvilli (Lieber et al., 1976). However there remains a significant issue with the A549 in vitro systems as a model for ATII cells in that they do not adopt ATII barrier or architectural properties (Godfrey, 1997). Furthermore, unlike primary ATII cells, cultured A549 cells do not undergo a transition to express the ATI-like phenotype. Lastly, A549 cells cultured in vitro are relatively inconsistent at expressing ATII-specific markers which has led to debate as to the suitability of A549 cells to model ATII cells (Mason and Williams, 1980). Cell lines are often derived from cancerous tissue or through immortalization of primary cells through retroviral transfection or transduction (O'Hare et al., 2001). The main disadvantages of cell lines however can be they show phenotypic expression irregularities deviating too far from the original primary cell (Clover and Gowen, 1994). TT1 cells were immortalized through the culturing of freshly isolated ATII cells using transduction with the catalytic subunit of telomerase (human telomerase reverse transcriptase; hTERT) and a temperature-sensitive mutant of simian virus 40 large-tumour antigen (Kemp et al., 2008). TT1 epithelial cells are the first to be produced and represent an ideal choice for in vitro genotoxicity testing given their close resemblance to primary alveolar cells.

TTI cells were tested for the expression of ATII-specific markers including alkaline phosphatase, prosurfactant protein C, and ATI-specific caveolin; and were negative for ATII-specific markers; prosurfactant protein C, alkaline phosphatase and thyroid transcription factor-1. Additionally, the TT1 cell line does not contain lamellar bodies and display a thin attenuated morphology containing caveolae vesicles (Sweeney et al., 2015). TT1 cells were previously characterised via Raman micro-spectroscopy to differentiate between ATII and ATI phenotypes, the latter morphology of which the TT1 cells adopt in cultured conditions (Kemp et al., 2008). Undifferentiated ATII cells were cuboidal, TT1 morphology was like that of ATI cells but less squamous and thin cytoplasmic attenuations were less prevalent. TT1 cells did show caveolin-1 expression albeit to a lesser extent than ATI cells. This could prove to be vital when determining the route of uptake when compared to the $16 \mathrm{HBE}^{-140^{-}}$cell line investigated in Chapter 4. 
As in Chapter 4, the concentration of ENMs used in TT1 and d.THP-1 monocultures was $0-100 \mu \mathrm{g} / \mathrm{ml}$ within which all three FLG samples induced a cytotoxic response, at the higher concentrations initially seen at $20 \mu \mathrm{g} / \mathrm{ml}$ for Neutral- and Amine-FLG and $10 \mu \mathrm{g} / \mathrm{ml}$ with cells treated with Carboxyl-FLG. CB particle exposures revealed no cytotoxic effects however. The cytotoxicity of GO NPs has recently been assessed in the works by Lategan and colleagues where the RAW 264.7 macrophage immune cell line was cultured in 48 well-plates and treated with ENMs at a concentration range between 15.6 and $500 \mu \mathrm{g} / \mathrm{ml}$. Viability and (pro)-inflammatory mediators were investigated (Lategan et al., 2018). The authors noted a significant reduction in cell viability of RAW 264.7 cells initially at $62.5 \mu \mathrm{g} / \mathrm{ml}$ which decreased further to $60 \%$ of the negative control at the maximum concentration. In the present study, given that the cell viability does not significantly alter between the lowest observed effect level and the final concentration it could be suggested that the d.THP-1 cells have reached their maximum capacity for uptake at this cytotoxicity plateau. The greater depletion in cell viability of d.THP-1 as compared to the $16 \mathrm{HBE} 140^{-}$or $\mathrm{TT} 1$ cells may be attributed to the greater capacity of immune cells to radically alter their actin cytoskeleton to facilitate uptake (Feito et al., 2014, Zhang et al., 2016).

The genotoxic effects of CB particles upon THP-1 immune cells was investigated by Don Porto Carero and colleagues alongside DEPs and urban particulate matter (UPM). Whilst displaying particle characterisation based on CB particle diameter ( 100nm) and when exposed to THP-1 immune cells between $16 \mathrm{ng} / \mathrm{ml}$ and $16 \mu \mathrm{g} / \mathrm{ml}$, the authors reported significant genotoxicity using the comet assay. Through the Alamar Blue assay, the cells showed no significant cytotoxicity whereby the authors hypothesised that particle composition and size were the contributing factors (Don Porto Carero et al., 2001). More recently, in the work by Sahu and colleagues, the human monocytic cell line (THP-1) was exposed to fractions of $C B$. A nano-powder whereby particles primary diameter was $<50 \mathrm{~nm}$, and a carbon powder where particle size was $\sim 500 \mathrm{~nm}$ were evaluated. In this study $1 \times 10^{6} \mathrm{cells} / \mathrm{ml}$ were seeded into 24-well plates and exposed to either CB fraction for 24 hours over a range of $50-$ $800 \mu \mathrm{g} / \mathrm{ml}$ in liquid interface cultures (Sahu et al., 2014). The MTT assay was employed to evaluate cytotoxicity. The authors noted only minor significant cytotoxicity initially at $100 \mu \mathrm{g} / \mathrm{ml}$ with the $<50 \mathrm{~nm}$ particle fraction which over increasing doses, was concentration-dependent and reduced viability to sub- $50 \%$ of the negative control at the highest concentration. The $500 \mathrm{~nm}$ fraction of CB presented its first significant response at $400 \mu \mathrm{g} / \mathrm{ml}$ before the viability depleted to $\sim 60 \%$ at $800 \mu \mathrm{g} / \mathrm{ml}$ revealing a striking difference between the nano and bulk fractions. The work by Sahu and colleagues have demonstrated that at very high concentrations of $C B$, far exceeding 100 $\mu \mathrm{g} / \mathrm{ml}$ (the highest used concentration in the present study) there is potential for CB particles to elicit cytotoxic and genotoxic effects, however this is also in conjunction with the primary particle size being sub-50nm in diameter. 
In the present study, the primary particle size as determined in Chapter 3 averaged $129 \mathrm{~nm}$, three times as large as the particles used in the work by Sahu and colleagues 2014, which is indicative of size playing an intricate role on the cytotoxic effects of CB upon THP-1 cells. The d.THP-1 macrophages showed significant IL-8 activation ( $1000 \mathrm{pg} / \mathrm{ml}$ ) across all tested ENMs at $100 \mu \mathrm{g} / \mathrm{ml}$ however only Carboxyl-FLG and CB particles were able to promote significant IL-8 responses at $50 \mu \mathrm{g} / \mathrm{ml}$ after 24 hours of exposure (Figure 5.3). IL-1 $\beta$ was also investigated however no response was observed, this was unexpected as the literature strongly suggests a link between carbon-based ENMs and IL-1 $\beta$ release linked to caspase-1 and the Nlrp3 inflammasome complex of monocytes (Meunier et al., 2012). Since the work by Meunier and colleagues conclusively demonstrated the link between double-walled carbon nanotubes (DWCNTs) and IL-1 $\beta$ release through caspase- 1 and the NIrp3 inflammasome, it is possible the FLG and CB particles do not interact with the d.THP-1 inflammasome complex in the present study. This could be supported given the low d.THP-1 IL-8response between the concentrations of $2-20 \mu \mathrm{g} / \mathrm{ml}$.

\subsubsection{Monocultured TT1 Cytotoxicity and Genotoxicity}

Evaluating the TT1 cell line grown as a monoculture was necessary to provide an indicator of background micronuclei frequency. Consequently, the ability of the ENMs to promote a cytotoxic and genotoxic response was investigated with RPD and the CBMN assay respectively. Following this cell supernatants were harvested for (pro)-inflammatory mediators IL-6 and IL-8. Gaining an understanding of the metabolic function and mitochondrial stress of these cells was also paramount given the nature of the oxidising potential of these ENMs observed in the bronchial cell line used in Chapter 4. As the data suggests, each ENM can induce a weak, but statistically significant reduction in cell viability (Amine-FLG/Carboxyl-FLG >CB $>$ Neutral-FLG). This demonstrated early on that surface functionalisation plays a major role in the interaction of FLG and alveolar epithelial cells. This has been conclusively highlighted in the study by Ruenraroengsak and Tetley (2015) whereby the bioreactivity of three types of polystyrene latex nanoparticles of 50 and $100 \mathrm{~nm}$ bearing neutral, anionic and cationic charges, upon TT1 cells, primary human alveolar macrophages (MAC) and ATII cells was evaluated. The experimental hypothesis stated NP bioreactivity would be greatly dependent on surface chemistry, charge, size as well as functional interactions. The study evaluated several biological endpoints including ROS production, oxidised glutathione levels, mitochondrial membrane integrity, cell morphology and particle uptake over a $1-100 \mu \mathrm{g} / \mathrm{ml}$ concentration range. Amine-NPs induced cell death in all three cell types inducing oxidative stress and mitochondrial disruption through cytochrome c release indicating apoptotic cell death. Unmodified and Carboxyl-modified NPs 
exhibited little cytotoxicity or mitochondrial damage, however ROS was still induced in ATII and MACS (Ruenraroengsak and Tetley, 2015). TT1 cells also showed the most resistance to the effects of unmodified and carboxyl-modified NPs even whilst internalising all three particle types. Two particle sizes were compared in their cytotoxic inducing effects in TT1 cells with the $50 \mathrm{~nm}$ particles bearing a cationic charge resulted in a $40 \%$ viability compared to negative control whilst the particles bearing neutral charge produced an $80 \%$ viability total at $100 \mu \mathrm{g} / \mathrm{ml}$. The $100 \mathrm{~nm}$ particles however only produced a significant cell viability decrease at $100 \mu \mathrm{g} / \mathrm{ml}$ with each particle type eliciting similar trends of concentration-dependent decrease (Ruenraroengsak and Tetley, 2015).

The present study however contradicts this with $\Pi 11$ cells showing greater genotoxic sensitivity to the Amine- and Carboxyl-FLG with earlier significant responses occurring at $8 \mu \mathrm{g} / \mathrm{ml}$, this was not observed in $16 \mathrm{HBE} 140^{\circ}$ cells. Instead $16 \mathrm{HBE} 140^{\circ}$ cells displayed significant genotoxicity to Amine-FLG at $50 \mu \mathrm{g} / \mathrm{ml}$ and no significant response to Carboxyl-FLG. The response in general to the FLG ENMs specifically demonstrates the importance of shape, size and chemical properties. The FLG materials are highly dynamic and range in size and shape which could directly influence their uptake-dependent cytotoxicity through frustrated phagocytosis. Given lung epithelial cells possess immune function, ENM phagocytosis could be possible, and in turn damaged epithelial cells may also provide targets for phagocytosis by neighbouring cells (Monks et al., 2005). The possibility of secondary mediators being transmitted extracellularly is very high given these ENMs are capable of emitting superoxide radicals, Ruenraroengsak and Tetley utilised the DCFH-DA to detect peroxide and singlet oxygen (Ruenraroengsak and Tetley, 2015). They discovered the 50nm amine-modified NPs significantly elevated ROS levels within TT1 cells in a concentration-dependent manner initiated at 4 hours of exposure. The ROS fluorescence detected by the DCFH was maintained over the 24-hour period indicative of significant cell death at higher concentrations. NAC manged to prevent ROS formation in TT1 cells treated with unmodified NPs and carboxyl-modified NPs however only limited protection was offered to TT1 cells treated with amine-modified NPs. The flux of glutathione in healthy cells is vital for maintaining a redox balance, commonly however NP treatments can often offset this healthy balance in favour of oxidants over antioxidants (Ruenraroengsak and Tetley, 2015). 


\subsubsection{Uptake in TT1}

In the present study the mechanism of uptake has not been elucidated however it is believed to be phagocytosis or macropinocytosis. The size of the intracellular vesicles bearing the encapsulated ENMs suggest phagolysosomes $(0.5 \mu \mathrm{m})$ or macropinosomes $(0.2-5 \mu \mathrm{m})$ (Conner and Schmid, 2003). Future work should focus on, classifying and inhibiting these pathways independently with cytochalasin D or amiloride for phagocytosis and macropinocytosis respectively, to more fully understand the uptake mechanism. This future work would require a repeat of the Seahorse bioanalysis in the presence of the targeted uptake inhibitors to test the hypothesis that uptake was responsible for the extra oxygen demands on the cell. TT1 cellular uptake has been characterised in the extensive study carried out by Thorley and colleagues (2014). TT1 cellular uptake was investigated with TEM where previously the authors noted that uptake in TT1 cells was dependent upon passive and active transport at 4 hours of exposure (Thorley et al., 2014). The 50nm latex NPs entered primarily via passive transport in TT1 cells whilst the $100 \mathrm{~nm}$ NPs entered through clathrin and caveolin mediated endocytosis and that aminemodified NPs were internalised more rapidly than unmodified and carboxyl-modified NPs. In the same study by Thorley and colleagues the authors noted that 3-8\% of the unmodified and carboxyl-modified NPs translocated across the monolayer of TT1 cells without interfering with monolayer integrity (Thorley et al., 2014). The authors reported that $40-60 \%$ of TT1 cells internalised NPs at a concentration of $50 \mu \mathrm{g} / \mathrm{ml}$ at which cell viability was respectively high at $90-92 \%$ but also the concentration in which a significant change in mitochondrial membrane potential was observed. This correlates with the ENMs investigated in the present study whereby at $20 \mu \mathrm{g} / \mathrm{ml}$ uptake was confirmed by TEM whilst also displaying cell viability $>90 \%$. Secondly, Thorley and colleagues reported surface functionalization had little effect on the ability of TT1 cells to internalize NPs, again this result was observed in the present study where no difference in uptake was observed from FLG ENMs, all three of which differed in surface chemistry. Lastly, Thorley and colleagues reported were that NPs were located within TT1 vesicles indicating active uptake, another result observed in the present study. ENMs in the present study were internalised successfully by the TT1 cells likely via phagocytosis or macropinocytosis. Since this is (to the authors knowledge) the first attempt to understand TT1 uptake of FLG and CB particles, similar ENMs have been explored which may indicate the type of internalisation mechanism. In a recent study Ruenraroengsak and colleagues assessed the ability of short functionalised MWCNTs to translocate across the pulmonary respiratory epithelial barrier of TT1 and ATII cells. The MWCNTs used in the study measured both 300 and 700nm in length, coated with poly(4-vinylpyridine). The uptake assessment was performed via spectroscopy and quantitative imaging (high resolution TEM). Whist the MWCNTs exhibited no toxicity to the epithelial barrier model, a significant depletion in barrier integrity was noted and uptake of MWCNTs was observed in 
$70 \%$ of TT1 cells scored (Ruenraroengsak et al., 2016). This is highly reflective of the present study which, although not quantitative in design or approach, did reveal a large population of cells which showed interaction or internalisation of FLG regardless of surface charge. The internalized ENMs were always contained within endosomes indicative of endocytic uptake and not passive diffusion into the cytoplasm. Ruenraroengsak and colleagues (2016) also reported 300nm MWCNTs showed significantly greater potential for translocation compared to the $700 \mathrm{~nm}$ variant. Comparatively, only $8 \%$ of ATII cells displayed successful internalization of MWCNTs, which accounted for only $17 \%$ of the total applied concentration (Ruenraroengsak et al., 2016).

\subsection{3 (Pro)-inflammatory Activation}

Under in vivo conditions, the structural alveolar region of the lung is maintained by predominantly ATI cells which form tight junctions (van den Bogaard et al., 2009, Crapo et al., 1982). Aside from providing structural integrity to the alveolar region, AT-I cells also play a role in ion and protein transport acting to regulate lung liquid homeostasis (Dodoo et al., 2000). A major problem with in vitro-modelled alveolar cell lines is their inadequacy in forming tight polarised junctions which closely mimic in vivo tissue (Blank et al., 2006, Koslowski et al., 2004, Luhrmann et al., 2007), (Steele et al., 1992). For this reason, many research groups utilise primary AT-II cells and await their morphological and phenotypic change into AT-I cells, however this can be time-consuming, expensive and reliant on patient surgery and ethical commitments, hence the importance of the TT1 cell line. In the study by van den Bogaard and colleagues, $\mathrm{T} 1$ cells were evaluated for their use in toxicity assays by challenging the cells seeded at i) varying densities, ii) with incremental LPS concentrations and iii) in liquid and air cultures (van den Bogaard et al., 2009). The IL-6, IL-8, TNF- $\alpha$ and antiproteinases (secretory leukoprotease inhibitor (SLP1) and elafin) were then quantified and these results compared against the performance of A549 cells. The barrier formation was monitored by TEER measurements. TT1 cells were stimulated with $0.1,1,10$ and $100 \mathrm{ng} / \mathrm{ml}$ of LPS and the formation of a tight monolayer was monitored by measuring TEER as a function of time 1 hour after equilibration after which the permeability properties of the cell layer were stable as detailed by (Grainger et al., 2006). TT1 cells released significantly more IL-8 upon LPS activation and responded similarly with A549 cells in serum rich media. Both cell lines showed a dose-dependent release of cytokine/chemokine and were sensitised by serum in the media and TT1 cells did not secrete SLP1 or elafin under any experimental condition whilst LPs stimulation of A549 cells did secrete these markers. This result concludes that TT1

cells may be used to evaluate toxicology of inhaled particles under in vitro conditions, but further development is required on the cell line itself. Optimisation of better culture conditions and 
maintaining a low passage number is paramount (van den Bogaard et al., 2009). Comparing data to the present study, the FLG and CB particles showed a similar level of IL- 6 activation as Grainger and colleagues reported with protein levels reaching $\sim 450 \mathrm{pg} / \mathrm{ml}$ at the highest concentration of $100 \mu \mathrm{g} / \mathrm{ml}$ (Grainger et al., 2006). Numerous ENMs have been linked with the activation of TLR receptors in monocytes and macrophages (Meunier et al., 2012, Qu et al., 2013) respectively. It has been proven that internalised PEGylated reduced graphene oxide (PrGO), one within A549 cells, triggered NF-k $\beta$ activation. In the same study, reduced graphene oxide ( $r G O)$ which did not enter the alveolar cells interacted with TLRs at the membrane surface again triggering NF-k $\beta$ activation. Immunohistochemistry of A549 cells exposed to PrGO at 25 and $50 \mu \mathrm{g} / \mathrm{ml}$, the intensity of NF-k $\beta$ in the cell nucleus was found to be enhanced suggesting ROS involvement, quite a probable link to the concentration-dependent immune activation seen in the present study (Reshma et al., 2016).

It is likely that the activation of TLRs by FLG and CB particles activated myeloid differentiation factor (MyD88) contributing largely to the (pro)-inflammatory response observed in the following manner. At low concentrations $2-8 \mu \mathrm{g} / \mathrm{ml}$ extracellular FLG and CB particles enter the cells via phagocytosis and macropinocytosis, this enhances the ROS production via the mitochondria and contributes to the activation of intracellular NF-k $\beta$ which diffuses into the nucleus to upregulate mRNA for (pro)inflammatory mediators IL-6 and IL-8. At higher concentrations where the cell may have exhausted ATP stores and uptake is no longer possible or at least diminished, the ENMs at the extracellular cell surface bind to TLR4 signalling the MyD88-TLR pathway again inducing NF-k $\beta$ activation and upregulated transcription of mRNA for IL-6 and IL-8. This signalling pathway was first demonstrated by Chen and colleagues using RAW 264.7 during which GO promoted TLR4/9 downstream signalling to MyD88 and TNF associated factor-6 (TRAF6) (Chen et al., 2012b). This was demonstrated secondly by Capasso and colleagues in 2014 (Capasso et al., 2014). Capasso and colleagues used BEAS-2B cells exposed to nickel NPs to investigate the activity and signalling of NF-k $\beta$. The authors reported signalling was dependent upon mitogen activated protein kinases (MAPK). Furthermore, the effects were duplicated in A549 cells with the only exception in the results being that whilst A549 cells showed uptake and signalling, the BEAS-2B cells showed little-to-no uptake and were also immune activated (Capasso et al., 2014). 


\subsubsection{ENM effects upon Co-Cultured Cells}

To determine the action of secondary genotoxicity the alveolar co-culture was constructed using TT1 and d.THP-1 macrophages in a 10:1 ratio adapted from the original Rothen-Rutishauser and colleagues (2005) model. To date, there has been substantial effort in the development of advanced cell models such as lung-on-a-chip, and multicellular cultures (Choi et al., 2016, Blom et al., 2016). These models however, often encompass low reproducibility are time-consuming and require expertise where trior quad cell-culture models are concerned. Whilst the aim in lung nanotoxicology would be to model the entire respiratory tract, this still pertains to over 40 cell types and realistically cannot be achieved with current technology and hazard endpoint applications with any measure of certainty or reproducibility. To assess the quality of the co-culture structural integrity in the present study, confocal microscopy was utilised to display fluorescent properties of the actin cytoskeleton stained with Phalloidin 633, identifying cell-cell boundaries. Confocal imaging in the present study revealed differentiated THP-1 cells (displaying FITC fluorescence) not just bound to the apical side of the epithelial monolayer but also growing between the alveolar cells indicating similarities to in vivo cellular architecture. Any areas of excess FITC fluorescence identified with confocal microscopy may also be evidence of a trans-epithelial network being established between the differentiated macrophages and the TT1 monolayer, this could have been aided by cytoplasmic mechanisms. One issue which was avoided through careful addition of the macrophages to the $\Pi 11$ cells established on the transwell insert was the d.THP-1 cells forming small colonies, an issue highlighted in the work by Klein and colleagues where small clumps of differentiated THP-1 cells formed upon A549 cells (Klein et al., 2013). In comparison to monocultured TT1 cells, the addition of d.THP-1 macrophages appeared to have little effect on the background frequency of \%Mn/BN, however upon addition of ENMs to the co-culture a noticeable elevation in \% $\mathrm{Mn} / \mathrm{BN}$ was observed. When co-cultured, each ENM displayed a similar DNA-damaging potential with all but Carboxyl-FLG inducing initial significant responses at $10 \mu \mathrm{g} / \mathrm{ml}$ elevating the $\% \mathrm{BN} / \mathrm{Mn}$ to approximately $3.5 \%$. Crucially no ENM induced significant cytotoxicity however the overall increase in micronuclei frequency when compared to monocultured cell lines is clearly higher which could suggest secondary mechanisms of genotoxicity, as initially hypothesised. Recently, Evans and colleagues investigated secondary mechanisms of genotoxicity with two types of superparamagnetic iron oxide $\left(\mathrm{Fe}_{2} \mathrm{O}_{3}\right.$ and $\left.\mathrm{Fe}_{3} \mathrm{O}_{4}\right) \mathrm{NPs}$ (SPIONs). In this study, primaryindirect genotoxicity was observed only with the $\mathrm{Fe}_{2} \mathrm{O}_{3}$ exposed to $16 \mathrm{HBE}^{-140^{-}}$cells, however when cocultured following the same SOP as used in the present study, both SPION types promoted genotoxicity, indicative of secondary mechanisms (Evans et al., 2019). In the present study, primaryindirect genotoxicity was however noted in the 2D monoculture model, likely because of oxidative stress. The co-culture genotoxicity data exhibited an elevated \%Mn/BN over monocultured TT1 
$\% \mathrm{Mn} / \mathrm{BN}$ following ENM exposures, thereby indicating the role of both primary-indirect oxidative stress coupled to secondary mechanisms of genotoxicity. Further experiments evaluating the genotoxic impact of each ENM upon d.THP-1 cells could help provide more clarity on this conclusion.

Secondary mechanisms are crucial to highlight due to the prevailing in vivo genotoxicity being caused through specific cell-to-cell interplay following immune cell recruitment (Evans et al., 2017, Downs et al., 2012). Whilst oxidative stress can be observed in both primary-indirect and secondary genotoxicity, in the latter it is a result of inflammatory mediators released by immune cells resulting in the oxidative response from surrounding epithelial cells. Numerous secondary mediators can be attributed to this response however given macrophages act through reactive nitrogen species in the removal of pathogenic infection, NADPH oxidase and RNS are likely to be responsible in the present study. The data from the NAC-treated cultures revealed no statistically significant micronuclei frequencies at any tested concentration for any ENM. While many modes of action have been proposed for NAC, it seems likely the prevailing factor in this study may have been its influence and inhibition of p38 MAP kinase and subsequent signalling to NF-k $\beta$ transcription factors (Zafarullah et al., 2003). This would in turn down-regulate the expression of key (pro)inflammatory mediators IL-6 and IL-8. This overarching depletion in the (pro)-inflammatory response would therefore have a knockon effect limiting the chronic response, especially over a short 24-hour period whereas a longer incubation or exposure time could test this theory. Respiratory burst could be one of many oxidative stress mechanisms playing an integral role in the increased genotoxicity of co-cultured $T T 1$ cells, one of the sources of this phenomena is reactive oxygen species, specifically NADPH oxidase (NOX2) however there have been strong indicators that other sources of oxygen radicals sourced from the mitochondria play a role in phagocytic respiratory burst in vitro (Vorobjeva et al., 2017). Whilst respiratory burst is typically isolated to neutrophils it is clear NOX2 could provide a useful target for future investigations into secondary genotoxicity (Abrikossova et al., 2012). This small cyclic process of oxidative stress and a (pro)-inflammatory response coupled with the bio-persistence of the test ENMs may lead to irreversible inflammation over a period greater than 24 hours. A second oxidative stress mediator influenced by (pro)-inflammatory mediators is nitric oxide synthase which can be stimulated and released in epithelial cells triggered by TNF $\alpha$ and IL-1 $\beta$, however given that suspension THP-1 cells did not express IL-1 $\beta$ during the monoculture exposures it seems unlikely that IL-1 $\beta$ would play a large role in this scenario (Robbins et al., 1997). 


\subsection{Chapter 5 Conclusions}

The work presented in Chapter 5 has explored the potential of industrially-relevant ENMs to promote secondary genotoxic mechanisms in an alveolar co-culture of TT1/d.THP-1 cells. Each test ENM was able to induce primary-indirect genotoxicity within monocultured TT1 cells, upon investigating the coculture model the $\% \mathrm{Mn} / \mathrm{BN}$ frequency became elevated from monocultured TT1 cells demonstrating secondary genotoxic mechanisms. This response would therefore be attributed to an alreadyestablished primary-indirect genotoxic response being heightened in the co-culture model by an immune response from the d.THP-1 macrophages. Oxidative stress was strongly linked to the ENM genotoxicity, demonstrated by the pre-incubation of the co-culture with $1.5 \mathrm{mM}$ of NAC which reduced the $\% \mathrm{Mn} / \mathrm{BN}$ to non-significant levels. Uptake was confirmed in monocultured TT1 cells with TEM/STEM and whilst uptake could not be confirmed within the alveolar co-culture (due to sectioning issues), it is believed to have occurred in d.THP-1 macrophages rather than in co-cultured TT1 cells. To elucidate the presence of secondary genotoxicity further, an extensive investigation into the (pro)inflammatory mediators would be required to better understand the expanse of activation within the co-culture which contributed to the observed genotoxic response. One such method to investigate the role of secreted (pro)-inflammatory mediators from d.THP-1 cells upon epithelial cell genotoxicity would be the conditioned medium approach, pending an appropriate methodology to remove the ENMs from the cell supernatant prior to transfer. 


\section{Chapter 6 General Discussion}

The ENMs investigated in the present study are intended to enhance consumer and industrial products (conductive inks, reinforced nanocomposites, flexible touchscreen technology) in the coming future. Consequently, due to the increasing demand and increased population occupational risk is therefore a priority. Recent findings have revealed that occupational exposure to ENMs including titanium NPs in a manufacturing plant in China resulted in cardiopulmonary effects within 83 exposed workers compared to 85 workers in the control group. Lung biomarkers such as lung surfactant protein-D (SPD) and malondialdehyde (MDA) as well as inflammatory markers were all significantly affected in a time- and dose-dependent pattern (Zhao et al., 2018). Zhao and colleagues concluded that occupational pulmonary assessments may provide useful health surveillance for workers exposed to ENMs.

A common factor in nanotoxicology publications is the rigorous characterisation of ENM physicochemical features. These features of the ENMs divided into primary (e.g. size distribution, shape, surface area, and surface charge) and secondary characteristics (how the primary characteristics have changed upon suspension in a diluent (typically serum-containing media)) must undergo evaluation. The physico-chemical features of ENMs are highly dynamic and so noting the characteristics of both the primary and secondary features is crucial to understanding their behaviour in vitro and in vivo. The primary physico-chemical characteristics will likely change when suspended in water or cell culture media, ultimately warranting the characterisation of novel ENMs whilst suspended in cell culture media where possible. One issue faced with modern characterisation techniques of secondary features is the suitability of the technique, i.e. does the technique provide a means of accurately describing one specific physico-chemical feature of the ENM. For instance, graphene-based ENMs do not obey the mathematical laws of spherical particles which underpin DLS analysis; however DLS remains the go-to technique for rapid hydrodynamic diameter predictions in nanotoxicology (Bengtson et al., 2016, Muthoosamy et al., 2015). DLS of ENMs however, remains a powerful tool for screening hydrodynamic diameters of agglomerates. This has been highlighted by Wills and colleagues whereby the authors report that whilst the absolute quantification of agglomerate state is difficult to reach with DLS, the technique does identify 'tipping points' in agglomeration states which informs the dose-response. DLS data can often be misinterpreted due to high biomolecule concentrations masking the ENMs present at low concentrations (Wills et al., 2017). These limitations can be overcome with competing or complimentary techniques again highlighting the importance of a multi-modal approach to ENM characterisation. ENM characterisation ultimately aims to combine several synergistic 
technologies to generate supportive data to provide a better understanding of the overall picture. Following comprehensive physico-chemical characterisation, toxicity testing may begin utilising a relevant cell line, often pertaining to the most likely exposure site of the human body. In vitro studies are preferred in the current climate of scientific endeavour to avoid the use of animal models. This can be achieved through the construction of more complex in vitro cell models whereby multiple cell lines are cultured to represent in vivo tissues. These cultures can either permit direct cell-cell contact or grown near to resemble in vivo tissue, architecture and cell-cell signalling (Evans et al., 2017). This work aimed to assess the in vitro cytotoxic and genotoxic potential of Neutral-, Amine-, Carboxyl-FLG and $C B$ particles in relation to respiratory models focusing on the bronchial and alveolar region. The observed toxicity was then investigated further focusing upon oxidative stress, (pro)-inflammatory responses and mitochondrial stress. Furthermore, how the primary and secondary physico-chemical characteristics (evaluated with a multi-modal approach) may have contributed to the genotoxic effects.

The initial focus of this thesis was to characterise the physico-chemical features of Neutral-, Amine-, Carboxyl-FLG and CB particles. This characterisation process was undertaken in Chapter $\mathbf{3}$ of this thesis and aimed to analyse both primary and secondary features of the ENMs utilising several key techniques such as; DLS, BET, AFM, Raman spectroscopy and plunge-freeze SEM. Within Chapter 3, there was also dosimetry data to provide an understanding the behaviour of the graphene-based ENMs in an exposure test system. This simple model offered an insight into the ENM sedimentation and reveal how much, if any, of the administered concentration of ENMs would reach the adherent cell lines. The rest of the methodologies then offer competing data as to the actual size of the ENM particle size and agglomerate sizes, which lead to the conclusion that when performing physicochemical characterisation on novel ENMs a panel of techniques is required which are relevant to the ENM under investigation. Following the comprehensive characterisation of ENM physico-chemical features, the potential of these ENMs to promote cytotoxicity and genotoxicity was evaluated with monocultured bronchial epithelial (16HBE140-) cells in Chapter 4 of this thesis. Additionally, a deeper investigation into the role of oxidative stress was conducted, a key process in the promotion of toxicity of ENMs. Mitochondrial stress, (pro)-inflammatory mediators and glutathione were therefore measured following a 24-hour exposure period. Cellular uptake was evaluated using TEM to understand if these ENMs were capable to being internalised by the cells. Chapter $\mathbf{5}$ of this thesis assessed the cytotoxic and genotoxic impact of each test ENM upon an advanced co-culture model of TT1/d.THP-1 cells. The alveolar co-culture model with full cell-to-cell contact between TT1 and d.THP1 cells could therefore depict a more representative in vivo structure. Therefore, this model could 
incorporate the detection of secondary genotoxic mechanisms, a process which is overlooked in monoculture models.

Physico-chemical characterisation data obtained in Chapter 3 revealed differences between the ENMs agglomerate size. Neutral-FLG appeared approximately half the size of Amine-FLG and Carboxyl-FLG (lateral diameter) when suspended in cell culture media measured by plunge freeze SEM. Whilst each ENM was shown to be endotoxin-free, they all had a unique chemical constituency with fluctuating levels of oxygen and nitrogen saturation. Perhaps the most striking difference were the proteins found attached to the surface of the ENMs. For the FLG materials contained nine proteins whilst the CB particles contained just one corona protein, likely BSA. SEM images following plunge-freezing revealed FLG agglomerates to be similar in agglomerate structure regardless of surface charge. FLG particles dispersed in water appeared more jagged at the edge asperities whereas particle edge asperities; when suspended in $10 \%$ supplemented culture media each FLG material appeared smoother. NeutralFLG whilst having the smallest agglomerate diameter $583.7 \mathrm{~nm}$ appeared to have the greatest depth of the FLG materials with an average of $94.73 \mathrm{~nm}$. Neutral-FLG was also revealed to possess the greatest surface area of $55.2 \mathrm{~m}^{2} / \mathrm{g}$; these features may have contributed to the observed genotoxicity in Chapter 4 with 16HBE140 cells given that Neutral-FLG induced the highest level of micronuclei of each FLG material. Whilst uptake was not quantified for each ENM under investigation, it is reasonable to assume the smaller agglomerate size of Neutral-FLG would have facilitated uptake more so than the larger sized agglomerates of Amine- and Carboxyl-FLG, demonstrated in the work by Zhang and colleagues (Zhang et al., 2009). Amine-FLG whilst appearing genotoxic at high concentrations of 50 and $100 \mu \mathrm{g} / \mathrm{ml}$ may have promoted a genotoxic response through a different mode of action which can be hypothesised through the EPR data acquired in Chapter 3. As the theory put forward by Li and colleagues in 2018, a hydrated graphene oxide structure can contain carbon radicals (Figure 6.1) (Li et al., 2018b). Hence, the Amine-FLG used in the present study may promote superoxide radical formation, causing heightened oxidative stress through the chemical structure containing carbon radicals. The surface reactivity of the carbon radical could also play a crucial role in the mechanisms behind the observed genotoxic response elicited by Amine-FLG, introduced via hydration of graphene ENMs. 


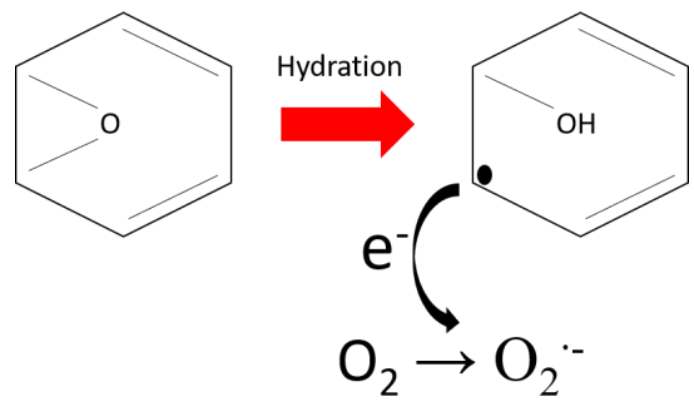

Figure 6.1 Hypothesised chemical structure of Amine-FLG. Image adapted from Li and colleagues ( $\mathrm{Li}$ et al., 2018b).

When suspended in media and introduced into a cellular environment, carbon radicals have the potential to facilitate superoxide production through the donation of an electron (Li et al., 2018b). The site at which this process would manifest strongly, as described by Li and colleagues, would be lipid peroxidation at the plasma membrane ( $\mathrm{Li}$ et al., 2018b). This process of lipid peroxidation can ultimately contribute to oxidative stress. Whilst not investigated in the current study, lipid peroxidation is a common theme among particle toxicology publications (Ayala et al., 2014, Schins, 2002, Alarifi et al., 2014). The production of MDA could provide an ideal future experiment for the Amine-FLG evaluated in the present study given how $\mathrm{Li}$ and colleagues reported a strong MDA production from hydrated graphene ENMs.

Carboxyl-FLG interestingly did not promote significant $(p<0.05)$ genotoxicity in $16 \mathrm{HBE}^{-140^{-}}$cells however it was not chemically inert as it significantly elevated IL-8 levels, depleted GSH and had the strongest effect upon mitochondrial metabolic function. Carboxyl-FLG appeared to be the fastest acting of all tested ENMs with regards to ROS production in cells with peak levels appearing at 1 hour of exposure, as compared to Neutral-, Amine-FLG and CB particles which presented maximal ROS production near the 2-hour time point. The effects of oxidative stress induced by carboxyl-graphene ENMs have been published prior to this study, with some studies reporting oxidative stress and cellular damage with low-level toxicity (Lammel et al., 2013, Guo and Mei, 2014b). The carboxyl groups appear therefore to offer some form of antioxidant biocompatibility where in vitro exposures are concerned. In the present study, the ATP depletion by Carboxyl-FLG may also represent the $16 \mathrm{HBE}^{-14 \mathrm{o}^{-}}$cells favouring this material over the others for cellular uptake, with the greater degree of uptake then contributing to a greater depletion in ATP. 
CB particles meanwhile, with only one serum protein at the corona interface, expectedly showed the smallest degree of uptake and yet elevated micronuclei to significant levels. However, as observed with EDX and ROS generation via the DCFH-DA assay, CB particles have a large oxygen saturation percentage which contributed largely to metabolic function depletion, measure using the Seahorse XFe24 bioanalyzer. How exactly the CB particles cause oxidative stress is currently unknown however isolating the Mn-SOD enzyme and investigating its activity may provide answers in a future investigation. Given the chain reaction displayed in Figure 6.2 there are numerous potential enzymes which could be affected by CB particle exposure. Chapter 4 indicated that from monitoring the GSH level depletion indicates the $G_{P x}$ enzyme functions adequately, therefore, an oversaturation or damaged Fe-catalase enzyme would result in $\mathrm{H}_{2} \mathrm{O}_{2}$ accumulation and subsequent oxidative stress. The other possibility being the oversaturation of Mn-SOD leading to an accumulation of superoxide radicals, which is likely the case here as each ENM has been shown to promote this radical by EPR. The ENMs in the present study may therefore be imparting oxidative stress and genotoxicity in specific mechanisms (Figure 6.2) based upon their physico-chemical features as originally hypothesised; however, to clearly elucidate each pathway some further experiments could be performed to confirm this theory.

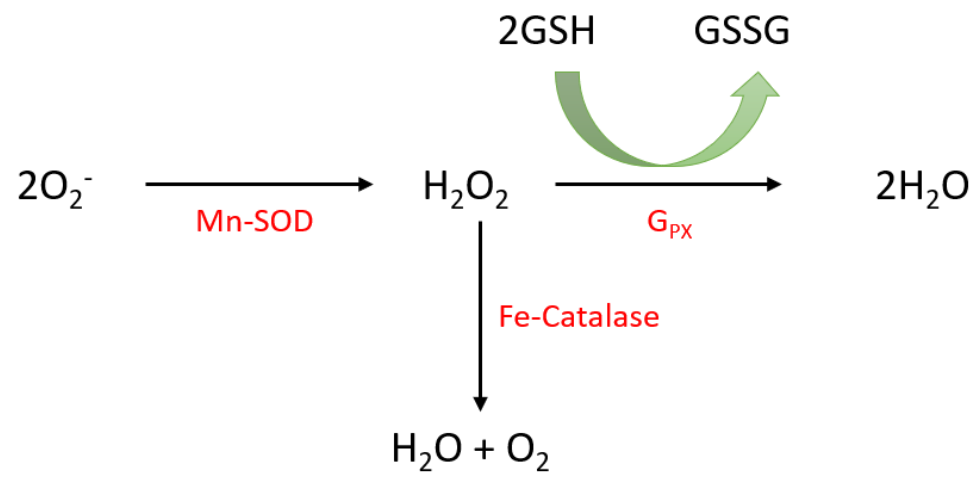

Figure 6.2 Mitochondrial enzymatic reactions. Carboxyl-FLG and CB particles are hypothesised to act within this series of enzymatic reactions linked to the oxidant: antioxidant balance within the cells.

Cytotoxicity was evaluated with d.THP-1 cells as an indicator of how immune cells would respond to 24-hour exposure to the ENMs. The graphene ENMs all promoted significant cytotoxicity to d.THP-1 cells whilst CB particle exposures did not invoke a cytotoxic response over the concentration range 0$100 \mu \mathrm{g} / \mathrm{ml}$. The cytotoxic effects of graphene oxide nanoparticles on murine macrophages coupled to IL-6 elevation have been observed in the literature by Lategan and colleagues (Lategan et al., 2018). In their investigation, IL-6 production was not concentration-dependent and only spiked significantly 
at 15.6 and $31.25 \mu \mathrm{g} / \mathrm{ml}$ whilst cytotoxicity reached significant levels at $62.5 \mu \mathrm{g} / \mathrm{ml}$. In the present study the (pro)-inflammatory response of THP-1 cells was quantified with ELISAs for IL-8 and IL-1ß, which are commonly expressed mediators of inflammation. IL-8 revealed only significant activation at high concentrations of 50 and $100 \mu \mathrm{g} / \mathrm{ml}$; in contrast IL-1 $\beta$ production was not observed. This is in contradiction to a recent study by Armstead and Li in which the authors exposed macrophages and lung A549 cells to tungsten carbide-cobalt (WC-Co) NPs and discovered an elevation in IL-1 (Armstead and $\mathrm{Li}, 2016)$. The interaction of $\mathrm{GO}$ nanosheets with macrophages was also investigated by Mukherjee and colleagues in 2018 whereby IL-1 $\beta$ expression was reported to be related to caspase signalling, a trademark of inflammasome activation (Mukherjee et al., 2018). The lack of IL-1 $\beta$ expression in the present study may therefore hint at a different inflammatory activation pathway which is independent of the inflammasome complex. This has been investigated by Hoyle and colleagues recently in 2018 whereby the authors demonstrated the ability of small-thin GO dosedependently inhibited the release of IL-1 $\beta$ and IL-6 in macrophages (Hoyle et al., 2018).

Upon investigating the cytotoxic and genotoxic impact of each ENM in TT1 cells, there was an immediate difference in the observed toxicity when compared to $16 \mathrm{HBE} 140^{-}$cells. TT1 cells demonstrated greater sensitivity to the ENMs with all materials promoting significant cytotoxicity and genotoxicity, initially appearing at relatively low concentrations of $8 \mu \mathrm{g} / \mathrm{ml}$. This suggests that a genotoxic response could be promoted regardless of physico-chemical characterisation, which differs to the response with $16 \mathrm{HBE} 14 \mathrm{O}^{-}$cells, whereby the bronchial epithelial cell line showed greater sensitivity to CB and Neutral-FLG exposures. This effect was supported largely by the extracellular concentration of (pro)-inflammatory mediators of both cell lines. The 16HBE140 cells showed lowlevel IL-6 activation following both 6 and 24-hour exposures to ENMs and significant IL-8 levels were only observed at 16 -hours of exposure to 50 and $100 \mu \mathrm{g} / \mathrm{ml}$ concentrations. TT1 cells however, following a 24-hour exposure to ENMs displayed concentration-dependent increases in both IL-6 (approximately 500 pg/ml) and IL-8 (approximately $1500 \mathrm{pg} / \mathrm{ml}$ ) initially at 10 and $20 \mu \mathrm{g} / \mathrm{ml}$ for each (pro)-inflammatory mediator respectively. The (pro)-inflammatory profiles for all test ENMs following TT1 exposure also follows the results observed by Ruenraroengsak and Tetley (2015) in which the authors exposed polystyrene NPs of different charge; neutral, anionic and cationic NPs to relevant lung cell types; AT2, TT1 and alveolar macrophages. The authors noted no difference in IL-6 and IL-8 between the ENMs and that all tested NPs produced respective IL-6 and IL-8 levels of 2000 and $3000 \mathrm{pg} / \mathrm{ml}$ (Ruenraroengsak and Tetley, 2015). Thus, and as shown in the present study the TT1 cells appear to show equal sensitivity to the test particle and do not discriminate according to surface charge. This effect appears therefore to carry over from spherical polystyrene NPs to sheet-like 
graphene ENMs. In conjunction with (pro)-inflammatory mediator release, Ruenraroengsak and Tetley also reported stronger cytotoxicity via cytochrome $\mathrm{C}$ release and apoptotic cell death with aminemodified polystyrene NPs compared to unmodified and carboxyl-NPs. This corresponds to observations in the present study where Amine-FLG promoted the highest significant genotoxic response with a $3.15 \% \mathrm{Mn} / \mathrm{BN}$. The ENMs in the present study were also evaluated for their capacity to induce oxidative stress upon TT1 mitochondria whereby each ENM displayed similar potency; Ruenraroengsak and Tetley however report little effect of unmodified and carboxyl-NPs upon mitochondria function whilst each test ENM induced ROS (Ruenraroengsak and Tetley, 2015). The only striking difference between the ENM exposures on both 16HBE140 and TT1 monocultures were the mitochondrial and bioenergetic profiles. ENMs reduce OCR/ECAR and metabolic function in 16HBE140 cells whilst in TT1 cells the opposite effect was observed. TT1 cells also displayed a smaller energetic profile (OCR) compared to the $16 \mathrm{HBE}^{-140^{-}}$cells however this may be attributable to the difference in seeding density or the number of mitochondria differing between cell lines. Given the difference, it could be assumed that the two cell lines have distinct mechanisms of coping with the ENM exposures. Testing this hypothesis could form an excellent future study by including endocytosis inhibitors (Cytochalasin D, Amiloride, Chlorpromazine, Filipin) which could selectively inhibit pathways of uptake in both cell lines. By pre-incubating the cells with an inhibitor of each endocytic pathway, the ATP depletion could then be monitored in the standard Seahorse XFe24 bioanalyzer thus proving if ENM uptake into the cells is the cause of the energetic demand on the cells. The confirmation of graphene ENM uptake has been recently investigated in the study by Xu and colleagues (2018), in which the authors exposed aminated graphene quantum dots (AG-QDs) at 50, 100 and $200 \mu \mathrm{g} / \mathrm{ml}$ to rat alveolar macrophages (NR8383). To monitor the active routes of ENM internalisation, the authors utilised; $\mathrm{NaN}_{3}(3 \mathrm{mM})$, genistein $(200 \mu \mathrm{M})$, chlorpromazine $(10 \mu \mathrm{g} / \mathrm{ml})$, amiloride $(50 \mu \mathrm{g} / \mathrm{ml})$ and cytochalasin $\mathrm{D}(10 \mu \mathrm{M})$ to selectively inhibit; energy-dependent endocytosis, caveolae-dependent uptake, clathrin-dependent uptake, macropinocytosis and phagocytosis respectively. The authors reported a significant reduction in fluorescence within NR8383 cells following the inhibition of energydependent endocytosis, phagocytosis and caveolae-dependent uptake indicative of those being the most prominent uptake routes (Xu et al., 2018). Other than ENM uptake, oxidative stress is the likely cause in the observed genotoxic response for which numerous enzymes can be investigated as alluded to previously such as Mn-SOD and Fe-catalase (Figure 6.3). 


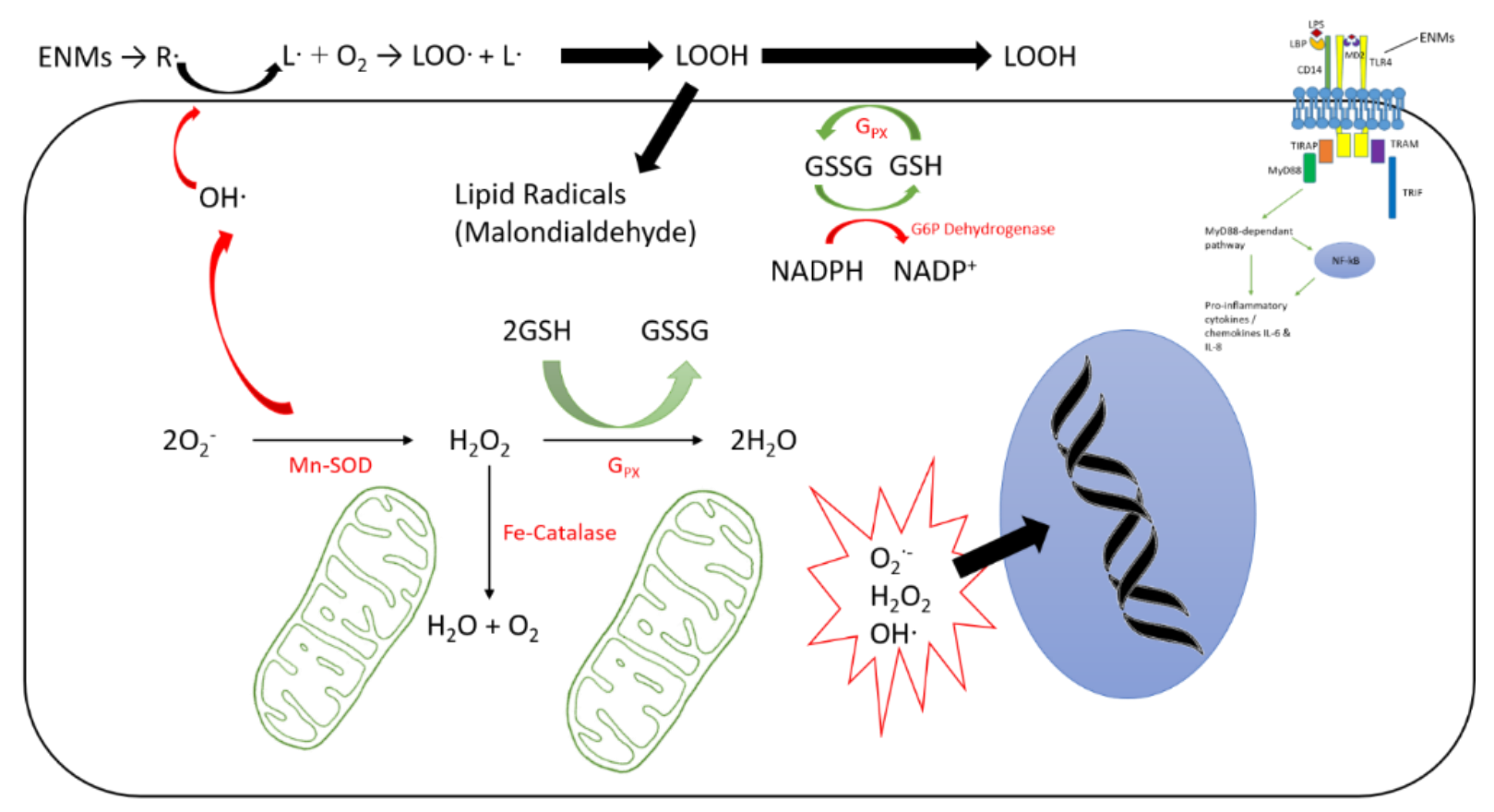

Figure 6.3 Potential mechanisms contributing to the genotoxicity observed in monocultured 16HBE140 and TT1 cells. A gradient will exist between the extracellular and intracellular space, thus promoting radical attack on the lipid membrane. Lipid radicals $\left(L^{\cdot} \cdot\right)$ together with $\mathrm{O}_{2}$ form Lipid Peroxy Radicals (LOO·), combining with excess lipid radicals then generates Lipid Hydroperoxide (LOOH). From here $\mathrm{G}_{\mathrm{PX}}$ can catalyse the conversion of $\mathrm{LOOH}$ into a stable Lipid Alcohol (LOH), depleting GSH and reducing the electron carrier NADP'. The binding of ENMs to TLRs on the surface membrane meanwhile triggers a series of phosphorylation activating NF-kB resulting in the expression of key (pro)-inflammatory mediators such as IL8. Superoxide generated by the ENMs in excess (particularly Neutral- and Carboxyl-FLG), plays a key role in the perturbation of mitochondrial function and overall depletion in GSH with Mn-SOD likely becoming over saturated, producing $\mathrm{OH} \cdot$.

Given that monocultured cell lines do not permit the detection of secondary genotoxic mechanisms, it was crucial to investigate the potential of each test ENM in the present study to promote secondary genotoxicity. To investigate secondary mechanisms of genotoxicity a minimum of two cell lines were required; TT1 cells and the second, differentiated THP-1 macrophages. This setup would therefore permit secondary signalling of (pro)-inflammatory mediators and important cell-to-cell interplay. Since data also existed prior to co-culture exposures pertaining to both cell types exposed to ENMs as monocultures, comparisons could be drawn between the data sets. Given that oxidative stress has played such a large role in both epithelial cell lines biological responses thus far, it was essential to investigate oxidative stress within the co-culture system. As detailed by Evans and colleagues in 2017, 
the construction of relevant lung co-cultures permits the transfer of (pro)-inflammatory mediators and highly reactive radicals between cell types (Evans et al., 2017). ENM exposure concentrations were reduced in the interest of time and as a result, significant genotoxicity was observed initially at $10 \mu \mathrm{g} / \mathrm{ml}$ for Neutral-, Amine-FLG and CB particles whilst the LOGEL for Carboxyl-FLG was $20 \mu \mathrm{g} / \mathrm{ml}$. The \%Mn/BN in TT1 cells co-cultured with differentiated THP-1 macrophages was also slightly elevated when compared to monocultured TT1 cells, rising from a maximum value of $3.15 \%$ to $4 \%$ in the coculture following CB exposure. Interestingly, no significant cytotoxicity was observed in the co-culture over the concentration-range applied, whilst monocultured $\Pi 11$ cells exhibited some degree of cytotoxicity which was consistent with the works by Ruenraroengsak and Tetley (2015). Whether or not the incorporation of differentiated THP-1 cells alleviated the TT1 cytotoxicity through macrophage-phagocytosis of the ENMs is unclear, however that remains the prevailing hypothesis in the co-culture model of this study.

The role of oxidative stress in the co-culture model may however involve the transfer of secondary inflammatory mediators; NADPH and NO. As described in the publication by Tan and colleagues, macrophages once activated by pathogens trigger the bactericidal response which produces NO, reliant upon the Nox2 gene (Tan et al., 2016). This process of ROS and NO production by NADPH oxidase and NO synthase relies heavily upon the pentose phosphate pathway (Tan et al., 2016). This systemic signalling of ROS and NO may be the source of the elevated genotoxic response observed in the co-culture model as compared to monocultured TT1 cells which would be absent of immuneactivated ROS and NO signalling. NO has been shown to be mutagenic both in vitro and in vivo and heavily relies upon the activation and subsequent signalling through p53 and the mitochondria (Li et al., 2002). The latter of which has been heavily affected by the ENMs under investigation in the present study, through both an up- and down-regulation in OCR and ECAR. Whilst NO signalling has not been fully elucidated, p53 activation and subsequent cellular apoptosis has been hypothesised in the literature (Messmer and Brune, 1996). Mitochondrial function including the translocation of cytochrome $\mathrm{C}$ has also been referenced with caspase activation through protease activating factor-1 (Apaf-1) (Li et al., 2002, Hortelano et al., 1997). NO does indeed possess immune function, at low levels in immune cells it has roles in immunoregulation such as moderating inflammation, immunesuppression and tissue restoration. Overproduction of this radical however can contribute to chronic inflammation, a hallmark of secondary genotoxicity (Li et al., 2002). One final possibility of oxidative stress contributing to the elevation in micronucleus frequency (and thereby chromosomal damage) would be the complexing of NO with other biomolecules such as superoxide. NO and superoxide may react to form peroxynitrite (ONOO-) this newly formed molecule can be stabilised however by tyrosine 
residues present in proteins resulting in nitrotyrosine. Testing this hypothesis may in fact be plausible in future experiments. NO can be converted into stable end products, those being nitrite and nitrate $\left(\mathrm{NO}_{2}{ }^{-}\right.$and $\left.\mathrm{NO}_{3}{ }^{-}\right)$which can be measured using fluorometric assays (Dekhuijzen and van Beurden, 2006). Lung co-cultures have become exceedingly common for screening ENM toxicity in vitro, in a recent publication by Fukai and colleagues (2018) the toxicity of MWCNTs were assessed in a co-culture of murine lung resident (GDL1) cells and RAW 264.7 macrophages. The authors reported the mutation frequency was greater in co-cultures than they were in monocultured GDL1 cells, with IL1- $\alpha$ and IL-1 $\beta$ produced by the RAW 264.7 cells in the co-culture. The frequency with which the DNA adduct 8oxodeoxyguanosine in GLD1 cells co-cultured with immune cells was greater than monocultured lung cells indicative of secondary mechanisms which was confirmed with a strong elevation in ROS (Fukai et al., 2018).

Whilst the mechanisms of NAC redox are unclear, it is reasonable to assume superoxide radicals would provide an obvious choice given it is consistently produced via oxidative phosphorylation in healthy cells (Kerksick and Willoughby, 2005). Oxidative stress is a complex process, often cross-linked with numerous cellular processes however NAC, a biproduct of glutathione metabolism, is believed to be central to immune regulation, antioxidant defence, cellular proliferation and membrane transporters and receptors (Kerksick and Willoughby, 2005). NAC and glutathione in particular, could be playing a pivotal role in the observed genotoxic response of the co-culture model given that NF-kB may be upregulated in response to oxidative stress, and NF-kB is a well-established redox-sensitive transcription factor (Sen and Packer, 1996, Sen, 1998). Whilst uptake into the nucleus often depends on extremely small size $(<5 \mathrm{~nm})$, it is doubtful the ENMs investigated in the present study would access the nucleus. Certainly, the TEM images acquired in the present study do not confirm the presence of ENMs within the cell nucleus. There is one study that has demonstrated AG-QDs were able to access the nucleus through imaging and analysis of the nuclear entry complexes pore genes Kap $\beta 2$ and Nup98 (Xu et al., 2018). NAC may provide a source of antioxidant metabolism which catalyses the complexing of NO and superoxide into stable metabolites such as nitrites. This represents a testable theory whereby the cells could be incubated with nitrite reductase to form nitrite in the extracellular supernatant and the subsequent levels of which measured using a fluorometric assay. A summary of potential causes of the secondary mechanisms of genotoxicity can be seen in Figure 6.4. 


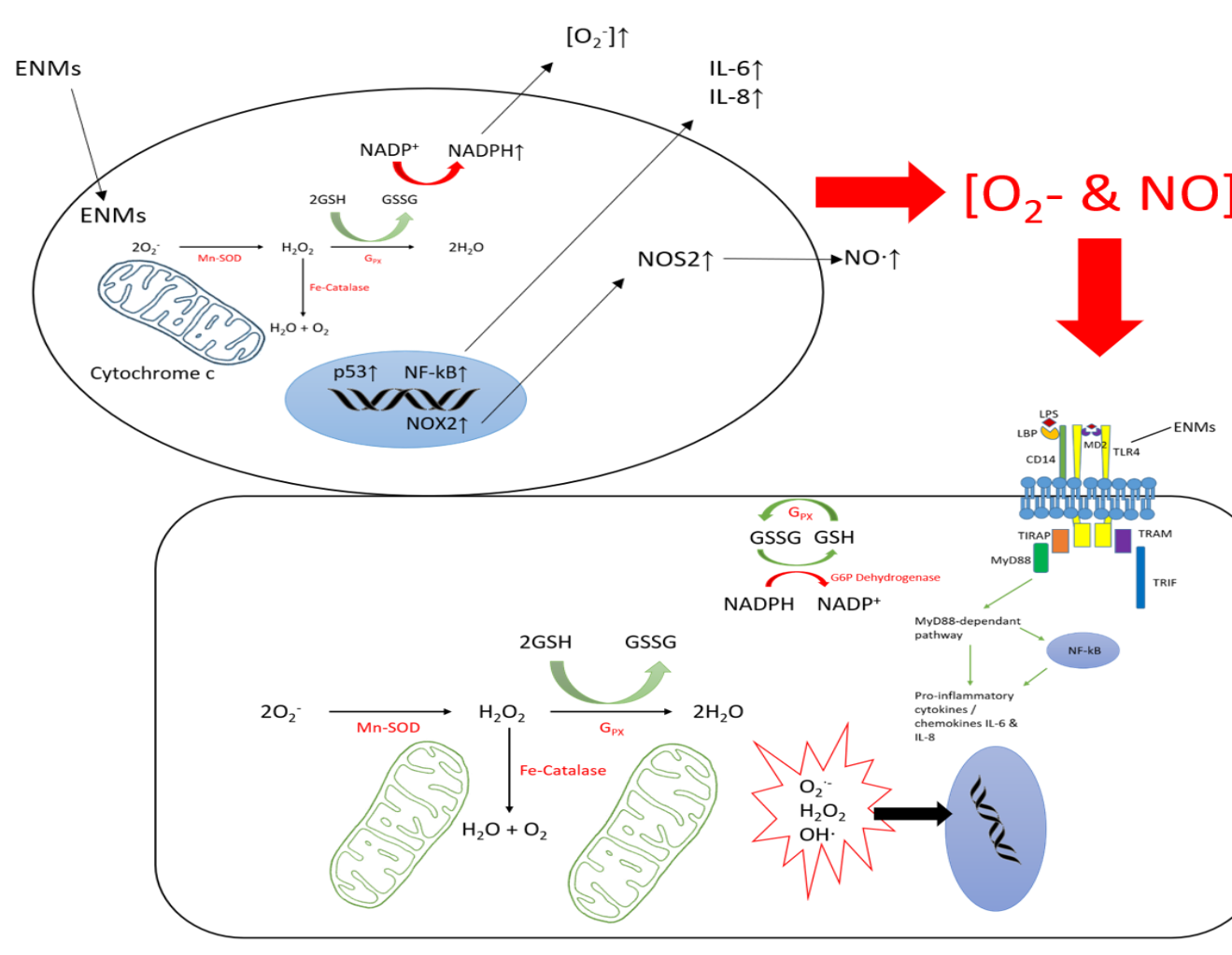

Figure 6.4 Potential co-culture secondary mechanisms of genotoxicity. Initial uptake of ENMs by THP-1 macrophages proteins such as CD14. Following phagocytosis, superoxide depletes mitochondrial function leading to oversatı Cytochrome $\mathrm{C}$ is released into the cell cytosol triggering apoptosis in some THP-1 macrophages. An increase in th consequently raises extracellular trafficking of (pro)-inflammatory mediators and NO. Superoxide and NO freely in peroxidation. Uptake of ENMs by TT1 cells would likely be reduced in this model however the depletion of glutathione to play a large role given that the significant genotoxicity was mitigated with the inclusion of NAC, a powerful antioxic 


\subsection{Thesis conclusions}

This study has demonstrated the potential of Neutral-, Amine-FLG and CB particles to promote significant genotoxicity in monocultured $16 \mathrm{HBE} 14 \mathrm{O}^{-}$cells through predominantly clastogenic mechanisms. Whilst in TT1 alveolar cells all tested ENMs promoted significant cytotoxicity and a greater genotoxic response. Given the awkward 3D dimensions of graphene-based ENMs, a strong emphasis was placed on characterising these materials, to which this study has concluded that multiple techniques should be applied to ascertain the particle and agglomerate sizes in situ. Plungefreeze SEM proved to be the most reliable source of agglomerate and particle size analysis providing both accurate means of particle sizing data and a snap-freeze image of how agglomerates appear in situ. Additionally, the images revealed how through electrostatic interactions, smaller agglomerates can form larger micron-sized aggregates. Cytotoxic and genotoxic evaluation of each ENM in monocultured $16 \mathrm{HBE}^{-140^{-}}$cells revealed a potency ranking of; $\mathrm{CB} \geq$ Neutral-FLG $>$ Amine-FLG. However, all tested ENMs promoted an IL-8 response at 16 hours of exposure during which all ENMs were internalised. Oxidative stress was observed with GSH depletion at 6 hours of exposure and a significant depletion in mitochondrial metabolic output.

When assessing the mechanistic toxicity of these ENMs in an alveolar model of TT1 cells, cytotoxicity was observed at low concentrations of $8 \mu \mathrm{g} / \mathrm{ml}$ with significant genotoxicity occurring at the same concentrations (Amine-FLG $\geq$ Carboxyl-FLG $>C B$ $>$ Neutral-FLG). Similarly, uptake was observed with all ENMs and a concentration-dependent increase in (pro)-inflammatory mediators observed. However, in contradiction to the OCR/ECAR and metabolic parameters measured in 16HBE140 cells (which were depleted by ENMs), TT1 cell metabolic function was elevated. Thus, each ENM was capable of primaryindirect genotoxicity in monocultured epithelial cells, the exception being Carboxyl-FLG in 16HBE140 cells. Each ENM was able to promote significant genotoxicity in a co-culture model of TT1/d.THP-1 macrophages ( $C B>A$ mine-FLG $>$ Neutral-FLG $>$ Carboxyl-FLG). This response indicates the capacity of each test ENM to promote secondary genotoxicity from an already-established primary-indirect mechanism which has then been increased by secondary mediators originating from the d.THP-1 macrophages. A pre-incubation with $1.5 \mathrm{mM}$ of NAC however did reduce the $\% \mathrm{Mn} / \mathrm{BN}$ in co-cultured $\Pi T 1$ cells indicative of a strong oxidative stress response. This study concludes a final potency ranking for the FLG materials as; Amine-FLG >Neutral-FLG >Carboxyl-FLG based upon the consistency with which cytotoxic and genotoxic data was collected and interpreted. Thus, the alveolar co-culture constructed in this study represented an ideal model for highlighting secondary genotoxic mechanisms which would have otherwise gone undetected using standard 2D culturing techniques. 


\section{Bibliography}

ABRIKOSSOVA, N., SKOGLUND, C., AHREN, M., BENGTSSON, T. \& UVDAL, K. 2012. Effects of gadolinium oxide nanoparticles on the oxidative burst from human neutrophil granulocytes. Nanotechnology, 23, 275101.

ADEREM, A. \& UNDERHILL, D. M. 1999. Mechanisms of phagocytosis in macrophages. Annu Rev Immunol, 17, 593-623.

AGgARWAL, P., HALL, J. B., MCLELAND, C. B., DOBROVOLSKAIA, M. A. \& MCNEIL, S. E. 2009. Nanoparticle interaction with plasma proteins as it relates to particle biodistribution, biocompatibility and therapeutic efficacy. Adv Drug Deliv Rev, 61, 428-37.

AGRAWAL, M., SARAF, S., ANTIMISIARIS, S. G., HAMANO, N., LI, S. D., CHOUGULE, M., SHOYELE, S. A., GUPTA, U., AJAZUDDIN \& ALEXANDER, A. 2018. Recent advancements in the field of nanotechnology for the delivery of anti-Alzheimer drug in the brain region. Expert Opin Drug Deliv, 15, 589-617.

AHMAD, R. \& KUPPUSAMY, P. 2010. Theory, instrumentation, and applications of electron paramagnetic resonance oximetry. Chem Rev, 110, 3212-36.

ALARIFI, S., ALI, D., VERMA, A., ALMAJHDI, F. N. \& AL-QAHTANI, A. A. 2014. Single-walled carbon nanotubes induce cytotoxicity and DNA damage via reactive oxygen species in human hepatocarcinoma cells. In Vitro Cell Dev Biol Anim, 50, 714-22.

ALLEN, A., HUTTON, D. A., PEARSON, J. P. \& SELLERS, L. A. 1984. Mucus glycoprotein structure, gel formation and gastrointestinal mucus function. Ciba Found Symp, 109, 137-56.

ALTMAN, E. I., BAYKARA, M. Z. \& SCHWARZ, U. D. 2015. Noncontact Atomic Force Microscopy: An Emerging Tool for Fundamental Catalysis Research. Acc Chem Res, 48, 2640-8.

AN, F. F. \& ZHANG, X. H. 2017. Strategies for Preparing Albumin-based Nanoparticles for Multifunctional Bioimaging and Drug Delivery. Theranostics, 7, 3667-3689.

ARMSTEAD, A. L. \& LI, B. 2016. In vitro inflammatory effects of hard metal (WC-Co) nanoparticle exposure. Int J Nanomedicine, 11, 6195-6206.

ARORA, S., RAJWADE, J. M. \& PAKNIKAR, K. M. 2012. Nanotoxicology and in vitro studies: the need of the hour. Toxicol Appl Pharmacol, 258, 151-65.

ARVIDSSON, R., BOHOLM, M., JOHANSSON, M. \& DE MONTOYA, M. L. 2018. "Just Carbon": Ideas About Graphene Risks by Graphene Researchers and Innovation Advisors. Nanoethics, 12, 199-210.

AYALA, A., MUNOZ, M. F. \& ARGUELLES, S. 2014. Lipid peroxidation: production, metabolism, and signaling mechanisms of malondialdehyde and 4-hydroxy-2-nonenal. Oxid Med Cell Longev, 2014, 360438.

BAALOUSHA, M. 2009. Aggregation and disaggregation of iron oxide nanoparticles: Influence of particle concentration, pH and natural organic matter. Sci Total Environ, 407, 2093-101.

BAALOUSHA, M. \& LEAD, J. R. 2012. Rationalizing nanomaterial sizes measured by atomic force microscopy, flow field-flow fractionation, and dynamic light scattering: sample preparation, polydispersity, and particle structure. Environ Sci Technol, 46, 6134-42.

BAE, S., KIM, H., LEE, Y., XU, X., PARK, J. S., ZHENG, Y., BALAKRISHNAN, J., LEI, T., KIM, H. R., SONG, Y. I., KIM, Y. J., KIM, K. S., OZYILMAZ, B., AHN, J. H., HONG, B. H. \& IIJIMA, S. 2010. Roll-to-roll production of 30-inch graphene films for transparent electrodes. Nat Nanotechnol, 5, 574-8.

BAGGIOLINI, M. \& CLARK-LEWIS, I. 1992. Interleukin-8, a chemotactic and inflammatory cytokine. FEBS Lett, 307, 97-101.

BAHARVAND, H., HASHEMI, S. M., KAZEMI ASHTIANI, S. \& FARROKHI, A. 2006. Differentiation of human embryonic stem cells into hepatocytes in 2D and 3D culture systems in vitro. Int J Dev Biol, 50, 645-52.

BALANDIN, A. A. 2011. Thermal properties of graphene and nanostructured carbon materials. Nat Mater, 10, 569-81. 
BASTACKY, J., LEE, C. Y., GOERKE, J., KOUSHAFAR, H., YAGER, D., KENAGA, L., SPEED, T. P., CHEN, Y. \& CLEMENTS, J. A. 1995. Alveolar lining layer is thin and continuous: low-temperature scanning electron microscopy of rat lung. J Appl Physiol (1985), 79, 1615-28.

BENDRE, V., GAUTAM, M., CARR, R., SMITH, J. \& MALLOY, A. 2011. Characterisation of nanoparticle size and concentration for toxicological studies. J Biomed Nanotechnol, 7, 195-6.

BENGTSON, S., KLING, K., MADSEN, A. M., NOERGAARD, A. W., JACOBSEN, N. R., CLAUSEN, P. A., ALONSO, B., PESQUERA, A., ZURUTUZA, A., RAMOS, R., OKUNO, H., DIJON, J., WALLIN, H. \& VOGEL, U. 2016. No cytotoxicity or genotoxicity of graphene and graphene oxide in murine lung epithelial FE1 cells in vitro. Environ Mol Mutagen, 57, 469-82.

BENGTSON, S., KNUDSEN, K. B., KYJOVSKA, Z. O., BERTHING, T., SKAUG, V., LEVIN, M., KOPONEN, I. K., SHIVAYOGIMATH, A., BOOTH, T. J., ALONSO, B., PESQUERA, A., ZURUTUZA, A., THOMSEN, B. L., TROELSEN, J. T., JACOBSEN, N. R. \& VOGEL, U. 2017. Differences in inflammation and acute phase response but similar genotoxicity in mice following pulmonary exposure to graphene oxide and reduced graphene oxide. PLoS One, 12, e0178355.

BHATTACHARJEE, S., ERSHOV, D., FYTIANOS, K., VAN DER GUCHT, J., ALINK, G. M., RIETJENS, I. M., MARCELIS, A. T. \& ZUILHOF, H. 2012. Cytotoxicity and cellular uptake of tri-block copolymer nanoparticles with different size and surface characteristics. Part Fibre Toxicol, 9, 11.

BIANCO, A. 2013. Graphene: safe or toxic? The two faces of the medal. Angew Chem Int Ed Engl, 52, 4986-97.

BICKEL, M. 1993. The role of interleukin-8 in inflammation and mechanisms of regulation. $J$ Periodontol, 64, 456-60.

BLANK, F., ROTHEN-RUTISHAUSER, B. M., SCHURCH, S. \& GEHR, P. 2006. An optimized in vitro model of the respiratory tract wall to study particle cell interactions. J Aerosol Med, 19, 392-405.

BLEEKER, E. A., DE JONG, W. H., GEERTSMA, R. E., GROENEWOLD, M., HEUGENS, E. H., KOERSJACQUEMIJNS, M., VAN DE MEENT, D., POPMA, J. R., RIETVELD, A. G., WIJNHOVEN, S. W., CASSEE, F. R. \& OOMEN, A. G. 2013. Considerations on the EU definition of a nanomaterial: science to support policy making. Regul Toxicol Pharmacol, 65, 119-25.

BLOM, R. A., ERNI, S. T., KREMPASKA, K., SCHAERER, O., VAN DIJK, R. M., AMACKER, M., MOSER, C., HALL, S. R., VON GARNIER, C. \& BLANK, F. 2016. A Triple Co-Culture Model of the Human Respiratory Tract to Study Immune-Modulatory Effects of Liposomes and Virosomes. PLoS One, 11, e0163539.

BOURDON, J. A., SABER, A. T., JACOBSEN, N. R., JENSEN, K. A., MADSEN, A. M., LAMSON, J. S., WALLIN, H., MOLLER, P., LOFT, S., YAUK, C. L. \& VOGEL, U. B. 2012. Carbon black nanoparticle instillation induces sustained inflammation and genotoxicity in mouse lung and liver. Part Fibre Toxicol, 9, 5.

BOYLES, M. S., YOUNG, L., BROWN, D. M., MACCALMAN, L., COWIE, H., MOISALA, A., SMAIL, F., SMITH, P. J., PROUDFOOT, L., WINDLE, A. H. \& STONE, V. 2015. Multi-walled carbon nanotube induced frustrated phagocytosis, cytotoxicity and pro-inflammatory conditions in macrophages are length dependent and greater than that of asbestos. Toxicol In Vitro, 29, 1513-28.

BRAAKHUIS, H. M., PARK, M. V., GOSENS, I., DE JONG, W. H. \& CASSEE, F. R. 2014. Physicochemical characteristics of nanomaterials that affect pulmonary inflammation. Part Fibre Toxicol, 11, 18.

BRESSAN, E., FERRONI, L., GARDIN, C., SBRICOLI, L., GOBBATO, L., LUDOVICHETTI, F. S., TOCCO, I., CARRARO, A., PIATTELLI, A. \& ZAVAN, B. 2014. Graphene based scaffolds effects on stem cells commitment. J Transl Med, 12, 296.

BUNCH, J. S., VERBRIDGE, S. S., ALDEN, J. S., VAN DER ZANDE, A. M., PARPIA, J. M., CRAIGHEAD, H. G. \& MCEUEN, P. L. 2008. Impermeable atomic membranes from graphene sheets. Nano Lett, 8, 2458-62.

BURGUM, M. J., EVANS, S. J., JENKINS, G. J., DOAK, S. H. \& CLIFT, M. J. D. 2018. Considerations for the Human Health Implications of Nanotheranostics. In: CONDE, J. (ed.) Handbook of Nanomaterials for Cancer Theranostics. Elsevier. 
BUSSY, C., ALI-BOUCETTA, H. \& KOSTARELOS, K. 2013. Safety considerations for graphene: lessons learnt from carbon nanotubes. Acc Chem Res, 46, 692-701.

CAPASSO, L., CAMATINI, M. \& GUALTIERI, M. 2014. Nickel oxide nanoparticles induce inflammation and genotoxic effect in lung epithelial cells. Toxicol Lett, 226, 28-34.

CASALS, E., PFALLER, T., DUSCHL, A., OOSTINGH, G. J. \& PUNTES, V. 2010. Time evolution of the nanoparticle protein corona. ACS Nano, 4, 3623-32.

CASUSO, I., KODERA, N., LE GRIMELLEC, C., ANDO, T. \& SCHEURING, S. 2009. Contact-mode highresolution high-speed atomic force microscopy movies of the purple membrane. Biophys $J$, 97, 1354-61.

CEDERVALL, T., LYNCH, I., LINDMAN, S., BERGGARD, T., THULIN, E., NILSSON, H., DAWSON, K. A. \& LINSE, S. 2007. Understanding the nanoparticle-protein corona using methods to quantify exchange rates and affinities of proteins for nanoparticles. Proc Natl Acad Sci U S A, 104, 20505.

CHANG, Y., YANG, S. T., LIU, J. H., DONG, E., WANG, Y., CAO, A., LIU, Y. \& WANG, H. 2011. In vitro toxicity evaluation of graphene oxide on A549 cells. Toxicol Lett, 200, 201-10.

CHATTERJEE, N., YANG, J. S., PARK, K., OH, S. M., PARK, J. \& CHOI, J. 2015. Screening of toxic potential of graphene family nanomaterials using in vitro and alternative in vivo toxicity testing systems. Environ Health Toxicol, 30, e2015007.

CHEN, G. Y., PANG, D. W., HWANG, S. M., TUAN, H. Y. \& HU, Y. C. 2012a. A graphene-based platform for induced pluripotent stem cells culture and differentiation. Biomaterials, 33, 418-27.

CHEN, G. Y., YANG, H. J., LU, C. H., CHAO, Y. C., HWANG, S. M., CHEN, C. L., LO, K. W., SUNG, L. Y., LUO, W. Y., TUAN, H. Y. \& HU, Y. C. 2012b. Simultaneous induction of autophagy and toll-like receptor signaling pathways by graphene oxide. Biomaterials, 33, 6559-69.

CHNG, E. L. \& PUMERA, M. 2013. The toxicity of graphene oxides: dependence on the oxidative methods used. Chemistry, 19, 8227-35.

CHOI, J. H., LEE, J., SHIN, W., CHOI, J. W. \& KIM, H. J. 2016. Priming nanoparticle-guided diagnostics and therapeutics towards human organs-on-chips microphysiological system. Nano Converg, $3,24$.

CHRISTENSEN, F. M., JOHNSTON, H. J., STONE, V., AITKEN, R. J., HANKIN, S., PETERS, S. \& ASCHBERGER, K. 2010. Nano-silver - feasibility and challenges for human health risk assessment based on open literature. Nanotoxicology, 4, 284-95.

CHUA, C. K., SOFER, Z. \& PUMERA, M. 2012. Graphite oxides: effects of permanganate and chlorate oxidants on the oxygen composition. Chemistry, 18, 13453-9.

CLIFT, M. J., ENDES, C., VANHECKE, D., WICK, P., GEHR, P., SCHINS, R. P., PETRI-FINK, A. \& ROTHENRUTISHAUSER, B. 2014. A comparative study of different in vitro lung cell culture systems to assess the most beneficial tool for screening the potential adverse effects of carbon nanotubes. Toxicol Sci, 137, 55-64.

CLIFT, M. J., GEHR, P. \& ROTHEN-RUTISHAUSER, B. 2011. Nanotoxicology: a perspective and discussion of whether or not in vitro testing is a valid alternative. Arch Toxicol, 85, 723-31.

CLOVER, J. \& GOWEN, M. 1994. Are MG-63 and HOS TE85 human osteosarcoma cell lines representative models of the osteoblastic phenotype? Bone, 15, 585-91.

COIN, P. G., ROGGLI, V. L. \& BRODY, A. R. 1994. Persistence of long, thin chrysotile asbestos fibers in the lungs of rats. Environ Health Perspect, 102 Suppl 5, 197-9.

CONG, C., LI, K., ZHANG, X. X. \& YU, T. 2013. Visualization of arrangements of carbon atoms in graphene layers by Raman mapping and atomic-resolution TEM. Sci Rep, 3, 1195.

CONG, C., YU, T. \& WANG, H. 2010. Raman study on the g mode of graphene for determination of edge orientation. ACS Nano, 4, 3175-80.

CONNER, S. D. \& SCHMID, S. L. 2003. Regulated portals of entry into the cell. Nature, 422, 37-44.

CRAPO, J. D., BARRY, B. E., GEHR, P., BACHOFEN, M. \& WEIBEL, E. R. 1982. Cell number and cell characteristics of the normal human lung. Am Rev Respir Dis, 126, 332-7.

CROUCH, E. C. 1998. Collectins and pulmonary host defense. Am J Respir Cell Mol Biol, 19, 177-201. 
DAVIES, P. \& ALLISON, A. C. 1978. Effects of cytochalasin B on endocytosis and exocytosis. Front Biol, $46,143-60$.

DE PABLO, P. J. 2011. Introduction to atomic force microscopy. Methods Mol Biol, 783, 197-212.

DEKHUIJZEN, P. N. \& VAN BEURDEN, W. J. 2006. The role for N-acetylcysteine in the management of COPD. Int J Chron Obstruct Pulmon Dis, 1, 99-106.

DELOID, G., COHEN, J. M., DARRAH, T., DERK, R., ROJANASAKUL, L., PYRGIOTAKIS, G., WOHLLEBEN, W. \& DEMOKRITOU, P. 2014. Estimating the effective density of engineered nanomaterials for in vitro dosimetry. Nat Commun, 5, 3514.

DELOID, G. M., COHEN, J. M., PYRGIOTAKIS, G., PIRELA, S. V., PAL, A., LIU, J., SREBRIC, J. \& DEMOKRITOU, P. 2015. Advanced computational modeling for in vitro nanomaterial dosimetry. Part Fibre Toxicol, 12, 32.

DIABATE, S., MULHOPT, S., PAUR, H. R. \& KRUG, H. F. 2008. The response of a co-culture lung model to fine and ultrafine particles of incinerator fly ash at the air-liquid interface. Altern Lab Anim, 36, 285-98.

DIKALOV, S., SKATCHKOV, M. \& BASSENGE, E. 1997. Quantification of peroxynitrite, superoxide, and peroxyl radicals by a new spin trap hydroxylamine 1-hydroxy-2,2,6,6-tetramethyl-4-oxopiperidine. Biochem Biophys Res Commun, 230, 54-7.

DOAK, S., LIU, Y. \& CHEN, C. 2012a. Adverse Effects of Engineered Nanomaterials, Elsevir.

DOAK, S. H., GRIFFITHS, S. M., MANSHIAN, B., SINGH, N., WILLIAMS, P. M., BROWN, A. P. \& JENKINS, G. J. 2009. Confounding experimental considerations in nanogenotoxicology. Mutagenesis, 24, 285-93.

DOAK, S. H., MANSHIAN, B., JENKINS, G. J. \& SINGH, N. 2012b. In vitro genotoxicity testing strategy for nanomaterials and the adaptation of current OECD guidelines. Mutat Res, 745, 104-11.

DOAK, S. H. \& PFUHLER, S. 2012. Nanotoxicology. Preface. Mutat Res, 745, 1-3.

DOBROVOLSKAIA, M. A., GERMOLEC, D. R. \& WEAVER, J. L. 2009. Evaluation of nanoparticle immunotoxicity. Nat Nanotechnol, 4, 411-4.

DODOO, A. N., BANSAL, S. S., BARLOW, D. J., BENNET, F., HIDER, R. C., LANSLEY, A. B., LAWRENCE, M. J. \& MARRIOTT, C. 2000. Use of alveolar cell monolayers of varying electrical resistance to measure pulmonary peptide transport. J Pharm Sci, 89, 223-31.

DOHERTY, A. T. 2012. The in vitro micronucleus assay. Methods Mol Biol, 817, 121-41.

DON PORTO CARERO, A., HOET, P. H., VERSCHAEVE, L., SCHOETERS, G. \& NEMERY, B. 2001. Genotoxic effects of carbon black particles, diesel exhaust particles, and urban air particulates and their extracts on a human alveolar epithelial cell line (A549) and a human monocytic cell line (THP1). Environ Mol Mutagen, 37, 155-63.

DONALDSON, K. \& STONE, V. 2003. Current hypotheses on the mechanisms of toxicity of ultrafine particles. Ann Ist Super Sanita, 39, 405-10.

DONALDSON, K. \& TRAN, C. L. 2002. Inflammation caused by particles and fibers. Inhal Toxicol, 14, 527.

DORESWAMY, V. \& PEDEN, D. B. 2011. Modulation of asthma by endotoxin. Clin Exp Allergy, 41, 9-19.

DOWNS, T. R., CROSBY, M. E., HU, T., KUMAR, S., SULLIVAN, A., SARLO, K., REEDER, B., LYNCH, M., WAGNER, M., MILLS, T. \& PFUHLER, S. 2012. Silica nanoparticles administered at the maximum tolerated dose induce genotoxic effects through an inflammatory reaction while gold nanoparticles do not. Mutat Res, 745, 38-50.

DUAN, G., KANG, S. G., TIAN, X., GARATE, J. A., ZHAO, L., GE, C. \& ZHOU, R. 2015. Protein corona mitigates the cytotoxicity of graphene oxide by reducing its physical interaction with cell membrane. Nanoscale, 7, 15214-24.

DUCH, M. C., BUDINGER, G. R., LIANG, Y. T., SOBERANES, S., URICH, D., CHIARELLA, S. E., CAMPOCHIARO, L. A., GONZALEZ, A., CHANDEL, N. S., HERSAM, M. C. \& MUTLU, G. M. 2011. Minimizing oxidation and stable nanoscale dispersion improves the biocompatibility of graphene in the lung. Nano Lett, 11, 5201-7. 
EDMONDSON, R., BROGLIE, J. J., ADCOCK, A. F. \& YANG, L. 2014. Three-dimensional cell culture systems and their applications in drug discovery and cell-based biosensors. Assay Drug Dev Technol, 12, 207-18.

ELESPURU, R., PFUHLER, S., AARDEMA, M. J., CHEN, T., DOAK, S. H., DOHERTY, A., FARABAUGH, C. S., KENNY, J., MANJANATHA, M., MAHADEVAN, B., MOORE, M. M., OUEDRAOGO, G., STANKOWSKI, L. F., JR. \& TANIR, J. Y. 2018. Genotoxicity Assessment of Nanomaterials: Recommendations on Best Practices, Assays, and Methods. Toxicol Sci, 164, 391-416.

ELINGARAMIL, S., LI, X. \& HE, N. 2013. Applications of nanotechnology, next generation sequencing and microarrays in biomedical research. J Nanosci Nanotechnol, 13, 4539-51.

EVANS, S. J., CLIFT, M. J., SINGH, N., DE OLIVEIRA MALLIA, J., BURGUM, M., WILLS, J. W., WILKINSON, T. S., JENKINS, G. J. \& DOAK, S. H. 2017. Critical review of the current and future challenges associated with advanced in vitro systems towards the study of nanoparticle (secondary) genotoxicity. Mutagenesis, 32, 233-241.

EVANS, S. J., CLIFT, M. J. D., SINGH, N., WILLS, J. W., HONDOW, N., WILKINSON, T. S., BURGUM, M. J., BROWN, A. P., JENKINS, G. J. \& DOAK, S. H. 2019. In vitro detection of in vitro secondary mechanisms of genotoxicity induced by engineered nanomaterials. Part Fibre Toxicol, 16, 8.

FADEEL, B., BUSSY, C., MERINO, S., VAZQUEZ, E., FLAHAUT, E., MOUCHET, F., EVARISTE, L., GAUTHIER, L., KOIVISTO, A. J., VOGEL, U., MARTIN, C., DELOGU, L. G., BUERKI-THURNHERR, T., WICK, P., BELOIN-SAINT-PIERRE, D., HISCHIER, R., PELIN, M., CANDOTTO CARNIEL, F., TRETIACH, M., CESCA, F., BENFENATI, F., SCAINI, D., BALLERINI, L., KOSTARELOS, K., PRATO, M. \& BIANCO, A. 2018. Safety Assessment of Graphene-Based Materials: Focus on Human Health and the Environment. ACS Nano, 12, 10582-10620.

FAHY, J. V. \& DICKEY, B. F. 2010. Airway mucus function and dysfunction. N Eng/J Med, 363, 2233-47. FEITO, M. J., VILA, M., MATESANZ, M. C., LINARES, J., GONCALVES, G., MARQUES, P. A., VALLET-REGI, M., ROJO, J. M. \& PORTOLES, M. T. 2014. In vitro evaluation of graphene oxide nanosheets on immune function. J Colloid Interface Sci, 432, 221-8.

FENECH, M. 2007. Cytokinesis-block micronucleus cytome assay. Nat Protoc. England.

FENOGLIO, I., TOMATIS, M., LISON, D., MULLER, J., FONSECA, A., NAGY, J. B. \& FUBINI, B. 2006. Reactivity of carbon nanotubes: free radical generation or scavenging activity? Free Radic Biol Med, 40, 1227-33.

FERIN, J., OBERDORSTER, G. \& PENNEY, D. P. 1992. Pulmonary retention of ultrafine and fine particles in rats. Am J Respir Cell Mol Biol, 6, 535-42.

FERNANDEZ-IGLESIAS, N. \& BETTMER, J. 2015. Complementary mass spectrometric techniques for the quantification of the protein corona: a case study on gold nanoparticles and human serum proteins. Nanoscale, 7, 14324-31.

FOROOZANDEH, P. \& AZIZ, A. A. 2015. Merging worlds of nanomaterials and biological environment: factors governing protein corona formation on nanoparticles and its biological consequences. Nanoscale Res Lett, 10, 221.

FRANKLIN, N. M., ROGERS, N. J., APTE, S. C., BATLEY, G. E., GADD, G. E. \& CASEY, P. S. 2007. Comparative toxicity of nanoparticulate $\mathrm{ZnO}$, bulk $\mathrm{ZnO}$, and $\mathrm{ZnCl} 2$ to a freshwater microalga (Pseudokirchneriella subcapitata): the importance of particle solubility. Environ Sci Technol, 41, 8484-90.

FROHLICH, E. 2013. Cellular targets and mechanisms in the cytotoxic action of non-biodegradable engineered nanoparticles. Curr Drug Metab, 14, 976-88.

FROHLICH, E. \& SALAR-BEHZADI, S. 2014. Toxicological assessment of inhaled nanoparticles: role of in vivo, ex vivo, in vitro, and in silico studies. Int J Mol Sci, 15, 4795-822.

FUCHS, S., HOLLINS, A. J., LAUE, M., SCHAEFER, U. F., ROEMER, K., GUMBLETON, M. \& LEHR, C. M. 2003. Differentiation of human alveolar epithelial cells in primary culture: morphological characterization and synthesis of caveolin-1 and surfactant protein-C. Cell Tissue Res, 311, 31 45. 
FUKAI, E., SATO, H., WATANABE, M., NAKAE, D. \& TOTSUKA, Y. 2018. Establishment of an in vivo simulating co-culture assay platform for genotoxicity of multi-walled carbon nanotubes. Cancer Sci, 109, 1024-1031.

GABAY, C. 2006. Interleukin-6 and chronic inflammation. Arthritis Res Ther, 8 Suppl 2, S3.

GAI, P. L. \& BOYES, E. D. 2009. Advances in atomic resolution in situ environmental transmission electron microscopy and $1 \mathrm{~A}$ aberration corrected in situ electron microscopy. Microsc Res Tech, 72, 153-64.

GATOO, M. A., NASEEM, S., ARFAT, M. Y., DAR, A. M., QASIM, K. \& ZUBAIR, S. 2014. Physicochemical properties of nanomaterials: implication in associated toxic manifestations. Biomed Res Int, 2014, 498420.

GEHR, P., BACHOFEN, M. \& WEIBEL, E. R. 1978. The normal human lung: ultrastructure and morphometric estimation of diffusion capacity. Respir Physiol, 32, 121-40.

GEIM, A. K. \& NOVOSELOV, K. S. 2007. The rise of graphene. Nat Mater, 6, 183-91.

GEISER, M. \& KREYLING, W. G. 2010. Deposition and biokinetics of inhaled nanoparticles. Part Fibre Toxicol, 7, 2.

GODFREY, R. W. 1997. Human airway epithelial tight junctions. Microsc Res Tech, 38, 488-99.

GOERKE, J. 1998. Pulmonary surfactant: functions and molecular composition. Biochim Biophys Acta, $1408,79-89$.

GRAF, D., MOLITOR, F., ENSSLIN, K., STAMPFER, C., JUNGEN, A., HIEROLD, C. \& WIRTZ, L. 2007. Spatially resolved Raman spectroscopy of single- and few-layer graphene. Nano Lett, 7, 238-42.

GRAINGER, C. I., GREENWELL, L. L., LOCKLEY, D. J., MARTIN, G. P. \& FORBES, B. 2006. Culture of Calu3 cells at the air interface provides a representative model of the airway epithelial barrier. Pharm Res, 23, 1482-90.

GRIFFITHS, S. M., SINGH, N., JENKINS, G. J., WILLIAMS, P. M., ORBAEK, A. W., BARRON, A. R., WRIGHT, C. J. \& DOAK, S. H. 2011. Dextran coated ultrafine superparamagnetic iron oxide nanoparticles: compatibility with common fluorometric and colorimetric dyes. Anal Chem, 83, 3778-85.

GROSS, L., MOHN, F., MOLL, N., LILJEROTH, P. \& MEYER, G. 2009. The chemical structure of a molecule resolved by atomic force microscopy. Science, 325, 1110-4.

GUO, F., KIM, F., HAN, T. H., SHENOY, V. B., HUANG, J. \& HURT, R. H. 2011. Hydration-responsive folding and unfolding in graphene oxide liquid crystal phases. ACS Nano, 5, 8019-25.

GUO, X. \& MEI, N. 2014a. Assessment of the toxic potential of graphene family nanomaterials. J Food Drug Anal, 22, 105-15.

GUO, X. \& MEI, N. 2014b. Assessment of the toxic potential of graphene family nanomaterials. J Food Drug Anal, 22, 105-115.

HAN, S. G., KIM, J. K., SHIN, J. H., HWANG, J. H., LEE, J. S., KIM, T. G., LEE, J. H., LEE, G. H., KIM, K. S., LEE, H. S., SONG, N. W., AHN, K. \& YU, I. J. 2015. Pulmonary Responses of Sprague-Dawley Rats in Single Inhalation Exposure to Graphene Oxide Nanomaterials. Biomed Res Int, 2015, 376756.

HARTWIG, J. H., DAVIES, W. A. \& STOSSEL, T. P. 1977. Evidence for contractile protein translocation in macrophage spreading, phagocytosis, and phagolysosome formation. J Cell Biol, 75, 956-67.

HERMANNS, M. I., UNGER, R. E., KEHE, K., PETERS, K. \& KIRKPATRICK, C. J. 2004. Lung epithelial cell lines in coculture with human pulmonary microvascular endothelial cells: development of an alveolo-capillary barrier in vitro. Lab Invest, 84, 736-52.

HERZOG, F., CLIFT, M. J., PICCAPIETRA, F., BEHRA, R., SCHMID, O., PETRI-FINK, A. \& ROTHENRUTISHAUSER, B. 2013. Exposure of silver-nanoparticles and silver-ions to lung cells in vitro at the air-liquid interface. Part Fibre Toxicol, 10, 11.

HIEMSTRA, P. S., MCCRAY, P. B., JR. \& BALS, R. 2015. The innate immune function of airway epithelial cells in inflammatory lung disease. Eur Respir J, 45, 1150-62.

HOET, P. H., BRUSKE-HOHLFELD, I. \& SALATA, O. V. 2004. Nanoparticles - known and unknown health risks. J Nanobiotechnology, 2, 12. 
HOLE, P., SILLENCE, K., HANNELL, C., MAGUIRE, C. M., ROESSLEIN, M., SUAREZ, G., CAPRACOTTA, S., MAGDOLENOVA, Z., HOREV-AZARIA, L., DYBOWSKA, A., COOKE, L., HAASE, A., CONTAL, S., MANO, S., VENNEMANN, A., SAUVAIN, J. J., STAUNTON, K. C., ANGUISSOLA, S., LUCH, A., DUSINSKA, M., KORENSTEIN, R., GUTLEB, A. C., WIEMANN, M., PRINA-MELLO, A., RIEDIKER, M. \& WICK, P. 2013. Interlaboratory comparison of size measurements on nanoparticles using nanoparticle tracking analysis (NTA). J Nanopart Res, 15, 2101.

HOLGATE, S. T. 2010. Exposure, uptake, distribution and toxicity of nanomaterials in humans. J Biomed Nanotechnol, 6, 1-19.

HONG, B. J., COMPTON, O. C., AN, Z., ERYAZICI, I. \& NGUYEN, S. T. 2012. Successful stabilization of graphene oxide in electrolyte solutions: enhancement of biofunctionalization and cellular uptake. ACS Nano, 6, 63-73.

HORTELANO, S., DALLAPORTA, B., ZAMZAMI, N., HIRSCH, T., SUSIN, S. A., MARZO, I., BOSCA, L. \& KROEMER, G. 1997. Nitric oxide induces apoptosis via triggering mitochondrial permeability transition. FEBS Lett, 410, 373-7.

HOYLE, C., RIVERS-AUTY, J., LEMARCHAND, E., VRANIC, S., WANG, E., BUGGIO, M., ROTHWELL, N. J., ALLAN, S. M., KOSTARELOS, K. \& BROUGH, D. 2018. Small, Thin Graphene Oxide Is Antiinflammatory Activating Nuclear Factor Erythroid 2-Related Factor 2 via Metabolic Reprogramming. ACS Nano, 12, 11949-11962.

HSIAO, I. L. \& HUANG, Y. J. 2013. Effects of serum on cytotoxicity of nano- and micro-sized ZnO particles. J Nanopart Res, 15, 1829.

HU, W., PENG, C., LV, M., LI, X., ZHANG, Y., CHEN, N., FAN, C. \& HUANG, Q. 2011. Protein coronamediated mitigation of cytotoxicity of graphene oxide. ACS Nano, 5, 3693-700.

HUANG, X., JAIN, P. K., EL-SAYED, I. H. \& EL-SAYED, M. A. 2007. Gold nanoparticles: interesting optical properties and recent applications in cancer diagnostics and therapy. Nanomedicine (Lond), 2, 681-93.

HUHN, D., KANTNER, K., GEIDEL, C., BRANDHOLT, S., DE COCK, I., SOENEN, S. J., RIVERA GIL, P., MONTENEGRO, J. M., BRAECKMANS, K., MULLEN, K., NIENHAUS, G. U., KLAPPER, M. \& PARAK, W. J. 2013. Polymer-coated nanoparticles interacting with proteins and cells: focusing on the sign of the net charge. ACS Nano, 7, 3253-63.

HURST, S. M., WILKINSON, T. S., MCLOUGHLIN, R. M., JONES, S., HORIUCHI, S., YAMAMOTO, N., ROSEJOHN, S., FULLER, G. M., TOPLEY, N. \& JONES, S. A. 2001. II-6 and its soluble receptor orchestrate a temporal switch in the pattern of leukocyte recruitment seen during acute inflammation. Immunity, 14, 705-14.

HUSSAIN, S., THOMASSEN, L. C., FERECATU, I., BOROT, M. C., ANDREAU, K., MARTENS, J. A., FLEURY, J., BAEZA-SQUIBAN, A., MARANO, F. \& BOLAND, S. 2010. Carbon black and titanium dioxide nanoparticles elicit distinct apoptotic pathways in bronchial epithelial cells. Part Fibre Toxicol, $7,10$.

ILIAKIS, G., WANG, H., PERRAULT, A. R., BOECKER, W., ROSIDI, B., WINDHOFER, F., WU, W., GUAN, J., TERZOUDI, G. \& PANTELIAS, G. 2004. Mechanisms of DNA double strand break repair and chromosome aberration formation. Cytogenet Genome Res, 104, 14-20.

ITOH, H., NISHINO, M. \& HATABU, H. 2004. Architecture of the lung: morphology and function. J Thorac Imaging, 19, 221-7.

JACOBSEN, N. R., POJANA, G., WHITE, P., MOLLER, P., COHN, C. A., KORSHOLM, K. S., VOGEL, U., MARCOMINI, A., LOFT, S. \& WALLIN, H. 2008. Genotoxicity, cytotoxicity, and reactive oxygen species induced by single-walled carbon nanotubes and $C(60)$ fullerenes in the FE1-Mutatrade markMouse lung epithelial cells. Environ Mol Mutagen, 49, 476-87.

JANTZEN, K., ROURSGAARD, M., DESLER, C., LOFT, S., RASMUSSEN, L. J. \& MOLLER, P. 2012. Oxidative damage to DNA by diesel exhaust particle exposure in co-cultures of human lung epithelial cells and macrophages. Mutagenesis, 27, 693-701. 
JAROSZ, A., SKODA, M., DUDEK, I. \& SZUKIEWICZ, D. 2016. Oxidative Stress and Mitochondrial Activation as the Main Mechanisms Underlying Graphene Toxicity against Human Cancer Cells. Oxid Med Cell Longev, 2016, 5851035.

JIANG, W., KIM, B. Y., RUTKA, J. T. \& CHAN, W. C. 2008. Nanoparticle-mediated cellular response is size-dependent. Nat Nanotechnol. England.

JONES, C. F. \& GRAINGER, D. W. 2009. In vitro assessments of nanomaterial toxicity. Adv Drug Deliv Rev, 61, 438-56.

JOURNEAY, W. S., SURI, S. S., FENNIRI, H. \& SINGH, B. 2008. High-aspect ratio nanoparticles in nanotoxicology. Integr Environ Assess Manag, 4, 128-9.

KAGAN, V. E., KONDURU, N. V., FENG, W., ALLEN, B. L., CONROY, J., VOLKOV, Y., VLASOVA, II, BELIKOVA, N. A., YANAMALA, N., KAPRALOV, A., TYURINA, Y. Y., SHI, J., KISIN, E. R., MURRAY, A. R., FRANKS, J., STOLZ, D., GOU, P., KLEIN-SEETHARAMAN, J., FADEEL, B., STAR, A. \& SHVEDOVA, A. A. 2010. Carbon nanotubes degraded by neutrophil myeloperoxidase induce less pulmonary inflammation. Nat Nanotechnol, 5, 354-9.

KAMENCIC, H., LYON, A., PATERSON, P. G. \& JUURLINK, B. H. 2000. Monochlorobimane fluorometric method to measure tissue glutathione. Anal Biochem, 286, 35-7.

KEMP, S. J., THORLEY, A. J., GORELIK, J., SECKL, M. J., O'HARE, M. J., ARCARO, A., KORCHEV, Y., GOLDSTRAW, P. \& TETLEY, T. D. 2008. Immortalization of human alveolar epithelial cells to investigate nanoparticle uptake. Am J Respir Cell Mol Biol, 39, 591-7.

KERKSICK, C. \& WILLOUGHBY, D. 2005. The antioxidant role of glutathione and N-acetyl-cysteine supplements and exercise-induced oxidative stress. J Int Soc Sports Nutr, 2, 38-44.

KETTLER, K., KRYSTEK, P., GIANNAKOU, C., HENDRIKS, A. J. \& DE JONG, W. H. 2016. Exploring the effect of silver nanoparticle size and medium composition on uptake into pulmonary epithelial 16HBE14o-cells. J Nanopart Res, 18, 182.

KISHORE, U., GREENHOUGH, T. J., WATERS, P., SHRIVE, A. K., GHAI, R., KAMRAN, M. F., BERNAL, A. L., REID, K. B., MADAN, T. \& CHAKRABORTY, T. 2006. Surfactant proteins SP-A and SP-D: structure, function and receptors. Mol Immunol, 43, 1293-315.

KLEIN, S. G., SERCHI, T., HOFFMANN, L., BLOMEKE, B. \& GUTLEB, A. C. 2013. An improved 3D tetraculture system mimicking the cellular organisation at the alveolar barrier to study the potential toxic effects of particles on the lung. Part Fibre Toxicol, 10, 31.

KOHANE, D. S. 2007. Microparticles and nanoparticles for drug delivery. Biotechnol Bioeng, 96, 203-9. KOKKINOPOULOU, M., SIMON, J., LANDFESTER, K., MAILANDER, V. \& LIEBERWIRTH, I. 2017. Visualization of the protein corona: towards a biomolecular understanding of nanoparticlecell-interactions. Nanoscale, 9, 8858-8870.

KOPF, M., BAUMANN, H., FREER, G., FREUDENBERG, M., LAMERS, M., KISHIMOTO, T., ZINKERNAGEL, R., BLUETHMANN, H. \& KOHLER, G. 1994. Impaired immune and acute-phase responses in interleukin-6-deficient mice. Nature, 368, 339-42.

KOSLOWSKI, R., BARTH, K., AUGSTEIN, A., TSCHERNIG, T., BARGSTEN, G., AUFDERHEIDE, M. \& KASPER, M. 2004. A new rat type I-like alveolar epithelial cell line R3/1: bleomycin effects on caveolin expression. Histochem Cell Biol, 121, 509-19.

KOSTOGLOU NIKOLAOS, T. V., KONTOS. G ATHANASSIOS, GIANNAKOPOULOS KONSTANTINOS, TAMPAXIS CHRISTOS, PAPAVASILIOU AGGELIKI, CHARALAMBOPOULOU GEORGIA, STERIOTIS THEODORE, YUANGING LI, LIAO KIN, POLYCHRONOPOULOU KYRIAKI, MITTERER CHRISTIAN, REBHOLZ CLAUS. 2015. Synthesis of nanoporous graphene oxide adsorbents by freeze drying or microwave radiation: Characterization and hydrogen storage properties. International Journal of Hydrogen Energy: ELSEVIER.

KOTCHEY, G. P., ALLEN, B. L., VEDALA, H., YANAMALA, N., KAPRALOV, A. A., TYURINA, Y. Y., KLEINSEETHARAMAN, J., KAGAN, V. E. \& STAR, A. 2011. The enzymatic oxidation of graphene oxide. ACS Nano, 5, 2098-108.

KOTCHEY, G. P., ZHAO, Y., KAGAN, V. E. \& STAR, A. 2013. Peroxidase-mediated biodegradation of carbon nanotubes in vitro and in vivo. Adv Drug Deliv Rev, 65, 1921-32. 
KREWSKI, D., ACOSTA, D., JR., ANDERSEN, M., ANDERSON, H., BAILAR, J. C., 3RD, BOEKELHEIDE, K., BRENT, R., CHARNLEY, G., CHEUNG, V. G., GREEN, S., JR., KELSEY, K. T., KERKVLIET, N. I., LI, A. A., MCCRAY, L., MEYER, O., PATTERSON, R. D., PENNIE, W., SCALA, R. A., SOLOMON, G. M., STEPHENS, M., YAGER, J. \& ZEISE, L. 2010. Toxicity testing in the 21st century: a vision and a strategy. J Toxicol Environ Health B Crit Rev, 13, 51-138.

KUILA, T., BOSE, S., KHANRA, P., MISHRA, A. K., KIM, N. H. \& LEE, J. H. 2011. Recent advances in graphene-based biosensors. Biosens Bioelectron, 26, 4637-48.

KUMAR, R., MEHTA, B. R., BHATNAGAR, M., S, R., MAHAPATRA, S., SALKALACHEN, S. \& JHAWAR, P. 2014. Graphene as a transparent conducting and surface field layer in planar Si solar cells. Nanoscale Res Lett, 9, 349.

KURAPATI, R., MUKHERJEE, S. P., MARTIN, C., BEPETE, G., VAZQUEZ, E., PENICAUD, A., FADEEL, B. \& BIANCO, A. 2018. Degradation of Single-Layer and Few-Layer Graphene by Neutrophil Myeloperoxidase. Angew Chem Int Ed Engl, 57, 11722-11727.

LAMMEL, T., BOISSEAUX, P., FERNANDEZ-CRUZ, M. L. \& NAVAS, J. M. 2013. Internalization and cytotoxicity of graphene oxide and carboxyl graphene nanoplatelets in the human hepatocellular carcinoma cell line Hep G2. Part Fibre Toxicol, 10, 27.

LATEGAN, K., ALGHADI, H., BAYATI, M., DE CORTALEZZI, M. F. \& POOL, E. 2018. Effects of Graphene Oxide Nanoparticles on the Immune System Biomarkers Produced by RAW 264.7 and Human Whole Blood Cell Cultures. Nanomaterials (Basel), 8.

LAURENT, S., BURTEA, C., THIRIFAYS, C., REZAEE, F. \& MAHMOUDI, M. 2013. Significance of cell "observer" and protein source in nanobiosciences. J Colloid Interface Sci, 392, 431-45.

LEE, C., WEI, X., KYSAR, J. W. \& HONE, J. 2008. Measurement of the elastic properties and intrinsic strength of monolayer graphene. Science, 321, 385-8.

LEGLEITER, J. 2009. The effect of drive frequency and set point amplitude on tapping forces in atomic force microscopy: simulation and experiment. Nanotechnology, 20, 245703.

LI, C. Q., TRUDEL, L. J. \& WOGAN, G. N. 2002. Nitric oxide-induced genotoxicity, mitochondrial damage, and apoptosis in human lymphoblastoid cells expressing wild-type and mutant p53. Proc Natl Acad Sci U S A, 99, 10364-9.

LI, J., LIU, Q., HO, D., ZHAO, S., WU, S., LING, L., HAN, F., WU, X., ZHANG, G., SUN, R. \& WONG, C. P. 2018a. Three-Dimensional Graphene Structure for Healable Flexible Electronics Based on Diels-Alder Chemistry. ACS Appl Mater Interfaces, 10, 9727-9735.

LI, N., GEORAS, S., ALEXIS, N., FRITZ, P., XIA, T., WILLIAMS, M. A., HORNER, E. \& NEL, A. 2016. A work group report on ultrafine particles (American Academy of Allergy, Asthma \& Immunology): Why ambient ultrafine and engineered nanoparticles should receive special attention for possible adverse health outcomes in human subjects. J Allergy Clin Immunol, 138, 386-96.

LI, N., SIOUTAS, C., CHO, A., SCHMITZ, D., MISRA, C., SEMPF, J., WANG, M., OBERLEY, T., FROINES, J. \& NEL, A. 2003. Ultrafine particulate pollutants induce oxidative stress and mitochondrial damage. Environ Health Perspect, 111, 455-60.

LI, R., GUINEY, L. M., CHANG, C. H., MANSUKHANI, N. D., JI, Z., WANG, X., LIAO, Y. P., JIANG, W., SUN, B., HERSAM, M. C., NEL, A. E. \& XIA, T. 2018b. Surface Oxidation of Graphene Oxide Determines Membrane Damage, Lipid Peroxidation, and Cytotoxicity in Macrophages in a Pulmonary Toxicity Model. ACS Nano, 12, 1390-1402.

LI, S. L., MIYAZAKI, H., SONG, H., KURAMOCHI, H., NAKAHARAI, S. \& TSUKAGOSHI, K. 2012. Quantitative Raman spectrum and reliable thickness identification for atomic layers on insulating substrates. ACS Nano, 6, 7381-8.

LI, Y., YUAN, H., VON DEM BUSSCHE, A., CREIGHTON, M., HURT, R. H., KANE, A. B. \& GAO, H. 2013. Graphene microsheets enter cells through spontaneous membrane penetration at edge asperities and corner sites. Proc Natl Acad Sci U S A, 110, 12295-300.

LIAO, K. H., LIN, Y. S., MACOSKO, C. W. \& HAYNES, C. L. 2011. Cytotoxicity of graphene oxide and graphene in human erythrocytes and skin fibroblasts. ACS Appl Mater Interfaces, 3, 2607-15. 
LIEBER, M., SMITH, B., SZAKAL, A., NELSON-REES, W. \& TODARO, G. 1976. A continuous tumor-cell line from a human lung carcinoma with properties of type II alveolar epithelial cells. Int I Cancer, 17, 62-70.

LIU, X., SEN, S., LIU, J., KULAOTS, I., GEOHEGAN, D., KANE, A., PURETZKY, A. A., ROULEAU, C. M., MORE, K. L., PALMORE, G. T. \& HURT, R. H. 2011. Antioxidant deactivation on graphenic nanocarbon surfaces. Small, 7, 2775-85.

LIU, Z., SUENAGA, K., HARRIS, P. J. \& IIJIMA, S. 2009. Open and closed edges of graphene layers. Phys Rev Lett, 102, 015501.

LU, Y. C., YEH, W. C. \& OHASHI, P. S. 2008. LPS/TLR4 signal transduction pathway. Cytokine, 42, 14551.

LUHRMANN, A., BARGSTEN, G., KUZU, M., KOSLOWSKI, R., PABST, R. \& TSCHERNIG, T. 2007. The alveolar epithelial type I-like cell line as an adequate model for leukocyte migration studies in vitro. Exp Toxicol Pathol, 58, 277-83.

LUI, C. H., LI, Z., CHEN, Z., KLIMOV, P. V., BRUS, L. E. \& HEINZ, T. F. 2011. Imaging stacking order in fewlayer graphene. Nano Lett, 11, 164-9.

LUX, H., EDLING, M., SIEMROTH, P. \& SCHRADER, S. 2018. Fast and Cost-Effective Synthesis of HighQuality Graphene on Copper Foils Using High-Current Arc Evaporation. Materials (Basel), 11.

LUYTS, K., NAPIERSKA, D., DINSDALE, D., KLEIN, S. G., SERCHI, T. \& HOET, P. H. 2015. A coculture model of the lung-blood barrier: the role of activated phagocytic cells. Toxicol In Vitro, 29, 234-41.

MACLEAN-FLETCHER, S. \& POLLARD, T. D. 1980. Mechanism of action of cytochalasin B on actin. Cell, 20, 329-41.

MAJEED, W., BOURDO, S., PETIBONE, D. M., SAINI, V., VANG, K. B., NIMA, Z. A., ALGHAZALI, K. M., DARRIGUES, E., GHOSH, A., WATANABE, F., CASCIANO, D., ALI, S. F. \& BIRIS, A. S. 2017. The role of surface chemistry in the cytotoxicity profile of graphene. J Appl Toxicol, 37, 462-470.

MANSHIAN, B. B., SOENEN, S. J., AL-ALI, A., BROWN, A., HONDOW, N., WILLS, J., JENKINS, G. J. \& DOAK, S. H. 2015. Cell type-dependent changes in CdSe/ZnS quantum dot uptake and toxic endpoints. Toxicol Sci, 144, 246-58.

MASON, R. J., GREENE, K. \& VOELKER, D. R. 1998. Surfactant protein A and surfactant protein D in health and disease. Am J Physiol, 275, L1-13.

MASON, R. J. \& WILLIAMS, M. C. 1980. Phospholipid composition and ultrastructure of A549 cells and other cultured pulmonary epithelial cells of presumed type II cell origin. Biochim Biophys Acta, 617, 36-50.

MATERA, M. G., CALZETTA, L. \& CAZZOLA, M. 2016. Oxidation pathway and exacerbations in COPD: the role of NAC. Expert Rev Respir Med, 10, 89-97.

MERLOT, A. M., KALINOWSKI, D. S. \& RICHARDSON, D. R. 2014. Unraveling the mysteries of serum albumin-more than just a serum protein. Front Physiol, 5, 299.

MESSMER, U. K. \& BRUNE, B. 1996. Nitric oxide-induced apoptosis: p53-dependent and p53independent signalling pathways. Biochem J, 319 ( Pt 1), 299-305.

MEUNIER, E., COSTE, A., OLAGNIER, D., AUTHIER, H., LEFEVRE, L., DARDENNE, C., BERNAD, J., BERAUD, M., FLAHAUT, E. \& PIPY, B. 2012. Double-walled carbon nanotubes trigger IL-1beta release in human monocytes through NIrp3 inflammasome activation. Nanomedicine, 8, 987-95.

MILLER, E. J., TREWBY, W., FAROKH PAYAM, A., PIANTANIDA, L., CAFOLLA, C. \& VOITCHOVSKY, K. 2016. Sub-nanometer Resolution Imaging with Amplitude-modulation Atomic Force Microscopy in Liquid. J Vis Exp.

MILLER, M. R., BORTHWICK, S. J., SHAW, C. A., MCLEAN, S. G., MCCLURE, D., MILLS, N. L., DUFFIN, R., DONALDSON, K., MEGSON, I. L., HADOKE, P. W. \& NEWBY, D. E. 2009. Direct impairment of vascular function by diesel exhaust particulate through reduced bioavailability of endothelium-derived nitric oxide induced by superoxide free radicals. Environ Health Perspect, 117, 611-6.

MITTAL, M., SIDDIQUI, M. R., TRAN, K., REDDY, S. P. \& MALIK, A. B. 2014. Reactive oxygen species in inflammation and tissue injury. Antioxid Redox Signal, 20, 1126-67. 
MITTAL, S., KUMAR, V., DHIMAN, N., CHAUHAN, L. K., PASRICHA, R. \& PANDEY, A. K. 2016. Physicochemical properties based differential toxicity of graphene oxide/reduced graphene oxide in human lung cells mediated through oxidative stress. Sci Rep, 6, 39548.

MOGHARABI, M., ABDOLLAHI, M. \& FARAMARZI, M. A. 2014. Safety concerns to application of graphene compounds in pharmacy and medicine. Daru, 22, 23.

MOLLER, C., ALLEN, M., ELINGS, V., ENGEL, A. \& MULLER, D. J. 1999. Tapping-mode atomic force microscopy produces faithful high-resolution images of protein surfaces. Biophys J, 77, 11508.

MOLLER, P., DANESHVAR, B., LOFT, S., WALLIN, H., POULSEN, H. E., AUTRUP, H., RAVN-HAREN, G. \& DRAGSTED, L. O. 2003. Oxidative DNA damage in vitamin C-supplemented guinea pigs after intratracheal instillation of diesel exhaust particles. Toxicol Appl Pharmacol, 189, 39-44.

MOLLER, P., FOLKMANN, J. K., FORCHHAMMER, L., BRAUNER, E. V., DANIELSEN, P. H., RISOM, L. \& LOFT, S. 2008. Air pollution, oxidative damage to DNA, and carcinogenesis. Cancer Lett, 266, 84-97.

MONKS, J., ROSNER, D., GESKE, F. J., LEHMAN, L., HANSON, L., NEVILLE, M. C. \& FADOK, V. A. 2005. Epithelial cells as phagocytes: apoptotic epithelial cells are engulfed by mammary alveolar epithelial cells and repress inflammatory mediator release. Cell Death Differ, 12, 107-14.

MONOPOLI, M. P., PITEK, A. S., LYNCH, I. \& DAWSON, K. A. 2013. Formation and characterization of the nanoparticle-protein corona. Methods Mol Biol, 1025, 137-55.

MONTES-NAVAJAS, P., ASENJO, N. G., SANTAMARIA, R., MENENDEZ, R., CORMA, A. \& GARCIA, H. 2013. Surface area measurement of graphene oxide in aqueous solutions. Langmuir, 29, 13443-8.

MORGILLO, F., DALLIO, M., DELLA CORTE, C. M., GRAVINA, A. G., VISCARDI, G., LOGUERCIO, C., CIARDIELLO, F. \& FEDERICO, A. 2018. Carcinogenesis as a Result of Multiple Inflammatory and Oxidative Hits: a Comprehensive Review from Tumor Microenvironment to Gut Microbiota. Neoplasia, 20, 721-733.

MUKHERJEE, S. P., BOTTINI, M. \& FADEEL, B. 2017. Graphene and the Immune System: A Romance of Many Dimensions. Front Immunol, 8, 673.

MUKHERJEE, S. P., KOSTARELOS, K. \& FADEEL, B. 2018. Cytokine Profiling of Primary Human Macrophages Exposed to Endotoxin-Free Graphene Oxide: Size-Independent NLRP3 Inflammasome Activation. Adv Healthc Mater, 7.

MUKHERJEE, S. P., LOZANO, N., KUCKI, M., DEL RIO-CASTILLO, A. E., NEWMAN, L., VAZQUEZ, E., KOSTARELOS, K., WICK, P. \& FADEEL, B. 2016. Detection of Endotoxin Contamination of Graphene Based Materials Using the TNF-alpha Expression Test and Guidelines for EndotoxinFree Graphene Oxide Production. PLoS One, 11, e0166816.

MULLER, J., DECORDIER, I., HOET, P. H., LOMBAERT, N., THOMASSEN, L., HUAUX, F., LISON, D. \& KIRSCH-VOLDERS, M. 2008. Clastogenic and aneugenic effects of multi-wall carbon nanotubes in epithelial cells. Carcinogenesis, 29, 427-33.

MULLICK CHOWDHURY, S., LALWANI, G., ZHANG, K., YANG, J. Y., NEVILLE, K. \& SITHARAMAN, B. 2013. Cell specific cytotoxicity and uptake of graphene nanoribbons. Biomaterials, 34, 283-93.

MUTHOOSAMY, K., BAI, R. G., ABUBAKAR, I. B., SUDHEER, S. M., LIM, H. N., LOH, H. S., HUANG, N. M., CHIA, C. H. \& MANICKAM, S. 2015. Exceedingly biocompatible and thin-layered reduced graphene oxide nanosheets using an eco-friendly mushroom extract strategy. Int J Nanomedicine, 10, 1505-19.

MUZYKA, R., DREWNIAK, S., PUSTELNY, T., CHRUBASIK, M. \& GRYGLEWICZ, G. 2018. Characterization of Graphite Oxide and Reduced Graphene Oxide Obtained from Different Graphite Precursors and Oxidized by Different Methods Using Raman Spectroscopy. Materials (Basel), 11.

NAIR, R. R., BLAKE, P., GRIGORENKO, A. N., NOVOSELOV, K. S., BOOTH, T. J., STAUBER, T., PERES, N. M. \& GEIM, A. K. 2008. Fine structure constant defines visual transparency of graphene. Science, $320,1308$.

NAIR, R. R., REN, W., JALIL, R., RIAZ, I., KRAVETS, V. G., BRITNELL, L., BLAKE, P., SCHEDIN, F., MAYOROV, A. S., YUAN, S., KATSNELSON, M. I., CHENG, H. M., STRUPINSKI, W., BULUSHEVA, L. G., 
OKOTRUB, A. V., GRIGORIEVA, I. V., GRIGORENKO, A. N., NOVOSELOV, K. S. \& GEIM, A. K. 2010. Fluorographene: a two-dimensional counterpart of Teflon. Small, 6, 2877-84.

NAIR, R. R., WU, H. A., JAYARAM, P. N., GRIGORIEVA, I. V. \& GEIM, A. K. 2012. Unimpeded permeation of water through helium-leak-tight graphene-based membranes. Science, 335, 442-4.

NAKATA, T. 1993. Destruction of typical endotoxins by dry heat as determined using LAL assay and pyrogen assay. J Parenter Sci Technol, 47, 258-64.

NAZARENUS, M., ZHANG, Q., SOliMAN, M. G., DEL PINO, P., PELAZ, B., CARREGAL-ROMERO, S., REJMAN, J., ROTHEN-RUTISHAUSER, B., CLIFT, M. J., ZELLNER, R., NIENHAUS, G. U., DELEHANTY, J. B., MEDINTZ, I. L. \& PARAK, W. J. 2014. In vitro interaction of colloidal nanoparticles with mammalian cells: What have we learned thus far? Beilstein J Nanotechnol, 5, 1477-90.

NEL, A., XIA, T., MADLER, L. \& LI, N. 2006. Toxic potential of materials at the nanolevel. Science, 311, 622-7.

NEL, A. E., MADLER, L., VELEGOL, D., XIA, T., HOEK, E. M., SOMASUNDARAN, P., KLAESSIG, F., CASTRANOVA, V. \& THOMPSON, M. 2009. Understanding biophysicochemical interactions at the nano-bio interface. Nat Mater, 8, 543-57.

NEMMAR, A., HOYLAERTS, M. F., HOET, P. H., DINSDALE, D., SMITH, T., XU, H., VERMYLEN, J. \& NEMERY, B. 2002. Ultrafine particles affect experimental thrombosis in an in vivo hamster model. Am J Respir Crit Care Med, 166, 998-1004.

NI, Z. H., WANG, H. M., KASIM, J., FAN, H. M., YU, T., WU, Y. H., FENG, Y. P. \& SHEN, Z. X. 2007. Graphene thickness determination using reflection and contrast spectroscopy. Nano Lett, 7, 2758-63.

NICHOLLS, D. G. 2002. Mitochondrial function and dysfunction in the cell: its relevance to aging and aging-related disease. Int J Biochem Cell Biol, 34, 1372-81.

NOVOSELOV, K. S., FAL'KO, V. I., COLOMBO, L., GELLERT, P. R., SCHWAB, M. G. \& KIM, K. 2012. A roadmap for graphene. Nature, 490, 192-200.

NOVOSELOV, K. S., GEIM, A. K., MOROZOV, S. V., JIANG, D., ZHANG, Y., DUBONOS, S. V., GRIGORIEVA, I. V. \& FIRSOV, A. A. 2004. Electric field effect in atomically thin carbon films. Science, 306, 666-9.

NOWACK, B., BOLDRIN, A., CABALLERO, A., HANSEN, S. F., GOTTSCHALK, F., HEGGELUND, L., HENNIG, M., MACKEVICA, A., MAES, H., NAVRATILOVA, J., NEUBAUER, N., PETERS, R., ROSE, J., SCHAFFER, A., SCIFO, L., VAN LEEUWEN, S., VON DER KAMMER, F., WOHLLEBEN, W., WYRWOLL, A. \& HRISTOZOV, D. 2016. Meeting the Needs for Released Nanomaterials Required for Further Testing-The SUN Approach. Environ Sci Technol, 50, 2747-53.

O'HARE, M. J., BOND, J., CLARKE, C., TAKEUCHI, Y., ATHERTON, A. J., BERRY, C., MOODY, J., SILVER, A. R., DAVIES, D. C., ALSOP, A. E., NEVILLE, A. M. \& JAT, P. S. 2001. Conditional immortalization of freshly isolated human mammary fibroblasts and endothelial cells. Proc Natl Acad Sci U S A, 98, 646-51.

OBERDORSTER, G., MAYNARD, A., DONALDSON, K., CASTRANOVA, V., FITZPATRICK, J., AUSMAN, K., CARTER, J., KARN, B., KREYLING, W., LAI, D., OLIN, S., MONTEIRO-RIVIERE, N., WARHEIT, D. \& YANG, H. 2005a. Principles for characterizing the potential human health effects from exposure to nanomaterials: elements of a screening strategy. Part Fibre Toxicol, 2, 8.

OBERDORSTER, G., OBERDORSTER, E. \& OBERDORSTER, J. 2005b. Nanotoxicology: an emerging discipline evolving from studies of ultrafine particles. Environ Health Perspect, 113, 823-39.

$\mathrm{OH}$, N. \& PARK, J. H. 2014. Endocytosis and exocytosis of nanoparticles in mammalian cells. Int J Nanomedicine, 9 Suppl 1, 51-63.

ONG, K. J., MACCORMACK, T. J., CLARK, R. J., EDE, J. D., ORTEGA, V. A., FELIX, L. C., DANG, M. K., MA, G., FENNIRI, H., VEINOT, J. G. \& GOSS, G. G. 2014. Widespread nanoparticle-assay interference: implications for nanotoxicity testing. PLoS One, 9, e90650.

PANARITI, A., MISEROCCHI, G. \& RIVOLTA, I. 2012. The effect of nanoparticle uptake on cellular behavior: disrupting or enabling functions? Nanotechnol Sci Appl, 5, 87-100. 
PARK, E. J., LEE, S. J., LEE, K., CHOI, Y. C., LEE, B. S., LEE, G. H. \& KIM, D. W. 2017a. Pulmonary persistence of graphene nanoplatelets may disturb physiological and immunological homeostasis. J Appl Toxicol, 37, 296-309.

PARK, M., BLEEKER, E. A. J., BRAND, W., CASSEE, F. R., VAN ELK, M., GOSENS, I., DE JONG, W. H., MEESTERS, J. A. J., PEIJNENBURG, W., QUIK, J. T. K., VANDEBRIEL, R. J. \& SIPS, A. 2017b. Considerations for Safe Innovation: The Case of Graphene. ACS Nano, 11, 9574-9593.

PARKER, D. \& PRINCE, A. 2011. Innate immunity in the respiratory epithelium. Am J Respir Cell Mol Biol, 45, 189-201.

PATLOlLA, A. K., HUSSAIN, S. M., SCHLAGER, J. J., PATLOLLA, S. \& TCHOUNWOU, P. B. 2010. Comparative study of the clastogenicity of functionalized and nonfunctionalized multiwalled carbon nanotubes in bone marrow cells of Swiss-Webster mice. Environ Toxicol, 25, 608-21.

PATON, K. R., VARRLA, E., BACKES, C., SMITH, R. J., KHAN, U., O'NEILL, A., BOLAND, C., LOTYA, M., ISTRATE, O. M., KING, P., HIGGINS, T., BARWICH, S., MAY, P., PUCZKARSKI, P., AHMED, I., MOEBIUS, M., PETTERSSON, H., LONG, E., COELHO, J., O'BRIEN, S. E., MCGUIRE, E. K., SANCHEZ, B. M., DUESBERG, G. S., MCEVOY, N., PENNYCOOK, T. J., DOWNING, C., CROSSLEY, A., NICOLOSI, V. \& COLEMAN, J. N. 2014. Scalable production of large quantities of defect-free few-layer graphene by shear exfoliation in liquids. Nat Mater, 13, 624-30.

PATTON, J. S. \& BYRON, P. R. 2007. Inhaling medicines: delivering drugs to the body through the lungs. Nat Rev Drug Discov, 6, 67-74.

PELKMANS, L. 2005. Secrets of caveolae- and lipid raft-mediated endocytosis revealed by mammalian viruses. Biochim Biophys Acta, 1746, 295-304.

PFUHLER, S., FAUTZ, R., OUEDRAOGO, G., LATIL, A., KENNY, J., MOORE, C., DIEMBECK, W., HEWITT, N. J., REISINGER, K. \& BARROSO, J. 2014. The Cosmetics Europe strategy for animal-free genotoxicity testing: project status up-date. Toxicol In Vitro, 28, 18-23.

QIAN, L., WANG, W., ZHOU, Y. \& MA, J. 2017. Effects of reduced glutathione therapy on chronic hepatitis B. Cent Eur J Immunol, 42, 97-100.

QU, G., LIU, S., ZHANG, S., WANG, L., WANG, X., SUN, B., YIN, N., GAO, X., XIA, T., CHEN, J. J. \& JIANG, G. B. 2013. Graphene oxide induces toll-like receptor 4 (TLR4)-dependent necrosis in macrophages. ACS Nano, 7, 5732-45.

RAMACHANDRAIAH, K., HAN, S. G. \& CHIN, K. B. 2015. Nanotechnology in meat processing and packaging: potential applications - a review. Asian-Australas J Anim Sci. Korea South.

RAMOS, I. R., MALKIN, A. \& LYNG, F. M. 2015. Current Advances in the Application of Raman Spectroscopy for Molecular Diagnosis of Cervical Cancer. Biomed Res Int, 2015, 561242.

RASHIDI, L. \& KHOSRAVI-DARANI, K. 2011. The applications of nanotechnology in food industry. Crit Rev Food Sci Nutr, 51, 723-30.

REJMAN, J., OBERLE, V., ZUHORN, I. S. \& HOEKSTRA, D. 2004. Size-dependent internalization of particles via the pathways of clathrin- and caveolae-mediated endocytosis. Biochem J, 377, $159-69$.

REN, H., WANG, C., ZHANG, J., ZHOU, X., XU, D., ZHENG, J. \& GUO, S. 2010. DNA cleavage system of nanosized graphene oxide sheets and copper ions. ACS Nano, 4, 7169-74.

RESHMA, S. C., SYAMA, S. \& MOHANAN, P. V. 2016. Nano-biointeractions of PEGylated and bare reduced graphene oxide on lung alveolar epithelial cells: A comparative in vitro study. Colloids Surf B Biointerfaces, 140, 104-116.

RIDENE, M., KHA, C. S. \& FLIPSE, C. F. 2016. Role of silicon dangling bonds in the electronic properties of epitaxial graphene on silicon carbide. Nanotechnology, 27, 125705.

RIEDINGER, A., ZHANG, F., DOMMERSHAUSEN, F., ROCKER, C., BRANDHOLT, S., NIENHAUS, G. U., KOERT, U. \& PARAK, W. J. 2010. Ratiometric optical sensing of chloride ions with organic fluorophore-gold nanoparticle hybrids: a systematic study of design parameters and surface charge effects. Small, 6, 2590-7.

RISOM, L., MOLLER, P. \& LOFT, S. 2005. Oxidative stress-induced DNA damage by particulate air pollution. Mutat Res, 592, 119-37. 
ROACH, P., FARRAR, D. \& PERRY, C. C. 2005. Interpretation of protein adsorption: surface-induced conformational changes. J Am Chem Soc, 127, 8168-73.

ROBBINS, R. A., SISSON, J. H., SPRINGALL, D. R., NELSON, K. J., TAYLOR, J. A., MASON, N. A., POLAK, J. M. \& TOWNLEY, R. G. 1997. Human lung mononuclear cells induce nitric oxide synthase in murine airway epithelial cells in vitro: role of TNFalpha and IL-1beta. Am J Respir Crit Care Med, 155, 268-73.

ROBSON, A. L., DASTOOR, P. C., FLYNN, J., PALMER, W., MARTIN, A., SMITH, D. W., WOLDU, A. \& HUA, S. 2018. Advantages and Limitations of Current Imaging Techniques for Characterizing Liposome Morphology. Front Pharmacol, 9, 80.

ROITER, Y. \& MINKO, S. 2005. AFM single molecule experiments at the solid-liquid interface: in situ conformation of adsorbed flexible polyelectrolyte chains. J Am Chem Soc, 127, 15688-9.

ROMANO, M., SIRONI, M., TONIATTI, C., POLENTARUTTI, N., FRUSCELLA, P., GHEZZI, P., FAGGIONI, R., LUINI, W., VAN HINSBERGH, V., SOZZANI, S., BUSSOLINO, F., POLI, V., CILIBERTO, G. \& MANTOVANI, A. 1997. Role of IL-6 and its soluble receptor in induction of chemokines and leukocyte recruitment. Immunity, 6, 315-25.

ROTHEN-RUTISHAUSER, B. M., KIAMA, S. G. \& GEHR, P. 2005. A three-dimensional cellular model of the human respiratory tract to study the interaction with particles. Am J Respir Cell Mol Biol, 32, 281-9.

ROUSE, R. L., MURPHY, G., BOUDREAUX, M. J., PAULSEN, D. B. \& PENN, A. L. 2008. Soot nanoparticles promote biotransformation, oxidative stress, and inflammation in murine lungs. Am J Respir Cell Mol Biol, 39, 198-207.

RUENRAROENGSAK, P., CHEN, S., HU, S., MELBOURNE, J., SWEENEY, S., THORLEY, A. J., SKEPPER, J. N., SHAFFER, M. S., TETLEY, T. D. \& PORTER, A. E. 2016. Translocation of Functionalized MultiWalled Carbon Nanotubes across Human Pulmonary Alveolar Epithelium: Dominant Role of Epithelial Type 1 Cells. ACS Nano, 10, 5070-85.

RUENRAROENGSAK, P. \& TETLEY, T. D. 2015. Differential bioreactivity of neutral, cationic and anionic polystyrene nanoparticles with cells from the human alveolar compartment: robust response of alveolar type 1 epithelial cells. Part Fibre Toxicol, 12, 19.

RUGE, C. A., SCHAEFER, U. F., HERRMANN, J., KIRCH, J., CANADAS, O., ECHAIDE, M., PEREZ-GIL, J., CASALS, C., MULLER, R. \& LEHR, C. M. 2012. The interplay of lung surfactant proteins and lipids assimilates the macrophage clearance of nanoparticles. PLoS One, 7, e40775.

SAHOO, S. K., PARVEEN, S. \& PANDA, J. J. 2007. The present and future of nanotechnology in human health care. Nanomedicine, 3, 20-31.

SAHU, D., KANNAN, G. M. \& VIJAYARAGHAVAN, R. 2014. Carbon black particle exhibits size dependent toxicity in human monocytes. Int J Inflam, 2014, 827019.

SANCHEZ, V. C., JACHAK, A., HURT, R. H. \& KANE, A. B. 2012. Biological interactions of graphene-family nanomaterials: an interdisciplinary review. Chem Res Toxicol, 25, 15-34.

SAVOLAINEN, K., ALENIUS, H., NORPPA, H., PYLKKANEN, L., TUOMI, T. \& KASPER, G. 2010. Risk assessment of engineered nanomaterials and nanotechnologies--a review. Toxicology, 269, 92-104.

SAYES, C. M. \& WARHEIT, D. B. 2009. Characterization of nanomaterials for toxicity assessment. Wiley Interdiscip Rev Nanomed Nanobiotechnol, 1, 660-70.

SCASSELLATI SFORZOLINI, G., MORETTI, M., VILLARINI, M., FATIGONI, C. \& PASQUINI, R. 2004. [Evaluation of genotoxic and/or co-genotoxic effects in cells exposed in vitro to extremely-low frequency electromagnetic fields]. Ann $I g, 16,321-40$.

SCHINS, R. P. 2002. Mechanisms of genotoxicity of particles and fibers. Inhal Toxicol, 14, 57-78.

SCHINS, R. P. \& KNAAPEN, A. M. 2007. Genotoxicity of poorly soluble particles. Inhal Toxicol, 19 Suppl 1, 189-98.

SCHINWALD, A., MURPHY, F. A., JONES, A., MACNEE, W. \& DONALDSON, K. 2012. Graphene-based nanoplatelets: a new risk to the respiratory system as a consequence of their unusual aerodynamic properties. ACS Nano, 6, 736-46. 
SCOTT-FORDSMAND, J. J., PEIJNENBURG, W., SEMENZIN, E., NOWACK, B., HUNT, N., HRISTOZOV, D., MARCOMINI, A., IRFAN, M. A., JIMENEZ, A. S., LANDSIEDEL, R., TRAN, L., OOMEN, A. G., BOS, P. M. J. \& HUND-RINKE, K. 2017. Environmental Risk Assessment Strategy for Nanomaterials. Int J Environ Res Public Health, 14.

SEATON, B. A., CROUCH, E. C., MCCORMACK, F. X., HEAD, J. F., HARTSHORN, K. L. \& MENDELSOHN, R. 2010. Review: Structural determinants of pattern recognition by lung collectins. Innate Immun, 16, 143-50.

SEN, C. K. 1998. Redox signaling and the emerging therapeutic potential of thiol antioxidants. Biochem Pharmacol, 55, 1747-58.

SEN, C. K. \& PACKER, L. 1996. Antioxidant and redox regulation of gene transcription. Faseb j, 10, 70920.

SENGUPTA, B., GREGORY, W. E., ZHU, J., DASETTY, S., KARAKAYA, M., BROWN, J. M., RAO, A. M., BARROWS, J. K., SARUPRIA, S. \& PODILA, R. 2015. Influence of carbon nanomaterial defects on the formation of protein corona. RSC Adv, 5, 82395-82402.

SERRANO, A. G. \& PEREZ-GIL, J. 2006. Protein-lipid interactions and surface activity in the pulmonary surfactant system. Chem Phys Lipids, 141, 105-18.

SHAH, U. K., MALLIA, J. O., SINGH, N., CHAPMAN, K. E., DOAK, S. H. \& JENKINS, G. J. S. 2018. Reprint of: A three-dimensional in vitro HepG2 cells liver spheroid model for genotoxicity studies. Mutat Res, 834, 35-41.

SHEARER, C. J., SLATTERY, A. D., STAPLETON, A. J., SHAPTER, J. G. \& GIBSON, C. T. 2016. Accurate thickness measurement of graphene. Nanotechnology, 27, 125704.

SHTEIN, M., PRI-BAR, I., VARENIK, M. \& REGEV, O. 2015. Characterization of graphene-nanoplatelets structure via thermogravimetry. Anal Chem, 87, 4076-80.

SIMBERG, D., PARK, J. H., KARMALI, P. P., ZHANG, W. M., MERKULOV, S., MCCRAE, K., BHATIA, S. N., SAILOR, M. \& RUOSLAHTI, E. 2009. Differential proteomics analysis of the surface heterogeneity of dextran iron oxide nanoparticles and the implications for their in vivo clearance. Biomaterials, 30, 3926-33.

SINGH, N., MANSHIAN, B., JENKINS, G. J., GRIFFITHS, S. M., WILLIAMS, P. M., MAFFEIS, T. G., WRIGHT, C. J. \& DOAK, S. H. 2009. NanoGenotoxicology: the DNA damaging potential of engineered nanomaterials. Biomaterials, 30, 3891-914.

SINGH, Z. 2016. Applications and toxicity of graphene family nanomaterials and their composites. Nanotechnol Sci Appl, 9, 15-28.

SMULDERS, S., KAISER, J. P., ZUIN, S., VAN LANDUYT, K. L., GOLANSKI, L., VANOIRBEEK, J., WICK, P. \& HOET, P. H. 2012. Contamination of nanoparticles by endotoxin: evaluation of different test methods. Part Fibre Toxicol, 9, 41.

SOARES, S., SOUSA, J., PAIS, A. \& VITORINO, C. 2018. Nanomedicine: Principles, Properties, and Regulatory Issues. Front Chem, 6, 360.

SOROKIN, S. P. 1970. Properties of alveolar cells and tissues that strengthen alveolar defenses. Arch Intern Med, 126, 450-63.

SPEIT, G. 2009. How to assess the mutagenic potential of cosmetic products without animal tests? Mutat Res, 678, 108-12.

STEELE, M. P., LEVINE, R. A., JOYCE-BRADY, M. \& BRODY, J. S. 1992. A rat alveolar type II cell line developed by adenovirus 12SE1A gene transfer. Am J Respir Cell Mol Biol, 6, 50-6.

SU, W. C., KU, B. K., KULKARNI, P. \& CHENG, Y. S. 2016. Deposition of graphene nanomaterial aerosols in human upper airways. J Occup Environ Hyg, 13, 48-59.

SU, X., FRICKE, J., KAVANAGH, D. G. \& IRVINE, D. J. 2011. In vitro and in vivo mRNA delivery using lipidenveloped pH-responsive polymer nanoparticles. Mol Pharm, 8, 774-87.

SUBBIAH, R., VEERAPANDIAN, M. \& YUN, K. S. 2010. Nanoparticles: functionalization and multifunctional applications in biomedical sciences. Curr Med Chem, 17, 4559-77. 
SUKHANOVA, A., BOZROVA, S., SOKOLOV, P., BERESTOVOY, M., KARAULOV, A. \& NABIEV, I. 2018. Dependence of Nanoparticle Toxicity on Their Physical and Chemical Properties. Nanoscale Res Lett, 13, 44.

SUN, H. J., LIU, B., PENG, T. J. \& ZHAO, X. L. 2018. Effect of Reaction Temperature on Structure, Appearance and Bonding Type of Functionalized Graphene Oxide Modified P-Phenylene Diamine. Materials (Basel), 11.

SUSI, T., PICHLER, T. \& AYALA, P. 2015. X-ray photoelectron spectroscopy of graphitic carbon nanomaterials doped with heteroatoms. Beilstein J Nanotechnol, 6, 177-92.

SWAIN, R. J., KEMP, S. J., GOLDSTRAW, P., TETLEY, T. D. \& STEVENS, M. M. 2010. Assessment of cell line models of primary human cells by Raman spectral phenotyping. Biophys J, 98, 1703-11.

SWEENEY, S., BERHANU, D., RUENRAROENGSAK, P., THORLEY, A. J., VALSAMI-JONES, E. \& TETLEY, T. D. 2015. Nano-titanium dioxide bioreactivity with human alveolar type-l-like epithelial cells: Investigating crystalline phase as a critical determinant. Nanotoxicology, 9, 482-92.

TAATJES, D. J., QUINN, A. S., RAND, J. H. \& JENA, B. P. 2013. Atomic force microscopy: High resolution dynamic imaging of cellular and molecular structure in health and disease. J Cell Physiol, 228, 1949-55.

TABET, L., BUSSY, C., SETYAN, A., SIMON-DECKERS, A., ROSSI, M. J., BOCZKOWSKI, J. \& LANONE, S. 2011. Coating carbon nanotubes with a polystyrene-based polymer protects against pulmonary toxicity. Part Fibre Toxicol, 8, 3.

TAN, H. Y., WANG, N., LI, S., HONG, M., WANG, X. \& FENG, Y. 2016. The Reactive Oxygen Species in Macrophage Polarization: Reflecting Its Dual Role in Progression and Treatment of Human Diseases. Oxid Med Cell Longev, 2016, 2795090.

TAYLOR, E. L., ROSSI, A. G., SHAW, C. A., DAL RIO, F. P., HASLETT, C. \& MEGSON, I. L. 2004. GEA 3162 decomposes to co-generate nitric oxide and superoxide and induces apoptosis in human neutrophils via a peroxynitrite-dependent mechanism. Br J Pharmacol, 143, 179-85.

TENZER, S., DOCTER, D., KUHAREV, J., MUSYANOVYCH, A., FETZ, V., HECHT, R., SCHLENK, F., FISCHER, D., KIOUPTSI, K., REINHARDT, C., LANDFESTER, K., SCHILD, H., MASKOS, M., KNAUER, S. K. \& STAUBER, R. H. 2013. Rapid formation of plasma protein corona critically affects nanoparticle pathophysiology. Nat Nanotechnol, 8, 772-81.

TENZER, S., DOCTER, D., ROSFA, S., WLODARSKI, A., KUHAREV, J., REKIK, A., KNAUER, S. K., BANTZ, C., NAWROTH, T., BIER, C., SIRIRATTANAPAN, J., MANN, W., TREUEL, L., ZELLNER, R., MASKOS, M., SCHILD, H. \& STAUBER, R. H. 2011. Nanoparticle size is a critical physicochemical determinant of the human blood plasma corona: a comprehensive quantitative proteomic analysis. ACS Nano, 5, 7155-67.

TETZ, L. M., KAMAU, P. W., CHENG, A. A., MEEKER, J. D. \& LOCH-CARUSO, R. 2013. Troubleshooting the dichlorofluorescein assay to avoid artifacts in measurement of toxicant-stimulated cellular production of reactive oxidant species. J Pharmacol Toxicol Methods, 67, 56-60.

THIRUVENGADAM, M., RAJAKUMAR, G. \& CHUNG, I. M. 2018. Nanotechnology: current uses and future applications in the food industry. 3 Biotech, 8, 74.

THORLEY, A. J., RUENRAROENGSAK, P., POTTER, T. E. \& TETLEY, T. D. 2014. Critical determinants of uptake and translocation of nanoparticles by the human pulmonary alveolar epithelium. ACS Nano, 8, 11778-89.

THORLEY, A. J. \& TETLEY, T. D. 2007. Pulmonary epithelium, cigarette smoke, and chronic obstructive pulmonary disease. Int J Chron Obstruct Pulmon Dis, 2, 409-28.

TOBE, M., OGURA, N., ABIKO, Y. \& NAGURA, H. 2002. Interleukin-1beta stimulates interleukin-8 production and gene expression in synovial cells from human temporomandibular joint. J Oral Maxillofac Surg, 60, 741-7.

TRACHE, A. \& MEININGER, G. A. 2008. Atomic force microscopy (AFM). Curr Protoc Microbiol, Chapter 2, Unit 2C.2.

TRIPATHI, P. 2007. Nitric oxide and immune response. Indian J Biochem Biophys, 44, 310-9. 
TROTT, O. \& OLSON, A. J. 2010. AutoDock Vina: improving the speed and accuracy of docking with a new scoring function, efficient optimization, and multithreading. J Comput Chem, 31, 455-61.

TSUJI, K. \& HARRISON, S. J. 1979. Limulus amebocyte lysate - a means to monitor inactivation of lipopolysaccharide. Prog Clin Biol Res, 29, 367-78.

VAN DEN BOGAARD, E. H., DAILEY, L. A., THORLEY, A. J., TETLEY, T. D. \& FORBES, B. 2009. Inflammatory response and barrier properties of a new alveolar type 1-like cell line (TT1). Pharm Res, 26, $1172-80$.

VICARELLI, L., HEEREMA, S. J., DEKKER, C. \& ZANDBERGEN, H. W. 2015. Controlling defects in graphene for optimizing the electrical properties of graphene nanodevices. ACS Nano, 9, 3428-35.

VOROBJEVA, N., PRIKHODKO, A., GALKIN, I., PLETJUSHKINA, O., ZINOVKIN, R., SUD'INA, G., CHERNYAK, B. \& PINEGIN, B. 2017. Mitochondrial reactive oxygen species are involved in chemoattractant-induced oxidative burst and degranulation of human neutrophils in vitro. Eur J Cell Biol, 96, 254-265.

WALKEY, C. D. \& CHAN, W. C. 2012. Understanding and controlling the interaction of nanomaterials with proteins in a physiological environment. Chem Soc Rev, 41, 2780-99.

WANG, A., PU, K., DONG, B., LIU, Y., ZHANG, L., ZHANG, Z., DUAN, W. \& ZHU, Y. 2013. Role of surface charge and oxidative stress in cytotoxicity and genotoxicity of graphene oxide towards human lung fibroblast cells. J Appl Toxicol, 33, 1156-64.

WANG, W. L., BHANDARI, S., YI, W., BELL, D. C., WESTERVELT, R. \& KAXIRAS, E. 2012. Direct imaging of atomic-scale ripples in few-layer graphene. Nano Lett, 12, 2278-82.

WANG, Z., TIRUPPATHI, C., MINSHALL, R. D. \& MALIK, A. B. 2009. Size and dynamics of caveolae studied using nanoparticles in living endothelial cells. ACS Nano, 3, 4110-6.

WHITSETT, J. A., NOGEE, L. M., WEAVER, T. E. \& HOROWITZ, A. D. 1995. Human surfactant protein B: structure, function, regulation, and genetic disease. Physiol Rev, 75, 749-57.

WILLS, J. W., SUMMERS, H. D., HONDOW, N., SOORESH, A., MEISSNER, K. E., WHITE, P. A., REES, P., BROWN, A. \& DOAK, S. H. 2017. Characterizing Nanoparticles in Biological Matrices: Tipping Points in Agglomeration State and Cellular Delivery In Vitro. ACS Nano, 11, 11986-12000.

WINEY, M., MEEHL, J. B., O'TOOLE, E. T. \& GIDDINGS, T. H., JR. 2014. Conventional transmission electron microscopy. Mol Biol Cell, 25, 319-23.

WOJCIK, M., HAUSER, M., LI, W., MOON, S. \& XU, K. 2015. Graphene-enabled electron microscopy and correlated super-resolution microscopy of wet cells. Nat Commun, 6, 7384.

WRIGHT, J. R. 2005. Immunoregulatory functions of surfactant proteins. Nat Rev Immunol, 5, 58-68.

WU, H., CHEN, M., SHANG, M., LI, X., MU, K., FAN, S., JIANG, S. \& LI, W. 2018. Insights into the binding behavior of bovine serum albumin to black carbon nanoparticles and induced cytotoxicity. Spectrochim Acta A Mol Biomol Spectrosc, 200, 51-57.

XIA, T., KOVOCHICH, M., LIONG, M., MENG, H., KABEHIE, S., GEORGE, S., ZINK, J. I. \& NEL, A. E. 2009. Polyethyleneimine coating enhances the cellular uptake of mesoporous silica nanoparticles and allows safe delivery of siRNA and DNA constructs. ACS Nano, 3, 3273-86.

XU, L., YANHUI, D., ZHENYU, W., JIAN, Z., FEI, L., WHITE, J. C. \& BAOSHAN, X. 2018. Graphene quantum dots in alveolar macrophage: uptake-exocytosis, accumulation in nuclei, nuclear responses and DNA cleavage. Particle and Fibre Toxicology.

YANG, K., WAN, J., ZHANG, S., TIAN, B., ZHANG, Y. \& LIU, Z. 2012. The influence of surface chemistry and size of nanoscale graphene oxide on photothermal therapy of cancer using ultra-low laser power. Biomaterials, 33, 2206-14.

YANG, K., ZHANG, S., ZHANG, G., SUN, X., LEE, S. T. \& LIU, Z. 2010. Graphene in mice: ultrahigh in vivo tumor uptake and efficient photothermal therapy. Nano Lett, 10, 3318-23.

YAO, T., XIN, G., SCOTT, S. M., GONG, B. \& LIAN, J. 2018. Thermally-Conductive and MechanicallyRobust Graphene Nanoplatelet Reinforced UO2 Composite Nuclear Fuels. Sci Rep, 8, 2987.

YOKEL, R. A. \& MACPHAIL, R. C. 2011. Engineered nanomaterials: exposures, hazards, and risk prevention. J Occup Med Toxicol, 6, 7. 
ZAFARULLAH, M., LI, W. Q., SYLVESTER, J. \& AHMAD, M. 2003. Molecular mechanisms of Nacetylcysteine actions. Cell Mol Life Sci, 60, 6-20.

ZENSI, A., BEGLEY, D., PONTIKIS, C., LEGROS, C., MIHOREANU, L., BUCHEL, C. \& KREUTER, J. 2010. Human serum albumin nanoparticles modified with apolipoprotein $A-I$ cross the blood-brain barrier and enter the rodent brain. J Drug Target, 18, 842-8.

ZENSI, A., BEGLEY, D., PONTIKIS, C., LEGROS, C., MIHOREANU, L., WAGNER, S., BUCHEL, C., VON BRIESEN, H. \& KREUTER, J. 2009. Albumin nanoparticles targeted with Apo E enter the CNS by transcytosis and are delivered to neurones. J Control Release, 137, 78-86.

ZHANG, B., WEI, P., ZHOU, Z. \& WEI, T. 2016. Interactions of graphene with mammalian cells: Molecular mechanisms and biomedical insights. Adv Drug Deliv Rev, 105, 145-162.

ZHANG, F., ALI, Z., AMIN, F., FELTZ, A., OHEIM, M. \& PARAK, W. J. 2010. Ion and pH sensing with colloidal nanoparticles: influence of surface charge on sensing and colloidal properties. Chemphyschem, 11, 730-5.

ZHANG, F., LEES, E., AMIN, F., RIVERA GIL, P., YANG, F., MULVANEY, P. \& PARAK, W. J. 2011. Polymercoated nanoparticles: a universal tool for biolabelling experiments. Small, 7, 3113-27.

ZHANG, S., GAO, H. \& BAO, G. 2015. Physical Principles of Nanoparticle Cellular Endocytosis. ACS Nano, 9, 8655-71.

ZHANG, S., LI, J., LYKOTRAFITIS, G., BAO, G. \& SURESH, S. 2009. Size-Dependent Endocytosis of Nanoparticles. Adv Mater, 21, 419-424.

ZHANG, Y., TAN, Y. W., STORMER, H. L. \& KIM, P. 2005. Experimental observation of the quantum Hall effect and Berry's phase in graphene. Nature, 438, 201-4.

ZHAO, L., ZHU, Y., CHEN, Z., XU, H., ZHOU, J., TANG, S., XU, Z., KONG, F., LI, X., ZHANG, Y., ZHANG, J. \& JIA, G. 2018. Cardiopulmonary effects induced by occupational exposure to titanium dioxide nanoparticles. Nanotoxicology, 12, 169-184.

ZHI, X., FANG, H., BAO, C., SHEN, G., ZHANG, J., WANG, K., GUO, S., WAN, T. \& CUI, D. 2013. The immunotoxicity of graphene oxides and the effect of PVP-coating. Biomaterials, 34, 5254-61.

ZHOU, H., ZHANG, B., ZHENG, J., YU, M., ZHOU, T., ZHAO, K., JIA, Y., GAO, X., CHEN, C. \& WEI, T. 2014. The inhibition of migration and invasion of cancer cells by graphene via the impairment of mitochondrial respiration. Biomaterials, 35, 1597-607. 
7.1 Total Protein Levels assessed by the DC Protein Assay (required for normalisation)
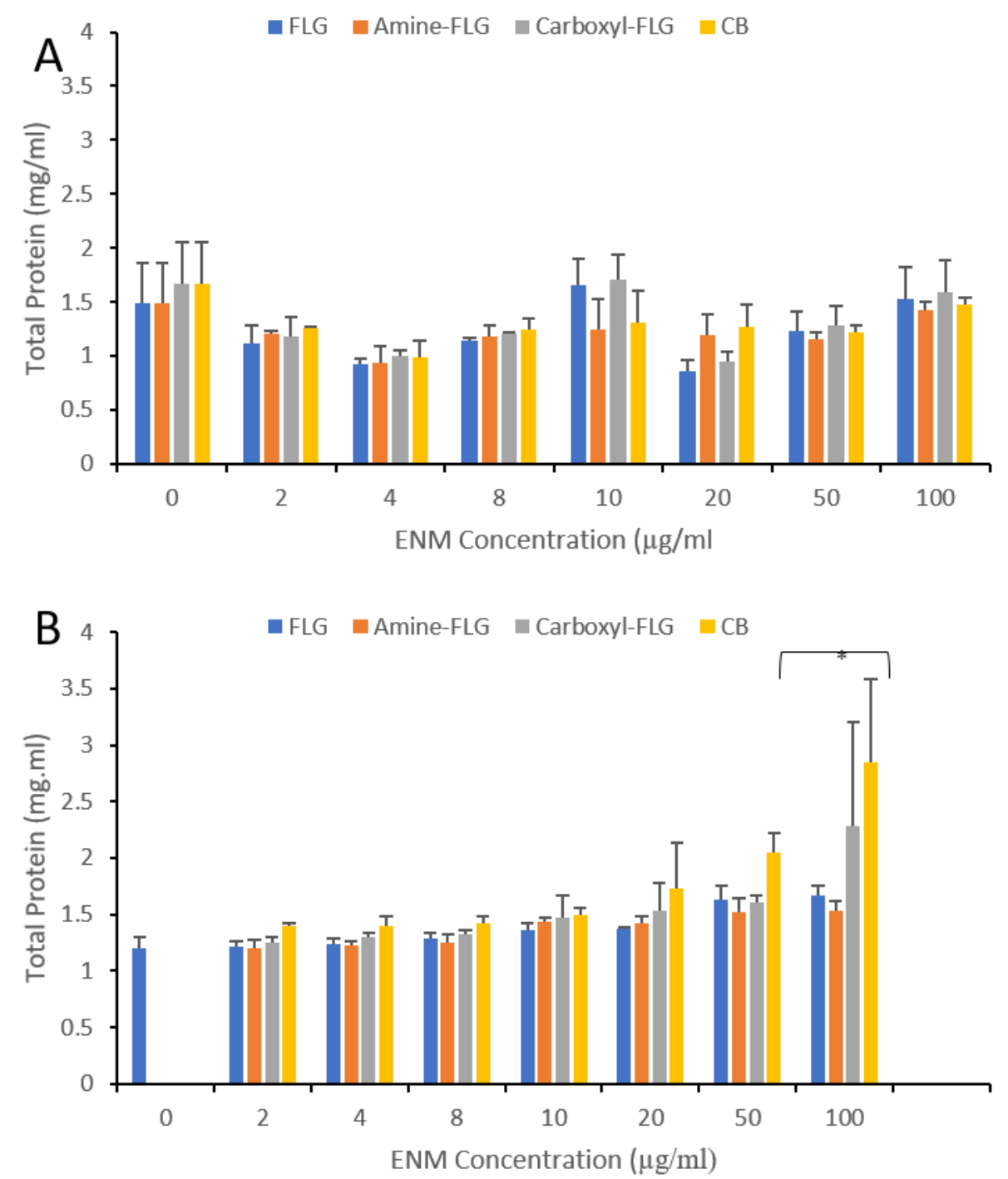

Figure 7.1 16HBE total protein levels following 6-hour (A) and 24-hour (B) exposures to ENMs. Protein levels were converted from optical densities (OD) to protein concentration $(\mathrm{mg} / \mathrm{ml})$ for each material to normalise glutathione data sets. Results were considered statistically significant $\left({ }^{*}\right)$ when $p<0.05 . \mathrm{N}=3$ 

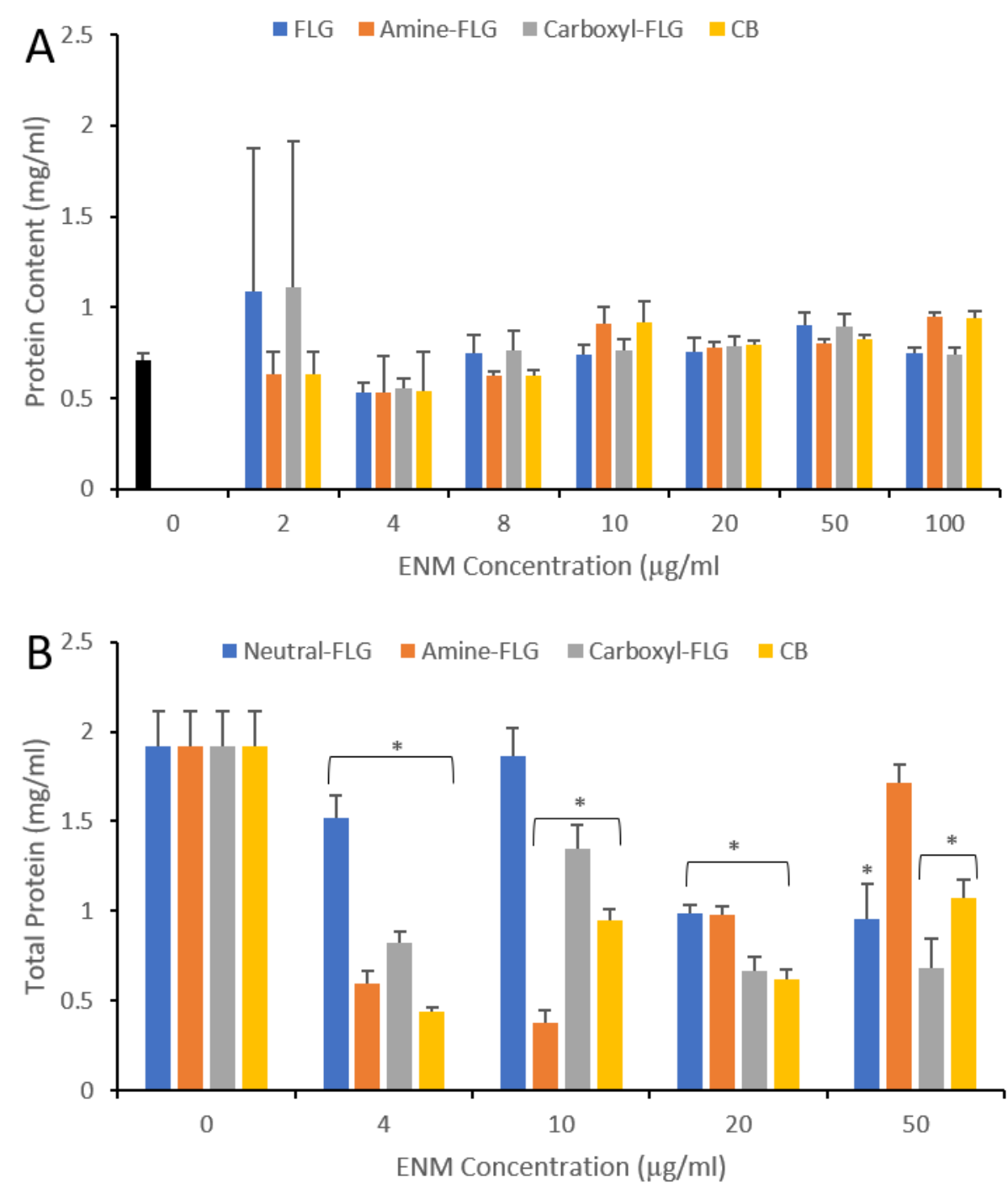

Figure 7.2 Total protein levels of $16 \mathrm{HBE}^{-140^{-}}$cells (A) and TT1 cells (B) following 24 -hour exposure to ENMs followed by bioenergetic analysis by the Seahorse Bioanalyzer XFe24. Protein levels were converted from optical densities (OD) to protein concentration $(\mathrm{mg} / \mathrm{ml})$ for each material to normalise Seahorse bioenergetic data sets. Results were considered statistically significant $\left({ }^{*}\right)$ when $p<0.05 . \mathrm{N}=3$ 


\subsection{OCR/ECAR Data Sets}
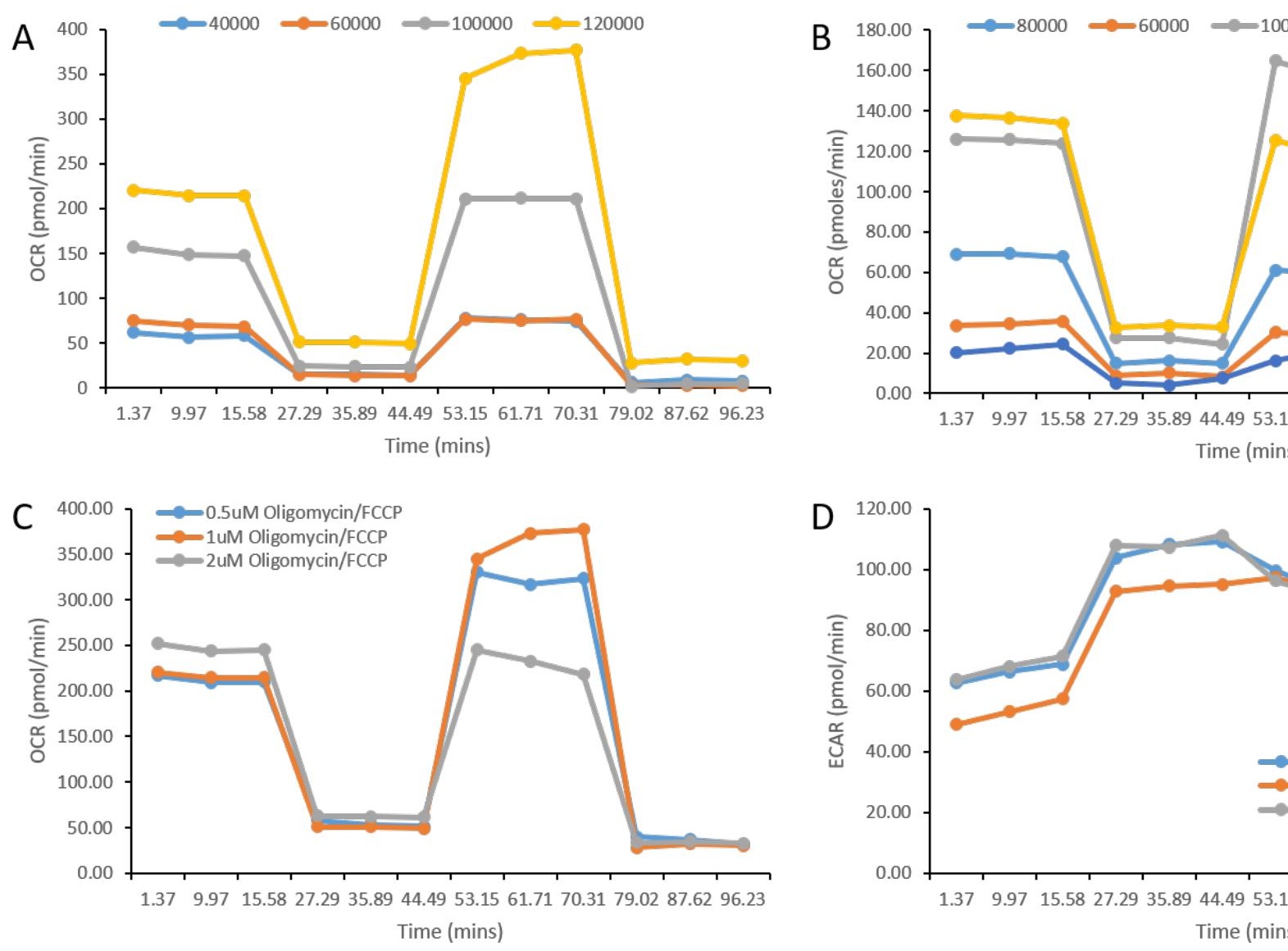

Figure 7.3 Cell line optimisation required prior to Seahorse XFe24 analysis. Cell titration to determine optimal 16HBE14o- cells (A), TT1 cells (B) and optimal compound injection concentrations demonstrating OCR profile (C) and 
Table 7.1 16HBE140` OCR Data

\begin{tabular}{|c|c|c|c|c|c|c|c|c|c|c|c|c|}
\hline $\begin{array}{l}\text { rage Time (minutes) } \\
\text { utral-FLG] }\end{array}$ & 1.37 & .98 & 8.55 & 7.26 & 5.86 & 44.47 & 3.18 & 1.79 & 70.39 & 07 & 68 & $6.2+2>$ \\
\hline 2.00 & $0.792->2$ & 87 & 369.1827 & 702 & 151 & 032 & & 368 & & & & 5 \\
\hline $\begin{array}{l}2.00 \\
4.00\end{array}$ & 6.5201 & 209696 & & & sen & & & & & & & \\
\hline $\begin{array}{l}4.00 \\
8.00\end{array}$ & $\begin{array}{l}0.6236 \\
2.3962\end{array}$ & $\begin{array}{l}490.8686 \\
303.4316\end{array}$ & $\begin{array}{l}495.5676 \\
268.1086\end{array}$ & 06.4215 & 109.3455 & 103.4379 & 954.358 & 935.3315 & 930.7178 & 46.83196 & 55.04477 & 242 \\
\hline 10.00 & 3.2111 & $\begin{array}{l}303.4316 \\
300.0103\end{array}$ & $\begin{array}{l}268.1086 \\
292.0712\end{array}$ & $\begin{array}{l}.36235 \\
.68988\end{array}$ & $\begin{array}{l}\begin{array}{l}9.355144 \\
2.33532\end{array} \\
\end{array}$ & $\begin{array}{l}67.519977 \\
58.88208\end{array}$ & $\begin{array}{l}580.4 \\
584.8\end{array}$ & $\begin{array}{l}564.07774 \\
563.2725\end{array}$ & 38 & $\begin{array}{l}40.52839 \\
25.65682\end{array}$ & $\begin{array}{l}99 \\
41\end{array}$ & $\begin{array}{l}67 \\
02\end{array}$ \\
\hline 20.00 & 2 & 312.3 & 304. & 2 & 33 & 63.7 & 554. & 35 & 9 & 3 & 6 & 43 \\
\hline $\begin{array}{r}50.00 \\
100.00\end{array}$ & $\begin{array}{l}45.4672 \\
53.9449\end{array}$ & $\begin{array}{r}234.4712 \\
341.506\end{array}$ & $\begin{array}{l}230.4075 \\
334.5081\end{array}$ & $\begin{array}{l}3.50449 \\
7.20211\end{array}$ & $\begin{array}{l}52.08992 \\
70.89728\end{array}$ & $\begin{array}{l}49.45499 \\
64.92081\end{array}$ & 36 & $\begin{array}{l}433 . \\
657\end{array}$ & $\begin{array}{r}42 \\
647\end{array}$ & $\begin{array}{l}55 \\
57\end{array}$ & $\begin{array}{l}29.4 \\
35.0\end{array}$ & 289 \\
\hline \multicolumn{13}{|l|}{$\begin{array}{l}\text { stDev } \\
\text { [Neutral-FLG] }\end{array}$} \\
\hline & 2.57608 & 36.84524 & 29.97788 & 24.71784 & 28.06563 & 24.43878 & 40.72902 & 37.20363 & 37.20303 & 18.55185 & 21.27841 & 18.84825 \\
\hline 2.00 & 34.8695 & 220.6817 & 214.4714 & 48.43015 & 46.4549 & 41.05816 & 493.9069 & 483.1821 & & & & \\
\hline $\begin{array}{l}4.00 \\
8.00\end{array}$ & $\begin{array}{l}.5 .17241 \\
.036363\end{array}$ & $\begin{array}{l}31.99621 \\
73.00184\end{array}$ & $\begin{array}{l}41.4 \\
45.3\end{array}$ & $\begin{array}{l}26.4 \\
32.2\end{array}$ & $\begin{array}{l}31.1 \\
36.3\end{array}$ & 54 & 16 & 242 & & & & \\
\hline 10.00 & 8.2815 & 104.4731 & 98.90786 & & 27.44851 & 23.4 & 318.6 & 299.2806 & 02 & 20.6 & 26.7 & \\
\hline & & & 83.38401 & & 20.34764 & 17.14349 & 259.0936 & 254.6261 & & 23.33585 & 25.72331 & \\
\hline $\begin{array}{l}50.00 \\
00.00\end{array}$ & .96294 & 58.10201 & 56.82187 & 25 & $\begin{array}{l}12.34375 \\
25.99267\end{array}$ & 13.27666 & $\begin{array}{l}84.51002 \\
263.1995\end{array}$ & $\begin{array}{l}71.82268 \\
45.84624\end{array}$ & 170.3657 & 11.09476 & 14.21325 & 152 \\
\hline 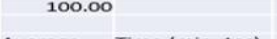 & 18.1754 & 111.4312 & 109.8 & & & & & & & & & \\
\hline ge-FLGI $_{\text {ef }}$ & 1.37 & 9.98 & 18.55 & 27.26 & 35.86 & 44.47 & 53.18 & 61.79 & 70.39 & 9.07 & 87.68 & 96.2 \\
\hline & 390.792 & 78.3887 & 369.1827 & 9.87702 & 71.1517 & 68.20324 & 57.8227 & 726.0368 & 720.8072 & & 94569 & 56 \\
\hline 2.00 & & 398.6914 & $\begin{array}{l}389.3675 \\
4592935\end{array}$ & & 77.99547 & 71.30811 & & 1184 & & 2921 & & \\
\hline $\begin{array}{l}4.00 \\
8.00\end{array}$ & 83 & $\begin{array}{l}476 . \\
400\end{array}$ & $\begin{array}{l}459 . \\
394 .\end{array}$ & $\begin{array}{l}742 \\
834\end{array}$ & $\begin{array}{l}106 \\
797\end{array}$ & $\begin{array}{l}693 \\
383\end{array}$ & $\begin{array}{l}33 \\
53\end{array}$ & $\begin{array}{l}775 \\
317\end{array}$ & & & & \\
\hline 10.00 & 297.8801 & 287.2968 & 282.6337 & 361 & 11 & & & & & & & \\
\hline 20.00 & 339.5724 & 328.9264 & 322.6341 & 72 & 76.7 & 73.8 & 43 & 634 & 32 & 196 & & \\
\hline & 8 & 26 & 249 & 6 & 59. & 57 & 2 & & & 4 & & \\
\hline \multicolumn{13}{|l|}{$\begin{array}{l}\text { tDev } \\
\text { Amine-FLG] }\end{array}$} \\
\hline & 2.5761 & 136.8452 & 129.9779 & 784 & 28.06563 & 878 & 9 & 36 & & 185 & 11 & 325 \\
\hline $\begin{array}{l}2.00 \\
4.00\end{array}$ & 06 & & 77 & 51 & 7 & & & 627 & & & & \\
\hline 8. & $\begin{array}{l}0.246817 \\
110.2729\end{array}$ & 108.4354 & 101.5913 & 23.41873 & 22.86606 & 30.09863 & & $\begin{array}{l}16 \\
09\end{array}$ & & 684 & & \\
\hline 10 & 29 & 109.3849 & 106.6594 & 13.3 & 12.61851 & 8.5 & 320. & 44 & & & & \\
\hline 20 & 7 & 128.3967 & 124. & 5 & & & & & & & & \\
\hline $\begin{array}{l}50.00 \\
\text { oo.oo }\end{array}$ & $\begin{array}{l}6708 \\
9378\end{array}$ & $\begin{array}{l}93.004 \\
30639\end{array}$ & $\begin{array}{l}87259 \\
76431\end{array}$ & & 10026 & & & & & & & \\
\hline 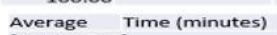 & 1.3 & 9.98 & 18.55 & 20 & 35.86 & 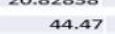 & .18 & & & & & \\
\hline & & & & & & & & & & & & \\
\hline 2.00 & & & & & & & & & & & & \\
\hline & 28 & & & & & & & & & & & \\
\hline & & 20 & & & 54. & & & & & & & \\
\hline 10. & 20 & 212 & 3 & 52 & 18 & & & & & & & \\
\hline 20 & & & & & & & & & & & & \\
\hline & & & & & & & & & & & & \\
\hline \multicolumn{13}{|l|}{, } \\
\hline & & & & & & & & & & & & \\
\hline & & & & & & & & & & & & \\
\hline & 25 . 12 & & & & & & & & & & & \\
\hline & & & & & & & & & & & & \\
\hline & & & & & & & & & & & & \\
\hline & & & & & & & & & & & & \\
\hline 00 & 9 & 12 & & 4.2 & 2.41 & & & & & 12 & & \\
\hline $\begin{array}{l}\text { Average }{ }^{T i} \\
\text { [Amine-FLG] }\end{array}$ & & 9.98 & & & 35.86 & 44.47 & & & & 17 & 87.68 & \\
\hline 2.00 & & & & & & & & & & & & \\
\hline & & & & & & & & & & & & \\
\hline & & & & & & & & & & & & \\
\hline & & & & & & & & & & & & \\
\hline & & & & & & & & & & & & \\
\hline $\begin{array}{r}50.00 \\
100.00\end{array}$ & $\begin{array}{r}194.5922 \\
176.259\end{array}$ & $\begin{array}{l}205 . \\
183.6\end{array}$ & & $\begin{array}{l}62 \\
50\end{array}$ & 507,7 & & & & & $\begin{array}{l}982 \\
564\end{array}$ & 626 & \\
\hline \multicolumn{13}{|l|}{$\begin{array}{l}\text { StDev } \\
\text { [Amine-FLG] }\end{array}$} \\
\hline & & & & & & & & & & & & \\
\hline & & & & & & & & & & & & \\
\hline & & & & & & & & & & & & \\
\hline & & & & & & & & & & & & \\
\hline & & & & & & & & & & & & \\
\hline 8 & 815 & & & & & 4 & 19 & 13 & 69 & $\begin{array}{l}41 \\
55 \\
\end{array}$ & $\begin{array}{l}4.46477 \\
431733\end{array}$ & $\begin{array}{l}5.29446 \\
8.66574\end{array}$ \\
\hline & & & & & & & & & & & & \\
\hline
\end{tabular}


Table 7.2 16HBE140` ECAR Data

\begin{tabular}{|c|c|c|c|c|c|c|c|c|c|c|c|c|}
\hline $\begin{array}{l}\text { Average Time (minutes) } \\
\text { [Neutral-FLG] }\end{array}$ & 1.37 & 9.98 & 18.55 & 27.26 & 35.86 & 4.47 & 53.18 & 1.79 & 70.39 & 07 & .68 & 9 \\
\hline 2.00 & 68.08692 & 70.59604 & 73.05615 & 108.0111 & 110.2448 & 110.0033 & 117.7325 & 115.4806 & 113.94 & 104.9594 & 104.8631 & 104.5921 \\
\hline .00 & $\begin{array}{l}1.58196 \\
7.87346\end{array}$ & $\begin{array}{l}63.88802 \\
99.26215\end{array}$ & $\begin{array}{l}65.18836 \\
104.2944\end{array}$ & $\begin{array}{l}90.54887 \\
147.5642\end{array}$ & $\begin{array}{r}92.76287 \\
153.689\end{array}$ & $\begin{array}{r}94.9488 \\
156.2084\end{array}$ & $\begin{array}{l}100.5848 \\
163.4545\end{array}$ & $\begin{array}{l}87.1 \\
151\end{array}$ & $\begin{array}{l}81.65844 \\
157.19007\end{array}$ & 87.0 & 90.77715 & 90.1063 \\
\hline 8.00 & $\begin{array}{r}97.87346 \\
66.61525\end{array}$ & $\begin{array}{l}99.26215 \\
70.14026\end{array}$ & $\begin{array}{l}104.2944 \\
75.09393\end{array}$ & 100.3011 & $\begin{array}{r}153.689 \\
101.1645\end{array}$ & $\begin{array}{l}156.2 \\
100.8\end{array}$ & $\begin{array}{l}163.4 \\
106.7\end{array}$ & $\begin{array}{l}151 . \\
106.5\end{array}$ & $\begin{array}{l}157.1907 \\
106.0285\end{array}$ & $\begin{array}{l}140.2198 \\
96.26387\end{array}$ & $\begin{array}{l}149.3727 \\
97.49874\end{array}$ & $\begin{array}{r}149.1724 \\
96.82485\end{array}$ \\
\hline 10.00 & 62.82903 & 64.52568 & 66.3119 & 92.5317 & 94.0921 & 94.4363 & $\begin{array}{r}106.7333 \\
101.442\end{array}$ & $\begin{array}{l}106.9006 \\
99.77523\end{array}$ & $\begin{array}{r}106.0285 \\
98.508\end{array}$ & $\begin{array}{l}96.26387 \\
90.05955\end{array}$ & $\begin{array}{l}97.49874 \\
90.38518\end{array}$ & $\begin{array}{l}6.82485 \\
9.89861\end{array}$ \\
\hline 20.00 & 59.95601 & 59.35539 & 61.89922 & 90.00556 & 90.67803 & 91.13744 & 99.36773 & 89.66299 & 84.72962 & $\mathbf{8 4 . 8 4 3 3 6 5}$ & $\begin{array}{l}92.99885 \\
\end{array}$ & $\begin{array}{l}89.89861 \\
\mathbf{8 2 . 9 8 5 0 4}\end{array}$ \\
\hline 50.00 & 50.45473 & 50.69917 & 0547 & 75.49889 & 78.66131 & & 81.75134 & 74.1 & & 73.9 & 75.7 & \\
\hline $\begin{array}{l}100.00 \\
\text { ev } \\
\text { utral-FLG] }\end{array}$ & 63.69135 & 65.05226 & 67.40 & 101.8 & 104.65 & 104. & 106. & 106. & 105.5 & 98.8 & 99 & 99. \\
\hline $\begin{array}{c}\text { ral-FLG] } \\
\text { o }\end{array}$ & 7.66496 & 5.990176 & 5.146459 & 3.08428 & 4.295628 & 3.537057 & 12.795 & 10.777772 & 10.52405 & 2.906657 & 2.546034 & 2.904207 \\
\hline 2.00 & 31.12099 & 31.61158 & 31.84971 & 40.77401 & 40.83237 & 42.3 & $\begin{array}{r}51.49014 \\
2253886\end{array}$ & 44. & 39.73482 & & & \\
\hline $\begin{array}{l}4.00 \\
8.00\end{array}$ & $\begin{array}{r}11.93398 \\
8.216018\end{array}$ & $\begin{array}{l}11.35624 \\
10.67046\end{array}$ & $\begin{array}{r}11.17803 \\
15.86334\end{array}$ & $\begin{array}{l}8.046756 \\
8.832581\end{array}$ & $\begin{array}{l}8.552649 \\
7.702231\end{array}$ & $\begin{array}{l}4.48 \\
7.81\end{array}$ & $\begin{array}{l}22.53886 \\
17.27104\end{array}$ & 16.0 & $\begin{array}{r}18.2204 \\
\end{array}$ & 4.91 & 5.8 & 26 \\
\hline $\begin{array}{r}8.00 \\
10.00\end{array}$ & $\begin{array}{l}8.216018 \\
8.433344\end{array}$ & $\begin{array}{l}10.67046 \\
8.506398\end{array}$ & $\begin{array}{r}15.86334 \\
8.4474\end{array}$ & $\begin{array}{r}8.832581 \\
5.426738\end{array}$ & $\begin{array}{r}7.702231 \\
5.38285\end{array}$ & $\begin{array}{l}7.814999 \\
3.859137\end{array}$ & $\begin{array}{l}17.27104 \\
18.44516\end{array}$ & $\begin{array}{l}20.28802 \\
17.47141\end{array}$ & $\begin{array}{l}19.18751 \\
16.27299\end{array}$ & $\begin{array}{l}8.665107 \\
4.851614\end{array}$ & 22 & $\begin{array}{l}006 \\
197\end{array}$ \\
\hline 20.00 & 7.695118 & 6.455134 & 6.257197 & 7.488643 & 6.507716 & $\mathbf{8 . 3 7 9 2 3 8}$ & 14.91075 & $\begin{array}{l}1.4714 \\
10.9208\end{array}$ & 7.544191 & $\begin{array}{l}4.85153844 \\
5.31584\end{array}$ & 3.001172 & $\begin{array}{l}5.674197 \\
3.290845\end{array}$ \\
\hline 50.00 & 9.696914 & 8.261172 & 7.55 & 9.794001 & & 8.36 & & 12.00708 & & 9.948568 & 43 & 10.2 \\
\hline 100.00 & 4.750971 & 5.824016 & 7.326655 & 11.2106 & 12.57238 & 14.06715 & 16.35997 & 17.25935 & 17.64082 & 14.54512 & 13.72959 & 15.10579 \\
\hline $\begin{array}{l}\text { erage Time (minutes) } \\
\text { nine-FLG] }\end{array}$ & 1.37 & 9.98 & 18.55 & 27.26 & 35.86 & 44.47 & 53.18 & 61.79 & 70.39 & 79.07 & 87.68 & 96.29 \\
\hline & 68.08692 & 70.59604 & 73.05615 & 108.0111 & 110.2448 & 110.0033 & 117.7325 & 115.4806 & 113.94 & 104.9594 & 104.8631 & 104.5921 \\
\hline 2.00 & 76.12731 & 78.52422 & 81.49889 & 116.3359 & 121.2838 & 124.1522 & 127.0449 & 112.6253 & 109.2044 & 113.661 & 118.1714 & \\
\hline & & 112.8215 & & & & & & & 3422 & 191 & 18 & \\
\hline 8.00 & 72.8 & $\begin{array}{c}75.1 \\
50\end{array}$ & $\begin{array}{r}77.9 \\
53\end{array}$ & 111. & 114. & 115 & 122 & 126 & 119 & 54 & 62 & \\
\hline $\begin{array}{l}10.00 \\
20.00\end{array}$ & $\begin{array}{l}50.31438 \\
61.41579\end{array}$ & $\begin{array}{r}50.84332 \\
61.48638\end{array}$ & $\begin{array}{r}53.1745 \\
63.47788\end{array}$ & $\begin{array}{l}7.55723 \\
91.76378\end{array}$ & $\begin{array}{l}76.48205 \\
91.71506\end{array}$ & $\begin{array}{l}79.17534 \\
93.60418\end{array}$ & $\begin{array}{r}87.02111 \\
100.263\end{array}$ & $\begin{array}{r}76.8619 \\
90.07074\end{array}$ & $\begin{array}{l}72.377484 \\
87.29933\end{array}$ & $\begin{array}{l}73.85194 \\
\mathbf{8 7 . 9 9 5 4 5}\end{array}$ & $\begin{array}{r}75.28261 \\
88.6618\end{array}$ & $\begin{array}{r}75.60597 \\
89.5177\end{array}$ \\
\hline 50.00 & 53.58114 & 55.9258 & 58.0175 & 81.88891 & 82.94534 & 83.65766 & 87.67925 & 82.59209 & 80.87008 & 77.61798 & 78.8545 & 79.39451 \\
\hline $\begin{array}{l}100.00 \\
\text { pev } \\
\text { mine-FLG] }\end{array}$ & 51.56633 & 51.27609 & 53.3112 & 75.92877 & 77.34319 & 78.3319 & 79.32882 & 73.43958 & 74.64058 & 73.21299 & 74.79274 & 74.65815 \\
\hline 2.00 & $\begin{array}{r}7.66496 \\
29777487\end{array}$ & 5.990176 & 5.146459 & 08428 & 4.295628 & 3.537057 & 2.795 & 77772 & 10.52405 & 906657 & 6034 & 04207 \\
\hline $\begin{array}{l}2.00 \\
4.00\end{array}$ & $\begin{array}{l}29.77487 \\
45.77271\end{array}$ & $\begin{array}{l}28.840444 \\
49.65472\end{array}$ & $\begin{array}{l}29.09698 \\
48.78039\end{array}$ & $\begin{array}{r}33.07985 \\
90.0148\end{array}$ & $\begin{array}{r}32.99437 \\
90.956\end{array}$ & $\begin{array}{l}33.18968 \\
92.04296\end{array}$ & $\begin{array}{l}48.07416 \\
63.45915\end{array}$ & $\begin{array}{l}39.16211 \\
71.29242\end{array}$ & $\begin{array}{l}34.93296 \\
70.98012\end{array}$ & $\begin{array}{l}31.771112 \\
88.54603\end{array}$ & 63 & \\
\hline $\begin{array}{l}8.00 \\
8.00\end{array}$ & 7.502833 & 6.262321 & 4.791957 & 8.981073 & 8.574645 & 7.738181 & 7.114932 & 5.221383 & 4.736846 & $\begin{array}{l}3.677339 \\
3.67393\end{array}$ & $\begin{array}{l}84.78882 \\
3.338556\end{array}$ & $\begin{array}{l}83.76688 \\
3.555738\end{array}$ \\
\hline 10.00 & 7.877831 & 6.292607 & 5.33 & 0.761062 & 1.256634 & 1.96 & 12.64017 & 8.9 & 5.2 & & & \\
\hline $\begin{array}{l}20.00 \\
50.00\end{array}$ & $\begin{array}{l}10.22012 \\
11.26655\end{array}$ & $\begin{array}{r}7.122721 \\
12.0053\end{array}$ & $\begin{array}{r}6.07024 \\
12.43665\end{array}$ & $\begin{array}{l}0.962313 \\
8.915801\end{array}$ & $\begin{array}{l}1.022883 \\
8.770092\end{array}$ & $\begin{array}{l}1.214 \\
8.056\end{array}$ & $\begin{array}{l}14.15295 \\
17.58362\end{array}$ & $\begin{array}{l}10 . \\
15 .\end{array}$ & $\begin{array}{l}7.230813 \\
14.07581\end{array}$ & $\begin{array}{l}0.717587 \\
8.689002\end{array}$ & $\begin{array}{l}1.36 \\
9.075\end{array}$ & 813 \\
\hline 100.00 & 2.02052 & 1.718959 & 3.730675 & 6.139678 & 7.707477 & 7.73 & 4.25468 & 2.644366 & 2.715098 & 5.81098 & 5.946973 & 5.906192 \\
\hline $\begin{array}{l}\text { Average Time (minutes) } \\
\text { [Carboxyl-FLG] }\end{array}$ & 1.37 & 9.98 & 18.55 & 27.26 & 35.86 & 44.47 & 53.18 & 61.79 & 70.39 & 79.07 & 87.68 & 96.29 \\
\hline 0 & 92 & 70.59604 & $73 . c$ & 108. & 110. & 110 & 117 & & & & & \\
\hline $\begin{array}{l}2.00 \\
4.00\end{array}$ & $\begin{array}{l}47.1 \\
95.5\end{array}$ & $\begin{array}{l}47.7 \\
91.8\end{array}$ & $\begin{array}{l}49.2 \\
94.3\end{array}$ & $\begin{array}{l}86.1 \\
156 .\end{array}$ & $\begin{array}{l}90.7 \\
162\end{array}$ & & & & & & & \\
\hline 8.00 & 61.41868 & 63.69364 & 64.7 & 107.9829 & 110.1976 & 111 & $\begin{array}{l}141 . \\
95 .\end{array}$ & $\begin{array}{l}132 \\
96 .\end{array}$ & $\begin{array}{l}142 \\
98.2\end{array}$ & & & 212 \\
\hline 10.00 & 6. & 63 & 65.8 & 112. & 116 & & & & & & & \\
\hline & & & & & & & & & & & & \\
\hline $\begin{array}{l}50.00 \\
00.00 \\
\text { on }\end{array}$ & $\begin{array}{r}61.79752 \\
56.3464\end{array}$ & $\begin{array}{l}62.079999 \\
58.80391\end{array}$ & $\begin{array}{l}64.86628 \\
61.19325\end{array}$ & $\begin{array}{l}107.2083 \\
108.7732\end{array}$ & $\begin{array}{l}112.0514 \\
110.4783\end{array}$ & $\begin{array}{l}116.6517 \\
110.3484\end{array}$ & $\begin{array}{r}99.52227 \\
95.97773\end{array}$ & $\begin{array}{r}93.50147 \\
94.55152\end{array}$ & $\begin{array}{l}95.65488 \\
96.29352\end{array}$ & $\begin{array}{l}103 . \\
97.4\end{array}$ & $\begin{array}{l}105 \\
97.5\end{array}$ & $\begin{array}{l}25 \\
66 \\
\end{array}$ \\
\hline $\begin{array}{l}\text { stDev } \\
\text { [Carboxyl-FL }\end{array}$ & 56.3464 & 58.80391 & 61.19325 & 108.7732 & 110.4783 & 110.3484 & $95.97 / 3$ & 94.55152 & 96.29352 & & & \\
\hline 2.00 & $\begin{array}{r}7.66496 \\
26.000079\end{array}$ & $\begin{array}{l}5.990 \\
26.54\end{array}$ & $\begin{aligned} 5.146 \\
27\end{aligned}$ & & 4.2 & & & & & & & \\
\hline & & 27. & & & & & & & & & & \\
\hline 8.00 & 6 & 16. & & & 23. & & & & & & & \\
\hline $\begin{array}{l}10.00 \\
20.00\end{array}$ & & & & & & & & & & & & \\
\hline $\begin{array}{l}20.00 \\
50.00\end{array}$ & $\begin{array}{l}12.60776 \\
13.21635\end{array}$ & $\begin{array}{r}12.8944 \\
13.76587\end{array}$ & $\begin{array}{l}14.41153 \\
14.13984\end{array}$ & $\begin{array}{l}27.51765 \\
25.35328\end{array}$ & $\begin{array}{r}28.07432 \\
26.5396\end{array}$ & $\begin{array}{l}30.57 \\
27.14\end{array}$ & $\begin{array}{l}26 . \\
26 .\end{array}$ & $\begin{array}{l}26.7 \\
26.3\end{array}$ & & 882 & & 24.4 \\
\hline 100.00 & 30.35075 & 29.18726 & 29.66822 & 55.07461 & 53.42079 & 53.73826 & 46.53957 & 48.14937 & 49.58157 & 46.87469 & 47.98569 & 47.34699 \\
\hline (e) & 1.37 & 9. & 18 & 27 & 35. & 44.4 & 53. & & 70 & & .68 & 6.29 \\
\hline & & & & & & & & & & & & \\
\hline 2.00 & & & & & & & & & & & & \\
\hline & & & & & & & & & & & & \\
\hline $\begin{array}{l}8.00 \\
10.00\end{array}$ & $\begin{array}{l}64.7152 \\
47.6696\end{array}$ & $\begin{array}{l}65.90111 \\
49.54839\end{array}$ & $\begin{array}{l}68.3 \\
51.4\end{array}$ & $\begin{array}{l}113.7958 \\
86.34117\end{array}$ & $\begin{array}{l}118 . \\
84.5\end{array}$ & $\begin{array}{l}119 . \\
89.7\end{array}$ & $\begin{array}{l}10 \\
78\end{array}$ & & 104 & & 43 & \\
\hline 20 & 54.6 & 54.12194 & 56.5 & 96.5 & 97.9 & 10 & 89. & 84. & 84. & & 24 & 28 \\
\hline 50.00 & 53.4 , & $54.7:$ & 56.56 & 96 & 98.7 & & 85. & 83. & 83.3 & 87. & & \\
\hline$e^{100.00}$ & 45.88579 & 46.10669 & 48.11809 & 80.68029 & 82.52462 & 84.47856 & 72.17069 & 68.82863 & 69.11095 & 74.3866 & 74.9881 & 74.81566 \\
\hline & & & & & & & & & & & & \\
\hline & & & & & & & & & & & & \\
\hline & & & & & & & 6 & & & & & \\
\hline & & & & & & & & 45 & 45.8 & 45 & & \\
\hline & & & & & & & & & & & & \\
\hline & & & & & & & & & & & & \\
\hline $\begin{array}{r}50.00 \\
100.00\end{array}$ & $\begin{array}{r}11.45968 \\
19.8175\end{array}$ & $\begin{array}{r}11.29428 \\
20.80934\end{array}$ & $\begin{array}{l}11.42126 \\
21.28359\end{array}$ & $\begin{array}{l}18.20641 \\
35.20625\end{array}$ & $\begin{array}{l}19.05182 \\
36.69564\end{array}$ & $\begin{array}{r}17.76109 \\
36.33992\end{array}$ & $\begin{array}{r}19.29662 \\
34.03025\end{array}$ & $\begin{array}{r}19.47015 \\
32.4331\end{array}$ & $\begin{array}{l}18.35027 \\
32.36593\end{array}$ & $\begin{array}{r}15.44812 \\
33.02857\end{array}$ & $\begin{array}{l}15.86153 \\
33.05621\end{array}$ & $\begin{array}{l}15.17326 \\
32.17296\end{array}$ \\
\hline
\end{tabular}


Table 7.3 TT1 OCR Data

\begin{tabular}{|c|c|c|c|c|c|c|c|c|c|c|c|c|c|}
\hline \multicolumn{14}{|c|}{ [Neutral-FLG] } \\
\hline & & 102.2733 & 97.64667 & 96.09667 & 19.53 & 19.12 & 19.41333 & 136.45 & 134.7467 & 133.21 & 8.356667 & 8.136667 & 18.46667 \\
\hline 4.00 & & 113.7667 & 110.6633 & 111.0933 & 34.89667 & 36.40667 & 37.72 & 150.9867 & 153.4967 & 153.08 & 27.98667 & 32.94 & 32.80667 \\
\hline 10.00 & & 97.74333 & 96.65667 & 96.93 & 30.66 & 32.15667 & 32.81667 & 138.68 & 138.0467 & 135.8133 & 20.30667 & 21.89667 & 18.91333 \\
\hline 20.00 & & 197.7267 & 198.27 & 200.75 & 69.74667 & 70.71 & 72.41333 & 280.4333 & 274.55 & 269.2733 & 45.93667 & 50.34667 & 54.06333 \\
\hline 50.00 & & 184.79 & 180.5667 & 179.87 & 58.65 & 41.47667 & 39.53667 & 297.5433 & 296.7867 & 299.0467 & 19.32667 & 20.63 & 20.31667 \\
\hline \multicolumn{14}{|l|}{ 5tDev } \\
\hline \multicolumn{14}{|l|}{ [Neutral-FLG] } \\
\hline 0 & & 53.46 & 51.26 & 51.57 & 12.08 & 10.14 & 10.16 & 89.72 & 82.15 & 78.95 & 4.22 & 4.84 & 23.36 \\
\hline 4.00 & & 87.14 & 87.25 & 91.00 & 32.15 & 33.22 & 36.01 & 129.90 & 129.53 & 127.75 & 32.08 & 40.27 & 38.32 \\
\hline 10.00 & & 70.87163 & 68.57228 & 68.68959 & 21.10461 & 20.35631 & 20.04771 & 112.3934 & 106.1103 & 102.4012 & 13.75247 & 13.89506 & 15.35645 \\
\hline 20.00 & & 108.7933 & 100.5016 & 100.8551 & 29.02055 & 30.81752 & 29.87658 & 179.9621 & 166.8374 & 158.1938 & 23.92943 & 26.7416 & 18.60086 \\
\hline 50.00 & & 120.0402 & 123.2967 & 125.5163 & 65.80931 & 36.7219 & 31.58935 & 61.77285 & 244.8089 & 247.9368 & 16.8103 & 19.78886 & 17.19548 \\
\hline \multicolumn{14}{|c|}{ [Amine-FLG] } \\
\hline & & 30.83 & 35.60333 & 40.75667 & 25.82 & 31.42667 & 37.2 & 75.27 & 81.09 & 86.12 & 57.01333 & 62.68 & 67.9 \\
\hline 4.00 & & 107.77 & 103.2133 & 101.33 & 20.76 & 20.59667 & 21.34 & 145.2733 & 143.6433 & 141.8333 & 8.53 & 8.49 & 7.98 \\
\hline 10.00 & & 111.1033 & 109.91 & 110.3367 & 33.34333 & 34.67667 & 34.60333 & 158.4233 & 157.2567 & 155.1533 & 23.92667 & 24.80667 & 23.29333 \\
\hline 20.00 & & 135.55 & 133.17 & 127.6033 & 35.83333 & 36.77 & 37.73667 & 208.1967 & 205.4933 & 204.9933 & 33.43333 & 33.70333 & 34.15 \\
\hline 50.00 & & 206.6133 & 199.5933 & 197.83 & 70.24 & 60.11667 & 57.9 & 309.1867 & 303.7367 & 302.3567 & 27.26 & 29.75333 & 27.98333 \\
\hline \multicolumn{14}{|l|}{ stDev } \\
\hline \multicolumn{14}{|l|}{ [Amine-FLG] } \\
\hline & & 51.03 & 44.38 & 38.46 & 2.49 & 7.68 & 12.59 & 38.26 & 33.43 & 27.25 & 38.20 & 43.30 & 49.17 \\
\hline 4.00 & & 52.36 & 50.41 & 50.69 & 12.77 & 11.16 & 11.54 & 88.50 & 81.14 & 77.80 & 4.50 & 5.35 & 5.40 \\
\hline 10.00 & & 81.42932 & 80.51012 & 81.6761 & 29.81869 & 30.25339 & 29.15655 & 127.5111 & 124.3442 & 121.5444 & 13.69268 & 17.25082 & 14.87411 \\
\hline 20.00 & & 13.75839 & 13.07376 & 19.73442 & 19.06881 & 19.73784 & 19.01241 & 54.25091 & 58.01907 & 58.21454 & 25.22913 & 23.44943 & 22.93014 \\
\hline 50.00 & & 134.353 & 132.7633 & 134.7123 & 69.83088 & 53.93965 & 48.49162 & 231.2023 & 219.9905 & 215.2957 & 35.04401 & 38.20908 & 37.90429 \\
\hline \multicolumn{14}{|c|}{ [Carboxyl-FLG] } \\
\hline & & 102.2733 & 97.64667 & 96.09667 & 19.53 & 19.12 & 19.41333 & 136.45 & 134.7467 & 133.21 & 8.356667 & 8.136667 & 7.24 \\
\hline 4.00 & & 123.5533 & 120.04 & 119.1833 & 34.61 & 35.49667 & 37.06667 & 192.0267 & 187.4567 & 185.94 & 18.26667 & 21.42667 & 21.85333 \\
\hline 10.00 & & 99.42 & 96.51 & 96.08667 & 24.30667 & 25.94 & 27.38667 & 143.22 & 140.18 & 138.3967 & 13.68667 & 14.36 & 14.93 \\
\hline 20.00 & & 233.57 & 218.5767 & 207.62 & 48.63667 & 47.79333 & 47.87667 & 383.4867 & 366.8867 & 364.0633 & 19.72 & 22.57333 & 23.51333 \\
\hline 50.00 & & 122.4867 & 141.9167 & 132.6833 & 35.81667 & 30.32 & 31.26333 & 167.4833 & 170.1967 & 170.9333 & 20.83333 & 19.23 & 21.26 \\
\hline \multicolumn{14}{|l|}{ StDev } \\
\hline \multicolumn{14}{|c|}{ [Carboxyl-FLG] } \\
\hline & & 53.46 & 51.26 & 51.57 & 12.08 & 10.14 & 10.16 & 89.72 & 82.15 & 78.95 & 4.22 & 4.84 & 3.87 \\
\hline 4.00 & & 71.50 & 71.38 & 71.14 & 20.13 & 19.77 & 19.18 & 118.88 & 114.35 & 110.20 & 10.32 & 11.22 & 12.34 \\
\hline 10.00 & & 66.11738 & 65.99565 & 66.53807 & 21.87603 & 21.75336 & 21.94088 & 108.4153 & 100.3852 & 98.12253 & 12.2686 & 15.86278 & 16.2276 \\
\hline 20.00 & & 136.0399 & 145.9659 & 160.8331 & 39.34034 & 38.77461 & 39.11539 & 258.9732 & 240.8675 & 232.3613 & 21.50714 & 24.61911 & 23.27824 \\
\hline 50.00 & & 51.63787 & 20.11696 & 34.55195 & 17.01456 & 20.85732 & 20.92945 & 107.2323 & 104.3469 & 102.3675 & 17.33267 & 17.12037 & 21.04801 \\
\hline \multirow{2}{*}{$\begin{array}{l}\text { Average } \\
\text { [CB] }\end{array}$} & Time (minutes) & 1.37 & 9.98 & 18.55 & 27.26 & 35.86 & 44.47 & 53.18 & 61.79 & 70.39 & 79.07 & 87.68 & 96.29 \\
\hline & 0 & 30.83 & 35.60333 & 40.75667 & 25.82 & 31.42667 & 37.2 & 75.27 & 81.09 & 86.12 & 57.01333 & 62.68 & 67.9 \\
\hline 4.00 & & 119.04 & 115.1767 & 114.4433 & 31.37 & 32.25667 & 32.31333 & 161.5667 & 159.1167 & 156.94 & 20.65667 & 20.82667 & 19.83 \\
\hline 10.00 & & 124.9833 & 118.7633 & 114.5233 & 25.31 & 26.35333 & 26.41333 & 171.1733 & 166.6333 & 163.5667 & 15.35 & 17.17 & 16.59333 \\
\hline 20.00 & & 98.44333 & 94.91667 & 93.69 & 33.21 & 22.03667 & 42.13 & 191.88 & 174.8833 & 158.7233 & 13.733333 & 16.63333 & 16.13 \\
\hline 50.00 & & 155.2 & 144.28 & 141.4633 & 37.21 & 31.19333 & 50.55333 & 280.56 & 274.78 & 303.0633 & 19.20333 & 19.61667 & 18.99667 \\
\hline \multicolumn{14}{|l|}{ StDev } \\
\hline [CB] & & & & & & & & & & & & & \\
\hline 0 & & 51.03 & 44.38 & 38.46 & 2.49 & 7.68 & 12.59 & 38.26 & 33.43 & 27.25 & 38.20 & 43.30 & 49.17 \\
\hline 4.00 & & 55.42 & 54.61 & 55.38 & 26.22 & 27.50 & 26.98 & 93.06 & 86.19 & 82.52 & 25.28 & 26.07 & 25.72 \\
\hline 10.00 & & 86.74188 & 87.9919 & 93.22989 & 38.70217 & 37.80973 & 38.57294 & 129.6459 & 119.4627 & 115.4784 & 22.65094 & 24.1614 & 22.24536 \\
\hline 20.00 & & 68.67184 & 68.95724 & 70.63476 & 26.25145 & 24.88313 & 34.05919 & 144.8949 & 124.2166 & 109.029 & 12.73095 & 15.22531 & 14.81506 \\
\hline 50.00 & & 121.775 & 121.1047 & 120.7384 & 31.13458 & 30.67394 & 35.48012 & 183.6186 & 179.0389 & 186.3675 & 15.13086 & 13.60329 & 10.93324 \\
\hline
\end{tabular}


Table 7.4 TT1 ECAR Data

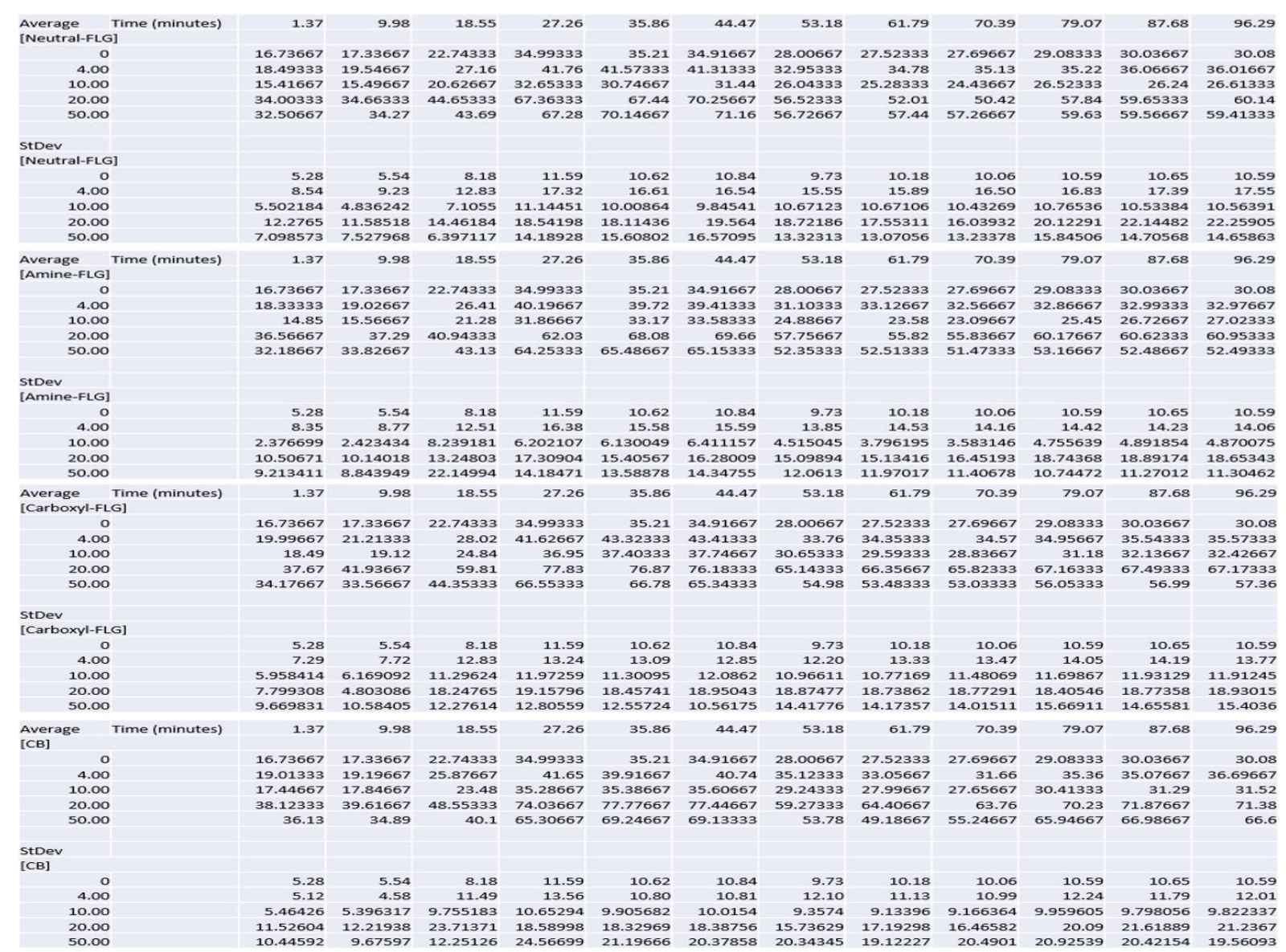

\subsection{SDS-PAGE of BSA}

\section{MW}

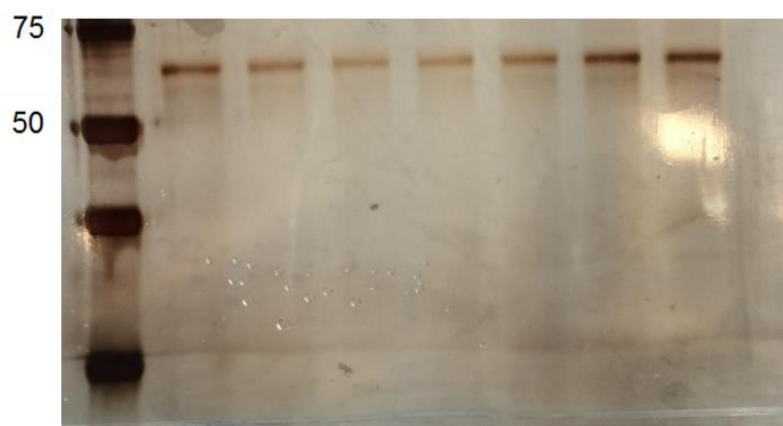

Figure 7.4 SDS-PAGE of BSA only. BSA (Sigma, UK) was run on a separate gel to confirm the molecular weight of the bands observed between $50-75 \mathrm{kDa}$. 
7.4 Raw IL-1 $\beta$ ELISA Data

\begin{tabular}{|c|c|c|c|c|c|c|c|c|c|c|c|}
\hline 1 & 2 & 3 & 4 & 5 & 6 & 7 & 8 & 9 & 10 & 11 & 12 \\
\hline 2.09 & 2.091 & 2.19 & 0.017 & 0.014 & 0.02 & 0.028 & 0.036 & 0.049 & 0.022 & 0.021 & 0.022 \\
\hline 1.682 & 1.68 & 1.653 & 0.028 & 0.027 & 0.028 & 0.027 & 0.026 & 0.025 & 0.024 & 0.029 & 0.022 \\
\hline 0.853 & 0.86 & 0.727 & 0.023 & 0.018 & 0.024 & 0.023 & 0.021 & 0.031 & 0.039 & 0.049 & 0.019 \\
\hline 0.301 & 0.497 & 0.386 & 0.028 & 0.018 & 0.027 & 0.026 & 0.025 & 0.022 & 0.026 & 0.001 & 0.014 \\
\hline 0.127 & 0.117 & 0.112 & 0.025 & 0.025 & 0.024 & 0.025 & 0.023 & 0.02 & 0.022 & 0.022 & 0.026 \\
\hline 0.074 & 0.076 & 0.069 & 0.024 & 0.03 & 0.03 & 0.026 & 0.021 & 0.02 & 0.023 & 0.026 & 0.023 \\
\hline 0.059 & 0.064 & 0.057 & 0.029 & 0.03 & 0.032 & 0.028 & 0.026 & 0.027 & 0.025 & 0.027 & 0.027 \\
\hline 0.026 & 0.017 & 0.033 & 0.028 & 0.025 & 0.027 & 0.027 & 0.024 & 0.026 & 0.028 & 0.027 & 0.019 \\
\hline 1 & 2 & 3 & 4 & 5 & 6 & 7 & 8 & 9 & 10 & 11 & 12 \\
\hline 2.233 & 2.203 & 2.173 & 0.009 & 0.004 & 0.012 & 0.016 & 0.024 & 0.039 & 0.017 & 0.011 & 0.015 \\
\hline 1.692 & 1.68 & 1.661 & 0.03 & 0.034 & 0.025 & 0.024 & 0.028 & 0.016 & 0.013 & 0.025 & 0.017 \\
\hline 0.87 & 0.759 & 0.729 & 0.02 & 0.016 & 0.018 & 0.012 & 0.018 & 0.018 & 0.015 & 0.044 & 0.013 \\
\hline 0.205 & 0.203 & 0.192 & 0.023 & 0.014 & 0.024 & 0.025 & 0.025 & 0.022 & 0.029 & -0.007 & 0.004 \\
\hline 0.131 & 0.12 & 0.113 & 0.022 & 0.021 & 0.02 & 0.022 & 0.022 & 0.017 & 0.02 & 0.014 & 0.018 \\
\hline 0.075 & 0.081 & 0.072 & 0.021 & 0.027 & 0.032 & 0.026 & 0.022 & 0.022 & 0.024 & 0.024 & 0.014 \\
\hline 0.058 & 0.06 & 0.055 & 0.027 & 0.025 & 0.027 & 0.023 & 0.021 & 0.022 & 0.018 & 0.022 & 0.019 \\
\hline 0.03 & 0.026 & 0.035 & 0.03 & 0.026 & 0.03 & 0.026 & 0.027 & 0.026 & 0.026 & 0.026 & 0.014 \\
\hline 1 & 2 & 3 & 4 & 5 & 6 & 7 & 8 & 9 & 10 & 11 & 12 \\
\hline 2.228 & 2.187 & 2.162 & 0.01 & 0.008 & 0.012 & 0.018 & 0.028 & 0.043 & 0.017 & 0.013 & 0.016 \\
\hline 1.692 & 1.685 & 1.663 & 0.031 & 0.036 & 0.026 & 0.025 & 0.029 & 0.019 & 0.015 & 0.026 & 0.018 \\
\hline 0.774 & 0.762 & 0.832 & 0.023 & 0.019 & 0.019 & 0.013 & 0.02 & 0.02 & 0.016 & 0.047 & 0.016 \\
\hline 0.206 & 0.206 & 0.194 & 0.026 & 0.016 & 0.026 & 0.025 & 0.027 & 0.023 & 0.029 & -0.005 & 0.005 \\
\hline 0.133 & 0.121 & 0.116 & 0.024 & 0.023 & 0.022 & 0.022 & 0.023 & 0.019 & 0.021 & 0.018 & 0.019 \\
\hline 0.078 & 0.082 & 0.074 & 0.024 & 0.03 & 0.034 & 0.026 & 0.024 & 0.024 & 0.026 & 0.025 & 0.014 \\
\hline 0.059 & 0.062 & 0.057 & 0.029 & 0.025 & 0.028 & 0.023 & 0.024 & 0.023 & 0.02 & 0.022 & 0.021 \\
\hline 0.033 & 0.027 & 0.037 & 0.031 & 0.027 & 0.03 & 0.027 & 0.029 & 0.028 & 0.027 & 0.027 & 0.016 \\
\hline
\end{tabular}

Figure 7.5 Raw IL-1 $\beta$ ELISA Data. Supernatant tested for IL-1 $\beta$ proved unsuccessful and thus could not be converted to $\mathrm{pg} / \mathrm{ml}$ as $\mathrm{OD}$ values fell below standard curve detection. 